\title{
Metagenomic approaches to discover lipolytic enzymes
}

\author{
Dissertation
}

for the award of the degree

“Doctor rerum naturalium" (Dr. rer. nat.)

of the Georg-August-Universität Göttingen

\author{
within the doctoral program in Biology \\ of the Georg-August University School of Science (GAUSS) \\ Submitted by
}

Mingji Lu

from ChongQing, China

Göttingen 2021 


\section{Thesis Committee}

Prof. Dr. Rolf Daniel, Dept. of Genomic and Applied Microbiology \& Göttingen

Genomics Laboratory, Georg August University Göttingen

PD Dr. Michael Hoppert, Dept. of General Microbiology, Georg August

University Göttingen

\section{Members of the Examination Board}

Reviewer: Prof. Dr. Rolf Daniel, Dept. of Genomic and Applied Microbiology \&

Göttingen Genomics Laboratory, Georg August University Göttingen

Second Reviewer: PD Dr. Michael Hoppert, Dept. of General Microbiology,

Georg August University Göttingen

\section{Further members of the Examination Board:}

Prof. Dr. Kai Heimel, Dept. of Molecular Microbiology and Genetics, Georg

August University Göttingen

Prof. Dr. Stefanie Pöggeler, Dept. Genetics of Eukaryotic Microorganisms, Georg

August University Göttingen

Prof. Dr. Gerhard Braus, Dept. of Molecular Microbiology and Genetics, Georg

August University Göttingen

Prof. Dr. Dieter Heineke, Assistance of the Dean and Dean of Studies, Georg

August University Göttingen

Date of the oral examination: .2021.4.12 


\section{Promovierenden-Erklärung der Georg-August-Universität Göttingen}

Name

(Name, Vorname)

Anschrift

(Straße, PLZ, Wohnort)

Ich beabsichtige, eine Dissertation zum Thema

an der Georg-August-Universität Göttingen anzufertigen. Dabei werde ich von betreut.

Ich gebe folgende Erklärung ab:

1. Die Gelegenheit zum vorliegenden Promotionsvorhaben ist mir nicht kommerziell vermittelt worden. Insbesondere habe ich keine Organisation eingeschaltet, die gegen Entgelt Betreuerinnen und Betreuer für die Anfertigung von Dissertationen sucht oder die mir obliegenden Pflichten hinsichtlich der Prüfungsleistungen für mich ganz oder teilweise erledigt.

2. Hilfe Dritter wurde bis jetzt und wird auch künftig nur in wissenschaftlich vertretbarem und prüfungsrechtlich zulässigem Ausmaß in Anspruch genommen. Insbesondere werden alle Teile der Dissertation selbst angefertigt; unzulässige fremde Hilfe habe ich dazu weder unentgeltlich noch entgeltlich entgegengenommen und werde dies auch zukünftig so halten.

3. Die Ordnung zur Sicherung der guten wissenschaftlichen Praxis an der Universität Göttingen wird von mir beachtet.

4. Eine entsprechende Promotion wurde an keiner anderen Hochschule im In- oder Ausland beantragt; die eingereichte Dissertation oder Teile von ihr wurden/werden nicht für ein anderes Promotionsvorhaben verwendet.

Mir ist bekannt, dass unrichtige Angaben die Zulassung zur Promotion ausschließen bzw. später zum Verfahrensabbruch oder zur Rücknahme des erlangten Grades führen können.

(Ort), (Datum) 


\section{Contents}

Chapter I: General Introduction ..............................................................1

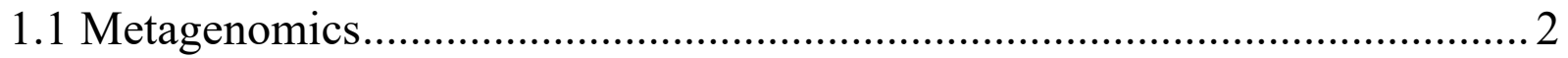

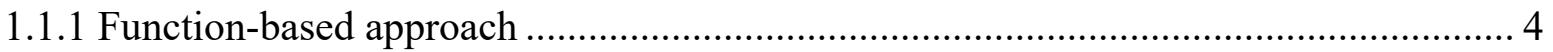

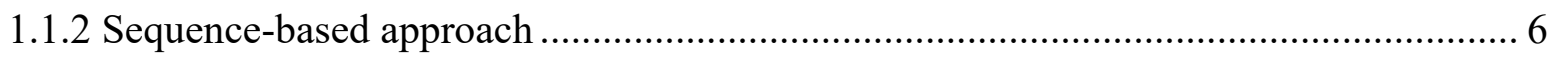

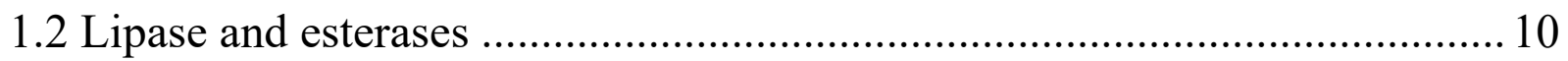

1.2.1 General properties and biological functions .......................................................... 10

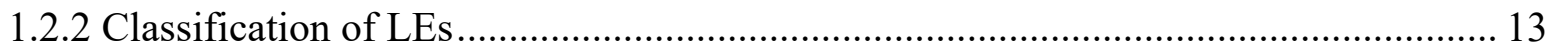

1.2.3 Microbial lipases/esterases potential for industrial applications ................................ 19

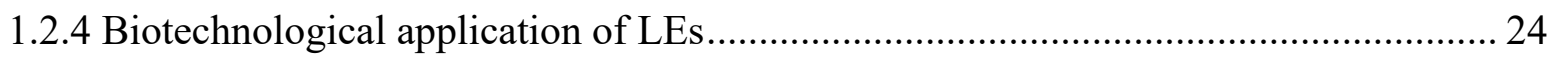

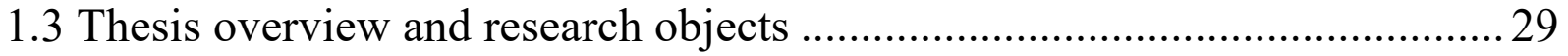

1.3.1 Thermophilic composts for extremophilic LEs isolation............................................. 29

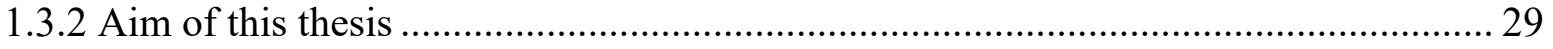

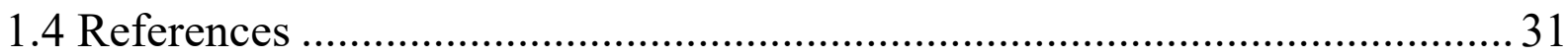

Chapter II: Metagenomic Screening for lipolytic enzymes reveals an ecology-clustered distribution pattern .............................................................58

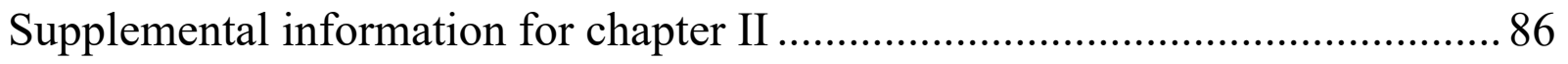

\section{Chapter III: Biochemical profiles of two thermostable and organic} solvent-tolerant esterases derived from a compost metagenome............186

Supplemental information for chapter III....................................................204

\section{Chapter IV: A novel caboxylesterase derived from a compost} metagenome exhibiting high stability and activity towards high salinity 


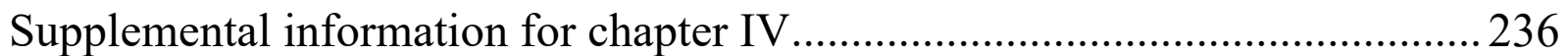

\section{Chapter V: General discussion .................................................................268}

5.1 Microbial composition in compost consortia ........................................269

5.2 Screening for lipolytic enzymes through metagenomic approaches ...........272

5.3 Comparative analysis of distribution of putative lipolytic genes across

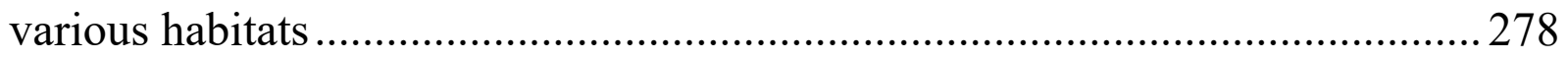

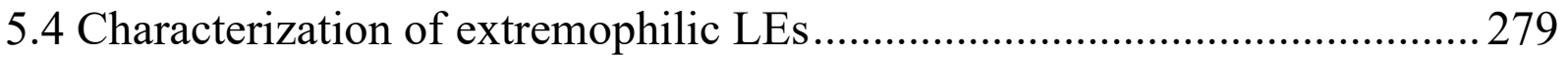

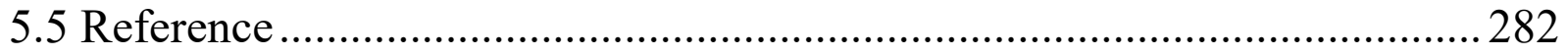

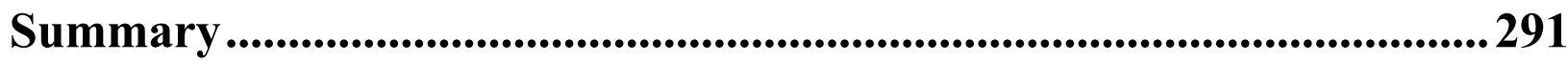

Acknowledgements...................................................................295 


\section{Chapter I}

\section{General Introduction}




\subsection{Metagenomics}

We are living on a "Planet of microbes", with microorganisms occupying almost every environmental niche and representing the largest part of the global biodiversity. It is estimated that the total number of microbial cells is more than $10^{30}$ (Turnbaugh et al. 2009). Prokaryotes represent the largest proportion of individual organisms. The total amount of bacterial and archaeal carbon is estimated to be almost equal to the total carbon of plants (Uritskiy and Di Ruggiero 2019) showing that microorganisms also play an essential role in biogeochemical cycles on this planet. Moreover, the genomes of microorganisms present enormous untapped genetic reservoir of novel enzymes and biomolecules promising for industrial applications (Simon and Daniel 2009; Dukunde et al. 2017).

Despite the obvious importance of microbes, the knowledge of their diversity is largely limited by the traditional culture-based methods. It is widely accepted that less than $1 \%$ microorganisms can be cultivated with standard laboratory protocols (Kumar et al. 2015). To circumvent the difficulties and limitations in cultivation techniques, a new discipline, metagenomics, has emerged as a strategic approach for direct exploring the genetic material from so far uncultured organisms (Berini et al. 2017; Laudadio et al. 2019; Almeida et al. 2019).

Metagenomics can be defined as the genomic analysis of the collective microbial assemblage found in an environmental sample (Hugenholtz and Tyson 2008). Through function-based and sequence-based approaches (Simon and Daniel 2011; Madhavan et al. 2017), metagenomics provides new insights into taxonomic and metabolic diversity of microbial communities (Fig. 1-1). According to the bibliographic analysis (Fig. 1-2), an increasing number of studies have applied metagenomic approaches to study the microbial assemblages from as many as approximately 2,192 different sites distributed across the planet (Ferrer et al. 2015). 
They include terrestrial habitats (topsoil, forest soil, plant rhizosphere soil, deserts, acid mine site, etc.) (Li et al. 2009; Fang et al. 2015; Dornelas et al. 2017), aquatic environments (deep sea sediments, superficial and deep seawater, river sediment, pond water, lake water, etc.) (Minegishi et al. 2013; Biver and Vandenbol 2013; Rabausch et al. 2013; Lee et al. 2017; Zhang et al. 2017), and eukaryotic-associated microbiomes (marine sponge, termite, rumen, human microbiota, etc.) (Qin et al. 2007; Pehrsson et al. 2016; Pratama et al. 2019; Almeida et al. 2019).

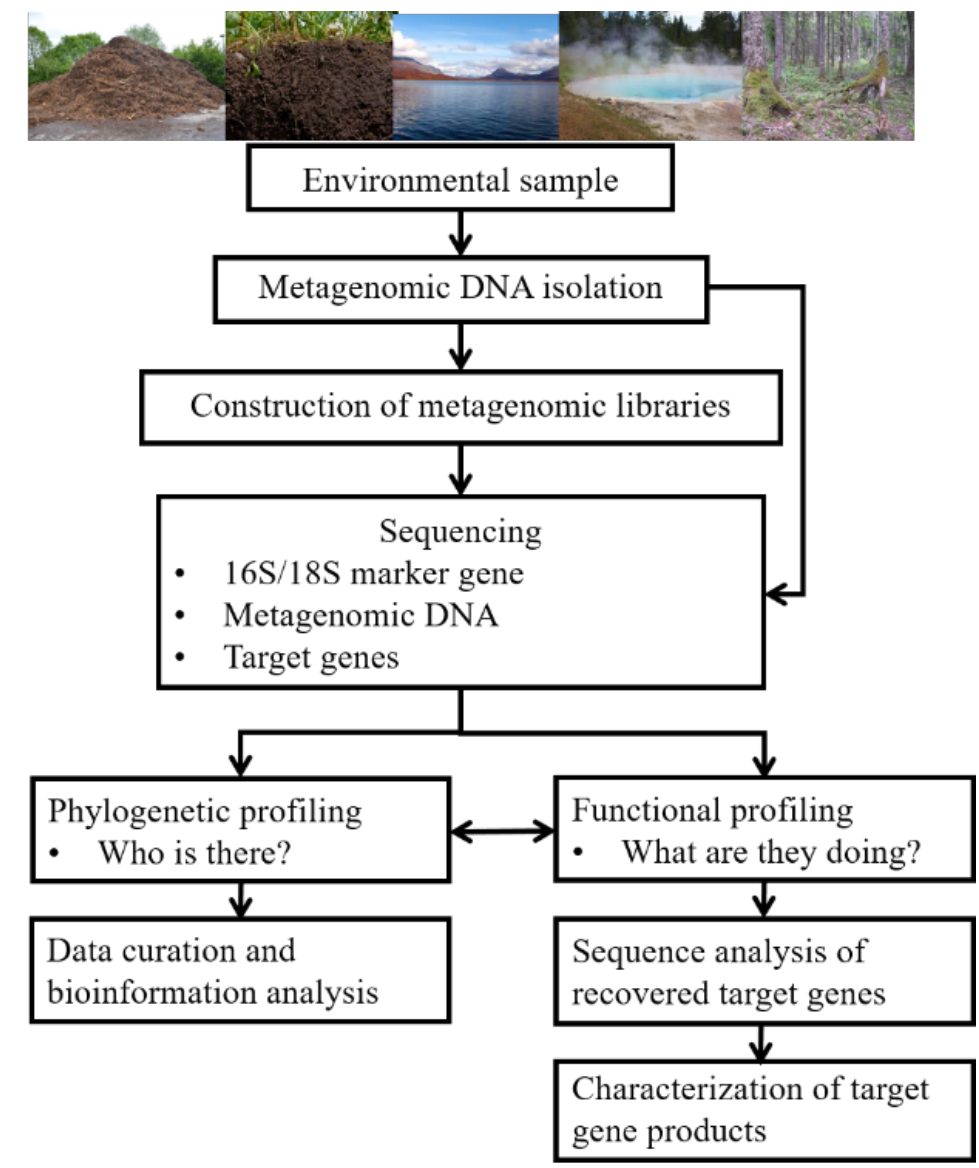

Fig. 1-1: Steps involved in metagenomic approaches (modified from Simon \& Daniel, 2009). DNA is first isolated from the habitat of interest. Next, metagenomic DNA can be analyzed through different strategies, including direct sequencing and/or screening of libraries to investigate the phylogenetic and functional diversity of microorganisms. 


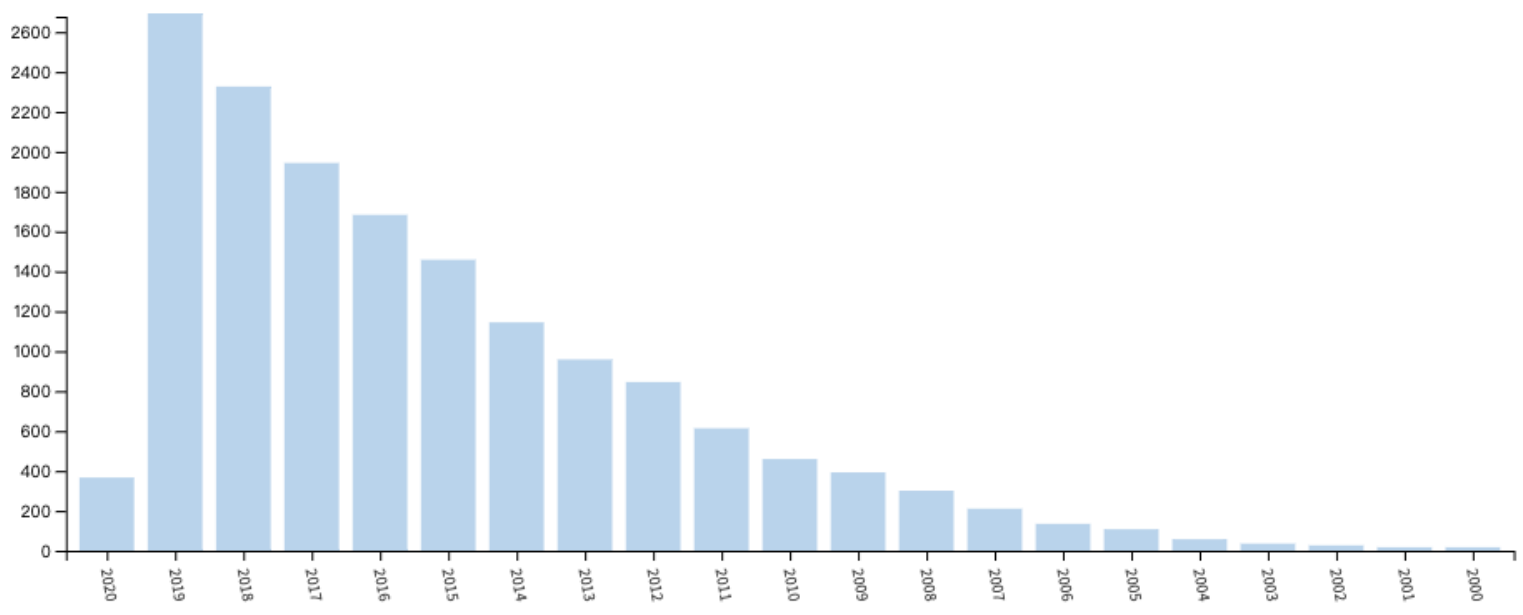

Fig. 1-2: Bibliographic analysis on metagenomics-related studies. "metagenom*" was used as keyword for searching in title and abstract in the Web of knowledge (http://apps.webofknowledge.com/). Time of data collection was 01-03-2020.

\subsubsection{Function-driven approach}

The function-driven approach involves screening for enzymatic activities expressed from environmental DNA in a surrogate host (Lam et al. 2015). The advantage of this approach is the certainty that the target protein is synthesized in an active form and produced by the host cell (Armour et al. 2019). In principle, it consists of cloning DNA fragments, expressing genes in a surrogate host, and screening for enzymatic activities.

First, environmental DNA is extracted, purified, size-selected and ligated into a vector. The extraction methods are divided into two types: direct and indirect (Miller et al. 1999). In comparison to the indirect isolation, the direct lysis of microbial cells is more commonly used due to the higher yields of nucleic acids (Devi et al. 2015; Satyanarayana et al. 2017). The selection of a vector depends largely on the proteins of interest during heterogeneous expression. For example, plasmids have high copy numbers and strong promotor, hence, usually used in screens in which a single gene 
is responsible for the activity. Plasmids are suitable for small-insert library with inserts sizes smaller than $10 \mathrm{~kb}$ (Lu et al. 2019). Vectors like fosmids (Fu et al. 2011; De Santi et al. 2016a), cosmids (Craig et al. 2010; Lam et al. 2015), and bacterial artificial chromosomes (BACs) (Ufarté et al. 2015b; Berini et al. 2017) are applied to construct large-insert libraries for identifying complex activities such as multigene encoded products, operons and entire biochemical pathways. After ligating the environmental DNA fragments into suitable vectors, the recombinant vectors are transferred into host cells, e.g. various Escherichia coli strains, for heterogenous expression. Finally, novel biomolecules are identified by the following strategies: (i) phenotypic trait detection, in which positive clones are identified by the reaction or interaction of an added substance with the expressed gene product (Brady 2007; Tasse et al. 2010; Maruthamuthu et al. 2016); (ii) Heterologous complementation, which relies on the expression of foreign gene product that is vital for host strains or mutants of host strains growing under selective conditions (Donato et al. 2010; Lei et al. 2018); and (iii) induced gene expression, also called substrate-induced gene expression (SIGEX) or metabolite-regulated expression (METREX), which is a high-throughput screening methods particularly suitable for the detection of catabolic genes (Uchiyama and Miyazaki 2009). In Table 1-1, we listed examples of biomolecules identified by the three different methods.

In short, the success of function-based approach significantly depends on the expression of target genes in a foreign host. However, due to the biased expression of foreign genes in host strains such as E. coli (Uchiyama and Miyazaki 2009; ReyesDuarte et al. 2012; Vargas-Albores et al. 2019), the hit rate is relatively low in function-driven screens. McMahon et al. (2012) proved that only about $40 \%$ of foreign genes were expressed in the most popular host cell (E. coli) in metagenomic studies. To overcome these problems, E. coli was improved as a screening host at 
the level of both transcription and translation (Lam et al. 2015). In addition, alternative hosts such as Streptomyces lividans (McMahon et al. 2012), Thermus thermophilus (Angelov et al. 2009), Sulfolobus solfataricus (Albers et al. 2006), Aspergillus oryzae (Nagamine et al. 2019) and diverse Proteobacteria (Craig et al. 2010; Hao et al. 2019) have been developed to allow detection of more and diverse target enzymes (Tripathi and Shrivastava 2019). Other technological bottlenecks such as a low proportion of metagenomic DNA accessible for expression (Guazzaroni et al. 2014), a lack of relevant substrates for screening (FernándezArrojo et al. 2010) and a poor performance of enzymatic activities under screening conditions (Fernández-Arrojo et al. 2010) also hamper the identification of novel molecules by function-based screening (Wang et al. 2019).

Nevertheless, function-based approach is still widely used, as it allows for discovery of novel enzymes even having no known homologous structures or sequences. Particularly, new methods such as fluorescence-activated cell sorting (FACS)-driven screening, and microfluidics-driven screening have been developed to improve the sensibility and throughput of function-based screen approaches (Ngara and Zhang 2018). For example, Scanlon and his colleagues (2014) described an ultra-high-throughput screening platform employing microfluidic gel microdroplets for discovery and/or engineering of natural product antibiotics.

\subsubsection{Sequence-based approach}

The recent advances in high-throughput DNA sequencing technologies have significantly reduced the sequencing costs and paved the way for sequence-based screening by direct sequencing of metagenomes. This approach is often applied in two ways: targeted metagenomics or shotgun metagenomics (Bharagava et al. 2018). 


\section{Targeted metagenomics}

The targeted metagenomics is employed to explore both the phylogenetic diversity and relative abundance of a particular gene in a sample (Techtmann and Hazen 2016; Bharagava et al. 2018; Awasthi et al. 2020b). Normally, targeted genes are investigated using PCR-based or hybridization-based techniques with primers/probes designed from conserved regions of known genes or gene products (Bender and Bard 2018; Dulanto Chiang and Dekker 2020). To reveal the taxonomic composition of a given community, taxonomic marker gene analysis such as $16 \mathrm{~S}$ and 18S rRNA gene, has been applied to different types of habitats (Forbes et al. 2017; Varma et al. 2018; Schulz et al. 2019; Meng et al. 2019; Egelkamp et al. 2019). It allows in-depth comparative analysis of microbial community composition in a set of samples, and could reveal, e.g. shifts in microbial diversity before and after a perturbation (Schloss et al. 2003; Turnbaugh and Gordon 2009; Aylward et al. 2012; Garrido-Cardenas and Manzano-Agugliaro 2017). Target genes such as genes encoding lipases (López-López et al. 2015), xylose isomerases (Parachin and Gorwa-Grauslund, 2011), dioxygenases (Iwai et al. 2010; Zaprasis et al. 2010), nitrite reductases (Bartossek et al. 2010), dimethylsulfoniopropionate-degrading gene (Varaljay et al. 2010) and nitrite reductases (Bartossek et al. 2010) were also identified based on PCR amplification. Despite these proven efficiency in amplicon surveys, this approach is limited by the universality of primers (Simon and Daniel, 2009). Moreover, inherited are also the amplification related errors and artifacts, such as biases introduced by the primers and chimeric sequences resulted from coamplification of homologous genes (Suzuki and Giovannoni, 1996; Wang and Wang 1996). 


\section{Whole genome shotgun metagenomics}

In contrast to amplicon-based approach, the direct metagenomic shotgun sequencing theoretically allows profiling the taxonomic composition and the function of the entire microbial community.

Studies based on direct sequencing can be divided into two groups: read-based and assembly-based. By classifying single reads with regard to taxonomy and function, the read-based approach is suited to answer questions related to shifts of microbial community composition, and abundances of genes or metabolic pathways (Jünemann et al. 2017). There are well established tools for reference-based classification of short reads. For example, the taxonomic and functional annotation of short reads using the MG-RAST pipeline (Keegan et al. 2016) are performed by mapping representative sequences against a custom M5nr database (Wilke et al. 2012) and a BLAST-like alignment tool (Kent 2002). MEGAN (Huson et al. 2016) uses a lowest common ancestor method to assign the taxonomy/function to each read, after searches with BLAST against a reference database. CARMA3 (Zhang and Lin 2019) implements a reciprocal BLAST search and a HMMER3-based variant (against the Pfam database) for annotation. Taxator-tk (Drö Ge et al. 2015) uses a combined approach of sequence-segmented similarities to a reference dataset and an approximated phylogenetic tree for taxonomic classification.

In assembly-based metagenomics, quality-checked reads are first assembled to long, contiguous sequences (contigs) and/or scaffolds. By now, a range of assemblers were developed specialized for metagenome short reads, such as MetaVelvet (Namiki et al. 2012), Meta-IDBA (Peng et al. 2011), IDBA-UD (Peng et al. 2012), MEGAHIT (Li et al. 2015a) and metaSPAdes (Nurk et al. 2017). The resulting assemblies were clustered into so-called genomic bins for taxonomic classification (Berini et al. 2017; Almeida et al. 2019; Guo et al. 2019; Awasthi et 
al. 2020b; Moreno-Indias and Tinahones 2020). To gain insights beyond taxonomic composition, a gene prediction, functional annotation, and metabolic reconstruction are done on assembled contigs. Predicted protein-encoding genes are annotated by similarity/homology searches against: 1) general protein database such as UniProt (Consortium 2018) and NCBI non-redundant database (Consortium 2018); 2) conserved domain databases such as Pfam (Finn et al. 2014) and CATH (Knudsen and Wiuf 2010); 3) metabolic databases such as COG (Clusters of Orthologous Groups of proteins) (Tatusov et al. 2000), eggNOG (evolutionary genealogy of genes: Non-supervised Orthologous Groups) (Huerta-Cepas et al. 2019), and KEGG (Kyoto Encyclopedia of Genes and Genomes) (Kanehisa et al. 2016); 4) motif databases such as MOTIF (Boeva 2016), MEME suite (Bailey et al. 2009) and HOMER (Heinz et al. 2010); and 5) special enzyme sequence resources such as Carbohydrate-Active Enzyme (CAZyme) (Cantarel et al. 2009), PeroxiBase (Fawal et al. 2013), antibiotic resistance genes (McArthur et al. 2013; Alcock et al. 2020) and lactamase (LacED) (Thai et al. 2009). In addition, several analysis platforms like MG-RAST(Keegan et al. 2016), IMG/M(Chen et al. 2017), and CAMERA (Seshadri et al. 2007) are available for the process and deposit of metagenomic data. 


\subsection{Lipase and esterase}

\subsubsection{General properties and biological functions}

Lipolytic enzymes (LEs) are a diverse group of water-soluble hydrolases that catalyze the cleavage and formation of ester and even non-ester bonds (Rao et al. 2009b; Thakur 2012; Kovacic et al. 2019; Samoylova et al. 2019). They have been studied for more than 150 years since Bernard (1856) first reported the degradation of fats by mammalian pancreatic fluids. LEs can be divided into two groups with respect to the substrate specificity: lipases (EC 3.1.1.1, triacylglycerol hydrolases) and esterases (EC 3.1.1.3, carboxyl ester hydrolases) (Bornscheuer 2002). Briefly, esterases preferentially hydrolyze water soluble esters and triacylglycerols with a fatty acid chain length shorter than $\mathrm{C}_{10}$. Lipases prefer water insoluble substrates, typically triacylglycerols with medium to long chain fatty acids ( $\geqslant \mathrm{C} 10)$ (Arpigny and Jaeger 1999; Neves Petersen et al. 2001). In addition, lipases are also distinguished from esterases by the feature of interfacial activation mediated by the hydrophobic domain (lid) covering the active site of the enzyme (Khan et al. 2017; Parapouli et al. 2018).

LEs are ubiquitous in all domains of life (Kovacic et al. 2019), and common in microorganisms. Some microbial strains are industrially important lipase/esterase producers (Rajendran et al. 2009). For example, bacterial species such as Pseudomonas cepacian (Secundo and Carrea 2002), P. aeruginosa (Ogino et al. 2004), P. fluorescens (Kiran et al. 2014), P. fragi (Sayali et al. 2013), Bacillus thermocatenulatus (Schmidt-Dannert et al. 1996), B. amyloliquefaciens (Musa et al. 2018), Staphylococcus hyicus (Lee et al. 2012), S. epidermidis (Abd Rahman et al. 2010), S. arlettae (Chauhan and Garlapati 2013) are used in industrial lipase/esterase production processes. Moreover, due to the catalytic versatility such as esterificatios, 
transesterification and inter-esterification in aqueous and non-aqueous media, LEs have been widely applied in a variety of biotechnological applications

\section{The structure of LEs}

Winkler et al. (1990) reported the first 3D structure of a human pancreatic lipase determined by X-ray crystallography. Subsequently, a great number of LEs have been evaluated by X-ray crystallography and nuclear magnetic resonance (NMR) (Mandrich et al. 2008; López-López et al. 2015; Kim 2017). These studies have proved that almost all LEs shared a canonical $\alpha / \beta$ hydrolase fold, despite low sequence similarities (Kim 2017). As shown in Fig. 1-3, the parallel strands display a left-handed super helical twist, $\beta 3$ to $\beta 8$ are connected by $\alpha$ helices which pack on either side of the central $\beta$ sheet (Dukunde et al. 2017). This topology provides a stable scaffold for positioning catalytic sites, of which the spatial positioning of side chains is remarkably well conserved (Glogauer et al. 2011).

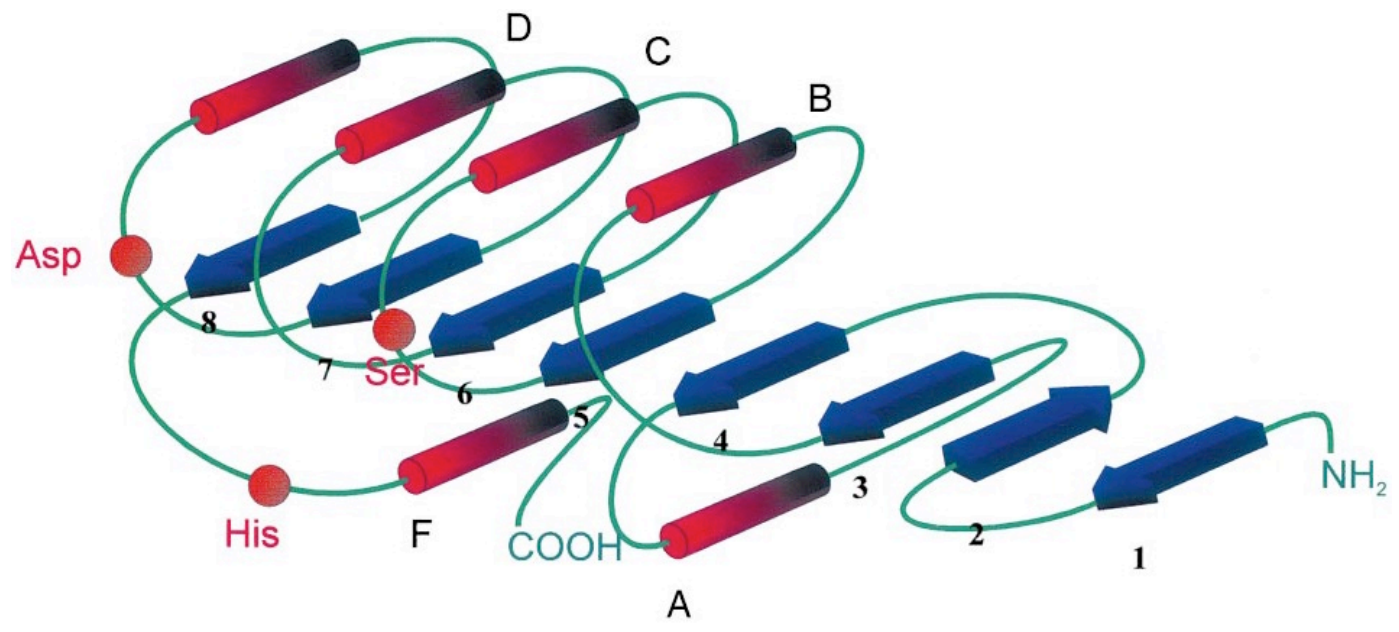

Fig. 1-3: The canonical structure of the $\alpha / \beta$ hydrolase fold. L-Sheets (1-8) are shown as blue arrows, Khelices (A-F) as red cylinders. The catalytic triad are indicated as red circles. The nucleophile serine residue locates immediately after $\beta 5$, the aspartic/glutamic acid and histidine residues after $\beta 7$ and $\beta 8$, respectively (Ollis et al. 1992; Jaeger et al. 1999). The picture is taken from Bornscheuer (2002). 
Additionally, the structural unique feature for lipases is the presence of a flexible domain (referred to as a 'flap' or 'lid') at the protein surface(Ramnath et al. 2016). The lid may consist of a single $\alpha$ helix or two helices flanked by a loop region. In the presence of a minimum substrate concentration, the lid moves apart, making the active site accessible to the substrate (Bornscheuer 2002), and thus, the catalytic activity of the enzyme increases. This phenomenon is designated as interfacial activation (Glogauer et al. 2011; Adlercreutz 2013; Ramnath et al. 2016).

\section{The catalytic triad}

The catalytic triad of LEs is composed of three residues: a nucleophilic residue (serine), a catalytic acid residue (aspartate or glutamate acid) and a histidine residue (Fig. 1-3). They are far apart in the primary sequence but spatially close in the folded protein (Brumlik and Buckley 1996). The serine residue is usually embedded in a highly conserved pentapeptide G-X-S-X-G. According to the 3D structure, the serine residue locates in a tight turn between sheet $\beta 5$ and helix $\alpha \mathrm{C}$, which form a highly conserved $\beta$-turn- $\alpha$ motif termed as 'nucleophilic elbow'. Moreover, in the immediate vicinity of the serine residue, there is an oxyanion hole donating backbone amide protons to stabilize the transition state of the substrate in the enzyme (Mandrich et al. 2008). Two main types of oxyanion holes, GX and GGGX, have been found in LEs (Lu et al. 2019).

\section{Catalytic versatility}

LEs catalyze a wide range of reactions, such as ester exchange, alcoholysis, acidolysis, aminolysis, hydrolysis, esterification, intramolecular esterification, and synthesis of estolides and other polymers.

\section{$>$ Transesterification:}

(a) Ester exchange 
$\mathrm{R}-\mathrm{COO}-\mathrm{R}^{\prime}+\mathrm{R}^{\prime \prime}-\mathrm{COO}-\mathrm{R}^{*} \rightarrow$ R-COO-R${ }^{*}+\mathrm{R}^{\prime \prime}-\mathrm{COO}-\mathrm{R}^{\prime}$

(b) Alcoholysis

$\mathrm{R}-\mathrm{COO}-\mathrm{R}^{\prime}+\mathrm{R}^{\prime \prime}-\mathrm{OH} \rightarrow \mathrm{R}-\mathrm{COO}-\mathrm{R}^{\prime \prime}+\mathrm{R}^{\prime}-\mathrm{OH}$

(c) Acidolysis

$\mathrm{R}-\mathrm{COO}-\mathrm{R}^{\prime}+\mathrm{R}$ "-COOH $\rightarrow$ R"-COO-R' + R-COOH

(d) Aminolysis

$\mathrm{R}-\mathrm{COO}-\mathrm{R}^{\prime}+\mathrm{R}^{\prime \prime}-\mathrm{NH}_{2} \rightarrow \mathrm{R}-\mathrm{CONH}-\mathrm{R}^{\prime \prime}+\mathrm{R}^{\prime}-\mathrm{OH}$

Hydrolysis:

$\mathrm{R}-\mathrm{COO}-\mathrm{R}^{\prime}+\mathrm{H}_{2} \mathrm{O} \rightarrow \mathrm{R}-\mathrm{COOH}+\mathrm{R}^{\prime}-\mathrm{OH}$

$>$ Esterification:

$\mathrm{R}-\mathrm{COOH}+\mathrm{R}^{\prime}-\mathrm{OH} \rightarrow \mathrm{R}-\mathrm{COO}-\mathrm{R}^{\prime}+\mathrm{H} 2 \mathrm{O}$

> Intramolecular esterification:

$\mathrm{HO}-\mathrm{R}-\mathrm{COOH} \rightarrow \mathrm{R}-\mathrm{COO}-\mathrm{R}$ (Lactones)

$>$ Synthesis of estolides and other polymers:

$\mathrm{R}-\mathrm{COOH}+\mathrm{HO}-\mathrm{R}$ '-COOH $\rightarrow$ R-COO-R'-COOH

\subsubsection{Classification of LEs}

For a long period of time, LEs were grouped simply by their substrate specificities. However, this method is too vague due to the lack of consistency for substrates and methods used in different laboratories. The increasing sequence information in public databases enables the comparison of amino acid sequences, which provides a picture about the similarity and evolutionary relationships among LEs. Thus, LEs are commonly classified based on sequence similarity, i.e. the classification system 
of Arpigny and Jaeger (1999). They grouped bacterial LEs into eight families (family I-VIII) according to conserved amino acid sequence motifs and biochemical properties. A recent update to this system was an addition of 11 families (IX to XIX) (Kovacic et al. 2019; Table 1-1). Besides the nineteen families, there are claims of novel families, such as Est22 (Li et al. 2017b), Est9X (Jeon et al. 2009), LipSM54 (Li et al. 2016) and EstDZ2 (Zarafeta et al. 2016). 
Table 1-1: Current classification of lipolytic enzymes.

\begin{tabular}{|c|c|c|c|}
\hline Family & Sub-family & General description & Reference \\
\hline \multirow[t]{8}{*}{ I } & I. 1 & $\begin{array}{l}>\quad \text { Similar to Pseudomonas aeruginosa lipase; } \\
>\quad \text { Have a mass between } 30-32 \mathrm{kDa} \\
>\quad \text { Require chaperone proteins (Lifs) for expression. }\end{array}$ & Arpigny and Jaeger, 1999 \\
\hline & I. 2 & $\begin{array}{l}\text { Similar to Burkholderia glumae lipase; } \\
\quad \text { Have a mass larger than } 32 \mathrm{kDa} \\
\quad \text { Need Lifs for expression. }\end{array}$ & Arpigny and Jaeger, 1999 \\
\hline & I. 3 & $\begin{array}{l}>\quad \text { Lipases from Pseudomonas fluorescens and Serratia marcescens; } \\
>\quad \text { Have a mass between } 50 \text { to } 65 \mathrm{kDa} .\end{array}$ & Arpigny and Jaeger, 1999 \\
\hline & I. 4 & $\begin{array}{l}\text { The smallest lipases, with a molecular mass of less than } 20 \mathrm{kDa} \text {; } \\
\text { Several with the alternative pentapeptide motif AXSXG. }\end{array}$ & Arpigny and Jaeger, 1999 \\
\hline & $\mathrm{I} .5$ & $\begin{array}{l}\text { Commonly from Gram-positive prokaryotes; } \\
>\quad \text { Have a mass of approximately } 46 \mathrm{kDa} \text {. }\end{array}$ & Arpigny and Jaeger, 1999 \\
\hline & I.6 & $\begin{array}{l}\text { Start as preproproteins (around } 75 \mathrm{kDa} \text { ) due to an amino acid N-terminal domain of } 200 \text { amino } \\
\text { acids used as a translocator signal through the cell membrane. }\end{array}$ & Rosenstein and Götz 2000 \\
\hline & I.7 & $\begin{array}{l}\text { Have a wide range of substrates, both tri- and mono-glycerides of varying fatty acid chain } \\
\text { lengths. }\end{array}$ & Jaeger and Eggert 2002 \\
\hline & I.8 & $\begin{array}{l}\quad \text { From Pseudoalteromonas haloplanktis; } \\
\quad \text { Lacks the lid structure and } \mathrm{Ca}^{2+} \text { pockets. }\end{array}$ & de Pascale et al. 2008 \\
\hline II & - & $\begin{array}{l}>\quad \text { Modified pentapeptide motif around the active serine: Gly-Asp-Ser-(Leu) [GDS(L)]; } \\
>\quad \text { Secreted and membrane-bound esterases. }\end{array}$ & $\begin{array}{l}\text { Mølgaard et al. } 2000 \\
\text { Akoh et al. } 2004 \\
\text { Li et al. } 2019\end{array}$ \\
\hline III & - & $\begin{array}{l}\text { Extracellular esterases; } \\
\text { Show sequence similarity }(\sim 20 \%) \text { to human platelet activating factor acetylhydrolase (PAF- } \\
\text { AH). }\end{array}$ & Arpigny and Jaeger, 1999 \\
\hline \multirow[t]{2}{*}{ IV } & $\begin{array}{l}\text { GTSAG } \\
\text { motif }\end{array}$ & $\begin{array}{l}\quad \text { Also called hormone-sensitive lipase (HSL); } \\
\quad \text { Have typical motifs of HGG and GTSAG. }\end{array}$ & Li et al. 2014 \\
\hline & $\begin{array}{l}\text { GDSAG } \\
\text { motif }\end{array}$ & Another subfamily in family IV, but with GDSAG motif. & Li et al. 2014 \\
\hline $\mathrm{V}$ & $\mathrm{V}-1$ & $\begin{array}{l}\text { Have conserved motif HGGG locates upstream of the pentapeptide motif GxSxG; } \\
\text { High sequence similarity with non-lipolytic enzymes: epoxide hydrolases, dehalogenases and } \\
\text { haloperoxidases; }\end{array}$ & $\begin{array}{l}\text { Verschueren et al. 1993; } \\
\text { Misawa et al. } 1998\end{array}$ \\
\hline
\end{tabular}


Also known as ABHD6 hydrolases.

$\mathrm{V}-2>$ Known as carboxymethylbutenolide lactonase.

Arpigny and Jaeger, 1999

V-3 $>$ Uncharacterised conserved protein UCP031982, XabL type.

> Consists of both phospholipases and carboxylesterases with broad substrate specificity;

$>$ Also featured by the small molecular masses $(23-26 \mathrm{kDa})$

VII

$>\quad$ Large bacterial esterases, with a molecular mass of $\sim 55 \mathrm{kDa}$;

$>$ Sequence homology with eukaryotic acetylcholine esterases and intestinelliver carboxylesterases.

VIII $\quad>\quad>$ Show high sequence similar to class C $\beta$-lactamases;

$>$ Have a molecular mass of $\sim 42 \mathrm{kDa}$;

$>$ The serine residue in the SXXK tetrapeptide.

New type of thermoalkalophilic lipase (PhaZ7) from

$>$ Sshows high specificity for amorphous polyesters.

$>$ New type of thermostable esterase (EstD) from Thermotoga maritima;

$>$ Have a molecular mass of $44.5 \mathrm{kDa}$;

$>$ Optimal activity around $95{ }^{\circ} \mathrm{C}$ and at $\mathrm{pH} 7$.

X-2 $>$ Secretory lipase from Rhodococcus sp. strain CR-53;

$>\quad$ Display an unusual Y-type 0xyanion hole, similar to the Candida antarctica lipase clan.

$\begin{array}{ll}\text { XI } & - \\ \text { XII } & -\end{array}$

$>$ Presence of an Arg-Gly sequence in oxyanion hole instead of His-Gly;

$>$ A signature sequence distinctive of filamentous fungal lipases (LipG).

$>\quad$ Novel cold-adapted alkaline lipase from an intertidal flat metagenome;

$>$ Contain an extra domain in N- or C-terminal: Bacterial Ig-like domain.

Bassegoda et al. 2012

Lee et al. 2006

\section{Park et al. 2007}

Arpigny and Jaeger, 1999

Arpigny and Jaeger, 1999

Wagner et al. 2002;

Hausmann and Jaeger 2010

Handrick et al. 2001

Levisson et al. 2007

Kim et al. 2009

Montoro-García et al. 2009

Charbonneau

and

Beauregard 2013

Rao et al. 2011 


\begin{tabular}{|c|c|c|c|c|}
\hline $\mathrm{XV}$ & - & $>$ & Esterases Est10, EstGK1, EstZ3, EstD2, EstGtA2, Est5S and EstWSD belong to this family. & $\begin{array}{l}\text { Bayer et al. 2010; Lee et al. } \\
\text { 2010; Kim et al. 2012; } \\
\text { Charbonneau and } \\
\text { Beauregard 2013; Wang et } \\
\text { al. 2013; Rodríguez et al. } \\
\text { 2015 }\end{array}$ \\
\hline XVI & - & $>$ & A novel cold-adapted esterase from an Arctic intertidal metagenomic library. & Fu et al. 2013 \\
\hline XVII & - & $>$ & A novel thermophilic and halophilic esterase from Janibacter sp. R02. & Castilla et al. 2017 \\
\hline XVIII & - & $>$ & $\begin{array}{l}\text { Esterase estUT1 isolated from Ureibacillus thermosphaericus; } \\
\text { Have a typical catalytic triad and the active serine is included in a pentapeptide (GGSVG). }\end{array}$ & Samoylova et al. 2018 \\
\hline XIX & - & $>$ & $\begin{array}{l}\text { The novel, thermostable lipase (LipSm) from Stenotrophomonas maltophilia; } \\
\text { Lack the requirement for interfacial activation for small substrates. }\end{array}$ & Parapouli et al. 2018 \\
\hline EstA & - & $>$ & Related to family III but different conserved motifs (pentapeptide GHSMG). & Chu et al. 2008 \\
\hline EstF & - & $>$ & $\begin{array}{l}\text { Related to family V but with a modified pentapeptide, GTSXG, and different flanking regions } \\
\text { around the HG motif. }\end{array}$ & Fu et al. 2011 \\
\hline EstY & - & $\begin{array}{l}> \\
>\end{array}$ & $\begin{array}{l}\text { Derived from pathogenic bacteria; } \\
\text { First possible lipolytic virulence factors that do not belong to the GDSL family; } \\
\text { Isolated from surface river water. }\end{array}$ & Wu and Sun 2009 \\
\hline EM3L4 & - & $>$ & $\begin{array}{l}\text { A new esterase derived from a metagenomic library of deep-sea sediment; } \\
\text { show only } 33-58 \% \text { amino acid identities to known proteins. }\end{array}$ & Jeon et al. 2011 \\
\hline Est9x & - & $>$ & $\begin{array}{l}\text { A new esterase from a marine microbial metagenome of the South China Sea; } \\
\text { Show lower than } 27 \% \text { sequence identities with the characterized lipolytic enzymes. }\end{array}$ & Fang et al. 2014 \\
\hline Est10 & - & $>$ & $\begin{array}{l}\text { A cold-adapted and salt-tolerant esterase from a psychrotrophic bacterium Psychrobacter } \\
\text { pacificensis. }\end{array}$ & Wu et al. $2013 b$ \\
\hline Est12 & - & $>$ & A novel esterase Est12 from a genomic library of a psychrotrophic Psychrobacter celer 3Pb1. & Wu et al. $2013 b$ \\
\hline EstGH & - & $>$ & $\begin{array}{l}\text { A novel esterase EstGH from a metagenomic library of soil sample; } \\
\text { Show low similarity }(29 \%) \text { to known esterases. }\end{array}$ & Nacke et al. 2011 \\
\hline EstJ & - & $\vec{\nu}$ & $\begin{array}{l}\text { A novel alkaliphilic esterase (EstJ) from a soil metagenome of Jeju Island; } \\
\text { Show low similarity (32-45 \%) to putative } \alpha / \beta \text { hydrolases, and unique motifs of WMVSGG. }\end{array}$ & Choi et al. 2013 \\
\hline
\end{tabular}




\begin{tabular}{|c|c|c|c|c|}
\hline EstL28 & - & $>$ & $\begin{array}{l}\text { A novel cold-active esterase (EstL28) from swamp sediment metagenome, with a molecular } \\
\text { mass of } 31.3 \mathrm{kDa} \text {. }\end{array}$ & Seo et al. 2014 \\
\hline LipC & - & $>$ & A halophilic esterolytic enzyme LipC from archeaon Haloarcula marismortui. & Rao et al. 2009 \\
\hline LipS & - & $>$ & A metagenome-derived lipase (LipS) with an optimum temperature at $70^{\circ} \mathrm{C}$. & Chow et al. 2012 \\
\hline LipT & - & $>$ & A metagenome-derived lipase (LipT) with an optimum temperature at $75^{\circ} \mathrm{C}$. & Chow et al. 2012 \\
\hline lp_3505 & - & $>$ & $\begin{array}{l}\text { A novel esterase from Lactobacillus plantarum; } \\
\text { Cold-active and salt-tolerant and show potential application for cheese ripening. }\end{array}$ & Esteban-Torres et al. 2014 \\
\hline PE10 & - & $>$ & A halotolerant esterase from a marine bacterium Pelagibacterium halotolerans $\mathrm{B} 2 \mathrm{~T}$. & Jiang et al. 2012 \\
\hline FLS18 & - & $>$ & $\begin{array}{l}\text { Two novel esterases FLS18C and FLS18D were derived from a metagenomic library of the } \\
\text { South China Sea marine sediment. }\end{array}$ & Hu et al. 2010 \\
\hline RlipE1 & - & $>$ & A novel esterase from a metagenomic library of China Holstein cow rumen. & Liu et al. 2009 \\
\hline
\end{tabular}


Additionally, several databases are available that group LEs into different families: ESTHER database (Lenfant et al. 2013) (http://bioweb.ensam.inra.fr/ESTHER/general?what=index), microbial esterases and lipases database (MELDB) (Kang et al. 2006), Carbohydrate Active Enzymes database (CAZy) (Cantarel et al. 2009) (www.cazy.org) and Lipase Engineering Database (LED) (Fischer and Pleiss 2003)( $\underline{\text { http://www.led.uni-stuttgart.de/). }}$

\subsubsection{Microbial lipases/esterases potential for industrial applications}

\section{Thermostability}

Thermostability is always one the most desirable features of LEs for industrial applications. Most of the industrial processes are carried out at temperatures above $45^{\circ} \mathrm{C}$, generally to reduce the contaminations and accelerate the reaction rates (Vieille and Zeikus 2001; Gotor-Fernández et al. 2006; Ramnath et al. 2016). Thus, the enzymes need to be stable at this temperature and above. Thermostable/thermophilic LEs have successfully been isolated from microbial strains mainly from Bacillus and Pseudomonas, as well as metagenomes such as compost, hot spring and hydrothermal deep-sea sediment (Table 1-2). 
Table 1-2: Examples of thermostable LEs isolated from cultured microorganisms and metagenomic libraries.

\begin{tabular}{llll}
\hline Source of LEs & \multicolumn{2}{l}{ Enzyme properties } & Reference \\
\cline { 2 - 3 } & $\begin{array}{l}\text { Optimal } \\
\text { temp. }\left({ }^{\circ} \mathrm{C}\right)\end{array}$ & Optimal pH & \\
\hline Microorganism & & & \\
Bacillus sp. strain L2 & 70 & 8.0 & Sabri et al. 2009 \\
B. thermoleovorans ID-1 & $70-75$ & 7.5 & Lee et al. 1999 \\
Geobacillus sp. & 70 & 9.0 & Abdel-Fattah and Gaballa 2008 \\
Pseudomonas sp. & 90 & 11.0 & Rathi et al. 2000 \\
Pyrobaculum calidifontis & 90 & 7.0 & Hotta et al. 2002 \\
Pyrococcus furiosus & 100 & - & Ikeda and Clark 1998 \\
Pyrococcus horikoshii & 95 & 7.0 & Feng et al. 2000 \\
Alicyclobacillus acidocaldarius & 65 & 6 & Mandrich et al. 2008 \\
Janibacter sp. R02 & 80 & 8 & Castilla et al. 2017 \\
Sulfolobus tokodaii & 80 & 9 & Wei et al. 2013 \\
Fervidobacterium changbaicum & 75 & 8 & Cai et al. 2011 \\
Thermus thermophilus & 80 & 8 & Leis et al. 2015 \\
Metagenome & & & \\
Thermal environmental samples & $>95$ & 6.0 & Rhee et al. 2005 \\
Hot spring & 70 & 9 & Tirawongsaroj et al. 2008 \\
Activated sludge & 60 & 8 & Zarafeta et al. 2016 \\
Hydrothermal deep-sea sediment & 60 & 8.5 & Shao et al. 2013 \\
Enrichment cultures maintained at & 70,75 & 8.0 & Zhu et al. 2013 \\
65 to 75 ${ }^{\circ}$ C & - & Chow et al. 2012 \\
Red sea brine pool & 65 & 8.5 & \\
Composts at thermophilic stage & 80 & 7 & Mohamed et al. 2013 \\
& 70 & 9 & Lu et al. 2019 \\
\hline
\end{tabular}




\section{Organic solvent tolerance}

Most of the industrial relevant reactions are performed in the presence of organic solvents. It is advantageous to carry out the reactions under a water-restricted environment, due to the shifting of thermodynamic equilibria in favor of synthesis (esterification and transesterification), increasing solubility of substrates and product, improved thermal stability of the enzymes since, simpler removal of solvent (most organic solvents have lower boiling point than water), and reducing chemical waste (Dandavate et al. 2009; Ahmed et al. 2010; Ebrahimpour et al. 2011; Salihu and Alam 2015; Kumar et al. 2016). However, enzymes tend to denature or loose activity in the presence of organic solvents, as a result of removal of water molecules at the enzyme surface and the active sites by organic solvents. Thus, LEs that function in the presence of organic solvents are of particular interest for industrial applications. In recent years, a focus is to find new LEs with excellent activity, selectivity and stability in organic solvents (examples were listed in Table 1-3). 
Table 1-3: Examples of organic solvent tolerant LEs isolated from microorganisms and metagenomic libraries.

\begin{tabular}{|c|c|c|c|}
\hline \multirow[t]{2}{*}{ Source of LEs } & \multicolumn{2}{|l|}{ Enzyme properties } & \multirow[t]{2}{*}{ Reference } \\
\hline & $\begin{array}{l}\text { Incubation } \\
\text { condition }\end{array}$ & Stable in ${ }^{\mathrm{a}}$ & \\
\hline \multicolumn{4}{|l|}{ Microorganism } \\
\hline $\begin{array}{l}\text { Pseudomonas } \\
\text { aeruginosa san-ai }\end{array}$ & $30^{\circ} \mathrm{C}, 48 \mathrm{~h}$ & $25 \%(\mathrm{v} / \mathrm{v})$ chloroform and $n$-hexane & Karadzic et al. 2006 \\
\hline $\begin{array}{l}\text { Bacillus sphaericus } \\
205 y\end{array}$ & $37^{\circ} \mathrm{C}, 30 \mathrm{~min}$ & $25 \%(\mathrm{v} / \mathrm{v}) n$-hexane and $p$-xylene. & Hun et al. 2003 \\
\hline Bacillus megaterium & $29^{\circ} \mathrm{C}, 1 \mathrm{~h}$ & $\begin{array}{l}25-80 \%(\mathrm{v} / \mathrm{v}) \text { ethanol and acetone } 100 \% \\
\text { (v/v) 2-propanol, 1-butanol, Tol, } n \text {-hexane } \\
\text { and } n \text {-heptane }\end{array}$ & Lima et al. 2004 \\
\hline $\begin{array}{l}\text { Sulfolobus solfataricus } \\
\text { PI }\end{array}$ & $\begin{array}{l}30^{\circ} \mathrm{C} \text { or } 70^{\circ} \mathrm{C}, 1 \\
\mathrm{~h}\end{array}$ & $\begin{array}{l}40 \%(\mathrm{v} / \mathrm{v}) \text { methanol, ethanol and } 2- \\
\text { propanol. }\end{array}$ & Mandrich et al. 2005 \\
\hline $\begin{array}{l}\text { Stenotrophomonas } \\
\text { maltophilia CGMCC } \\
4254\end{array}$ & $30^{\circ} \mathrm{C}, 24 \mathrm{~h}$ & $\begin{array}{l}20 \text { and } 50 \%(\mathrm{v} / \mathrm{v}) \text { benzene, toluene, } \mathrm{n}- \\
\text { hexane and } \mathrm{n} \text {-heptane. }\end{array}$ & Li et al. 2013 \\
\hline $\begin{array}{l}\text { Pseudomonas } \\
\text { aeruginosa } \mathrm{MH} 38\end{array}$ & $25^{\circ} \mathrm{C}, 1 \mathrm{~h}$ & 30 and $50 \%(\mathrm{v} / \mathrm{v})$ benzene and hexane & Jang et al. 2014 \\
\hline Monascus purpureus & $40^{\circ} \mathrm{C}, 24 \mathrm{~h}$ & $\begin{array}{l}20 \%(\mathrm{v} / \mathrm{v}) \text { methanol, ethanol, acetonitrile, } \\
\text { glycerol, acetone, } n \text {-Hexane, toluene and } \\
\text { chloroform }\end{array}$ & Kang et al. 2017 \\
\hline Chromohalobacter $s p$. & $35^{\circ} \mathrm{C}, 30 \mathrm{~min}$ & 20 and $50 \%(\mathrm{v} / \mathrm{v})$ benzene and hexane & Ai et al. 2018 \\
\hline $\begin{array}{l}\text { Psychrobacter } \quad s p . \\
\text { ZY124 }\end{array}$ & $37^{\circ} \mathrm{C}, 5 \mathrm{~h}$ & $\begin{array}{l}10,30 \text { and } 50 \%(\mathrm{v} / \mathrm{v}) \mathrm{DMSO} \text {, methanol, } \\
\text { ethanol, acetone, acetonitrile, toluene, } \\
\text { pentane, hexane and octane }\end{array}$ & Zhang et al. $2018 \mathrm{~b}$ \\
\hline \multicolumn{4}{|l|}{ Metagenome } \\
\hline Marine mud & $30^{\circ} \mathrm{C}, 12 \mathrm{~h}$ & $\begin{array}{l}20 \%(\mathrm{v} / \mathrm{v}) \text { ethanol, acetonitrile, DMF and } \\
\text { cyclohexane }\end{array}$ & Gao et al. 2016 \\
\hline Forest soil sample. & $37^{\circ} \mathrm{C}, 2 \mathrm{~h}$ & $25 \%$ (v/v) DMSO, Benzene and p-xylene & Berlemont et al. 2013 \\
\hline $\begin{array}{l}\text { Wastewater treatment } \\
\text { plant of a meat packing } \\
\text { and dairy industry }\end{array}$ & $4{ }^{\circ} \mathrm{C}, 48 \mathrm{~h}$ & $\begin{array}{l}15 \text { and } 30 \%(\mathrm{v} / \mathrm{v}) \text { methanol, ethanol, 1- } \\
\text { propanol, 2-propanol, glycerol, THF, } \\
\text { dioxane, DMSO }\end{array}$ & Glogauer et al. 2011 \\
\hline Soil & $30{ }^{\circ} \mathrm{C}, 2 \mathrm{~h}$ & $\begin{array}{l}15 \text { and } 30 \%(\mathrm{v} / \mathrm{v}) \mathrm{DMSO}, \mathrm{DMF}, p \text {-xylene, } \\
\text { hexane, heptane, and octane }\end{array}$ & Wang et al. 2013 \\
\hline Compost & $\begin{array}{l}\text { Room temp., } 26 \\
\text { d }\end{array}$ & $\begin{array}{l}30 \%(\mathrm{v} / \mathrm{v}) \text { methanol, ethanol, isopropanol, } \\
\mathrm{DMSO} \text {, acetone }\end{array}$ & Lu et al., 2019 \\
\hline $\begin{array}{l}\text { Soil contaminated with } \\
\text { petroleum } \\
\text { hydrocarbons }\end{array}$ & No data & $30 \%(v / v)$ DMF and DMSO & Pereira et al. 2015 \\
\hline
\end{tabular}

${ }^{a}$ Abbreviations for the organic solvents are: DMF, N,N-dimethylformamide; DMSO, dimethylsulfoxide; THF, tetrahydrofuran.

\section{Halotolerance}

Among extremophilic LEs, halophilic/halotolerant LEs are another major group of industrial relevant enzymes. In comparison to the non-halophilic/non-halotolerant 
counterparts, halophilic/halotolerant LEs are able to successfully compete with salt ions for hydration and maintain their functional conformation in the presence of high ionic concentration (Karan et al. 2012). Thus, halophilic/halotolerant LEs can generally maintain their function in processes in which water activity is low. Some of them are also thermostable and tolerant to a wide range organic solvents (Delgado-García et al. 2012). These properties make halophilic/halotolerant LEs an option for new enzymatic processes in various products (pharmaceuticals, foods, textiles, chemicals, etc). In principle, mechanisms that contribute to halophilic/halotolerant enzyme stability are a high proportion of negatively charged residues (mainly aspartic and glutamic acid) located on the protein surface, conglomeration of slightly hydrophobic groups in the presence of high salt concentrations, and hydration of the protein surface due to amino acid residues with carboxylic groups (Lanyi 1974; Coquelle et al. 2010; Delgado-García et al. 2012; Munawar and Engel 2013). The examples of recently identified halophilic/halotolerant LEs are listed in Table 1-4. 
Table 1-4: Examples of organic halophilic/halotolerant LEs isolated from microorganisms and metagenomic libraries.

\begin{tabular}{|c|c|c|c|}
\hline \multirow[t]{2}{*}{ Source of LEs } & \multicolumn{2}{|c|}{ Enzyme properties } & \multirow[t]{2}{*}{ Reference } \\
\hline & Salt range & Maximum activity $(\%)^{\mathrm{a}}$ & \\
\hline \multicolumn{4}{|l|}{ Microorganism } \\
\hline Pelagibacterium halotolerans & $0-4 \mathrm{M}$ & $\sim 160 \%$ at $3 \mathrm{M}$ & Jiang et al. 2012 \\
\hline Alcanivorax borkumensis & $0-3.5 \mathrm{M}$ & $100 \%$ at $0 \mathrm{M}$ & Tchigvintsev et al. 2015 \\
\hline Serratia sp. & $0-4 \mathrm{M}$ & $100 \%$ at $0 \mathrm{M}$ & Jiang et al. 2016 \\
\hline Alkalibacterium sp. & $0-4 \mathrm{M}$ & $\sim 105 \%$ at $2 \mathrm{M}$ & Wang et al. 2016 \\
\hline Psychrobacter pacificensis & $0-5 \mathrm{M}$ & $143.2 \%$ at $2 \mathrm{M}$ & Wu et al. 2013a \\
\hline Lactobacillus plantarum & $0-25 \%$ & $\sim 250 \%$ at $1 \mathrm{M}$ & $\begin{array}{l}\text { Esteban-Torres et al. } \\
2014\end{array}$ \\
\hline Zunongwangia profunda & $0-4.5 \mathrm{M}$ & $100 \%$ at $0 \mathrm{M}$ & Rahman et al. 2016 \\
\hline Haloarcula marismortui & $0-5 \mathrm{M}$ & $800 \mathrm{mU}$ at $3 \mathrm{M}$ & Rao et al. 2009 \\
\hline Thalassospira sp. & $0-4 \mathrm{M}$ & $283 \%$ at $3 \mathrm{M}$ & De Santi et al. 2016b \\
\hline $\begin{array}{l}\text { Bacillus licheniformis } \\
\text { Metagenome }\end{array}$ & $0-5 \mathrm{M}$ & $588 \%$ at $3.5 \mathrm{M}$ & Zhang et al. 2018 \\
\hline Deep sea sponge & $0-24 \%$ & $100 \%$ at $0 \mathrm{M}$ & Borchert et al. 2017 \\
\hline Marine sponge & $0-4 \mathrm{M}$ & $234 \%$ at $5 \mathrm{M}$ & Selvin et al. 2012 \\
\hline Soil & $0-5 \mathrm{M}$ & $155 \%$ at $1 \mathrm{M}$ & Jayanath et al. 2018 \\
\hline Marine arctic sediment & $0-4 \mathrm{M}$ & $675 \%$ at $3 \mathrm{M}$ & De Santi et al. 2016a \\
\hline Deep-sea shrimp & $\begin{array}{l}0-4 \mathrm{M} \\
0-4 \mathrm{M}\end{array}$ & $\begin{array}{l}\sim 250 \% \text { at } 3.2 \mathrm{M} \\
\sim 250 \% \text { at } 3.6 \mathrm{M}\end{array}$ & Alcaide et al. 2015b \\
\hline Marine water & $0-3.5 \mathrm{M}$ & $\sim 250 \%$ at $3.5 \mathrm{M}$ & Tchigvintsev et al. 2015 \\
\hline Desert basin soil & $0-5 \mathrm{M}$ & $\sim 140 \%$ at $1 \mathrm{M}$ & Wang et al. 2013 \\
\hline Marine water & $0-4 \mathrm{M}$ & $\sim 190 \%$ at $4 \mathrm{M}$ & Fang et al. 2014 \\
\hline
\end{tabular}

a The activity measured without salt $(\mathrm{NaCl}$, if not mentioned) was taken as $100 \%$

\subsubsection{Biotechnological application of LEs}

Due to the catalytic versatility, LEs are widely used in various biotechnological applications (Table 1-5).

\section{Biosensor application}

Qualitative and quantitative determination of lipids and lipid-binding proteins can be used either directly or indirectly in biosensor applications. These biosensors are cheaper and less time-consuming comparing to the chemical methods.

\section{Bioremediation}

LEs are used in the treatment of industrial waste and direct bioremediation of contaminated environments. 


\section{Food and beverage industry}

LEs are used in situ to improve the shelf life and rheological properties and produce aromas and emulgents. In addition, LEs are employed ex situ to produce flavors and improve the taste and textures of food.

\section{Detergent industry}

LEs are one of the most important additives in powder and liquid detergents.

\section{$>$ Paper industry}

Since early 1990, LEs are used to control the pitch in the large-scale paper-making process (Koseki et al. 2009).

\section{Medical and pharmaceutical application}

Products of lipolysis (free fatty acids and diacylglycerols, etc.) can be used for diagnosing diseases, such as development of atherosclerosis, hyperlipidemia, and development of tumors (Verma $\mathrm{N}$ et al. 2012; Coughlan et al. 2015; Jensen et al. 2016).

\section{Cosmetic industry}

LEs are involved in the synthesis of surfactants and aroma products (Guerrand 2017).

\section{Agriculture}

LEs are used to synthesize intermediates for pesticides, insecticides and other agrochemical compounds.

\section{Biodiesel production}

Biodiesel is an environmentally friendly alternative fuel to petroleum-based diesel. Generally, biodiesel is produced by digesting the substrates such as vegetable oils, 
jatropha oil, animal fat, waste edible oil and industrial acid oil with the cocktail of enzymes (Wang et al. 2017).

\section{$>$ Textile industry}

Desizing is an important process in textile industry. Traditional desizing uses acidic or oxidizing agents, which damages the cellulose material in the fabric. In comparison, the enzymatic process is more eco-friendly than the traditional method (www.wipo.int). 
Table 1-5: Potential applications of LEs

\begin{tabular}{|c|c|c|}
\hline Application fields & Examples & Reference \\
\hline Biosensor & $\begin{array}{l}>\text { Erythrocyte cholinesterases for exposure to nerve agents and other chemical weapons } \\
>\quad \text { Acetylcholinesterases for detecting insecticides } \\
>\quad \text { Amperometric biosensors }\end{array}$ & $\begin{array}{l}\text { Hasan et al. 2006; } \\
\text { Camacho et al. } 2013\end{array}$ \\
\hline Bioremediation & $\begin{array}{l}>\quad \text { Breaking down pollutants such as fats generated from generating leather products } \\
>\quad \text { Prokaryotic isoesterase breaking down dimethylpthalate } \\
>\quad \text { Biofilm deposits, oil contaminated soils, lipid-rich wastewater treatment }\end{array}$ & $\begin{array}{l}\text { Cammarota et al. 2013; } \\
\text { Kim 2017; Rigoldi et al. } \\
\text { 2018; Laudadio et al. } 2019\end{array}$ \\
\hline $\begin{array}{l}\text { Food and } \\
\text { beverage industry }\end{array}$ & $\begin{array}{ll}> & \text { Production of flavors in cheese } \\
> & \text { Interesterification of fats and oils } \\
> & \text { Accelerating the ripening of cheese and lipolysis of butter, fats, and cream } \\
> & \text { Removal of fat from meat and fish products } \\
> & \text { Mediating the flavor by releasing different lengths of fatty acids } \\
> & \text { Emulsifiers } \\
> & \text { Production of maltose and lactose like sugar fatty acid esters }\end{array}$ & $\begin{array}{l}\text { Jaeger and Reetz 1998; } \\
\text { Rajendran et al. 2009; } \\
\text { Meng et al. 2015; Coughlan } \\
\text { et al. 2015; De Filippis et } \\
\text { al. 2017; Khan and Sathya } \\
\text { 2017; Kuddus } 2018\end{array}$ \\
\hline Detergent industry & Cocktails of enzymes to enhancing the detergents ability to remove stains & $\begin{array}{l}\text { Romdhane et al. 2010; } \\
\text { Nerurkar et al. 2013; Bora } \\
2014\end{array}$ \\
\hline Paper industry & $\begin{array}{l}>\quad \text { Eco-friendly degradation of lignin in pulp } \\
>\quad \text { Removal of pitch and lipid stains } \\
>\quad \text { Avoiding the formation of sticky materials } \\
>\quad \text { Modification of raw starch }\end{array}$ & $\begin{array}{l}\text { Koseki et al. 2009; Verma } \\
\mathrm{N} \text { et al. 2012; Ramnath et } \\
\text { al. } 2016\end{array}$ \\
\hline $\begin{array}{l}\text { Medical and } \\
\text { pharmaceutical } \\
\text { application }\end{array}$ & 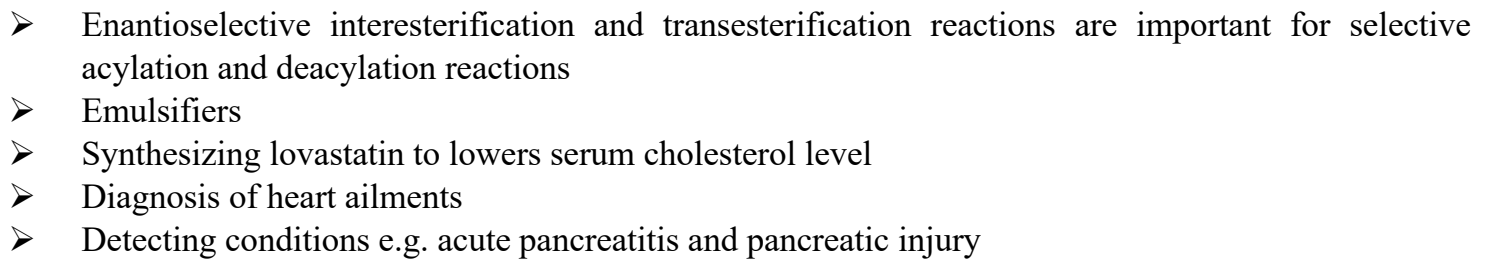 & $\begin{array}{l}\text { Gotor-Fernández et al. } \\
\text { 2006; Gaur and Khare } \\
\text { 2011; Coughlan et al. 2015; } \\
\text { Luan et al. 2016; Dornelas } \\
\text { et al. } 2017\end{array}$ \\
\hline $\begin{array}{l}\text { Cosmetics } \\
\text { industry }\end{array}$ & 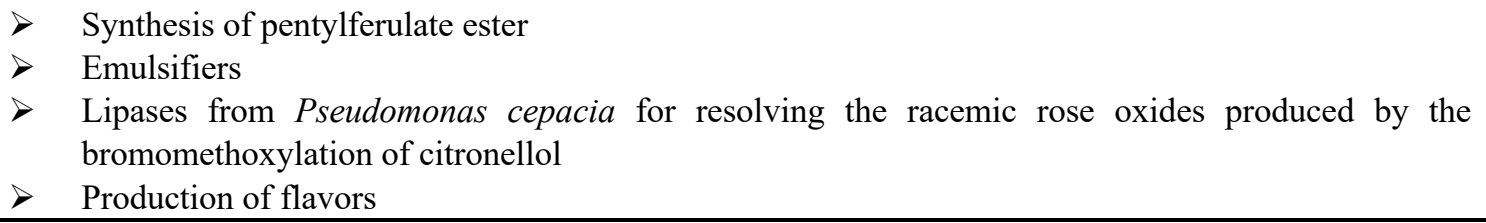 & $\begin{array}{l}\text { Chandel et al. 2011; } \\
\text { Garlapati and Banerjee } \\
\text { 2013; Rigoldi et al. } 2018\end{array}$ \\
\hline
\end{tabular}


$>$ Esters of cinnamic acid, ellagic acid and ferulic acid for fragrance compound

Agriculture

Biodiesel

production

Textile industry

$>$ Removing size lubricants

$>$ Desizing of the denim and other cotton fabrics at the commercial scale

$>$ Improving the ability of polyester fabric to uptake chemical compounds
Horne et al. 2002;

Guerrand 2017

Gaur and Khare 2011;

Ramnath et al. 2016;

Dornelas et al. 2017; Pate

et al. 2018

Andualema and Gessesse

2012; Xiao et al. 2017;

Rigoldi et al. 2018 


\subsection{Thesis overview and research objects}

\subsubsection{Thermophilic composts for extremophilic LEs isolation}

Composting is an aerobic process that accelerates the degradation of organic waste under controlled conditions (Rebollido et al. 2008). The composting process is mainly carried out by a succession of microorganisms that break down complex organic particles into simpler products. According to the temperature change, the composting process can be generally divided into three stages: mesophilic stage, thermophilic stage, and a curing/maturing stage. During the thermophilic phase, heat generated by microbial succession can raise temperatures to above $50{ }^{\circ} \mathrm{C}$ (Dougherty et al. 2012). Correspondingly, compost is a potential source for recovery of extremophilic enzymes. Recently, extremophilic enzymes, such as thermophilic, organic solvent tolerant and alkaliphilic lipolytic enzymes, have been successfully identified from compost metagenomes (Lämmle et al. 2007; Kang et al. 2011; Leis et al. 2015; Ufarté et al. 2015a; Wang et al. 2016a; Lu et al. 2019).

In this study, compost samples were collected at a composting company (Göttingen GmbH, Göttingen, Germany, 51³4' 25.1" N $9^{\circ} 139$ 54' 33.0" E). The sampling piles were the fermentation product of fresh tree branches or household waste. To ensure using mainly thermophilic microorganisms as a source for analysis, compost at the core zone of compost pile was collected.

\subsubsection{Aim of this thesis}

The aim of this study was to explore LEs in compost microbial consortia through function-driven and sequence-based approaches. 
Firstly, to answer the question "who is there", phylogenetic analyses of the two composts microbial communities were performed based on the pyrotag sequence data of 16S rRNA genes and transcripts.

Then, to identify novel lipolytic genes, functional screening of the constructed metagenomic libraries were performed. A sequence-based screening strategy based on profile HMMs were also developed. Moreover, to explore the distribution of LEs across various ecological niches, comparative analysis of performed based on the screening results of different metagenomes.

Finally, to exhibit the potential of LEs for potentially industrial application, the characterization of three LEs from different lipolytic families were carried out. 


\subsection{References}

Abd Rahman RNZR, Ahmad Kamarudin NH, Yunus J, Salleh AB, Basri M (2010) Expression of an organic solvent stable lipase from Staphylococcus epidermidis AT2. Int J Mol Sci 11:3195-3208 . doi: 10.3390/ijms11093195

Abdel-Fattah YR, Gaballa AA (2008) Identification and over-expression of a thermostable lipase from Geobacillus thermoleovorans Toshki in Escherichia coli. Microbiol Res 163:13-20 . doi: 10.1016/j.micres.2006.02.004

Adlercreutz P (2013) Immobilisation and application of lipases in organic media. Chem Soc Rev 42:6406-6436 . doi: 10.1039/c3cs35446f

Ahmed EH, Raghavendra T, Madamwar D (2010) An alkaline lipase from organic solvent tolerant Acinetobacter sp. EH28: Application for ethyl caprylate synthesis. Bioresour Technol 101:3628-3634 . doi: 10.1016/J.BIORTECH.2009.12.107

Ai L, Huang Y, Wang C (2018) Purification and characterization of halophilic lipase of Chromohalobacter sp . from ancient salt well. J Basic Microbiol 58:647-657 . doi: 10.1002/jobm.201800116

Akoh CC, Lee GC, Liaw YC, Huang TH, Shaw JF (2004) GDSL family of serine esterases/lipases. Prog Lipid Res 43:534-552 . doi: 10.1016/j.plipres.2004.09.002

Albers S V., Jonuscheit M, Dinkelaker S, Urich T, Kletzin A, Tampé R, Driessen AJM, Schleper C (2006) Production of recombinant and tagged proteins in the hyperthermophilic archaeon Sulfolobus solfataricus. Appl Environ Microbiol 72:102-111 . doi: 10.1128/AEM.72.1.102111.2006

Alcaide M, Tchigvintsev A, Martínez-Martínez M, Popovic A, Reva ON, Lafraya Á, Bargiela R, Nechitaylo TY, Matesanz R, Cambon-Bonavita M-A, Jebbar M, Yakimov MM, Savchenko A, Golyshina O V, Yakunin AF, Golyshin PN, Ferrer M, MAMBA Consortium (2015) Identification and characterization of carboxyl esterases of gill chamber-associated microbiota in the deep-sea shrimp Rimicaris exoculata by using functional metagenomics. Appl Environ Microbiol 81:2125-2136 . doi: 10.1128/AEM.03387-14 
Alcock BP, Raphenya AR, Lau TTY, Tsang KK, Bouchard M, Edalatmand A, Huynh W, Nguyen AL V., Cheng AA, Liu S, Min SY, Miroshnichenko A, Tran HK, Werfalli RE, Nasir JA, Oloni M, Speicher DJ, Florescu A, Singh B, Faltyn M, Hernandez-Koutoucheva A, Sharma AN, Bordeleau E, Pawlowski AC, Zubyk HL, Dooley D, Griffiths E, Maguire F, Winsor GL, Beiko RG, Brinkman FSL, Hsiao WWL, Domselaar G V., McArthur AG (2020) CARD 2020: Antibiotic resistome surveillance with the comprehensive antibiotic resistance database. Nucleic Acids Res 48:D517-D525 . doi: 10.1093/nar/gkz935

Almeida A, Mitchell AL, Boland M, Forster SC, Gloor GB, Tarkowska A, Lawley TD, Finn RD (2019) A new genomic blueprint of the human gut microbiota. Nature 568:499-504 . doi: 10.1038/s41586-019-0965-1

Andualema B, Gessesse A (2012) Microbial lipases and their industrial applications: Review. Biotechnology 11:100-118 . doi: 10.3923/biotech.2012.100.118

Angelov A, Mientus M, Liebl S, Liebl W (2009) A two-host fosmid system for functional screening of (meta)genomic libraries from extreme thermophiles. Syst Appl Microbiol 32:177-185 . doi: 10.1016/j.syapm.2008.01.003

Armour CR, Nayfach S, Pollard KS, Sharpton TJ (2019) A metagenomic meta-analysis reveals functional signatures of health and disease in the human gut microbiome. mSystems 4:e00332-18 . doi: 10.1128/msystems.00332-18

Arpigny JL, Jaeger K-E (1999) Bacterial lipolytic enzymes: classification and properties. Biochem J 343:177-183 . doi: 10.1042/0264-6021:3430177

Awasthi MK, Ravindran B, Sarsaiya S, Chen H, Wainaina S, Singh E, Liu T, Kumar S, Pandey A, Singh L, Zhang Z (2020) Metagenomics for taxonomy profiling: tools and approaches. Bioengineered 11:356-374 . doi: 10.1080/21655979.2020.1736238

Aylward FO, Burnum KE, Scott JJ, Suen G, Tringe SG, Adams SM, Barry KW, Nicora CD, Piehowski PD, Purvine SO, Starrett GJ, Goodwin LA, Smith RD, Lipton MS, Currie CR (2012) Metagenomic and metaproteomic insights into bacterial communities in leaf-cutter ant fungus gardens. ISME J 6:1688-1701 . doi: 10.1038/ismej.2012.10 
Bailey TL, Boden M, Buske FA, Frith M, Grant CE, Clementi L, Ren J, Li WW, Noble WS (2009) MEME SUITE: tools for motif discovery and searching. Nucleic Acids Res 37:W202-W208 . doi: $10.1093 /$ nar/gkp335

Bassegoda A, Pastor FIJ, Diaz P (2012) Rhodococcus sp. strain CR-53 lipr, the first member of a new bacterial lipase family (Family X) displaying an unusual Y-type oxyanion hole, similar to the Candida antarctica lipase clan. Appl Environ Microbiol 78:1724-1732 . doi: 10.1128/AEM.06332-11

Bayer S, Kunert A, Ballschmiter M, Greiner-Stoeffele T (2010) Indication for a new lipolytic enzyme family: Isolation and characterization of two esterases from a metagenomic library. J Mol Microbiol Biotechnol 18:181-187 . doi: 10.1159/000315459

Bender JM, Bard JDi (2018) Metagenomics in pediatrics: Using a shotgun approach to diagnose infections. Curr Opin Pediatr 30:125-130 . doi: 10.1097/MOP.0000000000000577

Berini F, Casciello C, Marcone GL, Marinelli F (2017) Metagenomics: novel enzymes from nonculturable microbes. FEMS Microbiol Lett 364: . doi: 10.1093/femsle/fnx211

Berlemont R, Spee O, Delsaute M, Lara Y, Schuldes J, Simon C, Power P, Daniel R, Galleni M (2013) Novel organic solvent-tolerant esterase isolated by metagenomics: insights into the lipase/esterase classification. Rev Argent Microbiol 45:3-12

Bharagava RN, Purchase D, Saxena G, Mulla SI (2018) Applications of metagenomics in microbial bioremediation of pollutants: from genomics to environmental cleanup. In: Das $\mathrm{S}$, Dash HR (eds) Microbial Diversity in the Genomic Era. Elsevier Inc., Oxford, UK, pp 459477

Biver S, Vandenbol M (2013) Characterization of three new carboxylic ester hydrolases isolated by functional screening of a forest soil metagenomic library. J Ind Microbiol Biotechnol 40:191-200 . doi: 10.1007/s 10295-012-1217-7

Boeva V (2016) Analysis of genomic sequence motifs for deciphering transcription factor binding and transcriptional regulation in Eukaryotic cells. Front. Genet. 7:24

Bora L (2014) Purification and characterization of highly alkaline lipase from bacillus licheniformis MTCC 2465: And study of its detergent compatibility and applicability. J Surfactants Deterg 17:889-898 . doi: 10.1007/s11743-013-1517-6 
Borchert E, Selvin J, Kiran SG, Jackson SA, O’Gara F, Dobson ADW (2017) A novel cold active esterase from a deep sea sponge Stelletta normani metagenomic library. Front Mar Sci 4:113 . doi: 10.3389/fmars.2017.00287

Bornscheuer UT (2002) Microbial carboxyl esterases: classification, properties and application in biocatalysis. FEMS Microbiol Rev 26:73-81 . doi: 10.1111/j.1574-6976.2002.tb00599.x

Brady SF (2007) Construction of soil environmental DNA cosmid libraries and screening for clones that produce biologically active small molecules. Nat Protoc 2:1297-1305 . doi: 10.1038/nprot.2007.195

Brumlik MJ, Buckley JT (1996) Identification of the catalytic triad of the lipase/acyltransferase from Aeromonas hydrophila. J Bacteriol 178:2060-2064 . doi: 10.1128/jb.178.7.20602064.1996

Cai J, Xie Y, Song B, Wang Y, Zhang Z, Feng Y (2011) Fervidobacterium changbaicum Lip1: Identification, cloning, and characterization of the thermophilic lipase as a new member of bacterial lipase family V. Appl Microbiol Biotechnol 89:1463-1473 . doi: 10.1007/s00253$010-2971-y$

Cammarota MC, Rosa DR, Duarte ICS, Saavedra NK, Varesche MBA, Zaiat M, Freire DMG (2013) The effect of enzymatic pre-hydrolysis of dairy wastewater on the granular and immobilized microbial community in anaerobic bioreactors. Environ Technol 34:417-428 . doi: 10.1080/09593330.2012.698649

Cantarel BL, Coutinho PM, Rancurel C, Bernard T, Lombard V, Henrissat B (2009) The carbohydrate-active enZymes database (CAZy): an expert resource for glycogenomics. Nucleic Acids Res 37:233-238

Castilla A, Panizza P, Rodríguez D, Bonino L, Díaz P, Irazoqui G, Rodríguez Giordano S (2017) A novel thermophilic and halophilic esterase from Janibacter sp. R02, the first member of a new lipase family (Family XVII). Enzyme Microb Technol 98:86-95 . doi: 10.1016/J.ENZMICTEC.2016.12.010

Chandel C, Kumar A, Kanwar SS (2011) Enzymatic synthesis of butyl ferulate by silicaimmobilized lipase in a non-aqueous medium. J Biomater Nanobiotechnol 2:400-408 . doi: 10.4236/jbnb.2011.24049 
Charbonneau DM, Beauregard M (2013) Role of key salt bridges in thermostability of $G$. thermodenitrificans EstGtA2: distinctive patterns within the new bacterial lipolytic enzyme family XV. PLoS One 8:e76675 . doi: 10.1371/journal.pone.0076675

Charoenpanich J, Soongrung T, Chinnasri S, Suebchuea N, Suppoontong M, Thiemsawait S (2018) A novel broad-temperature active and solvent stable esterase from a newly isolated Bacillus aerophilus. Biocatal Agric Biotechnol 13:116-122 . doi: 10.1016/j.bcab.2017.12.004

Chauhan M, Garlapati VK (2013) Production and characterization of a halo-, solvent-, thermotolerant alkaline lipase by Staphylococcus arlettae JPBW-1, isolated from rock salt mine. Appl Biochem Biotechnol 171:1429-1443 . doi: 10.1007/s12010-013-0433-6

Chen IMA, Markowitz VM, Chu K, Palaniappan K, Szeto E, Pillay M, Ratner A, Huang J, Andersen E, Huntemann M, Varghese N, Hadjithomas M, Tennessen K, Nielsen T, Ivanova NN, Kyrpides NC (2017) IMG/M: Integrated genome and metagenome comparative data analysis system. Nucleic Acids Res 45:507-516 . doi: 10.1093/nar/gkw929

Choi JE, Kwon MA, Na HY, Hahm DH, Song JK (2013) Isolation and characterization of a metagenome-derived thermoalkaliphilic esterase with high stability over a broad $\mathrm{pH}$ range. Extremophiles 17:1013-1021 . doi: 10.1007/s00792-013-0583-z

Chow J, Kovacic F, Dall Antonia Y, Krauss U, Fersini F, Schmeisser C, Lauinger B, Bongen P, Pietruszka J, Schmidt M, Menyes I, Bornscheuer UT, Eckstein M, Thum O, Liese A, MuellerDieckmann J, Jaeger KE, Streit WR (2012) The metagenome-derived enzymes LipS and LipT increase the diversity of known lipases. PLoS One 7:e47665 . doi: 10.1371/journal.pone.0047665

Chu X, He H, Guo C, Sun B (2008) Identification of two novel esterases from a marine metagenomic library derived from South China Sea. Appl Microbiol Biotechnol 80:615-625 . doi: 10.1007/s00253-008-1566-3

The UniProt Consortium (2018) UniProt: The universal protein knowledgebase. Nucleic Acids Res 45:D158-D169 . doi: 10.1093/nar/gkw1099

Coquelle N, Talon R, Juers DH, Girard É, Kahn R, Madern D (2010) Gradual adaptive changes of a protein facing high salt concentrations. J Mol Biol 404:493-505 . doi: 10.1016/j.jmb.2010.09.055 
Coughlan LM, Cotter PD, Hill C, Alvarez-Ordóñez A (2015) Biotechnological applications of functional metagenomics in the food and pharmaceutical industries. Front Microbiol 6:672 . doi: $10.3389 /$ fmicb.2015.00672

Craig JW, Chang FY, Kim JH, Obiajulu SC, Brady SF (2010) Expanding small-molecule functional metagenomics through parallel screening of broad-host-range cosmid environmental DNA libraries in diverse Proteobacteria. Appl Environ Microbiol 76:16331641 . doi: 10.1128/AEM.02169-09

Dandavate V, Jinjala J, Keharia H, Madamwar D (2009) Production, partial purification and characterization of organic solvent tolerant lipase from Burkholderia multivorans V2 and its application for ester synthesis. Bioresour Technol 100:3374-3381 . doi: 10.1016/j.biortech.2009.02.011

De Filippis F, Parente E, Ercolini D (2017) Metagenomics insights into food fermentations. Microb Biotechnol 10:91-102 . doi: 10.1111/1751-7915.12421

de Pascale D, Cusano AM, Autore F, Parrilli E, di Prisco G, Marino G, Tutino ML (2008) The cold-active Lip1 lipase from the Antarctic bacterium Pseudoalteromonas haloplanktis TAC125 is a member of a new bacterial lipolytic enzyme family. Extremophiles 12:311-323 . doi: 10.1007/s00792-008-0163-9

De Santi C, Altermark B, Pierechod MM, Ambrosino L, de Pascale D, Willassen N-P (2016a) Characterization of a cold-active and salt tolerant esterase identified by functional screening of Arctic metagenomic libraries. BMC Biochem 17: . doi: 10.1186/s12858-016-0057-x

De Santi C, Leiros HKS, Di Scala A, de Pascale D, Altermark B, Willassen NP (2016b) Biochemical characterization and structural analysis of a new cold-active and salt-tolerant esterase from the marine bacterium Thalassospira sp. Extremophiles 20:323-336 . doi: $10.1007 / \mathrm{s} 00792-016-0824-\mathrm{z}$

Delgado-García M, Valdivia-Urdiales B, Aguilar-González CN, Contreras-Esquivel JC, Rodríguez-Herrera R (2012) Halophilic hydrolases as a new tool for the biotechnological industries. J Sci Food Agric 92:2575-2580 . doi: 10.1002/jsfa.5860 
Devi SG, Fathima AA, Radha S, Arunraj R, Curtis WR, Ramya M (2015) A rapid and economical method for efficient DNA extraction from diverse soils suitable for metagenomic applications. PLoS One 10:e0132441 . doi: 10.1371/journal.pone.0132441

Donato JJ, Moe LA, Converse BJ, Smart KD, Berklein EC, McManus PS, Handelsman J (2010) Metagenomic analysis of apple orchard soil reveals antibiotic resistance genes encoding predicted bifunctional proteins. Appl Environ Microbiol 76:4396-4401 . doi: 10.1128/AEM.01763-09

Dornelas JCM, Figueiredo JEF, De Abreu CS, Lana UGP, Oliveira CA, Marriel IE (2017) Characterization and phylogenetic affiliation of Actinobacteria from tropical soils with potential uses for agro-industrial processes. Genet Mol Res 16:1-16 . doi: $10.4238 /$ gmr 16039703

Dougherty MJ, D’haeseleer P, Hazen TC, Simmons BA, Adams PD, Hadi MZ (2012) Glycoside hydrolases from a targeted compost metagenome, activity-screening and functional characterization. BMC Biotechnol 12:38 . doi: 10.1186/1472-6750-12-38

Drö Ge J, Gregor I, Mchardy AC (2015) Taxator-tk: precise taxonomic assignment of metagenomes by fast approximation of evolutionary neighborhoods. Bioinformatics 31:817824 . doi: 10.1093/bioinformatics/btu745

Dukunde A, Schneider D, Lu M, Brady S, Daniel R (2017) A novel, versatile family IV carboxylesterase exhibits high stability and activity in a broad $\mathrm{pH}$ spectrum. Biotechnol Lett 39:577-587 . doi: 10.1007/s10529-016-2282-1

Dulanto Chiang A, Dekker JP (2020) From the pipeline to the bedside: advances and challenges in clinical metagenomics. J Infect Dis 221:S331-S340 . doi: 10.1093/infdis/jiz151

Ebrahimpour A, Rahman RNZRA, Basri M, Salleh AB (2011) High level expression and characterization of a novel thermostable, organic solvent tolerant, 1,3-regioselective lipase from Geobacillus sp. strain ARM. Bioresour Technol 102:6972-6981 . doi: 10.1016/j.biortech.2011.03.083

Egelkamp R, Zimmermann T, Schneider D, Hertel R, Daniel R (2019) Impact of nitriles on bacterial communities. Front Environ Sci 7:103 . doi: 10.3389/fenvs.2019.00103 
Esteban-Torres M, Santamaría L, de las Rivas B, Muñoz R (2014) Characterisation of a cold-active and salt-tolerant esterase from Lactobacillus plantarum with potential application during cheese ripening. Int Dairy J 39:312-315 . doi: 10.1016/j.idairyj.2014.08.004

Fang Y, Wang S, Liu S, Jiao Y (2015) Discovery a novel organic solvent tolerant esterase from Salinispora arenicola CNP193 through genome mining. Int J Biol Macromol 80:334-340 . doi: 10.1016/j.ijbiomac.2015.06.045

Fang Z, Li J, Wang Q, Fang W, Peng H, Zhang X, Xiao Y (2014) A novel esterase from a marine metagenomic library exhibiting salt tolerance ability. J Microbiol Biotechnol 24:771-780 . doi: $10.4014 / j m b .1311 .11071$

Fawal N, Li Q, Savelli B, Brette M, Passaia G, Fabre M, Mathé C, Dunand C (2013) PeroxiBase: A database for large-scale evolutionary analysis of peroxidases. Nucleic Acids Res 41:441444 . doi: 10.1093/nar/gks1083

Feng Y, Joh Y-G, Ishikawa K, Ishida H, Ando S, Yamagaki T, Nakanishi H, Cao S, Matsui I, Kosugi Y (2000) Thermophilic phospholipase A2 in the cytosolic fraction from the archaeon Pyrococcus horikoshii. J Am Oil Chem Soc 77:1147-1152 . doi: 10.1007/s11746-000-01808

Ferrer M, Martínez-Martínez M, Bargiela R, Streit WR, Golyshina O V, Golyshin PN (2015) Estimating the success of enzyme bioprospecting through metagenomics: current status and future trends. Microb Biotechnol 9:22-34 . doi: 10.1111/1751-7915.12309

Finn RD, Bateman A, Clements J, Coggill P, Eberhardt RY, Eddy SR, Heger A, Hetherington K, Holm L, Mistry J, Sonnhammer ELL, Tate J, Punta M (2014) Pfam: the protein families database. Nucleic Acids Res 42:D222-D230 . doi: 10.1093/nar/gkt1223

Fischer M, Pleiss J (2003) The Lipase Engineering Database: a navigation and analysis tool for protein families. Nucleic Acids Res 31:319-321

Forbes JD, Knox NC, Ronholm J, Pagotto F, Reimer A (2017) Metagenomics: The next cultureindependent game changer. Front Microbiol 8:1069 . doi: 10.3389/fmicb.2017.01069

Fu C, Hu Y, Xie F, Guo H, Ashforth EJ, Polyak SW, Zhu B, Zhang L (2011) Molecular cloning and characterization of a new cold-active esterase from a deep-sea metagenomic library. Appl Microbiol Biotechnol 90:961-970 . doi: 10.1007/s00253-010-3079-0 
Fu J, Leiros HKS, De Pascale D, Johnson KA, Blencke HM, Landfald B (2013) Functional and structural studies of a novel cold-adapted esterase from an Arctic intertidal metagenomic library. Appl Microbiol Biotechnol 97:3965-3978 . doi: 10.1007/s00253-012-4276-9

Gao W, Wu K, Chen L, Fan H, Zhao Z, Gao B, Wang H, Wei D (2016) A novel esterase from a marine mud metagenomic library for biocatalytic synthesis of short-chain flavor esters. Microb Cell Fact 15:41 . doi: 10.1186/s12934-016-0435-5

Garlapati VK, Banerjee R (2013) Solvent-free synthesis of flavour esters through immobilized lipase mediated transesterification. Enzyme Res 2013: . doi: 10.1155/2013/367410

Garrido-Cardenas JA, Manzano-Agugliaro F (2017) The metagenomics worldwide research. Curr Genet 63:819-829 . doi: 10.1007/s00294-017-0693-8

Gaur R, Khare SK (2011) Solvent tolerant Pseudomonads as a source of novel lipases for applications in non-aqueous systems. Biocatal Biotransformation 29:161-171 . doi: $10.3109 / 10242422.2011 .609588$

Gilbert EJ (1993) Pseudomonas lipases: Biochemical properties and molecular cloning. Enzyme Microb. Technol. 15:634-645

Glogauer A, Martini VP, Faoro H, Couto GH, Müller-Santos M, Monteiro RA, Mitchell DA, de Souza EM, Pedrosa FO, Krieger N (2011) Identification and characterization of a new true lipase isolated through metagenomic approach. Microb Cell Fact 10:54 . doi: 10.1186/14752859-10-54

Gotor-Fernández V, Brieva R, Gotor V (2006) Lipases: Useful biocatalysts for the preparation of pharmaceuticals. J Mol Catal B Enzym 40:111-120 . doi: 10.1016/j.molcatb.2006.02.010

Guerrand D (2017) Lipases industrial applications: Focus on food and agroindustries. OCL 24:D403 . doi: 10.1051/ocl/2017031

Guo J, Quensen JF, Sun Y, Wang Q, Brown CT, Cole JR, Tiedje JM (2019) Review, evaluation, and directions for gene-targeted assembly for ecological analyses of metagenomes. Front Genet 10:957 . doi: 10.3389/fgene.2019.00957

Handrick R, Reinhardt S, Focarete ML, Scandola M, Adamus G, Kowalczuk M, Jendrossek D (2001) A new type of thermoalkalophilic hydrolase of Paucimonas lemoignei with high 
specificity for amorphous polyesters of short chain-length hydroxyalkanoic acids. J Biol Chem 276:36215-36224 . doi: 10.1074/jbc.M101106200

Hao T, Xie Z, Wang M, Liu L, Zhang Y, Wang W, Zhang Z, Zhao X, Li P, Guo Z, Gao S, Lou C, Zhang G, Merritt J, Horsman GP, Chen Y (2019) An anaerobic bacterium host system for heterologous expression of natural product biosynthetic gene clusters. Nat Commun 10:3665 . doi: 10.1038/s41467-019-11673-0

Hasan F, Shah AA, Hameed A (2006) Industrial applications of microbial lipases. Enzyme Microb Technol 39:235-251 . doi: 10.1016/J.ENZMICTEC.2005.10.016

Hausmann S, Jaeger K-E (2010) Lipolytic Enzymes from Bacteria. In: Timmis KN (ed) Handbook of Hydrocarbon and Lipid Microbiology. Springer Berlin Heidelberg, Berlin, Heidelberg, pp 1099-1126

Heinz S, Benner C, Spann N, Bertolino E, Lin YC, Laslo P, Cheng JX, Murre C, Singh H, Glass CK (2010) Simple combinations of lineage-determining transcription factors prime cisregulatory elements required for macrophage and B cell identities. Mol Cell 38:576-589 . doi: 10.1016/j.molcel.2010.05.004

Horne I, Harcourt RL, Sutherland TD, Russell RJ, Oakeshott JG (2002) Isolation of a Pseudomonas monteilli strain with a novel phosphotriesterase. FEMS Microbiol Lett 206:5155 . doi: 10.1111/j.1574-6968.2002.tb10985.x

Hotta Y, Ezaki S, Atomi H, Imanaka T (2002) Extremely stable and versatile carboxylesterase from a hyperthermophilic Archaeon. Appl Environ Microbiol 68:3925-3931 . doi: 10.1128/AEM.68.8.3925-3931.2002

Hu Y, Fu C, Huang Y, Yin Y, Cheng G, Lei F, Lu N, Li J, Ashforth EJ, Zhang L, Zhu B (2010) Novel lipolytic genes from the microbial metagenomic library of the South China Sea marine sediment. FEMS Microbiol Ecol 72:228-37 . doi: 10.1111/j.1574-6941.2010.00851.x

Huerta-Cepas J, Szklarczyk D, Heller D, Hernández-Plaza A, Forslund SK, Cook H, Mende DR, Letunic I, Rattei T, Jensen LJ, Mering C von, Bork P (2019) eggNOG 5.0: a hierarchical, functionally and phylogenetically annotated orthology resource based on 5090 organisms and 2502 viruses. Nucleic Acids Res 47:D309-D314

Hugenholtz P, Tyson GW (2008) Microbiology: Metagenomics. Nature 455:481-483 
Hun CJ, Rahman RNZA, Salleh AB, Basri M (2003) A newly isolated organic solvent tolerant Bacillus sphaericus 205y producing organic solvent-stable lipase. Biochem Eng J 15:147151 . doi: 10.1016/S1369-703X(02)00185-7

Huson DH, Beier S, Flade I, Górska A, El-Hadidi M, Mitra S, Ruscheweyh H-J, Tappu R (2016) MEGAN community edition - interactive exploration and analysis of large-scale microbiome sequencing data. PLOS Comput Biol 12:e1004957 . doi: 10.1371/journal.pcbi.1004957

Ikeda M, Clark DS (1998) Molecular cloning of extremely thermostable esterase gene from hyperthermophilic archaeon Pyrococcus furiosus in Escherichia coli. Biotechnol Bioeng 57:624-629 . doi: 10.1002/(SICI)1097-0290(19980305)57:5<624::AID-BIT15>3.0.CO;2-B

Jaeger K-E, Dijkstra BW, Reetz MT (1999) Bacterial biocatalysts: Molecular biology, threedimensional structures, and biotechnological applications of lipases. Annu Rev Microbiol 53:315-351 . doi: 10.1146/annurev.micro.53.1.315

Jaeger KE, Eggert T (2002) Lipases for biotechnology. Curr Opin Biotechnol 13:390-397 . doi: 10.1016/S0958-1669(02)00341-5

Jaeger KE, Reetz MT (1998) Microbial lipases form versatile tools for biotechnology. Trends Biotechnol 16:396-403 . doi: 10.1016/S0167-7799(98)01195-0

Jang E, Ryu BH, Kim TD (2014) Identification, characterization, and immobilization of an organic solvent-stable alkaline hydrolase (PA27) from Pseudomonas aeruginosa MH38. Molecules 19:14396-14405 . doi: 10.3390/molecules 190914396

Jayanath G, Mohandas SP, Kachiprath B, Solomon S, Sajeevan TP, Bright Singh IS, Philip R (2018) A novel solvent tolerant esterase of GDSGG motif subfamily from solar saltern through metagenomic approach: Recombinant expression and characterization. Int J Biol Macromol 119:393-401 . doi: 10.1016/j.ijbiomac.2018.06.057

Jensen MB V., Horsfall LE, Wardrope C, Togneri PD, Marles-Wright J, Rosser SJ (2016) Characterisation of a new family of carboxyl esterases with an OsmC domain. PLoS One 11:e0166128 . doi: 10.1371/journal.pone.0166128

Jeon JH, Kim JT, Kim YJ, Kim HK, Lee HS, Kang SG, Kim SJ, Lee JH (2009) Cloning and characterization of a new cold-active lipase from a deep-sea sediment metagenome. Appl Microbiol Biotechnol 81:865-874 . doi: 10.1007/s00253-008-1656-2 
Jeon JH, Kim S-J, Lee HS, Cha S-S, Lee JH, Yoon S-H, Koo B-S, Lee C-M, Choi SH, Lee SH, Kang SG, Lee J-H (2011) Novel metagenome-derived carboxylesterase that hydrolyzes $\beta$ lactam antibiotics. Appl Environ Microbiol 77:7830-6 . doi: 10.1128/AEM.05363-11

Jiang H, Zhang S, Gao H, Hu N (2016) Characterization of a cold-active esterase from Serratia sp. and improvement of thermostability by directed evolution. BMC Biotechnol 16: . doi: $10.1186 / \mathrm{s} 12896-016-0235-3$

Jiang X, Huo Y, Cheng H, Zhang X, Zhu X, Wu M (2012) Cloning, expression and characterization of a halotolerant esterase from a marine bacterium Pelagibacterium halotolerans B2T. Extremophiles 16:427-435 . doi: 10.1007/s00792-012-0442-3

Jünemann S, Kleinbölting N, Jaenicke S, Henke C, Hassa J, Nelkner J, Stolze Y, Albaum SP, Schlüter A, Goesmann A, Sczyrba A, Stoye J (2017) Bioinformatics for NGS-based metagenomics and the application to biogas research. J Biotechnol 261:10-23 . doi: 10.1016/j.jbiotec.2017.08.012

Kamarudin NHA, Rahman RNZRA, Ali MSM, Leow TC, Basri M, Salleh AB (2014) A new coldadapted, organic solvent stable lipase from mesophilic Staphylococcus epidermidis AT2. Protein J 33:296-307 . doi: 10.1007/s10930-014-9560-3

Kanehisa M, Sato Y, Kawashima M, Furumichi M, Tanabe M (2016) KEGG as a reference resource for gene and protein annotation. Nucleic Acids Res 44:D457-D462 . doi: 10.1093/nar/gkv1070

Kang CH, Oh KH, Lee MH, Oh TK, Kim BH, Yoon JH (2011) A novel family VII esterase with industrial potential from compost metagenomic library. Microb Cell Fact 10:41 . doi: $10.1186 / 1475-2859-10-41$

Kang HY, Kim JF, Kim MH, Park SH, Oh TK, Hur CG (2006) MELDB: A database for microbial esterases and lipases. FEBS Lett 580:2736-2740 . doi: 10.1016/j.febslet.2006.04.034

Kang LJ, Meng ZT, Hu C, Zhang Y, Guo HL, Li Q, Li M (2017) Screening, purification, and characterization of a novel organic solvent-tolerant esterase, Lip2, from Monascus purpureus strain M7. Extremophiles 21:345-355 . doi: 10.1007/s00792-016-0907-x 
Karadzic I, Masui A, Zivkovic LI, Fujiwara N (2006) Purification and characterization of an alkaline lipase from Pseudomonas aeruginosa isolated from putrid mineral cutting oil as component of metalworking fluid. J Biosci Bioeng 102:82-89 . doi: 10.1263/jbb.102.82

Karan R, Capes MD, DasSarma S (2012) Function and biotechnology of extremophilic enzymes in low water activity. Aquat Biosyst 8:4 . doi: 10.1186/2046-9063-8-4

Keegan KP, Glass EM, Meyer F (2016) MG-RAST, a metagenomics service for analysis of microbial community structure and function. In: Methods in Molecular Biology. Humana Press Inc., pp 207-233

Kent WJ (2002) BLAT---The BLAST-Like Alignment Tool. Genome Res 12:656-664 . doi: $10.1101 /$ gr.229202

Khan FI, Lan D, Durrani R, Huan W, Zhao Z, Wang Y (2017) The lid domain in lipases: structural and functional determinant of enzymatic properties. Front Bioeng Biotechnol 5:16 . doi: 10.3389/fbioe. 2017.00016

Khan M, Sathya TA (2018) Extremozymes from metagenome: Potential applications in food processing. Crit Rev Food Sci Nutr 58:2017-2025 . doi: 10.1080/10408398.2017.1296408

Kim MK, Kang TH, Kim J, Kim H, Yun HD (2012) Cloning and identification of a new group esterase (Est5S) from noncultured rumen bacterium. J Microbiol Biotechnol 22:1044-1053

Kim S, Joo S, Yoon S, Kim S, Moon J, Ryu Y, Kim KK, Kim TD (2009) Purification, crystallization and preliminary crystallographic analysis of Est-Y29: A novel oligomeric $\beta$ lactamase. Acta Crystallogr Sect F Struct Biol Cryst Commun 65:310-312 . doi: $10.1107 / \mathrm{S} 1744309109005442$

Kim TD (2017) Bacterial hormone-sensitive lipases (bHSLs): emerging enzymes for biotechnological applications. J Microbiol Biotechnol 27:1907-1915 . doi: 10.4014/jmb.1708.08004

Knudsen M, Wiuf C (2010) The CATH database. Hum Genomics 4:207-212 . doi: 10.1186/14797364-4-3-207 
Koseki T, Fushinobu S, Ardiansyah, Shirakawa H, Komai M (2009) Occurrence, properties, and applications of feruloyl esterases. Appl Microbiol Biotechnol 84:803-810 . doi: $10.1007 / \mathrm{s} 00253-009-2148-8$

Kovacic F, Babic N, Krauss U, Jaeger K (2019) Classification of lipolytic enzymes from bacteria. In: Rojo F (ed) Aerobic Utilization of Hydrocarbons, Oils and Lipids. Handbook of Hydrocarbon and Lipid Microbiology. Springer, Cham, pp 1-35

Kuddus M (2018) Cold-active enzymes in food biotechnology: An updated mini review. J Appl Biol Biotechnol 6:58-63 . doi: 10.7324/JABB.2018.60310

Kumar A, Dhar K, Singh Kanwar S, Arora PK (2016) Lipase catalysis in organic solvents: advantages and applications. Biol Proced Online 18:2 . doi: 10.1186/s12575-016-0033-2

Kumar S, Krishnani KK, Bhushan B, Brahmane MP (2015) Metagenomics: Retrospect and prospects in high throughput age. Biotechnol Res Int 2015:1-13 . doi: 10.1155/2015/121735

Lam KN, Cheng J, Engel K, Neufeld JD, Charles TC (2015) Current and future resources for functional metagenomics. Front Microbiol 6:1196 . doi: 10.3389/fmicb.2015.01196

Lämmle K, Zipper H, Breuer M, Hauer B, Buta C, Brunner H, Rupp S (2007) Identification of novel enzymes with different hydrolytic activities by metagenome expression cloning. $\mathrm{J}$ Biotechnol 127:575-592 . doi: 10.1016/j.jbiotec.2006.07.036

Lanyi JK (1974) Salt-dependent properties of proteins from extremely halophilic bacteria. Bacteriol Rev 38:272-290

Laudadio I, Fulci V, Stronati L, Carissimi C (2019) Next-generation metagenomics: methodological challenges and opportunities. Omi A J Integr Biol 23:327-333 . doi: 10.1089/omi.2019.0073

Lee CW, Kwon S, Park S-H, Kim B-Y, Yoo W, Ryu BH, Kim H-W, Shin SC, Kim S, Park H, Kim TD, Lee JH (2017) Crystal structure and functional characterization of an esterase (EaEST) from Exiguobacterium antarcticum. PLoS One 12:e0169540 . doi: 10.1371/journal.pone.0169540 
Lee D-W, Koh Y-S, Kim K-J, Kim B-C, Choi H-J, Kim D-S, Suhartono MT, Pyun Y-R (1999) Isolation and characterization of a thermophilic lipase from Bacillus thermoleovorans ID-1. FEMS Microbiol Lett 179:393-400 . doi: 10.1111/j.1574-6968.1999.tb08754.x

Lee MH, Hong KS, Malhotra S, Park J-H, Hwang EC, Choi HK, Kim YS, Tao W, Lee S-W (2010) A new esterase EstD2 isolated from plant rhizosphere soil metagenome. Appl Microbiol Biotechnol 88:1125-1134 . doi: 10.1007/s00253-010-2729-6

Lee MH, Lee CH, Oh TK, Song JK, Yoon JH (2006) Isolation and characterization of a novel lipase from a metagenomic library of tidal flat sediments: Evidence for a new family of bacterial lipases. Appl Environ Microbiol 72:7406-7409 . doi: 10.1128/AEM.01157-06

Lei J, Miao Y, Lan Y, Han X, Liu H, Gan Y, Niu L, Wang Y, Zheng Z (2018) A novel complementation assay for quick and specific screen of genes encoding glycerol-3-phosphate acyltransferases. Front Plant Sci 9:353 . doi: 10.3389/fpls.2018.00353

Leis B, Angelov A, Mientus M, Li H, Pham VTT, Lauinger B, Bongen P, Pietruszka J, Gonçalves LG, Santos H, Liebl W (2015) Identification of novel esterase-active enzymes from hot environments by use of the host bacterium Thermus thermophilus. Front Microbiol 6:275 . doi: $10.3389 /$ fmicb.2015.00275

Lenfant N, Hotelier T, Velluet E, Bourne Y, Marchot P, Chatonnet A (2013) ESTHER, the database of the $\alpha / \beta$-hydrolase fold superfamily of proteins: tools to explore diversity of functions. Nucleic Acids Res 41:423-429 . doi: 10.1093/nar/gks1154

Levisson M, Van Der Oost J, Kengen SWM (2007) Characterization and structural modeling of a new type of thermostable esterase from Thermotoga maritima. FEBS J 274:2832-2842 . doi: 10.1111/j.1742-4658.2007.05817.x

Li D, Liu CM, Luo R, Sadakane K, Lam TW (2015) MEGAHIT: An ultra-fast single-node solution for large and complex metagenomics assembly via succinct de Bruijn graph. Bioinformatics 31:1674-1676 . doi: 10.1093/bioinformatics/btv033

Li H, Han X, Qiu W, Xu D, Wang Y, Yu M, Hu X, Zhuo R (2019) Identification and expression analysis of the GDSL esterase/lipase family genes, and the characterization of SaGLIP8 in Sedum alfredii Hance under cadmium stress. PeerJ 7:e6741 . doi: 10.7717/peerj.6741 
Li LL, McCorkle SR, Monchy S, Taghavi S, van der Lelie D (2009) Bioprospecting metagenomes: Glycosyl hydrolases for converting biomass. Biotechnol Biofuels 2:1-11 . doi: 10.1186/1754$6834-2-10$

Li M, Yang LR, Xu G, Wu JP (2016) Cloning and characterization of a novel lipase from Stenotrophomonas maltophilia GS11: The first member of a new bacterial lipase family XVI. J Biotechnol 228:30-36 . doi: 10.1016/j.jbiotec.2016.04.034

Li M, Yang LR, Xu G, Wu JP (2013) Screening, purification and characterization of a novel coldactive and organic solvent-tolerant lipase from Stenotrophomonas maltophilia CGMCC 4254. Bioresour Technol 148:114-120 . doi: 10.1016/j.biortech.2013.08.101

Li PY, Ji P, Li CY, Zhang Y, Wang GL, Zhang XY, Xie B Bin, Qin QL, Chen XL, Zhou BC, Zhang YZ (2014) Structural basis for dimerization and catalysis of a novel sterase from the GTSAG motif subfamily of the bacterial hormone-sensitive lipase family. J Biol Chem 289:19031-19041 . doi: 10.1074/jbc.M114.574913

Li PY, Yao QQ, Wang P, Zhang Y, Li Y, Zhang YQ, Hao J, Zhou BC, Chen XL, Shi M, Zhang YZ, Zhang XY (2017) A novel subfamily esterase with a homoserine transacetylase-like fold but no transferase activity. Appl Environ Microbiol 83:e00131-17 . doi: 10.1128/AEM.00131-17

Lima VMG, Krieger N, Mitchell DA, Fontana JD (2004) Activity and stability of a crude lipase from Penicillium aurantiogriseum in aqueous media and organic solvents. Biochem Eng J 18:65-71 . doi: 10.1016/S1369-703X(03)00165-7

Liu K, Wang J, Bu D, Zhao S, McSweeney C, Yu P, Li D (2009) Isolation and biochemical characterization of two lipases from a metagenomic library of China Holstein cow rumen. Biochem Biophys Res Commun 385:605-611 . doi: 10.1016/j.bbrc.2009.05.110

López-López O, Knapik K, Cerdán ME, González-Siso MI (2015) Metagenomics of an alkaline hot spring in Galicia (Spain): Microbial diversity analysis and screening for novel lipolytic enzymes. Front Microbiol 6:1291 . doi: 10.3389/fmicb.2015.01291

Lu M, Dukunde A, Daniel R (2019) Biochemical profiles of two thermostable and organic solventtolerant esterases derived from a compost metagenome. Appl Microbiol Biotechnol 103:3421-3437 . doi: 10.1007/s00253-019-09695-1 
Luan Z-J, Yu H-L, Ma B-D, Qi Y-K, Chen Q, Xu J-H (2016) Dramatically improved performance of an esterase for cilastatin synthesis by cap domain engineering. Ind Eng Chem Res 55:12167-12172 . doi: 10.1021/acs.iecr.6b02440

Madhavan A, Sindhu R, Parameswaran B, Sukumaran RK, Pandey A (2017) Metagenome analysis: a powerful tool for enzyme bioprospecting. Appl Biochem Biotechnol 183:636-651 . doi: $10.1007 / \mathrm{s} 12010-017-2568-3$

Mandrich L, Menchise V, Alterio V, De Simone G, Pedone C, Rossi M, Manco G (2008) Functional and structural features of the oxyanion hole in a thermophilic esterase from Alicyclobacillus acidocaldarius. Proteins Struct Funct Genet 71:1721-1731 . doi: $10.1002 /$ prot. 21877

Mandrich L, Merone L, Pezzullo M, Cipolla L, Nicotra F, Rossi M, Manco G (2005) Role of the $\mathrm{N}$ terminus in enzyme activity, stability and specificity in thermophilic esterases belonging to the HSL family. J Mol Biol 345:501-512 . doi: 10.1016/j.jmb.2004.10.035

Maruthamuthu M, Jiménez DJ, Stevens P, van Elsas JD (2016) A multi-substrate approach for functional metagenomics-based screening for (hemi)cellulases in two wheat straw-degrading microbial consortia unveils novel thermoalkaliphilic enzymes. BMC Genomics 17:1-16 . doi: 10.1186/s12864-016-2404-0

McArthur AG, Waglechner N, Nizam F, Yan A, Azad MA, Baylay AJ, Bhullar K, Canova MJ, De Pascale G, Ejim L, Kalan L, King AM, Koteva K, Morar M, Mulvey MR, O’Brien JS, Pawlowski AC, Piddock LJV, Spanogiannopoulos P, Sutherland AD, Tang I, Taylor PL, Thaker M, Wang W, Yan M, Yu T, Wright GD (2013) The comprehensive antibiotic resistance database. Antimicrob Agents Chemother 57:3348-3357 . doi: 10.1128/AAC.00419-13

McMahon MD, Guan C, Handelsman J, Thomas MG (2012) Metagenomic analysis of Streptomyces lividans reveals host-dependent functional expression. Appl Environ Microbiol 78:3622-3629 . doi: 10.1128/AEM.00044-12

Meng Q, Yang W, Men M, Bello A, Xu X, Xu B, Deng L, Jiang X, Sheng S, Wu X, Han Y, Zhu H (2019) Microbial community succession and response to environmental variables during 
cow manure and corn straw composting. Front Microbiol 10:529 . doi: $10.3389 /$ fmicb.2019.00529

Meng Y, Li S, Yuan H, Zou D, Liu Y, Zhu B, Li X (2015) Effect of lipase addition on hydrolysis and biomethane production of Chinese food waste. Bioresour Technol 179:452-459 . doi: 10.1016/j.biortech.2014.12.015

Miller DN, Bryant JE, Madsen EL, Ghiorse WC (1999) Evaluation and optimization of DNA extraction and purification procedures for soil and sediment samples. Appl Environ Microbiol 65:4715-4724 . doi: 10.1128/aem.65.11.4715-4724.1999

Minegishi H, Shimane Y, Echigo A, Ohta Y, Hatada Y, Kamekura M, Maruyama T, Usami R (2013) Thermophilic and halophilic $\beta$-agarase from a halophilic archaeon Halococcus sp. 197A. Extremophiles 17:931-939 . doi: 10.1007/s00792-013-0575-z

Misawa E, Chan Kwo Chion CKC, Archer I V., Woodland MP, Zhou N-Y, Carter SF, Widdowson DA, Leak DJ (1998) Characterisation of a catabolic epoxide hydrolase from a Corynebacterium sp. Eur J Biochem 253:173-183 . doi: 10.1046/j.14321327.1998.2530173.x

Mohamed YM, Ghazy MA, Sayed A, Ouf A, El-Dorry H, Siam R (2013) Isolation and characterization of a heavy metal-resistant, thermophilic esterase from a Red Sea Brine Pool. Sci Rep 3:3358 . doi: 10.1038/srep03358

Mølgaard A, Kauppinen S, Larsen S (2000) Rhamnogalacturonan acetylesterase elucidates the structure and function of a new family of hydrolases. Structure 8:373-383 . doi: 10.1016/S0969-2126(00)00118-0

Montoro-García S, Martínez-Martínez I, Navarro-Fernández J, Takami H, García-Carmona F, Sánchez-Ferrer Á (2009) Characterization of a novel thermostable carboxylesterase from Geobacillus kaustophilus HTA426 shows the existence of a new carboxylesterase family. J Bacteriol 191:3076-3085 . doi: 10.1128/JB.01060-08

Moreno-Indias I, Tinahones FJ (2020) Metagenomics. Princ Nutr Nutr 81-87 . doi: 10.1016/b9780-12-804572-5.00011-2 
Munawar N, Engel PC (2013) Halophilic enzymes: Characteristics, structural adaptation and potential applications for biocatalysis. Curr Biotechnol 2:334-344 . doi: $10.2174 / 18722083113076660033$

Musa H, Kasim FH, Gunny AAN, Gopinath SCB, Ahmad MA (2018) Biosecretion of higher halophilic lipase by a novel Bacillus amyloliquefaciens AIKK2 using agro-waste as supporting substrate. Process Biochem 72:55-62 . doi: 10.1016/j.procbio.2018.06.022

Nacke H, Will C, Herzog S, Nowka B, Engelhaupt M, Daniel R (2011) Identification of novel lipolytic genes and gene families by screening of metagenomic libraries derived from soil samples of the German Biodiversity Exploratories. FEMS Microbiol Ecol 78:188-201 . doi: 10.1111/j.1574-6941.2011.01088.x

Nagamine S, Liu C, Nishishita J, Kozaki T, Sogahata K, Sato Y, Minami A, Ozaki T, SchmidtDannert C, Maruyama J ichi, Oikawa H (2019) Ascomycete Aspergillus oryzae is an efficient expression host for production of basidiomycete terpenes by using genomic DNA sequences. Appl Environ Microbiol 85:e00409-19 . doi: 10.1128/AEM.00409-19

Namiki T, Hachiya T, Tanaka H, Sakakibara Y (2012) MetaVelvet: An extension of Velvet assembler to de novo metagenome assembly from short sequence reads. Nucleic Acids Res 40:e155 . doi: 10.1093/nar/gks678

Nerurkar M, Joshi M, Pariti S, Adivarekar R (2013) Application of lipase from marine bacteria Bacillus sonorensis as an additive in detergent formulation. J Surfactants Deterg 16:435-443 . doi: 10.1007/s11743-012-1434-0

Neves Petersen MT, Fojan P, Petersen SB (2001) How do lipases and esterases work: The electrostatic contribution. J. Biotechnol. 85:115-147

Ngara TR, Zhang H (2018) Recent advances in function-based metagenomic screening. Genomics, Proteomics Bioinforma. 16:405-415

Nurk S, Meleshko D, Korobeynikov A, Pevzner PA (2017) MetaSPAdes: A new versatile metagenomic assembler. Genome Res 27:824-834 . doi: 10.1101/gr.213959.116

Ollis DL, Cheah E, Cygler M, Dijkstra B, Frolow F, Franken SM, Harel M, Remington SJ, Silman I, Schrag J, Sussman JL, Verschueren KHG, Goldman A (1992) The $\alpha / \beta$ hydrolase fold. Protein Eng Des Sel 5:197-211 . doi: 10.1093/protein/5.3.197 
Parapouli M, Foukis A, Stergiou P-Y, Koukouritaki M, Magklaras P, Gkini OA, Papamichael EM, Afendra A-S, Hatziloukas E (2018) Molecular, biochemical and kinetic analysis of a novel, thermostable lipase (LipSm) from Stenotrophomonas maltophilia Psi-1, the first member of a new bacterial lipase family (XVIII). J Biol Res 25:4 . doi: 10.1186/s40709-018-0074-6

Park SY, Kim JT, Kang SG, Woo JH, Lee JH, Choi HT, Kim SJ (2007) A new esterase showing similarity to putative dienelactone hydrolase from a strict marine bacterium, Vibrio sp. GMD509. Appl Microbiol Biotechnol 77:107-115 . doi: 10.1007/s00253-007-1134-2

Patel U, Chandpura J, Chauhan K, Gupte S (2018) Screening and isolation of an organic solvent tolerant lipase producing bacteria from various oil contaminated sites. Indian J Appl Microbiol 21:22-36

Pehrsson EC, Tsukayama P, Patel S, Mejía-Bautista M, Sosa-Soto G, Navarrete KM, Calderon M, Cabrera L, Hoyos-Arango W, Bertoli MT, Berg DE, Gilman RH, Dantas G (2016) Interconnected microbiomes and resistomes in low-income human habitats. Nature 533:212216 . doi: $10.1038 /$ nature 17672

Peng Y, Leung HCM, Yiu SM, Chin FYL (2011) Meta-IDBA: A de Novo assembler for metagenomic data. Bioinformatics 27:94-101 . doi: 10.1093/bioinformatics/btr216

Peng Y, Leung HCM, Yiu SM, Chin FYL (2012) IDBA-UD: A de novo assembler for single-cell and metagenomic sequencing data with highly uneven depth. Bioinformatics 28:1420-1428 . doi: 10.1093/bioinformatics/bts174

Pereira MR, Mercaldi GF, Maester TC, Balan A, de Macedo Lemos EG (2015) Est16, a new esterase isolated from a metagenomic library of a microbial consortium specializing in diesel oil degradation. PLoS One 10:e0133723 . doi: 10.1371/journal.pone.0133723

Pratama R, Schneider D, Böer T, Daniel R (2019) First insights into bacterial gastrointestinal tract communities of the Eurasian beaver (Castor fiber). Front Microbiol 10:1646 . doi: 10.3389/fmicb.2019.01646

Qin Q-L, Zhao D-L, Wang J, Chen X-L, Dang H-Y, Li T-G, Zhang Y-Z, Gao P-J (2007) Wangia profunda gen. nov., sp. nov., a novel marine bacterium of the family Flavobacteriaceae isolated from southern Okinawa Trough deep-sea sediment. FEMS Microbiol Lett 271:5358 . doi: 10.1111/j.1574-6968.2007.00694.x 
Rabausch U, Juergensen J, Ilmberger N, Böhnke S, Fischer S, Schubach B, Schulte M, Streit W V. (2013) Functional screening of metagenome and genome libraries for detection of novel flavonoid-modifying enzymes. Appl Environ Microbiol 79:4551-4563 . doi: 10.1128/AEM.01077-13

Rahman MA, Culsum U, Tang W, Zhang SW, Wu G, Liu Z (2016) Characterization of a novel cold active and salt tolerant esterase from Zunongwangia profunda. Enzyme Microb Technol 85:1-11 . doi: 10.1016/j.enzmictec.2015.12.013

Rajendran A, Palanisamy A, Thangavelu V (2009) Lipase catalyzed ester synthesis for food processing industries. Brazilian Arch Biol Technol 52:207-219 . doi: 10.1590/S151689132009000100026

Ramnath L, Sithole B, Govinden R (2016) Classification of lipolytic enzymes and their biotechnological applications in the pulping industry. Can J Microbiol 63:179-192 . doi: 10.1139/cjm-2016-0447

Rao L, Xue Y, Zhou C, Tao J, Li G, Lu JR, Ma Y (2011) A thermostable esterase from Thermoanaerobacter tengcongensis opening up a new family of bacterial lipolytic enzymes. Biochim Biophys Acta - Proteins Proteomics 1814:1695-1702 . doi: 10.1016/j.bbapap.2011.08.013

Rao L, Zhao X, Pan F, Li Y, Xue Y, Ma Y, Lu JR (2009) Solution behavior and activity of a halophilic esterase under high salt concentration. PLoS One 4:e6980 . doi: 10.1371/journal.pone.0006980

Rathi P, Bradoo S, Saxena RK, Gupta R (2000) A hyper-thermostable, alkaline lipase from Pseudomonas sp. with the property of thermal activation. Biotechnol Lett 22:495-498 . doi: 10.1023/A:1005604617440

Rebollido R, Martínez J, Aguilera Y, Melchor K, Koerner I, Stegmann R (2008) Microbial populations during composting process of organic fraction of municipal solid waste. Appl Ecol Environ Res 6:61-67

Reyes-Duarte D, Ferrer M, García-Arellano H (2012) Functional-based screening methods for lipases, esterases, and phospholipases in metagenomic libraries. Methods Mol Biol 861:101113 . doi: 10.1007/978-1-61779-600-5_6 
Rhee J, Ahn D, Kim Y, Oh J (2005) New thermophilic and thermostable esterase with sequence similarity to the hormone-sensitive lipase family, cloned from a metagenomic library. Appl Environ Microbiol 71:817-825 . doi: 10.1128/AEM.71.2.817

Rigoldi F, Donini S, Redaelli A, Parisini E, Gautieri A (2018) Review: Engineering of thermostable enzymes for industrial applications. APL Bioeng 2:011501 . doi: $10.1063 / 1.4997367$

Rodríguez MC, Loaces I, Amarelle V, Senatore D, Iriarte A, Fabiano E, Noya F (2015) Est10: A novel alkaline esterase isolated from bovine rumen belonging to the new family XV of lipolytic enzymes. PLoS One 10:e0126651 . doi: 10.1371/journal.pone.0126651

Romdhane IB-B, Fendri A, Gargouri Y, Gargouri A, Belghith H (2010) A novel thermoactive and alkaline lipase from Talaromyces thermophilus fungus for use in laundry detergents. Biochem Eng J 53:112-120 . doi: 10.1016/J.BEJ.2010.10.002

Rosenstein R, Götz F (2000) Staphylococcal lipases: Biochemical and molecular characterization. Biochimie 82:1005-1014

Sabri S, Rahman RNZRA, Leow TC, Basri M, Salleh AB (2009) Secretory expression and characterization of a highly Ca2+-activated thermostable L2 lipase. Protein Expr Purif 68:161-166 . doi: 10.1016/j.pep.2009.08.002

Salihu A, Alam MZ (2015) Solvent tolerant lipases: A review. Process Biochem 50:86-96

Samoylova Y V., Sorokina KN, Piligaev A V., Parmon VN (2019) Application of bacterial thermostable lipolytic enzymes in the modern biotechnological processes: A review. Catal Ind 11:168-178 . doi: 10.1134/S2070050419020107

Samoylova Y V., Sorokina KN, Romanenko M V., Parmon VN (2018) Cloning, expression and characterization of the esterase estUT1 from Ureibacillus thermosphaericus which belongs to a new lipase family XVIII. Extremophiles 22:271-285 . doi: 10.1007/s00792-018-0996-9

Satyanarayana SDV, Krishna MSR, Kumar PP (2017) Optimization of high-yielding protocol for DNA extraction from the forest rhizosphere microbes. 3 Biotech 7:91 . doi: 10.1007/s13205017-0737-2 
Schloss PD, Hay AG, Wilson DB, Walker LP (2003) Tracking temporal changes of bacterial community fingerprints during the initial stages of composting. FEMS Microbiol Ecol 46:19. doi: 10.1016/S0168-6496(03)00153-3

Schulz G, Schneider D, Brinkmann N, Edy N, Daniel R, Polle A, Scheu S, Krashevska V (2019) Changes in trophic groups of protists with conversion of rainforest into rubber and oil palm plantations. Front Microbiol 10:240 . doi: 10.3389/fmicb.2019.00240

Selvin J, Kennedy J, Lejon DPH, Kiran GS, Dobson ADW (2012) Isolation identification and biochemical characterization of a novel halo-tolerant lipase from the metagenome of the marine sponge Haliclona simulans. Microb Cell Fact 11:72 . doi: 10.1186/1475-2859-11-72

Seo S, Lee YS, Yoon SH, Kim SJ, Cho JY, Hahn BS, Koo BS, Lee CM (2014) Characterization of a novel cold-active esterase isolated from swamp sediment metagenome. World $\mathrm{J}$ Microbiol Biotechnol 30:879-886 . doi: 10.1007/s11274-013-1496-9

Seshadri R, Kravitz SA, Smarr L, Gilna P, Frazier M (2007) CAMERA: A community resource for metagenomics. PLoS Biol 5:e75 . doi: 10.1371/journal.pbio.0050075

Shao H, Xu L, Yan Y (2013) Isolation and characterization of a thermostable esterase from a metagenomic library. J Ind Microbiol Biotechnol 40:1211-1222 . doi: 10.1007/s10295-013$1317-\mathrm{z}$

Simon C, Daniel R (2009) Achievements and new knowledge unraveled by metagenomic approaches. Appl Microbiol Biotechnol 85:265-276 . doi: 10.1007/s00253-009-2233-z

Simon C, Daniel R (2011) Metagenomic analyses: Past and future trends. Appl Environ Microbiol 77:1153-1161 . doi: 10.1128/AEM.02345-10

Tasse L, Bercovici J, Pizzut-Serin S, Robe P, Tap J, Klopp C, Cantarel BL, Coutinho PM, Henrissat B, Leclerc M, Doré J, Monsan P, Remaud-Simeon M, Potocki-Veronese G (2010) Functional metagenomics to mine the human gut microbiome for dietary fiber catabolic enzymes. Genome Res 20:1605-1612 . doi: 10.1101/gr.108332.110

Tatusov RL, Galperin MY, Natale DA, Koonin E V (2000) The COG database: a tool for genomescale analysis of protein functions and evolution 
Tchigvintsev A, Tran H, Popovic A, Kovacic F, Brown G, Flick R, Hajighasemi M, Egorova O, Somody JC, Tchigvintsev D, Khusnutdinova A, Chernikova TN, Golyshina O V., Yakimov MM, Savchenko A, Golyshin PN, Jaeger KE, Yakunin AF (2015) The environment shapes microbial enzymes: five cold-active and salt-resistant carboxylesterases from marine metagenomes. Appl Microbiol Biotechnol 99:2165-2178 . doi: 10.1007/s00253-014-6038-3

Techtmann SM, Hazen TC (2016) Metagenomic applications in environmental monitoring and bioremediation. J. Ind. Microbiol. Biotechnol. 43:1345-1354

Thai QK, Bös F, Pleiss J (2009) The lactamase engineering database: A critical survey of TEM sequences in public databases. BMC Genomics 10:390 . doi: 10.1186/1471-2164-10-390

Thakur S (2012) Lipases, its sources, properties and applications: A review. Int J Sci Eng Res 3:129

Tirawongsaroj P, Sriprang R, Harnpicharnchai P, Thongaram T, Champreda V, Tanapongpipat S, Pootanakit K, Eurwilaichitr L (2008) Novel thermophilic and thermostable lipolytic enzymes from a Thailand hot spring metagenomic library. J Biotechnol 133:42-49 . doi: 10.1016/J.JBIOTEC.2007.08.046

Tripathi NK, Shrivastava A (2019) Recent developments in bioprocessing of recombinant proteins: expression hosts and process development. Front Bioeng Biotechnol 7:420 . doi: 10.3389/fbioe. 2019.00420

Turnbaugh PJ, Gordon JI (2009) The core gut microbiome, energy balance and obesity. J Physiol 587:4153-4158 . doi: 10.1113/jphysiol.2009.174136

Turnbaugh PJ, Hamady M, Yatsunenko T, Cantarel BL, Ley RE, Sogin ML, Jones WJ, Roe B a, Jason P, Egholm M, Henrissat B, Heath AC, Knight R, Gordon JI, Rey FE, Manary MJ, Trehan I, Dominguez-Bello MG, Contreras M, Magris M, Hidalgo G, Baldassano RN, Anokhin AP, Heath AC, Warner B, Reeder J, Kuczynski J, Caporaso JG, Lozupone C a., Lauber C, Clemente JC, Knights D, Knight R, Gordon JI (2009) A core gut microbiome between lean and obesity twins. Nature 457:480-484 . doi: 10.1038/nature07540

Uchiyama T, Miyazaki K (2009) Functional metagenomics for enzyme discovery: challenges to efficient screening. Curr Opin Biotechnol 20:616-622 . doi: 10.1016/j.copbio.2009.09.010 
Ufarté L, Laville É, Duquesne S, Potocki-Veronese G (2015a) Metagenomics for the discovery of pollutant degrading enzymes. Biotechnol Adv 33:1845-1854 . doi: 10.1016/j.biotechadv.2015.10.009

Ufarté L, Potocki-Veronese G, Laville É (2015b) Discovery of new protein families and functions: New challenges in functional metagenomics for biotechnologies and microbial ecology. Front. Microbiol. 6:563

Uritskiy G, Di Ruggiero J (2019) Applying genome-resolved metagenomics to deconvolute the halophilic microbiome. Genes (Basel) 10:220 . doi: 10.3390/genes10030220

Vargas-Albores F, Martínez-Córdova LR, Martínez-Porchas M, Calderón K, Lago-Lestón A (2019) Functional metagenomics: a tool to gain knowledge for agronomic and veterinary sciences. Biotechnol Genet Eng Rev 35:69-91 . doi: 10.1080/02648725.2018.1513230

Varma VS, Dhamodharan K, Kalamdhad AS (2018) Characterization of bacterial community structure during in-vessel composting of agricultural waste by $16 \mathrm{~S}$ rRNA sequencing. 3 Biotech 8:301 . doi: 10.1007/s13205-018-1319-7

Verma N, Thakur S, Bhatt AK (2012) Microbial lipases: Industrial applications and properties (a review). Int Res J Biol Sci Int Res J Biol Sci 1:2278-3202 . doi: 10.4172/2375-4338.1000156

Verschueren KHG, Seljee F, Rozeboom HJ, Kalk KH, Dijkstra BW (1993) Crystallographic analysis of the catalytic mechanism of haloalkane dehalogenase. Nature 363:693-698 . doi: $10.1038 / 363693 \mathrm{a} 0$

Vieille C, Zeikus GJ (2001) Hyperthermophilic enzymes: sources, uses, and molecular mechanisms for thermostability. Microbiol Mol Biol Rev 65:1-43 . doi: 10.1128/MMBR.65.1.1-43.2001

Wagner UG, Petersen EI, Schwab H, Kratky C (2002) EstB from Burkholderia gladioli: A novel esterase with a $\beta$-lactamase fold reveals steric factors to discriminate between esterolytic and $\beta$-lactam cleaving activity. Protein Sci 11:467-478 . doi: 10.1110/ps.33002

Wang C, Dong D, Wang H, Müller K, Qin Y, Wang H, Wu W (2016a) Metagenomic analysis of microbial consortia enriched from compost: new insights into the role of Actinobacteria in lignocellulose decomposition. Biotechnol Biofuels 9:22 . doi: 10.1186/s13068-016-0440-2 
Wang G, Wang Q, Lin X, Ng TB, Yan R, Lin J, Ye X (2016b) A novel cold-adapted and highly salt-tolerant esterase from Alkalibacterium sp. SL3 from the sediment of a soda lake. Sci Rep 6:19494 . doi: 10.1038/srep19494

Wang H, Hart DJ, An Y (2019) Functional metagenomic technologies for the discovery of novel enzymes for biomass degradation and biofuel production. Bioenergy Res 12:457-470 . doi: $10.1007 / \mathrm{s} 12155-019-10005-\mathrm{w}$

Wang S, Wang K, Li L, Liu Y (2013) Isolation and characterization of a novel organic solventtolerant and halotolerant esterase from a soil metagenomic library. J Mol Catal B Enzym 95:1-8 . doi: 10.1016/j.molcatb.2013.05.015

Wang X, Qin X, Li D, Yang B, Wang Y (2017) One-step synthesis of high-yield biodiesel from waste cooking oils by a novel and highly methanol-tolerant immobilized lipase. Bioresour Technol 235:18-24 . doi: 10.1016/j.biortech.2017.03.086

Wei T, Feng S, Shen Y, He P, Ma G, Yu X, Zhang F, Mao D (2013) Characterization of a novel thermophilic pyrethroid-hydrolyzing carboxylesterase from Sulfolobus tokodaii into a new family. J Mol Catal B Enzym 97:225-232 . doi: 10.1016/j.molcatb.2013.07.022

Wilke A, Harrison T, Wilkening J, Field D, Glass EM, Kyrpides N, Mavrommatis K, Meyer F (2012) The M5nr: A novel non-redundant database containing protein sequences and annotations from multiple sources and associated tools. BMC Bioinformatics 13:141 . doi: $10.1186 / 1471-2105-13-141$

Wu C, Sun B (2009) Identification of novel esterase from metagenomic library of Yangtze River. J Microbiol Biotechnol 19:187-193 . doi: 10.4014/jmb.0804.292

Wu G, Wu G, Zhan T, Shao Z, Liu Z (2013a) Characterization of a cold-adapted and salt-tolerant esterase from a psychrotrophic bacterium Psychrobacter pacificensis. Extremophiles 17:809819 . doi: 10.1007/s00792-013-0562-4

Wu G, Zhang S, Zhang H, Zhang S, Liu Z (2013b) A novel esterase from a psychrotrophic bacterium Psychrobacter celer 3Pb1 showed cold-adaptation and salt-tolerance. J Mol Catal B Enzym 98:119-126 . doi: 10.1016/j.molcatb.2013.10.012

Xiao F, Li Z, Pan L (2017) Application of microbial lipase and its research progress. Prog Appl Microbiol 8-14 
Zarafeta D, Moschidi D, Ladoukakis E, Gavrilov S, Chrysina ED, Chatziioannou A, Kublanov I, Skretas G, Kolisis FN (2016) Metagenomic mining for thermostable esterolytic enzymes uncovers a new family of bacterial esterases. Sci Rep 6:38886 . doi: 10.1038/srep38886

Zhang S, Lin X (2019) CARMA3: Scaffold protein involved in NF- $\kappa$ B signaling. Front Immunol 10:176 . doi: 10.3389/fimmu.2019.00176

Zhang W, Xu H, Wu Y, Zeng J, Guo Z, Wang L, Shen C, Qiao D, Cao Y (2018a) A new coldadapted, alkali-stable and highly salt-tolerant esterase from Bacillus licheniformis. Int J Biol Macromol 111:1183-1193 . doi: 10.1016/J.IJBIOMAC.2018.01.152

Zhang Y, Hao J, Zhang YQ, Chen XL, Xie B Bin, Shi M, Zhou BC, Zhang YZ, Li PY (2017) Identification and characterization of a novel salt-tolerant esterase from the deep-sea sediment of the South China Sea. Front Microbiol 8:441 . doi: 10.3389/fmicb.2017.00441

Zhang Y, Ji F, Wang J, Pu Z, Jiang B, Bao Y (2018b) Purification and characterization of a novel organic solvent-tolerant and cold-adapted lipase from Psychrobacter sp. ZY124. Extremophiles 22:287-300 . doi: 10.1007/s00792-018-0997-8

Zhu Y, Li J, Cai H, Ni H, Xiao A, Hou L (2013) Characterization of a new and thermostable esterase from a metagenomic library. Microbiol Res 168:589-597 . doi: 10.1016/J.MICRES.2013.04.004 


\section{Chapter II}

\section{Metagenomic Screening for lipolytic enzymes reveals an ecology-clustered distribution pattern}

Mingji Lu; Dominik Schneider; Rolf Daniel 
Article

\title{
Metagenomic screening for lipolytic genes reveals an ecology-clustered distribution pattern
}

\author{
Mingji Lu ${ }^{1}$, Dominik Schneider ${ }^{1}$, Rolf Daniel ${ }^{1 *}$ \\ ${ }^{1}$ Department of Genomic and Applied Microbiology, Institute of Microbiology and Genetics \\ Georg-August-University of Göttingen, Grisebachstraße 8, 37077, Göttingen, Germany \\ *Correspondence: Rolf Daniel, Genomic and Applied Microbiology \& Göttingen Genomics Laboratory, Institute of \\ Microbiology and Genetics, University of Göttingen, Grisebachstr. 8, 37077 Göttingen, Germany. Phone: +49-551-3933827,
} Fax: +49-551-3912181, Email: rdaniel@gwdg.de

\begin{abstract}
Lipolytic enzymes are one of the most important enzyme types for application in various industrial processes. Despite the continuously increasing demand, only a small portion of the so far encountered lipolytic enzymes exhibit adequate stability and activities for biotechnological applications. To explore novel and/or extremophilic lipolytic enzymes, microbial consortia in two composts at thermophilic stage were analyzed using function-driven and sequence-based metagenomic approaches. Analysis of community composition by ampliconbased 16S rRNA genes and transcripts, and direct metagenome sequencing revealed that the communities of the compost samples were dominated by members of the phyla Actinobacteria, Proteobacteria, Firmicutes, Bacteroidetes and Chloroflexi. Function-driven screening of the metagenomic libraries constructed from the two samples yielded 115 unique genes encoding lipolytic enzymes. The family assignment of these enzymes was conducted by analyzing the phylogenetic relationship and generation of a protein sequence similarity network according to an integral classification system. The sequence-based screening was performed by using a newly developed database, containing a set of profile Hidden Markov models, highly sensitive and specific for detection of lipolytic enzymes. By comparing the lipolytic enzymes identified through both approaches, we demonstrated that the activity-directed complements sequence-based detection, and vice versa. The sequence-based comparative analysis of lipolytic genes regarding diversity, function and taxonomic origin derived from 175 metagenomes indicated significant differences between habitats. Analysis of the prevalent and distinct microbial groups providing the lipolytic genes revealed characteristic patterns and groups driven by ecological factors. The here presented data suggests that the diversity and distribution of lipolytic genes in metagenomes of various habitats are largely constrained by ecological factors.
\end{abstract}

Keywords: lipolytic enzymes, function-driven metagenomics, sequence-based metagenomics, profile HMM, lipolytic enzyme classification, comparative analysis, compost

\section{Introduction}

Lipolytic enzymes (LEs) acting on carboxyl ester bonds in lipids, include esterases (EC 3.1.1.3, carboxylesterases) and true lipases (EC 3.1.1.1, triacylglycerol acyl hydrolases). Due to the catalytic versatility, LEs have remarkable applications in various processes relevant to food, paper, medical, detergent, and pharmaceutical industries (Hita et al. 2009; Romdhane et al. 2010; Ferrer et al. 2015; Sarmah et al. 2018). Nowadays, LEs are considered to be one of the most important biocatalysts for biotechnological applications.

In principle, LEs can be classified on the basis of the substrate preference (Sarmah et al. 2018) and sequence similarity (Chen et al. 2016). The latter provides an easy-to-perform way for classification and indication of the similarity and evolutionary relationship between LEs. Arpigny and Jaeger (1999) have elaborated the most widely accepted classification of bacterial lipases into eight families (I to VIII). The classification system was based on conserved sequence motifs and biological properties of 53 LEs. A recent update of this system resulted in addition of 11 families (IX to XIX) (Kovacic et al. 2019). Besides the nineteen families, there are claims of novel families, such as Est22 (Li et al. 2017), Est9X(Jeon et al. 2009), LipSM54 (Li et al. 2016) and EstDZ2 (Zarafeta et al. 2016). To avoid an artificial inflation of the number of families, these novel families were mostly excluded during classification of newly identified lipolytic enzymes in previous studies (Hitch and Clavel 2019). To the best of our knowledge, we aimed to incorporate all the 'so-called' novel families during classification to avoid false 'novelty'.

LEs are ubiquitous among all aspects of life, with most of them originating from microorganisms (Kovacic et al. 2019). Environmental microbes, including the so far uncultured species, encode a largely untapped reservoir of novel LEs. Metagenomic function-driven and sequence-based approaches provided access to the genetic resources from so far uncultured and uncharacterized microorganisms (Simon and Daniel 2009; Simon and Daniel 2011). LEs are among the most frequent targets in function-based screens of metagenomic libraries derived from diverse habitats, such as compost (Kang et al. 2011; Lu et al. 2019), landfill leachate (Rashamuse et al. 2009), 
marine sediment (Peng et al. 2011; Zhang et al. 2017), activated sludges (Liaw et al. 2010) and hot springs (LópezLópez et al. 2015).

In contrast to the easy access to metagenome-derived sequencing data, most published metagenomic screenings for LEs were enzyme activity-driven and not sequence-based (Ferrer et al. 2015; Berini et al. 2017). Only a few studies explored LEs by sequence-driven approaches, including analysis based on regular expression patterns (Masuch et al. 2015), ancestral sequence reconstruction (Verma et al. 2019) and conserved motifs (Zhang et al. 2009; Barriuso and Jesús Martínez 2015; Zarafeta et al. 2016). For various reasons, only a very limited number of LEs were identified by these strategies. Sequence-based approaches primarily rely on the reference database to infer functions of newly-discovered genes and the corresponding enzymes (Hugenholtz and Tyson 2008; Quince et al. 2017; Berini et al. 2017; Ngara and Zhang 2018). With protein-of-interest-specific databases, biomolecules such as antibiotic resistance genes (Gibson et al. 2015; Willmann et al. 2015; Pehrsson et al. 2016) and CAZymes (Wang et al. 2016; Montella et al. 2017; Wang et al. 2019) were successfully profiled across habitats. Recently, with the rapid accumulation of genomic/metagenomic data in public repositories (Keegan et al. 2016; Chen et al. 2017; Eric Sayers et al. 2019), it is likely that current knowledge regarding LEs only reflects the tip of the iceberg and that the full diversity of these enzymes is far from being completely described.

In order to quantitatively analyze LEs distributed in environmental samples, we developed a LE-specific profile Hidden Markov Model (HMM) database. Profile HMMs have been widely adopted for detection of remote homologs (Gibson et al. 2015; Walsh et al. 2017; Berglund et al. 2017) and annotation of general functions in microbial genomes and metagenomes (Skewes-Cox et al. 2014; Reyes et al. 2017; Bzhalava et al. 2018). However, they have not yet been specifically applied to LEs. Once developed and validated, the database was applied to profile the lipolytic genes in metagenomes from various habitats. Profiling the distribution of LEs among various habitats provides researchers a straightforward approach for their downstream analysis. In this study, two composts were sampled and LEs identified through function-based and sequence-based approaches were compared. The distribution of lipolytic genes in 175 metagenomes was also investigated by sequence-based screening.

\section{Material and Methods}

\section{Sample collection}

Compost samples were collected as described previously (Lu et al. 2019). Briefly, two compost piles fermenting mainly wood chips (Pile_1) or kitchen waste (Pile_2) were sampled. Temperatures at the sampling spots were measured, and the two samples were designated as compst55 $\left(55^{\circ} \mathrm{C}\right.$ for Pile_1) and compst $76\left(76^{\circ} \mathrm{C}\right.$ for Pile_2). Approximately $50 \mathrm{~g}$ compost per sample was collected in sterile plastic tubes and stored at -20 or $-80{ }^{\circ} \mathrm{C}$ until further use.

\section{Isolation of nucleic acids}

Metagenomic DNA of the compost sample was isolated by using the phenol-chloroform method (Zhou et al. 1996) and MoBio Power Soil DNA extraction kit as recommended by the manufacturer (MO BIO Laboratories, Hilden, Germany). DNA obtained from these two methods was pooled per sample and stored at $-20^{\circ} \mathrm{C}$ until use.

RNA was extracted by employing the MoBio PowerSoil RNA isolation kit as recommended by the manufacturer (MO BIO Laboratories). Residual DNA was removed by treatment with 2 U Turbo DNase (Applied Biosystems, Darmstadt, Germany) at $37{ }^{\circ} \mathrm{C}$ for $1 \mathrm{~h}$ and recovered by using RNeasy MinElute Cleanup kit as recommended by the manufacturer (Qiagen, Hilden, Germany). RNA yields were estimated by employing a Qubit $^{\circledR}$ Fluorometer as recommended by the manufacturer (Thermo Fisher Scientific, Schwerte, Germany). A PCR reaction targeting the 16S rRNA gene was performed to verify the complete removal of DNA as described by Schneider et al. (2015). Subsequently, the DNA-free RNA was converted to cDNA using the SuperScript ${ }^{\mathrm{TM}}$ III reverse transcriptase (Thermo Fisher Scientific). Briefly, a mixture $(14 \mu \mathrm{l})$ containing $100 \mathrm{ng}$ of DNA-free RNA in DEPC-treated water, $2 \mu \mathrm{M}$ of reverse primer (5' - CCGTCAATTCMTTTGAGT-') and $10 \mathrm{mM}$ dNTP mix was incubated at $65^{\circ} \mathrm{C}$ for $5 \mathrm{~min}$ and chilled on ice for at least $1 \mathrm{~min}$. Then, $10 \mu \mathrm{l}$ of cDNA synthesis mix including reaction buffer, $5 \mathrm{mM} \mathrm{MgCl}, 0.01 \mathrm{M}$ DTT, $1 \mu \mathrm{l}$ 40U RiboLock ${ }^{\mathrm{TM}}$ RNase inhibitor (Thermo Fisher Scientific) and $200 \mathrm{U}$ SuperScript ${ }^{\mathrm{TM}}$ III reverse transcriptase (Thermo Fisher Scientific) was added to each $\mathrm{RNA} /$ primer mixture in the previous step, and incubated at $55^{\circ} \mathrm{C}$ for $90 \mathrm{~min}$. The reaction was terminated at 70 ${ }^{\circ} \mathrm{C}$ for $15 \mathrm{~min}$.

\section{Sequencing of 16S rRNA genes and transcripts}

The PCR amplification of the V3-V5 regions of bacterial 16S rRNA genes and transcripts were performed with the following set of primers comprising the Roche 454 pyrosequencing adaptors (underlined), a key (TCAG), a unique 10-bp multiplex identifier (MID), and template-specific sequence per sample: the forward primer V3for_B (5'-CGTATCGCCTCCCTCGCGCCATCAG-MID-TACGGRAGGCAGCAG-3'), (Liu et al. 2007), and reverse primer V5rev_B 5'-CTATGCGCCTTGCCAGCCCGCTCAG-MID-CCGTCAATTCMTTTGAGT-3' (Wang and 
Qian 2009). The PCR reaction mixture (50 $\mu \mathrm{l})$ contained $10 \mu \mathrm{l}$ of fivefold reaction buffer, $200 \mu \mathrm{M}$ of each of the four deoxynucleoside triphosphates, $0.2 \mu \mathrm{M}$ of each primer, $5 \% \mathrm{DMSO}, 1 \mathrm{U}$ of Phusion hot start high-fidelity DNA Polymerase (Finnzymes, Vantaa, Finland) and $50 \mathrm{ng}$ template (DNA or cDNA). The thermal cycling scheme comprised initial denaturation at $98{ }^{\circ} \mathrm{C}$ for $5 \mathrm{~min}, 25$ cycles of denaturation at $98^{\circ} \mathrm{C}$ for $45 \mathrm{~s}$, annealing for $45 \mathrm{~s}$ at $60{ }^{\circ} \mathrm{C}$, and extension at $72{ }^{\circ} \mathrm{C}$ for $30 \mathrm{~s}$, followed by a final extension period at $72{ }^{\circ} \mathrm{C}$ for $5 \mathrm{~min}$. All amplicon PCR reactions were performed in triplicate and pooled in equimolar amounts for sequencing. The Göttingen Genomics Laboratory determined the sequences of the partial 16S rRNA gene and transcript amplicons by using a 454 GSFLX sequencer and titanium chemistry as recommended by the manufacturer (Roche, Mannheim, Germany).

Quality-filtering and denoising of the recovered 16S rRNA pyrotag reads were performed with the QIIME (1.9.1) software package (Bolyen et al. 2019) by employing the scheme outlined by Schneider et al. (2015). Forward and reverse primer sequences were removed with the split_libraries.py script. Pyrosequencing noise and chimeric sequences were removed with UCHIME (Edgar et al. 2011). Operational taxonomic unit (OTU) determination was performed by employing the pick_open_reference_otus.py script at genetic divergence level of $3 \%$. Taxonomic classification of OTUs was performed by parallel_assign_taxonomy_blast.py script against the Silva SSU database release 128 (Quast et al. 2013). The filter otu table.py script was used to remove singletons, chloroplast sequences, extrinsic domain OTUs, and unclassified OTUs. Rarefaction curves was calculated with QIIME software by using alpha-rarefaction.py.

\section{Metagenomic sequencing and data processing}

The sequencing libraries were constructed and indexed with Nextera DNA Sample Preparation kit and Index kit as recommended by the manufacturer (Illumina, San Diego, CA, USA). Paired-end sequencing was performed using a HiSeq 4000 instrument $(2 \times 150 \mathrm{bp})$ as recommended by the manufacturer (Illumina). Raw reads were trimmed with Trimmomatic version 0.36 (Bolger et al. 2014) and verified with FastQC version 0.11.5 (Andrew, 2010). Then, reads were submitted to MG-RAST metagenomics analysis server and processed by the default quality control pipeline (Keegan et al. 2016). Microbial composition analysis was performed using MG-RAST best hit classification tool against the databases of M5RNA (Non-redundant multisource ribosomal RNA annotation) and M5NR (M5 non-redundant protein) with default settings. Functional classification was performed based on clusters of orthologous groups (COGs) and Subsystem categories with default settings. Since we mainly focused on the bacterial community, the baseline for all fractions reported referred to the reads assigned to the bacterial domain.

\section{Construction of metagenomic plasmid libraries and function-based screening for lipolytic activity}

Lipolytic genes were screened by constructing small-insert plasmid libraries as described by Lu et al. (2019). Briefly, DNA was sheared by sonication for $3 \mathrm{~s}$ at $30 \%$ amplitude and cycle 0.5 (UP200S Sonicator, Stuttgart, Germany), and size-separated using a $0.8 \%$ low-melting point agarose gel. DNA fragments from 6 to $12 \mathrm{~kb}$ were recovered by gel extraction using the peqGold Gel Extraction kit as recommended by the manufacturer (Peqlab Biotechnologie $\mathrm{GmbH}$, Erlangen, Germany). The metagenomic small-insert library was constructed using the vectors $\mathrm{pFLD}$ or $\mathrm{pCR}-\mathrm{XL}-\mathrm{TOPO}$ (Thermo Fisher Scientific).

Vector pFLD was digested with $P m l I$ at $37^{\circ} \mathrm{C}$ for $2 \mathrm{~h}$ and dephosphorylated with $5 \mathrm{U}$ Antarctic phosphatase at $37^{\circ} \mathrm{C}$ for $30 \mathrm{~min}$ as recommended by the manufacturer (NEB, Ipswich, MA). Subsequently, the ends of DNA fragments were blunt-ended and phosphorylated by employing the Fast DNA End Repair kit (Thermo Fisher Scientific). SureClean was applied to purify DNA or vector between steps as described by the manufacturer (Bioline GmbH, Luckenwalde, Germany). Finally, metagenomic fragments and $\mathrm{pFLD}$ vector were ligated using T4 DNA ligase (Thermo Fisher Scientific) at $16{ }^{\circ} \mathrm{C}$, overnight. Metagenomic DNA fragments were cloned into vector pCR-XL-TOPO following the protocol of the manufacturer recommended in the TOPO-XL-PCR cloning kit (Thermo Fisher Scientific).

To screen for lipolytic activity, Escherichia coli TOP10 was used as the host (Dukunde et al. 2017). Librarybearing cells were plated onto LB agar plates $(15 \mathrm{~g} / \mathrm{L})$ containing $1 \%(\mathrm{v} / \mathrm{v})$ emulsified tributyrin (Sigma) as the indicator substrate and the appropriate antibiotic (pFLD, $100 \mu \mathrm{g} / \mathrm{ml}$ Ampicillin; pCR-XL-TOPO, $50 \mu \mathrm{g} / \mathrm{m}$ Kanamycin). The quality of the libraries was controlled by checking the average insert sizes and the percentage of insert-bearing E. coli clones (Table 1). Cells were incubated on indicator agar at $37^{\circ} \mathrm{C}$ for $24 \mathrm{~h}$ and subsequently for 1 to $7 \mathrm{~d}$ at $30^{\circ} \mathrm{C}$. Lipolytic-positive $E$. coli clones were identified by the formation of clear zones (halos) around individual colonies.

The recombinant plasmid DNA derived from positive clones was isolated by using the QIAGEN plasmid mini kit (QIAGEN) and digested by PmlI (vector PFLD) or EcoRI (vector pCR-XL-TOPO) at $37{ }^{\circ} \mathrm{C}$ for $2 \mathrm{~h}$. The digestion pattern was analyzed, and phenotype of positive clones was confirmed by transformation of the selected plasmids (from previous step) into the host and rescreening on indicator agar plates. In addition, lipolytic activity towards different triacylglycerides was measured qualitatively by incubating the confirmed lipolytic positive clones on agar plates emulsified with tributyrin $(\mathrm{C} 4)$, tricaproin $(\mathrm{C} 6)$, tricaprylin $(\mathrm{C} 8)$, tricaprin $(\mathrm{C} 10)$, trilaurin 
(C12), trimyristin (C14), or tripalmitin (C16). Formation of clearing zones (halos) on agar plates indicated lipolytic activity.

\section{Analysis of lipolytic genes from function-based screenings}

The plasmids recovered from the confirmed positive clones were pooled in equal amounts (50 $\mathrm{ng}$ of each clone) for compost55 and compost76. Then, the two plasmid DNA mixtures were sequenced using an Illumina MiSeq instrument with reagent kit version 3 ( $2 \times 300$ cycles) as recommended by the manufacturer (Illumina)). To remove the vector sequences, raw reads were initially mapped against vector sequences (pFLD or pCR-XL-TOPO) using Bowtie 2 (Langmead and Salzberg 2012). The unmapped reads were quality-filtered by Trimmomatic v0.30 (Bolger et al. 2014) and assembled into contigs by Metavelvet v1.2.01 (Namiki et al. 2012) and MIRA 4 (Chevreux et al. 1999). In addition, both ends of the inserts of each plasmid were sequenced using Sanger technology and the following primers: pFLD504_F (5'-GCCTTACCTGATCGCAATCAGGATTTC-3') and pFLD706_R (5'-CGAGGAGAGGGTTAGGGATÄGGCTTAC-3') for vector pFLD, and M13_Forward (5'GTAAAACGACGGCCAG-3') and M13 Reverse (5'-CAGGAAACAGCTATGAC-3') for vector pCR-XLTOPO . The raw Sanger reads were processed with the Staden package (Staden et al. 2003). Finally, the full insert sequence for each plasmid was reconstructed by mapping the processed Sanger reads on the contigs assembled from the Illumina reads. Open reading frames (ORFs) were predicted by MetaGeneMark (Zhu et al. 2010) using default parameters. Lipolytic genes were annotated by searches against NCBI Non-redundant sequence database (http://www.ncbi.nlm.nih.gov/gorf/gorf.html).

\section{Family classification of lipolytic enzymes revealed from function-based screening}

LEs were clustered according to the classification standard defined by Arpigny and Jaeger (1999). In order to classify LEs identified from function-based screening, we have integrated all the so far reported lipolytic families, including families I to XIX, and potential novel families reported in recent studies (Supplementary Table S1). A neighbor-joining tree was constructed with LEs identified from this study and reference proteins (Supplementary Table S2) using MEGA version 7 (Tamura et al. 2013). The robustness of the tree was tested by bootstrap analysis using 500 replications. The phylogenetic tree was depicted by GraPhlAn (Asnicar et al. 2015). To confirm the classification and group proteins in clusters, a protein sequence similarity network was generated. In a protein sequence similarity network, members in a potential isofunctional group consist of nodes (symbol) that share a sequence similarity larger than a selected value and are connected by edges (line). As similarity increases, edges decrease and finally proteins can be separated into defined clusters (Gerlt et al. 2015). In this study, a protein sequence similarity network was generated by submitting the same sequence dataset used in the phylogenetic analysis to the Enzyme Function Initiative-Enzyme Similarity Tool web server (EFI-EST; http://efi.igb.illinois.edu/efi-est/index.php) (Atkinson et al. 2009) with an E-value cutoff of $\leq 1 \mathrm{e}^{-10}$ and alignment score $\geqslant 16$. The resulting network was visualized in Cytoscape 3.2.1 using the organic layout (Shannon et al. 2003). In addition, multiple-sequence alignments were conducted to explore the presence of catalytic residues, and conservative and distinct motifs in each lipolytic family by employing ClustalW (Larkin et al. 2007).

\section{Building profile Hidden Markov Model (HMM) database for sequence-based screening}

A search method based on profile HMMs was developed to identify and annotate putative lipolytic genes in metagenomes (Supplementary Figure 1). In order to target homologous sequences, profile HMMs were built from multiple sequence alignments, which requires relatedness in the input protein sequences. Thus, consistent to the classification of functional-derived LEs, we generally followed the clustering system of Arpigny and Jaeger (1999).

With the exception of LEs belonging to families II and VIII, and patatin-like-proteins, LEs in the other families generally share a conserved $\alpha / \beta$-hydrolase fold and a canonical G-x-S-x-G pentapetide around the catalytic serine (Kovacic et al. 2019). ESTHER is a database dedicated to proteins with $\alpha / \beta$-hydrolase-fold and their classifications (Lenfant et al. 2013), containing approximately $60,000 \alpha / \beta$ hydrolases grouped in 214 clusters so far (as of November 2019). In ESTHER, families I-XIX were integrated into an own classification with corresponding entries (http://bioweb.supagro.inra.fr/ESTHER/Arpigny_Jaeger.table). We thereby designated lipolytic families that were classified and named according to ESTHER database as ELFs (abbreviation of ESTHER Lipolytic Families). For lipolytic families that were not incorporated into the 19 families (I-XIX), their corresponding ELFs were determined by searching LEs against ESTHER database. Generally, a LE was assigned to an ELF if its BLASTp top hit (with lowest e-value) had $\geqslant 60 \%$ amino acid identity and $\geqslant 80 \%$ query coverage. Protein sequences in all of the determined ELFs were downloaded from ESTHER database for profile HMM construction.

Firstly, multiple sequence alignments were performed with protein sequences in each ELF, using the following three algorithms and default settings: ClustalW (Thompson et al. 1994), Clustal Omega (Sievers et al. 2011) and Muscle (Edgar 2004). Subsequently, the three alignment sets were run through hmmbuild in HMMER3 (Eddy 2018) to create three sets of profile HMMs. Moreover, profile HMMs supplied in the ESTHER database were downloaded. Finally, four profile HMM databases were constructed by concatenating and compressing the 
respective set of profile HMMs using hmmpress. Thereafter, we designated the four profile HMM databases with respect to the corresponding alignment algorithm (clustalw-pHMMs, omega-pHMMs and muscle-pHMMs) or source (ESTHER-pHMMs).

For families II, VIII and patatin-like-proteins, profile HMMs were retrieved directly from Pfam database (Finn et al. 2014) using the searching keywords of "GDSL", "beta-lactamase" and "patatin", respectively. The profile HMM database was constructed as described above and designated as pfam-pHMMs, specifying for LEs in families II and VIII, and patatin-like-protein.

\section{Validating profile HMM database}

The prediction sensitivity and specificity of the profile HMM databases were evaluated using four datasets. Dataset 1, LEs recruited in the UniProtKB database (as of November 2019) using as search strategy the EC numbers 3.1.1.1 or 3.1.1.3, and protein length between 200 to 800 amino acids. Only the prokaryotic LEs were selected for analysis (Supplementary Table S3a). Dataset 2, LEs reported in literature. Most of these enzymes were obtained through metagenomic approaches and biochemically characterized, and with a confirmed lipolytic family assignment by constructing a multiple sequence alignment and/or phylogenetic tree (Supplementary Table S3b). Dataset 3, protein sequences predicted by MetaGeneMark (Zhu et al. 2010) from identified inserts harboring functional lipolytic genes (Supplementary Table S3c). Dataset 4, randomly selected protein sequences (not recruited from ESTHER database) that were annotated in Uniprot or NCBI database as non-lipolytic proteins but with sequence homology to LEs (Supplementary Table S3d). Proteins in the four datasets were screened against the profile HMM databases successively with hmmscan using an E-value cutoff of $\leqslant 1 \mathrm{e}^{-10}$. The sensitivity and specificity of each database were evaluated by the recalls and false positive returns. In addition, we compared our homology-based method (profile HMMs) with the similarity-based pairwise sequence alignment method (BLAST; Altschul et al. 1990). The database for BLAST-based searching was built with the same dataset used for profile HMM construction. BLASTp was performed at an E-value cutoff of $\leqslant 1 \mathrm{e}^{-10}$.

In order to improve the accuracy for assigning proteins to lipolytic families and distinguishing "true" LEs from the non-lipolytic proteins, protein sequences were annotated by two methods and combined for final assignment. Briefly, putative lipolytic proteins (PLPs) identified by screening against the selected profile HMM database (one from clustalw-pHMMs, omega-pHMMs, muscle-pHMMs and ESTHER-pHMMs) were further searched against the ESTHER database (all entries in the database were included; as of November 2019) by BLASTp using an Evalue cutoff of $\leqslant 1 \mathrm{e}^{-10}$ (Supplementary Figure 1). A PLP was assigned to a lipolytic family only if it was annotated into the same ELF by hmmscan and BLASTp. Otherwise, according to the BLAST results, the remaining PLPs were either annotated as "unassigned" PLPs or non-lipolytic proteins (Supplementary Figure 1). In principle, PLPs with the best Blast hits were affiliated to the miscellaneous ESTHER families (functions were not determined, including 5_AlphaBeta_hydrolase, 6_AlphaBeta_hydrolase, Abhydrolase_7 and AlphaBeta_hydrolase), or other ESTHER families (with $<60 \%$ identity or $<70 \%$ query coverage) were classified as unassigned PLPs. The remaining PLPs with the best Blast hits showing $\geqslant 60 \%$ amino acid identity and $\geqslant 70 \%$ query coverage to the non-lipolytic ESTHER families were classified as non-lipolytic proteins.

Family annotation of PLPs obtained by screening against pfam-phmms were confirmed by a further scan against the CATH HMMs database (Knudsen and Wiuf 2010) using the Github repository cath-tools-genomescan (https://github.com/UCLOrengoGroup/cath-tools-genomescan). PLPs were assigned to lipolytic families VIII and II, or patatin-like-proteins only if the PLP was assigned to the specific Funfams (functional families) dedicated to lipolytic-related activities, which were inferred from the functionally characterized LEs and gene ontology (GO) annotations (Supplementary Table S4). Additionally, based on our literature search, the LEs in family VIII were generally restricted to PLPs with sequence length between 350 to 450 amino acids. In other cases, the PLP was grouped into non-lipolytic proteins.

For the unassigned PLPs, these sequences show low similarity to any ESTHER family with known function or CATH Funfams, and hence, could contain novel lipolytic or non-lipolytic proteins. Non-lipolytic proteins were excluded from the downstream analysis.

\section{Sequence-based screening for putative lipolytic genes}

Sequence-based screening for putative lipolytic genes in the two compost metagenomes were performed as described above. Briefly, the processed metagenomic short reads were assembled into contigs with SPADES version 3.10 (Bankevich et al. 2012). Then, protein sequences were deduced from PROKKA v1.14.5 annotation (Seemann 2014). In order to obtain full-length lipolytic genes, only proteins with amino acid sequence length between 200 and 800 amino acids were retained. Subsequently, the resulting protein sequences were screened against the selected profile HMM databases using hmmscan (Eddy 2011) with an E-value cutoff of $\leq 1 \mathrm{e}^{-10}$. Identified PLPs were further assigned into different lipolytic families as described above (Supplementary Figure 1). Moreover, the lipolytic family classification of assigned PLPs was confirmed by constructing the protein sequence similarity network (Atkinson et al. 2009). The phylogenetic origins of PLP-encoding genes and their 
corresponding contigs were determined using Kaiju web server (http://kaiju.binf.ku.dk/server; Menzel et al. 2016). Phylogenetic distributions of assigned PLPs in each lipolytic family were visualized via Circos software (Krzywinski et al. 2009).

\section{Comparative analysis of metagenomic datasets}

A total of 175 assembled metagenomes from 15 different habitats were retrieved from the Integrated Microbial Genomes and Microbiomes database (IMG/M). These included metagenomes from anaerobic digestor active sludges (ADAS, $n=9$ ), agriculture soils ( $\mathrm{AS}, \mathrm{n}=10$ ), composts (COM, $n=18$ ), grassland soils ( $\mathrm{GS}, \mathrm{n}=11$ ), human gut systems $(\mathrm{HG}, \mathrm{n}=16)$, hypersaline mats $(\mathrm{HM}, \mathrm{n}=7)$, hydrocarbon resource environments $(\mathrm{HRE}, \mathrm{n}=6)$, hot springs (HS, $n=14)$, landfill leachates ( $L L, n=10$ ), marine sediments (MS, $n=12)$, marine waters (MW, $n=10)$, oil reservoirs $(\mathrm{OR}, \mathrm{n}=13)$, river waters $(\mathrm{RW}, \mathrm{n}=11)$, tropical forest soils $(\mathrm{TFS}, \mathrm{n}=14)$ and wastewater bioreactors (WB $\mathrm{n}=13$ ) (Supplementary Table S5). Data processing including open reading frame prediction in assembled contigs and taxonomic assignment of the corresponding deduced protein sequences were conducted by the IMG/M builtin pipelines (Chen et al. 2017). The protein sequences were downloaded from IMG/M database and used in the sequence-based screening as described above (Supplementary Figure 1).

For comparative analysis, the abundance of PLP-encoding genes in each metagenome were normalized according to the method described by Kaminski et al. (2015). The normalized count is in units of LPGM (Lipolytic hits Per Gigabase per Million mapped genes). Unless otherwise stated, LPGM values were used for all calculations Heatmap was built in R v3.5.2 (R Core Team, 2016) with the function heatmap.2 using the "Heatplus" package (Ploner et al., 2020). The heatmap hierarchical clustering was performed with "vegan" package (vegdist = "bray", data.dist = "ward.D"). Non-metric multidimensional scaling (NMDS) was also performed with the "vegan", package (Oksanen et al. 2018). The analysis of similarities (ANOSIM) was performed with 9,999 permutations using PAST 4 (Hammer et al. 2001). The phylogenetic annotation of PLPs was retrieved from IMG/M. Association networks between habitats and phylogenetic distribution of PLPs at genus level were generated by mapping significant point biserial correlation values with the "indicspecies" package in R (Cáceres 2013). Only genera with significant correlation coefficients $(P=0.05)$ were included. The resulting bipartite networks were visualized with Cytoscape v3.5 by using the edge-weighted spring embedded layout algorithm, whereby the habitats were source nodes, genera target nodes and edges (lines connecting nodes) weighted positive associations between genera and specific habitat or habitats combinations.

In addition, due to the ambiguity of unassigned PLPs, all analyses were performed successively using two datasets: (1) only assigned PLPs, as the consideration of excluding the potential non-lipolytic ones, (2) assigned and unassigned PLPs combined (total PLPs), in order to include all the possible lipolytic ones. This paper mainly focuses on the assigned PLPs for the sake of accuracy, but the comparative analysis of total PLPs was also performed.

\section{Data availability}

The short reads and insert sequences were submitted to NCBI databases. Metagenomic short reads are available at in the NCBI sequence read archive (SRA) under accession numbers SRR13115019 (compost55) and SRR13115018 (compost76) and 16S rRNA pyrotag reads under SAMN06859928 (compost55 genes), SAMN06859946 (compost55 transcripts), SAMN06859935 (compost76 genes) and SAMN06859953 (compost76 transcripts). The insert sequences of the plasmids are available in GenBank under accession numbers MW408002--MW408112 (Supplementary Table S9).

\section{Results and Discussion}

\section{Phylogenetic and functional profile of microbes in the compost metagenomes}

During the heating-up process of composting, the succession of microorganisms plays a key role in degrading organic matter (Dougherty et al. 2012). In this study, the bacterial community compositions in two compost samples with different pile core temperatures of 55 (compost55) and $76{ }^{\circ} \mathrm{C}$ (compost76) were revealed by amplicon-based sequencing of 16S rRNA genes (DNA-based, total community) and transcripts (RNA-based, active community) (Supplementary Figures S2 and S3). To extend the taxonomic analysis, the environmental DNA from both metagenomes were also directly sequenced (Supplementary Table S6). Generally, the bacterial community determined by direct sequencing were consistent with that derived from 16S rRNA gene-based analysis. The bacterial phyla Actinobacteria, Proteobacteria, Firmicutes, Bacteroidetes and Chloroflexi were predominant (relative abundance $>5 \%$ ) in compost 55 and compost76 (Supplementary Figures S3 and S4). This is in agreement with previous studies of bacterial communities in thermophilic composts (Ryckeboer et al., 2003; Antunes et al., 2016; Zhen et al., 2018; Zhou et al., 2018). Differences were detected, which were derived mainly from the different feedstock composition (wood chips vs. kitchen waste) and composting conditions (core temperature 55 vs. $76^{\circ} \mathrm{C}$ ). Actinobacteria was the most abundant phylum (>25\%) in compost55 (Supplementary Figures S3 and S4), which is accordance with the bacterial communities in composts using mainly plant material 
as feedstock (Yu et al. 2007; Zhang et al. 2014). In compost76, members of the Firmicutes were most abundant ( $>55 \%$ ), which was also reported for composts harboring high-nitrogen feedstock, such as animal manure and kitchen waste (Niu et al. 2013; Antunes et al. 2016; Ma et al. 2018; Zhou et al. 2019). The 16S rRNA gene and transcript analysis (Supplementary Table S7) revealed genera that were present (>1\%) in compost55 such as Brockia, Rhodothermus, Thermobispora, Longispora, Geobacillus, Filomicrobium and Thermomonospora, and in compost76 such as Symbiobacterium, Calditerricola and Thermaerobacter were among the typical bacterial taxa previously identified in composting processes (Ryckeboer et al. 2003; Antunes et al. 2016; Yu et al. 2018; Zhou et al. 2018).

Additionally, the metagenomic data were searched against the COG and subsystem databases to assess the functions prominent in compost microbes (Supplementary Figure S5). In principle, compost55 and compost76 share similar metabolic patterns (Supplementary Figure S5). Particularly, the broad diversity and abundance of gene functions in carbohydrate metabolism and transport (COG) and carbohydrates (subsystems) indicated that composts were potential candidates for exploring biocatalysts (Hu et al. 2010a; Leis et al. 2015; Wang et al. 2016; Egelkamp et al. 2019). Notably, the COG category of lipid transport and metabolism as well as the subsystems category of fatty acids, lipids, and isoprenoids were more abundant in the compost55 community than in the compost76 community, suggesting a higher possibility to identify lipolytic genes in the compost 55 metagenome.

\section{Function-based screening of LEs in compost metagenomes}

In this study, four metagenomic libraries were prepared to probe the diversity of LEs from compost microbes by the function-driven approach using tributyrin-containing indicator agar (Table 1). Overall, approximately 4.89 and $2.56 \mathrm{~Gb}$ of cloned compost DNA were screened, yielding 199 and 51 positive clones for compost55 and compost76, respectively. Previous studies have used various vectors such as BACs, fosmids and plasmids for function-based screening of LEs from different bioresources (Lee et al. 2004; Lämmle et al. 2007; Kim et al. 2010; Nacke 2011; Berlemont et al. 2013; Shao et al. 2013; Leis et al. 2015; Jia et al. 2019). The hit rate to recover a lipolytic-positive clone ranged from 0.714 to 208 per $\mathrm{Gb}$ of cloned DNA (Table 1). Among the compost metagenomic libraries, the targeting probability towards a LE in our study ranged from 16.1 to 43.6 per $\mathrm{Gb}$ and is generally consistent with the values from other studies (Lämmle et al. 2007; Kim et al. 2010; Leis et al. 2015). Also notably, the targeting probabilities in metagenomic libraries from compost and sludge are generally higher than those from other environments, such as grassland, forest soil and river water (Wu and Sun 2009; Nacke 2011; Berlemont et al. 2013). According to Liaw et al. (2010), the targeting probability and/or hit rate for discovering a lipolytic clone is largely attributed to the sample source.

Table 1. Summary of metagenomic libraries used for lipolytic activity screening in this and other studies

\begin{tabular}{|c|c|c|c|c|}
\hline Environmental sample $^{\mathrm{b}}$ & $\begin{array}{l}\text { Vector type } \\
\text { (average insert } \\
\text { size in } \mathrm{kb} \text { ) }\end{array}$ & $\begin{array}{l}\text { No. of library-containing } \\
\text { clones (confirmed } \\
\text { positive hits, No. of hits } \\
\text { per million of clones) }\end{array}$ & $\begin{array}{l}\text { Probability } \\
\text { (No. of hits per Gb } \\
\text { of DNA screened) }\end{array}$ & Reference \\
\hline$\overline{\text { Compost }}$ & Plasmid (5.3) & $675,200(156,213)$ & 43.6 & compost55 (this study) \\
\hline Compost & Plasmid (5.6) & $234,912(43,183)$ & 32.7 & compost55 (this study) \\
\hline Compost & Plasmid (6) & $281,281(37,132)$ & 21.9 & compost 76 (this study) \\
\hline Compost & Plasmid (6.2) & $140,747(14,100)$ & 16.1 & compost76 (this study) \\
\hline Compost & Plasmid (3.2) & $21,000(14,670)$ & 208 & Lämmle et al. 2007 \\
\hline Compost & Fosmid (35) & $23,400(19,810)$ & 23.2 & Kim et al. 2010 \\
\hline Compost & Fosmid (37.5) & $1,920(2,1040)$ & 27.8 & Leis et al. 2015 \\
\hline Compost & Fosmid $\left(-^{\mathrm{a}}\right)$ & $13,000(10,770)$ & $\mathrm{a}^{\mathrm{a}}$ & Kang et al. 2011 \\
\hline Compost & plasmid $\left(-^{a}\right)$ & $66,000(6,0.90)$ & $\mathrm{L}^{\mathrm{a}}$ & Popovic et al. 2017 \\
\hline Grassland soil & Plasmid (5.7) & $510,808(2,0.4)$ & 0.714 & Nacke et al. 2011 \\
\hline Grassland soil & Fosmid (27.8) & $50,952(2,40)$ & 1.41 & Nacke et al. 2011 \\
\hline Forest soil & Fosmid (35) & $33,700(8,240)$ & 6.78 & Lee et al. 2004 \\
\hline Forest soil & Plasmid (3.1) & $70,000(3,42)$ & 13.8 & Berlemont et al. 2013 \\
\hline River surface water & $\mathrm{BAC}(50)$ & $8,000(1,120)$ & 2.5 & Wu and Sun 2009 \\
\hline Hot spring biofilm & $\mathrm{BAC}(50)$ & $68,352(10,150)$ & 2.93 & Yan et al. 2017 \\
\hline Surface sea water & BAC (70) & $20,000(4,200)$ & 2.86 & Chu et al. 2008 \\
\hline Marine sediment & plasmid (4.5) & $29,000(6,200)$ & 46.0 & Ranjan et al. 2018 \\
\hline Marine sediment & Fosmid (36) & $40,000(19,480)$ & 13.2 & Hu et al. 2010 \\
\hline Marine mud & fosmid (40) & $40,000(5,120)$ & 3.12 & Gao et al. 2016 \\
\hline Deep-sea hydrothermal vent & fosmid (35) & $18,000(7,390)$ & 11.1 & Fu et al. 2015 \\
\hline Paper mill sludge & plasmid (5.1) & $15,000(13,870)$ & 170 & Jia et al. 2019 \\
\hline Activated sludge & plasmid (5.1) & $3,818(12,3140)$ & 616 & Liaw et al. 2010 \\
\hline Activated sludge & plasmid (2.5) & $40,000(1,24)$ & 10.0 & Shao et al. 2013 \\
\hline Solar saltern & fosmid (35) & $51,00(1,200)$ & 5.60 & Jayanath et al. 2018 \\
\hline Oil field soil & plasmid (3.9) & $83,000(1,12)$ & 3.09 & Fan et al. 2011 \\
\hline
\end{tabular}

${ }^{a}$ This information is not specified in the reference

${ }^{b}$ Except compost metagenomic libraries, only those included the full library information were listed. 
Other studies further suggested that samples subjected to specific enrichment processes, such as composting and waste treatment procedures, usually resulted in a high hit rate (Mayumi et al. 2008; Kang et al. 2011; Popovic et al. 2017).

The insert sizes of the recovered plasmids ( 250 in total) with a confirmed phenotype ranged from 1,038 to $12,587 \mathrm{bp}$. In all inserts, at least one putative gene showing similarities to known genes encoding lipolytic enzymes was detected. In total, 210 and 60 lipolytic genes were identified from compost55 and compost76 libraries, respectively. To identify unique and full-length LEs, the amino acid sequences deduced from the corresponding lipolytic genes were clustered at $100 \%$ identity. This resulted in 115 (92 for compost55, 23 for compost 76, with 7 shared by both samples) unique and full-length LEs (Supplementary Table S8). The length of the unique LEs ranged from 223 to 707 amino acids, with calculated molecular masses from 23.9 to $72.3 \mathrm{kDa}$ (Supplementary Table S9). Forty of these showed the highest similarity to esterases/lipases from uncultured bacteria, and one (EstC55-13) to an enzyme from an uncultured archaeon. Among them, seven LEs showed the highest identity (53 to $65 \%$ ) to lipolytic enzymes obtained during function-based screening of metagenomes derived from marine sediment (Hu et al. 2010b), forest topsoil (Lee et al. 2004), mountain soil (Ko et al. 2012), activated sludge (Liaw et al. 2010), wheat field (Stroobants et al. 2015) and compost (Okano et al. 2015). In the remaining 34 cases, the matching esterases/lipases were mainly detected by sequence-based metagenomic surveys of composts (15 LEs), soil (7 LEs), marine sediment (6 LEs) and marine water (3 LEs).

\section{Functionally derived LEs are affiliated with various LE families}

The LEs identified through function-based screening were grouped into families based on the classification system reported by Arpigny and Jaeger (1999). With the increasing amount of reports on LEs, claims of new families have been reported (Arpigny and Jaeger 1999; Jeon et al. 2011; Wang et al. 2013; Esteban-Torres et al. 2014; Fang et al. 2014; Rahman et al. 2016; Castilla et al. 2017). In this study, we integrated 29 so-called "novel" families into the classification system for phylogenetic analysis. As shown in the phylogenetic tree (Figure 1), LEs were assigned to 12 families, including families I, II, III, IV, V, VII, VIII, XVII, EM3L4 (Lee et al. 2011), FLS18 (Hu et al. 2010b), EstGS (Nacke et al. 2011), LipT (Chow et al. 2012), patatin-like-proteins and tannases (Supplementary Table S8). The majority of the LEs were affiliated to families V (25 LEs), VIII (21 LEs), IV (15 LEs), I (8 LEs) and patatin-like-proteins (9 LEs). Noteworthy, 7 LEs could not be classified into any known lipolytic family, indicating new branches of LEs. In agreement with previous studies (Arpigny and Jaeger 1999; Glogauer et al. 2011; Akmoussi-Toumi et al. 2018), the "true lipases", which can hydrolyze long-chain substrates ( $\geq \mathrm{C} 10$ ) were all affiliated to family I (Figure 1). The remaining LEs exhibiting a preference for short-chain substrates $(<\mathrm{C} 10)$ were esterases.

To verify the classification result, a protein sequence similarity network was built (Figure 1). The network visualizes relationships among evolutionarily related proteins and is usually considered as an approach complementary to the phylogenetic analysis (Atkinson et al. 2009; Gerlt et al. 2015). At a threshold of 1x10-16, the network produced clusters that almost matched all the lipolytic families, with the same classification results as obtained by phylogenetic analysis (Figure 1).

Multiple sequence alignments revealed the catalytic residues and conserved motifs in each family (Supplementary Figure S6). For LEs that harbor the canonical $\alpha / \beta$-hydrolase fold, the catalytic triad is consistently composed of a nucleophilic serine, an aspartic acid/glutamic acid and a histidine residue (Nardini \& Dijkstra, 1999). Most of these LEs contain the conserved motif Gly-x-Ser-x-Gly in which the catalytic serine is embedded (Supplementary Figure S6). Alternatively, three LEs in family I show variations of this conserved motif. The variations were Ala-x-Ser-x-Gly, Thr-x-Ser-x-Gly (Diamond et al. 2019) and Ser-x-Ser-x-Gly (Dalcin Martins et al. 2018) (Supplementary Figure S6).

Family II LEs share a canonical $\alpha / \beta / \alpha$-hydrolase fold, which is characterized by a conserved hydrophobic core consisting of five $\beta$-strands and at least four $\alpha$-helices (Akoh et al. 2004). As shown in Supplementary Figure 6a, there are four homology blocks and one conserved residue in each block (serine, glycine, asparagine, and histidine, respectively), which is essential for catalysis (Akoh et al. 2004; Hong et al. 2008). The structures of family VIII enzymes show remarkable sequence similarities to $\beta$-lactamases and penicillin-binding proteins (Bornscheuer 2002). Site-directed mutagenesis demonstrated that the catalytic triad is composed of serine and lysine located in a Ser-X-X-Lys motif, and a tyrosine (Supplementary Figure 6) (Biver and Vandenbol 2013; Kovacic et al. 2019). The patatin-like-proteins display an $\alpha / \beta / \alpha$-hydrolase fold, in which a central six-stranded beta-sheet is sandwiched between alpha-helices front and back (Banerji and Flieger 2004). Unlike the catalytic triad of Ser-Asp/Glu-His for most lipolytic proteins, the catalytic Ser-Asp dyad is responsible for the catalytic activity of patatin-likeproteins. In addition, they also contained the Gly-x-Ser-x-Gly motif with the catalytic serine embedded (Supplementary Figure 6). 


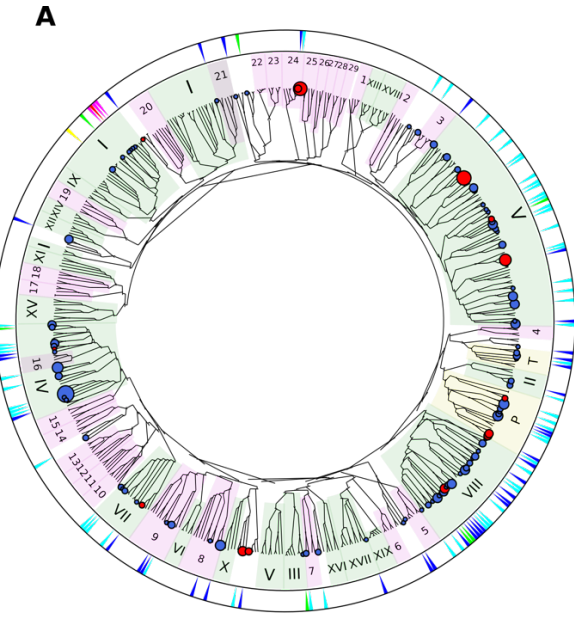

Inner tree: putative lipolytic proteins derived through function-driven screening

Outer ring: substrate specificity towards triglycerides $\begin{array}{lll}\nabla \text { Tributyrin (C4) } & \nabla \text { Tricaproin (C6) } & \nabla \text { Tricaprylin (C8) } \\ \nabla \text { Tricaprin (C10) } & \nabla \text { Trilaurin (C12) } & \nabla \text { Trimyristin (C14) }\end{array}$

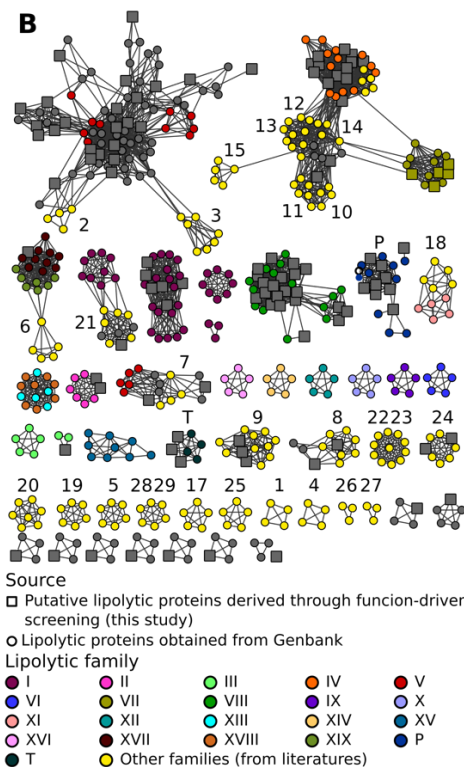

Figure 1. Classification of LEs identified through the function-driven approach. A, Unrooted phylogenetic tree was constructed using FA-identified LEs in this study obtained and references retrieved from GenBank (Supplementary Table S2). Phylogenetic tree was constructed using MEGA 7 with neighbor-joining method. The robustness of the tree was tested by bootstrap analysis with 500 replications. Inner tree: the circles represent LEs detected in compost55 (blue) and compost76 (red), sized by abundance (counts of replicates). LEs assigned to families of I-XIX were shaded in green background. Patatin-like-proteins and tannases (designated as P and T, respectively) were shaded in yellow. Other recent reported lipolytic families were shaded in magenta: 1, Est22 (Li et al. 2017); 2, EstL28 (Seo et al. 2014); 3, Rv0045c (Guo et al. 2010); 4, EstGX1 (Jiménez et al. 2012); 5, EstLiu (Rahman et al. 2016); 6, EstY (Wu and Sun 2009); 7; EstGS (Nacke et al. 2011); 8, EM3L4 (Lee et al. 2011); 9, FLS18 (Hu et al. 2010b); 10, Est903 (Jia et al. 2019); 11, EstJ (Choi et al. 2013); 12, PE10 (Jiang et al. 2012); 13, Est12 (Wu et al. 2013); 14, EstDZ2 (Zarafeta et al. 2016); 15, Est9x (Jeon et al. 2009); 16, Lip10 (Guo et al. 2016); 17, EstGH (Nacke et al. 2011); 18, EML1 (Jeon et al. 2009); 19, FnL (Yu et al. 2010); 20, EstP2K (Ouyang et al. 2013); 21, LipA (Couto et al. 2010); 22, LipSM54 (Li et al. 2016); 23, MtEst45 (Lee 2016); 24, LipT (Chow et al. 2012); 25, EstSt7 (Wei et al. 2013); 26, Rlip1 (Liu et al. 2009); 27, EstA (Chu et al. 2008); 28, FLS12 (Hu et al. 2010b); 29, lp_3505 (Esteban-Torres et al. 2014). Outer ring: substrate specificity of corresponding clones towards different carbon chain length $(\mathrm{C} 4-\mathrm{C} 14)$ of triglycerides. B, Protein sequence similarity network of LEs belonging to different families. Networks were generated from all-by-all BLAST comparisons of amino acid sequences from the same dataset used for the construction of the phylogenetic tree. Each node represents a sequence. Larger square nodes represent LEs derived from function-based screening performed in this study. Small circle nodes represent LEs retrieved from GenBank. Nodes were arranged using the $y$ Files organic layout provided in Cytoscape version 3.4.0. Each edge in the network represents a BLAST connection with an E-value cutoff of $\leqslant 1 \mathrm{e}^{-16}$. At this cut-off, sequences have a mean percent identity and alignment length of $36.3 \%$ and 273 amino acids, respectively.

\section{Development of a LE profile HMM database for sequence-based screening}

Profile HMMs are statistical models that convert patterns, motifs and other properties from a multiple sequence alignment into a set of position-specific hidden states, i.e. frequencies, insertions, and deletions (Reyes et al. 2017). Profile HMMs are sensitive in detecting remote homologs. Thus, they have been utilized to detect, e.g. viral protein sequences (Skewes-Cox et al. 2014; Bzhalava et al. 2018), antibiotic resistance genes (Gibson et al. 2015), GDSL esterase/lipase family genes ( $\mathrm{Li}$ et al. 2019) in metagenomes.

In this study, a total of 32 ELFs were determined for profile HMM database construction (Supplementary Table S10). Subsequently, four profile HMM databases (Omega-phmms, Muscle-phmms, Clustalw-phmms, ESTHERphmms) specific for LEs affiliated to a/ $\beta$ hydrolase superfamily were constructed. Each database consists of 32 profile HMMs (Supplementary Table S11). The prediction sensitivity and specificity of the four databases were evaluated using four datasets (Table 2). All of the four databases obtained high recalls for the datasets 1, 2 and 3 (Table 2), with the highest ones for omega-pHMMs $(4,446$ in total), followed by muscle-pHMMs $(4,444)$, clustalw-pHMMs $(4,425)$ and ESTHER-pHMMs $(4,425)$. Noteworthy, omega-pHMMs did not identify any false 
positive LEs for dataset 3. Thus, omega-pHMMs was chosen for downstream screening. In addition, we compared omega-pHMMs with the pairwise sequence alignment method (BLASTp) for their ability to predict LEs. The omega-pHMM database exhibited improved sensitivity for datasets 1, 2, and 3. In total, 135 more LEs were identified using omega-pHMMs than BLASTp (Table 2).

Table 2 Comparison of profile HMM databases based on different alignment tools to detect LEs

\begin{tabular}{|c|c|c|c|c|c|c|c|c|}
\hline \multirow{2}{*}{ Datasets $^{\mathrm{a}}$} & \multirow{2}{*}{$\begin{array}{l}\text { Nr. of LEs } \\
(\alpha / \beta \\
\text { hydrolase })\end{array}$} & \multirow{2}{*}{$\begin{array}{l}\text { Nr. of LEs } \\
\text { (non- } \alpha / \beta \\
\text { hydrolase) }\end{array}$} & \multicolumn{5}{|c|}{ Recall of LEs ( $\alpha / \beta$ hydrolase) } & \multirow{2}{*}{$\begin{array}{l}\text { Recall of LEs } \\
\text { (non- } \alpha / \beta \\
\text { hydrolase) }\end{array}$} \\
\hline & & & $\begin{array}{l}\text { Omega- } \\
\text { phmms }\end{array}$ & $\begin{array}{l}\text { Muscle- } \\
\text { phmms }\end{array}$ & $\begin{array}{l}\text { Clustalw- } \\
\text { phmms }\end{array}$ & $\begin{array}{l}\text { ESTHER- } \\
\text { phmms }\end{array}$ & BLASTp & \\
\hline Dataset 1 & 4382 & 554 & 4243 & 4244 & 4228 & 4225 & 4122 & 554 \\
\hline Dataset 2 & 130 & 32 & 125 & 125 & 121 & 124 & 117 & 32 \\
\hline Dataset 3 & 80 & 36 & 78 & 75 & 76 & 76 & 70 & 36 \\
\hline Dataset 4 & 68 & 0 & 56 & 55 & 53 & 53 & 51 & 0 \\
\hline
\end{tabular}

${ }^{a}$ Dataset 1, LEs from Uniprot database; Dataset 2, recently reported LEs; Dataset 3, MetaGeneMark-predicted proteins from inserts conferring lipolytic activity; Dataset 4, potential non-lipolytic proteins with homology to LEs.

The accuracy of omega-pHMMs for lipolytic family assignment was also assessed. For datasets 2 and 3, we achieved high precision of annotating LEs to the known lipolytic families, with the exception of LEs from novel families (Supplementary Table S12). Dataset 4 included non-lipolytic proteins, such as epoxide hydrolases, dehalogenases and haloperoxidases and exhibited significant homology with LEs in subfamilies V.1 and V.2 (Arpigny and Jaeger 1999). Our "homology-based" method only differentiated part of these non-lipolytic homologies from "true" LEs (Table 2).

To improve the annotation accuracy, putative lipolytic proteins (PLPs) were further searched against the entire ESTHER database by BLASTp. By combining the annotations from both methods (Supplementary Figure 1), these "novel" LEs in datasets 2 and 3 were correctly identified as "unassigned", in terms of not assigned to any known ELF (Supplementary Table S12). Moreover, almost all of the non-lipolytic proteins (> 92 \%) in dataset 4 were distinguished from LEs (Supplementary Table S12).

To identify LEs affiliated to families VIII and II, and patatin-like proteins, enzymes were successively screened against pfam-pHMMs and CATH HMMs database. For the first three datasets, all the LEs in the three families were correctly identified by screening against pfam-pHMMs (Table 2, Supplementary Table S13).

As demonstrated in other sequence-based metagenomic approaches (Liu et al. 2015b; Maimanakos et al. 2016; Azziz et al. 2019), our screening strategy is also vastly dependent on the completeness and accuracy of the reference databases (ESTHER and CATH database in this study). Hence, PLPs exhibiting closest similarity to members affiliated to the miscellaneous ESTHER families or no ESTHER/CATH hits returned, were classified into the "unassigned" group in this study (Supplementary Table S12). This might have resulted in an underestimation of assigned lipolytic proteins (Supplementary Table S13).

\section{Sequence-based screening confirmed compost metagenomes as reservoir for putative lipolytic genes}

Initial screening of the assembled metagenomes of compost55 and compost76 resulted in the identification of 4,157 and 2,234 PLPs, respectively. Among them, 1,234 and 759 were further assigned into 28 and 26 families, respectively. The assigned PLPs belonged mainly to family VIII, hormone-sensitive lipase-like proteins, patatinlike proteins, II, A85-Feruloyl-Esterase, Carb_B_Bacteria and homoserine transacetylase (Supplementary Figure S7). The family assignment was also verified by constructing a protein sequence similarity network (supplementary Figure S8). The large number of unassigned PLPs (2,460 for compost55 and 1,208 for compost76) indicated the presence of candidates for novel lipolytic families.

The assigned PLPs were generally of bacterial origin ( $>95 \%)$, and mainly affiliated to the phyla ( $>5 \%)$ Actinobacteria, Proteobacteria, Firmicutes and Bacteroidetes (Figure 2). The corresponding contigs were also taxonomically assigned and exhibited a similar phylogeny as seen for the embedded PLP-encoding gene sequences (Figure 2). 


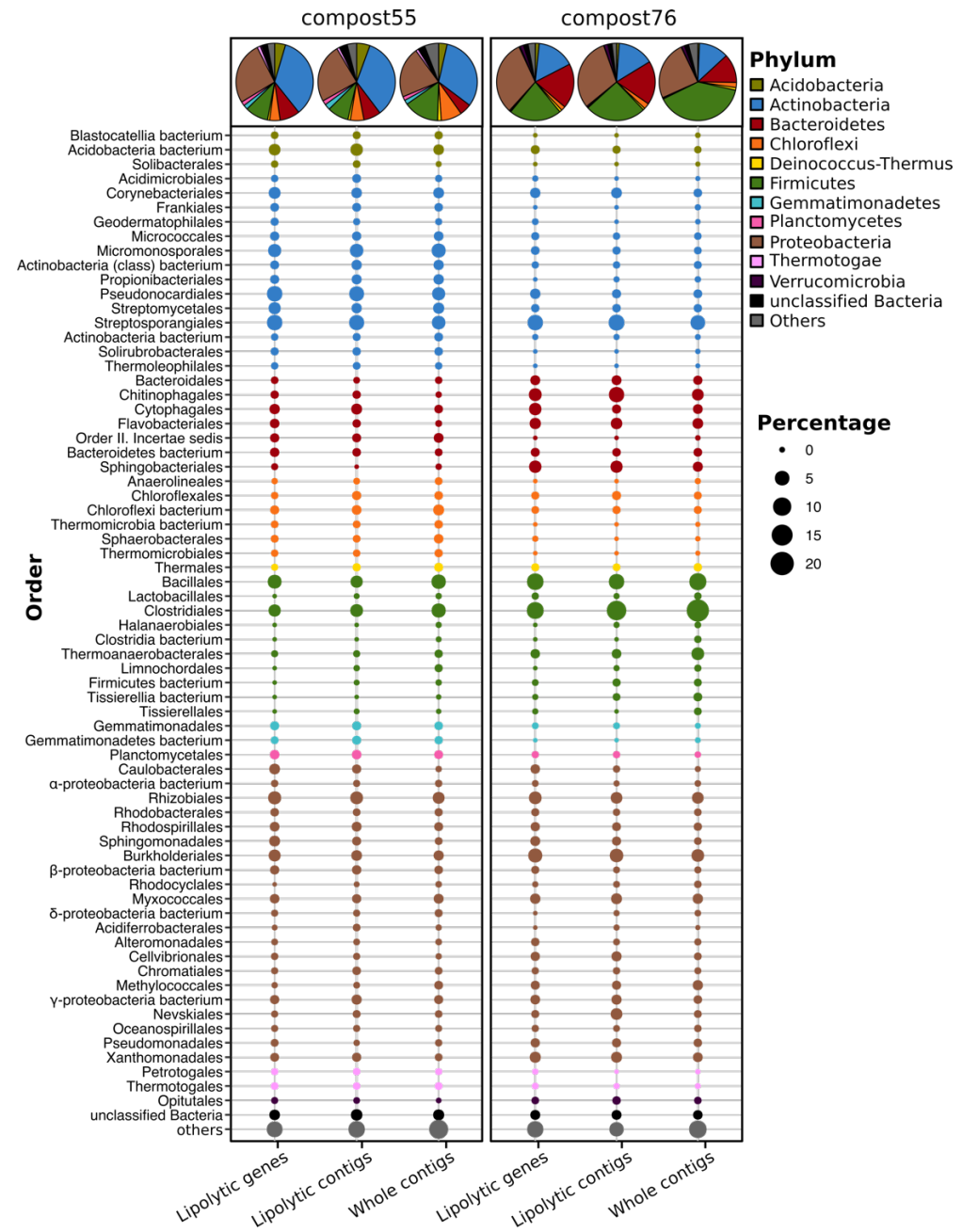

Figure 2. Phylogenetic distribution of assigned PLP-encoding genes identified in compost55 and compost76 metagenomes. The phylogenetic origin of PLP-encoding genes, the contigs harboring these genes, and the whole assembled contigs were annotated by Kaiju (Menzel et al. 2016), and expressed as the proportion of the respective total counts in each sample. The pie charts represent the taxonomic composition at phylum level. Taxa with an abundance of less than $1 \%$ were grouped into "others".

Members of the Actinobacteria have been reported as important biomass degraders (Ryckeboer et al. 2003; Hubbe et al. 2010; Wang et al. 2016; Lewin et al. 2016). In this study, 34.7 (compost55) and $15.8 \%$ (compost76) of the assigned PLPs originated from Actinobacteria. At genus level, the assigned PLPs were affiliated to Mycobacterium, Actinomadura, Thermomonospora, Streptomyces, Micromonospora, Pseudonocardia and Thermobifida (Supplementary Table S14). Members of these genera have been reported as producers for lipases/esterasses (Wei et al. 1998; Alisch et al. 2004; Chahinian et al. 2005; Guo et al. 2010; Hu et al. 2010a; Brault et al. 2012; Mander et al. 2014; Sriyapai et al. 2015). Moreover, some of the corresponding families, such as Micromonosporaceae, Streptomycetaceae and Thermomonosporaceae, are commonly found in thermophilic composts (Schloss et al. 2003; Blaya et al. 2016; Lima-Junior et al. 2016).

Proteobacteria are also an abundant source for the assigned PLPs in compost55 (26.2 \%) and compost76 (31.4\%) (Figure 2). Popovic et al., (2017) identified 80 LEs, of which, $65 \%$ were proteobacterial origin by screening of 16 metagenomic DNA libraries prepared from seawater, soils, compost and wastewater. In our study, 
lipolytic genes exhibited high taxonomic diversity at genus level, they were distributed across 97 and 111 genera for compost55 and compot76, respectively (Supplementary Table S14).

The assigned PLPs affiliated to Firmicutes originated mainly from Clostridiales and Bacillales (Figure 2). By analyzing the microbial diversity and metabolic potential of compost metagenomes, members of Clostridiales and Bacillales were shown to play key roles in degradation of different organic compounds (Martins et al. 2013; Antunes et al. 2016). Bacteroidetes is the fourth most abundant phylum for assigned PLPs in compost55 (8.4\%) and compost76 (18.8 \%) (Figure 2). At genus level, the assigned PLPs derived mainly from Rhodothermus in compost55, and Sphingobacterium, Flavobacterium, Niastella and Flavihumibacter in compost76 (Supplementary Table S14). Members of these genera are known as important fermenters during composting (Neher et al. 2013; Antunes et al. 2016; Lapébie et al. 2019).

Strikingly, the phylogenetic distribution of assigned PLPs in each sample, to some extent, corresponded well to the taxonomic composition revealed from the whole contigs (Figure 2) but with minor differences in the rank abundance order. The 16S rRNA amplicon (Supplementary Figure S3) and metagenomic datasets (Supplementary Figure S4) also showed a composition of dominant orders similar to that deduced from lipolytic genes/contigs (Figure 2). Wang et al. (2016) revealed that the phylogenetic distribution of CAZyme genes in the rice strawadapted compost consortia was in accordance to its microbial composition. By mapping resistance gene dissemination between humans and their environment, Pehrsson et al. (2016) found that resistomes across habitats were generally structured by bacterial phylogeny along ecological gradients.

\section{Comparison between function-driven and sequence-based screening of LEs}

Metagenomics allows tapping into the rich genetic resources of so far uncultured microorganisms (Simon and Daniel 2011) through function-driven or sequence-based approaches. The function-driven strategy targets a particular activity of metagenomic library-bearing hosts (Ngara and Zhang 2018). In this way, we identified 13 novel LEs (Supplementary Table S9, Supplementary Figure S6b), which confirmed functional screening as a valuable approach for discovering entirely novel classes of genes and enzymes, particularly when the function could not be predicted based on DNA sequence alone (Reyes-Duarte et al. 2012; Lam et al. 2015; Villamizar et al. 2017).

The sequence-based screening strategy is also frequently used due to the easy access to a wealth of metagenome sequence data and continuous advances in bioinformatics (Chan et al. 2010; Liu et al. 2015c; Maimanakos et al 2016). Based on the ESTHER and Pfam database, our profile HMM-based search approach efficiently provided an overview of PLP distribution in the two compost metagenomes (Supplementary Figure S7). The hit rate for LEs was higher by sequence-based than by function-based screening, but the sequence-based derived hits need to be functionally verified. In addition, we noticed that only part of the functional screening-derived lipolytic genes were identified during sequence-based screening. By mapping the metagenomic short reads to the functional screening-derived lipolytic genes, 63 genes (out of 115 lipolytic genes in total) had a coverage of $100 \%$ and 88 of $\geqslant 99 \%$ (Supplementary Table S15). The BLAST-based comparison between lipolytic genes derived from function-driven and sequence-based approaches indicated that 31 genes from each approach exhibited $100 \%$ sequence identity, and 64 over 99\% identity (Supplementary Table S16).

In summary, function-driven and sequence-based strategies have advantages and disadvantages. The functiondriven screenings are generally constrained by factors, such as labor-intensive operation, limitations of the employed host systems and low hit rate (Simon and Daniel 2011). However, function-based approaches are activity-directed, and sequence- and database-independent, thus, they bear the potential to discover entirely novel genes for proteins of interest (Rabausch et al. 2013; Lam et al. 2015). Sequencing-based screening, on the other hand, is effective in identifying sequences and potential genes encoding targeted biomolecules in metagenomes. Sequence-based screens largely rely on the used search algorithms, and quality and content of the reference databases to infer the functions of discovered candidate genes (Ngara and Zhang 2018). Thus, the best way to explore novel molecules is to combine the two approaches (Barriuso and Jesús Martínez 2015). Function-driven screens can be employed to complete and verify reference database entries on which sequence-based screening is dependent on. In addition, sequence-based approaches can serve as a pre-selection step for function-driven screens and analysis (Chan et al. 2010; Masuch et al. 2015; Pehrsson et al. 2016; Streit et al. 2018).. The known novel LEs identified by function-based approaches and the functional enzymes identified in this study were employed to expand the LE-specific profile HMM database and annotate the PLPs derived from sequence-based screening.

\section{Assigned PLPs are distributed by ecological factors}

In this study, 175 metagenomes representing various ecology niches were selected for sequence-based searching of PLPs. In total, we have screened approx. 1.23 billion genes in $65 \mathrm{Gbp}$ of assembled metagenomes and recovered approx. 0.22 million (absolute counts) PLP-encoding genes. The assigned PLPs ( $34 \%$ of the total counts) were normalized to LPGM values for comparative analysis. In accordance with the function-based screening, samples subjected to certain enrichment processes, particularly lipid-related, tend to have a higher hit rate (Figure 3). For example, samples with high LPGM values were derived from a hydrocarbon resource environment and oil 
reservoir that are enriched with oil-degrading microbes (Liu et al. 2015a; Hu et al. 2016; Vigneron et al. 2017; Liu et al. 2018), and composts and wastewater bioreactors that are reservoir for microbes decomposing organic compounds (Dougherty et al. 2012; Silva et al. 2012; Antunes et al. 2016; Berini et al. 2017). Intriguingly, samples from human gut systems were also candidates for LEs (LPGM values > 7500). The human intestinal microorganisms play an import role in degrading diet components into metabolizable molecules (Wang et al. 2015). The function- and sequence-based study of human gut metagenomes have proved that the human gut microbiome is a rich source for various carbohydrate active enzymes ( $\mathrm{Li}$ et al. 2009; Turnbaugh et al. 2009; Tasse et al. 2010; Moore et al. 2011).

Overall, the assigned PLPs were classified into 34 lipolytic families (Fig 3). Members of the Hormonesensitive lipase like and patatin-like-protein families were most abundant (average LPGM values across samples > 2000), followed by families of A85-EsteraseD-FGH, VIII and Bacterial_lip_FamI.1 (average LPGM values > 700) (Fig 3). However, no family was shared by all samples. Nevertheless, members from families of Hormonesensitive-lipase-like, patatin-like-proteins, VIII, homoserine transacetylase, II and A85-Feruloyl-Esterase were detected in more than $90 \%$ of samples (Figure 3). Enzymes belonging to families of PHAZ7_phb_depolymerase, Bact LipEH166 FamXII and Bacterial lip FamI.2 were not or only rarely detected $(<6 \%$ of all samples) and showed a low abundance (LPGM values $<1$ ). The prevalence and abundance of a lipolytic family revealed by the sequence-based screening are dependent on the distribution of corresponding target genes in the microbial consortia (Wang et al. 2016). Taking members from the "abundant" family Hormone-sensitive_lipase like as example, the corresponding genes are widely distributed in more than 1,200 species as recorded in the ESTHER database so far. This was, somehow, also reflected by the function-based screening, in which a large proportion of the identified LEs belonged to family Hormone-sensitive_lipase_like. In contrast, according to the ESTHER database, only 23, 8 and 6 species harboring LEs were affiliated to the "rare" families like PHAZ7_phb_depolymerase, PC-sterol_acyltransferase and Bact_LipEH166_FamXII, respectively (Supplementary Figure S12).

To investigate the distribution of assigned PLPs that cause the observed lipolytic family profiles across samples and habitats, a matrix with LPGM values representing the abundance of PLPs per lipolytic family identified in each metagenome was generated. The lipolytic family profiles clustered by habitats (Figure 3), which was confirmed by NMDS (stress level 0.2268; Supplementary Figure S9). ANOSIM (Clarke 1993) was used to pairwise compare the multivariate (group) differences of lipolytic family profiles between habitats. A R valuebased matrix was generated among habitats (Supplementary Figure S9), a high R value (between 0 to 1 ) indicated a high group dissimilarity between two habitats. Generally, each habitat exhibited a distinctive pattern of lipolytic family profiles (overall $\mathrm{R}$ value $=0.6168$; Supplementary Table S17). For example, PLPs detected in agricultural soils were only present in eight lipolytic families with low abundances. In contrast, PLPs in composts were detected in almost all lipolytic families, and with remarkably high abundance in families such as Hormonesensitive lipase like, patatin-like-protein and VIII (Supplementary Figure S10). Notably, the lowest group dissimilarity was observed between the habitats compost and wastewater bioreactor $(\mathrm{R}=0.1941, \mathrm{P}<0.001$, ANISOM; Supplementary Figure S9). The analysis of lipolytic profiles across habitats allows selecting suitable habitats for function-based screening, e.g. targeting LEs of a specific family or with some properties for desired applications. Metagenomes from composts are promising for recovering LEs in families LYsophospholipase_carboxylesterase (family VI), CarbLipBact_2 (family XIII-2/XVIII) and CarbLipBact_1 (family XIII-1) (Supplementary Figure S11). 


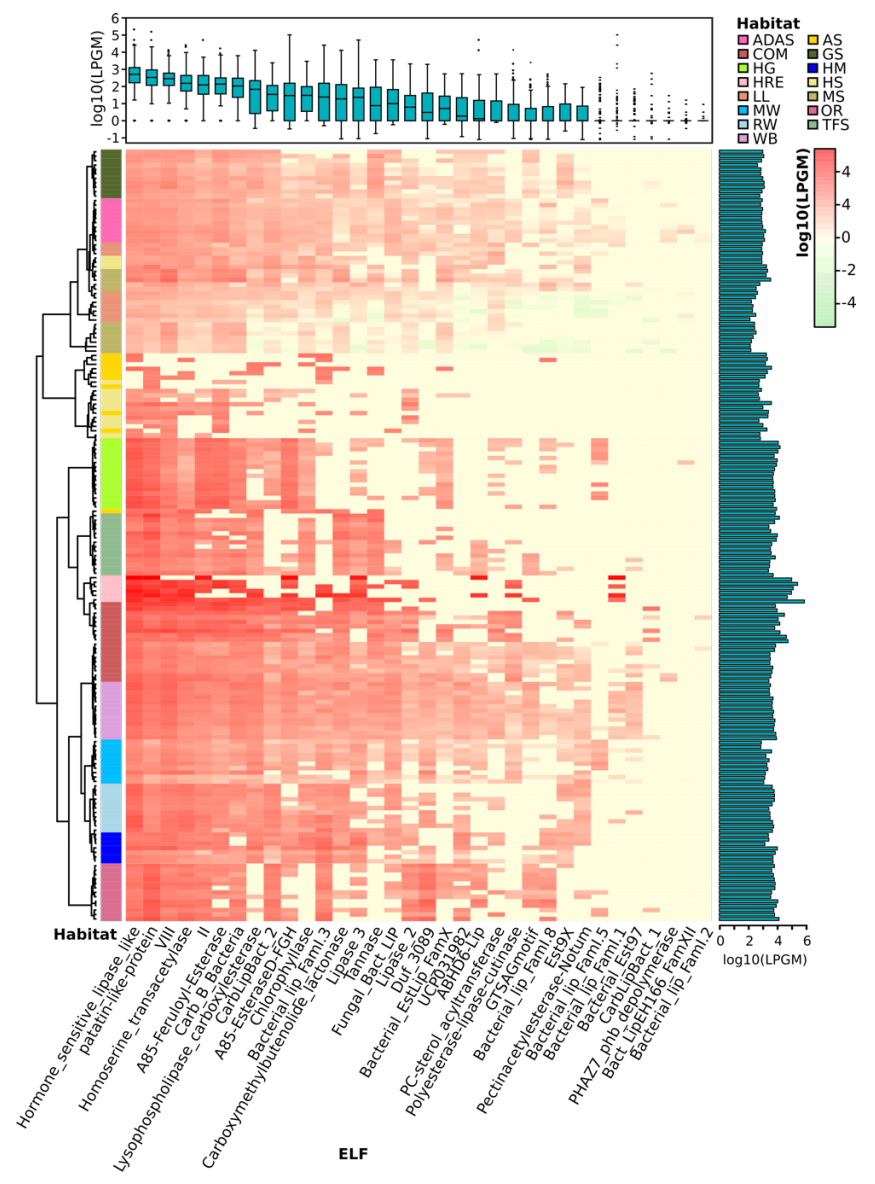

Figure 3. Lipolytic family profile of assigned PLPs across samples. Hierarchical clustering analysis of the lipolytic family profile in each sample was performed using the Ward.D clustering method and Bray-Curtis distance matrices. LPGM values were $\log _{10}$ transformed. The color intensity of the heat map (light green to red) indicates the change of LPGM values (low to high). The habitats are depicted by different colors. The lipolytic family profile in each sample was generally clustered by habitat (overall $\mathrm{R}$ value $=0.621, \mathrm{P}<0.001$, ANOSIM test). The boxplot (top) represents the distribution of the assigned PLPs in each ELF across samples. Mean values ( $\mathrm{n}=175$ samples) are given. The bar plot (right) shows the total abundance of assigned PLPs by summing up the abundance in each family of each sample. Abbreviations of habitats: ADAS, anaerobic digestor active sludge; AS, agricultural soil; COM, compost; GS grassland soil; HG, human gut; HM, hypersaline mat; HRE, hydrocarbon resource environment; HS, hot spring; LL, landfill leachate; MS, marine sediment; MW, marine water; OR, oil reservoir; $\mathrm{RW}$, river water; TFS, tropical forest soil; WB, wastewater bioreactor; ELF, ESTHER lipolytic family.

\section{The phylogenetic distribution of assigned PLPs}

More than $98 \%$ of the assigned PLPs were encoded by bacterial community members. Although LEs are widely encoded in various microbial genomes (Hausmann and Jaeger 2010; Ramnath et al. 2016; Kovacic et al. 2019a), the assigned PLPs were mainly derived from the bacterial phyla Proteobacteria $(66.5 \%)$, Bacteroidetes $(12.5 \%)$, Actinobacteria (7.7 \%), Firmicutes $(6.7 \%$ ) (Figure 4). This is consistent with the taxonomic origin of reference LEs in ESTHER database (Supplementary Figure S12). Moreover, enzymes from members of Proteobacteria were dominant in almost all lipolytic families (Figure 4). At genus level, the phylogenetic origins of assigned PLPs were scattered across approx. 2,000 bacterial genera, with enriched abundance in the genera Acinetobacter, Pseudomonas, Bacteroides, Bradyrhizobium and Mycobacterium (average LPGM values across samples > 180). Many of the LEs from these genera were described as exoenzymes (Rudek and Haque 1976; Gilbert 1993; 
Snellman and Colwell 2004; Guo et al. 2010; Liu et al. 2018). Notably, a similar taxonomic enrichment at genus level was also observed for the reference LEs in ESTHER database as 960 LEs were encoded by Mycobacterium, 410 by Pseudomonas, 260 by Bacteroides, 166 by Acinetobacter, and 164 by Bradyrhizobium species (Supplementary Table S18).
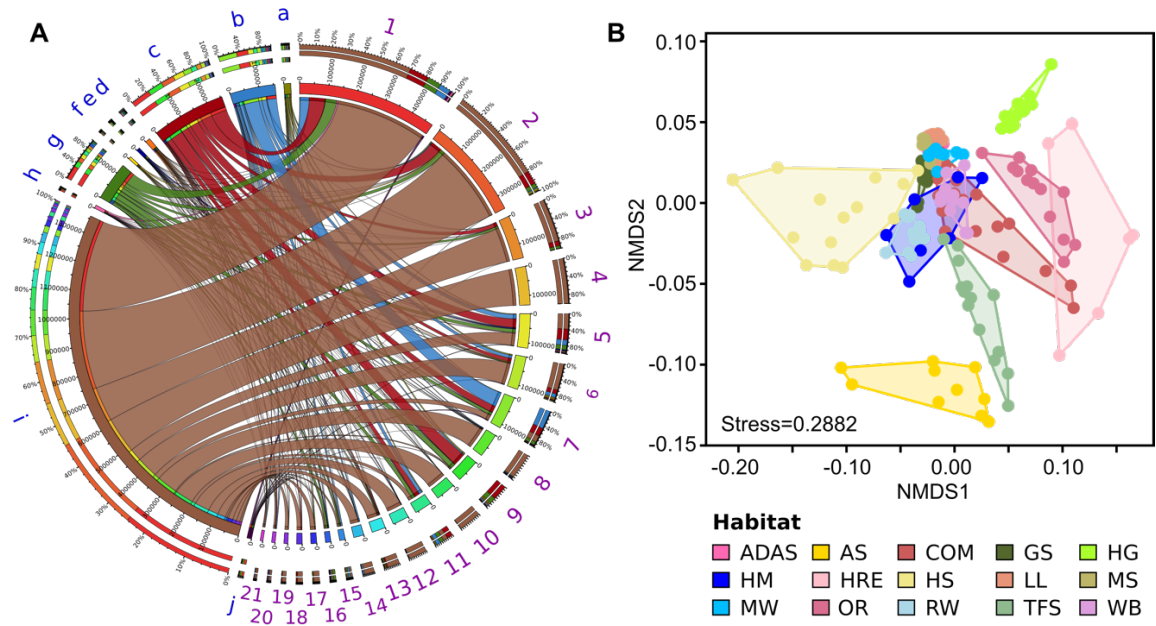

Figure 4. Phylogenetic distribution of assigned PLPs. A, phylogenetic distributions of assigned PLPs in abundant bacterial phyla possessing PLP-encoding genes across all the samples. The abundance inferred from LPGM values matrix of assigned PLPs per family identified in each bacterial phylum was generated by summing the corresponding LPGM values across all samples. The width of bars from each bacterial phylum and functional enzyme family indicates their relative abundances across all samples. a-j are bacterial phyla (in blue): a, Acidobacteria; b, Actinobacteria; c, Bacteroidetes; d, Chloroflexi; e, Cyanobacteria; f, Deinococcus-Thermus; g, Firmicutes; h, Planctomycetes; i, Proteobacteria; j, Verrucomicrobia. 1-21 are lipolytic families (in purple): 1, Hormone-sensitive_lipase_like; 2, patatin-like-protein; 3, A85-EsteraseD-FGH; 4, Bacterial_lip_FamI.1; 5, VIII; 6. Homoserine transacetylase; 7, II; 8, Lipase_3; 9, A85-Feruloyl-Esterase; 10, ABHD6-Lip; 11, Carb_B_Bacteria; 12, Bacterial lip FamI.3; 13, Lysophospholipase_carboxylesterase; 14, Carboxymethylbutenolide_lactonase; 15 , CarbLipBact_2; 16, Chlorophyllase; 17, Tannase; 18, Polyesterase-lipase-cutinase; 19, Duf_3089; 20, Fungal_Bact_LIP; 21, Lipase_2. Only phyla and lipolytic families with a relative abundance $>0.5 \%$ are shown. B, Non-metric multidimensional scaling (NMDS) analysis of phylogenetic distribution of assigned PLPs across samples based on Bray-Curtis distances at bacterial genus level. Only genera with a mean LPGM values of $\geqslant 0.5$ across all the samples were included. Abbreviations of habitats: ADAS, anaerobic digestor active sludge; AS, agricultural soil; COM, compost; GS grassland soil; HG, human gut; HM, hypersaline mat; HRE, hydrocarbon resource environment; HS, hot spring; LL, landfill leachate; MS, marine sediment; MW, marine water; OR, oil reservoir; RW, river water; TFS, tropical forest soil; WB, wastewater bioreactor; ELF, ESTHER lipolytic family.

The taxonomic origin of assigned PLPs at genus level varied significantly across habitats (overall $\mathrm{R}$ value = $0.821, \mathrm{P}<0.01$ ), especially for the human gut system, oil reservoir and hydrocarbon resource environment (Supplementary Figure S14). The average R value was 0.98, 0.97 and 0.94, respectively (Supplementary Table $\mathrm{S} 19)$. The lowest dissimilarity was observed between compost and wastewater bioreactor $(\mathrm{R}$ value $=0.2317, \mathrm{P}$ $<0.001$, ANISOM).

\section{Habitats harboring prevalent and distinct microbial clusters are main drivers of PLP distribution}

Bipartite association networks have been used to identify microbial taxa responsible for shifts in community structures (Hartmann et al. 2015; Dukunde et al. 2019). In this study, a bipartite association network was constructed to visualize the associations between bacterial members at genus level that harbor lipolytic genes and habitats or habitat combinations (Figure 5). 225 of the total 712 genera, were not significantly separated in abundance and frequency by habitat. These belonged mainly to Proteobacteria ( 82 genera), Bacteroidetes (43 genera), Firmicutes (33 genera), and Actinobacteria (25 genera) (Supplementary Table S20). These nonsignificant genera were conserved across different habitats, generally represented the "indigenous group" (Hartmann et al. 2015; Wemheuer et al. 2017), and formed the core microbiota harboring lipolytic genes. This core microbiota was also an indication of the prevalence of lipolytic genes across microbes and habitats (Bornscheuer 2002; Hasan et al. 2006; Barriuso and Jesús Martínez 2015; Berini et al. 2017). In contrast, the 
significant indicators, with respect to the "characteristic group" (Rime et al. 2016; Dukunde et al. 2019), highlighted the bacterial genera that were responsible for the change of assigned PLPs distribution across habitats (Figure 5). Particularly, the indicators associated with only one habitat defined the distinctiveness of microbiota in each habitat (Hartmann et al. 2015). In this study, the unique-associated indicators accounted for $76 \%$ of all significant indicators (Supplementary Table S20). This strongly resembled the ANISOM result, in which the high overall $\mathrm{R}$ value $(0.8199)$ suggested a significant distinctiveness of the phylogenetic origins of assigned PLPs across habitats (Supplementary Table S19). With respect to each habitat, a high ratio of unique-associated indicators to the total significant genera in a habitat generally indicated a high $\mathrm{R}$ value (Pearson's $\mathrm{r}$ correlation $=$ $0.6672, \mathrm{P}<0.01$, linear regression; Supplementary Figure S15). For example, out of the 75 indicators that were significantly associated to the habitat hydrocarbon resource environment, 65 were unique-associated indicators, with a mean R value of 0.93 (Supplementary Table 19). This is also the case for the habitats oil reservoir ( 60 out of 75 ; mean $R$ value $=0.96)$ and human gut system $(35$ out of 41 ; mean $R$ value $=0.97)$.

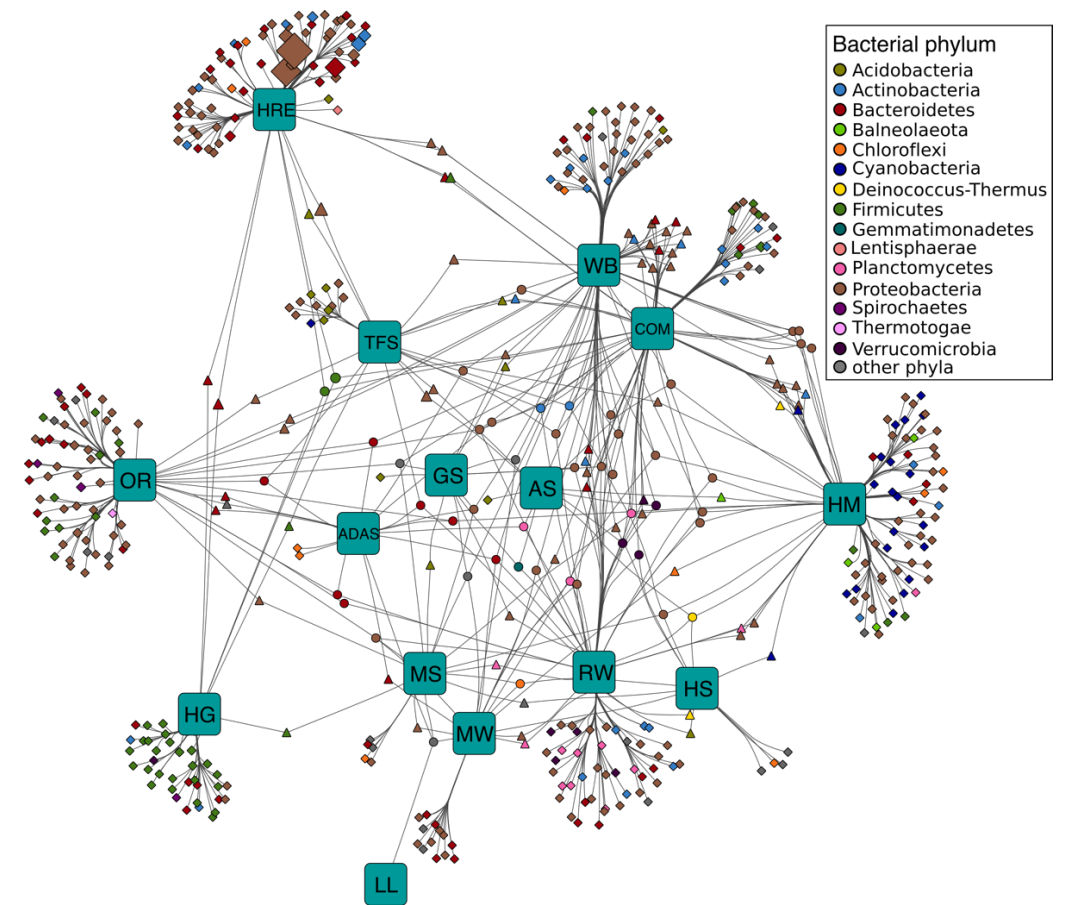

Figure 5. Association networks between bacterial origin of assigned PLPs at genus level and habitats. The abundance of PLPs in each genus per sample was presented by LPGM values, and only genera with mean LPGM values of $\geqslant 0.5$ across all the samples were used. Source nodes (rounded squares) represent habitats, target node represent bacterial genera (circles, diamonds and triangles), and edges represent associations between habitats and bacterial genera. Target node size represent its mean abundance inferred from LPGM values across habitats. Target node is colored according to its phylogenetic origin at phylum level. The length of edges is weighted according to association strength. Unique clusters, which associate with only one habitat, consist of nodes shaped as diamond. Triangle and circle nodes represent genera with significant cross association between two and more habitats, respectively. Data only represents genera that showed significant positive association with habitats $(\mathrm{P}=0.05)$. For ease of visualization, edges were bundled together, with a stress value of 3. Abbreviations of habitats: ADAS, anaerobic digestor active sludge; AS, agricultural soil; COM, compost; GS grassland soil; HG, human gut; HM, hypersaline mat; HRE, hydrocarbon resource environment; HS, hot spring; LL, landfill leachate; MS, marine sediment; MW, marine water; OR, oil reservoir; RW, river water; TFS, tropical forest soil; WB, wastewater bioreactor; ELF, ESTHER lipolytic family.

Only a small fraction of the indicators exhibited cross associations between two ( $14 \%$ of the total indicators) or more $(10 \%)$ habitats. Nevertheless, the 29 cross-associated indicators between habitats compost and wastewater bioreactor explained the low dissimilarity of phylogenetic distributions of assigned PLPs between the two habitats $(\mathrm{R}=0.2317, \mathrm{P}<0.001$, ANISOM $)$. 
Similar to the "indigenous group", the "characteristic group" consisted mainly of genera affiliated to Proteobacteria (224 genera), Bacteroidetes (72), Firmicutes (49) and Actinobacteria (36). Among them, proteobacterial genera largely characterized the major habitats, such as tropical forest soil $(83 \%)$, wastewater bioreactor $(67 \%)$, hypersaline mat (52\%), hydrocarbon resource environment (51\%), oil reservoir (51\%), compost $(50 \%)$, marine water (46\%), river water (45\%), and grassland soil (42\%), whereas Bacteroidetes and Firmicutes characterized the human gut system $(68 \%)$ and the active sludge of an anaerobic digestor $(53 \%)$ (Supplementary Table S20). Noteworthy, the unique-associated indicators affiliated to Cyanobacteria were primarily enriched in the hypersaline mat (95\% indicators), which is also the case for Planctomycetes and Verrucomicrobia in river water ( 88 and $80 \%$, respectively). Pehrsson et al (2015) detected a link between microbial community structure and functional gene repertoire. This link could be extended to the distribution pattern of indicators in our study. For example, various studies have proved that the microbes in human gut systems were dominated by Firmicutes (Mahowald et al. 2009; Vital et al. 2014; Rinninella et al. 2019), which in turn leads to the Firmicutes-dominated indicators for lipolytic genes (Figure 5). Among all the habitats, only hypersaline mats were featured by the Cyanobacteria-dominated oxygenic layer for photosynthesis (Sørensen et al. 2005; Lindemann et al. 2013), which explained that almost all the Cyanobacteria indicators were associated with the hypersaline mat (Figure 5).

\section{Conclusions}

In this study, two compost samples (compost55 and compost76) were used for metagenomic screening of potential lipolytic genes. Through the function-driven screening, 115 unique LEs were identified and assigned into 12 known lipolytic families. In addition, 7 LEs were not assigned to any known family, indicating new branches of lipolytic families. Our results show that functional screening is a promising approach to discover novel lipolytic genes, particularly for targeted genes, whose function is not predicted based on DNA sequence alone. For sequence-based screening, we have developed a search and annotation strategy specific for putative lipolytic genes in metagenomes (Supplementary Figure 1). Our profile HMM-based searching methods yielded higher sensitivity (recall) for LEs than the BLASTp-derived counterpart. The annotation method also remarkably increased the specificity and accuracy in distinguishing lipolytic from non-lipolytic proteins. With this sequence-based strategy, we identified the putative lipolytic genes within the two compost metagenomes. Analysis of the phylogenetic origin of these genes indicated a potential link between microbial taxa and their functional traits. By comparing the lipolytic hits identified by function-driven and sequence-based screening, we conclude that the best way for exploring and exploiting LEs is to combine both approaches.

In addition, assembled metagenomes from samples of various habitats were used for comparative analysis of the PLP distribution. We profiled the lipolytic family and phylogenetic origin of assigned PLPs for each sample. The two profiles were generally driven by ecological factors, i.e. the habitat. Moreover, the habitat also determined the conserved and distinctive microbial groups harboring the putative lipolytic genes.

PLPs were also mainly enriched in the bacterial phyla Proteobacteria, Bacteroidetes, Actinobacteria, Firmicutes (Supplementary Figure S16). The profile of the phylogenetic total PLPdistribution in each sample clustered also by habitats (Supplementary Figures S17, S18 and S19). The bipartite association network identified the conserved and distinctive microbial groups harboring PLP-encoding genes among the habitats (Supplementary Tables S21 and S22). Thus, our study provided a sequence-based strategy for effective identification and annotation of potential lipolytic genes in assembled metagenomes. More importantly, through this strategy, the overview of how the lipolytic genes distributed ecologically (in various habitats), functionally (in different lipolytic enzyme families) and phylogenetically (in diverse microbial groups) is an advantage for novel and/or industrially relevant LE identification.

Supplementary Materials: The supplementary materials are also available online. The Supplementary Figures S1-S19 and the Supplementary Tables S1-S22 are presented in two files (Supplementary Figures S1-S19.pdf and Supplementary Tables S1-S22.xlsx, respectively)

Acknowledgments: We thank Dr. Silja Brady and Mechthild Bömeke for providing technical assistance.

Funding: We acknowledge the support of Mingji Lu by "Erasmus Mundus Action 2 - Lotus I Project". The funders had no role in study design, data collection, and interpretation, or the decision to submit the work for publication.

Conflicts of Interest: The authors declare that the research was conducted in the absence of any commercial or financial relationships that could be construed as a potential conflict of interest.

Authors Approvals: All authors have seen and approved the manuscript. The manuscript has not been accepted or published elsewhere. 


\section{References}

Akmoussi-Toumi S, Khemili-Talbi S, Ferioune I, Kebbouche-Gana S (2018) Purification and characterization of an organic solvent-tolerant and detergent-stable lipase from Haloferax mediterranei CNCMM 50101. Int J Biol Macromol 116:817-830 . doi: 10.1016/j.ijbiomac.2018.05.087

Akoh CC, Lee GC, Liaw YC, Huang TH, Shaw JF (2004) GDSL family of serine esterases/lipases. Prog Lipid Res 43:534-552 . doi: 10.1016/j.plipres.2004.09.002

Alisch M, Feuerhack A, Müller H, Mensak B, Andreaus J, Zimmermann W (2004) Biocatalytic modification of polyethylene terephthalate fibres by esterases from actinomycete isolates. Biocatal Biotransformation 22:347-351 . doi: 10.1080/10242420400025877

Altschul SF, Gish W, Miller W, Myers EW, Lipman DJ (1990) Basic local alignment search tool. J Mol Biol 215:403-410 . doi: 10.1016/S0022-2836(05)80360-2

Antunes LP, Martins LF, Pereira RV, Thomas AM, Barbosa D, Lemos LN, Silva GMM, Moura LMS, Epamino GWC, Digiampietri LA, Lombardi KC, Ramos PL, Quaggio RB, De Oliveira JCF, Pascon RC, Da Cruz JB, Da Silva AM, Setubal JC (2016) Microbial community structure and dynamics in thermophilic composting viewed through metagenomics and metatranscriptomics. Sci Rep 6:38915 . doi: 10.1038/srep38915

Arpigny JL, Jaeger K-E (1999) Bacterial lipolytic enzymes: classification and properties. Biochem J 343:177183 . doi: 10.1042/0264-6021:3430177

Asnicar F, Weingart G, Tickle TL, Huttenhower C, Segata N (2015) Compact graphical representation of phylogenetic data and metadata with GraPhlAn. PeerJ 3:e1029 . doi: 10.7717/peerj.1029

Atkinson HJ, Morris JH, Ferrin TE, Babbitt PC (2009) Using sequence similarity networks for visualization of relationships across diverse protein superfamilies. PLoS One 4:e4345 . doi: 10.1371/journal.pone.0004345

Azziz G, Giménez M, Romero H, Valdespino-Castillo PM, Falcón LI, Ruberto LAM, Mac Cormack WP, Batista S (2019) Detection of presumed genes encoding beta-lactamases by sequence based screening of metagenomes derived from Antarctic microbial mats. Front Environ Sci Eng 13:44 . doi: 10.1007/s11783019-1128-1

Banerji S, Flieger A (2004) Patatin-like proteins: A new family of lipolytic enzymes present in bacteria? Microbiology 150:522-525 . doi: 10.1099/mic.0.26957-0

Bankevich A, Nurk S, Antipov D, Gurevich AA, Dvorkin M, Kulikov AS, Lesin VM, Nikolenko SI, Pham S, Prjibelski AD, Pyshkin A V., Sirotkin A V., Vyahhi N, Tesler G, Alekseyev MA, Pevzner PA (2012) SPAdes: A new genome assembly algorithm and its applications to single-cell sequencing. J Comput Biol 19:455-477 . doi: 10.1089/cmb.2012.0021

Barriuso J, Jesús Martínez M (2015) In silico metagenomes mining to discover novel esterases with industrial application by sequential search strategies. J Microbiol Biotechnol 25:732-737 . doi: 10.4014/jmb.1406.06049

Berglund F, Marathe NP, Österlund T, Bengtsson-Palme J, Kotsakis S, Flach CF, Larsson DGJ, Kristiansson E (2017) Identification of 76 novel B1 metallo- $\beta$-lactamases through large-scale screening of genomic and metagenomic data. Microbiome 5:134 . doi: 10.1186/s40168-017-0353-8

Berini F, Casciello C, Marcone GL, Marinelli F (2017) Metagenomics: novel enzymes from non-culturable microbes. FEMS Microbiol Lett 364: . doi: 10.1093/femsle/fnx211

Berlemont R, Spee O, Delsaute M, Lara Y, Schuldes J, Simon C, Power P, Daniel R, Galleni M (2013) Novel organic solvent-tolerant esterase isolated by metagenomics: insights into the lipase/esterase classification. Rev Argent Microbiol 45:3-12

Biver S, Vandenbol M (2013) Characterization of three new carboxylic ester hydrolases isolated by functional screening of a forest soil metagenomic library. J Ind Microbiol Biotechnol 40:191-200 . doi: $10.1007 / \mathrm{s} 10295-012-1217-7$

Blaya J, Marhuenda FC, Pascual JA, Ros M (2016) Microbiota characterization of compost using omics approaches opens new perspectives for phytophthora root rot control. PLoS One 11:e0158048 . doi: 10.1371/journal.pone.0158048

Bolger AM, Lohse M, Usadel B (2014) Trimmomatic: a flexible trimmer for Illumina sequence data. Bioinformatics 30:2114-2120 . doi: 10.1093/bioinformatics/btu170

Bolyen E, Rideout JR, Dillon MR, Bokulich NA, Abnet CC, Al-Ghalith GA, Alexander H, Alm EJ, Arumugam M, Asnicar F, Bai Y, Bisanz JE, Bittinger K, Brejnrod A, Brislawn CJ, Brown CT, Callahan BJ, CaraballoRodríguez AM, Chase J, Cope EK, Da Silva R, Diener C, Dorrestein PC, Douglas GM, Durall DM, Duvallet C, Edwardson CF, Ernst M, Estaki M, Fouquier J, Gauglitz JM, Gibbons SM, Gibson DL, Gonzalez A, Gorlick K, Guo J, Hillmann B, Holmes S, Holste H, Huttenhower C, Huttley GA, Janssen S, Jarmusch AK, Jiang L, Kaehler BD, Kang K Bin, Keefe CR, Keim P, Kelley ST, Knights D, Koester I, Kosciolek T, Kreps J, Langille MGI, Lee J, Ley R, Liu YX, Loftfield E, Lozupone C, Maher M, Marotz C, Martin BD, McDonald D, McIver LJ, Melnik A V., Metcalf JL, Morgan SC, Morton JT, Naimey AT, Navas-Molina JA, Nothias LF, Orchanian SB, Pearson T, Peoples SL, Petras D, Preuss ML, Pruesse E, Rasmussen LB, Rivers A, Robeson MS, Rosenthal P, Segata N, Shaffer M, Shiffer A, Sinha R, Song SJ, Spear JR, Swafford AD, 
Thompson LR, Torres PJ, Trinh P, Tripathi A, Turnbaugh PJ, Ul-Hasan S, van der Hooft JJJ, Vargas F, Vázquez-Baeza Y, Vogtmann E, von Hippel M, Walters W, Wan Y, Wang M, Warren J, Weber KC, Williamson CHD, Willis AD, Xu ZZ, Zaneveld JR, Zhang Y, Zhu Q, Knight R, Caporaso JG (2019) Reproducible, interactive, scalable and extensible microbiome data science using QIIME 2. Nat. Biotechnol. $37: 852-857$

Bornscheuer UT (2002) Microbial carboxyl esterases: classification, properties and application in biocatalysis. FEMS Microbiol Rev 26:73-81 . doi: 10.1111/j.1574-6976.2002.tb00599.x

Brault G, Shareck F, Hurtubise Y, Lépine F, Doucet N (2012) Isolation and characterization of EstC, a new coldactive esterase from Streptomyces coelicolor A3(2). PLoS One 7:e32041 . doi: 10.1371/journal.pone.0032041

Bzhalava Z, Hultin E, Dillner J (2018) Extension of the viral ecology in humans using viral profile hidden Markov models. PLoS One 13:e190938 . doi: 10.1371/journal.pone.0190938

Cáceres M De (2013) How to use the indicspecies package (ver. 1.7.1). R Proj. 29

Castilla A, Panizza P, Rodríguez D, Bonino L, Díaz P, Irazoqui G, Rodríguez Giordano S (2017) A novel thermophilic and halophilic esterase from Janibacter sp. R02, the first member of a new lipase family (Family XVII). Enzyme Microb Technol 98:86-95 . doi: 10.1016/J.ENZMICTEC.2016.12.010

Chahinian H, Ali Y Ben, Abousalham A, Petry S, Mandrich L, Manco G, Canaan S, Sarda L (2005) Substrate specificity and kinetic properties of enzymes belonging to the hormone-sensitive lipase family: Comparison with non-lipolytic and lipolytic carboxylesterases. Biochim Biophys Acta 1738:29-36 . doi: 10.1016/j.bbalip.2005.11.003

Chan WY, Wong M, Guthrie J, Savchenko A V., Yakunin AF, Pai EF, Edwards EA (2010) Sequence- and activitybased screening of microbial genomes for novel dehalogenases. Microb Biotechnol 3:107-120 . doi: $10.1111 / \mathrm{j} .1751-7915.2009 .00155 . \mathrm{x}$

Chen IMA, Markowitz VM, Chu K, Palaniappan K, Szeto E, Pillay M, Ratner A, Huang J, Andersen E, Huntemann M, Varghese N, Hadjithomas M, Tennessen K, Nielsen T, Ivanova NN, Kyrpides NC (2017) IMG/M: Integrated genome and metagenome comparative data analysis system. Nucleic Acids Res 45:507516. doi: 10.1093/nar/gkw929

Chen Y, Black DS, Reilly PJ (2016) Carboxylic ester hydrolases: Classification and database derived from their primary, secondary, and tertiary structures. Protein Sci 25:1942-1953 . doi: 10.1002/pro.3016

Chevreux B, Wetter T, Suhai S (1999) Genome sequence assembly using trace signals and additional sequence information. Comput Sci Biol Proc Ger Conf Bioinforma 99:45-56

Choi JE, Kwon MA, Na HY, Hahm DH, Song JK (2013) Isolation and characterization of a metagenome-derived thermoalkaliphilic esterase with high stability over a broad $\mathrm{pH}$ range. Extremophiles 17:1013-1021 . doi: $10.1007 / \mathrm{s} 00792-013-0583-\mathrm{Z}$

Chow J, Kovacic F, Dall Antonia Y, Krauss U, Fersini F, Schmeisser C, Lauinger B, Bongen P, Pietruszka J, Schmidt M, Menyes I, Bornscheuer UT, Eckstein M, Thum O, Liese A, Mueller-Dieckmann J, Jaeger KE, Streit WR (2012) The metagenome-derived enzymes LipS and LipT increase the diversity of known lipases. PLoS One 7:e47665 . doi: 10.1371/journal.pone.0047665

Chu X, He H, Guo C, Sun B (2008) Identification of two novel esterases from a marine metagenomic library derived from South China Sea. Appl Microbiol Biotechnol 80:615-625 . doi: 10.1007/s00253-008-1566-3

Clarke KR (1993) Non - parametric multivariate analyses of changes in community structure. Aust J Ecol 18:117143 . doi: $10.1111 /$ j.1442-9993.1993.tb00438.x

Couto GH, Glogauer A, Faoro H, Chubatsu LS, Souza EM, Pedrosa FO (2010) Isolation of a novel lipase from a metagenomic library derived from mangrove sediment from the south Brazilian coast. Genet Mol Res 9:514-523

Dalcin Martins P, Danczak RE, Roux S, Frank J, Borton MA, Wolfe RA, Burris MN, Wilkins MJ (2018) Viral and metabolic controls on high rates of microbial sulfur and carbon cycling in wetland ecosystems. Microbiome 6:138 . doi: 10.1186/s40168-018-0522-4

Diamond S, Andeer PF, Li Z, Crits-Christoph A, Burstein D, Anantharaman K, Lane KR, Thomas BC, Pan C, Northen TR, Banfield JF (2019) Mediterranean grassland soil C-N compound turnover is dependent on rainfall and depth, and is mediated by genomically divergent microorganisms. Nat Microbiol 4:1356-1367 . doi: 10.1038/s41564-019-0449-y

Dougherty MJ, D'haeseleer P, Hazen TC, Simmons BA, Adams PD, Hadi MZ (2012) Glycoside hydrolases from a targeted compost metagenome, activity-screening and functional characterization. BMC Biotechnol 12:38 doi: $10.1186 / 1472-6750-12-38$

Dukunde A, Schneider D, Lu M, Brady S, Daniel R (2017) A novel, versatile family IV carboxylesterase exhibits high stability and activity in a broad pH spectrum. Biotechnol Lett 39:577-587 . doi: 10.1007/s10529-0162282-1 
Dukunde A, Schneider D, Schmidt M, Veldkamp E, Daniel R (2019) Tree species shape soil bacterial community structure and function in temperate deciduous forests. Front Microbiol 10:1519 . doi: $10.3389 /$ fmicb.2019.01519

Eddy SR (2011) Accelerated Profile HMM Searches. PLoS Comput Biol 7:e1002195 . doi: 10.1371/journal.pcbi.1002195

Eddy SR (2018) HMMER User's Guide Biological sequence analysis using profile hidden Markov models

Edgar RC (2004) MUSCLE: multiple sequence alignment with high accuracy and high throughput. Nucleic Acids Res 32:1792-1797 . doi: 10.1093/nar/gkh340

Edgar RC, Haas BJ, Clemente JC, Quince C, Knight R (2011) UCHIME improves sensitivity and speed of chimera detection. Bioinformatics 27:2194-2200 . doi: 10.1093/bioinformatics/btr381

Egelkamp R, Zimmermann T, Schneider D, Hertel R, Daniel R (2019) Impact of nitriles on bacterial communities. Front Environ Sci 7:103 . doi: 10.3389/fenvs.2019.00103

Eric Sayers GW, Cavanaugh M, Clark K, Ostell J, Pruitt KD, Karsch-Mizrachi I (2019) GenBank. Nucleic Acids Res 47:94-99 . doi: 10.1093/nar/gky989

Esteban-Torres M, Santamaría L, de las Rivas B, Muñoz R (2014) Characterisation of a cold-active and salttolerant esterase from Lactobacillus plantarum with potential application during cheese ripening. Int Dairy J 39:312-315 . doi: 10.1016/j.idairyj.2014.08.004

Fan X, Liu X, Wang K, Wang S, Huang R, Liu Y (2011) Highly soluble expression and molecular characterization of an organic solvent-stable and thermotolerant lipase originating from the metagenome. J Mol Catal B Enzym 72:319-325 . doi: 10.1016/j.molcatb.2011.07.009

Fang Z, Li J, Wang Q, Fang W, Peng H, Zhang X, Xiao Y (2014) A novel esterase from a marine metagenomic library exhibiting salt tolerance ability. J Microbiol Biotechnol 24:771-780 . doi: 10.4014/jmb.1311.11071

Ferrer M, Martínez-Martínez M, Bargiela R, Streit WR, Golyshina O V, Golyshin PN (2015) Estimating the success of enzyme bioprospecting through metagenomics: current status and future trends. Microb Biotechnol 9:22-34 . doi: 10.1111/1751-7915.12309

Finn RD, Bateman A, Clements J, Coggill P, Eberhardt RY, Eddy SR, Heger A, Hetherington K, Holm L, Mistry J, Sonnhammer ELL, Tate J, Punta M (2014) Pfam: the protein families database. Nucleic Acids Res 42:D222-D230 . doi: 10.1093/nar/gkt1223

Fu L, He Y, Xu F, Ma Q, Wang F, Xu J (2015) Characterization of a novel thermostable patatin-like protein from a Guaymas basin metagenomic library. Extremophiles 19:829-840 . doi: 10.1007/s00792-015-0758-x

Gao W, Wu K, Chen L, Fan H, Zhao Z, Gao B, Wang H, Wei D (2016) A novel esterase from a marine mud metagenomic library for biocatalytic synthesis of short-chain flavor esters. Microb Cell Fact 15:41 . doi: 10.1186/s12934-016-0435-5

Gerlt JA, Bouvier JT, Davidson DB, Imker HJ, Sadkhin B, Slater DR, Whalen KL (2015) Enzyme Function Initiative-Enzyme Similarity Tool (EFI-EST): A web tool for generating protein sequence similarity networks. Biochim Biophys Acta 1854:1019-1037 . doi: 10.1002/cncr.27633.Percutaneous

Gibson MK, Forsberg KJ, Dantas G (2015) Improved annotation of antibiotic resistance determinants reveals microbial resistomes cluster by ecology. ISME J 9:207-216 . doi: 10.1038/ismej.2014.106

Gilbert EJ (1993) Pseudomonas lipases: Biochemical properties and molecular cloning. Enzyme Microb. Technol. 15:634-645

Glogauer A, Martini VP, Faoro H, Couto GH, Müller-Santos M, Monteiro RA, Mitchell DA, de Souza EM, Pedrosa FO, Krieger N (2011) Identification and characterization of a new true lipase isolated through metagenomic approach. Microb Cell Fact 10:54 . doi: 10.1186/1475-2859-10-54

Guo H, Zhang Y, Shao Y, Chen W, Chen F, Li M (2016) Cloning, expression and characterization of a novel cold-active and organic solvent-tolerant esterase from Monascus ruber M7. Extremophiles 20:451-459 . doi: $10.1007 / \mathrm{s} 00792-016-0835-9$

Guo J, Zheng X, Xu L, Liu Z, Xu K, Li S, Wen T, Liu S, Pang H (2010) Characterization of a novel esterase Rv0045c from Mycobacterium tuberculosis. PLoS One 5:4-10 . doi: 10.1371/journal.pone.0013143

Hammer DAT, Ryan PD, Hammer Ø, Harper DAT (2001) Past: Paleontological Statistics Software Package for Education and Data Analysis

Hartmann M, Frey B, Mayer J, Mäder P, Widmer F (2015) Distinct soil microbial diversity under long-term organic and conventional farming. ISME J 9:1177-1194 . doi: 10.1038/ismej.2014.210

Hasan F, Shah AA, Hameed A (2006) Industrial applications of microbial lipases. Enzyme Microb Technol 39:235-251 . doi: 10.1016/J.ENZMICTEC.2005.10.016

Hausmann S, Jaeger K-E (2010) Lipolytic enzymes from bacteria. In: Timmis KN (ed) Handbook of Hydrocarbon and Lipid Microbiology. Springer Berlin Heidelberg, Berlin, Heidelberg, pp 1099-1126

Hitch TCA, Clavel T (2019) A proposed update for the classification and description of bacterial lipolytic enzymes. PeerJ 7:e7249 . doi: 10.7717/peerj.7249 
Hong JK, Choi HW, Hwang IS, Kim DS, Kim NH, Choi DS, Kim YJ, Hwang BK (2008) Function of a novel GDSL-type pepper lipase gene, CaGLIP1, in disease susceptibility and abiotic stress tolerance. Planta 227:539-558 . doi: 10.1007/s00425-007-0637-5

Hu P, Tom L, Singh A, Thomas BC, Baker BJ, Piceno YM, Andersen GL, Banfield JF (2016) Genome-resolved metagenomic analysis reveals roles for candidate phyla and other microbial community members in biogeochemical transformations in oil reservoirs. MBio 7:e01669-15 . doi: 10.1128/mBio.01669-15

Hu X, Thumarat U, Zhang X, Tang M, Kawai F (2010a) Diversity of polyester-degrading bacteria in compost and molecular analysis of a thermoactive esterase from Thermobifida alba AHK119. Appl Microbiol Biotechnol 87:771-779 . doi: 10.1007/s00253-010-2555-x

Hu Y, Fu C, Huang Y, Yin Y, Cheng G, Lei F, Lu N, Li J, Ashforth EJ, Zhang L, Zhu B (2010b) Novel lipolytic genes from the microbial metagenomic library of the South China Sea marine sediment. FEMS Microbiol Ecol 72:228-37 . doi: 10.1111/j.1574-6941.2010.00851.x

Hubbe MA, Nazhad M, Sánchez C (2010) Composting as a way to convert cellulosic biomass and organic waste into high-value soil admendments: a review. BioResources 5:2808-2854

Hugenholtz P, Tyson GW (2008) Microbiology: Metagenomics. Nature 455:481-483

Jayanath G, Mohandas SP, Kachiprath B, Solomon S, Sajeevan TP, Bright Singh IS, Philip R (2018) A novel solvent tolerant esterase of GDSGG motif subfamily from solar saltern through metagenomic approach: Recombinant expression and characterization. Int $\mathrm{J}$ Biol Macromol 119:393-401 . doi: 10.1016/j.ijbiomac.2018.06.057

Jeon JH, Kim JT, Kim YJ, Kim HK, Lee HS, Kang SG, Kim SJ, Lee JH (2009) Cloning and characterization of a new cold-active lipase from a deep-sea sediment metagenome. Appl Microbiol Biotechnol 81:865-874 . doi: 10.1007/s00253-008-1656-2

Jeon JH, Kim S-J, Lee HS, Cha S-S, Lee JH, Yoon S-H, Koo B-S, Lee C-M, Choi SH, Lee SH, Kang SG, Lee J$\mathrm{H}$ (2011) Novel metagenome-derived carboxylesterase that hydrolyzes $\beta$-lactam antibiotics. Appl Environ Microbiol 77:7830-6 . doi: 10.1128/AEM.05363-11

Jia ML, Zhong XL, Lin ZW, Dong BX, Li G (2019) Expression and characterization of an esterase belonging to a new family via isolation from a metagenomic library of paper mill sludge. Int J Biol Macromol 126:1192_ 1200 . doi: $10.1016 /$ j.ijbiomac.2019.01.025

Jiang X, Huo Y, Cheng H, Zhang X, Zhu X, Wu M (2012) Cloning, expression and characterization of a halotolerant esterase from a marine bacterium Pelagibacterium halotolerans B2T. Extremophiles 16:427435 . doi: $10.1007 / \mathrm{s} 00792-012-0442-3$

Jiménez DJ, Montaña JS, Álvarez D, Baena S (2012) A novel cold active esterase derived from Colombian high Andean forest soil metagenome. World J Microbiol Biotechnol 28:361-370 . doi: 10.1007/s11274-0110828-x

Kaminski J, Gibson MK, Franzosa EA, Segata N, Dantas G, Huttenhower C (2015) High-specificity targeted functional profiling in microbial communities with ShortBRED. PLOS Comput Biol 11:e1004557 . doi: 10.1371/journal.pcbi.1004557

Kang CH, Oh KH, Lee MH, Oh TK, Kim BH, Yoon JH (2011) A novel family VII esterase with industrial potential from compost metagenomic library. Microb Cell Fact 10:41 . doi: 10.1186/1475-2859-10-41

Keegan KP, Glass EM, Meyer F (2016) MG-RAST, a metagenomics service for analysis of microbial community structure and function. In: Methods in Molecular Biology. Humana Press Inc., pp 207-233

Kim YH, Kwon EJ, Kim SK, Jeong YS, Kim J, Yun HD, Kim H (2010) Molecular cloning and characterization of a novel family VIII alkaline esterase from a compost metagenomic library. Biochem Biophys Res Commun 393:45-49 . doi: 10.1016/j.bbrc.2010.01.070

Knudsen M, Wiuf C (2010) The CATH database. Hum Genomics 4:207-212 . doi: 10.1186/1479-7364-4-3-207

Ko KC, Rim SO, Han Y, Shin BS, Kim GJ, Choi JH, Song JJ (2012) Identification and characterization of a novel cold-adapted esterase from a metagenomic library of mountain soil. J Ind Microbiol Biotechnol 39:681689 . doi: 10.1007/s10295-011-1080-y

Kovacic F, Babic N, Krauss U, Jaeger K (2019) Classification of Lipolytic Enzymes from Bacteria. In: Rojo F (ed) Aerobic Utilization of Hydrocarbons, Oils and Lipids. Handbook of Hydrocarbon and Lipid Microbiology. Springer, Cham, pp 1-35

Krzywinski M, Schein J, Birol I, Connors J, Gascoyne R, Horsman D, Jones SJ, Marra MA (2009) Circos: an information aesthetic for comparative genomics. Genome Res 19:1639-1645 . doi: 10.1101/gr.092759.109

Lam KN, Cheng J, Engel K, Neufeld JD, Charles TC (2015) Current and future resources for functional metagenomics. Front Microbiol 6:1196 . doi: 10.3389/fmicb.2015.01196

Lämmle K, Zipper H, Breuer M, Hauer B, Buta C, Brunner H, Rupp S (2007) Identification of novel enzymes with different hydrolytic activities by metagenome expression cloning. J Biotechnol 127:575-592 . doi: 10.1016/j.jbiotec.2006.07.036

Langmead B, Salzberg SL (2012) Fast gapped-read alignment with Bowtie 2. Nat Methods 9:357-359 . doi: 10.1038/nmeth.1923 
Lapébie P, Lombard V, Drula E, Terrapon N, Henrissat B (2019) Bacteroidetes use thousands of enzyme combinations to break down glycans. Nat Commun 10:1-7 . doi: 10.1038/s41467-019-10068-5

Larkin MA, Blackshields G, Brown NP, Chenna R, McGettigan PA, McWilliam H, Valentin F, Wallace IM, Wilm A, Lopez R, Thompson JD, Gibson TJ, Higgins DG (2007) Clustal W and Clustal X version 2.0. Bioinformatics 23:2947-2948 . doi: 10.1093/bioinformatics/btm404

Lee JH, Jeon JH, Kim JT, Lee HS, Kim SJ, Kang SG, Choi SH (2011) Novel lipolytic enzymes identified from metagenomic library of deep-sea sediment. Evidence-based Complement Altern Med 2011: . doi: $10.1155 / 2011 / 271419$

Lee SW, Won K, Lim HK, Kim JC, Choi GJ, Cho KY (2004) Screening for novel lipolytic enzymes from uncultured soil microorganisms. Appl Microbiol Biotechnol 65:720-726 . doi: 10.1007/s00253-004-17223

Lee Y-S (2016) Isolation and characterization of a novel cold-adapted esterase, MtEst45, from microbulbifer thermotolerans DAU221. Front Microbiol 7:218 . doi: 10.3389/fmicb.2016.00218

Leis B, Angelov A, Mientus M, Li H, Pham VTT, Lauinger B, Bongen P, Pietruszka J, Gonçalves LG, Santos H, Liebl W (2015) Identification of novel esterase-active enzymes from hot environments by use of the host bacterium Thermus thermophilus. Front Microbiol 6:275 . doi: 10.3389/fmicb.2015.00275

Lenfant N, Hotelier T, Velluet E, Bourne Y, Marchot P, Chatonnet A (2013) ESTHER, the database of the $\alpha / \beta$ hydrolase fold superfamily of proteins: tools to explore diversity of functions. Nucleic Acids Res 41:423429. doi: $10.1093 / \mathrm{nar} / \mathrm{gks} 1154$

Lewin GR, Carlos C, Chevrette MG, Horn HA, McDonald BR, Stankey RJ, Fox BG, Currie CR (2016) Evolution and ecology of Actinobacteria and their bioenergy applications. Annu Rev Microbiol 70:235-254 . doi: 10.1146/annurev-micro-102215-095748

Li H, Han X, Qiu W, Xu D, Wang Y, Yu M, Hu X, Zhuo R (2019) Identification and expression analysis of the GDSL esterase/lipase family genes, and the characterization of SaGLIP8 in Sedum alfredii Hance under cadmium stress. PeerJ 7:e6741 . doi: 10.7717/peerj.6741

Li LL, McCorkle SR, Monchy S, Taghavi S, van der Lelie D (2009) Bioprospecting metagenomes: Glycosyl hydrolases for converting biomass. Biotechnol Biofuels 2:1-11 . doi: 10.1186/1754-6834-2-10

Li M, Yang LR, Xu G, Wu JP (2016) Cloning and characterization of a novel lipase from Stenotrophomonas maltophilia GS11: The first member of a new bacterial lipase family XVI. J Biotechnol 228:30-36 . doi: 10.1016/j.jbiotec.2016.04.034

Li PY, Yao QQ, Wang P, Zhang Y, Li Y, Zhang YQ, Hao J, Zhou BC, Chen XL, Shi M, Zhang YZ, Zhang XY (2017) A novel subfamily esterase with a homoserine transacetylase-like fold but no transferase activity. Appl Environ Microbiol 83:e00131-17 . doi: 10.1128/AEM.00131-17

Liaw R-B, Cheng M-P, Wu M-C, Lee C-Y (2010) Use of metagenomic approaches to isolate lipolytic genes from activated sludge. Bioresour Technol 101:8323-8329 . doi: 10.1016/j.biortech.2010.05.091

Lima-Junior JD, Viana-Niero C, Conde Oliveira D V., Machado GE, Rabello MC da S, Martins-Junior J, Martins LF, Digiampietri LA, da Silva AM, Setubal JC, Russell DA, Jacobs-Sera D, Pope WH, Hatfull GF, Leão SC (2016) Characterization of mycobacteria and mycobacteriophages isolated from compost at the São Paulo Zoo Park Foundation in Brazil and creation of the new mycobacteriophage Cluster U. BMC Microbiol 16:111 . doi: 10.1186/s12866-016-0734-3

Lindemann SR, Moran JJ, Stegen JC, Renslow RS, Hutchison JR, Cole JK, Dohnalkova AC, Tremblay J, Singh K, Malfatti SA, Chen F, Tringe SG, Beyenal H, Fredrickson JK (2013) The epsomitic phototrophic microbial mat of Hot Lake, Washington: Community structural responses to seasonal cycling. Front Microbiol 4:323 . doi: 10.3389/fmicb.2013.00323

Liu JF, Sun XB, Yang GC, Mbadinga SM, Gu JD, Mu BZ (2015a) Analysis of microbial communities in the oil reservoir subjected to $\mathrm{CO} 2$-flooding by using functional genes as molecular biomarkers for microbial $\mathrm{CO} 2$ sequestration. Front Microbiol 6:236 . doi: 10.3389/fmicb.2015.00236

Liu K, Wang J, Bu D, Zhao S, McSweeney C, Yu P, Li D (2009) Isolation and biochemical characterization of two lipases from a metagenomic library of China Holstein cow rumen. Biochem Biophys Res Commun 385:605-611 . doi: 10.1016/j.bbrc.2009.05.110

Liu SP, Liu RX, Zhang L, Shi GY (2015b) Sequence-based screening and characterization of cytosolic mandelate oxidase using oxygen as electron acceptor. Enzyme Microb Technol 69:24-30 . doi: 10.1016/j.enzmictec.2014.11.001

Liu SP, Liu RX, Zhang L, Shi GY (2015c) Sequence-based screening and characterization of cytosolic mandelate oxidase using oxygen as electron acceptor. Enzyme Microb Technol 69:24-30 . doi: 10.1016/j.enzmictec.2014.11.001

Liu YF, Galzerani DD, Mbadinga SM, Zaramela LS, Gu JD, Mu BZ, Zengler K (2018) Metabolic capability and in situ activity of microorganisms in an oil reservoir. Microbiome 6:5 . doi: 10.1186/s40168-017-0392-1

Liu Z, Lozupone C, Hamady M, Bushman FD, Knight R (2007) Short pyrosequencing reads suffice for accurate microbial community analysis. Nucleic Acids Res 35:e120 . doi: 10.1093/nar/gkm541 
López-López O, Knapik K, Cerdán ME, González-Siso MI (2015) Metagenomics of an alkaline hot spring in Galicia (Spain): Microbial diversity analysis and screening for novel lipolytic enzymes. Front Microbiol 6:1291 . doi: 10.3389/fmicb.2015.01291

Lu M, Dukunde A, Daniel R (2019) Biochemical profiles of two thermostable and organic solvent-tolerant esterases derived from a compost metagenome. Appl Microbiol Biotechnol 103:3421-3437 . doi: 10.1007/s00253-019-09695-1

Ma S, Fang C, Sun X, Han L, He X, Huang G (2018) Bacterial community succession during pig manure and wheat straw aerobic composting covered with a semi-permeable membrane under slight positive pressure. Bioresour Technol 259:221-227 . doi: 10.1016/j.biortech.2018.03.054

Mahowald MA, Rey FE, Seedorf H, Turnbaugh PJ, Fulton RS, Wollam A, Shah N, Wang C, Magrini V, Wilson RK, Cantarel BL, Coutinho PM, Henrissat B, Crock LW, Russell A, Verberkmoes NC, Hettich RL, Gordon JI (2009) Characterizing a model human gut microbiota composed of members of its two dominant bacterial phyla. Proc Natl Acad Sci U S A 106:5859-5864 . doi: 10.1073/pnas.0901529106

Maimanakos J, Chow J, Gaßmeyer SK, Güllert S, Busch F, Kourist R, Streit WR (2016) Sequence-based screening for rare enzymes: new insights into the world of AMDases reveal a conserved motif and 58 novel enzymes clustering in eight distinct families. Front Microbiol 7:1332 . doi: 10.3389/fmicb.2016.01332

Mander P, Yoo HY, Kim SW, Choi YH, Cho SS, Yoo JC (2014) Transesterification of waste cooking oil by an organic solvent-tolerant alkaline lipase from Streptomyces sp. CS273. Appl Biochem Biotechnol 172:13771389 . doi: 10.1007/s12010-013-0610-7

Martins LF, Antunes LP, Pascon RC, de Oliveira JCF, Digiampietri LA, Barbosa D, Peixoto BM, Vallim MA, Viana-Niero C, Ostroski EH, Telles GP, Dias Z, da Cruz JB, Juliano L, Verjovski-Almeida S, da Silva AM, Setubal JC (2013) Metagenomic analysis of a tropical composting operation at the São Paulo zoo park reveals diversity of biomass degradation functions and organisms. PLoS One 8:e61928 . doi: 10.1371/journal.pone.0061928

Masuch T, Kusnezowa A, Nilewski S, Bautista JT, Kourist R, Leichert LI (2015) A combined bioinformatics and functional metagenomics approach to discovering lipolytic biocatalysts. Front Microbiol 6:1110 . doi: 10.3389/fmicb.2015.01110

Mayumi D, Akutsu-Shigeno Y, Uchiyama H, Nomura N, Nakajima-Kambe T (2008) Identification and characterization of novel poly(dl-lactic acid) depolymerases from metagenome. Appl Microbiol Biotechnol 79:743-750 . doi: 10.1007/s00253-008-1477-3

Menzel P, Ng KL, Krogh A (2016) Fast and sensitive taxonomic classification for metagenomics with Kaiju. Nat Commun 7:11257 . doi: 10.1038/ncomms 11257

Montella S, Ventorino V, Lombard V, Henrissat B, Pepe O, Faraco V (2017) Discovery of genes coding for carbohydrate-active enzyme by metagenomic analysis of lignocellulosic biomasses. Sci Rep 7:42623 . doi: $10.1038 /$ srep42623

Moore AM, Munck C;, Sommer MOA, Dantas G (2011) Functional metagenomic investigations of the human intestinal microbiota. Front Microbiol 2:188 . doi: 10.3389/fmicb.2011.00188

Nacke H (2011) Identification and characterization of microbial key functions in soils of the German Biodiversity Exploratories representing different land use and management types

Nacke H, Will C, Herzog S, Nowka B, Engelhaupt M, Daniel R (2011) Identification of novel lipolytic genes and gene families by screening of metagenomic libraries derived from soil samples of the German Biodiversity Exploratories. FEMS Microbiol Ecol 78:188-201 . doi: 10.1111/j.1574-6941.2011.01088.x

Namiki T, Hachiya T, Tanaka H, Sakakibara Y (2012) MetaVelvet: An extension of Velvet assembler to de novo metagenome assembly from short sequence reads. Nucleic Acids Res 40:e155 . doi: 10.1093/nar/gks678

Neher DA, Weicht TR, Bates ST, Leff JW, Fierer N (2013) Changes in bacterial and fungal communities across compost recipes, preparation methods, and composting times. PLoS One 8:e79512 . doi: 10.1371/journal.pone.0079512

Ngara TR, Zhang H (2018) Recent advances in function-based metagenomic screening. Genomics, Proteomics Bioinforma. 16:405-415

Niu Q, Qiao W, Qiang H, Li YY (2013) Microbial community shifts and biogas conversion computation during steady, inhibited and recovered stages of thermophilic methane fermentation on chicken manure with a wide variation of ammonia. Bioresour Technol 146:223-233 . doi: 10.1016/j.biortech.2013.07.038

Okano H, Hong X, Kanaya E, Angkawidjaja C, Kanaya S (2015) Structural and biochemical characterization of a metagenome-derived esterase with a long N-terminal extension. Protein Sci 24:93-104 . doi: $10.1002 /$ pro. 2591

Oksanen J, Blanchet FG, Friendly M, Kindt R, Legendre P, Mcglinn D, Minchin PR, O'hara RB, Simpson GL, Solymos P, Henry M, Stevens H, Szoecs E, Maintainer HW (2018) vegan: Community Ecology Package. https://cran.r-project.org/web/packages/vegan/vegan.pdf. Accessed 30 Oct 2018

Ouyang LM, Liu JY, Qiao M, Xu JH (2013) Isolation and biochemical characterization of two novel metagenomederived esterases. Appl Biochem Biotechnol 169:15-28 . doi: 10.1007/s12010-012-9949-4 
Pehrsson EC, Tsukayama P, Patel S, Mejía-Bautista M, Sosa-Soto G, Navarrete KM, Calderon M, Cabrera L, Hoyos-Arango W, Bertoli MT, Berg DE, Gilman RH, Dantas G (2016) Interconnected microbiomes and resistomes in low-income human habitats. Nature 533:212-216 . doi: 10.1038/nature17672

Peng Q, Zhang X, Shang M, Wang X, Wang G, Li B, Guan G, Li Y, Wang Y (2011) A novel esterase gene cloned from a metagenomic library from neritic sediments of the South China Sea. Microb Cell Fact 10:95 . doi: $10.1186 / 1475-2859-10-95$

Popovic A, Hai T, Tchigvintsev A, Hajighasemi M, Nocek B, Khusnutdinova AN, Brown G, Glinos J, Flick R, Skarina T, Chernikova TN, Yim V, Brüls T, Paslier D Le, Yakimov MM, Joachimiak A, Ferrer M, Golyshina O V., Savchenko A, Golyshin PN, Yakunin AF (2017) Activity screening of environmental metagenomic libraries reveals novel carboxylesterase families. Sci Rep 7:44103 . doi: 10.1038/srep44103

Quast C, Pruesse E, Yilmaz P, Gerken J, Schweer T, Yarza P, Peplies J, Glöckner FO (2013) The SILVA ribosomal RNA gene database project: Improved data processing and web-based tools. Nucleic Acids Res 41:D590-D596 . doi: 10.1093/nar/gks1219

Quince C, Walker AW, Simpson JT, Loman NJ, Segata N (2017) Shotgun metagenomics, from sampling to analysis. Nat Biotechnol 35:833-844 . doi: 10.1038/nbt.3935

Rabausch U, Juergensen J, Ilmberger N, Böhnke S, Fischer S, Schubach B, Schulte M, Streit W V. (2013) Functional screening of metagenome and genome libraries for detection of novel flavonoid-modifying enzymes. Appl Environ Microbiol 79:4551-4563 . doi: 10.1128/AEM.01077-13

Rahman MA, Culsum U, Tang W, Zhang SW, Wu G, Liu Z (2016) Characterization of a novel cold active and salt tolerant esterase from Zunongwangia profunda. Enzyme Microb Technol 85:1-11 . doi: 10.1016/j.enzmictec.2015.12.013

Ramnath L, Sithole B, Govinden R (2016) Classification of lipolytic enzymes and their biotechnological applications in the pulping industry. Can J Microbiol 63:179-192 . doi: 10.1139/cjm-2016-0447

Ranjan R, Yadav MK, Suneja G, Sharma R (2018) Discovery of a diverse set of esterases from hot spring microbial mat and sea sediment metagenomes. Int $\mathrm{J}$ Biol Macromol 119:572-581 . doi: 10.1016/j.ijbiomac.2018.07.170

Rashamuse K, Magomani V, Ronneburg T, Brady D (2009) A novel family VIII carboxylesterase derived from a leachate metagenome library exhibits promiscuous $\beta$-lactamase activity on nitrocefin. Appl Microbiol Biotechnol 83:491-500 . doi: 10.1007/s00253-009-1895-x

Reyes-Duarte D, Ferrer M, García-Arellano H (2012) Functional-based screening methods for lipases, esterases, and phospholipases in metagenomic libraries. Methods Mol Biol 861:101-113 . doi: 10.1007/978-1-61779$600-5 \_6$

Reyes A, Alves JMP, Durham AM, Gruber A (2017) Use of profile hidden Markov models in viral discovery: current insights. Adv Genomics Genet 7:29-45 . doi: 10.2147/AGG.S136574

Rime T, Hartmann M, Frey B (2016) Potential sources of microbial colonizers in an initial soil ecosystem after retreat of an alpine glacier. ISME J 10:1625-1641 . doi: 10.1038/ismej.2015.238

Rinninella E, Raoul P, Cintoni M, Franceschi F, Miggiano GAD, Gasbarrini A, Mele MC (2019) What is the healthy gut microbiota composition? A changing ecosystem across age, environment, diet, and diseases. Microorganisms 7:14 . doi: 10.3390/microorganisms7010014

Rudek W, Haque RU (1976) Extracellular enzymes of the genus Bacteroides. J Clin Microbiol 4:458-60

Ryckeboer J, Mergaert J, Vaes K, Klammer S, Clercq D, Coosemans J, Insam H, Swings J (2003) A survey of bacteria and fungi occurring during composting and self-heating processes. Ann Microbiol 53:349-410

Sarmah N, Revathi D, Sheelu G, Yamuna Rani K, Sridhar S, Mehtab V, Sumana C (2018) Recent advances on sources and industrial applications of lipases. Biotechnol Prog 34:5-28 . doi: 10.1002/btpr.2581

Schloss PD, Hay AG, Wilson DB, Walker LP (2003) Tracking temporal changes of bacterial community fingerprints during the initial stages of composting. FEMS Microbiol Ecol 46:1-9 . doi: 10.1016/S01686496(03)00153-3

Schneider D, Engelhaupt M, Allen K, Kurniawan S, Krashevska V, Heinemann M, Nacke H, Wijayanti M, Meryandini A, Corre MD, Scheu S, Daniel R (2015) Impact of lowland rainforest transformation on diversity and composition of soil prokaryotic communities in sumatra (Indonesia). Front Microbiol 6:1339. doi: $10.3389 /$ fmicb.2015.01339

Seemann T (2014) Prokka: rapid prokaryotic genome annotation. Bioinformatics 30:2068-2069 . doi: 10.1093/bioinformatics/btu153

Seo S, Lee YS, Yoon SH, Kim SJ, Cho JY, Hahn BS, Koo BS, Lee CM (2014) Characterization of a novel coldactive esterase isolated from swamp sediment metagenome. World J Microbiol Biotechnol 30:879-886 . doi: $10.1007 / \mathrm{s} 11274-013-1496-9$

Shannon P, Markiel A, Ozier O, Baliga NS, Wang JT, Ramage D, Amin N, Schwikowski B, Ideker T (2003) Cytoscape: A software environment for integrated models of biomolecular interaction networks. Genome Res 13:2498-2504 . doi: 10.1101/gr.1239303 
Shao H, Xu L, Yan Y (2013) Isolation and characterization of a thermostable esterase from a metagenomic library. J Ind Microbiol Biotechnol 40:1211-1222 . doi: 10.1007/s10295-013-1317-z

Sievers F, Wilm A, Dineen D, Gibson TJ, Karplus K, Li W, Lopez R, McWilliam H, Remmert M, Söding J, Thompson JD, Higgins DG (2011) Fast, scalable generation of high-quality protein multiple sequence alignments using Clustal Omega. Mol Syst Biol 7:539 . doi: 10.1038/msb.2011.75

Silva CC, Hayden H, Sawbridge T, Mele P, Kruger RH, Rodrigues MVN, Costa GGL, Vidal RO, Sousa MP, Torres APR, Santiago VMJ, Oliveira VM (2012) Phylogenetic and functional diversity of metagenomic libraries of phenol degrading sludge from petroleum refinery wastewater treatment system. AMB Express 2:18 . doi: $10.1186 / 2191-0855-2-18$

Simon C, Daniel R (2009) Achievements and new knowledge unraveled by metagenomic approaches. Appl Microbiol Biotechnol 85:265-276 . doi: 10.1007/s00253-009-2233-z

Simon C, Daniel R (2011) Metagenomic analyses: Past and future trends. Appl Environ Microbiol 77:1153-1161 . doi: 10.1128/AEM.02345-10

Skewes-Cox P, Sharpton TJ, Pollard KS, DeRisi JL (2014) Profile Hidden Markov Models for the detection of viruses within metagenomic sequence data. PLoS One 9:e105067 . doi: 10.1371/journal.pone.0105067

Snellman EA, Colwell RR (2004) Acinetobacter lipases: Molecular biology, biochemical properties and biotechnological potential. J. Ind. Microbiol. Biotechnol. 31:391-400

Sørensen KB, Canfield DE, Teske AP, Oren A (2005) Community composition of a hypersaline endoevaporitic microbial mat. Appl Environ Microbiol 71:7352-7365 . doi: 10.1128/AEM.71.11.7352-7365.2005

Sriyapai P, Kawai F, Siripoke S, Chansiri K, Sriyapai T (2015) Cloning, expression and characterization of a thermostable esterase HydS14 from Actinomadura sp. strain S14 in pichia pastoris. Int J Mol Sci 16:1357913594 . doi: $10.3390 /$ ijms 160613579

Staden R, Judge DP, Bonfield JK (2003) Managing sequencing projects in the GAP4 environment. In: Introduction to Bioinformatics. Humana Press, Totowa, NJ, pp 327-344

Streit W, Chow J, Jaeger K-E, Katzke N, Koch R, Borchert M, Bj'rnvad M, Roggenbuck M, Lippold F, Golyshin P, Golyshina O, Tran H, Ferrer M, Daniel R, Hollensteiner J (2018) A metagenomic collection of novel and highly efficient biocatalysts for industrial biotechnology. Impact 2018:82-84 . doi: 10.21820/23987073.2018.5.82

Stroobants A, Martin R, Roosens L, Portetelle D, Vandenbol M (2015) New lipolytic enzymes identified by screening two metagenomic libraries derived from the soil of a winter wheat field. Biotechnolgie, Agron Société Environ 19:125-131

Tamura K, Stecher G, Peterson D, Filipski A, Kumar S (2013) MEGA6: Molecular evolutionary genetics analysis version 6.0. Mol Biol Evol 30:2725-2729 . doi: 10.1093/molbev/mst197

Tasse L, Bercovici J, Pizzut-Serin S, Robe P, Tap J, Klopp C, Cantarel BL, Coutinho PM, Henrissat B, Leclerc M, Doré J, Monsan P, Remaud-Simeon M, Potocki-Veronese G (2010) Functional metagenomics to mine the human gut microbiome for dietary fiber catabolic enzymes. Genome Res 20:1605-1612 . doi: $10.1101 /$ gr. 108332.110

Thompson JD, Higgins DG, Gibson TJ (1994) CLUSTAL W: improving the sensitivity of progressive multiple sequence alignment through sequence weighting, position-specific gap penalties and weight matrix choice. Nucleic Acids Res 22:4673-80

Turnbaugh PJ, Hamady M, Yatsunenko T, Cantarel BL, Duncan A, Ley RE, Sogin ML, Jones WJ, Roe BA, Affourtit JP, Egholm M, Henrissat B, Heath AC, Knight R, Gordon JI (2009) A core gut microbiome in obese and lean twins. Nature 457:480-484 . doi: 10.1038/nature07540

Verma S, Kumar R, Meghwanshi GK (2019) Identification of new members of alkaliphilic lipases in archaea and metagenome database using reconstruction of ancestral sequences. 3 Biotech 9:1-8 . doi: 10.1007/s13205019-1693-9

Vigneron A, Alsop EB, Lomans BP, Kyrpides NC, Head IM, Tsesmetzis N (2017) Succession in the petroleum reservoir microbiome through an oil field production lifecycle. ISME J 11:2141-2154 . doi: 10.1038/ismej.2017.78

Villamizar GAC, Nacke H, Daniel R (2017) Function-based metagenomic library screening and heterologous expression strategy for genes encoding phosphatase activity. In: Methods in Molecular Biology. Humana Press Inc., pp 249-260

Vital M, Howe AC, Tiedje JM (2014) Revealing the bacterial butyrate synthesis pathways by analyzing (meta)genomic data. MBio 5:e00889-14. . doi: 10.1128/mBio.00889-14

Walsh CJ, Guinane CM, O' Toole PW, Cotter PD (2017) A profile Hidden Markov Model to investigate the distribution and frequency of LanB-encoding lantibiotic modification genes in the human oral and gut microbiome. PeerJ 5:e3254 . doi: 10.7717/peerj.3254

Wang C, Dong D, Wang H, Müller K, Qin Y, Wang H, Wu W (2016) Metagenomic analysis of microbial consortia enriched from compost : new insights into the role of Actinobacteria in lignocellulose decomposition. Biotechnol Biofuels 9:22 . doi: 10.1186/s13068-016-0440-2 
Wang L, Zhang G, Xu H, Xin H, Zhang Y (2019) Metagenomic analyses of microbial and carbohydrate-active enzymes in the rumen of holstein cows fed different forage-to-concentrate ratios. Front Microbiol 10:649 . doi: $10.3389 /$ fmicb.2019.00649

Wang S, Wang K, Li L, Liu Y (2013) Isolation and characterization of a novel organic solvent-tolerant and halotolerant esterase from a soil metagenomic library. J Mol Catal B Enzym 95:1-8 . doi: 10.1016/j.molcatb.2013.05.015

Wang W-L, Xu S-Y, Ren Z-G, Tao L, Jiang J-W, Zheng S-S (2015) Application of metagenomics in the human gut microbiome. World J Gastroenterol 21:803-814 . doi: 10.3748/wjg.v21.i3.803

Wang Y, Qian P-Y (2009) Conservative fragments in bacterial 16S rRNA genes and primer design for 16S ribosomal DNA amplicons in metagenomic studies. PLoS One 4:e7401 . doi: 10.1371/journal.pone.0007401

Wei T, Feng S, Shen Y, He P, Ma G, Yu X, Zhang F, Mao D (2013) Characterization of a novel thermophilic pyrethroid-hydrolyzing carboxylesterase from Sulfolobus tokodaii into a new family. J Mol Catal B Enzym 97:225-232 . doi: 10.1016/j.molcatb.2013.07.022

Wei Y, Swenson L, Castro C, Derewenda U, Minor W, Arai H, Aoki J, Inoue K, Servin-Gonzalez L, Derewenda ZS (1998) Structure of a microbial homologue of mammalian platelet-activating factor acetylhydrolases: Streptomyces exfoliatus lipase at $1.9 \AA$ resolution. Structure 6:511-519 . doi: 10.1016/S09692126(98)00052-5

Wemheuer F, Kaiser K, Karlovsky P, Daniel R, Vidal S, Wemheuer B (2017) Bacterial endophyte communities of three agricultural important grass species differ in their response towards management regimes. Sci Rep 7:40914 . doi: 10.1038/srep40914

Willmann M, El-Hadidi M, Huson DH, Schütz M, Weidenmaier C, Autenrieth IB, Peter S (2015) Antibiotic selection pressure determination through sequence-based metagenomics. Antimicrob Agents Chemother 59:7335-7345 . doi: 10.1128/AAC.01504-15

Wu C, Sun B (2009) Identification of novel esterase from metagenomic library of Yangtze River. J Microbiol Biotechnol 19:187-193 . doi: 10.4014/jmb.0804.292

Wu G, Zhang S, Zhang H, Zhang S, Liu Z (2013) A novel esterase from a psychrotrophic bacterium Psychrobacter celer $3 \mathrm{~Pb} 1$ showed cold-adaptation and salt-tolerance. J Mol Catal B Enzym 98:119-126 . doi: 10.1016/j.molcatb.2013.10.012

Yan W, Li F, Wang L, Zhu Y, Dong Z, Bai L (2017) Discovery and characterizaton of a novel lipase with transesterification activity from hot spring metagenomic library. Biotechnol Reports 14:27-33 . doi: 10.1016/j.btre.2016.12.007

Yu H, Zeng G, Huang H, Xi X, Wang R, Huang D, Huang G, Li J (2007) Microbial community succession and lignocellulose degradation during agricultural waste composting. Biodegradation 18:793-802 . doi: 10.1007/s10532-007-9108-8

Yu S, Yu S, Han W, Wang H, Zheng B, Feng Y (2010) A novel thermophilic lipase from Fervidobacterium nodosum Rt17-B1 representing a new subfamily of bacterial lipases. J Mol Catal B Enzym 66:81-89 . doi: 10.1016/j.molcatb.2010.03.007

Yu Z, Tang J, Liao H, Liu X, Zhou P, Chen Z, Rensing C, Zhou S (2018) The distinctive microbial community improves composting efficiency in a full-scale hyperthermophilic composting plant. Bioresour Technol 265:146-154 . doi: 10.1016/j.biortech.2018.06.011

Zarafeta D, Moschidi D, Ladoukakis E, Gavrilov S, Chrysina ED, Chatziioannou A, Kublanov I, Skretas G, Kolisis FN (2016) Metagenomic mining for thermostable esterolytic enzymes uncovers a new family of bacterial esterases. Sci Rep 6:38886 . doi: 10.1038/srep38886

Zhang X, Zhong Y, Yang S, Zhang W, Xu M, Ma A, Zhuang G, Chen G, Liu W (2014) Diversity and dynamics of the microbial community on decomposing wheat straw during mushroom compost production. Bioresour Technol 170:183-195 . doi: 10.1016/j.biortech.2014.07.093

Zhang Y, Hao J, Zhang YQ, Chen XL, Xie B Bin, Shi M, Zhou BC, Zhang YZ, Li PY (2017) Identification and characterization of a novel salt-tolerant esterase from the deep-sea sediment of the South China Sea. Front Microbiol 8:441 . doi: 10.3389/fmicb.2017.00441

Zhang Y, Pengjun S, Wanli L, Kun M, Yingguo B, Guozeng W, Zhichun Z, Bin Y (2009) Lipase diversity in glacier soil based on analysis of metagenomic DNA fragments and cell culture. J Microbiol Biotechnol 19:888-897 . doi: 10.4014/jmb.0812.695

Zhou G, Xu X, Qiu X, Zhang J (2019) Biochar influences the succession of microbial communities and the metabolic functions during rice straw composting with pig manure. Bioresour Technol 272:10-18 . doi: 10.1016/j.biortech.2018.09.135

Zhou H, Gu W, Sun W, Hay AG (2018) A microbial community snapshot of windrows from a commercial composting facility. Appl Microbiol Biotechnol 102:8069-8077 . doi: 10.1007/s00253-018-9201-4

Zhou J, Bruns MA, Tiedje JM (1996) DNA recovery from soils of diverse composition. Appl Environ Microbiol $62: 316-322$ 
Zhu W, Lomsadze A, Borodovsky M (2010) Ab initio gene identification in metagenomic sequences. Nucleic Acids Res 38:e132 . doi: 10.1093/nar/gkq275 


\section{Supplemental information for chapter II}

\section{Content}

Supplementary Figure S1. Overall workflow for identification of lipolytic enzymes (LEs) through function-driven and sequence-based approaches in this study.

Supplementary Figure S2. Rarefaction curves of subsampled OTUs for 16S rRNA genes (DNA level) and transcripts (RNA level) in compost55 and compost76 at 97\% similarity.

Supplementary Figure S3. Phylogenetic composition of bacterial communities in compost55 and compost76, revealed from 16S rRNA genes (DNA-level) and transcripts (RNA-level).

Supplementary Figure S4. Phylogenetic composition of bacterial communities in compost55 and compost76 as annotated by the MG-RAST platform.

Supplementary Figure S5. Functional distribution pattern in compost55 (blue filled circle) and compost76 (red filled circle) microbial consortia.

Supplementary Figure S6a. Multiple sequence alignments of partial amino acid sequences harboring homologous catalytic regions of homology. Lipolytic enzymes were from reported families.

Supplementary Figure S6b. Multiple sequence alignments of partial amino acid sequences harboring homologous catalytic regions of homology. Lipolytic enzymes were from putative novel families identified in this study.

Supplementary Figure S7. Phylogenetic distribution at phylum level of assigned PLPs in the most abundant lipolytic families.

Supplementary Figure S8. Protein Sequence similarity network for classification of assigned PLPs obtained by screening against from compost55 and compost76 assembled metagenomes.

Supplementary Figure S9. Functional lipolytic family profiles of assigned PLPs in different samples.

Supplementary Figure S10. Distribution of lipolytic families revealed from assigned PLPs of each habitat.

Supplementary Figure S11. Lipolytic families showing significant changes in abundance across different habitats.

Supplementary Figure S12. Phylogenetic origins of LEs in ESTHER database at phylum level in the most abundant lipolytic families.

Supplementary Figure S13. Taxonomic origins at genus level of the assigned PLPs across samples.

Supplementary Figure S14. Phylogenetic distribution of the assigned PLPs at phylum level in each habitat.

Supplementary Figure S15. Linear regression, the $x$ was the ratio of unique indicators to the total significant indicators in a habitat, as demonstrated by the bipartite association network shown in Figure 5. 
Supplementary Figure S16. Phylogenetic origin of the total PLPs (assigned and unassigned PLPs combined) at (A) domain and (B) phylum level.

Supplementary Figure S17. Heat map of the taxonomic origins at genus level of total PLPs across samples.

Supplementary Figure S18. Analysis of the phylogenetic profile at genus level of total PLPs across samples.

Supplementary Figure S19. Phylogenetic distribution of the total PLPs at phylum level of each habitat. 


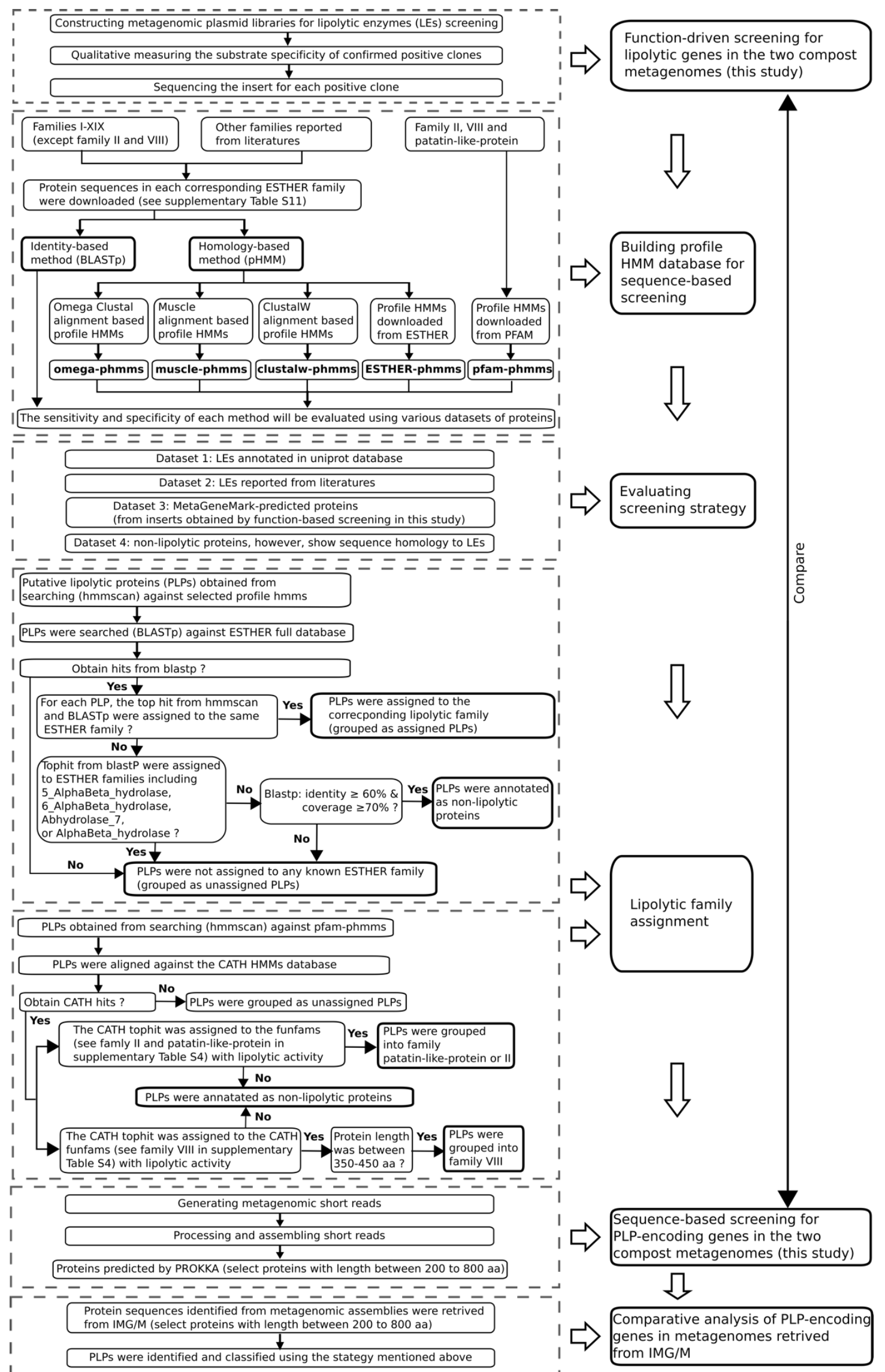


Supplementary Figure S1. Overall workflow for identification of lipolytic enzymes (LEs) through function-driven and sequence-based approaches in this study.

Firstly, LEs were identified by function-based screening of constructed metagenomic libraries. Positive clones were collected and inserts harboring lipolytic genes were sequenced. Lipolytic genes were subsequently revealed and classified. As for sequence-based screening, a search method based on the profile Hidden Markov Models (HMMs) was developed to identify and annotate the putative lipolytic proteins (PLPs) in assembled metagenomes. LEs can be generally divided into two major groups: $\alpha / \beta$ hydrolase or not $\alpha / \beta$ hydrolase. For LEs belong to the $\alpha / \beta$ hydrolase superfamily, four LE-specific profile HMM databases were retrieved (omega-phmms, muscle-phmms, clustalw-phmms and ESTHERphmms). For LEs that are not $\alpha / \beta$ hydrolases, profile HMMs were retrieved from the pfam database (pfam-phmms). The prediction sensitivity and specificity of each profile HMM database were evaluated using four datasets, and the best one was selected for subsequent analysis. The lipolytic family assignment of PLPs obtained by screening against the selected profile HMM database (one of omegaphmms, muscle-phmms, clustalw-phmms and ESTHER-phmms) were generally conducted by combining the annotations from hmmscan against the profile HMM database and blastp against the full ESTHER database. For PLPs obtained by screening against pfam-phmms, the annotation was performed by the subsequent screening against the CATH HMMs database. Based on the strategies for sequencebased screening and lipolytic family assignment, PLPs in the two compost assembled metagenomes were identified and annotated. The results from function-driven and sequence-based screening were also compared. Finally, assembled metagenomes in various habitats were retrieved from the IMG/M database, and PLPs were identified for comparative analysis. 


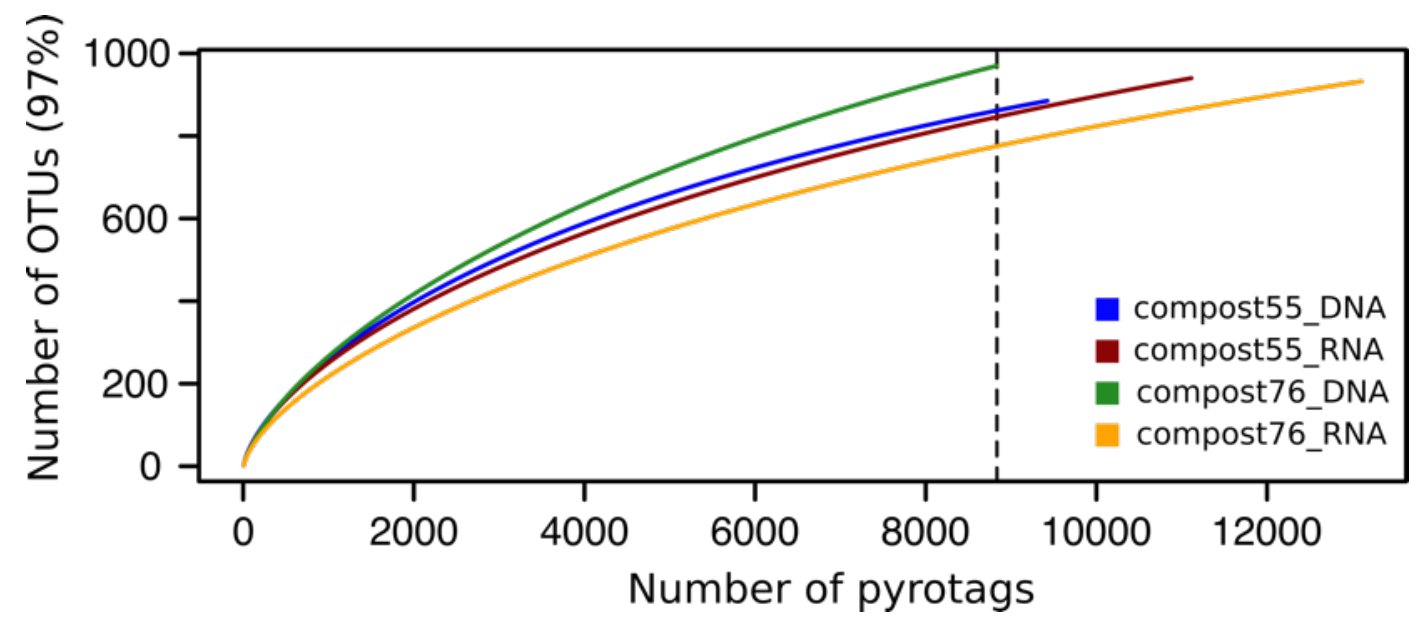

Supplementary Figure S2. Rarefaction curves of subsampled OTUs for 16S rRNA genes (DNA level) and transcripts (RNA level) in compost55 and compost76 at 97\% similarity. 


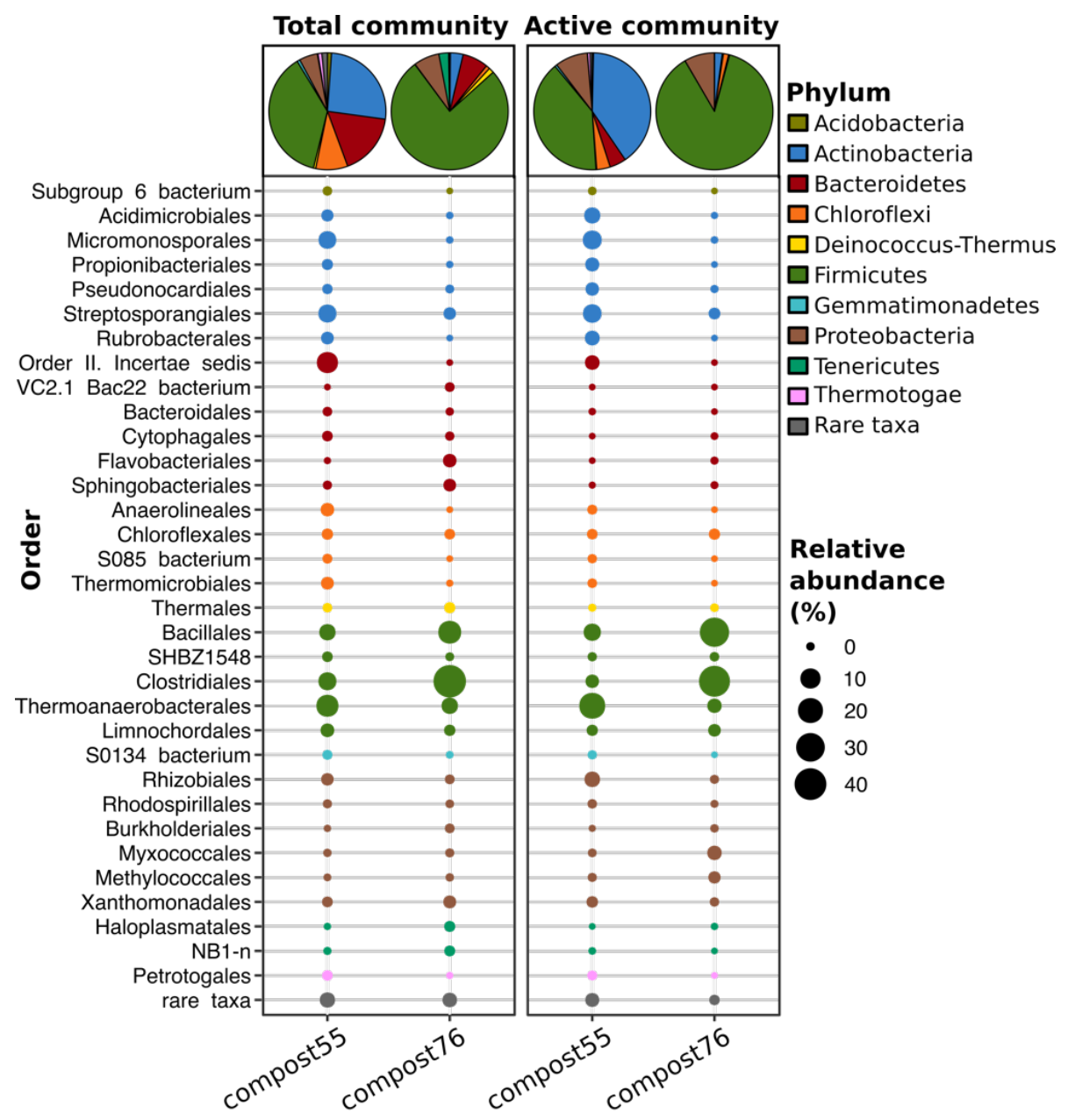

Supplementary Figure S3. Phylogenetic composition of bacterial communities in compost55 and compost76, revealed from 16S rRNA genes (DNA-level) and transcripts (RNA-level). Taxonomic specificity ranges from phylum level (pie chart) to order level (point chart) resolution when applicable. Taxonomic classification of DNA- or RNA-derived 16S rRNA gene sequences was performed according to SILVA SSU database 128 (Quast et al. 2013). Low relative abundant groups ( $<1 \%$ at phylum level or $<0.5 \%$ at order level) were summarized as artificial group "rare taxa". 


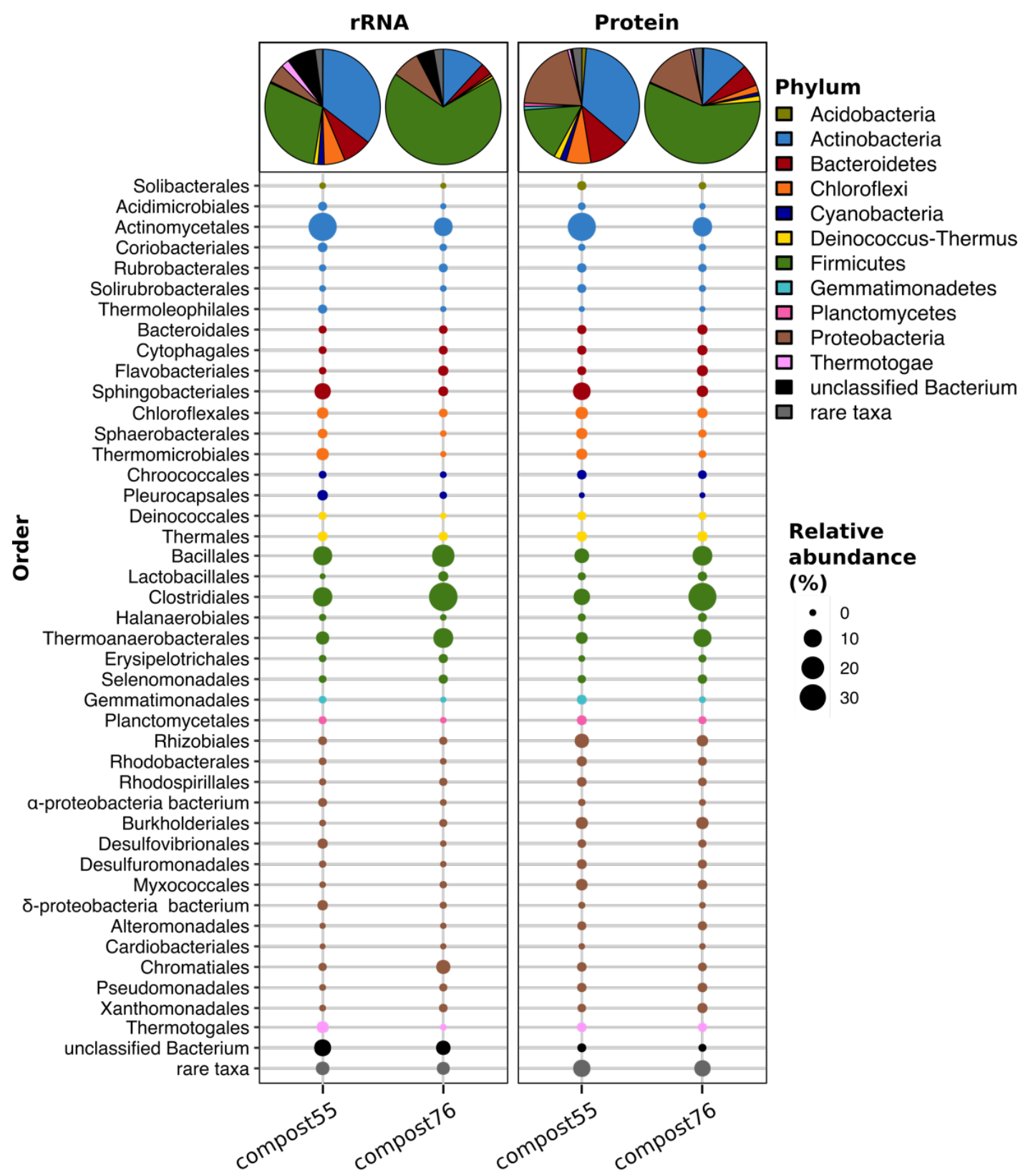

Supplementary Figure S4. Phylogenetic composition of bacterial communities in compost55 and compost76 as annotated by the MG-RAST platform (Keegan et al. 2016). Taxonomic specificity ranges from phylum level (pie chart) to order level (point chart) resolution when applicable. Microbial composition annotation was performed using MG-RAST best hit classification tool against the databases of M5RNA (Non-redundant multisource ribosomal RNA annotation) and M5NR (M5 non-redundant protein) available within MG-RAST with default settings. Low relative abundant groups ( $<1 \%$ at phylum level, $<0.5 \%$ at order level) were summarized as artificial group "rare taxa". 

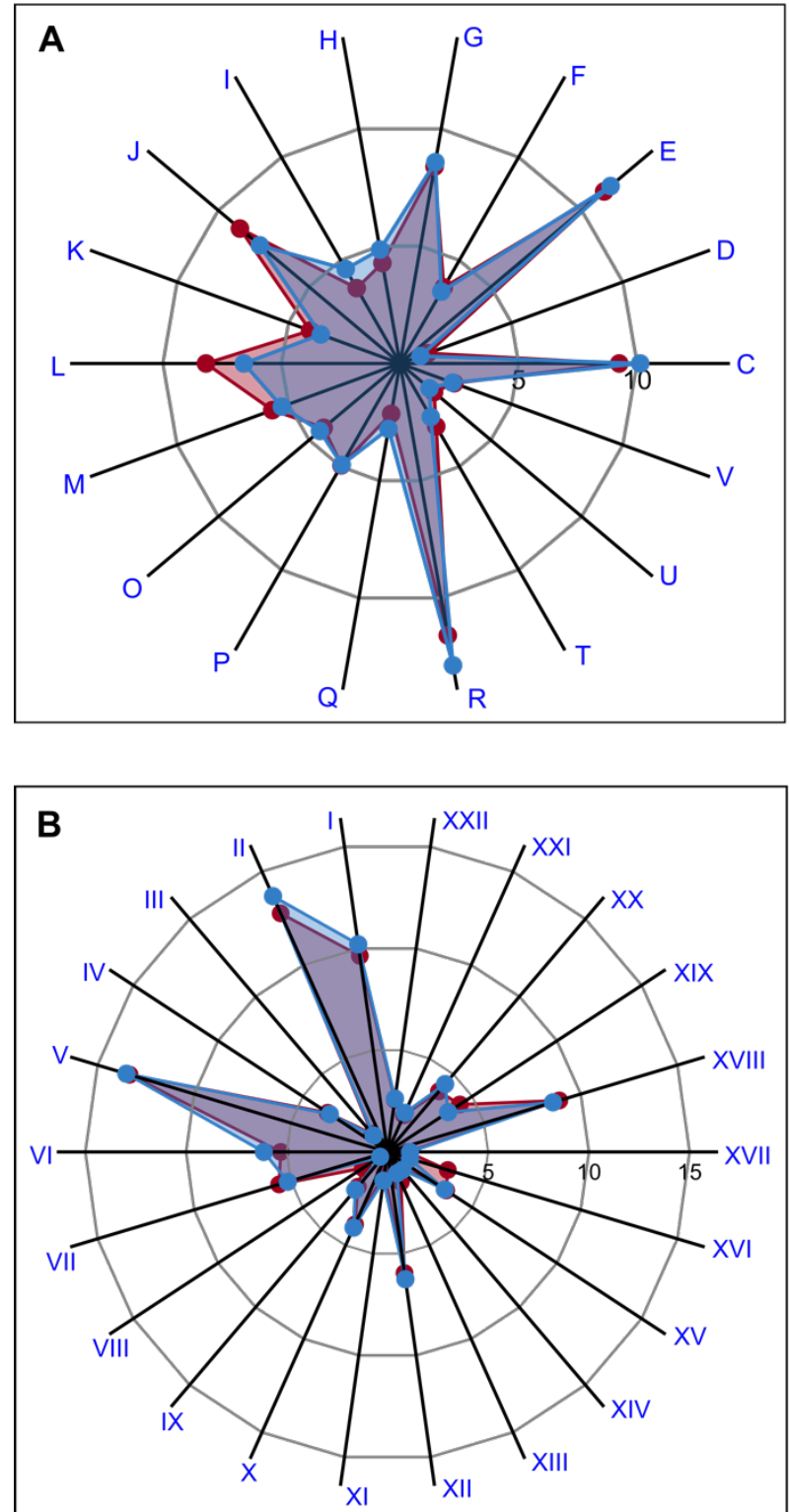

\section{COG catagory}

\section{C-Energy production and conversion}

D-Cell cycle control, cell division,

chromosome partitioning

E-Amino acid transport and metabolism

F-Nucleotide transport and metabolism

G-Carbohydrate transport and metabolism

$\mathrm{H}$-Coenzyme transport and metabolism

I-Lipid transport and metabolism

J-Translation, ribosomal structure and biogenesis

K-Transcription

L-Replication, recombination and repair

M-Cell wall/membrane/envelope biogenesis

O-Posttranslational modification,

protein turnover, chaperones

P-Inorganic ion transport and metabolism

Q-Secondary metabolites biosynthesis,

transport and catabolism

$\mathrm{R}$-General function prediction only

T-Signal transduction mechanisms

U-Intracellular trafficking, secretion,

and vesicular transport

V-Defense mechanisms

\section{Subsystems catagory}

I-Amino Acids and Derivatives

II-Carbohydrates

III-Cell Division and Cell Cycle

IV-Cell Wall and Capsule

$\mathrm{V}$-Clustering-based subsystems

VI-Cofactors, Vitamins, Prosthetic Groups, Pigments

VII-DNA Metabolism

VIII-Dormancy and Sporulation

IX-Fatty Acids, Lipids, and Isoprenoids

X-Membrane Transport

XI-Metabolism of Aromatic Compounds

XII-Miscellaneous

XIII-Motility and Chemotaxis

XIV-Nitrogen Metabolism

XV-Nucleosides and Nucleotides

XVI-Phages, Prophages, Transposable elements, Plasmids

XVII-Phosphorus Metabolism

XVIII-Protein Metabolism

XIX-RNA Metabolism

XX-Respiration

XXI-Stress Response

XXII-Virulence, Disease and Defense

Supplementary Figure S5. Functional distribution pattern in compost55 (blue filled circle) and compost76 (red filled circle) microbial consortia. MG-RAST annotation against COG (A) and (B) subsystems database are shown. Only functional categories with relative abundance of more than $1 \%$ were shown. The functional annotation of metagenomic reads was performed by MG-RAST pipeline (Keegan et al. 2016). 
Family I

EstC55-71

EstC55-88

EstC55-90

EstC55-105

EstC55-151

EstC76-177

EstC55-213

EstC55-235

AAB71210

CAA 67627

WP_03693241

145 DIVGHSOGG. MMPRYYT 238 TVIOTKYD ....EVVTP 275 PIDLSE....HLAIPFD

145 DIVGHSOGG. MMPRYYT 238 TVIOTKYD ... EVVTP 275 PLDLSE.... HLAIPFD

126 DIIGHSQGG. MMPRYYV 214 TVIASRYD . . . . . EVVTP 247 PLNPVE.... . HLAIIWD

99 DLVTHSMGG. LS SRWYV 174 GTFWSPCD . . . . EIIIP $203 \ldots \ldots$. . . . . . HISILAD

142 DIIGHSOGG. NVPMYWM 236 TVIMSRYD ..... VVVTP 269 PODPAG....HVGIFND

111 SVIAHSMGG. IVARRYM 195 GRPGR..DWGVLGELIVG 232 VIEGAV.....H. . . IAD

92 HLVAHSMGG. LDARYLI 198 GMAGPGTD. . . . . VPITP 237 WGRFLGTLRVDH. . . LR

160 DIVGHSQGG. MMPHYYI 252 TVIATRHD. ..... IVVTP 285 PDDPVG....HIGISFD

120 DIVGHSOGG. MLPRYYV 199 TVITTRYD . . . . EVVIP 234 PLDLYM.... HDQATKD

103 DIVAHSMGG ANTIYYT 157 TSIYSSAD *.................

164 DFVGHSOGGGI LPNAYI 260 TVISTRLD ..... MTVTP 291 PLDAYG...HGRIPYD

WP_012843686 92 HLIAHSMGG. LDARYLI 198 GMAGPGTD. . . . . VPITP 237 WGRFLGTLRVDH. . . LR

WP_071578729 103 DIVAHSMGG.ANTLYYI 157 TSIYSSAD . . . . MIVM 185 . . . VG . . . . HIGLLMN

WP_019713218 103 DIVAHSMGG ANTIYYT 157 TSTYSSAD ... MTVM $185 \ldots . . .25$

$\boldsymbol{\Lambda}$

Family II

EstC55-71

EstC55-88

WP_014065857

WP 072715438

WP 098062360

WP_103038013

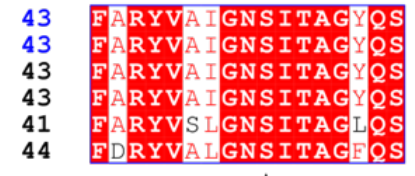

$\Delta$

EstC55-71

EstC55-88

WP_014065857

WP_072715438

WP_098062360

WP_103038013
$\boldsymbol{\Delta}$

$\Delta$

\section{Family III}

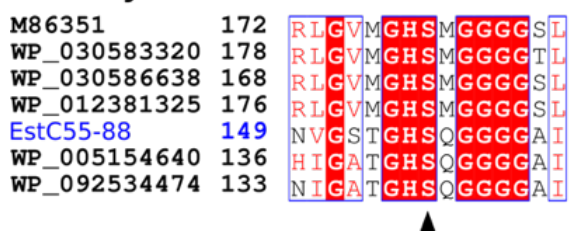

$\Delta$

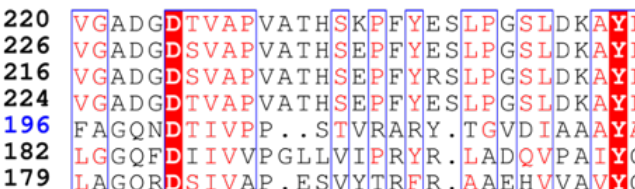

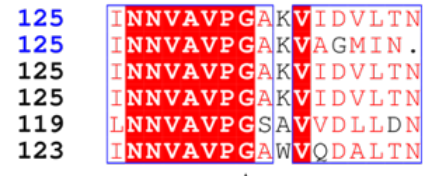

$\Delta$

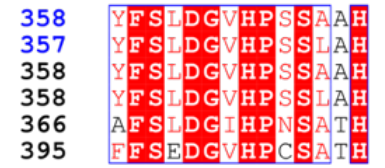

$\Delta$

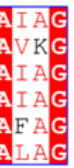

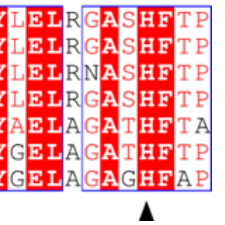


Family IV

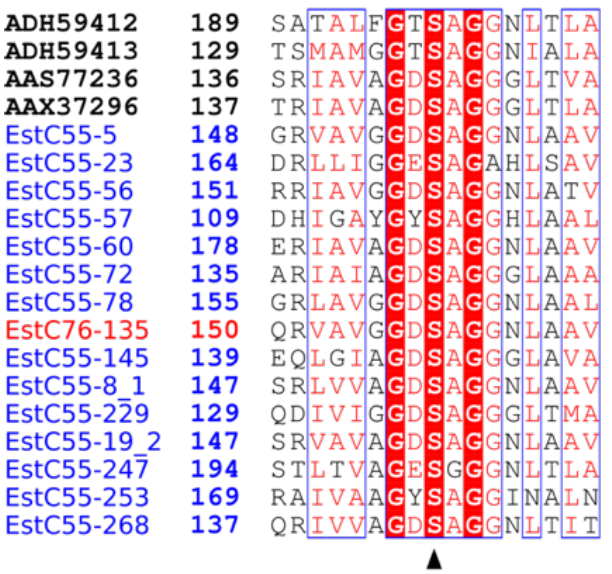

Family V

\begin{tabular}{|c|c|c|}
\hline & & \\
\hline CAA37863 & 137 & $\mathrm{GA} I \mathrm{SV}$ \\
\hline CAA47949 & 137 & SA I S V \\
\hline AAC 67392 & 140 & $\mathrm{~F} \vee \mathrm{AQ}$ \\
\hline EstC55-2 & 103 & L I. G \\
\hline EstC55-8_2 & 101 & $\mathrm{LV}$ \\
\hline EstC55-1 $\overline{2}$ & 176 & 4 \\
\hline EstC55-18 & 79 & W \\
\hline EstC55-19_1 & 90 & [" \\
\hline EstC55-20 & 146 & G GMT \\
\hline EstC55-25 & 158 & V LVG \\
\hline stC55-31 & 89 & SMI \\
\hline EstC55-34 & 97 & \\
\hline EstC55-43 & 90 & . \\
\hline EstC55-51 & 89 & 15 \\
\hline EstC55-76 & 173 & $\mathrm{~T}$ \\
\hline EstC76-28_1 & 81 & $\mathrm{~V} V \mathrm{P}$ \\
\hline EstC76-28_2 & 81 & \\
\hline EstC55-15 & 123 & $L$ \\
\hline EstC55-197 & 112 & $\mathrm{I}$ \\
\hline EstC55-215 & 90 & $\mathrm{I}$ \\
\hline EstC55-231 & 167 & $\mathrm{~T}$ \\
\hline EstC55-244 & 96 & GL $\mathrm{T}$ \\
\hline EstC76-248 & 81 & 0 \\
\hline EstC55-256 & 90 & MI \\
\hline EstC76-263 & 159 & 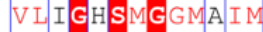 \\
\hline stC76-266 & 81 & $\mathrm{NV} \cdot$.GDS \\
\hline
\end{tabular}

290 232 236 237 250 264 253 210 279

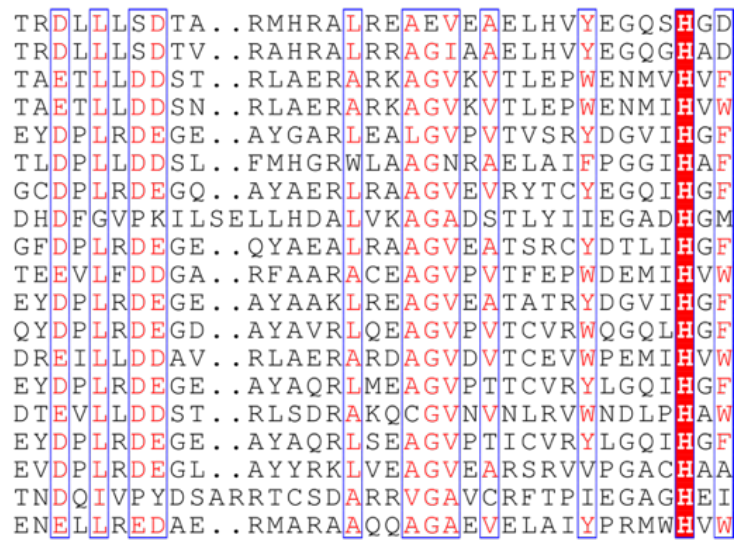

$\Delta$

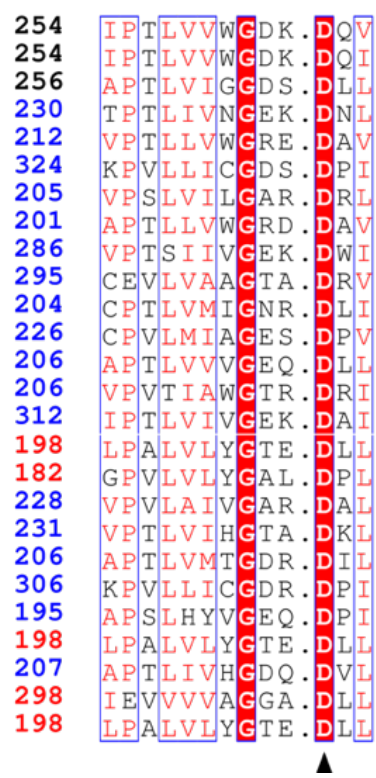

282 282 284 258 240 352 233 229 314 323 232 254 234 234 340 225 210 256 259

234 334 220 225 235 326 225

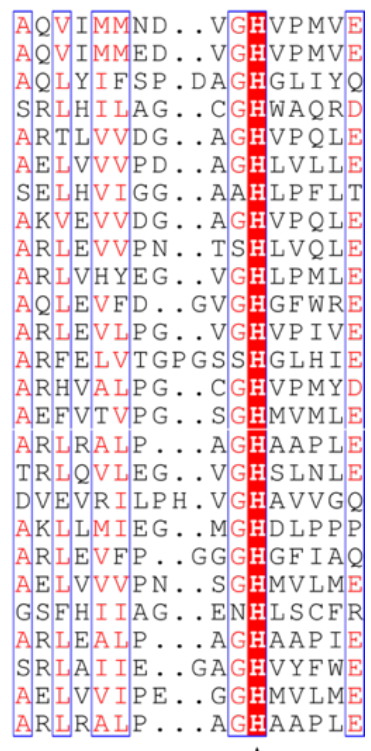
$\Delta$ 


\section{Family VII \\ Q01470 \\ P37967 \\ KJJ40755 175 \\ WP 064730418177 \\ EstC55-3 \\ EstC55-52 \\ EstC55-62 \\ EstC55-118 \\ EstC76-136}

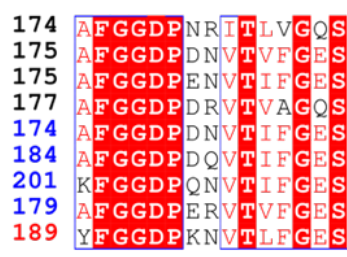

$\Delta$

Family VIII

WP 005474626

WP_014985987

WP_015576461

CDG54282

WP 093402197

WP 092376400

EstC55-4

EstC55-7

EstC55-40

EstC55-46

EstC55-53

EstC55-65

EstC55-66

EstC55-73

EstC55-80

EstC76-98

EstC55-110

EstC55-113

EstC76-123

EstC55-147

EstC55-164

EstC55-168

EstC76-174

EstC55-239

EstC55-245

EstC55-258
63 PWER T TVVNVWWTTKGĀTA. LCAHITADRGLIDLD

Family XVII

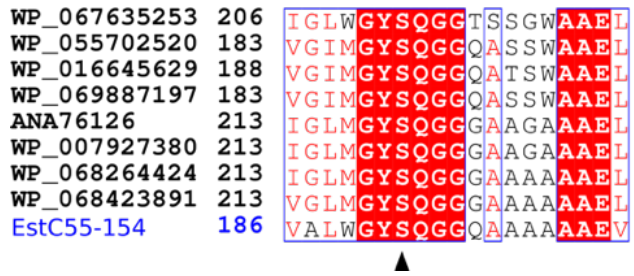

$\Delta$

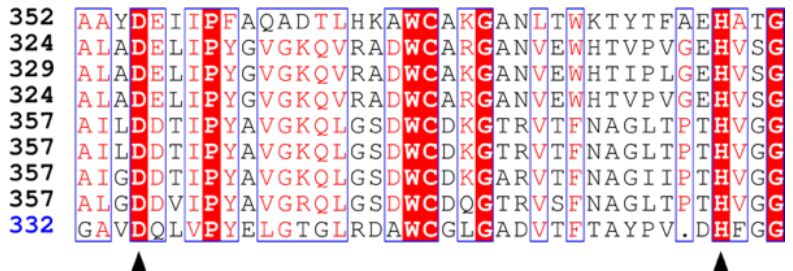


EM3L4

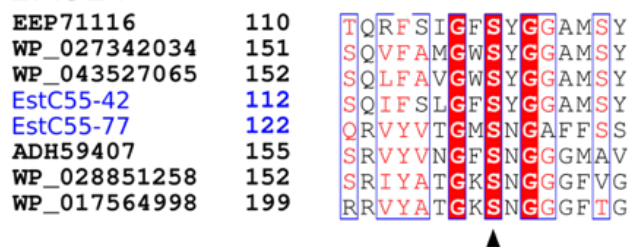

\section{EstGS}

AEM45109

OGO52417

EstC55-24

WP_052387799

WP_089246854
190
189
172
180
205
168

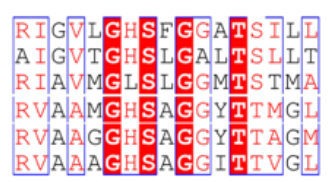

$\boldsymbol{\Delta}$

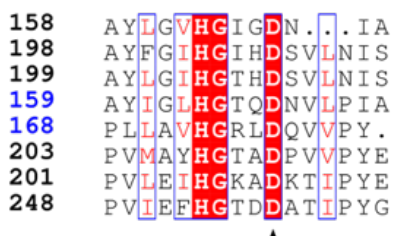

$\Delta$

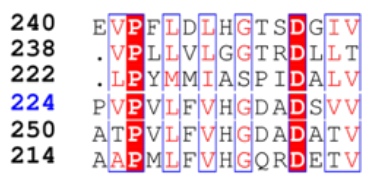

$\Delta$

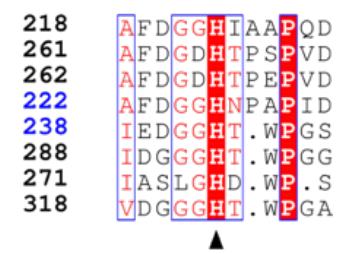

269 PRYRGDIVGGDHLGFL 266 PRYLVELLGANHIRFA 250 GAT LVISIDKASHTGEA

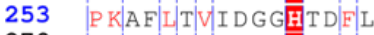
279 PKAF I T L LNGDHGG GI 243

FLS18

ACL67851 144 RIYLWGHSMGGAGTYH 196 LQGDQD. RLVTP TRQWVARMKELGMEHIYIEVPGGDHSLF ACL67852 169 RIYLMGHSMGGGGTLYL 221 VQGDQDR L VIVE IARRWVAKMKELGMTHEYIEIKDGNHVTS

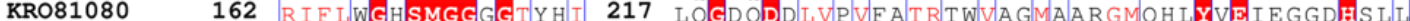
WP 014066117140 RVY TGLSMGGHGTWY 216 FHGADDPVVSVEA SRRMVAIRELGADVOYTEYEGVGHNAW

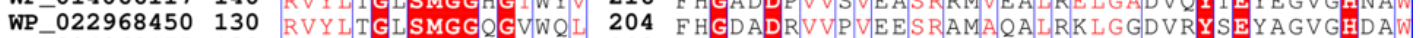

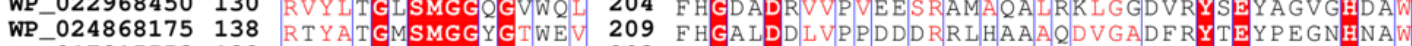
WP_017915553 132 RTYLTGMSMGGYGTWEI 203 FHGAODDVVLPHDDRKIVRAF KNLDADVRYTEYPQGNHNA W

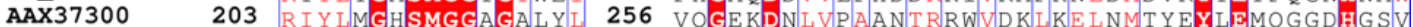
WP_050044108 151 RIYLAGHSMGGGGTIHI 202 VTGDKDTTVPVQMIRPFARKMKETNAKHVYKEIAGGNHGTT

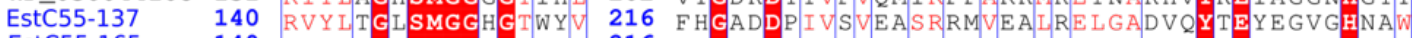

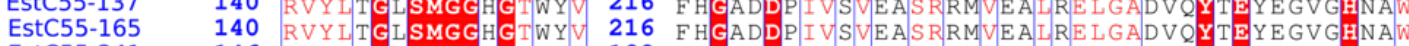

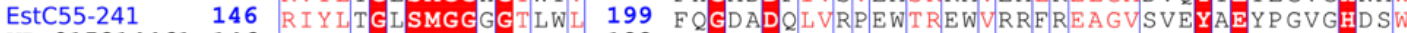
WP_015814461 146 RTYLTGLSMGGGGTLWI 199 FHGDADPVVPVAGT QKWVAQLQDLGAEVSYKEFVDVKHDSW

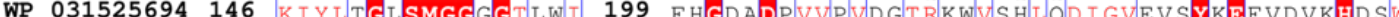
WP_026630869 146 KTYLTGIS SMGGGGTLWL

LipT

ADW21422 148 TDPEKVFVTGCSAGAYGAVF 234 WP_003047954 148 AQAERVEVTGCSAGAYGAIF 234 WP_038060347 148 PKAERVFVTGCSAGGYGAVF 234 $\begin{array}{llll}\text { AFS } 34517 & 148 & \text { TDPEKVFVTGCSAGAYGAVF } & 234\end{array}$ $\begin{array}{llll}\text { EStC76-179 } & 148 \text { TNPERVEVTGCSAGAYGAVI } 234\end{array}$ EstC76-218 148 TNPERVFVTGCSAGAYGAVL 234 LAQYTT LAOYTTLLDGTOI

$\boldsymbol{\Lambda}$

\begin{tabular}{ll|}
\hline LDGTQIFFY & 285 \\
LDGTQIFY & 285 \\
LDGTQIYFY & 285 \\
LDGTQIFFY & 285 \\
LDGTQIYFY & 285 \\
LDGTOIYFY & 285
\end{tabular}

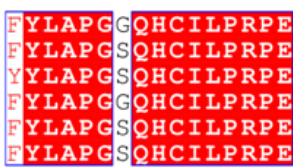

EstL28

EstC55-81

AFK29752

MBE13165

OON27855

WP 012642884

102
97
91
88
94
92

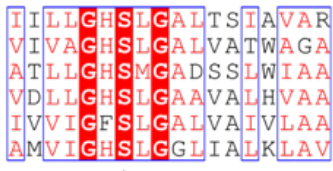

192
197
178
178
175
173

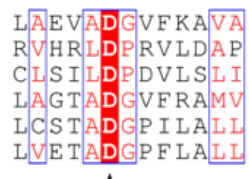

255

262

243

242

237

236

$\Delta$

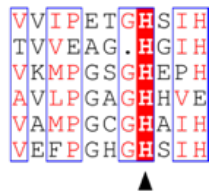



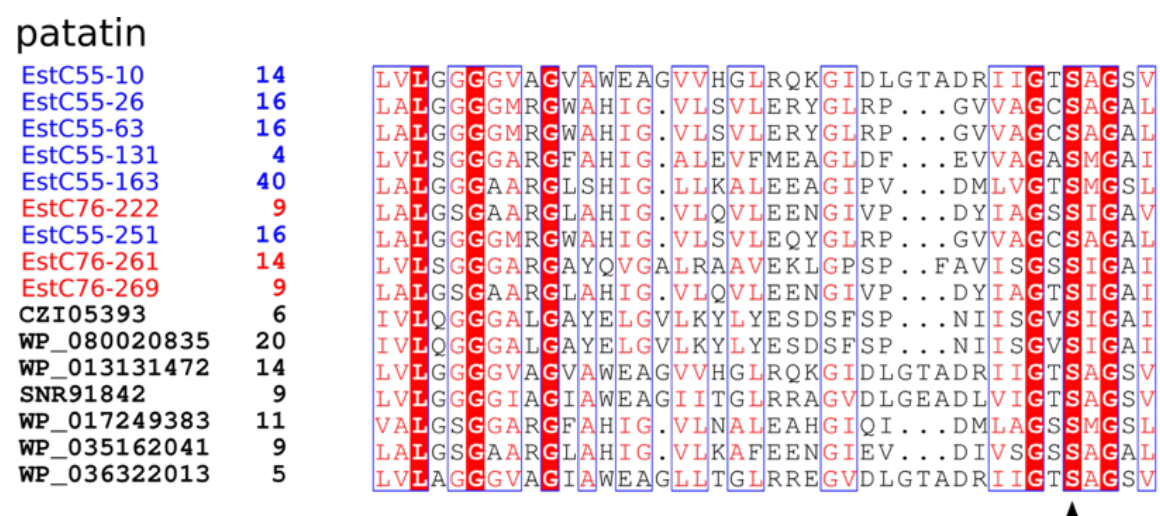

$\begin{array}{ll}\text { EstC55-10 } & 170 \\ \text { EstC55-26 } & 135 \\ \text { EstC55-63 } & 135 \\ \text { EstC55-131 } & 121 \\ \text { EstC55-163 } & 160 \\ \text { EstC76-222 } & 128 \\ \text { EstC55-251 } & 135 \\ \text { EstC76-261 } & 167 \\ \text { EstC76-269 } & 128 \\ \text { CZI05393 } & 159 \\ \text { WP_080020835 } & 173 \\ \text { WP_013131472 } & 170 \\ \text { SNR91842 } & 159 \\ \text { WP_017249383 } & 130 \\ \text { WP_035162041 } & 128 \\ \text { WP_036322013 } & 157\end{array}$

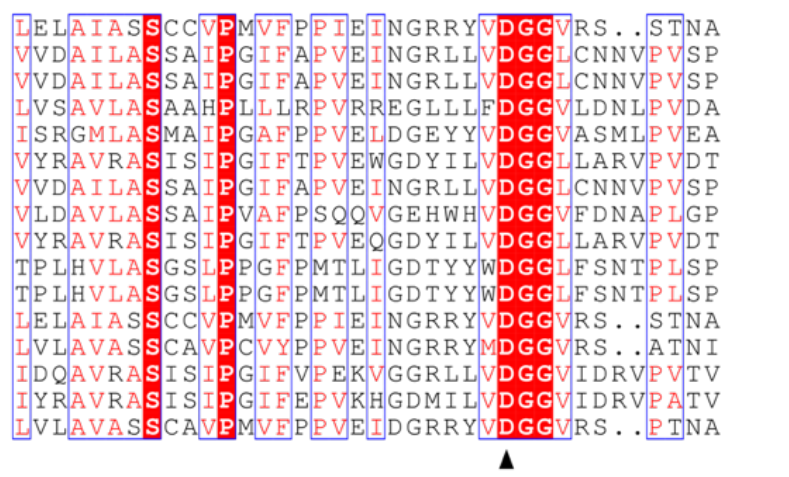

tannase
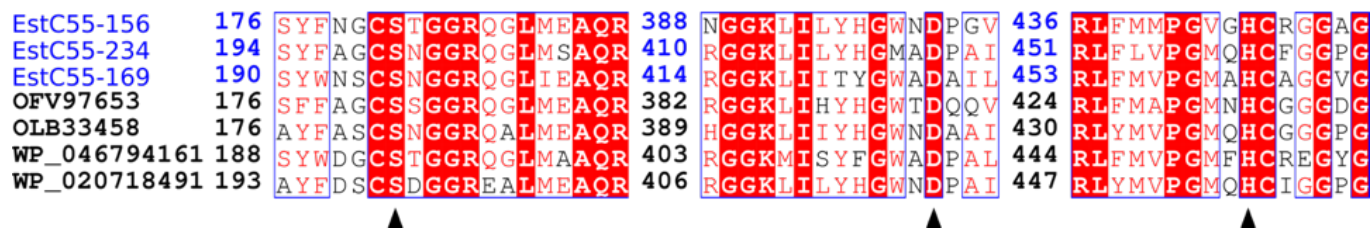

Supplementary Figure S6a. Multiple sequence alignments of partial amino acid sequences harboring homologous catalytic regions. Lipolytic enzymes were from reported families. Residues, which are partially consistent, are in frames. Identical residues are shaded in red. Triangles underneath residues indicate the catalytic triad. The functionally identified LEs were assigned to known lipolytic families. Family I: EstC55-71, EstC55-88, EstC55-90, EstC55-105, EstC55-151, EstC55-213 and EstC55-235, functionally derived LEs from sample compost55 (this study); EstC76-177, functionally derived LE from sample compost76 (this study); AAB71210, lipase LipA from Streptomyces cinnamoneus; AAA22574, lipase from Bacillus subtilis; CAA67627, triacylglycerol lipase from Cutibacterium acnes; WP_036932411, triacylglycerol lipase from Cutibacterium avidum; WP_012843686, alpha/beta fold hydrolase from Rhodothermus marinus; WP_071578729, triacylglycerol lipase from Bacillus sp. FMQ74; WP_019713218, triacylglycerol lipase from Bacillus subtilis. Family II: EstC55-111 and EstC55-150, functionally derived LEs from sample compost55 (this study); WP_014065857, SGNH/GDSL hydrolase family protein from Rhodothermus marinus; WP_072715438, hypothetical protein from Rhodothermus profundi; WP_098062360, hypothetical protein from Longimonas halophila; WP_103038013, SGNH/GDSL hydrolase family protein from Salinivenus iranica. Family III: EstC5595, functionally derived LE from sample compost55 (this study); M86351, triacylglycerol acylhydrolase from Streptomyces sp; WP_030583320, lipase from Streptomyces globisporus; WP_030586638, lipase from Streptomyces anulatus; WP_012381325, alpha/beta hydrolase from Streptomyces; WP_005154640, lipase from Amycolatopsis azurea; WP_092534474, acetylxylan esterase from Yuhushiella deserti. 
Family IV: EstC55-5, EstC55-23, EstC55-56, EstC55-57, EstC55-60, EstC55-72, EstC55-78, EstC55145, EstC55-8_1, EstC55-229, EstC55-19_2, EstC55-247, EstC55-253 and EstC55-268, functionally derived LEs from sample compost55 (this study); EstC76-135, F functionally derived LE from sample compost76 (this study); ADH59412, esterase from uncultured bacterium; ADH59413, esterase from uncultured bacterium; AAS77236, lipase/esterase from uncultured bacterium; AAX37296, lipase/esterase from uncultured bacterium. Family V: EstC55-2, EstC55-8_2, EstC55-12, EstC55-18, EstC55-19_1, EstC55-20, EstC55-25, EstC55-31, EstC55-34, EstC55-43, EstC55-51, EstC55-76, EstC55-159, EstC55-197, EstC55-215, EstC55-231, EstC55-244 and EstC55-256, functionally derived LEs from sample compost55 (this study); EstC76-28_1, EstC76-28_2, EstC76-248, EstC76-263 and EstC76-266, functionally derived LEs from sample compost76 (this study); CAA37863, triacylglycerol lipase from Moraxella sp.; CAA47949, triacylglycerol lipase from Psychrobacter immobilis; AAC67392, lipolytic enzyme from Sulfolobus acidocaldarius. Family VII: EstC55-3, EstC55-52, EstC55-62 and EstC55-118, functionally derived LEs from sample compost55 (this study); EstC76-136, functionally derived LE from sample compost76 (this study); Q01470, serine esterase from Pseudarthrobacter oxydans; P37967, para-nitrobenzyl esterase from Bacillus subtilis; KJJ40755, para-nitrobenzyl esterase from Bacillus subtilis; WP_064730418, carboxylesterase from Streptomyces parvulus. Family VIII: EstC55-4, EstC55-7, EstC55-40, EstC55-46, EstC55-53, EstC55-65, EstC55-66, EstC55-73, EstC55-80, EstC55-110, EstC55-113, EstC55-147, EstC55-164, EstC55-168, EstC55-239, EstC55-245 and EstC55258, functionally derived LEs from sample compost55 (this study); EstC76-98, EstC76-123 and EstC76174, functionally derived LEs from sample compost76 (this study); WP_005474626, esterase from Streptomyces bottropensis; WP_014985987, esterase from Nocardia brasiliensis; WP_015576461, esterase from Streptomyces; CDG54282, esterase EstB from Halomonas sp. A3H3; WP_093402197, carboxylesterase from Verrucosispora sediminis; WP_092376400, carboxylesterase from Xiangella phaseoli. Family XVII: EstC55-154, functionally derived LE from sample compost55 (this study); WP_067635253, triacylglycerol lipase from Actinomadura latina; WP_055702520, lipase from Streptomyces silaceus; WP_016645629, inactive lipase from Streptomyces aurantiacus; WP_069887197, lipase from Streptomyces luteocolor; ANA76126, secretory lipase LipJ2 from Janibacter sp. R02; WP_007927380, secretory lipase from Janibacter hoylei; WP_068264424, lipase from Janibacter limosus; WP_068423891, lipase from Janibacter terrae. EM3L4: EstC55-42 and EstC55-77, functionally derived LEs from sample compost55 (this study); EEP71116, ferruloyl esterase fee1B from Micromonospora sp. ATCC 39149; WP_027342034, cellulose-binding protein from Hamadaea tsunoensis; WP_043527065, cellulose-binding protein from Actinoplanes utahensis; ADH59407, esterase/lipase from uncultured bacterium; WP_028851258, hypothetical protein from Thermocrispum municipal; WP_017564998, hypothetical protein from Nocardiopsis synnemataformans. EstGS: EstC55-24, functionally derived LE from sample compost55 (this study); AEM45109, hypothetical protein from uncultured organism; OGO52417, hypothetical protein from Chloroflexi bacterium; WP_022959187, alpha/beta hydrolase from Spongiibacter tropicus; WP_052387799, alpha/beta hydrolase from Dactylosporangium aurantiacum; WP_089246854, chlorophyllase from Asanoa hainanensis. FLS18: EstC55-137, EstC55-165 and EstC55-241, functionally derived LEs from sample compost55 (this study); ACL67851, esterase/lipase from uncultured bacterium FLS18; ACL67852, esterase/lipase from uncultured bacterium FLS18; KRO81080, hypothetical protein from OM182 bacterium; WP_014066117, phospholipase from Rhodothermus marinus; WP_022968450, phospholipase from Arenimonas oryziterrae; WP_024868175, phospholipase from Pseudoxanthomonas suwonensis; WP_017915553, phospholipase from Xanthomonas sp. SHU 308; AAX37300, 
lipase/esterase from uncultured bacterium; WP_050044108, alpha/beta hydrolase from Verrucomicrobia bacterium SCGC AAA168-F10; WP_015814461, phospholipase/carboxylesterase from Dyadobacter fermentans; WP_031525694, phospholipase/carboxylesterase from Dyadobacter crusticola; WP_026630869, phospholipase/carboxylesterase from Dyadobacter alkalitolerans. LipT: EstC76-179 and EstC76-218, functionally derived LEs from sample compost76 (this study); ADW21422, putative esterase from Thermus scotoductus SA-01; WP_003047954, esterase from Thermus aquaticus; WP_038060347, esterase from Thermus filiformis; AFS34517, LipT from uncultured bacterium. EstL28: EstC55-81, functionally derived esterase from sample compost55 (this study); AFK29752, esterase from uncultured bacterium; MBE13165, hypothetical protein from Chloroflexi bacterium; OON27855, hypothetical protein from Micromonospora sp. Rc5; WP_012642884, alpha/beta hydrolase from Thermomicrobium roseum; WP_051913750, alpha/beta hydrolase from Thermorudis peleae; Patatinlike-protein: EstC55-10, EstC55-26, EstC55-63, EstC55-131, EstC55-163 and EstC55-251, functionally derived LEs from sample compost55 (this study); EstC76-222, EstC76-261 and EstC76-269 functionally derived LEs from sample compost76 (this study); CZI05393, patatin from Legionella pneumophila; WP_080020835, patatin-like phospholipase family protein from Legionella pneumophila; WP_013131472, patatin from Thermobispora bispora; SNR91842, NTE family protein from Streptosporangium subroseum; WP_017249383, esterase from Brevibacillus brevis; WP_035162041, esterase from Caloranaerobacter azorensis; WP_036322013, patatin-like phospholipase family protein from Microbispora sp.. Tannase: EstC55-156, EstC55-234 and EstC55-269, functionally derived LEs from sample compost55 (this study); OFV97653, hypothetical protein from Acidobacteria bacterium; OLB33458, feruloyl esterase from Acidobacteria bacterium; WP_046794161, tannase/feruloyl esterase family alpha/beta hydrolase from Rhizobium sp;; WP_020718491, tannase/feruloyl esterase family alpha/beta hydrolase from Acidobacteriaceae bacterium. 

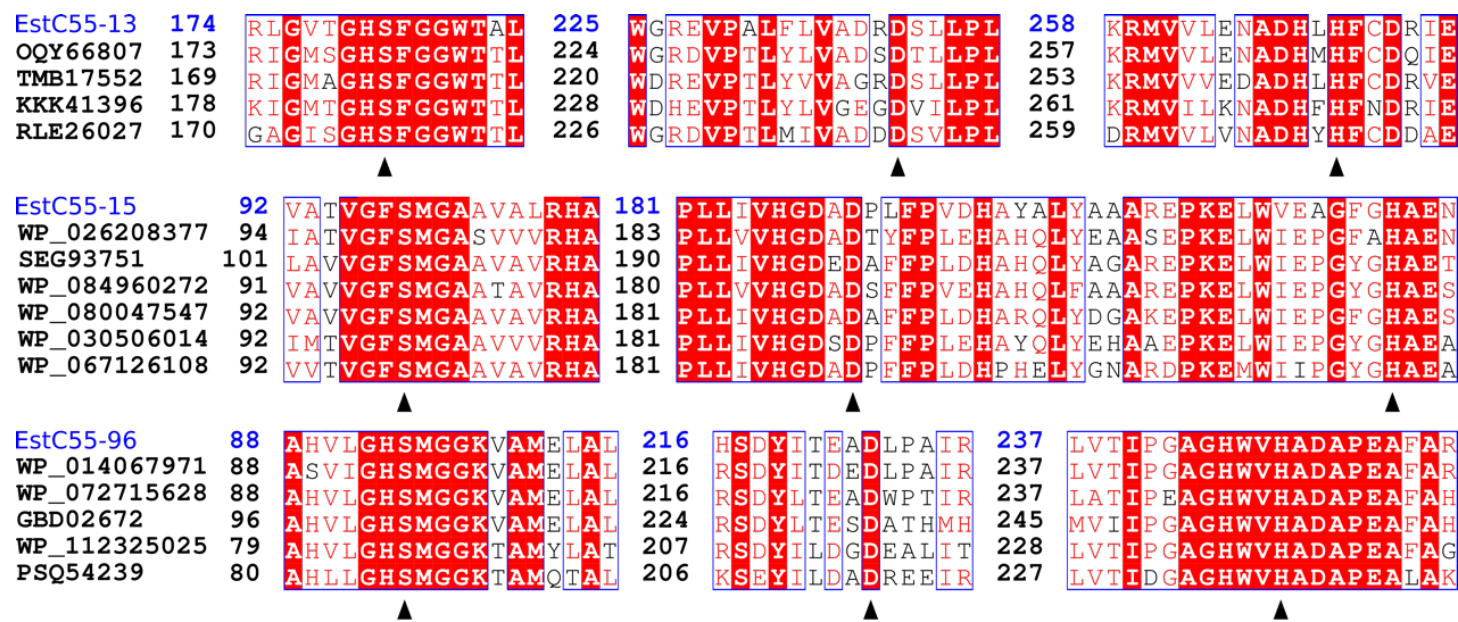

$\Delta$
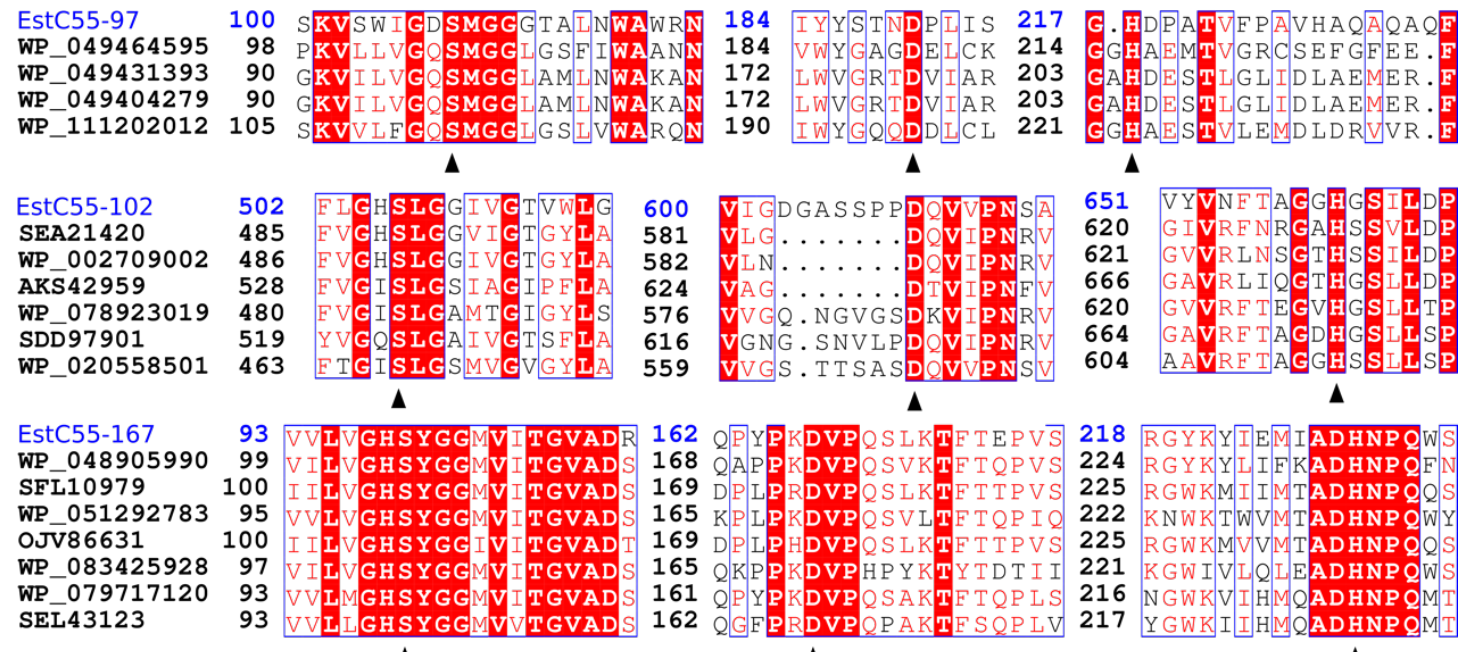

$\Delta$
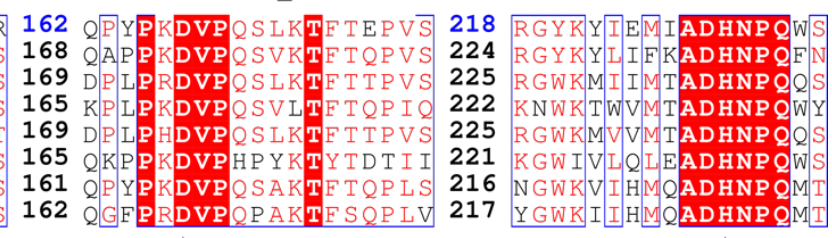

$\Delta$

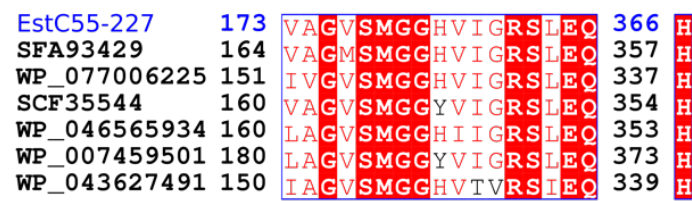
WP_043627491 150

$\Delta$

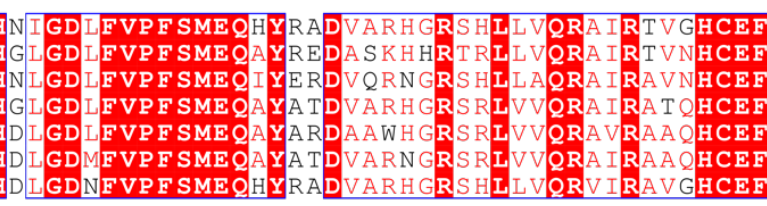

$\Delta$

$\Lambda$

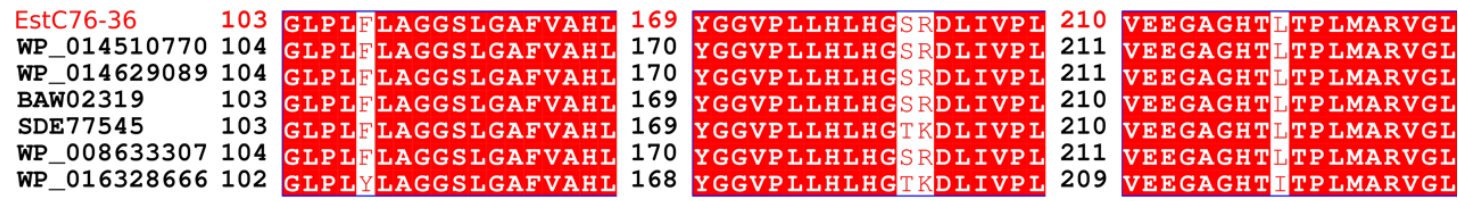

$\Delta$

$\Delta$

$\Lambda$

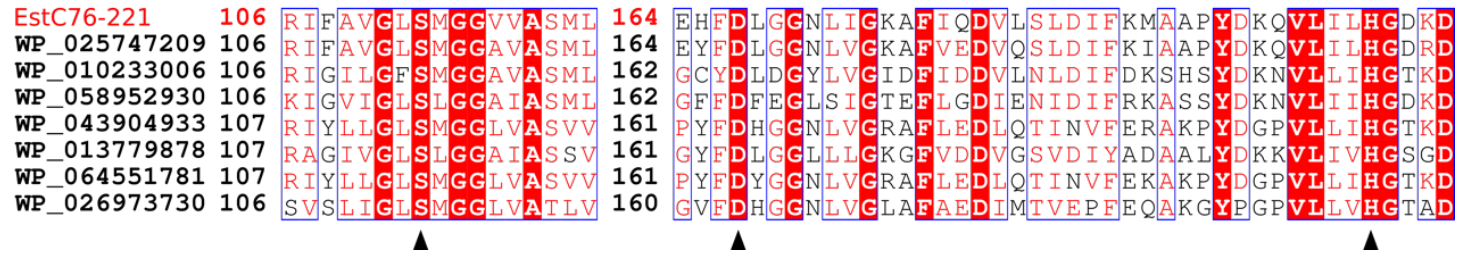

Supplementary Figure S6b. Multiple sequence alignments of partial amino acid sequences harboring homologous catalytic regions. Lipolytic enzymes were from putative novel families identified in this study. Residues, which are partially consistent, are in frames. Identical residues are shaded in red. Triangles underneath residues indicate the catalytic triad. Putative new family 1: EstC55-13, functionally derived LE from sample compost55 (this study); OQY66807, hypothetical protein from Polyangiaceae bacterium UTPRO1; TMB17552, hypothetical protein from Deltaproteobacteria 
bacterium; KKK41396, Alpha/beta hydrolase family protein from Lokiarchaeum sp. GC14_75; RLE26027, hypothetical protein from Actinobacteria bacterium. Putative new family 2: EstC55-15, functionally derived LE from sample compost55 (this study); WP_026208377, hydrolase from Catelliglobosispora koreensis; SEG93751, Alpha/beta hydrolase family protein from Nonomuraea solani; WP_084960272,alpha/beta hydrolase from Thermoactinospora rubra; WP_080047547, alpha/beta hydrolase from Nonomuraea sp. ATCC 55076; WP_030506014, alpha/beta hydrolase from Microbispora rosea; WP_067126108, hydrolase from Microtetraspora malaysiensis. Putative new family 3: EstC55-96, functionally derived LE from sample compost55 (this study); WP_014067971, alpha/beta fold hydrolase from Rhodothermus marinus; WP_072715628, alpha/beta fold hydrolase from Rhodothermus profundi; GBD02672, Esterase YbfF from bacterium HR18; WP_112325025, alpha/beta fold hydrolase from Rhodothermaceae bacterium; PSQ54239, alpha/beta hydrolase from Bacteroidetes bacterium QH_10_64_37. Putative new family 4: EstC55-97, functionally derived LE from sample compost55 (this study); WP_049464595, alpha/beta hydrolase from Stenotrophomonas maltophilia; WP_049431393, alpha/beta hydrolase from Stenotrophomonas maltophilia; WP_049404279, alpha/beta hydrolase from Stenotrophomonas maltophilia; WP_111202012.1, hypothetical protein from Stenotrophomonas maltophilia. Putative new family 5: EstC55-102, functionally derived LE from sample compost55 (this study); SEA21420, alpha/beta hydrolase family protein from Thiothrix caldifontis; WP_002709002, lipase from Thiothrix nivea; AKS42959, Extracellular lipase, Pla-1/cef family from Wenzhouxiangella marina; WP_078923019, lipase from Thiothrix eikelboomii; SDD97901, Alpha/beta hydrolase family protein from Aquimonas voraii; WP_020558501, hypothetical protein from Thiothrix flexilis. Putative new family 6: EstC55-167, functionally derived LE from sample compost55 (this study); WP_048905990, alpha/beta hydrolase from Pedobacter sp. V48; SFL10979, alpha/beta hydrolase family protein from Porphyromonadaceae bacterium KH3CP3RA; WP_051292783, alpha/beta hydrolase from Olivibacter sitiensis; OJV86631, alpha/beta hydrolase from Bacteroidia bacterium 44-10; WP_083425928, alpha/beta hydrolase from Zhouia amylolytica; WP_079717120, alpha/beta hydrolase from Parapedobacter luteus; SEL43123, Pimeloyl-ACP methyl ester carboxylesterase from Parapedobacter koreensis. Putative new family 7: EstC55-227, functionally derived LE from sample compost55 (this study); SFA93429, hypothetical protein SAMN05216266_102299 from Amycolatopsis marina; WP_077006225, hypothetical protein from Saccharothrix sp. ALI-22-I; SCF35544, Alpha/beta hydrolase family from Micromonospora saelicesensis; WP_046565934, alpha/beta hydrolase from Micromonospora sp. HK10; WP_007459501, alpha/beta hydrolase from Micromonospora lupini; WP_043627491, alpha/beta hydrolase from Nonomuraea candida. Putative new family 8: EstC76-36, functionally derived LE from sample compost76 (this study); WP_014510770, alpha/beta hydrolase from Thermus thermophilus; WP_014629089, phospholipase from Thermus thermophilus; BAW02319, esterase from Thermus thermophilus; SDE77545, hypothetical protein SAMN04488243_1105 from Thermus arciformis; WP_008633307, phospholipase from Thermus parvatiensis; WP_016328666, alpha/beta hydrolase from Thermus oshimai. Putative new family 9: EstC76-221, functionally derived LE from sample compost76 (this study); WP_025747209, alpha/beta hydrolase from Caldicoprobacter; WP_010233006, alpha/beta hydrolase from Clostridium arbusti; WP_058952930 alpha/beta fold hydrolase from Clostridium tyrobutyricum; WP_043904933 alpha/beta fold hydrolase from Parageobacillus genomosp.; WP_013779878 alpha/beta hydrolase from Mahella australiensis; WP_064551781 alpha/beta fold hydrolase from Parageobacillus thermoglucosidasius; WP_026973730 alpha/beta fold hydrolase from Alicyclobacillus contaminans. 

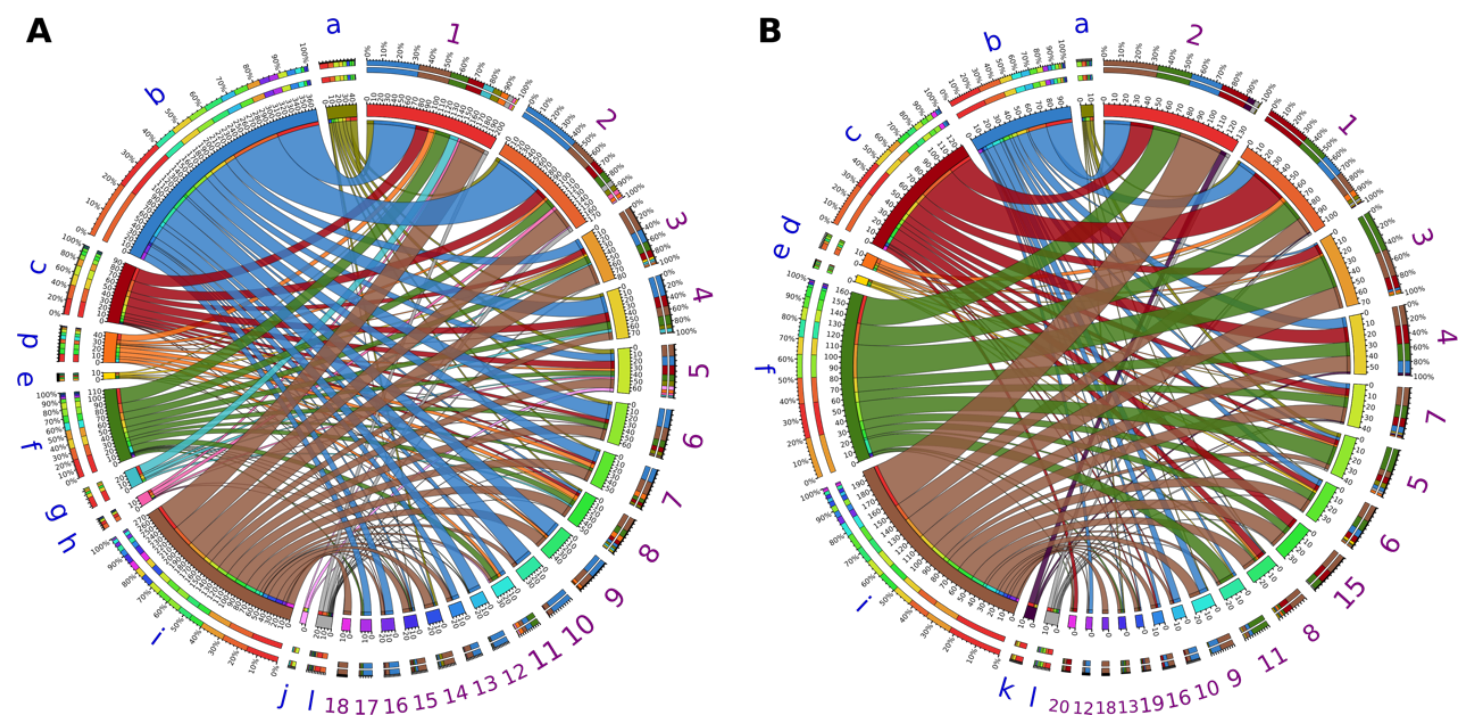

Supplementary Figure S7. Phylogenetic distribution at phylum level of assigned PLPs in the most abundant lipolytic families. Assigned PLPs were identified by screening the assembled metagenome of (A) compost55 and (B) compost76. Taxonomic information of PLP-encoding genes was annotated by KAIJU (Menzel et al. 2016). The data was visualized via Circos software. The width of bars from each phylum (blue) and ESTHER family (purple) indicates their relative abundance. Bacterial phyla: a, Acidobacteria; b, Actinobacteria; c, Bacteroidetes; d, Chloroflexi e, Deinococcus-Thermus; f, Firmicutes; g, Gemmatimonadetes; h, Planctomycetes; i, Proteobacteria; j, Thermotogae; k, Verrucomicrobia; 1, unclassified Bacteria. Lipolytic families in ESTHER databases: 1, VIII ; 2, Hormone-sensitive_lipase_like; 3, patatin-like-protein; 4, II; 5, A85-Feruloyl-Esterase; 6, Carb_B_Bacteria; 7, Homoserine_transacetylase; 8, Lysophospholipase_carboxylesterase; 9, Carboxymethyl-butenolide_lactonase; 10, Polyesterase-lipase-cutinase; 11, CarbLipBact_2; 12, Lipase_2; 13, Chlorophyllase; 14, Tannase; 15, A85-EsteraseD-FGH; 16, Fungal_Bact_LIP; 17, Est9X; 18, Bacterial_lip_FamI.3; 19, PC-sterol_acyltransferase; 20, Lipase_3. Only phyla and protein families with a relative abundance $>1 \%$ are shown. 


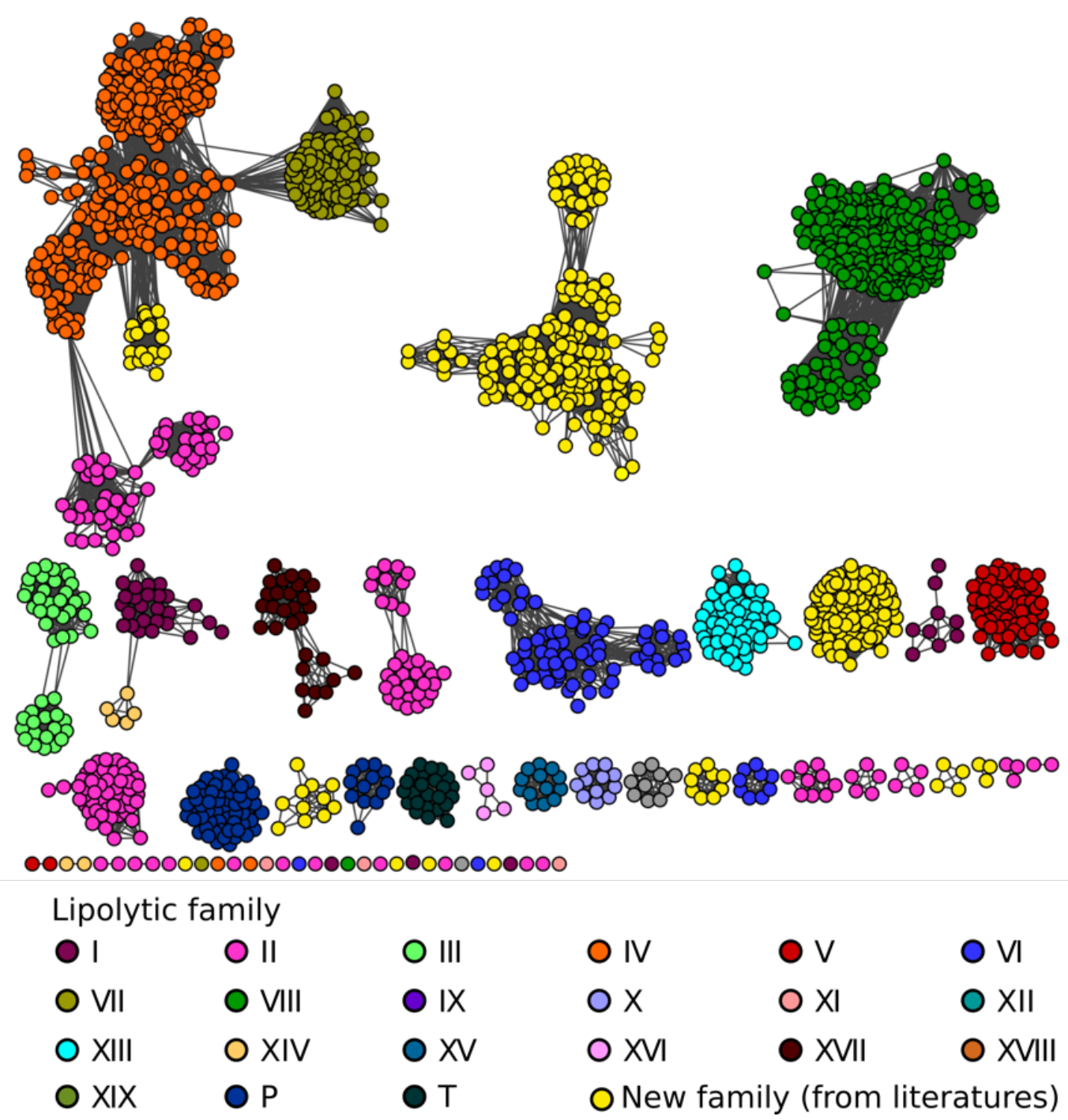

Supplementary Figure S8. Protein Sequence similarity network for classification of assigned PLPs obtained by screening against from compost 55 and compost 76 assembled metagenomes. Assigned PLPs were pooled and clustered at $100 \%$ identity using CD-HIT (Huang et al. 2010). Then, the resulting sequences were submitted to the EFI-EST (Gerlt et al. 2015) to generate the network. Each node represents an assigned PLP and is colored according to its lipolytic family. Each edge in the network represents a BLAST connection with an E-value cutoff of $\leq 1 \mathrm{e}^{-15}$. At this cut-off, sequences have a median percent identity and alignment length of $35 \%$ and 291 amino acids, respectively. Lengths of edges are not meaningful except that sequences in tightly clustered groups are relatively more similar to each other than sequences with few connections. Nodes were arranged using the $y$ Files organic layout provided with Cytoscape version 3.4.0 (Shannon et al. 2003). 

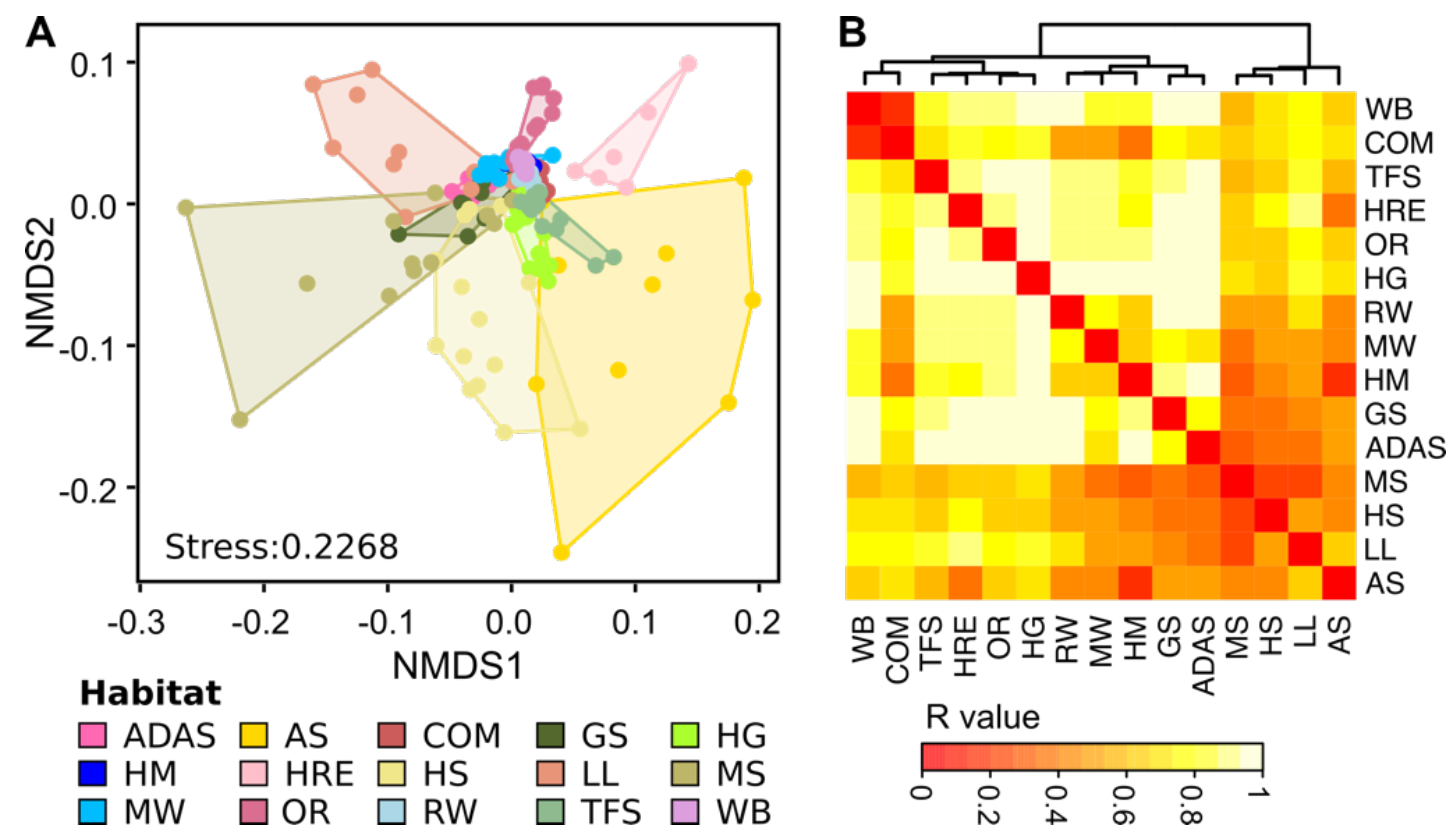

Supplementary Figure S9. Functional lipolytic family profiles of assigned PLPs in different samples. A, Non-metric multidimensional scaling (NMDS) analysis of the lipolytic family profiles across samples was performed. Samples were colored by its habitat source. B, ANOSIM test the group dissimilarity of lipolytic family profiles between habitats (9999 permutations, $\mathrm{p}<0.001)$. The resulting $\mathrm{R}$ values are shown by the heatmap, and the color intensity (red to light yellow) indicates the change of $\mathrm{R}$ values ( 0 to 1). Hierarchical clustering analysis of $\mathrm{R}$ values was performed to generate the cluster dendrogram using the Ward.D clustering method based on Bray-Curtis distance matrices. For all the analysis, LPGM values were $\log _{10}$ transformed. Abbreviations of habitats: ADAS, anaerobic digestor active sludge; AS, agricultural soil; COM, compost; GS grassland soil; HG, human gut; HM, hypersaline mat; HRE, hydrocarbon resource environment; HS, hot spring; LL, landfill leachate; MS, marine sediment; MW, marine water; OR, oil reservoir; RW, river water; TFS, tropical forest soil; WB, wastewater bioreactor. 


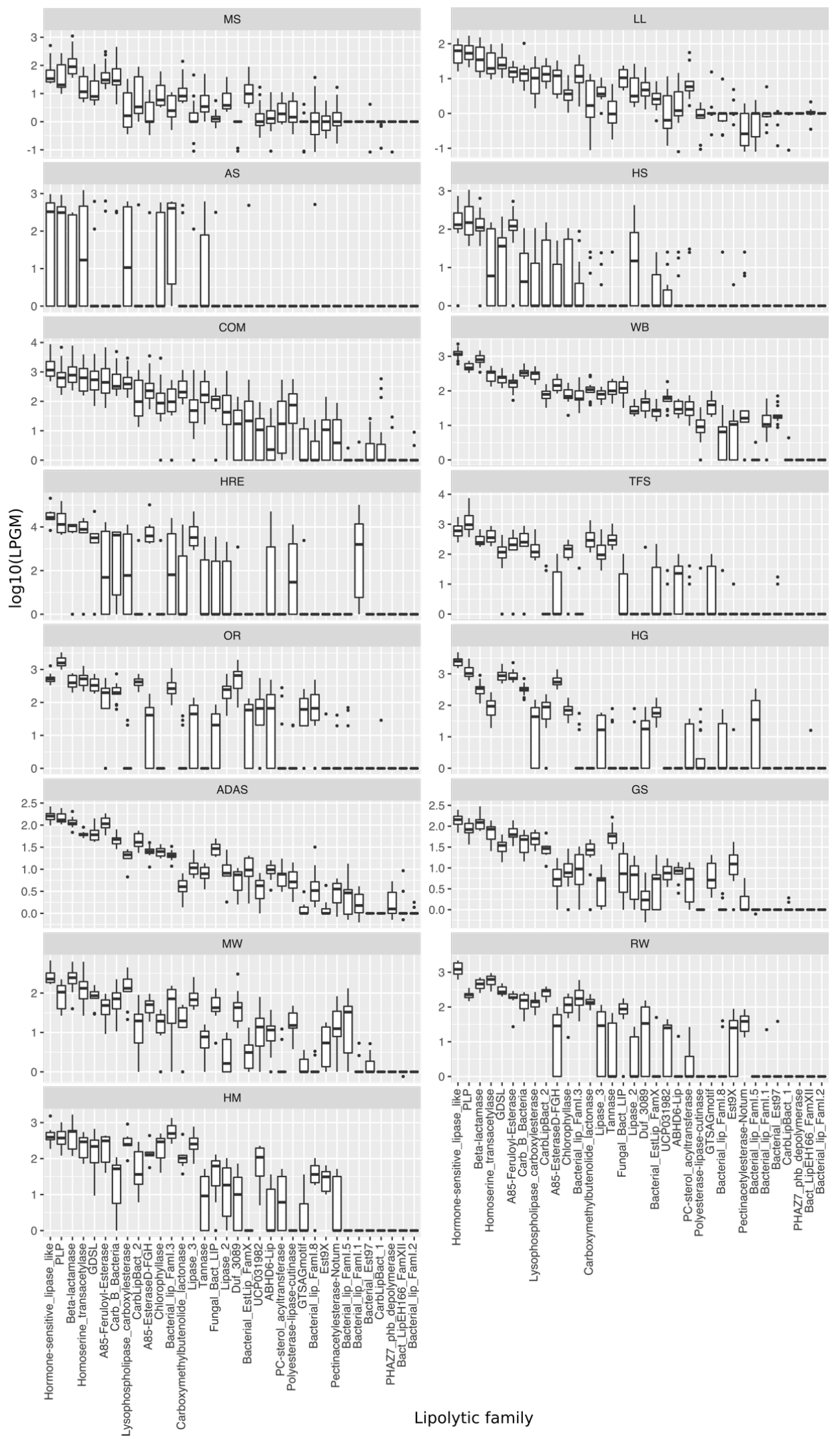

Supplementary Figure S10. Distribution of lipolytic families revealed from assigned PLPs of each habitat. The abundance was inferred from $\log _{10}$ scaled LPGM values. 


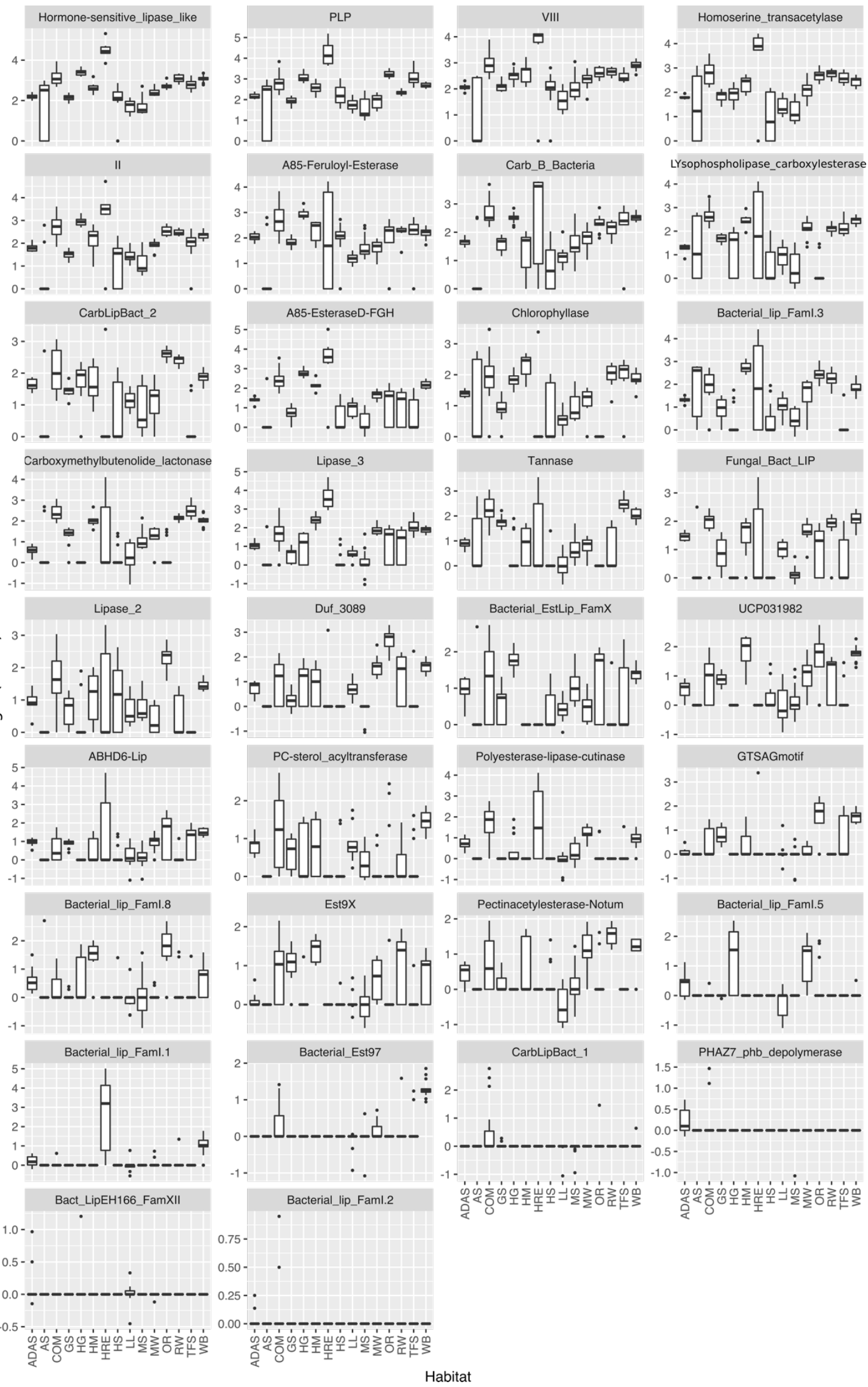

Supplementary Figure S11. Lipolytic families showing significant changes in abundance across different habitats. The abundance was inferred from $\log _{10}$ scaled LPGM values. 


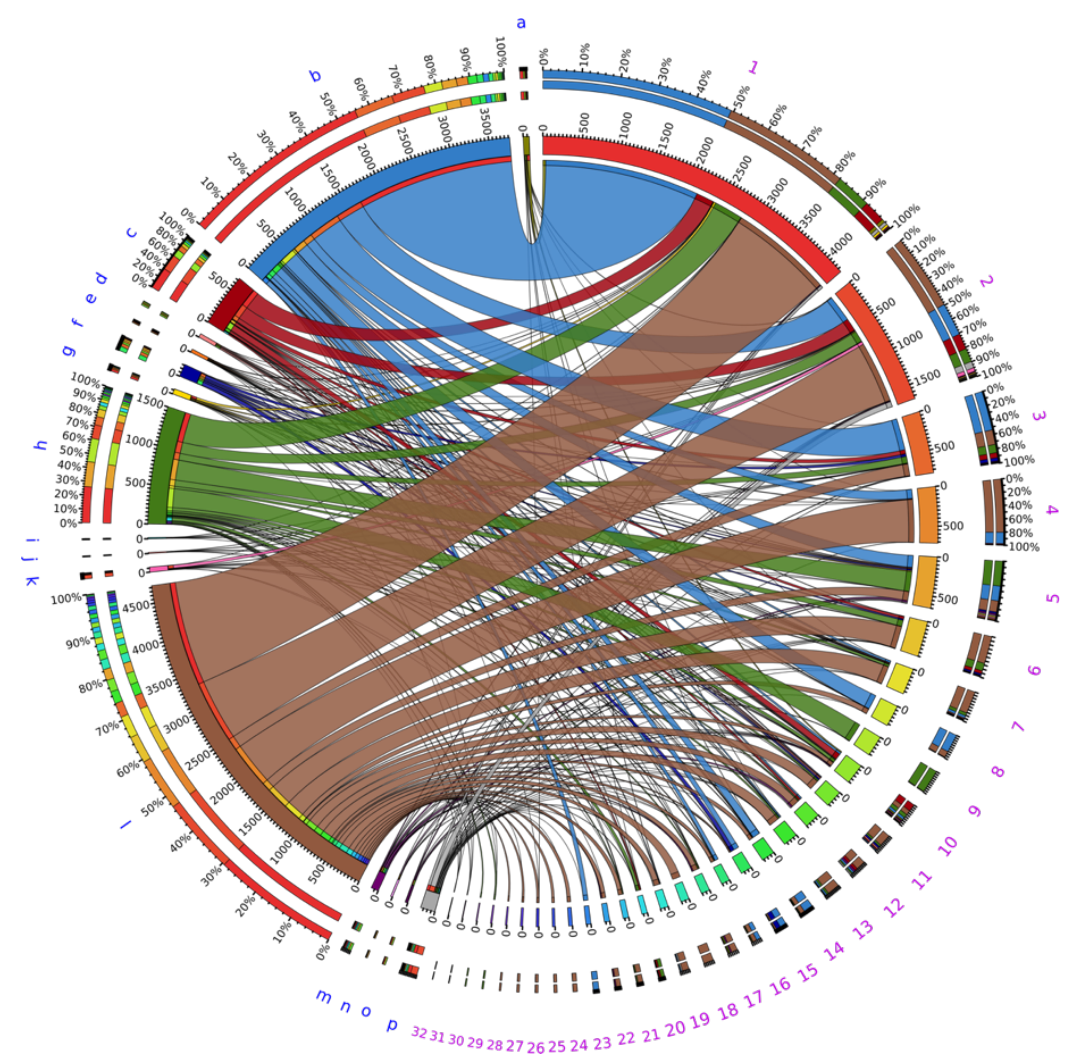

Supplementary Figure S12. Phylogenetic origins of LEs in ESTHER database at phylum level in the most abundant lipolytic families. Taxonomic information of lipolytic genes was retrieved from ESTHER database (Lenfant et al. 2013). Visualization was performed via Circos software. The width of bars from each phylum (blue) and lipolytic family (purple) indicates their relative abundance. Bacterial phyla: a, Acidobacteria; b, Actinobacteria; c, Bacteroidetes; d, Chlorobi; e, Chloroflexi; f, Cyanobacteria; g, Deinococcus-Thermus; h, Firmicutes; i, Gemmatimonadetes; j, Lentisphaerae; k, Planctomycetes; 1 , Proteobacteria; m, Spirochaetes; n, Thermotogae; o, Verrucomicrobia; p, unclassified Bacteria. Lipolytic family based on ESTHER database (Arpingy classification): 1, Carb_B_Bacteria (VII) ; 2, Hormone-sensitive_lipase_like (IV); 3, Chlorophyllase (EstGS); 4, Carboxymethylbutenolide_lactonase (V.2); 5, CarbLipBact_2 (XIII-2); 6, A85-EsteraseD-FGH (lp_3505/FLS12/EstA); 7, Homoserine_transacetylase (Est22); 8, Fungal-Bact_LIP (X-2/XVII/XIX); 9, CarbLipBact_1 (XIII1/XVIII); 10, A85-Feruloyl-Esterase (Rlip1/EstSt7); 11, LYsophospholipase_carboxylesterase (VI); 12, Duf_3089 (XV); 13, UCP031982 (V.3); 14, Est9X (Est9X); 15, Lipase_2 (I.4/I.7); 16, Polyesteraselipase-cutinase (III); 17, Pectinacetylesterase-Notum (LipT); 18, Bacterial_lip_FamI.8 (I.8); 19, GTSAGmotif (IV); 20, Bacterial_EstLip_FamX (X); 21, Lipase_3 (XI); 22, ABHD6-Lip (V.1); 23, Bacterial_Est97 (XVI); 24, Bacterial_lip_FamI.1 (I.1); 25, PHAZ7_phb_depolymerase (IX); 26, Tannase (Tannase); 27, Bacterial_lip_FamI.3 (I.3); 28, Bacterial_lip_FamI.2 (I.2); 29, Bacterial_lip_FamI.5 (I.5); 30, Bacterial_lip_FamI.6 (I.6); 31, PC-sterol_acyltransferase (XIV); 32, Bact_LipEH166_FamXII (XII). Only phyla and ESTHER families with a relative abundance $>1 \%$ are shown.. 


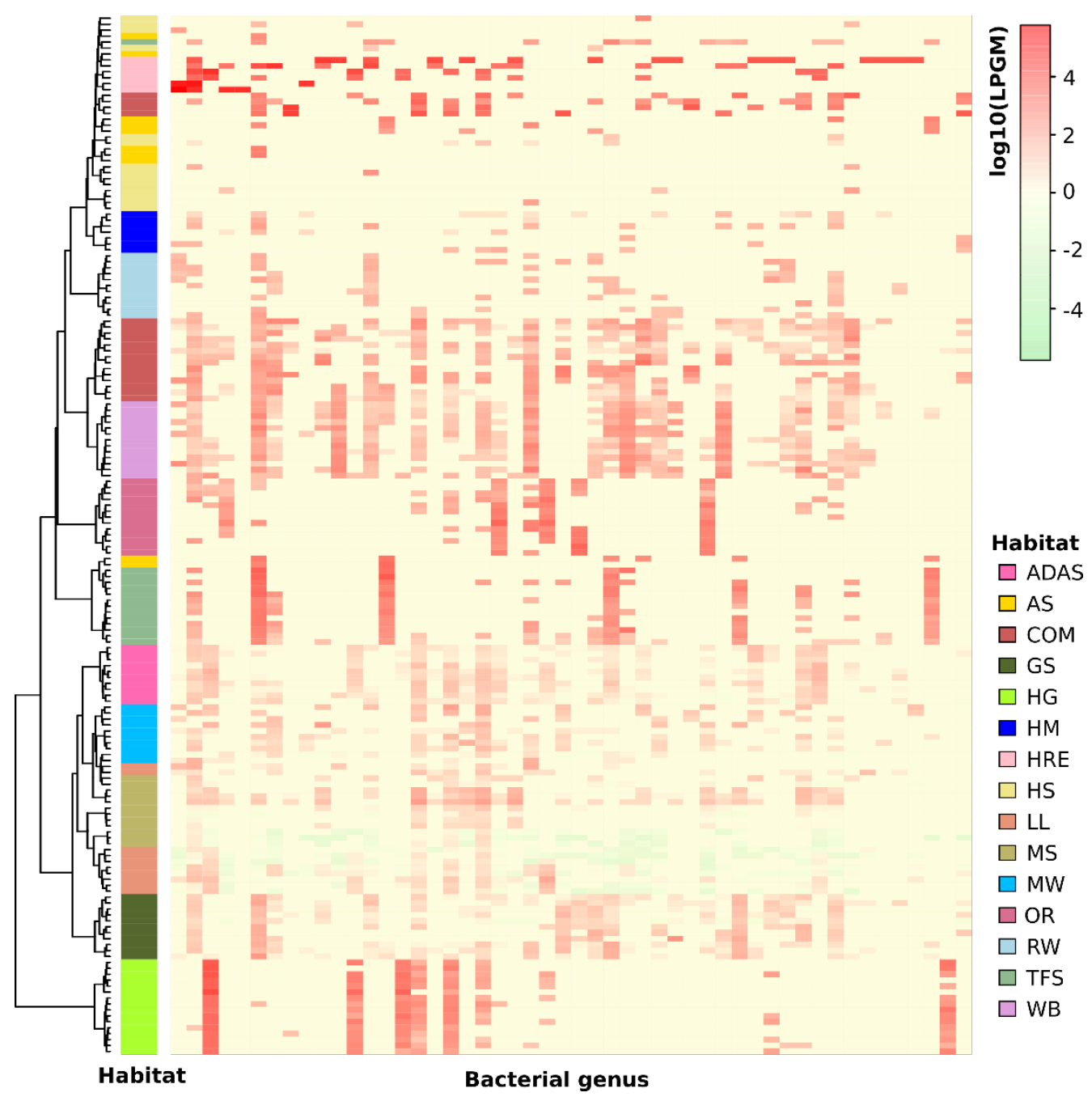

Supplementary Figure S13. Taxonomic origins at genus level of the assigned PLPs across samples. The abundance of assigned PLPs per each genus in each sample was inferred from LPGM values. Only genera with a mean LPGM value of $\geq 0.5$ across all the samples were used for analysis, only the top 50 genera areshown here (ranked by the mean LPGM values across samples). Hierarchical clustering analysis of the phylogenetic distribution profile in each sample was performed using the Ward.D clustering method based on Bray-Curtis distance matrices. The color intensity of the heat map (light green to red) indicates the change of abundance (low to high). The habitats were presented by different colors. The phylogenetic distribution of assigned PLPs in each sample was generally clustered by habitats (overall $\mathrm{R}$ value $=0.8199, \mathrm{P}<0.001$, ANOSIM test). Abbreviations: ADAS, anaerobic digestor active sludge; AS, agriculture soil; COM, compost; GS grassland soil; HG, human gut; HM, hypersaline mat; HRE, hydrocarbon resource environments; HS, hot spring; LL, landfill leachate; MS, marine sediment; MW, marine water; OR, oil reservoir; RW, river water; TFS, tropical forest soil; WB, wastewater bioreactor. 


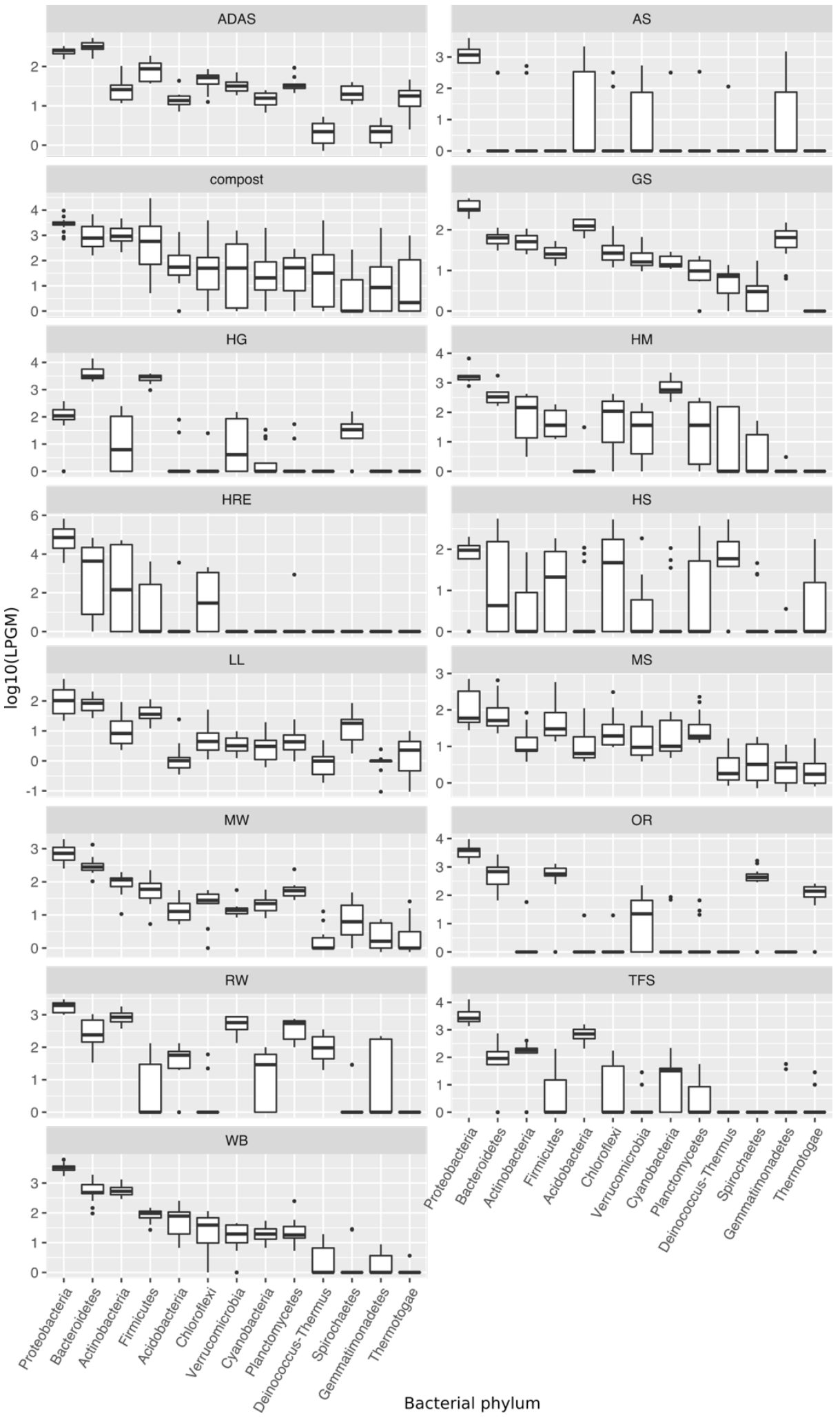

Supplementary Figure S14. Phylogenetic distribution of the assigned PLPs at phylum level in each habitat. The abundance was inferred from $\log _{10}$ scaled LPGM values. 


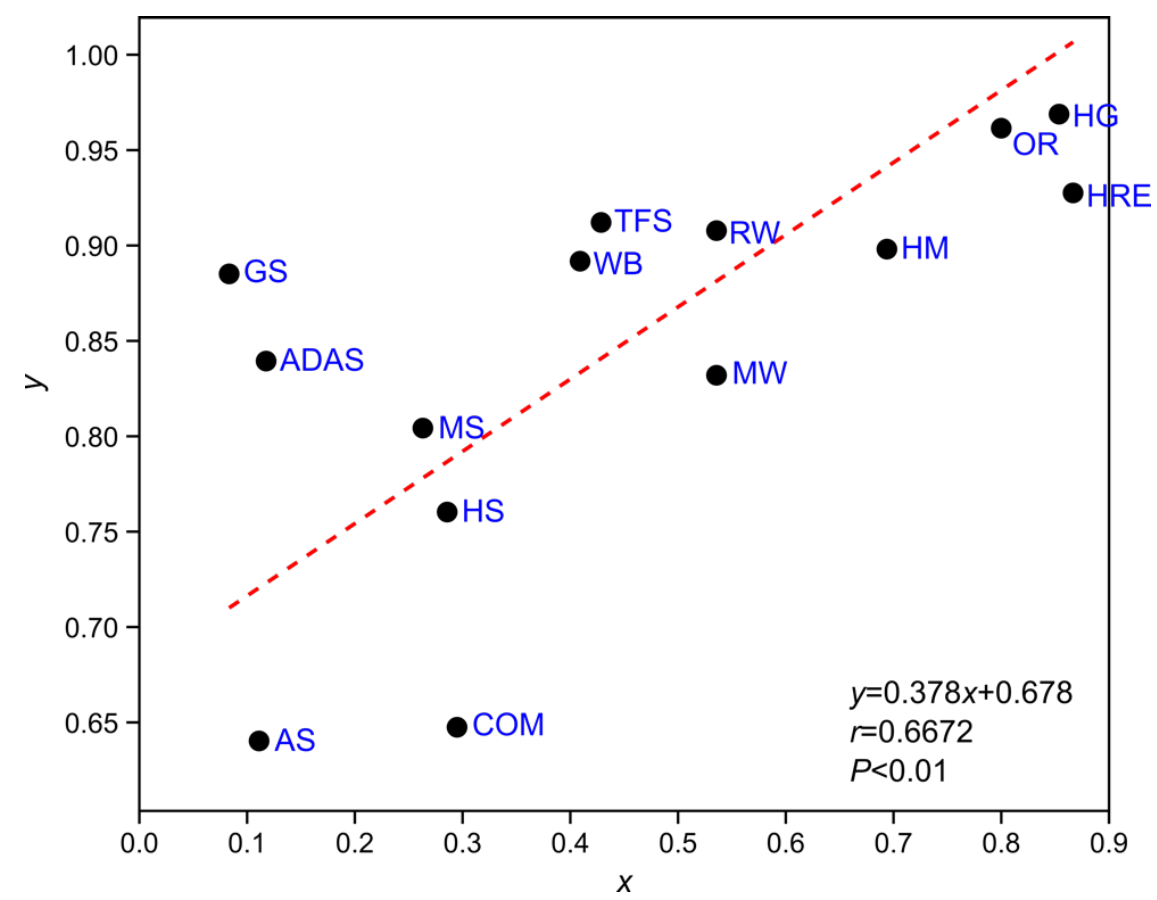

Supplementary Figure S15. Linear regression. The $\mathrm{x}$ was the ratio of unique indicators to the total significant indicators in a habitat, as demonstrated by the bipartite association network shown in Figure 5. The corresponding y was the mean dissimilarity of the taxonomic profile of assigned PLPs across habitats, in terms of averaged $\mathrm{R}$ values generated by the ANOSIM test $(\mathrm{P}<0.001$; see Supplementary Table S18). The Reduced Major Axis (RMA) algorithm was used for regression. The permutation test on correlation uses 9,999 replicates. Pearson's $r$ correlation $r=0.6672, p<0.01$. 

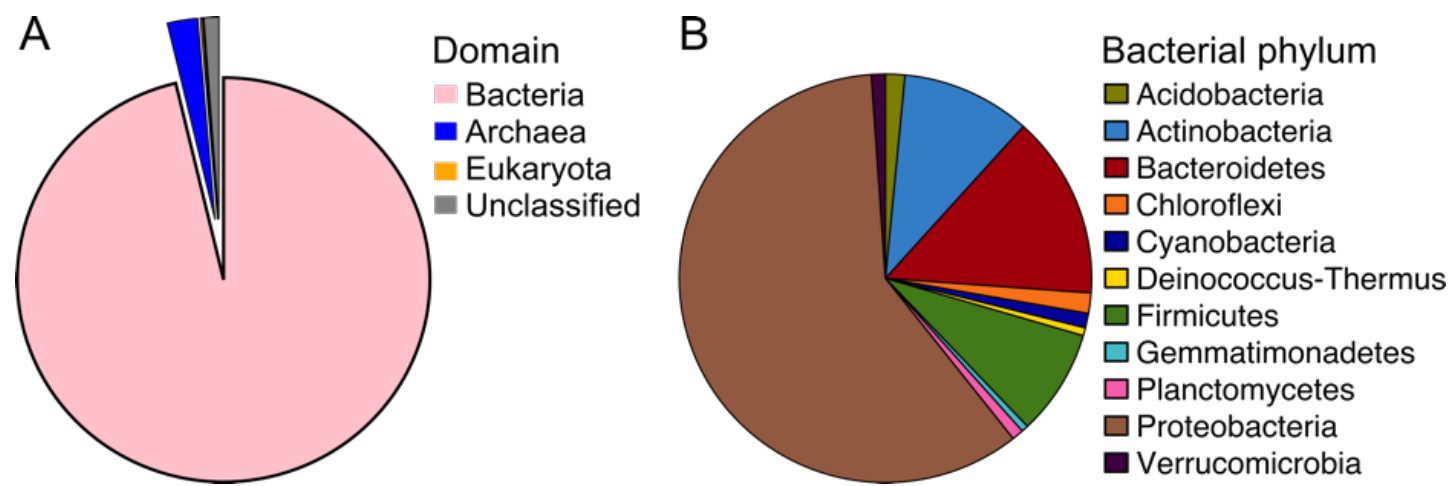

Supplementary Figure S16. Phylogenetic origin of the total PLPs (assigned and unassigned PLPs combined) at (A) domain and (B) phylum level. The abundance of PLPs in each domain or phylum was calculated by summing the LPGMs values across samples. 


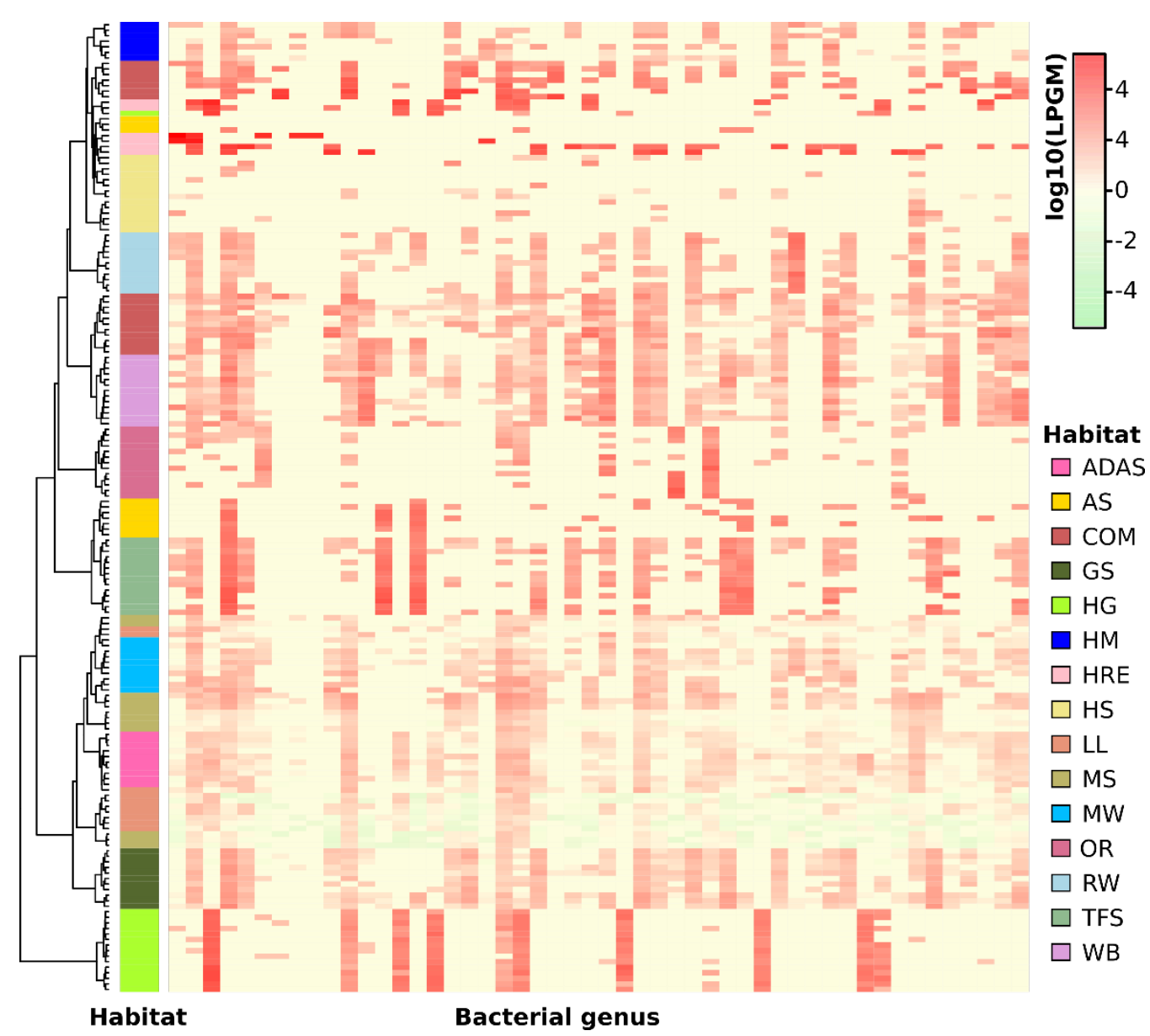

Supplementary Figure S17. Heat map of the taxonomic origins at genus level of total PLPs across samples. The abundance of total PLPs per genus in each sample was inferred from LPGM values. Only genera with a mean LPGM value of $\geq 0.5$ across all samples were used for analysis and only the top 50 genera are shown here (ranked by the mean LPGM values across samples). The clustering analysis was performed using the Ward.D clustering method based on Bray-Curtis distance matrices. The color intensity of the heat map (light green to red) indicates the change of abundance (low to high). The habitats are presented by different colors. The phylogenetic distribution of assigned PLPs in each sample clustered generally by habitats (overall $\mathrm{R}$ value $=0.821, \mathrm{P}<0.001$, ANOSIM test). Abbreviations: ADAS, anaerobic digestor active sludge; AS, agriculture soil; COM, compost; GS grassland soil; HG, human gut; HM, hypersaline mat; HRE, hydrocarbon resource environments; HS, hot spring; LL, landfill leachate; MS, marine sediment; MW, marine water; OR, oil reservoir; RW, river water; TFS, tropical forest soil; WB, wastewater bioreactor. 

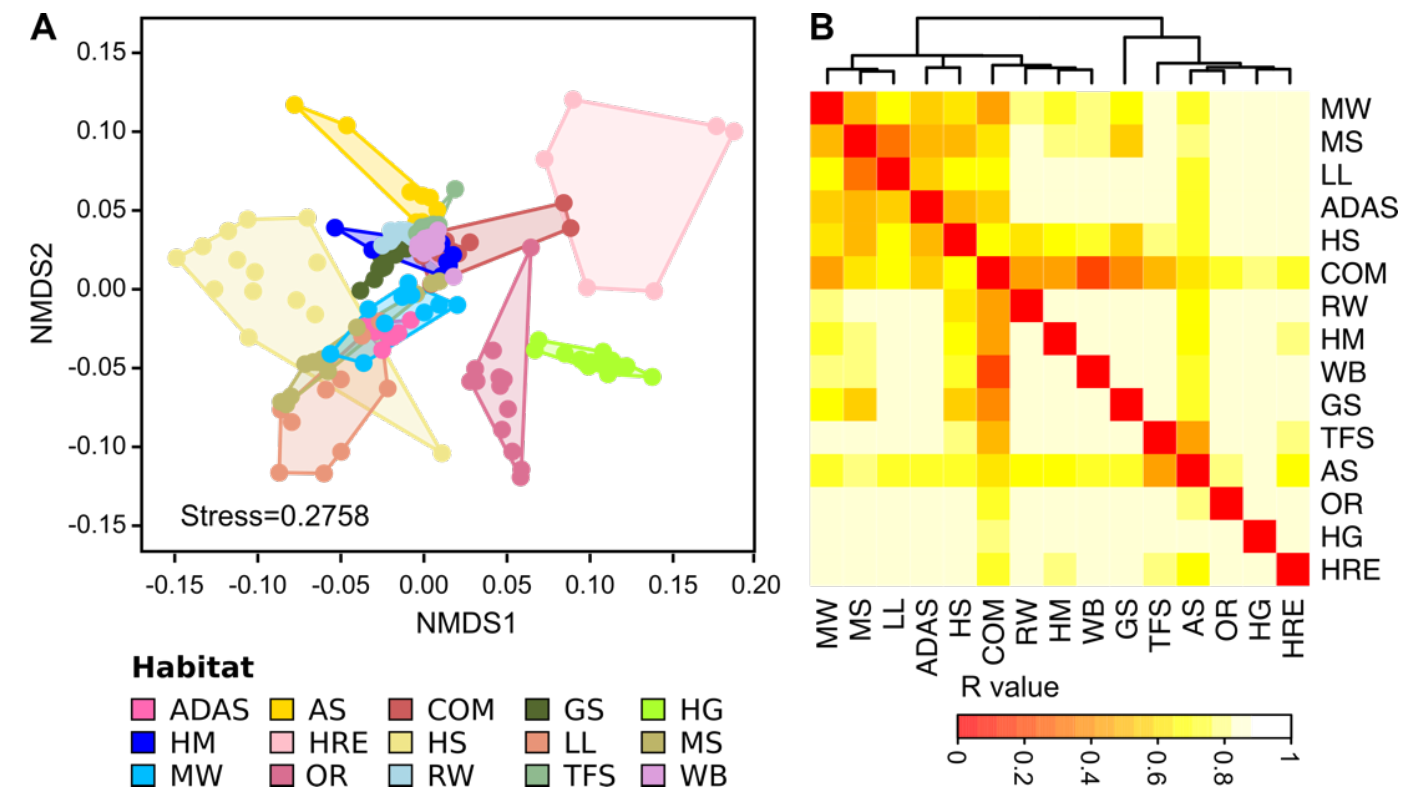

Supplementary Figure S18. Analysis of the phylogenetic profile at genus level of total PLPs across samples. A, Non-metric multidimensional scaling (NMDS) analysis of the phylogenetic profile of total PLPs across samples was performed. Samples were colored by its habitat origin. The abundance of PLPs in each genus per sample is presented by the LPGM values. Only genera with mean LPGM values of $\geq 0.5$ across all the samples were used for analysis. B, ANOSIM test the group dissimilarity of these phylogenetic profiles between habitats ( 9999 putations, $\mathrm{P}<0.001)$. The resulting $\mathrm{R}$ values are shown by the heatmap, and the color intensity (red to light yellow) indicates the change of $R$ values ( 0 to 1 ). Hierarchical clustering analysis of $\mathrm{R}$ values was performed to generate the cluster dendrogram using the Ward.D clustering method based on Bray-Curtis distance matrices 

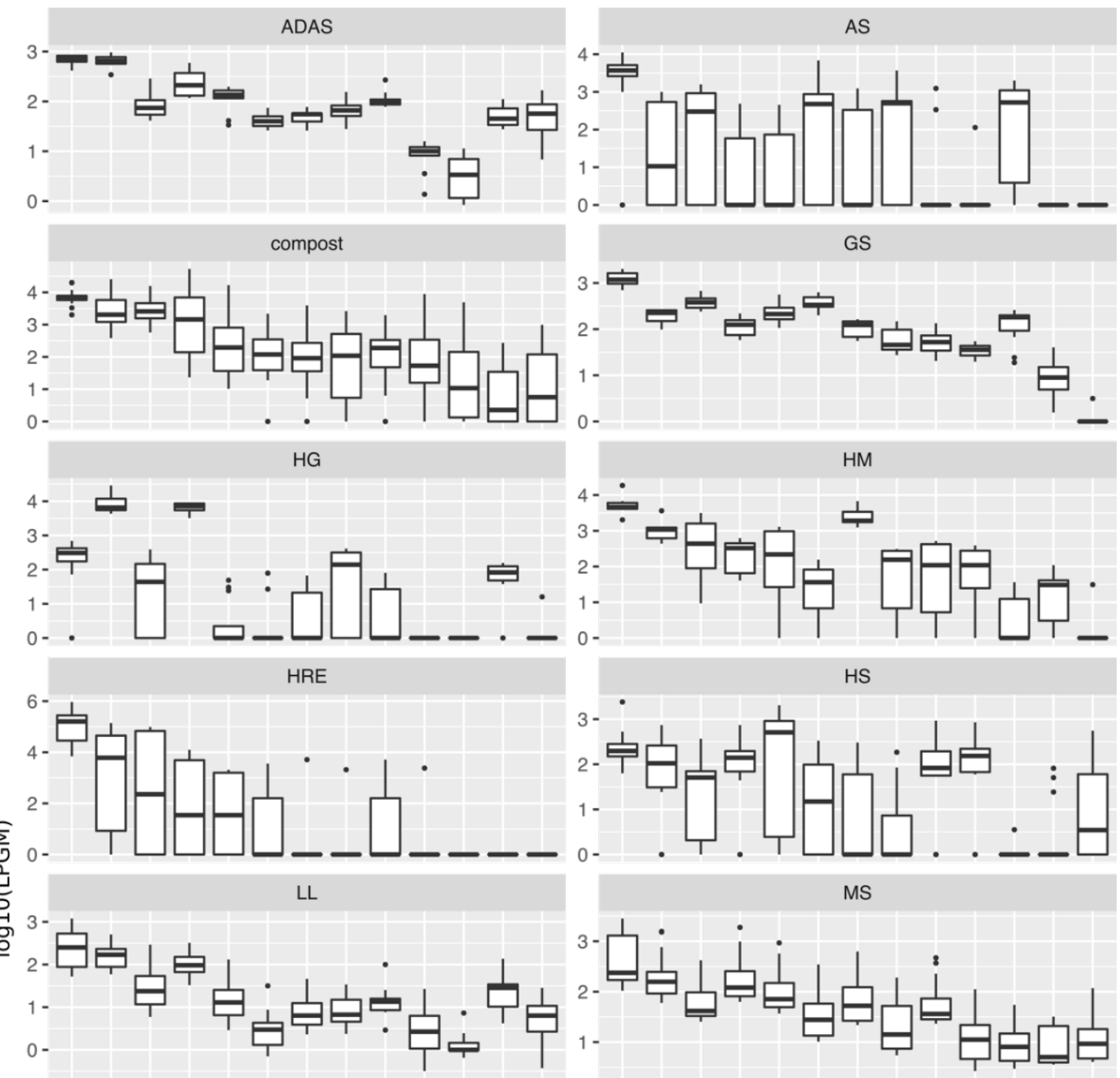

0 -

HS
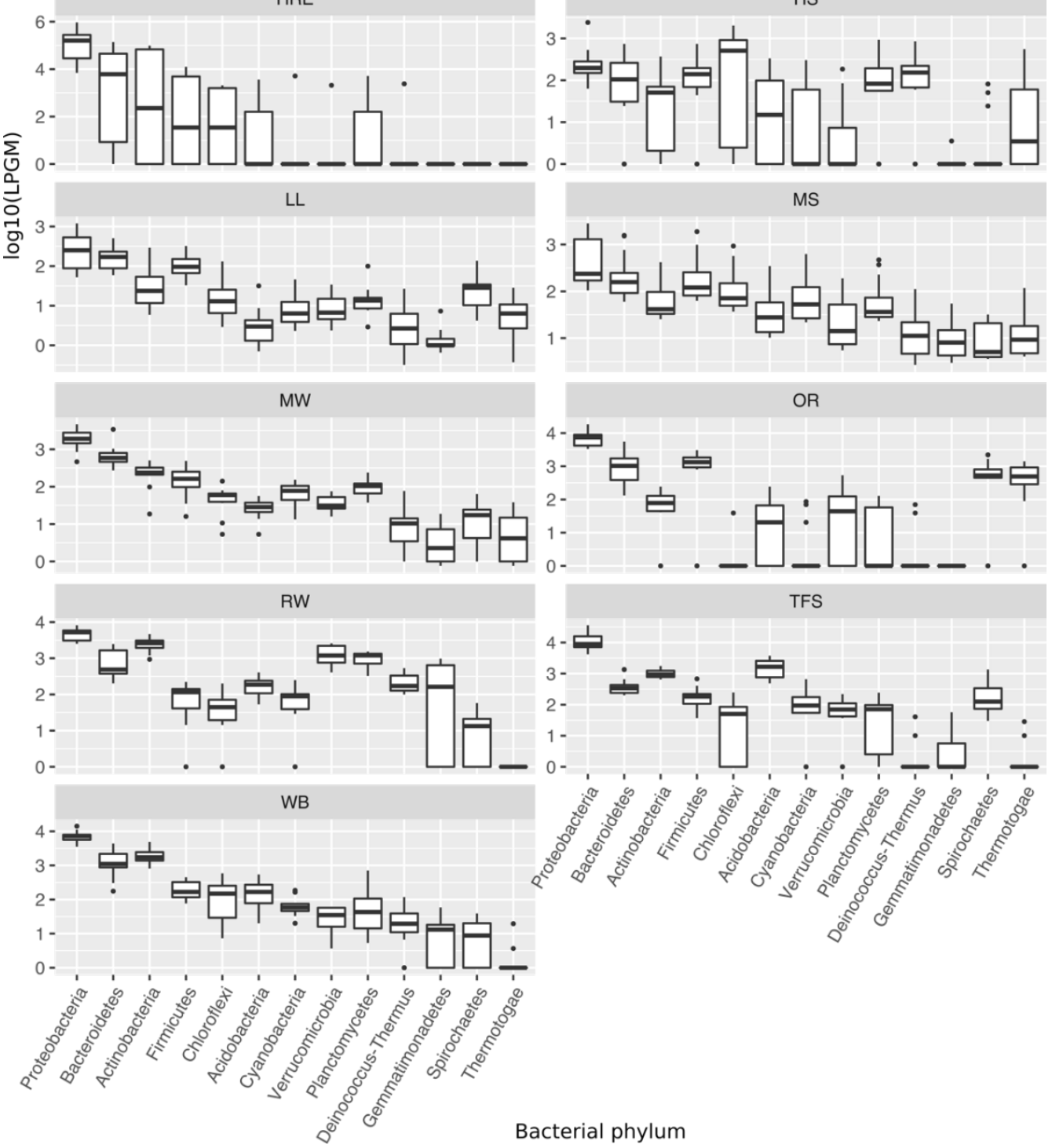

Supplementary Figure S19. Phylogenetic distribution of the total PLPs at phylum level of each habitat. The abundance was inferred from $\log _{10}$ scaled LPGM values. 


\section{References}

Gerlt JA, Bouvier JT, Davidson DB, Imker HJ, Sadkhin B, Slater DR, Whalen KL (2015) Enzyme Function Initiative-Enzyme Similarity Tool (EFI-EST): A web tool for generating protein sequence similarity networks. Biochim Biophys Acta 1854:10191037 . doi: 10.1002/cncr.27633.Percutaneous

Huang Y, Niu B, Gao Y, Fu L, Li W (2010) CD-HIT Suite: a web server for clustering and comparing biological sequences. Bioinformatics 26:680-692 . doi: 10.1093/bioinformatics/btq003

Keegan KP, Glass EM, Meyer F (2016) MG-RAST, a metagenomics service for analysis of microbial community structure and function. In: Methods in Molecular Biology. Humana Press Inc., pp 207-233

Lenfant N, Hotelier T, Velluet E, Bourne Y, Marchot P, Chatonnet A (2013) ESTHER, the database of the $\alpha / \beta$-hydrolase fold superfamily of proteins: tools to explore diversity of functions. Nucleic Acids Res 41:423-429 . doi: 10.1093/nar/gks1154

Menzel P, Ng KL, Krogh A (2016) Fast and sensitive taxonomic classification for metagenomics with Kaiju. Nat Commun 7:11257 . doi: 10.1038/ncomms11257

Quast C, Pruesse E, Yilmaz P, Gerken J, Schweer T, Yarza P, Peplies J, Glöckner FO (2013) The SILVA ribosomal RNA gene database project: Improved data processing and webbased tools. Nucleic Acids Res 41:D590-D596 . doi: 10.1093/nar/gks1219

Shannon P, Markiel A, Ozier O, Baliga NS, Wang JT, Ramage D, Amin N, Schwikowski B, Ideker T (2003) Cytoscape: A software environment for integrated models of biomolecular interaction networks. Genome Res 13:2498-2504 . doi: $10.1101 /$ gr.1239303 
Supplementary Table S1 Recent reported novel lipolytic enzymes/families

\begin{tabular}{llll}
\hline $\begin{array}{l}\text { Lipolytic } \\
\text { family }\end{array}$ & $\begin{array}{l}\text { Sequence } \\
\text { length (aa) }\end{array}$ & Function & Reference \\
\hline Est22 & 463 & Homoserine transacetylase & Li et al., 2017 \\
EstL28 & 290 & Esterase & Seo et al., 2013 \\
Rv0045c & 298 & Esterase & Guo et al., 2010 \\
EstGX1 & 201 & Lipase & Jime nez et al., 2012 \\
EstLiu & 293 & Esterase & Rahman et al., 2016 \\
EstY & 423 & Esterase & Wu \& Sun, 2009 \\
EstGS & 397 & Esterase & Nacke et al., 2011 \\
EM3L4 & 330 & Lipase & Jeon et al., 2011 \\
FLS18 & 259 & Esterase & Hu et al., 2010 \\
Est903 & 300 & Esterase & Jia et al., 2019 \\
EstJ & 317 & Esterase & Choi et al., 2013 \\
PE10 & 279 & Esterase & Jiang et al., 2012 \\
Est12 & 329 & Esterase & Wu et al., (2013) \\
EstDZ2 & 271 & Secreted carboxylesterase & Zarafeta et al., 2016 \\
Est9x & 294 & Lipase & Jeong et al., 2009 \\
Lip10 & 348 & Lipase & Guo et al., 2016 \\
EstGH & 448 & Esterase & Nacke et al., 2011 \\
EML1 & 304 & Esterase & Jeong et al., 2009 \\
FnL & 302 & Lipase & Yu et al., 2010 \\
EstP2K & 224 & Esterase & Ouyang et al., 2013 \\
LipA & 277 & Lipase & Couto et al., 2010 \\
LipSM54 & 526 & Lipase & Li et al., 2016 \\
MtEst45 & 516 & Acyl esterase & Lee et al., 2016 \\
LipT & 329 & Esterase & Chow et al., 2012 \\
EstSt7 & 322 & Esterase & Wei et al., 2013 \\
Rlip1 & 361 & Esterase & Liu et al., 2009 \\
EstA & 277 & Esterase & Chu et al., 2008 \\
FLS12 & 270 & Esterase & Hu et al., 2010 \\
lp_3505 & 263 & Acetyl esterase & Esteban-Torres et al., 2014 \\
\hline & & & \\
& & &
\end{tabular}




\section{Supplementary Table S2 Reference LEs used for constructing phylogentic tree}

\begin{tabular}{|c|c|c|c|}
\hline Accession Nr. & Description & Microbial organism & Lipolytic family \\
\hline SDT19935 & triacylglycerol lipase & Pseudomonas xinjiangensis & Family I \\
\hline AAB53647 & extracellular lipase & Pseudomonas wisconsinensis & Family I \\
\hline CAA56780 & lipase & Acinetobacter calcoaceticus & Family I \\
\hline AAC 15585 & lipase & Pseudomonas fluorescens & Family I \\
\hline BAA09135 & lipase & Pseudomonas aeruginosa & Family I \\
\hline Q05489 & $\begin{array}{l}\text { triacylglycerol lipase } \\
\text { triacylglycerol lipase }\end{array}$ & Burkholderia glumae & Family I \\
\hline AAC 05510 & precursor & Pseudomonas luteola & Family I \\
\hline AAA50466 & lipase & Burkholderia cepacia & Family I \\
\hline CAA49812 & lipase & Burkholderia glumae & Family I \\
\hline BAA02012 & triacylglycerol lipase & Pseudomonas fluorescens & Family I \\
\hline AAD09856 & thermostable lipase TliA & Pseudomonas fluorescens & Family I \\
\hline WP 025854682 & lipase & Pseudomonas sp. CHM02 & Family I \\
\hline AA $\bar{A} 22574$ & lipase & Bacillus subtilis & Family I \\
\hline WP_071578729 & triacylglycerol lipase & Bacillus sp. & Family I \\
\hline WP_019713218 & triacylglycerol lipase & Bacillus subtilis & Family I \\
\hline WP_059292026 & triacylglycerol lipase & Bacillus malacitensis & Family I \\
\hline $\mathrm{WP}^{-} 043021508$ & triacylglycerol lipase & Bacillus velezensis & Family I \\
\hline AAC 12257 & lipase & Geobacillus stearothermophilus & Family I \\
\hline AAF40217 & lipase & Geobacillus stearothermophilus & Family I \\
\hline CAA64621 & triacylglycerol lipase & Geobacillus thermocatenulatus & Family I \\
\hline AAD30278 & lipase & Geobacillus thermoleovorans & Family I \\
\hline CAA67627 & triacylglycerol lipase & Propionibacterium acnes & Family I \\
\hline AAB71210 & lipase LipA & Streptomyces cinnamoneus & Family I \\
\hline WP 036932411 & triacylglycerol lipase, partial & Propionibacterium avidum & Family I \\
\hline $\mathrm{WP}^{-} 002519511$ & triacylglycerol lipase & Propionibacterium acnes & Family I \\
\hline AAA 26633 & lipase precursor & Staphylococcus aureus & Family I \\
\hline AAF21294 & lipase & Staphylococcus haemolyticus & Family I \\
\hline AAC67547 & lipase precursor & Staphylococcus epidermidis & Family I \\
\hline AAG35723 & lipase precursor & Staphylococcus warneri & Family I \\
\hline WP_042532658 & alpha/beta hydrolase & Oceanobacillus oncorhynchi & Family II \\
\hline WP_071394859 & alpha/beta hydrolase & Bacillus tuaregi & Family II \\
\hline WP_013842524 & $\begin{array}{l}\text { alpha/beta hydrolase } \\
\text { triacylglycerol }\end{array}$ & Desulfotomaculum ruminis & Family II \\
\hline M86351 & acylhydrolase (lipA) & Streptomyces exfoliatus & Family III \\
\hline WP_030583320 & lipase & Streptomyces globisporus & Family III \\
\hline $\mathrm{WP}^{-} 030586638$ & lipase & Streptomyces anulatus & Family III \\
\hline $\mathrm{WP}^{-} 012381325$ & alpha/beta hydrolase & Streptomyces griseus & Family III \\
\hline WP_ 051831627 & alpha/beta hydrolase & Streptomyces violens & Family III \\
\hline WP_011196250 & alpha/beta hydrolase & Symbiobacterium thermophilum & Family IV \\
\hline $\mathrm{ABQ} 11271$ & lipase/esterase & uncultured bacterium & Family IV \\
\hline AAS77236 & lipase/esterase & uncultured bacterium & Family IV \\
\hline AAX37296 & lipase/esterase & uncultured bacterium & Family IV \\
\hline CAA37863 & triacylglycerol lipase & Moraxella sp. & Family V \\
\hline WP 002973797 & alpha/beta hydrolase & Leptospira terpstrae & Family V \\
\hline WP 002975917 & alpha/beta hydrolase & Leptospira vanthielii & Family V \\
\hline CAĀ47949 & triacylglycerol lipase & Psychrobacter immobilis & Family V \\
\hline WP 038681048 & 3-oxoadipate enol-lactonase & Rubrobacter radiotolerans & Family V \\
\hline $\mathrm{WP}^{-} 007576549$ & 3-oxoadipate enol-lactonase & Patulibacter medicamentivorans & Family V \\
\hline $\mathrm{AAC} 21862$ & esterase/lipase & Haemophilus influenzae Rd KW20 & Family V \\
\hline WP 010945144 & alpha/beta fold hydrolase & Haemophilus ducrevi & Family V \\
\hline $\mathrm{WP}^{-} 011147413$ & esterase & Photorhabdus luminescens & Family V \\
\hline
\end{tabular}


WP_006621073
WP_006617627
WP_023068329
OCQ99642
WP_015175368
Q01470
P37967
CAA22794
KJJ40755
WP_063638034
WP_064730418
WP_079426436
WP_076288838
WP_013412622
WP_005474626
WP_014985987
WP_015576461
KXK14623
ACZ40829
WP_059212061
WP_040337170
WP_056535681
WP_071051881

AAK07742

WP 056130558

WP 052107835

WP_012277824

WP_012143864

NP 228147

WP_041843998

WP 012057344

WP_052515459

APW'63053

ABE69172

GAK59442

WP_054029223

WP 051945529

GAD̄03196

ACB11220

SFR44003

WP_012136589

WP 007016102

WP_046019340

BAD̄77330

KYD25926

WP_081189860

WP 044893000

WP_024030776

WP 011026365

WP_049685837

WP_028991973 alpha/beta hydrolase

alpha/beta hydrolase

alpha/beta hydrolase

serine esterase

alpha/beta hydrolase

Phenmedipham hydrolase

Para-nitrobenzyl esterase

putative carboxylesterase

para-nitrobenzyl esterase

carboxylesterase/lipase

family protein

carboxylesterase

carboxylesterase

carboxylesterase

carboxylesterase

esterase

esterase

esterase

beta-lactamase

beta-lactamase

carboxylesterase

hypothetical protein

serine_hydrolase

serine_hydrolase

PHB depolymerase PhaZ7

precursor

alpha/beta hydrolase

alpha/beta hydrolase

lipase class 2

hypothetical protein

hypothetical protein

TM0336

alpha/beta hydrolase

DUF3887 domain-

containing protein

alpha/beta fold hydrolase

Esterase EstD

probable lipase

probable lipase

lipase family protein

lipase family protein

lipase

lipase

hypothetical protein

SAMN04488073_1267

hypothetical protein

hypothetical protein

hypothetical protein

carboxylesterase

carboxylesterase

carboxylesterase

alpha/beta fold hydrolase

alpha/beta fold hydrolase

acetyltransferase

acetyltransferase

acetyltransferase
Arthrospira platensis

Arthrospira platensis

Lyngbya aestuarii

Oscillatoriales cyanobacterium

Oscillatoria nigroviridis

Pseudarthrobacter oxydans

Bacillus subtilis

Streptomyces coelicolor

Bacillus subtilis

Bacillus atrophaeus

Streptomyces parvulus

Clostridium oryzae

Paenibacillus pabuli

Caldicellulosiruptor owensensis

Streptomyces bottropensis

Nocardia brasiliensis

Streptomyces sp.

Chloroflexi bacterium

Sphaerobacter thermophilus

Streptomyces canus

Candidatus Blastococcus massiliensis

Marmoricola sp.

Frankia sp.

Paucimonas lemoignei

Lysobacter sp.

Lysobacter daejeonensis

Shewanella halifaxensis

Shewanella sediminis

Thermotoga maritima

Thermotoga $\mathrm{sp}$.

Thermosipho melanesiensis

Dethiosulfatarculussandiegensis

Paludisphaera borealis

uncultured bacterium pFosLip

Candidatus Vecturithrix granuli

Desulfatitalea tepidiphila

Verrucomicrobium sp.

Agarivorans albus

uncultured bacterium

Marinobacter gudaonensis

Marinobacter lipolyticus

Bermanella marisrubri

Marinomonas sp.

Geobacillus kaustophilus

Geobacillus sp.

Geobacillus sp.

Bacillus alveayuensis

Bacillus vireti

Caldanaerobacter subterraneus

Thermoanaerobacter kivui

Thermoanaerobacter thermocopriae
Family VI

Family VI

Family VI

Family VI

Family VI

Family VII

Family VII

Family VII

Family VII

Family VII

Family VII

Family VII

Family VII

Family VII

Family VIII

Family VIII

Family VIII

Family VIII

Family VIII

Family VIII

Family VIII

Family VIII

Family VIII

Family IX

Family IX

Family IX

Family IX

Family IX

Family X

Family X

Family X

Family X

Family X

Family XI

Family XI

Family XI

Family XI

Family XI

Family XII

Family XII

Family XII

Family XII

Family XII

Family XIII

Family XIII

Family XIII

Family XIII

Family XIII

Family XIV

Family XIV

Family XIV 
WP_012995938
WP_041589396
ADE28719
ADE28720
WP_049895542
AFP50148
WP_077001818
WP_030038081
WP_018637498
WP_077040295
WP_068423891
WP_072625206
WP_068313207
WP_068264424
WP_007927380
ANA76126
WP_066165759
WP_042479182
WP_079523184
ASU50657
QBK24554
WP_124766500
WP_126293751
WP_118877586
ARJ54612
WP_049451625
WP_093999627
WP_106468945
WP_049430271
WP_057504400

CEO58279

BAE66557

EIT75442

OGE49037

OJJ29996

ALN44199

ADA70028

ADH59412

ADH59413

WP_073290055

WP 054509002

WP_076503926

WP 084842892

SFC64030

ADF51938

WP 022959187

WP 07599942

OGŌ52417

AEM45109

WP 011731534

WP_003898161

SBS76993 acetyltransferase

acetyltransferase

EstGK1

EstZ3

DUF3089 domain-

containing protein

esterase

hypothetical protein

alpha/beta hydrolase

alpha/beta hydrolase

alpha/beta hydrolase

lipase

lipase

lipase

lipase

secretory lipase

secretory lipase LipJ2

alpha/beta fold hydrolase

alpha/beta fold hydrolase

alpha/beta fold hydrolase

esterase

alpha/beta fold hydrolase

alpha/beta fold hydrolase

alpha/beta fold hydrolase

alpha/beta fold hydrolase

secreted lipase

lipase, partial

lipase

lipase

lipase

lipase

Putative Arylacetamide

deacetylase

unnamed protein product

arylacetamide deacetylase

hypothetical protein

hypothetical protein

Lip10

lipolytic enzyme precursor

esterase

esterase

alpha/beta hydrolase

alpha/beta hydrolase

esterase

esterase

Acetyl esterase/lipase

esterase/lipase-like protein

alpha/beta hydrolase

hypothetical_protein

hypothetical_protein

hypothetical protein

hypothetical protein

hypothetical protein

conserved exported

hypothetical protein
Thermoanaerobacter italicus

Thermoanaerobacterium

thermosaccharolyticum

uncultured bacterium

uncultured bacterium

Oribacterium sp.

uncultured microorganism

Variovorax sp.

Streptomyces resistomycificus

Frankia elaeagni

Rhodococcus sp.

Janibacter terrae

Janibacter terrae

Janibacter anophelis

Janibacter limosus

Janibacter hoylei

Janibacter sp. R02

Bacillus sp. KCTC 13219

Bacillus ndiopicus

Solibacillus isronensis

Ureibacillus thermosphaericus

Ureibacillus thermophilus

Lysinibacillus composti

Lysinibacillus telephonicus

Lysinibacillus yapensis

Stenotrophomonas maltophilia

Stenotrophomonas maltophilia

Stenotrophomonas sp. CC120222-04

Stenotrophomonas maltophilia

Stenotrophomonas maltophilia

Pseudomonas geniculata

Penicillium brasilianum

Aspergillus oryzae

Aspergillus oryzae

Penicillium arizonense

Aspergillus wentii

Monascus ruber

uncultured marine bacterium

uncultured bacterium

uncultured bacterium

Chryseobacterium polytrichastri

Chryseobacterium sp.

Chryseobacterium shigense

Zunongwangia atlantica

Zunongwangia mangrovi

Zunongwangia profunda

Spongiibacter tropicus

Halioglobus pacificus

Chloroflexi bacterium

uncultured organism

Mycobacterium smegmatis

Mycobacterium smegmatis

uncultured Mycobacterium sp.
Family XIV

Family XIV

Family XV

Family XV

Family XV

Family XVI

Family XVI

Family XVI

Family XVI

Family XVI

Family XVII

Family XVII

Family XVII

Family XVII

Family XVII

Family XVII

Family XVIII

Family XVIII

Family XVIII

Family XVIII

Family XVIII

Family XVIII

Family XVIII

Family XVIII

Family XIX

Family XIX

Family XIX

Family XIX

Family XIX

Family XIX

Lip10

Lip10

Lip10

Lip10

Lip10

Lip10

EstF

EstF

EstF

EstLiu

EstLiu

EstLiu

EstLiu

EstLiu

EstLiu

EstGS

EstGS

EstGS

EstGS

EstGH

EstGH

EstGH 


\begin{tabular}{|c|c|c|c|}
\hline WP_071947591 & hypothetical protein & Mycobacterium sp. WY10 & EstGH \\
\hline AEM45123 & hypothetical protein & hypothetical protein & EstGH \\
\hline AIT16227 & putative lipase & uncultured bacterium & EML1 \\
\hline WP_020471493 & lipase family protein & Zavarzinella formosa & EML1 \\
\hline WP_018291660 & lipase family protein & Verrucomicrobium sp. & EML1 \\
\hline WP_051926020 & lipase family protein & Leptolyngbya sp. & EML1 \\
\hline $\mathrm{ABB} 79948$ & class 3 lipase & uncultured bacterium & EML1 \\
\hline WP_047854544 & alpha/beta hydrolase & Archangium gephyra & EstL28 \\
\hline EGC̄ 22818 & alpha/beta domain protein & Streptococcus sanguinis SK353 & EstL28 \\
\hline AFK29752 & esterase & uncultured bacterium & EstL28 \\
\hline WP_082858528 & alpha/beta hydrolase & Planctomyces sp. SH-PL62 & EstJ \\
\hline WP_015246870 & alpha/beta hydrolase & Singulisphaera acidiphila & EstJ \\
\hline OHB78550 & esterase partial & Planctomycetes bacterium RBG 166410 & EstJ \\
\hline WP_086013385 & esterase & Schlesneria paludicola & EstJ \\
\hline WP_076349888 & esterase & Paludisphaera borealis & EstJ \\
\hline $\mathrm{AFG} 17170$ & EstJ & uncultured bacterium & EstJ \\
\hline WP_060986166 & esterase & Acidovorax delafieldii & EstY \\
\hline WP_069103995 & esterase & Acidovorax sp. RAC01 & EstY \\
\hline OGB̄ 09581 & esterase & Burkholderiales bacterium & EstY \\
\hline WP_019426758 & hypothetical protein & Limnohabitans sp. Rim 28 & EstY \\
\hline ABY 83635 & $\begin{array}{l}\text { esterase, partial } \\
\text { poly(3-hydroxybutyrate) }\end{array}$ & uncultured bacterium & EstY \\
\hline OUU14110 & depolymerase & Porticoccaceae bacterium & FLS18 \\
\hline ACL67852 & esterase/lipase & uncultured bacterium FLS18 & FLS18 \\
\hline WP_070116203 & hypothetical protein & $\begin{array}{l}\text { Pseudohongiella acticola } \\
\text { OM182 bacterium BACL3 MAG-120619- }\end{array}$ & FLS18 \\
\hline KRO81080 & hypothetical protein & bin3 & FLS18 \\
\hline ACL67851 & $\begin{array}{l}\text { esterase/lipase } \\
\text { polyhydroxybutyrate }\end{array}$ & uncultured bacterium & FLS18 \\
\hline WP_015250293 & depolymerase & Singulisphaera acidiphila & EM3L4 \\
\hline OGO16435 & hypothetical protein & Chloroflexi bacterium & EM3L4 \\
\hline OGO11912 & hypothetical protein & Chloroflexi bacterium & EM3L4 \\
\hline OGT27829 & hypothetical protein & Gammaproteobacteria bacterium & EM3L4 \\
\hline ADH59407 & esterase/lipase & uncultured bacterium & EM3L4 \\
\hline WP_019907066 & alpha/beta hydrolase & Methylobacterium sp. 77 & PE10 \\
\hline WP_056144266 & alpha/beta hydrolase & Methylobacterium sp. Leaf85 & PE10 \\
\hline WP_027174325 & alpha/beta hydrolase & Methylobacterium sp. 10 & PE10 \\
\hline WP_046141294 & alpha/beta hydrolase & Devosia soli & PE10 \\
\hline SDḠ73374 & Acetyl esterase/lipase & Pelagibacterium luteolum & PE10 \\
\hline AEV42214 & esterase & Pelagibacterium halotolerans & PE10 \\
\hline WP_077447883 & esterase & Psychrobacter sp. & Est12 \\
\hline WP_068405132 & esterase & Psychrobacter sp. & Est12 \\
\hline WP_011514506 & esterase & Psychrobacter cryohalolentis & Est12 \\
\hline OEН̈67893 & esterase & Psychrobacter sp. & Est12 \\
\hline AGQ21328 & esterase & Psychrobacter sp. & Est12 \\
\hline WP_033186083 & lipase & Pseudoalteromonas sp. & Est9x \\
\hline WP_051275252 & alpha/beta hydrolase & Aestuariibacter salexigens & Est9x \\
\hline WP_055012904 & lipase & Pseudoalteromonas sp. & Est9x \\
\hline WP_010179535 & putative_lipase & Glaciecola sp. & Est9x \\
\hline AFR79233 & esterase & Uncultured bacterium & Est9x \\
\hline YP_765030 & esterase & Rhizobium leguminosarum & EstA \\
\hline ZP_02167917 & esterase & Hoeflea phototrophica & EstA \\
\hline NP_354481 & esterase D & Agrobacterium fabrum & EstA \\
\hline WP_071219778 & esterase & Paenibacillus sp. & FLS12 \\
\hline ACL67846 & esterase & uncultured bacterium FLS12 & FLS12 \\
\hline EDV78171 & putative esterase & Geobacillus sp. & FLS12 \\
\hline
\end{tabular}




WP_024625887
WP_003640069
YP_004890987
AFS34518
BAD41030
AEN92268
EAR67363
EFV74766
WP_038060347
WP_003047954
WP_038056291
ADW21422
CZI05393
WP_013131472
WP_036322013
WP_071677097
EGK1 13482.1

WP 046794161

OLB̄B33458

WP 020718491

WP 043319646

WP_066959386

WP_078083231

WP_073276480

WP 074903882

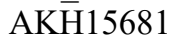

WP 008099315

WP_079555980

ACM91105

WP 067838338

WP 083066055

WP_085163092

WP 031683431

WP_003400489

ANQ̄80463

WP_049586806

WP_118495271

WP_025254722

WP_005409763

WP_097047631

WP 057499404

AGF̄ 29555

WP 019336523

WP 010980100

PVŪ70598

AAY79544

WP 069282843

WP_048099963

WP_128998162 alpha/beta fold hydrolase

alpha/beta fold hydrolase

acetyl esterase

LipS

esterase

EstGtA2

esterase

esterase

esterase

esterase

esterase

putative esterase

Patatin

Patatin

patatin-like phospholipase

family protein

phospholipase

patatin family phospholipase

tannase/feruloyl esterase

family alpha/beta hydrolase

feruloyl esterase

tannase/feruloyl esterase

family alpha/beta hydrolase

acyl esterase

acyl esterase

acyl esterase

acyl esterase

acyl esterase

acyl esterase

esterase

esterase

lipase

alpha/beta hydrolase

alpha/beta hydrolase

alpha/beta hydrolase

alpha/beta hydrolase

alpha/beta hydrolase

esterase

homoserine O-

acetyltransferase

homoserine $\mathrm{O}$ -

acetyltransferase

homoserine O-

acetyltransferase

alpha/beta fold hydrolase

alpha/beta fold hydrolase

alpha/beta fold hydrolase

lipase

alpha/beta fold hydrolase

esterase

esterase

conserved Archaeal esterase

esterase

esterase

acetyltransferase
Lactobacillus fabifermentans

Lactobacillus pentosus

Lactobacillus plantarum

uncultured bacterium

Symbiobacterium thermophilum

Geobacillus thermodenitrificans

Bacillus sp.

Bacillus sp.

Thermus filiformis

Thermus aquaticus

Thermus amyloliquefaciens

Thermus scotoductus

Legionella pneumophila

Thermobispora bispora

Microbispora sp.

Thermus brockianus

Desmospora sp.

Rhizobium sp.

Acidobacteria bacterium

Acidobacteriaceae bacterium

Microbulbifer sp. HZ11

Microbulbifer sp. Q7

Microbulbifer mangrovi

Microbulbifer donghaiensis

Microbulbifer thermotolerans

Microbulbifer thermotolerans

Verrucomicrobiae bacterium

Alkalitalea saponilacus

uncultured bacterium

Mycobacterium mantenii

Mycobacterium arosiense

Mycobacterium lacus

Mycobacterium tuberculosis

Mycobacterium tuberculosis complex

uncultured bacterium

Alteromonas macleodii

Alteromonas sp. BL110

Alteromonas sp. ALT199

Stenotrophomonas maltophilia

Stenotrophomonas sp. CC120223-11

Stenotrophomonas maltophilia

Stenotrophomonas maltophilia

Stenotrophomonas maltophilia

Sulfurisphaera tokodaii

Sulfolobus sp. SCGC AB-777 L09

Sulfolobus acidocaldarius DSM 639

Sulfolobus sp. A20

Candidatus Acidianus copahuensis

Fervidobacterium changbaicum lp_3505

lp_3505

lp_3505

LipS

LipS

LipS

LipS

LipS

LipT

LipT

LipT

LipT

patatin

patatin

patatin

patatin

patatin

tannase

tannase

tannase

MtEst45

MtEst45

MtEst45

MtEst45

MtEst45

MtEst45

Rlip1

Rlip1

Rlip1

Rv0045c

Rv0045c

Rv0045c

Rv0045c

Rv0045c

Est22

Est22

Est22

Est22

LipSM54

LipSM54

LipSM54

LipSM54

LipSM54

EstSt7

EstSt7

EstSt7

EstSt7

EstSt7

FnL 


ABS61180
SDG89761
WP_077197788
WP_143145293
WP_004103732
AEH57833
WP_092991953
TAJ91530
RUM94998
RPI52117
TDJ72546
RJQ49102
ADZ54162

HAM01484

OYV61387

WP 052604404

ANI 19854

BAL56305

KPJ55944

RLS54875

RLS60676

HAC 89685

RLS47763

RLS97429

AXG50964

AMV32280 conserved hypothetical protein

Triacylglycerol

esterase/lipase

esterase

alpha/beta hydrolase

esterase

putative lipase

alpha/beta fold hydrolase

alpha/beta fold hydrolase

alpha/beta hydrolase

alpha/beta fold hydrolase

alpha/beta hydrolase

alpha/beta fold hydrolase

EstGX1

MarR family transcriptional

regulator

hypothetical protein

B7Z69_00985

MarR family transcriptional

regulator

EstDZ2a

lipase

hypothetical protein

AMS16_03300

alpha/beta hydrolase

alpha/beta hydrolase

alpha/beta hydrolase

alpha/beta hydrolase

alpha/beta hydrolase

putative esterase

Acetylxylan esterase precursor
Fervidobacterium nodosum Rt17-B1

Fervidobacterium changbaicum

Thermosipho affectus

Fervidobacterium gondwanense

Thermosipho africanus

uncultured microorganism

Thiohalomonas denitrificans

Gammaproteobacteria bacterium

Thiothrix sp.

Deltaproteobacteria bacterium

Proteobacteria bacterium

Gammaproteobacteria bacterium

uncultured bacterium

Acidimicrobiaceae bacterium

Actinobacteria bacterium 21-73-9

Acidithrix ferrooxidans

uncultured Acetothermia bacterium

uncultured Acetothermia bacterium

Planctomycetes bacterium DG 58

Planctomycetes bacterium

Planctomycetes bacterium

Planctomycetaceae bacterium

Planctomycetes bacterium

Planctomycetes bacterium

uncultured bacterium

FnL

FnL

FnL

FnL

FnL

EstP2K

EstP2K

EstP2K

EstP2K

EstP2K

EstP2K

EstP2K

EstGX1

EstGX1

EstGX1

EstGX1

EstDZ2a

EstDZ2a

EstDZ2a

EstDZ2a

EstDZ2a

Est903

Est903

Est903

Est903

Pirellula sp. SH-Sr6A

Est903 
Supplementary Table S3a Lipolytic enzymes downloaded from Uniprot database for validating the profile (part of the table, the full table

see

the

link

https:/www.biorxiv.org/search/Metagenomic\%252Bscreening\%252Bfor\%252Blipolytic\%252Bgenes\%252Brevea 1s\%252Ban\%252Becology-clustered\%252Bdistribution\%252Bpattern)（Dataset 1）

\begin{tabular}{|c|c|c|c|c|c|c|c|}
\hline Entry & Protein names & Organism & $\begin{array}{l}\text { Super } \\
\text { kingdom }\end{array}$ & Phylum & Order & Length & $\mathrm{EC}$ number \\
\hline A0A009EQT3 & Lactonizing lipase (EC 3.1.1.3) & Acinetobacter baumannii 348935 & Bacteria & Proteobacteria & Pseudomonadales & 323 & 3.1 .1 .3 \\
\hline A0A009EUG9 & Lactonizing lipase (EC 3.1.1.3) & Acinetobacter baumannii 348935 & Bacteria & Proteobacteria & Pseudomonadales & 336 & 3.1.1.3 \\
\hline A0A009FQY3 & Esterase TesA (EC 3.1.1.1) & Acinetobacter baumannii 348935 & Bacteria & Proteobacteria & Pseudomonadales & 209 & 3.1.1.1 \\
\hline A0A009G8V4 & Lactonizing lipase (EC 3.1.1.3) & Acinetobacter baumannii 118362 & Bacteria & Proteobacteria & Pseudomonadales & 324 & 3.1.1.3 \\
\hline A0A009GCA4 & Lactonizing lipase (EC 3.1.1.3) & Acinetobacter sp. 1289694 & Bacteria & Proteobacteria & Pseudomonadales & 313 & 3.1.1.3 \\
\hline A0A009GH23 & Esterase TesA (EC 3.1.1.1) & Acinetobacter sp. 1289694 & Bacteria & Proteobacteria & Pseudomonadales & 207 & 3.1.1.1 \\
\hline A0A009GYY5 & Lactonizing lipase (EC 3.1.1.3) & Acinetobacter sp. 1289694 & Bacteria & Proteobacteria & Pseudomonadales & 269 & 3.1 .1 .3 \\
\hline A0A009HL94 & Esterase TesA (EC 3.1.1.1) & Acinetobacter sp. 1295259 & Bacteria & Proteobacteria & Pseudomonadales & 207 & 3.1.1.1 \\
\hline A0A009HLH8 & Lactonizing lipase (EC 3.1.1.3) & Acinetobacter sp. 1295259 & Bacteria & Proteobacteria & Pseudomonadales & 313 & 3.1 .1 .3 \\
\hline A0A009I9R9 & Lactonizing lipase (EC 3.1.1.3) & Acinetobacter baumannii 1295743 & Bacteria & Proteobacteria & Pseudomonadales & 307 & 3.1 .1 .3 \\
\hline A0A009JPY9 & Lactonizing lipase (EC 3.1.1.3) & Acinetobacter baumannii 1419130 & Bacteria & Proteobacteria & Pseudomonadales & 324 & 3.1 .1 .3 \\
\hline A0A009L278 & Esterase TesA (EC 3.1.1.1) & Acinetobacter baumannii 146457 & Bacteria & Proteobacteria & Pseudomonadales & 209 & 3.1.1.1 \\
\hline A0A009LIG6 & Lactonizing lipase (EC 3.1.1.3) & Acinetobacter baumannii 146457 & Bacteria & Proteobacteria & Pseudomonadales & 338 & 3.1.1.3 \\
\hline A0A009ML24 & Lactonizing lipase (EC 3.1.1.3) & Acinetobacter sp. 1475718 & Bacteria & Proteobacteria & Pseudomonadales & 313 & 3.1 .1 .3 \\
\hline A0A009RMH5 & Esterase TesA (EC 3.1.1.1) & Acinetobacter sp. 809848 & Bacteria & Proteobacteria & Pseudomonadales & 207 & 3.1.1.1 \\
\hline A0A009S415 & Lactonizing lipase (EC 3.1.1.3) & Acinetobacter baumannii 951631 & Bacteria & Proteobacteria & Pseudomonadales & 324 & 3.1 .1 .3 \\
\hline A0A009SG56 & Lactonizing lipase (EC 3.1.1.3) & Acinetobacter baumannii 99063 & Bacteria & Proteobacteria & Pseudomonadales & 324 & 3.1.1.3 \\
\hline A0A009YG53 & Esterase TesA (EC 3.1.1.1) & Acinetobacter sp. 479375 & Bacteria & Proteobacteria & Pseudomonadales & 211 & 3.1.1.1 \\
\hline A0A009Z6Z7 & Lactonizing lipase (EC 3.1.1.3) & Acinetobacter sp. 479375 & Bacteria & Proteobacteria & Pseudomonadales & 322 & 3.1.1.3 \\
\hline A0A010IZF7 & Esterase TesA (EC 3.1.1.1) & Acinetobacter sp. 1542444 & Bacteria & Proteobacteria & Pseudomonadales & 207 & 3.1.1.1 \\
\hline A0A010JDR7 & Lactonizing lipase (EC 3.1.1.3) & Acinetobacter sp. 1542444 & Bacteria & Proteobacteria & Pseudomonadales & 313 & 3.1.1.3 \\
\hline A0A010JU09 & Lactonizing lipase (EC 3.1.1.3) & Acinetobacter sp. 1566109 & Bacteria & Proteobacteria & Pseudomonadales & 324 & 3.1.1.3 \\
\hline A0A010L6U0 & Esterase TesA (EC 3.1.1.1) & Acinetobacter sp. 1566109 & Bacteria & Proteobacteria & Pseudomonadales & 207 & 3.1.1.1 \\
\hline A0A010ULU7 & Lactonizing lipase (EC 3.1.1.3) & Acinetobacter sp. $263903-2$ & Bacteria & Proteobacteria & Pseudomonadales & 334 & 3.1.1.3 \\
\hline A0A010V2A9 & Lactonizing lipase (EC 3.1.1.3) & Acinetobacter sp. $263903-2$ & Bacteria & Proteobacteria & Pseudomonadales & 313 & 3.1.1.3 \\
\hline A0A010WGV4 & Esterase TesA (EC 3.1.1.1) & Acinetobacter sp. $263903-2$ & Bacteria & Proteobacteria & Pseudomonadales & 207 & 3.1 .1 .1 \\
\hline A0A011IJ62 & Lactonizing lipase (EC 3.1.1.3) & Acinetobacter sp. 723929 & Bacteria & Proteobacteria & Pseudomonadales & 313 & 3.1.1.3 \\
\hline A0A011JX18 & Lactonizing lipase (EC 3.1.1.3) & Acinetobacter sp. 723929 & Bacteria & Proteobacteria & Pseudomonadales & 269 & 3.1.1.3 \\
\hline A0A011K3K9 & Esterase TesA (EC 3.1.1.1) & Acinetobacter sp. 723929 & Bacteria & Proteobacteria & Pseudomonadales & 207 & 3.1.1.1 \\
\hline A0A011M4E2 & Esterase TesA (EC 3.1.1.1) & Candidatus Accumulibacter sp. SK-12 & Bacteria & Proteobacteria & NA & 206 & 3.1.1.1 \\
\hline A0A011MGM8 & Lipase A (EC 3.1.1.3) & Candidatus Accumulibacter sp. SK-12 & Bacteria & Proteobacteria & NA & 307 & 3.1.1.3 \\
\hline A0A011MPS8 & Esterase TesA (EC 3.1.1.1) & Candidatus Accumulibacter sp. SK-12 & Bacteria & Proteobacteria & NA & 220 & 3.1.1.1 \\
\hline A0A011N3B7 & Carboxylesterase 2 (EC 3.1.1.1) & Candidatus Accumulibacter sp. BA-92 & Bacteria & Proteobacteria & NA & 234 & 3.1.1.1 \\
\hline A0A011NHS5 & Esterase EstA (EC 3.1.1.1) & Candidatus Accumulibacter sp. SK-11 & Bacteria & Proteobacteria & NA & 377 & 3.1.1.1 \\
\hline A0A011NNZ6 & Esterase EstA (EC 3.1.1.1) & Candidatus Accumulibacter sp. SK-12 & Bacteria & Proteobacteria & NA & 378 & 3.1.1.1 \\
\hline A0A011NRW2 & Esterase TesA (EC 3.1.1.1) & Candidatus Accumulibacter sp. BA-92 & Bacteria & Proteobacteria & NA & 205 & 3.1.1.1 \\
\hline A0A011NTI4 & Carboxylesterase 2 (EC 3.1.1.1) & Candidatus Accumulibacter sp. SK-12 & Bacteria & Proteobacteria & NA & 236 & 3.1.1.1 \\
\hline
\end{tabular}


A0A011NZS4 A0A011P1E4 A0A011P216 A0A011P6G5 A0A011P911

A0A011PQC4

A0A011PQE5

A0A011PZA1

A0A011Q348

A0A011Q6T2

A0A011QDG0

A0A011QEA9

A0A011QQI8

A0A011QTT9

A0A011RLC2

A0A013RM5

A0A013S8D5

A0A013TE29

A0A013TPS8

A0A013TXZ3

A0A014B4I3

A0A014BP77

A0A014BQ95

A0A014BVI3

A0A014C673

0A014CDI0

A0A014CG4

A0A014CNC5

A0A014CXJ8

A0A014DCG5

A0A014DNG3

A0A014DW77

A0A014E0S6

A0A014ELX8

A0A014F485

A0A014GFK6

A0A017HFW3

A0A021XAE1

0A021XH5

A0A022I9R1

A0A022IWK9

A0A022J7T0

$\mathrm{A} 0 \mathrm{~A} 022 \mathrm{~J} 8 \mathrm{~K} 7$

A0A022KIF

$\mathrm{A} 0 \mathrm{~A} 022 \mathrm{KN} 98$

A0A022KNS3

A0A023KQK9
Esterase EstA (EC 3.1.1.1)

Esterase TesA (EC 3.1.1.1)

Esterase TesA (EC 3.1.1.1)

Lipase 3 (EC 3.1.1.3)

Esterase TesA (EC 3.1.1.1)

Esterase TesA (EC 3.1.1.1)

Carboxylesterase 2 (EC 3.1.1.1)

Carboxylesterase 2 (EC 3.1.1.1)

Esterase TesA (EC 3.1.1.1)

Carboxylesterase (EC 3.1.1.1)

Lipase A (EC 3.1.1.3)

Carboxylesterase 2 (EC 3.1.1.1)

Lipase (EC 3.1.1.3)

Lipase A (EC 3.1.1.3)

Carboxylesterase (EC 3.1.1.1)

Lactonizing lipase (EC 3.1.1.3)

Esterase TesA (EC 3.1.1.1)

Lactonizing lipase (EC 3.1.1.3)

Esterase TesA (EC 3.1.1.1)

Lactonizing lipase (EC 3.1.1.3)

Esterase TesA (EC 3.1.1.1)

Esterase TesA (EC 3.1.1.1)

Lactonizing lipase (EC 3.1.1.3)

Esterase TesA (EC 3.1.1.1)

Lactonizing lipase (EC 3.1.1.3)

Lactonizing lipase (EC 3.1.1.3)

Esterase TesA (EC 3.1.1.1)

Lactonizing lipase (EC 3.1.1.3)

Lactonizing lipase (EC 3.1.1.3)

Esterase TesA (EC 3.1.1.1)

Esterase TesA (EC 3.1.1.1)

Esterase TesA (EC 3.1.1.1)

Lactonizing lipase (EC 3.1.1.3)

Lactonizing lipase (EC 3.1.1.3)

Esterase TesA (EC 3.1.1.1)

Lactonizing lipase (EC 3.1.1.3)

Lipase (EC 3.1.1.3)

Lipolytic enzyme (EC 3.1.1.3)

Carboxylesterase NlhH (EC 3.1.1.1)

Esterase TesA (EC 3.1.1.1)

Lactonizing lipase (EC 3.1.1.3)

Esterase TesA (EC 3.1.1.1)

Lactonizing lipase (EC 3.1.1.3)

Esterase TesA (EC 3.1.1.1)

Lactonizing lipase (EC 3.1.1.3)

Lactonizing lipase (EC 3.1.1.3)

Autotransporter domain-containing

$\begin{array}{ll}\text { protein } & \text { Autotransporter } \\ \text { membrane } & \text { beta-barrel } \\ \text { domain- }\end{array}$
Candidatus Accumulibacter sp. BA-92 Candidatus Accumulibacter sp. BA-93 Candidatus Accumulibacter sp. BA-9 Candidatus Accumulibacter sp. SK-1 Candidatus Accumulibacter sp. BA-9 Candidatus Accumulibacter sp. SK-12 Candidatus Accumulibacter sp. BA-94 Candidatus Accumulibacter sp. BA-94

Candidatus Accumulibacter sp. SK-11 Alkalibacterium sp. AK22

Candidatus Accumulibacter sp. BA-93

Candidatus Accumulibacter sp. BA-9 Candidatus Accumulibacter sp. BA-94 Candidatus Accumulibacter sp. BA-92

Alkalibacterium sp. AK22

Acinetobacter baumannii 573719

Acinetobacter sp. 742879

Acinetobacter sp. 742879

Acinetobacter sp. 826659

Acinetobacter sp. 826659

Acinetobacter sp. 259778

Acinetobacter sp. 259776

Acinetobacter sp. 25977 7

Acinetobacter sp. $25977^{-}$

Acinetobacter sp. $25977^{-} 8$

Acinetobacter sp. $25977^{-} 3$

Acinetobacter sp. 25977_7

Acinetobacter sp. 259776

Acinetobacter sp. $25977 \quad 2$

Acinetobacter sp. 259773

Acinetobacter sp. 25977-10

Acinetobacter sp. $25977^{-} 1$

Acinetobacter sp. 25977_1

Acinetobacter sp. 25977_4

Acinetobacter sp. 259772

Acinetobacter sp. 25977_10

Limimaricola hongkongensis DSM

17492

Shinella sp. DD12

Shinella sp. DD12

Acinetobacter sp. 1000160

Acinetobacter sp. 1000160

Acinetobacter sp. 1564232

Acinetobacter sp. 1564232

Acinetobacter sp. 478810

Acinetobacter sp. 478810

Acinetobacter sp. 478810

Escherichia coli
Bacteria

Bacteria

Bacteria

Bacteria

Bacteria

Bacteria

Bacteria

Bacteria

Bacteria

Bacteria

Bacteria

Bacteria

Bacteria

Bacteria

Bacteria

Bacteria

Bacteria

Bacteria

Bacteria

Bacteria

Bacteria

Bacteria

Bacteria

Bacteria

Bacteria

Bacteria

Bacteria

Bacteria

Bacteria

Bacteria

Bacteria

Bacteria

Bacteria

Bacteria

Bacteria

Bacteria

Bacteria

Bacteria

Bacteria

Bacteria

Bacteria

Bacteria

Bacteria
Proteobacteria

Proteobacteria

Proteobacteria

Proteobacteria

Proteobacteria

roteobacteria

Proteobacteria

Proteobacteria

Firmicutes

Proteobacteria

Proteobacteria

Firmicutes

Proteobacteria

Proteobacteria

Proteobacteria

Proteobacteria

acteria

roteobacteria

Proteobacteria

Proteobacteria

Proteobacteria

Proteobacteria
Proteobacteria

Proteobacteria

Proteobacteria

Proteobacteria

Proteobacteria

Proteobacteria

Proteobacteria

Proteobacteria

Proteobacteria

Proteobacteria

roteobacteria

roteobacteria

Proteobacteria

Proteobacteria

Proteobacteria

Proteobacteria
Lactobacillales

NA

Lactobacillales

Pseudomonadales

Pseudomonadales

Pseudomonadales

Pseudomonadales

Pseudomonadales

seudomonadales

Pseudomonadales

Pseudomonadales

Pseudomonadale

Pseudomonadales

Pseudomonadales

Pseudomonadales

Rhodobacterales

Rhizobiales

Rhizobiales

Pseudomonadales

Pseudomonadales

seudomonadales

Pseudomonadales

Pseudomo

Pseudomonadales

Enterobacterales

3.1.1.1

3.1.1.1

3.1.1.

3.1.1.3

3.1.1.1

3.1.1.1 
containing protein) (Lipase) (EC 3.1.1.3) (Lipase 1) (Outer membrane autotransporter barrel domain protein)

A0A023M61 A0A023T3X2 EstATII (EC 3.1.1.1)

Esterase/lipase (EC 3.1.1.1)

Beta-lactamase family protein (EC 3.1.1.1)

A0A024H3L

A0A024H702

Liver carboxylesterase 1 (EC 3.1.1.1)

A0A024HBB5

Carboxylesterase 1 (EC 3.1.1.1)

A0A024HG5

Chaperone protein clpB (EC 3.1.1.3)

A0A024HK50

Esterase TesA (EC 3.1.1.1)

A0A024HQ86

A0A024K9K1

A0A031GAI

A0A031GCM8

A0A031GJE5

A0A031ILE0

A0A031IPG8

A0A031ISI 4

A0A031IZU6

A0A031J7M0

A0A037YJP4

$\mathrm{A} 0 \mathrm{~A} 045 \mathrm{H} 1 \mathrm{~T} 3$

A0A045IS29

A0A045J0I3

A0A060L3K4

A0A061CR69

A0A061CYQ8

A0A061D1S0

A0A061D610

A0A061YC10

A0A061YGS

Carboxylesterase 2 (EC 3.1.1.1)

functionally coupled
Phosphoribulokinase (EC 3.1.1.1)

Putative 3-oxoadipate enol-lactonase
Esterase estA (EC 3.1.1.1)

Carboxylesterase (EC 3.1.1.1)

Hydrolase, alpha/beta fold family

functionally coupled

Phosphoribulokinase (EC 3.1.1.1)

Triacylglycerol lipase (EC 3.1.1.3)

Lipase LipA (EC 3.1.1.3)

Carboxylesterase (EC 3.1.1.1)

Lipase (EC 3.1.1.3)

Lipase (EC 3.1.1.3)

Hydrolase, alpha/beta fold family

Autotransporter outer membrane beta-

barrel domain-containing protein

(Lipase) (EC 3.1.1.3)

Carboxylesterase NlhH (Lipase) (EC

3.1.-.-) (EC 3.1.1.3)

Alpha/beta hydrolase (Lipase) (EC

3.1.-.-; EC 3.1.1.3)

Alpha/beta hydrolase (Esterase) (EC

3.1.1.-; EC 3.1.1.3)

Lipase (EC 3.1.1.3) (EC 3.1.1.1)

Carboxylesterase (EC 3.1.1.1) Pseudomonas pseudoalcaligenes

Arylesterase (EC 3.1.2.-)

(Lysophospholipase) (EC 3.1.1.1)

Carboxylesterase bioH (EC 3.1.1.1)

Alpha/beta hydrolase

(Carboxylesterase NlhH) (EC 3.1.1.1)

Alpha/beta

hydrolase

(Lipase) uncultured bacterium

Thalassospira sp. GB04J01

Pseudarthrobacter siccitolerans

Pseudarthrobacter siccitoleran

Pseudomonas knackmussii (strain DSM 6978 / LMG 23759 / B13)

Pseudomonas knackmussii (strain DSM

6978 / LMG 23759 / B13)

Pseudomonas knackmussii (strain DSM

6978 / LMG 23759 / B13)

Pseudomonas knackmussii (strain DSM

6978 / LMG 23759 / B13)

Devosia sp. DBB001

Pseudomonas sp. RIT288

Pseudomonas sp. RIT288

Janthinobacterium lividum

Pseudomonas sp. RIT357

Pseudomonas sp. RIT357

Pseudomonas sp. RIT357

Pseudomonas sp. RIT357

Pseudomonas sp. RIT357

Escherichia coli

Mycobacterium tuberculosis

Mycobacterium tuberculosis

Mycobacterium tuberculosis

Burkholderia sp. 30(2014)

Pseudomonas pseudoalcaligenes

Pseudomonas pseudoalcaligenes

Pseudomonas pseudoalcaligenes

Escherichia coli

Escherichia coli
Bacteria

Bacteria

Bacteria

Bacteria

Bacteria

Bacteria

Bacteria

Bacteria

Bacteria

Bacteria

Bacteria

Bacteria

Bacteria

Bacteria

Bacteria

Bacteria

Bacteria

Bacteria

Bacteria

Bacteria

Bacteria

Bacteria

Bacteria

Bacteria

Bacteria

Bacteria

Bacteria
NA

Proteobacteria

Actinobacteria

Actinobacteria

Proteobacteria

Proteobacteria

Proteobacteria

Proteobacteria

Proteobacteria

Proteobacteria

Proteobacteria

Proteobacteria

Proteobacteria

Proteobacteria

Proteobacteria

Proteobacteria

Proteobacteria

Proteobacteria

Actinobacteria

Actinobacteria

Actinobacteria

Proteobacteria

Proteobacteria

Proteobacteria

Proteobacteria

Proteobacteria

Proteobacteria

Proteobacteria
NA

Rhodospirillales

Micrococcales

Micrococcales

Pseudomonadales

Pseudomonadales

Pseudomonadales

Pseudomonadales

Rhizobiales

Pseudomonadales

Pseudomonadales

Burkholderiales

Pseudomonadales

Pseudomonadales

Pseudomonadales

Pseudomonadales

Pseudomonadales

Enterobacterales

Corynebacteriales

Corynebacteriales

Corynebacteriales

Burkholderiales

Pseudomonadales

Pseudomonadales

Pseudomonadales

Pseudomonadales

Enterobacterales

Enterobacterales
3.1.1.

3.1.1.1

3.1.1.1

3.1.1.1

3.1.1.3

3.1.1.1

3.1.1.1

3.1.1.1

3.1.1.1

3.1.1.3

3.1 .1 .3

3.1 .1$.

3.1.1.3

3.1.1.1

3.1.1.1

3.1.1.3

3.1.-.-; 3.1.1.3

3.1.-.-; 3.1.1.3

3.1.1.-;

3.1.1.3

3.1.1.3

3.1.1.1

3.1.1.1

3.1.2.-;

3.1 .1$.

3.1 .1 .1

3.1.1.1

3.1.1.1 


$\begin{array}{ll}\text { A0A062BV08 } & \text { Esterase TesA (EC 3.1.1.1) } \\ \text { A0A062BVN1 } & \text { Lactonizing lipase (EC 3.1.1.3) } \\ \text { A0A062C442 } & \text { Lactonizing lipase (EC 3.1.1.3) } \\ \text { A0A062DKE7 } & \text { Lactonizing lipase (EC 3.1.1.3) } \\ \text { A0A062FCR0 } & \text { Lactonizing lipase (EC 3.1.1.3) } \\ \text { A0A062FNQ7 } & \text { Lactonizing lipase (EC 3.1.1.3) } \\ \text { A0A062GEE3 } & \text { Lactonizing lipase (EC 3.1.1.3) } \\ \text { A0A062GRT9 } & \text { Esterase TesA (EC 3.1.1.1) } \\ \text { A0A062GV77 } & \text { Lactonizing lipase (EC 3.1.1.3) } \\ \text { A0A062IBD1 } & \text { Lactonizing lipase (EC 3.1.1.3) } \\ \text { A0A062IXY3 } & \text { Lactonizing lipase (EC 3.1.1.3) } \\ \text { A0A062K6R8 } & \text { Lactonizing lipase (EC 3.1.1.3) } \\ \text { A0A062KD00 } & \text { Lactonizing lipase (EC 3.1.1.3) } \\ \text { A0A062KY07 } & \text { Lactonizing lipase (EC 3.1.1.3) }\end{array}$

Acinetobacter sp. 263903-1

Acinetobacter sp. 263903-1

Acinetobacter sp. 263903-1

Acinetobacter baumannii 496487

Acinetobacter baumannii 754286

Acinetobacter baumannii 940793

Acinetobacter sp. 72431

Acinetobacter sp. 72431

Acinetobacter sp. 72431

Acinetobacter baumannii 21072

Acinetobacter baumannii 233846

Acinetobacter baumannii 1288284

Acinetobacter baumannii 1288284

Acinetobacter baumannii 1571545

\begin{tabular}{lllll} 
Bacteria & Proteobacteria & Pseudomonadales & & 3.1 .1 .1 \\
Bacteria & Proteobacteria & Pseudomonadales & & 3.1 .1 .3 \\
Bacteria & Proteobacteria & Pseudomonadales & 3.1 .1 .3 \\
Bacteria & Proteobacteria & Pseudomonadales & 3.1 .1 .3 \\
Bacteria & Proteobacteria & Pseudomonadales & 3.1 .1 .3 \\
Bacteria & Proteobacteria & Pseudomonadales & & 3.1 .1 .3 \\
Bacteria & Proteobacteria & Pseudomonadales & & 3.1 .1 .3 \\
Bacteria & Proteobacteria & Pseudomonadales & & 3.1 .1 .1 \\
Bacteria & Proteobacteria & Pseudomonadales & & 3.1 .1 .3 \\
Bacteria & Proteobacteria & Pseudomonadales & & 3.1 .1 .3 \\
Bacteria & Proteobacteria & Pseudomonadales & & 3.1 .1 .3 \\
Bacteria & Proteobacteria & Pseudomonadales & 239 & 3.1 .1 .3 \\
Bacteria & Proteobacteria & Pseudomonadales & 334 & 3.1 .1 .3 \\
Bacteria & Proteobacteria & Pseudomonadales & 324 & 3.1 .1 .3 \\
\hline & & &
\end{tabular}


Supplementary Table S3b Lipolytic enzymes reported from literatures for validating the profile HMM database (Dataset 2)

\begin{tabular}{|c|c|c|c|c|c|c|}
\hline Protein name & Length (aa) & Lipolytic family & Accession Nr. to Genebank & Description & Source of microorganis & Reference \\
\hline 499EST & 313 & IV & AIE44670.1 & esterase & Acidicaldus sp. USBA-GBX-499 & Lopez et al., 2014 \\
\hline $7 \mathrm{~N} 9$ & 296 & IV & AVP72281.1 & esterase & uncultured bacterium & Borchert et al., 2017 \\
\hline ABO_1197 & 318 & V & CAL16645.1 & $\begin{array}{l}\text { carboxylic } \\
\text { hydrolase }\end{array}$ & Alcanivorax borkumensis SK2 & \\
\hline ABO_1251 & 505 & VII & CAL16699.1 & carboxylesterase & Alcanivorax borkumensis SK2 & Schneiker et al., 2006 \\
\hline ABO0195 & 319 & IV & WP_011587492.1 & alpha/beta hydrolase & Alcanivorax & \\
\hline AcXE2 & 214 & II & ACM59679.1 & $\begin{array}{l}\text { lipolytic protein GDSL } \\
\text { family }\end{array}$ & Caldicellulosiruptor bescii DSM 6725 & Soni et al., 2017 \\
\hline CaesCCR11 & 250 & XIII & AHL67377.1 & carboxylesterase, partial & Geobacillus thermoleovorans & Espinosa-Luna et al., 2016 \\
\hline E69 & 274 & IV & AUD08548.1 & esterase & Erythrobacter seohaensis & Huo et al., 2017 \\
\hline EaEST & 272 & $\mathrm{~V}$ & WP_014970431.1 & alpha/beta hydrolase & Exiguobacterium antarcticum & Lee et zl., 2017 \\
\hline EM3L2 & 404 & $\mathrm{XV}$ & ADH̄59405 & esterase & uncultured bacterium & Jeon et al., 2011 \\
\hline EM3L4 & 330 & EM3L4 & ADH59407 & esterase/lipase & uncultured bacterium & Jeon et al., 2011 \\
\hline EML1 & 304 & XI & ABB79948 & class 3 lipase & uncultured bacterium & Jeong et al., 2009 \\
\hline Est_p1 & 296 & $\mathrm{~V}$ & ACF33459.1 & putative lipase/esterase & uncultured bacterium & Peng et al., 2011 \\
\hline Est01 & 397 & VIII & HQ444406 & cold-adapted esterase & uncultured bacterium & Cheng et al., 2014 \\
\hline Est1 & 270 & $\mathrm{~V}$ & KR149567 & esterase & uncultured bacterium & Lu et al., 2019 \\
\hline Est10 & 223 & VI & AGD81840.1 & esterase & Psychrobacter pacificensis & Rodríguez et al., 2015 \\
\hline Est12 & 329 & IV & AGQ21328.1 & esterase & Psychrobacter sp. & Wu et al., (2013) \\
\hline Est2 & 389 & VIII & KR149568 & esterase & uncultured bacterium & Lu et al., 2019 \\
\hline Est22 & 478 & Est22 & ANQ80463.1 & esterase & uncultured bacterium & Li et al., 2017 \\
\hline Est22_2 & 423 & VIII & KF052088 & Est22 & uncultured bacterium & Mokoena et al., 2013 \\
\hline Est $25^{-}$ & 340 & IV & AII23256.1 & esterase & uncultured bacterium & Li et al., 2014 \\
\hline Est30 & 247 & XIII & AAN81911.1 & $\begin{array}{l}\text { thermostable } \\
\text { carboxylesterase Est } 30\end{array}$ & Geobacillus stearothermophilus & Ewis et al., 2004 \\
\hline Est4 & 316 & $\mathrm{~V}$ & ALE67003.1 & esterase & uncultured bacterium & Gao et al., 2016 \\
\hline Est40 & 299 & IV & $5 \mathrm{GMR}$ & esterase & uncultured bacterium & Li et al., 2017 \\
\hline Est56 & 287 & IV & KR149569 & esterase & uncultured bacterium & our previous research \\
\hline Est700 & 208 & I & AQX17346.1 & esterase & Bacillus licheniformis & Zhang et al., 2018 \\
\hline Est7K & 411 & VIII & KP756684 & family VIII esterase & uncultured bacterium & Lee et al., 2016 \\
\hline Est903 & 300 & IV & AXG50964.1 & putative esterase & uncultured bacterium & Jia et al., 2019 \\
\hline Est97 & 247 & XVI & AFP50148 & esterase & uncultured microorganism & Fu et al., 2013 \\
\hline Est9x & 294 & Est9X & AFR79233 & esterase & uncultured bacterium & Jeong et al., 2009 \\
\hline EstA & 277 & EstA & ABY60416.1 & esterase & uncultured bacterium & Chu et al., 2008 \\
\hline EstA3 & 414 & XIV & WP_011026365.1 & acetyltransferase & Caldanaerobacter subterraneus & Lee et al., 2016 \\
\hline EstA3_2 & 396 & VIII & DQ022078 & beta-lactamase & uncultured bacterium & Rao et al., 2011 \\
\hline EstAM & 314 & IV & ACF04196.1 & lipase/esterase & uncultured bacterium & Rashamuse et al., 2009 \\
\hline EstATII & 314 & IV & AHN10469.1 & esterase & uncultured bacterium & Mohamed et al., 2013 \\
\hline EstBL & 398 & VIII & AAX78516.1 & EstBL & Burkholderia cepacia & Rashamuse et al., 2007 \\
\hline EstC & 427 & VIII & ACH88047.1 & carboxylesterase & uncultured bacterium & Rashamuse et al., 2009 \\
\hline
\end{tabular}




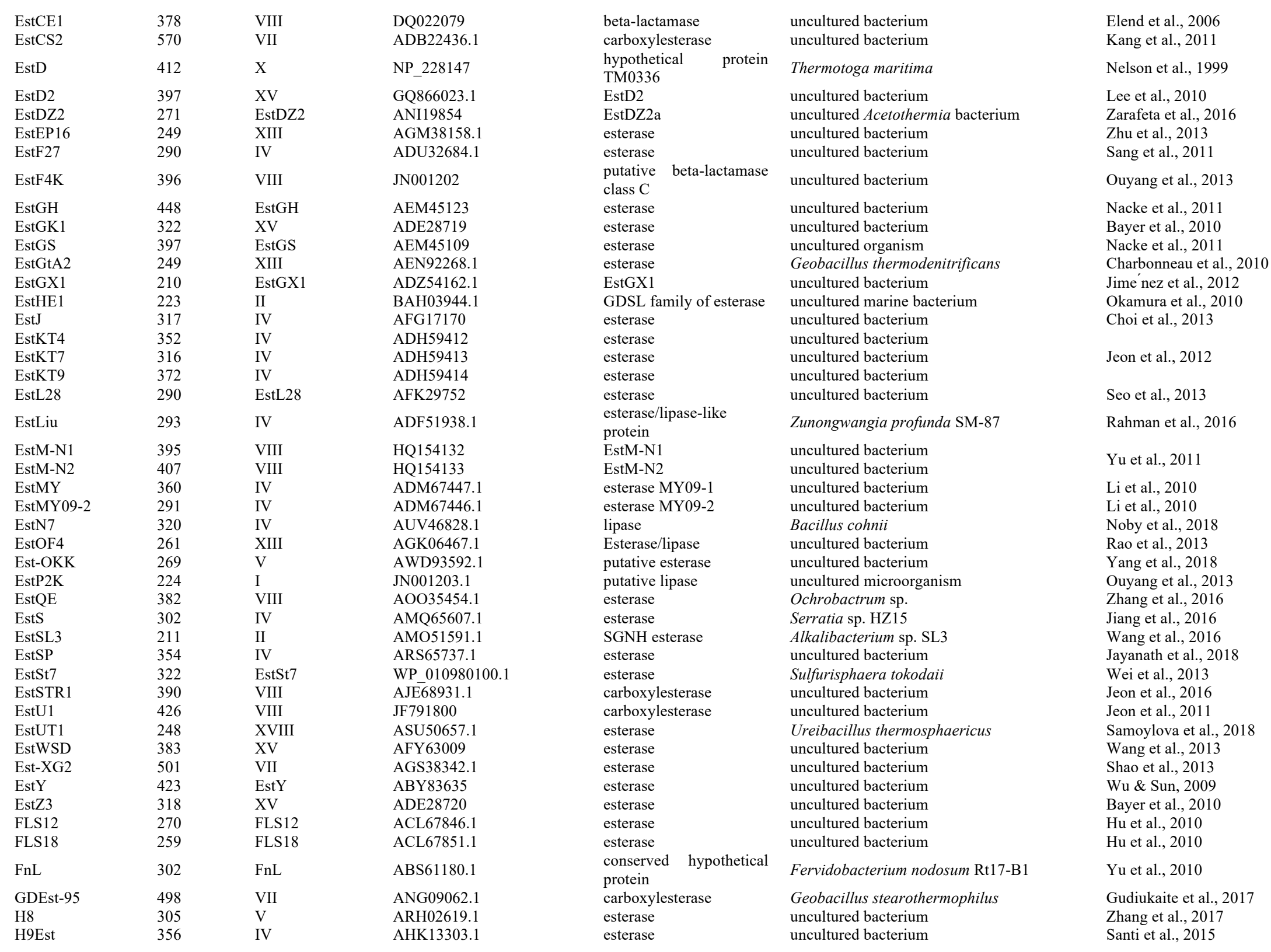




\begin{tabular}{|c|c|c|c|}
\hline LAE1 & 272 & VI & SRA059294 \\
\hline LAE2 & 324 & II & SRA059294 \\
\hline LAE3 & 300 & IV & SRA059294 \\
\hline LAE4 & 309 & IV & SRA059294 \\
\hline LAE5 & 217 & II & SRA059294 \\
\hline LAE6 & 315 & IV & SRA059294 \\
\hline LAE7 & 310 & II & SRA059294 \\
\hline Lip10 & 348 & IV & ALN44199.1 \\
\hline Lip-1452 & 483 & IV & ADB11056.1 \\
\hline Lip3 & 280 & XI & AHZ89331.1 \\
\hline Lip-948 & 315 & $\mathrm{~V}$ & ADB11055.1 \\
\hline LipA & 277 & I & ACJ13070.1 \\
\hline LipA9 & 404 & VIII & AYH52116.1 \\
\hline Lipab15 & 272 & IV & AID66450.1 \\
\hline Lipab18 & 272 & IV & AID66448.1 \\
\hline Lipab4 & 272 & IV & AID66447.1 \\
\hline Lipab8 & 272 & IV & AID66449.1 \\
\hline Lipag18 & 272 & IV & AID66446.1 \\
\hline LipBL & 404 & VIII & CBX87546.1 \\
\hline LipC & 327 & IV & AAV45166.1 \\
\hline $\mathrm{LipC} 12$ & 293 & I & AEK97793.1 \\
\hline LipEH166 & 381 & XII & ACB11220.1 \\
\hline LipG & 300 & XI & ABE69172.1 \\
\hline LipJ2 & 423 & XVII & ANA76126.1 \\
\hline Lipo1 & 308 & IV & ADC79133.1 \\
\hline Lipo10 & 432 & unclassified & ADC79144.1 \\
\hline Lipo11 & 291 & IV & ADC79145.1 \\
\hline Lipo12A & 291 & IV & ADC79146.1 \\
\hline Lipo12B & 506 & VIII & ADC79147.1 \\
\hline Lipo13 & 441 & II & ADC79148.1 \\
\hline Lipo2 & 282 & I & ADC79134.1 \\
\hline Lipo3 & 253 & $\mathrm{~V}$ & ADC79135.1 \\
\hline Lipo4A & 226 & VI & ADC79136.1 \\
\hline Lipo4B & 293 & $\begin{array}{l}\text { patatin-like- } \\
\text { protein }\end{array}$ & ADC79137.1 \\
\hline Lipo4C & 292 & $\begin{array}{l}\text { patatin-like- } \\
\text { protein }\end{array}$ & ADC79138.1 \\
\hline Lipo5 & 348 & IV & ADC79139.1 \\
\hline Lipo6 & 242 & VI & ADC79140.1 \\
\hline Lipo7A & 471 & unclassified & ADC79141.1 \\
\hline Lipo7B & 281 & unclassified & ADC79142.1 \\
\hline Lipo8 & 208 & II & ADC79143.1 \\
\hline LipR & 449 & $\mathrm{X}$ & ССC 86601.1 \\
\hline LipR 2 & 467 & $\mathrm{X}$ & WP 011331609.1 \\
\hline LipR $\overline{1}$ & 417 & I & AEL̄99900.1 \\
\hline LipR3 & 417 & I & AFV46383.1 \\
\hline LipS & 280 & XIII & AFS34518.1 \\
\hline LipSm & 400 & XIX & KX353755.1 \\
\hline LipSM54 & 271 & LipSM54 & AGF29555 \\
\hline
\end{tabular}

lipase
lipase
lipase
lipase
lipase
lipase
lipase
lipase
lipase
class 3 lipase
lipase
lipase
lipase
lipase
lipase
lipase
lipase
lipase
lipolytic enzyme
lipase/esterase
lipase
lipase
probable lipase
secretory lipase
lipase/esterase
lipase/esterase/thioestera
se family lipase
lipase/esterase
lipase/esterase
lipase/esterase
hypothetical protein
hypothetical protein
lipase/esterase
lipase/esterase
patatin-like
phospholipase
patatin-like
phospholipase
lipase/esterase
lipase/esterase
lipase/esterase
hypothetical protein
arylesterase
triacylglycerol hydrolase
triacylglycerol lipase
lipase
lipase
esterase/lipase
secreted lipase
lipase

uncultured bacterium

uncultured bacterium

uncultured bacterium

uncultured bacterium

uncultured bacterium

uncultured bacterium

ncultured bacterium

Monascus ruber

Psychrobacter sp. G

ncultured bacterium

Psychrobacter sp. G

uncultured bacterium

Marinobacter lipolyticus

Halomonas elongata

Halomonas eurihalina

Halomonas elongata

Chromohalobacter japonicus

Halomonas meridiana

Marinobacter lipolyticus

Haloarcula marismortui ATCC 43049

uncultured bacterium

uncultured bacterium

uncultured bacterium

Janibacter $\mathrm{sp}$. R02

uncultured sludge bacterium

uncultured sludge bacterium

uncultured sludge bacterium

uncultured sludge bacterium

uncultured sludge bacterium

uncultured sludge bacterium

uncultured sludge bacterium

uncultured sludge bacterium

uncultured sludge bacterium

uncultured sludge bacterium

uncultured sludge bacterium

uncultured sludge bacterium

uncultured sludge bacterium

uncultured sludge bacterium

uncultured sludge bacterium

uncultured sludge bacterium

Rhodococcus sp.

Rhodococcus erythropolis

uncultured bacterium

uncultured bacterium

uncultured bacterium

Stenotrophomonas maltophilia

Stenotrophomonas maltophilia
Martínez-Martínez et al., 2013

\author{
Guo et al., 2016 \\ Lin et al., 2010 \\ De Santi et al., 2016
Lin et al., 2010 \\ Lin et al., 2010
Couto et al., 2010 \\ Park et al., (2018)
}

Asyari et al., 2015

Pérez et al., 2012

Baliga et al., 2004

Glogauer et al., 2011

Kim et al., 2009

Castilla et al., (2017)

Liaw et al., (2010)

Bassegoda et al., 2012

Bassegoda et al., 2012

Kumar et al., 2013

Kumar et al., 2013

Chow et al., 2012

Parapouli et al., 2018

Li et al., 2016 


\begin{tabular}{|c|c|c|c|c|c|c|}
\hline LipT & 329 & LipT & AFS34517.1 & esterase/lipase & uncultured bacterium & Chow et al., 2012 \\
\hline LipYY31 & 470 & I & BAK52029.2 & lipase & Pseudomonas sp. YY31 & Yamashiro et al., 2013 \\
\hline lp 3505 & 263 & lp 3505 & YP 004890987.1 & acetyl esterase & Lactobacillus plantarum & Esteban-Torres et al., 2014 \\
\hline Lpc53E1 & 387 & VIIII & AFM̄M09717.1 & lipase & uncultured bacterium & Selvin et al., 2012 \\
\hline M37 & 340 & XI & AY527197.1 & lipase & Photobacterium sp. M37 & Ryu et al., 2006 \\
\hline MGS0010 & 414 & VIII & AHG30919.1 & esterase & gamma proteobacterium & \\
\hline MGS0018 & 277 & IV & AGT96416.1 & esterase & uncultured bacterium & Tohimintcous 1015 \\
\hline MGS0105 & 390 & VIII & AGT96414.1 & esterase & uncultured bacterium & I chiginintsev et al., zU15 \\
\hline MGS0153 & 318 & $\mathrm{~V}$ & WP 011588480.1 & esterase & Alcanivorax & \\
\hline MGS-B1 & 345 & IV & $\mathrm{KF} 831420.1$ & carboxylesterase & Tenericutes bacterium & \\
\hline MGS-K1 & 514 & VII & KF831421.1 & carboxylesterase & uncultured bacterium & \\
\hline MGS-M1 & 239 & IV & KF831414.1 & carboxylesterase & Firmicutes bacterium & \\
\hline MGS-M2 & 276 & $\mathrm{~V}$ & KF831415.1 & carboxylesterase & Firmicutes bacterium & \\
\hline MGS-MG1 & 261 & unclassified & KF831418.1 & carboxylesterase & Geobacillus sp. & Alcaide et al., 2015 \\
\hline MGS-MT1 & 348 & IV & KF831419.1 & carboxylesterase & uncultured gamma proteobacterium & \\
\hline MGS-RG1 & 222 & VI & KF831416.1 & carboxylesterase & uncultured gamma proteobacterium & \\
\hline MGS-RG2 & 225 & VI & KF831417.1 & carboxylesterase & uncultured gamma proteobacterium & \\
\hline MGS-RG3 & 277 & IV & KC986402.1 & esterase & uncultured bacterium & \\
\hline MtEst45 & 516 & MtEst45 & AKH15681.1 & acyl esterase & Microbulbifer thermotolerans & Lee et al., 2016 \\
\hline PE10 & 279 & IV & AEV42214.1 & esterase & Pelagibacterium halotolerans & Jiang et al., 2012 \\
\hline PhaZ7 & 380 & IX & AAK07742.1 & $\begin{array}{l}\text { PHB depolymerase } \\
\text { PhaZ7 precursor }\end{array}$ & Paucimonas lemoignei & Handrick et al., 2001 \\
\hline PLP & 302 & $\begin{array}{l}\text { patatin-like- } \\
\text { protein }\end{array}$ & AIT11629.1 & $\begin{array}{l}\text { patatin-like } \\
\text { phospholipase family } \\
\text { protein }\end{array}$ & uncultured bacterium & Fu et al., 2015 \\
\hline PMGL2 & 343 & IV & AMR72657.1 & esterase & uncultured bacterium & Petrovskaya et al., 2016 \\
\hline REst1 & 342 & IV & CBN72524.1 & esterase protein & Rheinheimera sp. Chandigarh & Virk et al., 2011 \\
\hline Rlip1 & 316 & Rlip1 & ACM91047.1 & esterase & uncultured bacterium & Liu et al., 2009 \\
\hline Rv0045c & 298 & $\mathrm{Rv} 0045 \mathrm{c}$ & NP 214559.1 & hydrolase & Mycobacterium tuberculosis $\mathrm{H} 37 \mathrm{Rv}$ & Guo et al., 2010 \\
\hline SAestA & 324 & IV & AIȲ29984.1 & esterase & Salinispora arenicola & Fang et al., 2015 \\
\hline SBLip1 & 445 & VIII & AFK83589.1 & lipolytic enzyme & uncultured bacterium & \\
\hline SBLip2 & 346 & IV & AFK83595.1 & lipolytic enzyme & uncultured bacterium & Biver et al., 2013 \\
\hline SBLip5.1 & 316 & IV & AFK83603.1 & lipolytic enzyme & uncultured bacterium & \\
\hline ThaEst2349 & 343 & IV & AHX83345.1 & esterase/lipase & Thalassospira sp. & De Santi et al., 2016 \\
\hline Vlip509 & 338 & $\mathrm{~V}$ & ABS72371.1 & alpha/beta hydrolases & Vibrio sp. GMD509 & Park et al., (2007) \\
\hline
\end{tabular}


Supplementary Table S3c. Protein sequences predicted from lipolytic inserts identified by function-driven screening (this study) for validating the profile HMM database ((part of the table, the full table see the link https:/www.biorxiv.org/search/Metagenomic\%252Bscreening\%252Bfor\%252Blip olytic\%252Bgenes\%252Breveals\%252Ban\%252Becologyclustered\%252Bdistribution\%252Bpattern) (Dataset 3)

\begin{tabular}{|c|c|c|c|c|c|c|}
\hline Gene name & $\begin{array}{l}\text { Protein } \\
\text { length (aa) }\end{array}$ & Strand & Start (bp) & $\begin{array}{l}\text { End } \\
\text { (bp) }\end{array}$ & $\begin{array}{l}\text { Corresonding } \\
\text { plasmid name }\end{array}$ & $\begin{array}{l}\text { Lipolytic } \\
\text { enzyme }^{\text {a }}\end{array}$ \\
\hline gene 1 & 143 & + & 1 & 432 & pE55-2 & $\mathrm{N}$ \\
\hline gene 2 & 284 & + & 434 & 1288 & pE55-2 & EstC55-2 \\
\hline gene 3 & 165 & - & 1320 & 1817 & pE55-2 & $\mathrm{N}$ \\
\hline gene_4 & 57 & + & 3 & 176 & pE55-3 & $\mathrm{N}$ \\
\hline gene 5 & 513 & + & 267 & 1808 & pE55-3 & EstC55-3 \\
\hline gene-6 6 & 232 & + & 1895 & 2593 & pE55-3 & $\mathrm{N}$ \\
\hline gene_7 & 142 & + & 2647 & 3075 & pE55-3 & $\mathrm{N}$ \\
\hline gene- 8 & 413 & - & 3098 & 4339 & pE55-3 & $\mathrm{N}$ \\
\hline gene 9 & 351 & - & 4435 & 5490 & pE55-3 & $\mathrm{N}$ \\
\hline gene 10 & 172 & - & 5567 & 6085 & pE55-3 & $\mathrm{N}$ \\
\hline gene_11 & 163 & - & 6195 & 6686 & pE55-3 & $\mathrm{N}$ \\
\hline gene_12 & 355 & - & 6761 & 7828 & pE55-3 & $\mathrm{N}$ \\
\hline gene 13 & 163 & - & 7825 & 8316 & pE55-3 & $\mathrm{N}$ \\
\hline gene_14 & 94 & - & 8313 & 8597 & pE55-3 & $\mathrm{N}$ \\
\hline gene_15 & 271 & + & 2 & 817 & pE55-4 & $\mathrm{N}$ \\
\hline gene 16 & 271 & + & 836 & 1651 & pE55-4 & EstC55-4 1 \\
\hline gene 17 & 390 & + & 1783 & 2955 & pE55-4 & EstC55-4_2 \\
\hline gene_18 & 131 & + & 3051 & 3446 & pE55-4 & $\mathrm{N}$ \\
\hline gene_19 & 136 & + & 3560 & 3970 & pE55-4 & $\mathrm{N}$ \\
\hline gene 20 & 153 & - & 4055 & 4516 & pE55-4 & $\mathrm{N}$ \\
\hline gene 21 & 487 & + & 4557 & 6020 & pE55-4 & $\mathrm{N}$ \\
\hline gene_22 & 230 & + & 6017 & 6709 & pE55-4 & $\mathrm{N}$ \\
\hline gene_23 & 325 & + & 6804 & 7781 & pE55-4 & $\mathrm{N}$ \\
\hline gene 24 & 109 & + & 7778 & 8104 & pE55-4 & $\mathrm{N}$ \\
\hline gene 25 & 58 & + & 3 & 179 & pE55-5 & $\mathrm{N}$ \\
\hline gene_26 & 310 & + & 265 & 1197 & pE55-5 & EstC55-5 \\
\hline gene_27 & 485 & - & 1267 & 2724 & pE55-5 & $\mathrm{N}$ \\
\hline gene 28 & 208 & + & 3025 & 3651 & pE55-5 & $\mathrm{N}$ \\
\hline gene_29 & 162 & + & 3749 & 4234 & pE55-5 & $\mathrm{N}$ \\
\hline gene_30 & 319 & + & 132 & 1091 & pE55-6 & EstC55-6 \\
\hline gene_31 & 407 & - & 1102 & 2325 & pE55-6 & $\mathrm{N}$ \\
\hline gene 32 & 160 & + & 2399 & 2881 & pE55-6 & $\mathrm{N}$ \\
\hline gene_33 & 164 & + & 2926 & 3420 & pE55-6 & $\mathrm{N}$ \\
\hline gene_34 & 316 & + & 3515 & 4465 & pE55-6 & $\mathrm{N}$ \\
\hline gene_35 & 258 & + & 4462 & 5238 & pE55-6 & $\mathrm{N}$ \\
\hline gene 36 & 126 & + & 5235 & 5612 & pE55-6 & $\mathrm{N}$ \\
\hline gene_37 & 109 & - & 3 & 329 & pE55-7 & $\mathrm{N}$ \\
\hline gene_38 & 340 & - & 335 & 1357 & pE55-7 & $\mathrm{N}$ \\
\hline gene 39 & 369 & + & 1607 & 2716 & pE55-7 & $\mathrm{N}$ \\
\hline gene 40 & 430 & + & 2947 & 4239 & pE55-7 & EstC55-7 \\
\hline gene_41 & 375 & - & 4331 & 5458 & pE55-7 & $\mathrm{N}$ \\
\hline
\end{tabular}




\begin{tabular}{|c|c|c|c|c|c|c|}
\hline gene_42 & 118 & + & 5907 & 6263 & pE55-7 & $\mathrm{N}$ \\
\hline gene 43 & 368 & - & 7020 & 8126 & pE55-7 & $\mathrm{N}$ \\
\hline gene-44 & 50 & - & 8126 & 8278 & pE55-7 & $\mathrm{N}$ \\
\hline gene_45 & 105 & + & 8659 & 8976 & pE55-7 & $\mathrm{N}$ \\
\hline gene-46 & 80 & - & 9108 & 9350 & pE55-7 & $\mathrm{N}$ \\
\hline gene 47 & 203 & + & 44 & 655 & pE55-8 & $\mathrm{N}$ \\
\hline gene_48 & 318 & - & 669 & 1625 & pE55-8 & EstC55-8_1 \\
\hline gene 49 & 246 & - & 1646 & 2386 & pE55-8 & EstC55-8_2 \\
\hline gene 50 & 136 & + & 2 & 412 & pE55-10 & $\mathrm{N}$ \\
\hline gene_51 & 296 & + & 492 & 1382 & pE55-10 & $\mathrm{N}$ \\
\hline gene_52 & 121 & + & 1405 & 1770 & pE55-10 & $\mathrm{N}$ \\
\hline gene_53 & 460 & + & 1880 & 3262 & pE55-10 & $\mathrm{N}$ \\
\hline gene_54 & 379 & + & 4416 & 5555 & pE55-10 & $\mathrm{N}$ \\
\hline gene_55 & 279 & - & 5464 & 6303 & pE55-10 & EstC55-10 \\
\hline gene_56 & 648 & + & 6510 & 8453 & pE55-10 & $\mathrm{N}$ \\
\hline gene_57 & 143 & + & 3 & 434 & pE55-12 & $\mathrm{N}$ \\
\hline gene_58 & 293 & + & 545 & 1426 & pE55-12 & $\mathrm{N}$ \\
\hline gene_59 & 212 & + & 1426 & 2064 & pE55-12 & $\mathrm{N}$ \\
\hline gene 60 & 384 & + & 2057 & 3211 & pE55-12 & EstC55-12 \\
\hline gene_61 & 157 & + & 3240 & 3713 & pE55-12 & $\mathrm{N}$ \\
\hline gene_62 & 230 & + & 3703 & 4395 & pE55-12 & $\mathrm{N}$ \\
\hline gene 63 & 148 & + & 4392 & 4838 & pE55-12 & $\mathrm{N}$ \\
\hline gene-64 & 362 & + & 4831 & 5919 & pE55-12 & $\mathrm{N}$ \\
\hline gene_65 & 41 & + & 5916 & 6038 & pE55-12 & $\mathrm{N}$ \\
\hline gene_66 & 975 & + & 3 & 2930 & pE55-13 & $\mathrm{N}$ \\
\hline gene_67 & 375 & - & 2911 & 4038 & pE55-13 & $\mathrm{N}$ \\
\hline gene_68 & 355 & - & 4049 & 5116 & pE55-13 & EstC55-13 \\
\hline gene_69 & 110 & + & 5208 & 5540 & pE55-13 & $\mathrm{N}$ \\
\hline gene 70 & 261 & + & 1 & 786 & pE55-15 & EstC55-15 \\
\hline gene-71 & 197 & + & 891 & 1484 & pE55-15 & $\mathrm{N}$ \\
\hline gene_72 & 48 & + & 1543 & 1686 & pE55-15 & $\mathrm{N}$ \\
\hline gene 73 & 267 & + & 1 & 804 & pE55-18 & EstC55-18 \\
\hline
\end{tabular}

${ }^{a} \mathrm{~N}$, Nonlipolytic proteins 


\section{Supplementary Table S3d Non-lipolytic proteins showing high homology to LEs} were retrieved from uniprot or NCBI database for validating the profile HMM databas (Dataset 4)

\begin{tabular}{|c|c|c|c|}
\hline $\begin{array}{l}\text { Entry (accession } \\
\text { Nr) }\end{array}$ & Database & Protein names & $\begin{array}{l}\text { Length } \\
\text { (aa) }\end{array}$ \\
\hline L7VEQ3 & uniprot & $\begin{array}{l}\text { 2-hydroxy-6-oxo-6-phenylhexa-2,4-dienoate hydrolase BphD } \\
\text { (EC 3.7.1.8) }\end{array}$ & 295 \\
\hline A0A0U0R506 & uniprot & $\begin{array}{l}\text { 2-hydroxy-6-oxo-6-phenylhexa-2,4-dienoate hydrolase BphD } \\
\text { (EC 3.7.1.8) }\end{array}$ & 294 \\
\hline A0A2X1S8R4 & uniprot & $\begin{array}{l}\text { 2-hydroxy-6-oxo-6-phenylhexa-2,4-dienoate hydrolase BphD } \\
\text { (EC 3.7.1.8) }\end{array}$ & 290 \\
\hline A0A0T9VGE3 & uniprot & $\begin{array}{l}\text { 2-hydroxy-6-oxo-6-phenylhexa-2,4-dienoate hydrolase BphD } \\
\text { (EC 3.7.1.8) }\end{array}$ & 295 \\
\hline F8GQY4 & uniprot & 2,6-dioxo-6-phenylhexa-3-enoate hydrolase $\mathrm{BphD}$ (EC 3.7.1.8) & 268 \\
\hline A0A375GEC5 & uniprot & 2,6-dioxo-6-phenylhexa-3-enoate hydrolase BphD (EC 3.7.1.8) & 263 \\
\hline A0A1S7N7P8 & uniprot & Non-heme haloperoxidase (EC 1.11.1.10) & 326 \\
\hline A0A1S7T7D7 & uniprot & Non-heme haloperoxidase (EC 1.11.1.10) & 326 \\
\hline A0A1S7T6S9 & uniprot & Non-heme haloperoxidase (EC 1.11.1.10) & 323 \\
\hline A0A1S7N8N9 & uniprot & Non-heme haloperoxidase (EC 1.11.1.10) & 323 \\
\hline A0A1S7N8S2 & uniprot & Non-heme haloperoxidase (EC 1.11.1.10) & 326 \\
\hline A0A1S7S3Y6 & uniprot & Non-heme haloperoxidase (EC 1.11.1.10) & 333 \\
\hline A0A1S7NDN6 & uniprot & Non-heme haloperoxidase (EC 1.11.1.10) & 325 \\
\hline A0A2Z5YBG9 & uniprot & $\begin{array}{l}\text { Non-heme chloroperoxidase (EC 1.11.1.10) (Non-heme } \\
\text { haloperoxidase Hpx) }\end{array}$ & 326 \\
\hline A0A2N9APQ2 & uniprot & $\begin{array}{l}\text { Arylesterase (Alpha/beta hydrolase fold), putative haloperoxidase } \\
\text { (EC 1.11.1.10) (EC 3.1.1.2) }\end{array}$ & 273 \\
\hline G4SWJ2 & uniprot & $\begin{array}{l}\text { Putative non-heme haloperoxidase, alpha/beta hydrolase fold (EC } \\
\text { 1.11.1.10) }\end{array}$ & 273 \\
\hline A0A0N7H8D0 & uniprot & $\begin{array}{l}\text { Alpha/beta hydrolase (Haloperoxidase) (Non-heme } \\
\text { haloperoxidase Hpx) (EC 1.11.1.10) }\end{array}$ & 308 \\
\hline A0A102S299 & uniprot & Alpha/beta hydrolase (EC 1.11.1.10) (Haloperoxidase) & 278 \\
\hline A0A375DNQ7 & uniprot & Non-heme haloperoxidase (EC 1.11.1.-) (EC 1.11.1.10) & 274 \\
\hline А0А375ЕC98 & uniprot & Non-heme haloperoxidase (EC 1.11.1.-) (EC 1.11.1.10) & 274 \\
\hline A0A375FEC6 & uniprot & Non-heme haloperoxidase (EC 1.11.1.-) (EC 1.11.1.10) & 274 \\
\hline A0A375D6M7 & uniprot & Non-heme haloperoxidase (EC 1.11.1.-) (EC 1.11.1.10) & 274 \\
\hline A0A375IUU4 & uniprot & Non-heme haloperoxidase (EC 1.11.1.-) (EC 1.11.1.10) & 269 \\
\hline A0A1S7RCN8 & uniprot & Non-heme haloperoxidase (EC 1.11.1.10) & 326 \\
\hline A0A1S7R0G9 & uniprot & Non-heme haloperoxidase (EC 1.11.1.10) & 278 \\
\hline A0A1S7RQ23 & uniprot & Non-heme haloperoxidase (EC 1.11.1.10) & 278 \\
\hline A0A1S7N1P6 & uniprot & Non-heme haloperoxidase (EC 1.11.1.10) & 278 \\
\hline A0A1S7R2U2 & uniprot & Non-heme haloperoxidase (EC 1.11.1.10) & 278 \\
\hline A0A1S7R186 & uniprot & Non-heme haloperoxidase (EC 1.11.1.10) & 278 \\
\hline A0A1S7S3E0 & uniprot & Non-heme haloperoxidase (EC 1.11.1.10) & 278 \\
\hline A0A1S7TAY0 & uniprot & Non-heme haloperoxidase (EC 1.11.1.10) & 278 \\
\hline A0A379M130 & uniprot & Non-heme haloperoxidase (EC 1.11.1.10) & 299 \\
\hline A0A1S7TZP1 & uniprot & Non-heme haloperoxidase (EC 1.11.1.10) & 278 \\
\hline A0A375DP21 & uniprot & $\begin{array}{l}\text { Putative Non-heme haloperoxidase, putative Alpha/beta } \\
\text { hydrolase domain (EC } 1.11 .1 .10 \text { ) }\end{array}$ & 276 \\
\hline A0A1S7RZV4 & uniprot & Non-heme haloperoxidase (EC 1.11.1.10) & 278 \\
\hline A0A1S7QF97 & uniprot & Non-heme haloperoxidase (EC 1.11.1.10) & 326 \\
\hline A0A1S7QYM3 & uniprot & Non-heme haloperoxidase (EC 1.11.1.10) & 280 \\
\hline A0A1S7UBZ3 & uniprot & Non-heme haloperoxidase (EC 1.11.1.10) & 278 \\
\hline
\end{tabular}




\begin{tabular}{|c|c|c|c|}
\hline A0A1X2DNB5 & uniprot & Non-heme haloperoxidase Hpx (EC 1.11.1.10) & 327 \\
\hline A0A1S7R6I3 & uniprot & Non-heme haloperoxidase (EC 1.11.1.10) & 278 \\
\hline A0A2X4UF21 & uniprot & Non-heme haloperoxidase (EC 1.11.1.10) & 279 \\
\hline A0A1S7N0Y4 & uniprot & Non-heme haloperoxidase (EC 1.11.1.10) & 278 \\
\hline A0A1S7RYV9 & uniprot & Non-heme haloperoxidase (EC 1.11.1.10) & 278 \\
\hline A0A1S7SHR8 & uniprot & Non-heme haloperoxidase (EC 1.11.1.10) & 325 \\
\hline A0A378W1U8 & uniprot & Non-heme haloperoxidase Hpx (EC 1.11.1.10) & 308 \\
\hline A0A1S7S3D1 & uniprot & Non-heme haloperoxidase (EC 1.11.1.10) & 278 \\
\hline A0A1S7MUS0 & uniprot & Non-heme haloperoxidase (EC 1.11.1.10) & 325 \\
\hline A0A1S7MPM6 & uniprot & Non-heme haloperoxidase (EC 1.11.1.10) & 278 \\
\hline A0A375EBJ1 & uniprot & $\begin{array}{l}\text { Putative Non-heme haloperoxidase, putative Alpha/beta } \\
\text { hydrolase domain (EC 1.11.1.10) }\end{array}$ & 276 \\
\hline F8JH88 & uniprot & Non-heme haloperoxidase (EC 1.11.1.10) & 279 \\
\hline F8JA98 & uniprot & Non-heme haloperoxidase (EC 1.11.1.10) & 324 \\
\hline A0A2N9AMF4 & uniprot & Non-heme haloperoxidase (EC 1.11.1.10) & 278 \\
\hline SFQ96535.1 & NCBI & epoxide hydrolase & 358 \\
\hline AWN43677.1 & NCBI & epoxide hydrolase & 384 \\
\hline ERK71245.1 & NCBI & epoxide hydrolase & 377 \\
\hline ALO47455.1 & NCBI & epoxide hydrolase & 410 \\
\hline ALO91291.1 & NCBI & epoxide hydrolase & 373 \\
\hline ALO98459.1 & NCBI & epoxide hydrolase & 411 \\
\hline PNG93099.1 & NCBI & epoxide hydrolase & 374 \\
\hline PNG95674.1 & NCBI & epoxide hydrolase & 391 \\
\hline PNG97285.1 & NCBI & epoxide hydrolase & 390 \\
\hline KDN82212.1 & NCBI & epoxide hydrolase & 372 \\
\hline KEP41268.1 & NCBI & epoxide hydrolase & 375 \\
\hline AGI60154.1 & NCBI & epoxide hydrolase & 388 \\
\hline AIT98391.1 & NCBI & epoxide hydrolase & 383 \\
\hline KIE51135.1 & NCBI & epoxide hydrolase & 329 \\
\hline KIE52625.1 & NCBI & epoxide hydrolase & 376 \\
\hline ART90207.1 & NCBI & epoxide hydrolase & 273 \\
\hline
\end{tabular}




\section{Supplementary Table S4 Functional families in CATH database used to identify LEs affiliate to family II, VIII}

\begin{tabular}{|c|c|c|c|}
\hline $\begin{array}{l}\text { Lipolytic } \\
\text { family }^{a}\end{array}$ & CATH superfamily & Functional family & Description \\
\hline \multirow[b]{2}{*}{ II } & $\begin{array}{l}\text { Autotransporter } \\
\text { superfamily }(2.40 .128 .130)\end{array}$ & FunFam 4295 & Autotransporting lipase, GDSL family \\
\hline & $\begin{array}{l}\text { SGNH hyd } \\
(3.40 .50 .1110)\end{array}$ & $\begin{array}{l}\text { FunFam } 15013 \\
\text { FunFam } 15022 \\
\text { FunFam } 15004 \\
\text { FunFam } 15024 \\
\text { FunFam } 14962 \\
\text { FunFam } 15007\end{array}$ & $\begin{array}{l}\text { Isoamyl acetate-hydrolyzing esterase } 1 \text { homolog } \\
\text { Acyl-CoA thioesterase I } \\
\text { GDSL esterase/lipase APG } \\
\text { Putative secreted hydrolase } \\
\text { Phospholipase B1, membrane-associated } \\
\text { Autotransporting lipase, GDSL family }\end{array}$ \\
\hline VIII $^{b}$ & $\begin{array}{l}\text { DD-peptidase/beta-lactamase } \\
\text { superfamily }(3.40 .710 .10)\end{array}$ & $\begin{array}{l}\text { FunFam } 22194 \\
\text { FunFam } 22219 \\
\text { FunFam } 4880 \\
\text { FunFam } 22208\end{array}$ & $\begin{array}{l}\text { Beta-lactamase (Penicillin-binding protein) (Penicillinase) } \\
\text { Extended spectrum beta-lactamase GES-11 } \\
\text { Low-affinity penicillin-binding protein } 2 \mathrm{X} \\
\text { Class C beta-lactamase CMY-10 }\end{array}$ \\
\hline \multirow{2}{*}{$\begin{array}{l}\text { patatin- } \\
\text { like- } \\
\text { protein }\end{array}$} & $\begin{array}{l}\text { Cytosolic phospholipase A2 catalytic } \\
\text { domain superfamily }(3.40 .1090 .10)\end{array}$ & $\begin{array}{l}\text { FunFam } 5858 \\
\text { FunFam } 6563 \\
\text { FunFam } 6569 \\
\text { FunFam } 6582 \\
\text { FunFam } 6604 \\
\end{array}$ & $\begin{array}{l}\text { YMR313Cp-like protein } \\
\text { Patatin group D-2 } \\
\text { Probable inactive patatin-like protein } 9 \\
\text { Patatin-like phospholipase domain-containing protein } 2 \\
\text { Patatin-like phospholipase domain-containing protein }\end{array}$ \\
\hline & $\begin{array}{l}\text { Alpha/Beta hydrolase fold, catalytic } \\
\text { domain }(3.40 .50 .1820)\end{array}$ & $\begin{array}{l}\text { FunFam } 115466 \\
\text { FunFam } 115488 \\
\text { FunFam } 115552 \\
\text { FunFam } 115703\end{array}$ & $\begin{array}{l}\text { Serine hydrolase-like protein } 2 \\
\text { Triacylglycerol lipase, pancreatic, putative } \\
\text { Hydrolase, alpha/beta fold family } \\
\text { Putative methylesterase } 13 \text {, chloroplastic }\end{array}$ \\
\hline
\end{tabular}

${ }^{a}$ Proteins with no CATH hits were grouped as putative family II, VIII, PLP candidates, respectively.

${ }^{\mathrm{b}}$ only proteins with sequence length between 350 and 450 aa were retrieved. 
Supplementary Table S5 Description of assembled metagenomes downloaded from Integrated Microbial Genomes database (IMG)

\begin{tabular}{|c|c|c|c|c|}
\hline $\begin{array}{l}\text { Sample } \\
\text { name }\end{array}$ & Habitat & $\begin{array}{l}\text { Accession } \mathrm{Nr} \text { in } \\
\mathrm{IMG}\end{array}$ & $\begin{array}{l}\text { Assembled } \\
\text { genome size } \\
\text { (bp) }\end{array}$ & $\begin{array}{l}\text { Assembled } \\
\text { gene count }\end{array}$ \\
\hline ADAS1 & Anaerobic digester active sludge & 3300009676 & 896231486 & 1557165 \\
\hline ADAS2 & Anaerobic digester active sludge & 3300009776 & 910918723 & 1761190 \\
\hline ADAS3 & Anaerobic digester active sludge & 3300025629 & 440662511 & 734719 \\
\hline ADAS4 & Anaerobic digester active sludge & 3300025867 & 804659355 & 1483826 \\
\hline ADAS5 & Anaerobic digester active sludge & 3300009655 & 593528173 & 1062322 \\
\hline ADAS6 & Anaerobic digester active sludge & 3300025861 & 767290509 & 1440611 \\
\hline ADAS7 & Anaerobic digester active sludge & 3300025784 & 689484126 & 1280091 \\
\hline ADAS8 & Anaerobic digester active sludge & 3300025714 & 559791615 & 1003218 \\
\hline ADAS9 & Anaerobic digester active sludge & 3300025859 & 779020248 & 1463790 \\
\hline ADAS10 & Anaerobic digester active sludge & 3300025748 & 640699893 & 1138626 \\
\hline AS1 & Agriculture soil & 3300026761 & 24453291 & 79694 \\
\hline AS2 & Agriculture soil & 3300026766 & 25781940 & 79948 \\
\hline AS3 & Agriculture soil & 3300027429 & 24424955 & 81886 \\
\hline AS4 & Agriculture soil & 3300027410 & 31302445 & 101087 \\
\hline AS5 & Agriculture soil & 3300026753 & 23078731 & 74587 \\
\hline AS6 & Agriculture soil & 3300027116 & 23624174 & 78960 \\
\hline AS7 & Agriculture soil & 3300026802 & 34836368 & 92819 \\
\hline AS8 & Agriculture soil & 3300026900 & 54610317 & 161548 \\
\hline AS9 & Agriculture soil & 3300027437 & 33318367 & 104972 \\
\hline AS10 & Agriculture soil & 3300027453 & 31838534 & 93132 \\
\hline COM1 & Compost & 3300001258 & 138073274 & 247533 \\
\hline COM2 & Compost & 2199352012 & 495813128 & 848933 \\
\hline COM3 & Compost & 3300017816 & 351196916 & 635693 \\
\hline COM4 & Compost & 3300027271 & 220508683 & 349813 \\
\hline COM5 & Compost & 3300017544 & 457787885 & 845994 \\
\hline COM6 & Compost & 3300017647 & 448939775 & 705231 \\
\hline COM7 & Compost & 3300020591 & 343503552 & 564868 \\
\hline COM8 & Compost & 3300001077 & 236465129 & 523041 \\
\hline COM9 & Compost & 3300003150 & 104091772 & 264942 \\
\hline COM10 & Compost & 3300000145 & 85065922 & 132297 \\
\hline COM11 & Compost & 3300001232 & 33898870 & 50489 \\
\hline COM12 & Compost & 2199352008 & 256665756 & 436647 \\
\hline COM13 & Compost & 2199352035 & 51405246 & 71460 \\
\hline COM14 & Compost & 3300000869 & 109516588 & 194772 \\
\hline COM15 & Compost & 3300005392 & 29361615 & 34669 \\
\hline COM16 & Compost & 3300009949 & 84449027 & 140672 \\
\hline COM17 & Compost & 3300009948 & 65120098 & 107383 \\
\hline COM18 & Compost & 3300009950 & 115845660 & 191034 \\
\hline GS1 & Grassland soil & 3300002916 & 214104574 & 539535 \\
\hline GS2 & Grassland soil & 3300002907 & 420592052 & 981120 \\
\hline GS3 & Grassland soil & 3300002915 & 165939417 & 439950 \\
\hline GS4 & Grassland soil & 3300002561 & 507792538 & 1099704 \\
\hline GS5 & Grassland soil & 3300002917 & 757346763 & 1678570 \\
\hline GS6 & Grassland soil & 3300002562 & 518915676 & 1127421 \\
\hline GS7 & Grassland soil & 3300002557 & 233865259 & 622655 \\
\hline GS8 & Grassland soil & 3300002908 & 967438113 & 2077504 \\
\hline GS9 & Grassland soil & 3300026296 & 548056717 & 951020 \\
\hline GS10 & Grassland soil & 3300026322 & 503750574 & 1023488 \\
\hline
\end{tabular}




\begin{tabular}{|c|c|c|c|c|}
\hline GS11 & Grassland soil & 3300026528 & 618069426 & 1070598 \\
\hline HG1 & Human gut system & 3300008571 & 163086704 & 220519 \\
\hline HG2 & Human gut system & 3300007356 & 126726456 & 161507 \\
\hline HG3 & Human gut system & 3300007210 & 160776829 & 231021 \\
\hline HG4 & Human gut system & 3300007361 & 173118880 & 229956 \\
\hline HG5 & Human gut system & 3300008672 & 200079075 & 256044 \\
\hline HG6 & Human gut system & 3300008744 & 208172635 & 293086 \\
\hline HG7 & Human gut system & 3300006463 & 212850221 & 293809 \\
\hline HG8 & Human gut system & 3300007796 & 173992781 & 238201 \\
\hline HG9 & Human gut system & 3300007804 & 100761432 & 131805 \\
\hline HG10 & Human gut system & 3300008622 & 152622707 & 215113 \\
\hline HG11 & Human gut system & 3300007717 & 164858816 & 204019 \\
\hline HG12 & Human gut system & 3300008299 & 209026199 & 284195 \\
\hline HG13 & Human gut system & 3300008479 & 194179397 & 270790 \\
\hline HG14 & Human gut system & 3300007109 & 141045918 & 188333 \\
\hline HG15 & Human gut system & 3300008750 & 98564025 & 116189 \\
\hline HG16 & Human gut system & 3300008100 & 94952779 & 133926 \\
\hline HM1 & Hypersaline mat & 3300005717 & 315943091 & 630229 \\
\hline HM2 & Hypersaline mat & 3300009133 & 380042747 & 844906 \\
\hline HM3 & Hypersaline mat & 3300005642 & 427721363 & 765179 \\
\hline HM4 & Hypersaline mat & 3300005143 & 124176687 & 221446 \\
\hline HM5 & Hypersaline mat & 3300025384 & 133680257 & 240521 \\
\hline HM6 & Hypersaline mat & 3300025377 & 125674444 & 244906 \\
\hline HM7 & Hypersaline mat & 3300025364 & 103340022 & 187339 \\
\hline HRE1 & $\begin{array}{l}\text { Hydrocarbon resource environments } \\
\text { (Oil reservoir) }\end{array}$ & 3300001422 & 23719607 & 35050 \\
\hline HRE2 & $\begin{array}{l}\text { Hydrocarbon resource environments } \\
\text { (Oil reservoir) }\end{array}$ & 3300001190 & 3612035 & 5365 \\
\hline HRE3 & $\begin{array}{l}\text { Hydrocarbon resource environments } \\
\text { (Oil reservoir) }\end{array}$ & 3300001393 & 11319761 & 17164 \\
\hline HRE4 & $\begin{array}{l}\text { Hydrocarbon resource environments } \\
\text { (Oil reservoir) }\end{array}$ & 3300001195 & 7016652 & 11041 \\
\hline HRE5 & $\begin{array}{l}\text { Hydrocarbon resource environments } \\
\text { (Oil reservoir) }\end{array}$ & 3300000507 & 28712273 & 40166 \\
\hline HRE6 & $\begin{array}{l}\text { Hydrocarbon resource environments } \\
\text { (Oil reservoir) }\end{array}$ & 3300000408 & 18846681 & 25476 \\
\hline HS1 & Hot spring & 3300025462 & 189101311 & 435914 \\
\hline HS2 & Hot spring & 3300025374 & 97476306 & 232572 \\
\hline HS3 & Hot spring & 3300025440 & 152818410 & 356023 \\
\hline HS4 & Hot spring & 3300025360 & 94027272 & 168331 \\
\hline HS5 & Hot spring & 3300000345 & 49094763 & 110064 \\
\hline HS6 & Hot spring & 3300031980 & 267257994 & 617247 \\
\hline HS7 & Hot spring & 3300031875 & 369211308 & 763354 \\
\hline HS8 & Hot spring & 3300033894 & 147463480 & 268501 \\
\hline HS9 & Hot spring & 3300007193 & 76574785 & 223595 \\
\hline HS10 & Hot spring & 3300005963 & 46964575 & 105967 \\
\hline HS11 & Hot spring & 3300005856 & 95778213 & 171593 \\
\hline HS12 & Hot spring & 3300007203 & 73453869 & 228153 \\
\hline HS13 & Hot spring & 3300007178 & 62396224 & 176450 \\
\hline HS14 & Hot spring & 3300005966 & 71045757 & 131700 \\
\hline LL1 & Landfill leachate & 3300028028 & 475545597 & 864354 \\
\hline LL2 & Landfill leachate & 3300029288 & 2066651250 & 3975154 \\
\hline LL3 & Landfill leachate & 3300015214 & 2151718941 & 3941155 \\
\hline LL4 & Landfill leachate & 3300028603 & 1729402186 & 3057430 \\
\hline LL5 & Landfill leachate & 3300028601 & 905611696 & 1440715 \\
\hline LL6 & Landfill leachate & 3300014205 & 2274189263 & 4713313 \\
\hline
\end{tabular}




\begin{tabular}{|c|c|}
\hline LL7 & Landfill leachate \\
\hline LL8 & Landfill leachate \\
\hline LL9 & Landfill leachate \\
\hline LL10 & Landfill leachate \\
\hline MS1 & Marine sediment \\
\hline MS2 & Marine sediment \\
\hline MS3 & Marine sediment \\
\hline MS4 & Marine sediment \\
\hline MS5 & Marine sediment \\
\hline MS6 & Marine sediment \\
\hline MS7 & Marine sediment \\
\hline MS8 & Marine sediment \\
\hline MS9 & Marine sediment \\
\hline MS10 & Marine sediment \\
\hline MS11 & Marine sediment \\
\hline MS12 & Marine sediment \\
\hline MW1 & Marine water \\
\hline MW2 & Marine water \\
\hline MW3 & Marine water \\
\hline MW4 & Marine water \\
\hline MW5 & Marine water \\
\hline MW6 & Marine water \\
\hline MW7 & Marine water \\
\hline MW8 & Marine water \\
\hline MW9 & Marine water \\
\hline MW10 & Marine water \\
\hline OR1 & Oil reservoir \\
\hline OR2 & Oil reservoir \\
\hline OR3 & Oil reservoir \\
\hline OR4 & Oil reservoir \\
\hline OR5 & Oil reservoir \\
\hline OR6 & Oil reservoir \\
\hline OR7 & Oil reservoir \\
\hline OR8 & Oil reservoir \\
\hline OR9 & Oil reservoir \\
\hline OR10 & Oil reservoir \\
\hline OR11 & Oil reservoir \\
\hline OR12 & Oil reservoir \\
\hline OR13 & Oil reservoir \\
\hline RW1 & River water \\
\hline RW2 & River water \\
\hline RW3 & River water \\
\hline RW4 & River water \\
\hline RW5 & River water \\
\hline RW6 & River water \\
\hline RW7 & River water \\
\hline RW8 & River water \\
\hline RW9 & River water \\
\hline RW10 & River water \\
\hline RW11 & River water \\
\hline TFS1 & Tropical forest soil \\
\hline TFS2 & Tropical forest soil \\
\hline TFS3 & Tropical forest soil \\
\hline TFS4 & Tropical forest soil \\
\hline TFS5 & Tropical forest soil \\
\hline TFS6 & Tropical forest soil \\
\hline
\end{tabular}

$\begin{array}{lll}3300014204 & 2297043378 & 4947135 \\ 3300014206 & 2497637605 & 5019461 \\ 3300028032 & 746937909 & 1379627 \\ 3300028602 & 1720483498 & 3252495 \\ 3300002053 & 799007063 & 857807 \\ 3300001782 & 449385852 & 488203 \\ 3300001752 & 546713840 & 1387307 \\ 3300002052 & 406438721 & 440211 \\ 3300001751 & 558305947 & 1410986 \\ 3300001855 & 303084940 & 795716 \\ 3300027901 & 2405913374 & 4986014 \\ 3300027888 & 2093100725 & 4192278 \\ 3300027893 & 2405770886 & 4638423 \\ 3300001753 & 719684507 & 1773993 \\ 3300001854 & 930812755 & 2122334 \\ 3300027814 & 762479598 & 1922283 \\ 3300028189 & 449905649 & 856501 \\ 3300031687 & 410396926 & 914988 \\ 3300028177 & 408634935 & 735188 \\ 3300028535 & 489316995 & 1087482 \\ 3300031629 & 806100132 & 1627096 \\ 3300003601 & 259650553 & 528258 \\ 3300025623 & 382735651 & 725551 \\ 3300000265 & 161683159 & 387725 \\ 3300000153 & 161992569 & 328376 \\ 3300000172 & 201262566 & 388542 \\ 3300035036 & 69829225 & 111598 \\ 3300035038 & 99252794 & 152636 \\ 3300035046 & 94073907 & 161691 \\ 3300036317 & 57638688 & 94156 \\ 3300035048 & 50068740 & 81530 \\ 3300035050 & 169884070 & 266430 \\ 3300035052 & 177248887 & 288315 \\ 3300035524 & 116600525 & 191865 \\ 3300035539 & 100086178 & 161409 \\ 3300035542 & 172062358 & 282985 \\ 3300035543 & 89955684 & 159016 \\ 3300035544 & 152691170 & 227435 \\ 3300035546 & 82893933 & 139497 \\ 3300028071 & 132265979 & 279140 \\ 3300027148 & 154072978 & 291628 \\ 3300027488 & 142548013 & 280720 \\ 3300024499 & 124449897 & 277128 \\ 3300024306 & 183398807 & 409005 \\ 3300028067 & 116036125 & 256146 \\ 3300027160 & 176666155 & 395671 \\ 3300026457 & 166921656 & 376394 \\ 3300024498 & 100335601 & 224516 \\ 3300027596 & 162454814 & 307205 \\ 3300027129 & 109047475 & 237601 \\ 3300026845 & 206272783 & 481964 \\ 3300026852 & 65261237 & 150920 \\ 3300026979 & 8192073 & 117856 \\ 3300026824 & 43775853 & 60571 \\ & & 197536 \\ 303675 & 103697\end{array}$




\begin{tabular}{lllll} 
TFS7 & Tropical forest soil & 3300027014 & 102013575 & 241417 \\
TFS8 & Tropical forest soil & 3300026908 & 76880979 & 188041 \\
TFS9 & Tropical forest soil & 3300026819 & 44903906 & 101777 \\
TFS10 & Tropical forest soil & 3300027063 & 156820484 & 368462 \\
TFS11 & Tropical forest soil & 3300027019 & 132250044 & 265708 \\
TFS12 & Tropical forest soil & 3300027010 & 104652154 & 259653 \\
TFS13 & Tropical forest soil & 3300027049 & 110067664 & 266329 \\
TFS14 & Tropical forest soil & 3300027042 & 103999555 & 260245 \\
WB1 & Wastewater bioreactor & 3300003757 & 256997933 & 400666 \\
WB2 & Wastewater bioreactor & 3300024984 & 355043206 & 529140 \\
WB3 & Wastewater bioreactor & 3300024992 & 490734981 & 617734 \\
WB4 & Wastewater bioreactor & 3300009070 & 473466628 & 577450 \\
WB5 & Wastewater bioreactor & 3300003484 & 218046762 & 321182 \\
WB6 & Wastewater bioreactor & 3300024990 & 476329968 & 648818 \\
WB7 & Wastewater bioreactor & 3300024998 & 350228177 & 459430 \\
WB8 & Wastewater bioreactor & 3300024991 & 412698899 & 554457 \\
WB9 & Wastewater bioreactor & 3300003418 & 171736237 & 298355 \\
WB10 & Wastewater bioreactor & 3300024986 & 306104122 & 372134 \\
WB11 & Wastewater bioreactor & 3300003407 & 264251597 & 417208 \\
WB12 & Wastewater bioreactor & 3300024987 & 280019008 & 367005 \\
WB13 & Wastewater bioreactor & 3300024988 & 341047186 & 437514 \\
\hline
\end{tabular}




\section{Supplementary Table S6 Compost55 and compost76 metagenome assembly} statistics

\begin{tabular}{llll}
\hline & Statistic & compost55 & compost76 \\
\hline Raw datasets & Raw reads (counts) & $60,423,492$ & $58,519,117$ \\
& Total bases (bp) & 16398254642 & 14115487744 \\
& Paired reads (counts) & $48,313,538$ & $49,098,115$ \\
Assembly & Unpaired reads (counts) & $2,201,461$ & $2,099,164$ \\
& N50 (bp) & 1,393 & 1,544 \\
& L50 (bp) & 104,761 & 109,695 \\
& Longest contig (bp) & $1,089,853$ & 563,504 \\
& Total bases in contigs (bp) & $988,194,787$ & $810,383,026$ \\
& Total bases in contigs & & $499,132,808$ \\
\hline
\end{tabular}


Supplementary Table S7 Summary of abundant bacterial genera in total and active bacterial community as revealed from 16 rRAN genes and transcripts, respectively

\begin{tabular}{|c|c|c|c|c|c|c|}
\hline \multirow{2}{*}{ Phylum } & \multirow{2}{*}{ Order } & \multirow{2}{*}{ Genus $^{\mathrm{a}}$} & \multicolumn{2}{|c|}{ compost55 } & \multicolumn{2}{|c|}{ compost76 } \\
\hline & & & DNA & RNA & DNA & RNA \\
\hline \multirow{12}{*}{ Actinobacteria } & Acidimicrobiales & (OM1 clade) & $0.81 \%$ & $1.50 \%$ & $0.00 \%$ & $0.00 \%$ \\
\hline & Acidimicrobiales & (uncultured) & $1.23 \%$ & $4.15 \%$ & $0.00 \%$ & $0.00 \%$ \\
\hline & Micromonosporales & Longispora & $5.76 \%$ & $4.51 \%$ & $0.00 \%$ & $0.00 \%$ \\
\hline & Micromonosporales & Salinispora & $1.88 \%$ & $4.80 \%$ & $0.01 \%$ & $0.03 \%$ \\
\hline & Propionibacteriales & Actinopolymorpha & $1.31 \%$ & $3.75 \%$ & $0.00 \%$ & $0.00 \%$ \\
\hline & Pseudonocardiales & Pseudonocardia & $0.64 \%$ & $2.80 \%$ & $0.08 \%$ & $0.01 \%$ \\
\hline & Streptosporangiales & Thermopolyspora & $1.24 \%$ & $1.08 \%$ & $1.24 \%$ & $1.31 \%$ \\
\hline & Streptosporangiales & Actinomadura & $0.80 \%$ & $1.58 \%$ & $0.17 \%$ & $0.01 \%$ \\
\hline & Streptosporangiales & Thermobispora & $6.15 \%$ & $3.25 \%$ & $0.05 \%$ & $0.06 \%$ \\
\hline & Streptosporangiales & Thermomonospora & $0.66 \%$ & $4.12 \%$ & $0.76 \%$ & $0.37 \%$ \\
\hline & Rubrobacterales & Rubrobacter & $2.54 \%$ & $4.66 \%$ & $0.00 \%$ & $0.00 \%$ \\
\hline & Bacteroidetes Incertae Sedis & & & & & \\
\hline \multirow[t]{2}{*}{ Bacteroidetes } & Order II & Rhodothermus & $15.11 \%$ & $4.49 \%$ & $0.00 \%$ & $0.00 \%$ \\
\hline & Flavobacteriales & (NS9 marine group) & $0.00 \%$ & $0.00 \%$ & $2.21 \%$ & $0.09 \%$ \\
\hline Chloroflexi & Chloroflexales & $(\mathrm{FFCH7168)}$ & $0.00 \%$ & $0.00 \%$ & $0.76 \%$ & $1.29 \%$ \\
\hline \multicolumn{7}{|l|}{ Deinococcus- } \\
\hline Thermus & Thermales & Thermus & $0.56 \%$ & $0.12 \%$ & $1.39 \%$ & $0.25 \%$ \\
\hline \multirow[t]{13}{*}{ Firmicutes } & Bacillales & Calditerricola & $0.04 \%$ & $0.00 \%$ & $15.47 \%$ & $35.30 \%$ \\
\hline & Bacillales & Geobacillus & $2.83 \%$ & $4.94 \%$ & $1.62 \%$ & $0.42 \%$ \\
\hline & Bacillales & Ureibacillus & $0.57 \%$ & $1.00 \%$ & $0.15 \%$ & $0.00 \%$ \\
\hline & Bacillales & Planifilum & $0.10 \%$ & $0.21 \%$ & $0.10 \%$ & $1.01 \%$ \\
\hline & Clostridiales & Thermaerobacter & $2.93 \%$ & $1.76 \%$ & $7.61 \%$ & $12.04 \%$ \\
\hline & Clostridiales & Symbiobacterium & $0.16 \%$ & $0.04 \%$ & $31.11 \%$ & $29.35 \%$ \\
\hline & Clostridiales & Hydrogenispora & $3.11 \%$ & $0.89 \%$ & $2.12 \%$ & $0.00 \%$ \\
\hline & Clostridiales & Ruminiclostridium & $0.87 \%$ & $0.11 \%$ & $1.38 \%$ & $0.00 \%$ \\
\hline & Thermoanaerobacterales & Thermosediminibacter & $0.01 \%$ & $0.00 \%$ & $2.05 \%$ & $0.61 \%$ \\
\hline & Thermoanaerobacterales & (Family III) & $0.00 \%$ & $0.00 \%$ & $3.56 \%$ & $2.03 \%$ \\
\hline & Thermoanaerobacterales & Brockia & $16.98 \%$ & $26.70 \%$ & $0.67 \%$ & $1.29 \%$ \\
\hline & Limnochordales & (Limnochordaceae) & $0.73 \%$ & $0.75 \%$ & $0.75 \%$ & $1.75 \%$ \\
\hline & Limnochordales & (Limnochordaceae) & $2.55 \%$ & $0.44 \%$ & $0.52 \%$ & $0.71 \%$ \\
\hline \multirow[t]{3}{*}{ Proteobacteria } & Rhizobiales & Filomicrobium & $1.97 \%$ & $5.17 \%$ & $0.01 \%$ & $0.01 \%$ \\
\hline & Myxococcales & Nannocystis & $0.00 \%$ & $0.00 \%$ & $0.23 \%$ & $3.46 \%$ \\
\hline & Methylococcales & Methylocaldum & $0.08 \%$ & $0.33 \%$ & $0.16 \%$ & $1.72 \%$ \\
\hline
\end{tabular}




\begin{tabular}{lllllll} 
& Xanthomonadales & Pseudoxanthomonas & $0.13 \%$ & $0.02 \%$ & $1.92 \%$ & $0.10 \%$ \\
Tenericutes & Xanthomonadales & (uncultured bacterium) & $0.64 \%$ & $1.12 \%$ & $0.06 \%$ & $0.01 \%$ \\
\hline
\end{tabular}

${ }^{a}$ Only genera from abundant orders (greater than $1 \%$ ) are given. In the case the order or genus could not be assigned, the taxonomic name at the highest determined taxonomic resolution is given in parenthesis. 
Supplementary Table S8 Annotation of functionally derived LEs to NCBI nr database

\begin{tabular}{|c|c|c|c|c|c|c|c|}
\hline $\begin{array}{l}\text { Name of } \\
\text { lipolytic } \\
\text { enzyme }\end{array}$ & $\begin{array}{l}\text { Query } \\
\text { length }\end{array}$ & $\begin{array}{l}\text { Subject } \\
\text { length }\end{array}$ & $\begin{array}{l}\text { Identity } \\
\%\end{array}$ & $\begin{array}{l}\text { Closest similar lipolytic } \\
\text { enzyme }\end{array}$ & Organism & Accession Nr. & E-value \\
\hline EstC55-2 & 291 & 291 & 99 & alpha/beta hydrolase & Chloroflexi bacterium & PZN58850 & $0.00 \mathrm{E}+00$ \\
\hline EstC55-3 & 513 & 525 & 50 & carboxylesterase & Acidimicrobiales bacterium & PZS19531 & $2.00 \mathrm{E}-128$ \\
\hline EstC55-4_1 & 271 & 271 & 83 & alpha/beta hydrolase & Pseudonocardia sp. CNS-004 & WP_075953220 & $9.00 \mathrm{E}-161$ \\
\hline EstC55-4 2 & 390 & 391 & 81 & serine hydrolase & Frankia sp. BMG5.36 & WP 071051881 & 0 \\
\hline EstC55-5 & 310 & 311 & 54 & lipolytic enzyme & uncultured bacterium & ACL̄ 67843 & $5.00 \mathrm{E}-102$ \\
\hline EstC55-6 & 345 & 343 & 32 & $\begin{array}{l}\text { patatin-like phospholipase } \\
\text { family protein }\end{array}$ & Halothermothrix orenii & WP_015923954 & $2.00 \mathrm{E}-44$ \\
\hline EstC55-7 & 430 & 417 & 58 & $\begin{array}{l}\text { beta-lactamase-related serine } \\
\text { hydrolase }\end{array}$ & Steroidobacter agariperforans & WP_129641015 & $1.00 \mathrm{E}-166$ \\
\hline EstC55-8_1 & 318 & 317 & 80 & lipase & Bacterium HR24 & GBD13261 & $6.00 \mathrm{E}-169$ \\
\hline EstC55-8_2 & 270 & 266 & 55 & alpha/beta fold hydrolase & Candidatus Entotheonella palauensis & WP 089934682 & $2.00 \mathrm{E}-99$ \\
\hline EstC55-10 & 288 & 288 & 100 & patatin & Thermobispora bispora & WP_013131472 & $0.00 \mathrm{E}+00$ \\
\hline EstC55-12 & 384 & 384 & 60 & alpha/beta hydrolase & Micromonospora pattaloongensis & WP_091555337 & $2.00 \mathrm{E}-143$ \\
\hline EstC55-13 & 355 & 360 & 46 & $\begin{array}{l}\text { Alpha/beta hydrolase family } \\
\text { protein }\end{array}$ & Lokiarchaeum sp. GC14_75 & KKK $\bar{K} 41396$ & $9.00 \mathrm{E}-111$ \\
\hline EstC55-15 & 253 & 243 & 62 & alpha/beta fold hydrolase & Catelliglobosispora koreensis & WP 026208377 & $1.00 \mathrm{E}-100$ \\
\hline EstC55-18 & 267 & 267 & 100 & $\begin{array}{l}\text { pimeloyl- methyl ester } \\
\text { esterase }\end{array}$ & Methylocaldum szegediense & WP_026611794 & 0 \\
\hline EstC55-19_1 & 270 & 266 & 57 & alpha/beta fold hydrolase & Candidatus Entotheonella palauensis & WP_089934682 & $1.00 \mathrm{E}-103$ \\
\hline EstC55-19_2 & 317 & 317 & 99 & Carboxylesterase NlhH & Bacterium HR24 & GBD̄ 13261 & $0.00 \mathrm{E}+00$ \\
\hline EstC55-20 & 350 & 350 & 100 & alpha/beta hydrolase & Actinobacteria bacterium & PZN40536 & 0 \\
\hline EstC55-23 & 322 & 327 & 62 & Acetyl esterase & bacterium HR29 & GBD24385 & $4.00 \mathrm{E}-130$ \\
\hline EstC55-24 & 325 & 342 & 51 & alpha/beta hydrolase & Dactylosporangium aurantiacum & WP 052387799 & $2.00 \mathrm{E}-79$ \\
\hline EstC55-25 & 367 & 367 & 89 & alpha/beta hydrolase & Pseudonocardia sp. CNS-004 & WP_-075951996 & 0 \\
\hline EstC55-26 & 317 & 317 & 97 & patatin & Rhodothermus marinus & WP_014066731 & 0 \\
\hline EstC55-31 & 265 & 263 & 76 & $\begin{array}{l}\text { alpha/beta fold family } \\
\text { hydrolase }\end{array}$ & Bacterium CSP1-2 & KRT̄ 66319 & $6.00 \mathrm{E}-149$ \\
\hline EstC55-34 & 290 & 290 & 92 & alpha/beta hydrolase & Rhodothermus marinus & WP 014067099 & 0 \\
\hline EstC55-38 & 329 & 329 & 97 & esterase & Thermus thermophilus & WP- 024119707 & 0 \\
\hline EstC55-40 & 444 & 454 & 53 & serine hydrolase & Sphaerobacter thermophilus & PZN̄ 60880 & $3.00 \mathrm{E}-152$ \\
\hline EstC55-42 & 373 & 363 & 62 & ferruloyl esterase & Micromonospora sp. ATCC 39149 & EEP71116 & $6.00 \mathrm{E}-148$ \\
\hline EstC55-46 & 415 & 410 & 73 & serine hydrolase & Micromonospora noduli & WP_112582259 & 0 \\
\hline EstC55-51 & 268 & 271 & 67 & alpha/beta fold hydrolase & Micromonospora pattaloongensis & WP_091559969 & $1.00 \mathrm{E}-111$ \\
\hline EstC55-52 & 503 & 504 & 87 & Carboxylesterase & Thermaerobacter marianensis & WP_013495056 & 0 \\
\hline
\end{tabular}




\begin{tabular}{|c|c|c|c|c|c|c|c|}
\hline EstC55-53 & 406 & 406 & 82 & $\begin{array}{l}\text { beta-lactamase-related serine } \\
\text { hydrolase }\end{array}$ & Steroidobacter cummioxidans & WP_116812916 & 0 \\
\hline EstC55-56 & 314 & 313 & 62 & alpha/beta hydrolase & Variovorax sp. BK460 & WP_130428336 & $3.00 \mathrm{E}-124$ \\
\hline EstC55-57 & 271 & 301 & 49 & lipase & Planctomycetaceae bacterium & MBN̄00417 & $1.00 \mathrm{E}-76$ \\
\hline EstC55-60 & 340 & 343 & 59 & alpha/beta hydrolase & Sandaracinaceae bacterium & RZO48478 & $2.00 \mathrm{E}-120$ \\
\hline EstC55-61 & 388 & 388 & 99 & beta-lactamase & Thermus & WP_008632158 & 0 \\
\hline EstC55-62 & 528 & 528 & 100 & carboxylesterase & Proteobacteria bacterium & PZN̄79242 & 0 \\
\hline EstC55-63 & 317 & 317 & 99 & patatin & Rhodothermus marinus & WP_014066731 & 0 \\
\hline EstC55-65 & 409 & 409 & 64 & $\begin{array}{l}\text { beta-lactamase family } \\
\text { protein }\end{array}$ & Chloroflexi bacterium & TMĒ11201 & $0.00 \mathrm{E}+00$ \\
\hline EstC55-66 & 429 & 431 & 69 & beta-lactamase & Thermomicrobium roseum & ACM07132 & 0 \\
\hline EstC55-71 & 317 & 317 & 98 & Extracellular esterase EstB & Bacterium HR41 & GBD45993 & ( \\
\hline EstC55-72 & 287 & 296 & 62 & lipase/esterase & uncultured bacterium & AAS77236 & $8.00 \mathrm{E}-115$ \\
\hline EstC55-73 & 385 & 393 & 71 & $\begin{array}{l}\text { beta-lactamase class } \mathrm{C} \\
\text { family }\end{array}$ & Paracoccus sp. J56 & WP_085501535 & 0 \\
\hline EstC55-76 & 373 & 357 & 77 & alpha/beta hydrolase & Actinobacteria bacterium & PZM88754 & $0.00 \mathrm{E}+00$ \\
\hline EstC55-77 & 278 & 308 & 36 & Lip3594 & uncultured bacterium & QCC19993.1 & $1.00 \mathrm{E}-45$ \\
\hline EstC55-78 & 319 & 311 & 53 & EST1 & uncultured microorganism & ADR31550 & $5.00 \mathrm{E}-103$ \\
\hline EstC55-80 & 408 & 375 & 70 & $\begin{array}{l}\text { beta-lactamase family } \\
\text { protein }\end{array}$ & Chloroflexi bacterium & TMF40125 & $0.00 \mathrm{E}+00$ \\
\hline EstC55-81 & 282 & 269 & 33 & alpha/beta hydrolase & Thermorudis peleae & WP_081886744 & $9.00 \mathrm{E}-27$ \\
\hline EstC55-88 & 317 & 317 & 98 & Extracellular esterase EstB & Bacterium HR41 & GBD̄̄45993 & 0 \\
\hline EstC55-90 & 286 & 286 & 100 & lipase & Thermomonospora curvata & WP_012852218 & 0 \\
\hline EstC55-95 & 288 & 283 & 51 & acetylxylan esterase & Yuhushiella deserti & WP_092534474 & $3.00 \mathrm{E}-85$ \\
\hline EstC55-96 & 267 & 267 & 97 & alpha/beta hydrolase & Rhodothermus marinus & WP_012844996 & 0 \\
\hline EstC55-97 & 240 & 234 & 28 & alpha/beta hydrolase & Stenotrophomonas maltophilia & WP_049464595 & $3.03 \mathrm{E}-07$ \\
\hline EstC55-100 & 376 & 363 & 57 & alpha/beta hydrolase & Dactylosporangium aurantiacum & WP_052386271 & $2.00 \mathrm{E}-131$ \\
\hline EstC55-102 & 707 & 699 & 47 & lipase & $\begin{array}{l}\text { Candidatus Competibacteraceae } \\
\text { bacterium }\end{array}$ & RUQ̄29580 & $5.00 \mathrm{E}-160$ \\
\hline EstC55-105 & 223 & 223 & 62 & triacylglycerol lipase & Chloroflexi bacterium & TMG05867 & $2.00 \mathrm{E}-92$ \\
\hline EstC55-110 & 434 & 436 & 66 & $\begin{array}{l}\text { beta-lactamase-related serine } \\
\text { hydrolase }\end{array}$ & Steroidobacter agariperforans & WP_129644730 & $0.00 \mathrm{E}+00$ \\
\hline EstC55-111 & 392 & 393 & 92 & $\begin{array}{l}\text { SGNH/GDSL hydrolase } \\
\text { family protein }\end{array}$ & Rhodothermus marinus & WP_014065857 & 0 \\
\hline EstC55-113 & 409 & 506 & 56 & beta-lactamase & uncultured sludge bacterium & ADC79147 & $2.00 \mathrm{E}-148$ \\
\hline EstC55-118 & 497 & 496 & 76 & $\begin{array}{l}\text { carboxylesterase family } \\
\text { protein }\end{array}$ & Thermaerobacter sp. FW80 & WP_135226007 & 0 \\
\hline EstC55-131 & 251 & 251 & 99 & phospholipase & Thermus thermophilus & WP_024119695 & $2.00 \mathrm{E}-170$ \\
\hline EstC55-145 & 305 & 307 & 56 & lipolytic enzyme & uncultured bacterium & ACL̄67849 & $1.00 \mathrm{E}-105$ \\
\hline
\end{tabular}




\begin{tabular}{|c|c|c|c|c|c|c|c|}
\hline EstC55-147 & 386 & 410 & 50 & $\begin{array}{l}\text { beta-lactamase-related serine } \\
\text { hydrolase }\end{array}$ & Acidimicrobiia bacterium & RTL09767 & $9.00 \mathrm{E}-106$ \\
\hline EstC55-150 & 393 & 393 & 97 & $\begin{array}{l}\text { SGNH/GDSL hydrolase } \\
\text { family protein }\end{array}$ & Rhodothermus marinus & WP_014065857 & 0 \\
\hline EstC55-151 & 311 & 315 & 66 & lipase (class 2) & Herbihabitans rhizosphaerae & RZS45231 & $8.00 \mathrm{E}-152$ \\
\hline EstC55-154 & 394 & 386 & 47 & lipase & Streptomyces sp. HST28 & WP_127910203 & $2.00 \mathrm{E}-96$ \\
\hline EstC55-156 & 517 & 520 & 51 & $\begin{array}{l}\text { tannase/feruloyl esterase } \\
\text { family alpha/beta hydrolase }\end{array}$ & Betaproteobacteria bacterium & TMḦ79627 & $1.00 \mathrm{E}-168$ \\
\hline EstC55-159 & 284 & 292 & 59 & alpha/beta hydrolase & Actinomadura amylolytica & WP_119727475 & $1.00 \mathrm{E}-94$ \\
\hline EstC55-163 & 303 & 267 & 39 & $\begin{array}{l}\text { patatin-like phospholipase } \\
\text { family protein }\end{array}$ & Orenia metallireducens & WP_068717466 & $2.00 \mathrm{E}-55$ \\
\hline EstC55-164 & 430 & 432 & 81 & serine hydrolase & Proteobacteria bacterium & PZN68398 & $0.00 \mathrm{E}+00$ \\
\hline EstC55-165 & 277 & 277 & 97 & phospholipase & Rhodothermus marinus & WP_014066117 & 0 \\
\hline EstC55-167 & 248 & 248 & 94 & alpha/beta hydrolase & Bacteroidetes bacterium & PZN50641 & $3.00 \mathrm{E}-162$ \\
\hline EstC55-168 & 513 & 598 & 55 & serine hydrolase & Blastocatellia bacterium & PWT89381 & 0 \\
\hline EstC55-169 & 533 & 544 & 51 & $\begin{array}{l}\text { tannase/feruloyl esterase } \\
\text { family alpha/beta hydrolase }\end{array}$ & Gammaproteobacteria bacterium & TAJ93847 & $0.00 \mathrm{E}+00$ \\
\hline EstC55-186 & 515 & 515 & 99 & carboxylesterase & Thermus thermophilus & WP_124105112 & 0 \\
\hline EstC55-188 & 237 & 238 & 99 & alpha/beta hydrolase & Thermus thermophilus & WP_014510770 & $3.00 \mathrm{E}-158$ \\
\hline EstC55-197 & 291 & 298 & 56 & alpha/beta hydrolase & Cyanobacterium TDX16 & OWY61995 & $5.00 \mathrm{E}-100$ \\
\hline EstC55-213 & 286 & 286 & 100 & esterase & Rhodothermus marinus & WP_014067193 & 0 \\
\hline EstC55-215 & 263 & 263 & 99 & alpha/beta hydrolase & Firmicutes bacterium & REJ37793 & $0.00 \mathrm{E}+00$ \\
\hline EstC55-227 & 472 & 455 & 69 & alpha/beta hydrolase & Micromonospora sp. HK10 & WP_046565934 & 0 \\
\hline EstC55-229 & 311 & 312 & 58 & esterase/lipase AS-Trib30 & uncultured bacterium & AIT69759 & $3.00 \mathrm{E}-99$ \\
\hline EstC55-231 & 366 & 384 & 68 & alpha/beta hydrolase & Micromonospora pattaloongensis & WP_091555337 & $7.00 \mathrm{E}-164$ \\
\hline EstC55-234 & 557 & 603 & 58 & $\begin{array}{l}\text { tannase/feruloyl esterase } \\
\text { family alpha/beta hydrolase }\end{array}$ & Steroidobacter sp. JW-3 & WP_129775856 & $0.00 \mathrm{E}+00$ \\
\hline EstC55-235 & 326 & 284 & 60 & alpha/beta fold hydrolase & Streptomyces yeochonensis & WP_107498754 & $7.00 \mathrm{E}-116$ \\
\hline EstC55-239 & 381 & 968 & 98 & Beta-lactamase & Rhodothermus marinus & WP_014067493 & 0 \\
\hline EstC55-241 & 567 & 569 & 61 & phospholipase & Acidobacteria bacterium & RPJ585855.1 & 0 \\
\hline EstC55-244 & 255 & 253 & 41 & esterase & Rhodospirillaceae bacterium & MAG96647 & $4.00 \mathrm{E}-49$ \\
\hline EstC55-245 & 462 & 446 & 53 & penicillin-binding protein & Acidobacteria bacterium & RLE30144 & $3 e-158$ \\
\hline EstC55-247 & 368 & 363 & 56 & alpha/beta hydrolase & Deltaproteobacteria bacterium & TMA57433 & $1.00 \mathrm{E}-143$ \\
\hline EstC55-251 & 317 & 317 & 97 & patatin & Rhodothermus marinus & WP_014066731 & 0 \\
\hline EstC55-253 & 304 & 287 & 29 & alpha/beta hydrolase & Clostridium felsineum & WP_077893977 & $7.00 \mathrm{E}-32$ \\
\hline EstC55-256 & 270 & 270 & 56 & alpha/beta hydrolase & Dehalococcoidia bacterium & TET95823.1 & $2.00 \mathrm{E}-102$ \\
\hline EstC55-258 & 389 & 388 & 82 & Esterase EstB & Bacterium HR29 & GBD24121.1 & 0 \\
\hline EstC55-268 & 303 & 303 & 65 & esterase LC-Est5 & uncultured bacterium & AIT56391 & $2.00 \mathrm{E}-121$ \\
\hline EstC76-21 & 288 & 288 & 100 & patatin & Thermobispora bispora & WP_013131472 & 0 \\
\hline EstC76-28_1 & 257 & 257 & 99 & alpha/beta hydrolase & Thermus thermophilus & WP_014629868 & $2.00 \mathrm{E}-175$ \\
\hline
\end{tabular}




\begin{tabular}{|c|c|c|c|c|c|c|c|}
\hline EstC76-28_2 & 243 & 243 & 97 & alpha/beta hydrolase & Thermus thermophilus & WP_014510102 & $1.83 \mathrm{E}-168$ \\
\hline EstC76-36 & 237 & 238 & 99 & alpha/beta hydrolase & Thermus thermophilus & WP_014510770 & $3.00 \mathrm{E}-158$ \\
\hline EstC76-98 & 425 & 431 & 43 & $\begin{array}{l}\text { beta-lactamase-related serine } \\
\text { hydrolase }\end{array}$ & Acidobacteria bacterium & TDI24993 & $1.00 \mathrm{E}-106$ \\
\hline EstC76-123 & 450 & 412 & 41 & serine hydrolase & Truepera radiovictrix & WP_013178639 & $1.00 \mathrm{E}-91$ \\
\hline EstC76-135 & 310 & 308 & 57 & Carboxylesterase NlhH & Bacterium HR25 & GBD̄ 14217 & $4.00 \mathrm{E}-113$ \\
\hline EstC76-136 & 515 & 515 & 99 & carboxylesterase & Thermus thermophilus & WP_014509791 & 0 \\
\hline EstC76-137 & 277 & 277 & 99 & phospholipase & Rhodothermus marinus & WP_014066117 & 0 \\
\hline EstC76-174 & 388 & 388 & 98 & beta-lactamase & Thermus & WP_008632158 & 0 \\
\hline EstC76-177 & 281 & 281 & 99 & alpha/beta hydrolase & Thermaerobacter sp. FW80 & WP_135226180 & $0.00 \mathrm{E}+00$ \\
\hline EstC76-179 & 329 & 329 & 97 & esterase & Thermus thermophilus & WP_024119707 & 0 \\
\hline EstC76-202 & 267 & 267 & 100 & $\begin{array}{l}\text { pimeloyl-[acyl-carrier } \\
\text { protein] methyl ester } \\
\text { esterase }\end{array}$ & Methylocaldum szegediense & WP_026611794 & 0 \\
\hline EstC76-218 & 329 & 329 & 99 & esterase & Thermus thermophilus & WP_014629382 & 0 \\
\hline EstC76-221 & 257 & 257 & 86 & alpha/beta hydrolase & Caldicoprobacter oshimai & WP_025747209 & $1.00 \mathrm{E}-164$ \\
\hline EstC76-222 & 260 & 260 & 91 & $\begin{array}{l}\text { patatin-like phospholipase } \\
\text { family protein }\end{array}$ & Caldicoprobacter & WP_025747046 & $2.00 \mathrm{E}-171$ \\
\hline EstC76-248 & 257 & 257 & 93 & alpha/beta hydrolase & Thermus thermophilus & WP_124104731 & $1.00 \mathrm{E}-162$ \\
\hline EstC76-250 & 251 & 251 & 99 & $\begin{array}{l}\text { patatin-like phospholipase } \\
\text { family protein }\end{array}$ & Thermus thermophilus & WP_024119695 & $4.00 \mathrm{E}-172$ \\
\hline EstC76-261 & 345 & 343 & 32 & $\begin{array}{l}\text { patatin-like phospholipase } \\
\text { family protein }\end{array}$ & Halothermothrix orenii & WP_015923954 & $2.00 \mathrm{E}-44$ \\
\hline EstC76-262 & 415 & 410 & 73 & serine hydrolase & Micromonospora noduli & WP_112582259 & 0 \\
\hline EstC76-263 & 376 & 363 & 57 & alpha/beta hydrolase & Dactylosporangium aurantiacum & WP_052386271 & $2.00 \mathrm{E}-131$ \\
\hline EstC76-266 & 257 & 257 & 94 & alpha/beta hydrolase & Thermus thermophilus & WP_014510103 & $2.00 \mathrm{E}-164$ \\
\hline EstC76-269 & 260 & 260 & 98 & $\begin{array}{l}\text { patatin-like phospholipase } \\
\text { family protein }\end{array}$ & Caldicoprobacter & WP_025747046 & 0 \\
\hline
\end{tabular}


Supplementary Table S9 Description of LEs from functional metagenomic selections

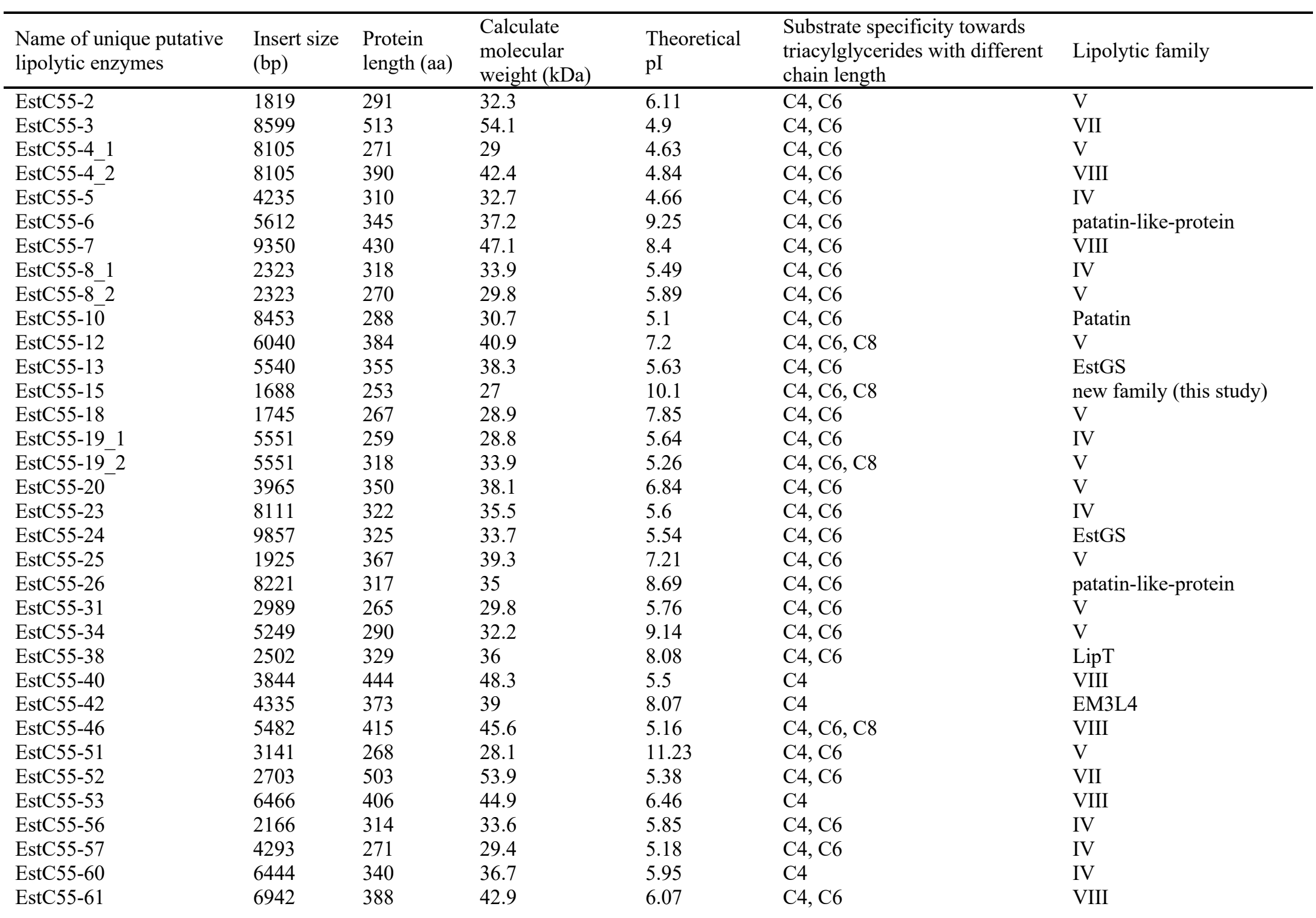




\begin{tabular}{|c|c|c|c|c|c|c|}
\hline EstC55-62 & 4505 & 528 & 56.7 & 8.2 & $\mathrm{C} 4, \mathrm{C} 6$ & VII \\
\hline EstC55-63 & 3924 & 317 & 34.9 & 7.74 & $\mathrm{C} 4$ & Patatin \\
\hline EstC55-65 & 3706 & 409 & 44.1 & 5.07 & $\mathrm{C} 4$ & VIII \\
\hline EstC55-66 & 5776 & 429 & 47 & 5.97 & $\mathrm{C} 4, \mathrm{C} 6$ & VIII \\
\hline EstC55-71 & 2814 & 317 & 33.2 & 8.99 & $\mathrm{C} 4, \mathrm{C} 6, \mathrm{C} 8, \mathrm{C} 10, \mathrm{C} 12, \mathrm{C} 14$ & I \\
\hline EstC55-72 & 6166 & 287 & 30.5 & 4.97 & $\mathrm{C} 4, \mathrm{C} 6$ & IV \\
\hline EstC55-73 & 4617 & 385 & 40.9 & 5.75 & $\mathrm{C} 4, \mathrm{C} 6$ & VIII \\
\hline EstC55-76 & 2896 & 373 & 40.9 & 9.82 & $\mathrm{C} 4, \mathrm{C} 6$ & $\mathrm{~V}$ \\
\hline EstC55-77 & 3392 & 278 & 30.1 & 5.07 & $\mathrm{C} 4$ & EM3L4 \\
\hline EstC55-78 & 6416 & 319 & 33.5 & 4.62 & $\mathrm{C} 4, \mathrm{C} 6$ & IV \\
\hline EstC55-80 & 3076 & 408 & 43.7 & 5.24 & $\mathrm{C} 4$ & VIII \\
\hline EstC55-81 & 3362 & 282 & 30.6 & 5 & $\mathrm{C} 4, \mathrm{C} 6$ & $\mathrm{~V}$ \\
\hline EstC55-88 & 8186 & 317 & 33.2 & 9.12 & $\mathrm{C} 4, \mathrm{C} 6, \mathrm{C} 8, \mathrm{C} 10, \mathrm{C} 12$ & $\mathrm{I}$ \\
\hline EstC55-90 & 4812 & 286 & 30.9 & 8.68 & $\mathrm{C} 4, \mathrm{C} 6, \mathrm{C} 8, \mathrm{C} 10, \mathrm{C} 12$ & I \\
\hline EstC55-95 & 6415 & 288 & 29.5 & 6.95 & $\mathrm{C} 4, \mathrm{C} 6, \mathrm{C} 8$ & III \\
\hline EstC55-96 & 5343 & 267 & 29.9 & 5.99 & $\mathrm{C} 4, \mathrm{C} 6$ & $\mathrm{~V}$ \\
\hline EstC55-97 & 4889 & 240 & 25.6 & 4.32 & $\mathrm{C} 4$ & new family (this study) \\
\hline EstC55-100 & 2878 & 376 & 40.8 & 5.23 & $\mathrm{C} 4, \mathrm{C} 6$ & $\mathrm{~V}$ \\
\hline EstC55-102 & 2936 & 707 & 72.3 & 4.75 & $\mathrm{C} 4$ & new family (this study) \\
\hline EstC55-105 & 2714 & 223 & 23.9 & 7 & $\mathrm{C} 4, \mathrm{C} 6, \mathrm{C} 8, \mathrm{C} 10$ & I \\
\hline EstC55-110 & 8074 & 434 & 48.2 & 6.85 & $\mathrm{C} 4, \mathrm{C} 6$ & VIII \\
\hline EstC55-111 & 2870 & 392 & 41.8 & 5.69 & $\mathrm{C} 4$ & II \\
\hline EstC55-113 & 5055 & 409 & 44.4 & 7.66 & $\mathrm{C} 4$ & VIII \\
\hline EstC55-118 & 2603 & 497 & 53.9 & 5.39 & $\mathrm{C} 4, \mathrm{C} 6$ & VII \\
\hline EstC55-131 & 5270 & 251 & 27.2 & 6.01 & $\mathrm{C} 4, \mathrm{C} 6$ & Patatin \\
\hline EstC55-145 & 3366 & 305 & 31.5 & 5.04 & $\mathrm{C} 4, \mathrm{C} 6$ & IV \\
\hline EstC55-147 & 4651 & 386 & 41.4 & 4.61 & $\mathrm{C} 4, \mathrm{C} 6$ & VIII \\
\hline EstC55-150 & 4567 & 393 & 41.7 & 5.23 & $\mathrm{C} 4, \mathrm{C} 6$ & II \\
\hline EstC55-151 & 5771 & 311 & 33.5 & 6.79 & $\mathrm{C} 4, \mathrm{C} 6, \mathrm{C} 8$ & I \\
\hline EstC55-154 & 5680 & 394 & 41.5 & 4.83 & $\mathrm{C} 4$ & XVII \\
\hline EstC55-156 & 4142 & 517 & 55.8 & 4.6 & $\mathrm{C} 4$ & Tannase \\
\hline EstC55-159 & 3057 & 284 & 30.5 & 10.87 & $\mathrm{C} 4$ & $\mathrm{~V}$ \\
\hline EstC55-163 & 2207 & 303 & 31.6 & 4.82 & $\mathrm{C} 4, \mathrm{C} 6$ & patatin-like-protein \\
\hline EstC55-164 & 6522 & 430 & 46 & 5.56 & $\mathrm{C} 4$ & VIII \\
\hline EstC55-165 & 3926 & 277 & 31.1 & 5.35 & $\mathrm{C} 4, \mathrm{C} 6$ & FLS18 \\
\hline EstC55-167 & 4015 & 248 & 27.2 & 6.09 & $\mathrm{C} 4$ & new family (this study) \\
\hline EstC55-168 & 5684 & 513 & 57.5 & 9.29 & $\mathrm{C} 4$ & VIII \\
\hline EstC55-169 & 5280 & 533 & 57.1 & 4.8 & $\mathrm{C} 4$ & Tannase \\
\hline EstC55-186 & 2955 & 515 & 56.2 & 5.83 & $\mathrm{C} 4$ & VII \\
\hline EstC55-188 & 6426 & 237 & 25.8 & 8.09 & $\mathrm{C} 4$ & new family (this study) \\
\hline
\end{tabular}




\begin{tabular}{|c|c|c|c|c|c|c|}
\hline EstC55-197 & 6388 & 291 & 30.7 & 4.73 & $\mathrm{C} 4, \mathrm{C} 6$ & $\mathrm{~V}$ \\
\hline EstC55-213 & 4812 & 286 & 31.9 & 10.12 & $\mathrm{C} 4$ & I (new subfamily) \\
\hline EstC55-215 & 5300 & 263 & 29.7 & 10.13 & $\mathrm{C} 4$ & $\mathrm{~V}$ \\
\hline EstC55-227 & 5479 & 472 & 50.7 & 9.45 & $\mathrm{C} 4$ & new family (this study) \\
\hline EstC55-229 & 7811 & 311 & 33.9 & 6.92 & $\mathrm{C} 4, \mathrm{C} 6$ & IV \\
\hline EstC55-231 & 6018 & 366 & 39.7 & 5.71 & $\mathrm{C} 4, \mathrm{C} 6$ & $\mathrm{~V}$ \\
\hline EstC55-234 & 7802 & 557 & 59.9 & 5.86 & $\mathrm{C} 4, \mathrm{C} 6$ & Tannase \\
\hline EstC55-235 & 3084 & 326 & 34.4 & 6.44 & $\mathrm{C} 4, \mathrm{C} 6, \mathrm{C} 8, \mathrm{C} 10, \mathrm{C} 12$ & I \\
\hline EstC55-239 & 6478 & 381 & 42.1 & 8.75 & $\mathrm{C} 4$ & VIII \\
\hline EstC55-241 & 3087 & 567 & 63 & 6.79 & $\mathrm{C} 4$ & FLS18 \\
\hline EstC55-244 & 4612 & 255 & 27.8 & 5.86 & $\mathrm{C} 4, \mathrm{C} 6$ & $\mathrm{~V}$ \\
\hline EstC55-245 & 9142 & 462 & 50 & 5.92 & $\mathrm{C} 4, \mathrm{C} 6$ & VIII \\
\hline EstC55-247 & 3706 & 368 & 39.4 & 5.12 & $\mathrm{C} 4, \mathrm{C} 6$ & IV \\
\hline EstC55-251 & 8621 & 317 & 34.9 & 8.34 & $\mathrm{C} 4$ & patatin-like-protein \\
\hline EstC55-253 & 1398 & 297 & 31.7 & 5.61 & $\mathrm{C} 4$ & IV \\
\hline EstC55-256 & 6473 & 270 & 29.8 & 5.63 & $\mathrm{C} 4, \mathrm{C} 6$ & $\mathrm{~V}$ \\
\hline EstC55-258 & 6696 & 389 & 42.1 & 6.02 & $\mathrm{C} 4, \mathrm{C} 6$ & VIII \\
\hline EstC55-268 & 7750 & 303 & 33.4 & 6.64 & $\mathrm{C} 4$ & IV \\
\hline EstC76-21 & 5941 & 288 & 30.7 & 5.1 & $\mathrm{C} 4, \mathrm{C} 6$ & patatin-like-protein \\
\hline EstC76-28_1 & 6905 & 257 & 28 & 6.24 & $\mathrm{C} 4, \mathrm{C} 6$ & $\mathrm{~V}$ \\
\hline EstC76-28_2 & 6905 & 243 & 26 & 5.98 & $\mathrm{C} 4, \mathrm{C} 6$ & $\mathrm{~V}$ \\
\hline EstC76-36 & 6361 & 237 & 25.8 & 8.09 & $\mathrm{C} 4$ & new family (this study) \\
\hline EstC76-98 & 6234 & 425 & 46.4 & 7.31 & $\mathrm{C} 4, \mathrm{C} 6, \mathrm{C} 8$ & VIII \\
\hline EstC76-123 & 4787 & 450 & 49.2 & 8.96 & $\mathrm{C} 4$ & VIII \\
\hline EstC76-135 & 6286 & 310 & 33.8 & 5.59 & $\mathrm{C} 4$ & IV \\
\hline EstC76-136 & 2955 & 515 & 56.2 & 5.83 & $\mathrm{C} 4$ & VII \\
\hline EstC76-137 & 1878 & 277 & 31.1 & 5.47 & $\mathrm{C} 4$ & FLS18 \\
\hline EstC76-174 & 5888 & 388 & 42.8 & 6.29 & $\mathrm{C} 4$ & VIII \\
\hline EstC76-177 & 4039 & 265 & 29.4 & 9.53 & $\mathrm{C} 4$ & I (new subfamily) \\
\hline EstC76-179 & 3556 & 329 & 36 & 8.08 & $\mathrm{C} 4, \mathrm{C} 6$ & LipT \\
\hline EstC76-202 & 1745 & 267 & 28.9 & 7.85 & $\mathrm{C} 4, \mathrm{C} 6$ & $\mathrm{~V}$ \\
\hline EstC76-218 & 2449 & 329 & 36.1 & 8.08 & $\mathrm{C} 4$ & LipT \\
\hline EstC76-221 & 3944 & 257 & 28.9 & 5.49 & $\mathrm{C} 4, \mathrm{C} 6$ & new family (this study) \\
\hline EstC76-222 & 2289 & 260 & 28.6 & 6.32 & $\mathrm{C} 4$ & patatin-like-protein \\
\hline EstC76-248 & 1575 & 257 & 28.1 & 6.25 & $\mathrm{C} 4$ & $\mathrm{~V}$ \\
\hline EstC76-250 & 5270 & 251 & 27.2 & 6.01 & $\mathrm{C} 4, \mathrm{C} 6$ & patatin-like-protein \\
\hline EstC76-261 & 5603 & 345 & 37.2 & 9.25 & $\mathrm{C} 4, \mathrm{C} 6$ & patatin-like-protein \\
\hline EstC76-262 & 5482 & 415 & 45.6 & 5.16 & $\mathrm{C} 4, \mathrm{C} 6, \mathrm{C} 8$ & VIII \\
\hline EstC76-263 & 2752 & 376 & 40.8 & 5.23 & $\mathrm{C} 4, \mathrm{C} 6$ & $\mathrm{~V}$ \\
\hline EstC76-266 & 11975 & 257 & 28.1 & 6.54 & $\mathrm{C} 4, \mathrm{C} 6$ & $\mathrm{~V}$ \\
\hline
\end{tabular}


Supplementary Table S10 Annotation of recent reported novel LEs (including these from literatures and this study) against ESTHER database

\begin{tabular}{|c|c|c|c|c|c|c|c|c|}
\hline $\begin{array}{l}\text { Protein } \\
\text { name }\end{array}$ & $\begin{array}{l}\text { Query } \\
\text { length }\end{array}$ & $\begin{array}{l}\text { Subject } \\
\text { length }\end{array}$ & Description & Acce. $\mathrm{Nr}$ & ESTHER Family & E-value & $\begin{array}{l}\text { Identity } \\
\%\end{array}$ & $\begin{array}{l}\text { Query } \\
\text { coverage } \\
\%\end{array}$ \\
\hline Est22 & 463 & 463 & $\begin{array}{l}\text { Alteromonas sp.; Homoserine O- } \\
\text { acetyltransferase }\end{array}$ & $\begin{array}{l}\text { 9alte- } \\
\text { a0a010qrx3 }\end{array}$ & Homoserine_transacetylase & 0 & 100 & 100 \\
\hline EstL28 & 290 & 322 & Dehalococcoides sp.; putative hydrolase & dehsc-q3zyd4 & 6_AlphaBeta_hydrolase & $2.00 \mathrm{E}-29$ & 28 & 100 \\
\hline Rv0045c & 298 & 298 & $\begin{array}{l}\text { Mycobacterium bovis; hypothetical protein } \\
\text { rv0045c }\end{array}$ & $\begin{array}{l}\text { myctu- } \\
\text { RV0045C }\end{array}$ & 6_AlphaBeta_hydrolase & e-171 & 100 & 100 \\
\hline EstGX1 & 201 & 230 & $\begin{array}{l}\text { Streptomyces bingchenggensis; } \\
\text { uncharacterized protein }\end{array}$ & strbb-d7bzy 9 & DLH-S & 0.073 & 27 & 40 \\
\hline EstLiu & 293 & 285 & Robiginitalea biformata; probable lipase & 9flao-a4cmr0 & $\begin{array}{l}\text { Hormone- } \\
\text { sensitive lipase like }\end{array}$ & $7.00 \mathrm{E}-48$ & 42 & 91 \\
\hline EstY & 423 & 423 & uncultured bacterium; Esterase & 9 bact-b0 $\ln 78$ & Fungal-Bact_LIP & 0 & 100 & 100 \\
\hline EstGS & 397 & 397 & $\begin{array}{l}\text { uncultured organism; Putative } \\
\text { uncharacterized protein }\end{array}$ & 9zzzz-g3crc6 & Chlorophyllase & 0 & 100 & 100 \\
\hline EM3L4 & 330 & 304 & $\begin{array}{l}\text { Mycobacterium leprae; putative secreted } \\
\text { hydrolase }\end{array}$ & mycle-LPQC & Esterase_phb & $5.00 \mathrm{E}-36$ & 32 & 97 \\
\hline FLS18 & 259 & 224 & $\begin{array}{l}\text { uncultured organism EH82; } \\
\text { Uncharacterized protein }\end{array}$ & 9zzzz-EH82 & 5_AlphaBeta_hydrolase & $5.00 \mathrm{E}-47$ & 44 & 84 \\
\hline Est903 & 300 & 300 & $\begin{array}{l}\text { uncultured bacterium; Est903 Putative } \\
\text { esterase }\end{array}$ & $\begin{array}{l}\text { 9bact- } \\
\text { a0a345g0q2 }\end{array}$ & $\begin{array}{l}\text { Hormone- } \\
\text { sensitive_lipase_like }\end{array}$ & e-176 & 100 & 100 \\
\hline EstJ & 317 & 310 & Planctomycetales bacterium; Esterase & $\begin{array}{l}\text { 9bact- } \\
\text { a0a1u7gdl0 }\end{array}$ & $\begin{array}{l}\text { Hormone- } \\
\text { sensitive_lipase_like }\end{array}$ & $2.00 \mathrm{E}-79$ & 52 & 85 \\
\hline PE10 & 279 & 279 & $\begin{array}{l}\text { Pelagibacterium; halotolerans } \\
\text { Phospholipase/carboxylesterase family } \\
\text { protein PE10 }\end{array}$ & pelhb-g4rec9 & $\begin{array}{l}\text { Hormone- } \\
\text { sensitive_lipase_like }\end{array}$ & 0 & 100 & 100 \\
\hline Est12 & 329 & 316 & $\begin{array}{l}\text { Psychrobacter cryohalolentis; alpha/beta } \\
\text { hydrolase }\end{array}$ & psyck-q1q8n0 & $\begin{array}{l}\text { Hormone- } \\
\text { sensitive lipase like }\end{array}$ & e-138 & 81 & 91 \\
\hline EstDZ2 & 271 & 271 & $\begin{array}{l}\text { uncultured Acetothermia bacterium; } \\
\text { estDZ2a esterase }\end{array}$ & $\begin{array}{l}\text { 9bact- } \\
\text { a0a112dxz2 }\end{array}$ & $\begin{array}{l}\text { Hormone- } \\
\text { sensitive lipase like }\end{array}$ & e-157 & 100 & 100 \\
\hline Est9x & 294 & 294 & uncultured bacterium; Esterase & 9 bact-j9vdv8 & Est9X & 0 & 100 & 100 \\
\hline Lip10 & 348 & 361 & $\begin{array}{l}\text { Aspergillus oryzae; arylacetamide } \\
\text { deacetylase }\end{array}$ & aspor-q2tw16 & $\begin{array}{l}\text { Hormone- } \\
\text { sensitive_lipase_like }\end{array}$ & e-155 & 71 & 97 \\
\hline EstGH & 448 & 437 & $\begin{array}{l}\text { Xanthomonas campestris; hypothetical } \\
\text { protein } \mathrm{xcc} 3623\end{array}$ & $\begin{array}{l}\text { xanca- } \\
\text { XCC3623 }\end{array}$ & Lipase_3 & $4.00 \mathrm{E}-06$ & 29 & 36 \\
\hline EML1 & 304 & 300 & uncultured bacterium; probable lipase & 9bact-q1paf1 & Lipase_3 & $4.00 \mathrm{E}-13$ & 34 & 53 \\
\hline FnL & 302 & 364 & Thermotoga maritima; esterase & thema-TM0053 & AlphaBeteta_hydrolase & $5.00 \mathrm{E}-31$ & 31 & 90 \\
\hline EstP2K & 224 & 218 & Uncultured prokaryote; esterase/lipase & $\begin{array}{l}\text { 9zzZZ- } \\
\text { KY203033 }\end{array}$ & 6_AlphaBeta_hydrolase & $2.00 \mathrm{E}-42$ & 45 & 91 \\
\hline
\end{tabular}




\begin{tabular}{|c|c|c|c|c|c|c|c|c|}
\hline LipA & 277 & 367 & Magnaporthe grisea; Triacylglycerol lipase & maggr-q0pnd5 & PGAP1 & $2.00 \mathrm{E}-24$ & 36 & 88 \\
\hline LipSM54 & 526 & 526 & $\begin{array}{l}\text { Xanthomonas gardneri; hypothetical } \\
\text { protein }\end{array}$ & $\begin{array}{l}\text { xanax- } \\
\text { XAC0753 }\end{array}$ & Cocaine_esterase & e-161 & 55 & 95 \\
\hline MtEst45 & 516 & 540 & Hahella chejuensis; predicted acylesterase & hahch-q2seh8 & Cocaine_esterase & e-114 & 42 & 95 \\
\hline LipT & 329 & 329 & uncultured bacterium; LipT & 9bact-k7qe48 & Pectinacetylesterase-Notum & 0 & 100 & 100 \\
\hline EstSt7 & 322 & 322 & $\begin{array}{l}\text { Sulfolobus tokodaii; hypothetical protein } \\
\text { st } 2026\end{array}$ & sulto-ST2026 & A85-Feruloyl-Esterase & 0 & 100 & 100 \\
\hline Rlip1 & 361 & 361 & uncultured bacterium; lipase & 9bact-c0k075 & A85-Feruloyl-Esterase & 0 & 100 & 100 \\
\hline EstA & 277 & 277 & uncultured bacterium; Esterase A & 9bact-b0fwn3 & A85-EsteraseD-FGH & e-164 & 100 & 100 \\
\hline FLS12 & 270 & 270 & uncultured bacterium; Esterase & 9bact-b8y562 & A85-EsteraseD-FGH & e-159 & 100 & 100 \\
\hline lp_3505 & 263 & 263 & Lactobacillus plantarum; acetylesterase & lacpl-EST2 & A85-EsteraseD-FGH & e-157 & 100 & 100 \\
\hline $\begin{array}{l}\text { EstC55- } \\
15\end{array}$ & 253 & 278 & Streptomyces coelicolor; putative hydrolase & strco-SCO4160 & AlphaBeta_hydrolase & $2.00 \mathrm{E}-69$ & 56 & 93 \\
\hline $\begin{array}{l}\text { EstC55- } \\
97\end{array}$ & 240 & 277 & $\begin{array}{l}\text { Sphingomonas wittichii; Alpha/beta } \\
\text { hydrolase fold }\end{array}$ & sphww-a5v750 & $\begin{array}{l}\text { Carbon- } \\
\text { carbon_bond_hydrolase }\end{array}$ & $1.00 \mathrm{E}-07$ & 35 & 82 \\
\hline $\begin{array}{l}\text { EstC55- } \\
102\end{array}$ & 707 & 430 & $\begin{array}{l}\text { Shewanella oneidensis; conserved } \\
\text { hypothetical protein }\end{array}$ & sheon-SO2934 & Lipase_bact_N_lipase & $7.00 \mathrm{E}-35$ & 29 & 80 \\
\hline $\begin{array}{l}\text { EstC55- } \\
167\end{array}$ & 248 & 262 & $\begin{array}{l}\text { Verrucomicrobiae bacterium; DG1235 } \\
\text { Putative uncharacterized protein }\end{array}$ & 9bact-b5jkk1 & HNLyase_Bact & $2.00 \mathrm{E}-66$ & 52 & 93 \\
\hline $\begin{array}{l}\text { EstC55- } \\
227\end{array}$ & 472 & 472 & $\begin{array}{l}\text { Deinococcus radiodurans; hypothetical } \\
\text { protein }\end{array}$ & deira-DR0553 & 6_AlphaBeta_hydrolase & $2.00 \mathrm{E}-57$ & 34 & 85 \\
\hline $\begin{array}{l}\text { EstC76- } \\
36\end{array}$ & 237 & 238 & $\begin{array}{l}\text { Thermus thermophilus; } \\
\text { AlphaBeta_hydrolase protein }\end{array}$ & theth-TT1662 & AlphaBeta_hydrolase & e-131 & 98 & 99 \\
\hline $\begin{array}{l}\text { EstC76- } \\
221\end{array}$ & 257 & 265 & $\begin{array}{l}\text { Clostridium acetobutylicum; alpha/beta } \\
\text { superfamily hydrolase }\end{array}$ & cloac-CAC3665 & AlphaBeta_hydrolase & $8.00 \mathrm{E}-74$ & 52 & 100 \\
\hline
\end{tabular}


Supplementary Table S11 Protein families used for building LE-specific profile hidden markov modle (HMM) database

\begin{tabular}{|c|c|c|c|c|c|}
\hline $\begin{array}{l}\text { Profile HMM name (ESTHER } \\
\text { family) }\end{array}$ & Database & $\begin{array}{l}\text { Lipolytic } \\
\text { family }\end{array}$ & Interpro annotation & Pfam annotation & Reference \\
\hline Bacterial_lip_FamI.1 & ESTHER & I.1 & IPR000734 (Lipase) & PF01764 (Lipase 3) & Nardini et al., 2000 \\
\hline Bacterial_lip_FamI.2 & ESTHER & I. 2 & IPR000734 (Lipase) & PF01764 (Lipase_3) & Arpigny \& Jaeger, (1999) \\
\hline Bacterial_lip_FamI.3 & ESTHER & I. 3 & IPR000734 (Lipase) & PF01764 (Lipase_3) & Angkawidjaja et al., 2010 \\
\hline Bacterial_lip_FamI.5 & ESTHER & I.5 & IPR000734 (Lipase) & PF01764 (Lipase_3) & Tyndall et al., 2002 \\
\hline Bacterial_lip_FamI.6 & ESTHER & I. 6 & IPR000734 (Lipase) & PF01764 (Lipase_3) & Tiesinga et al., 2007 \\
\hline Lipase_2 & ESTHER & $\begin{array}{l}\mathrm{I} .4 \\
\mathrm{I} .7\end{array}$ & IPR002918 (Lipase EstA/Esterase EstB) & PF01674 (Lipase_2) & Arpigny \& Jaeger, (1999) \\
\hline Bacterial lip_FamI.8 & ESTHER & I.8 & - & PF12146 (Hydrolase 4) & de Pascale et al., 2008 \\
\hline Lipase_GDSL & PFAM & II & IPR001087 (GDSL lipase/esterase) & PF00657 (Lipase_GDSL) & \\
\hline Lipase_GDSL_2 & PFAM & II & IPR013830 (SGNH hydrolase-type esterase domain) & PF13472 (Lipase_GDSL_2) & \\
\hline Lipase_GDSL_3 & PFAM & II & IPR013830 (SGNH hydrolase-type esterase domain) & PF14606 (Lipase_GDSL_3) & Molgaard A et al., 2000 \\
\hline Lipase_GDSL_lke & PFAM & II & $\begin{array}{l}\text { IPR032588 (Putative GDSL-like } \\
\text { Lipase/Acylhydrolase) }\end{array}$ & $\begin{array}{l}\text { PF16255 } \\
\text { (Lipase_GDSL_lke) }\end{array}$ & \\
\hline $\begin{array}{l}\text { Polyesterase-lipase } \\
\text {-cutinase }\end{array}$ & ESTHER & III & IPR041127 (Chlorophyllase enzyme) & $\begin{array}{l}\text { PF12695 (Abhydrolase_5), } \\
\text { PF12740 (Chlorophyllase2) }\end{array}$ & Sulaiman et al., (2012) \\
\hline $\begin{array}{l}\text { Hormone-sensitive } \\
\text { _lipase_like }\end{array}$ & ESTHER & IV & $\begin{array}{l}\text { IPR013094 (Alphabeta hydrolase fold-3), IPR002168 } \\
\text { (Lipolytic enzyme), IPR033140 } \\
\text { (Lipase_GDXG_put_SER_AS), IPR002168 } \\
\text { (Lipase_GDXG_HIS_AS) }\end{array}$ & PF07859 (Abhydrolase_3) & $\operatorname{Kim}(2017)$ \\
\hline GTSAGmotif & ESTHER & IV & IPR013094 (Alphabeta hydrolase fold-3) & PF07859 (Abhydrolase_3) & Li et al., 2014 \\
\hline ABHD6-Lip & ESTHER & V.1 & - & - & Pribasnig et al., (2015) \\
\hline $\begin{array}{l}\text { Carboxymethylbutenolide } \\
\text { lactonase }\end{array}$ & ESTHER & V.2 & $\begin{array}{l}\text { IPR000073 (Alpha/beta hydrolase fold-1), IPR026968 } \\
\text { (3-oxoadipate enol-lactonase) }\end{array}$ & PF12697 (Abhydrolase_6) & Arpigny \& Jaeger, (1999) \\
\hline ŪCP031982 & ESTHER & V.3 & $\begin{array}{l}\text { IPR016986 (Uncharacterised conserved protein } \\
\text { UCP031982, alpha/beta hydrolase, XabL), }\end{array}$ & & \\
\hline $\begin{array}{l}\text { LYsophospholipase } \\
\text { carboxylesterase }\end{array}$ & ESTHER & VI & $\begin{array}{l}\text { IPR005065 (Platelet-activating factor acetylhydrolase- } \\
\text { like) } \\
\text { IPR003140 } \\
\text { (Phospholipase/carboxylesterase/thioesterase } \\
\text { PLP Cesterase PLipase/COase/thioEstase) }\end{array}$ & PF02230 (Abhydrolase_2) & Park et al., (2007) \\
\hline Carb_B_Bacteria & ESTHER & VII & IPR002018 (Carboxylesterase, type B) & PF00135 (COesterase) & Ewis et al., (2004) \\
\hline Beta-lactamase & PFAM & VIII & IPR001466 (Beta-lactamase-related) & PF00144 (Beta-lactamase) & Hausmann \& Jaeger, 2010 \\
\hline PHAZ7_phb_depolymerase & ESTHER & IX & - & - & Handrick et al., (2001) \\
\hline Bacterial_EstLip_FamX & ESTHER & $\begin{array}{l}X .1 \\
X .2\end{array}$ & - & PF12695 (Abhydrolase_5) & $\begin{array}{l}\text { Levisson et al., (2007) } \\
\text { Bassegoda et al., (2012) }\end{array}$ \\
\hline Fungal-Bact_LIP & ESTHER & $\begin{array}{l}\text { XVII } \\
\text { XIX }\end{array}$ & IPR005152 (Lipase_secreted) & PF03583 (LIP) & $\begin{array}{l}\text { Castilla et al., } 2017 \\
\text { Parapouli et al., } 2018\end{array}$ \\
\hline
\end{tabular}


Lipase_3

Bact LipEH166 FamXII

CarbLipBact

CarbLipBact_2

PC-sterol acyltransferase

Duf_3089

Bacterial_Est97

Lipae

Patatin

DUF3734

Tannase

Est9X

A85-EsteraseD-FGH

Chlorophyllase

Pectinacetylesterase-Notum

A85-Feruloyl-Esterase

Homoserine_transacetylase
ESTHER

XI

ESTHER

ESTHER XIII-1

ESTHER XIII-2

ESTHER XIV

ESTHER XV

ESTHER

\section{XVI}

patatin-

like-

protein

ESTHER Tannase

ESTHER Est9X

lp_3505

ESTHER FLS12

EstA

ESTHER EstGS

ESTHER LipT

ESTHER Rlip

EstSt7

ESTHER Est22
IPR000734 (Lipase), IPR002921 (Fungal lipase-like domain), IPR005592 (Mono-/di-acylglycerol lipase, $\mathrm{N}$-terminal Mono/diacylglycerol_lipase_N)

IPR012354 (Esterase/lipase)

IPR012354 (Esterase/lipase)

IPR003386 (Lecithin:

cholesterol/phospholipid:diacylglycerol

acyltransferase)

IPR021440 (Protein of unknown function DUF3089)

IPR013818 (Lipase/vitellogenin)

IPR002641 (Patatin-like phospholipase domain)

IPR021095 (DUF3734)

IPR011118 (Tannase and feruloyl esterase)

IPR000801 (Esterase put), IPR014186 (S-

formylglutathione_hydrol)

IPR017395 (Chlorophyllase)

IPR004963 (Pectinacetylesterase/NOTUM

PAE/NOTUM)

IPR000801 (Esterase_put)

IPR008220 (Homoserine/serine acetyltransferase

MetX-like HAT MetX-like), IPR000073 (Alpha/beta hydrolase fold-1)
PF03893 (Lipase3 N), PF01764 (Lipase_ 3 )

Lee et al., (2006)

im et al., (2009)

Rao et al., (2013)

Chow et al., (2012)

Samoylova et al., 2018

PF02450 (LACT-Lecithin:

cholesterol acyltransferase)

PF11288 (DUF3089)

Rao et al., (2011)

Rodriguez et al., (2015);

Bayer et al., (2010)

PF00151(Lipase)

PF01734 (Patatin)

PF12536 (Patatin

phospholipase)

PF07519 (Tannase)

PF00756 (Esterase)

Fu et al., (2013)

Tirawongsaroj et al., 2008

Banerjee et al., 2012

Fang et al., 2014

Esteban-Torres et al., 2014

Hu et al., 2010

PF07224 (Chlorophyllase)

PF03283 (PAE)

PF00756 (Esterase)

Chow et al., 2012

Liu et al., 2009

Wei et al., 2013

Li et al., 2017

PF00561 (Abhydrolase 1) 
Supplementary Table S12 Validation of the liplolytic family assignment strategy with family/function-known LEs (with $\alpha / \beta$-hydrolase fold )

\begin{tabular}{|c|c|c|c|c|}
\hline Protein name & Blastp annotation ${ }^{\text {a }}$ & pHMM-omega annotation (lipolytic family) & $\begin{array}{l}\text { Lipolytic family (This } \\
\text { study) }{ }^{\mathrm{b}}\end{array}$ & $\begin{array}{l}\text { Lipolytic family } \\
\text { (literatures or database) }^{\mathrm{c}}\end{array}$ \\
\hline \multicolumn{5}{|l|}{ Dataset 2} \\
\hline 499EST & Hormone-sensitive_lipase_like & Hormone_sensitive_lipase_like (IV) & IV & IV \\
\hline 7N9 & Hormone-sensitive_lipase_like & Hormone_sensitive_lipase_like (IV) & IV & IV \\
\hline ABO_1197 & ABHD6-Lip & ABHD6-Lip (V) & $\mathrm{V}$ & $\mathrm{V}$ \\
\hline $\mathrm{ABO}_{-}^{-} 1251$ & Carb_B_Bacteria & Carb_B_Bacteria (VII) & VII & VII \\
\hline ABO0195 & Hormone-sensitive_lipase_like & Hormone_sensitive_lipase_like (IV) & IV & IV \\
\hline CaesCCR11 & CarbLipBact_2 & CarbLipBact_2 (XIII-2) & XIII & XIII \\
\hline E69 & GTSAGmotif & GTSAGmotif (IV) & IV & IV \\
\hline EaEST & Haloperoxidase & Carboxymethylbutenolide lactonase (V) & nonlipolytic protein & $\mathrm{V}$ \\
\hline EM3L2 & Duf 3089 & Duf $3089(\mathrm{XV})$ & $\mathrm{XV}$ & XV \\
\hline EM3L4 & Esterase phb & Lysophospholipase_carboxylesterase (VI) & unassigned & EM3L4 (novel family) \\
\hline EML1 & Lipase_ 3 & Lipase_3 (XI) & $\mathrm{XI}$ & $\mathrm{XI}$ \\
\hline Est_p1 & Aclacinomycin-methylesterase_RdmC & Carboxymethylbutenolide_lactonase (V) & nonlipolytic protein & $\mathrm{V}$ \\
\hline Est $\overrightarrow{1}$ & 6_AlphaBeta_hydrolase & Carboxymethylbutenolide_lactonase (V) & unassigned & $\mathrm{V}$ \\
\hline Est10 & LY'sophospholipase_carboxylesterase & Lysophospholipase_carboxylesterase (VI) & VI & VI \\
\hline Est12 & Hormone-sensitive_lipase_like & Hormone_sensitive_lipase_like (IV) & IV & IV \\
\hline Est22 & Homoserine transacetylase & Homoserine transacetylase (Est22) & Est22 & Est22 \\
\hline Est25 & GTSAGmotif & GTSAGmotif (IV) & IV & IV \\
\hline Est30 & CarbLipBact 1 & CarbLipBact 1 (XIII-1/XVIII) & XIII & XIII \\
\hline Est4 & 6 AlphaBeta hydrolase & ABHD6-Lip (V) & unassigned & $\mathrm{V}$ \\
\hline Est40 & Hormone-sensitive_lipase_like & Hormone_sensitive_lipase_like (IV) & IV & IV \\
\hline Est56 & Hormone-sensitive_lipase_like & Hormone_sensitive_lipase_like (IV) & IV & IV \\
\hline Est700 & Lipase_2 & Bacterial_lipase (I) & I & $\mathrm{I}$ \\
\hline Est903 & Hormone-sensitive lipase like & Hormone sensitive lipase like (IV) & IV & IV \\
\hline Est97 & Bacterial_Est97 & Bacterial Est97 (XVII) & XVI & XVI \\
\hline Est9x & Est9X & Est9X (Est9X) & Est9X & Est9X \\
\hline EstA & A85-EsteraseD-FGH & A85-EsteraseD-FGH (EstA/FLS12/lp_3505) & EstA & EstA \\
\hline EstA3 & PC-sterol_acyltransferase & Pcsterol_acyltransferase (XIV) & XIV & XIV \\
\hline EstAM & Hormone-sensitive lipase like & Hormone sensitive lipase like (IV) & IV & IV \\
\hline EstATII & Hormone-sensitive_lipase_like & Hormone_sensitive_lipase_like (IV) & IV & IV \\
\hline EstCS2 & Carb_B_Bacteria & Carb_B_Bacteria (VII) & VII & VII \\
\hline EstD & Bacterial_EstLip_FamX & Bacterial EstLip FamX (X) & $\mathrm{X}$ & $\mathrm{X}$ \\
\hline EstD2 & Duf 3089 & Duf $3089(\mathrm{XV})$ & XV & XV \\
\hline EstDZ2 & Hormone-sensitive_lipase_like & Hormone_sensitive_lipase_like (IV) & EstDZ2 & EstDZ2 \\
\hline EstEP16 & CarbLipBact_2 & CarbLipBact_2 (XIII-2) & XIII & XIII \\
\hline EstF27 & Hormone-sensitive_lipase_like & Hormone_sensitive_lipase_like (IV) & IV & IV \\
\hline EstGK1 & Duf_3089 & Duf_3089(XV) & XV & $\mathrm{XV}$ \\
\hline
\end{tabular}




\begin{tabular}{|c|c|}
\hline EstGS & Chlorophyllase \\
\hline EstGtA2 & CarbLipBact 2 \\
\hline EstJ & Hormone-sensitive_lipase_like \\
\hline EstKT4 & GTSAGmotif \\
\hline EstKT7 & GTSAGmotif \\
\hline EstKT9 & GTSAGmotif \\
\hline EstL28 & 6_AlphaBeta_hydrolase \\
\hline EstLiu & Hormone-sensitive_lipase_like \\
\hline EstMY & Hormone-sensitive_lipase_like \\
\hline EstMY09-2 & Hormone-sensitive_lipase_like \\
\hline EstN7 & Hormone-sensitive_lipase_like \\
\hline EstOF4 & CarbLipBact_1 \\
\hline Est-OKK & ABHD11-Acetyl_transferase \\
\hline EstP2K & 6_AlphaBeta_hydrolase \\
\hline EstS & Hormone-sensitive lipase like \\
\hline EstSP & Hormone-sensitive_lipase_like \\
\hline EstSt7 & A85-Feruloyl-Esterase \\
\hline EstUT1 & CarbLipBact_1 \\
\hline EstWSD & Duf_3089 \\
\hline Est-XG2 & Carb_B_Bacteria \\
\hline EstY & Fungal-Bact_LIP \\
\hline EstZ3 & Duf_3089 \\
\hline FLS12 & A85-EsteraseD-FGH \\
\hline FLS18 & 5_AlphaBeta_hydrolase \\
\hline FnL & ĀlphaBeta_hydrolase \\
\hline GDEst-95 & Carb_B_Bacteria \\
\hline H8 & Aclacinomycin-methylesterase_RdmC \\
\hline H9Est & Hormone-sensitive_lipase_like \\
\hline LAE3 & Hormone-sensitive_lipase_like \\
\hline LAE4 & Hormone-sensitive_lipase_like \\
\hline LAE6 & Hormone-sensitive_lipase_like \\
\hline Lip10 & Hormone-sensitive_lipase_like \\
\hline Lip-1452 & Hormone-sensitive_lipase_like \\
\hline Lip3 & Lipase_3 \\
\hline Lip-948 & ABHD6-Lip \\
\hline LipA & PGAP1 \\
\hline Lipab15 & Hormone-sensitive_lipase_like \\
\hline Lipab18 & Hormone-sensitive_lipase_like \\
\hline Lipab4 & Hormone-sensitive_lipase_like \\
\hline Lipab8 & Hormone-sensitive_lipase_like \\
\hline Lipag18 & Hormone-sensitive_lipase_like \\
\hline LipC & Hormone-sensitive lipase like \\
\hline LipC12 & Bacterial_lipase \\
\hline LipEH166 & Bact_LipËH166_FamXII \\
\hline LipG & Lipase_3 $3 \quad-$ \\
\hline
\end{tabular}

Chlorophyllase (EstGS)

CarbLipBact_2 (XIII-2)

Hormone_sensitive_lipase_like (IV)

GTSAGmotif (IV)

GTSAGmotif (IV)

GTSAGmotif (IV)

Carboxymethylbutenolide lactonase (V)

Hormone sensitive lipase like (IV)

Hormone_sensitive_lipase_like (IV)

Hormone_sensitive_lipase_like (IV)

Hormone sensitive lipase like (IV)

CarbLipBact 1 (XIII-1/XVIII)

ABHD6-Lip (V)

Bacterial lipase (I)

Hormone sensitive lipase like (IV)

Hormone_sensitive_lipase_like (IV)

A85-Feruloyl-Esterase (EstSt7/Rlip1)

CarbLipBact 1 (XIII-1/XVIII)

Duf_3089 (XV)

Carb_B_Bacteria (VII)

Fungal_Bact LIP (X-2/XVII/XIX)

Duf 3089 (XV)

A85-EsteraseD-FGH (EstA/FLS12/lp_3505)

A85-Feruloyl-Esterase (EstSt7/Rlip1)

Pcsterol acyltransferase (XIV)

Carb B Bacteria (VII)

Carboxymethylbutenolide_lactonase (V)

Hormone_sensitive_lipase_like (IV)

Hormone sensitive lipase like (IV)

Hormone sensitive lipase like (IV)

Hormone_sensitive_lipase_like (IV)

Hormone sensitive lipase like (IV)

Hormone sensitive lipase like (IV)

Lipase 3 (XI)

ABHD6-Lip (V)

Bacterial_lipase (I)

Hormone sensitive lipase like (IV)

Hormone_sensitive_lipase_like (IV)

Hormone sensitive lipase like (IV)

Hormone sensitive lipase like (IV)

Hormone_sensitive_lipase_like (IV)

Hormone_sensitive_lipase_like (IV)

Bacterial lipase (I)

Bact LipEH166 FamXII (XII)

Lipase_3 (XI)

\begin{tabular}{|c|c|}
\hline EstGS & EstGS \\
\hline XIII & XIII \\
\hline IV & IV \\
\hline IV & IV \\
\hline IV & IV \\
\hline IV & IV \\
\hline unassigned & EstL28 (novel family) \\
\hline IV & IV \\
\hline IV & IV \\
\hline IV & IV \\
\hline IV & IV \\
\hline XIII & XIII \\
\hline nonlipolytic protein & $\mathrm{V}$ \\
\hline unassigned & EstP2K (novel family) \\
\hline IV & IV \\
\hline IV & IV \\
\hline EstSt7 & EstSt7 \\
\hline XVIII & XVIII \\
\hline$X V$ & XV \\
\hline VII & VII \\
\hline $\mathrm{X}$ & $\mathrm{X}$ \\
\hline XV & XV \\
\hline FLS12 & FLS12 \\
\hline unassigned & FLS18 (novel family) \\
\hline unassigned & FnL (novel family) \\
\hline VII & VII \\
\hline nonlipolytic protein & $\mathrm{V}$ \\
\hline IV & IV \\
\hline IV & IV \\
\hline IV & IV \\
\hline IV & IV \\
\hline IV & IV \\
\hline IV & IV \\
\hline XI & XI \\
\hline V & $\mathrm{V}$ \\
\hline unassigned & I \\
\hline IV & IV \\
\hline IV & IV \\
\hline IV & IV \\
\hline IV & IV \\
\hline IV & IV \\
\hline IV & IV \\
\hline I & I \\
\hline XII & XII \\
\hline XI & XI \\
\hline
\end{tabular}




LipJ2
Lipo1
Lipo11
Lipo12A
Lipo2
Lipo3
Lipo4A
Lipo5
Lipo6
LipR
LipR_2
LipR1
LipR3
LipS
LipSm
LipSM54
LipT
LipYY31
lp_3505
M37
MGS0018
MGS0153
MGS-B1
MGS-K1
MGS-M1
MGS-M2
MGS-MG1
MGS-MT1
MGS-RG1
MGS-RG2
MGS-RG3
MtEst45
PE10
PhaZ7
PMGL2
REst1
Rlip1
Rv0045c
SAestA
SBLip2
SBLip5.1
ThaEst2349
Vlip509
EstGH
EstGX1

Fungal-Bact LIP

Hormone-sensitive_lipase_like

Hormone-sensitive_lipase_like

Hormone-sensitive lipase like

6-AlphaBeta hydrolase

ABHD11-Acetyl_transferase

LYsophospholipase_carboxylesterase

Hormone-sensitive lipase like

LYsophospholipase_carboxylesterase

Fungal-Bact_LIP

Fungal-Bact_LIP

Bacterial lipase

Bacterial_lipase

CarbLipBact_2

Fungal-Bact_LIP

Cocaine esterase

Pectinacetylesterase-Notum

Bacterial_lipase

A85-EsteraseD-FGH

Lipase_3

Hormone-sensitive_lipase_like

ABHD6-Lip

Hormone-sensitive lipase_like

Carb_B_Bacteria

Hormone-sensitive_lipase_like

6 AlphaBeta hydrolase

AlphaBeta hydrolase

GTSAGmotif

LYsophospholipase carboxylesterase

LYsophospholipase_carboxylesterase

Hormone-sensitive_lipase_like

Cocaine_esterase

Hormone-sensitive lipase like

PHAZ7_phb_depolymerase

GTSAGmotif

Hormone-sensitive lipase like

A85-Feruloyl-Esterase

6_AlphaBeta_hydrolase

Hormone-sensitive_lipase like

Hormone-sensitive lipase like

Hormone-sensitive_lipase_like

Hormone-sensitive_lipase_like

UCP031982

$\mathrm{ND}^{\mathrm{d}}$

$\mathrm{ND}^{\mathrm{d}}$
Fungal Bact LIP (X-2/XVII/XIX)

Hormone_sensitive_lipase_like (IV)

Hormone_sensitive_lipase_like (IV)

Hormone sensitive lipase like (IV)

Lipase 2 (I)

ABHD6-Lip (V)

Lysophospholipase carboxylesterase (VI)

Hormone sensitive lipase like (IV)

Lysophospholipase_carboxylesterase (VI)

Fungal_Bact_LIP (X-2/XVII/XIX)

Fungal Bact LIP (X-2/XVII/XIX)

Bacterial lipase (I)

Bacterial_lipase (I)

CarbLipBact_2 (XIII-2)

Fungal Bact LIP (X-2/XVII/XIX)

Hormone sensitive lipase like (IV)

Pectinacetylesterase-Notum (LipT)

Bacterial lipase (I)

A85-EsteraseD-FGH (EstA/FLS12/lp 3505)

Lipase_3 (XI)

Hormone_sensitive_lipase_like (IV)

ABHD6-Lip (V)

Hormone_sensitive_lipase_like (IV)

Carb_B_Bacteria (V̄II)

Hormone sensitive lipase like (IV)

Carboxymethylbutenolide lactonase (V)

Bacterial_EstLip_FamX $(\bar{X})$

GTSAGmotif (IV)

Lysophospholipase carboxylesterase (VI)

Lysophospholipase carboxylesterase (VI)

Hormone_sensitive_lipase_like (IV)

Chlorophyllase (EstGS)

Hormone sensitive lipase like (IV)

PHAZ7_phb_depolymerase (IX)

GTSAGmotif (IV)

Hormone sensitive lipase like (IV)

A85-Feruloyl-Esterase (EstSt7/Rlip1)

ABHD6-Lip (V)

Hormone_sensitive_lipase like (IV)

Hormone sensitive lipase like (IV)

Hormone_sensitive_lipase_like (IV)

Hormone_sensitive_lipase_like (IV)

UCP031982 (V)

$\mathrm{ND}^{\mathrm{d}}$

$\mathrm{ND}^{\mathrm{d}}$

\begin{tabular}{|c|c|}
\hline XVII & XVII \\
\hline IV & IV \\
\hline IV & IV \\
\hline IV & IV \\
\hline unassigned & I \\
\hline unassigned & $\mathrm{V}$ \\
\hline VI & VI \\
\hline IV & IV \\
\hline VI & VI \\
\hline $\mathrm{X}$ & $\mathrm{X}$ \\
\hline $\mathrm{X}$ & $\mathrm{X}$ \\
\hline I & I \\
\hline I & I \\
\hline XIII & XIII \\
\hline XIX & XIX \\
\hline unassigned & LipSM54 (novel family) \\
\hline LipT & LipT \\
\hline I & I \\
\hline lp 3505 & lp 3505 \\
\hline $\mathrm{XI}$ & $\mathrm{XI}$ \\
\hline IV & IV \\
\hline $\mathrm{V}$ & $\mathrm{V}$ \\
\hline IV & IV \\
\hline VII & VII \\
\hline IV & IV \\
\hline unassigned & $\mathrm{V}$ \\
\hline unassigned & unclassified \\
\hline IV & IV \\
\hline VI & VI \\
\hline VI & VI \\
\hline IV & IV \\
\hline unassigned & MtEst45 (novel family) \\
\hline IV & IV \\
\hline IX & IX \\
\hline IV & IV \\
\hline IV & IV \\
\hline Rlip1 & Rlip1 \\
\hline unassigned & Rv0045c (novel family) \\
\hline IV & IV \\
\hline IV & IV \\
\hline IV & IV \\
\hline IV & IV \\
\hline $\mathrm{V}$ & $\mathrm{V}$ \\
\hline $\mathrm{ND}^{\mathrm{d}}$ & EstGH (novel family) \\
\hline $\mathrm{ND}^{\mathrm{d}}$ & EstGX1 (novel family) \\
\hline
\end{tabular}




\begin{tabular}{|c|c|c|c|c|}
\hline $\begin{array}{l}\text { Lipo } 10 \\
\text { Lipo7A } \\
\text { Lipo7B }\end{array}$ & $\begin{array}{l}\operatorname{ND}^{d} \\
\text { ND }^{d} \\
\text { ND }^{d}\end{array}$ & $\begin{array}{l}\text { ND }^{d} \\
\text { ND }^{d} \\
\text { ND }^{d}\end{array}$ & $\begin{array}{l}\text { ND }^{d} \\
\text { ND }^{d} \\
\text { ND }^{d}\end{array}$ & $\begin{array}{l}\text { unclassified } \\
\text { unclassified } \\
\text { unclassified }\end{array}$ \\
\hline \multicolumn{5}{|l|}{ Dataset 3} \\
\hline EstC55-2 & Carbon-carbon bond hydrolase & ABHD6-Lip (V) & unassigned & $\mathrm{V}$ \\
\hline EstC55-3 & Carb_B_Bacteria & Carb_B_Bacteria (VII) & VII & VII \\
\hline EstC55-4_1 & 6_AlphāBeta_hydrolase & Carboxymethylbutenolide_lactonase (V) & unassigned & $\mathrm{V}$ \\
\hline EstC55-5 & Hormone-sensitive_lipase like & Hormone_sensitive lipase like (IV) & IV & IV \\
\hline EstC55-8_1 & Hormone-sensitive_lipase_like & Hormone_sensitive_lipase_like (IV) & IV & IV \\
\hline EstC55-8_2 & Epoxide_hydrolase & Carboxymethylbutenolide_lactonase (V) & unassigned & $\mathrm{V}$ \\
\hline EstC55-12 & 6_AlphaBeta_hydrolase & Carboxymethylbutenolide_lactonase (V) & unassigned & $\mathrm{V}$ \\
\hline EstC55-13 & PAF-Acetylhydrolase & Chlorophyllase (EstGS) & unassigned & $\begin{array}{l}\text { new subfamily (this } \\
\text { study) }\end{array}$ \\
\hline EstC55-15 & AlphaBeta_hydrolase & CarbLipBact_2 (XIII-2) & unassigned & new family (this study) \\
\hline EstC55-18 & $\mathrm{BioH}$ & Carboxymethylbutenolide_lactonase (V) & unassigned & $\mathrm{V}$ \\
\hline EstC55-19_1 & Hormone-sensitive_lipase_like & Hormone_sensitive_lipase_like (IV) & IV & IV \\
\hline EstC55-19_2 & Epoxide_hydrolase - & ABHD6-Lip (V) & unassigned & $\mathrm{V}$ \\
\hline EstC55-20 & 6_AlphaBeta_hydrolase & Carboxymethylbutenolide lactonase (V) & unassigned & $\mathrm{V}$ \\
\hline EstC55-23 & Hormone-sensitive lipase like & Hormone sensitive lipase like (IV) & IV & IV \\
\hline EstC55-24 & Chlorophyllase & Chlorophyllase (EstGS) & EstGS & EstGS \\
\hline EstC55-25 & 6_AlphaBeta_hydrolase & Carboxymethylbutenolide_lactonase (V) & unassigned & $\mathrm{V}$ \\
\hline EstC55-31 & 6 AlphaBeta hydrolase & Carboxymethylbutenolide lactonase (V) & unassigned & $\mathrm{V}$ \\
\hline EstC55-34 & Carboxymethylbutenolide_lactonase & Carboxymethylbutenolide_lactonase (V) & $\mathrm{V}$ & $\mathrm{V}$ \\
\hline EstC55-38 & Pectinacetylesterase-Notum & Pectinacetylesterase-Notum (LipT) & LipT & LipT \\
\hline EstC55-42 & Esterase_phb & Lysophospholipase_carboxylesterase (VI) & unassigned & EM3L4 \\
\hline EstC55-51 & 6_AlphaBeta_hydrolase & ABHD6-Lip (V) & unassigned & $\mathrm{V}$ \\
\hline EstC55-52 & Carb_B_Bacteria & Carb_B_Bacteria (VII) & VII & VII \\
\hline EstC55-56 & Hormo-̄e-sensitive_lipase_like & Hormone_sensitive_lipase_like (IV) & IV & IV \\
\hline EstC55-57 & Hormone-sensitive_lipase_like & Hormone_sensitive_lipase_like (IV) & IV & IV \\
\hline EstC55-60 & Hormone-sensitive_lipase_like & Hormone_sensitive_lipase_like (IV) & IV & IV \\
\hline EstC55-62 & Carb_B_Bacteria - & Carb_B_Bacteria $(\overline{\mathrm{V}} \mathrm{II})$ & VII & VII \\
\hline EstC55-71 & Lipase 2 & Bacterial lipase (I) & I & I \\
\hline EstC55-72 & Hormone-sensitive lipase like & Hormone sensitive lipase like (IV) & IV & IV \\
\hline EstC55-76 & 6_AlphaBeta_hydrolase & Carboxymethylbutenolide_lactonase (V) & unassigned & $\mathrm{V}$ \\
\hline EstC55-77 & Esterase_phb & Lysophospholipase_carboxylesterase (VI) & unassigned & EM3L4 \\
\hline EstC55-78 & Hormone-sensitive_lipase_like & Hormone_sensitive_lipase_like (IV) & IV & IV \\
\hline EstC55-81 & 6 AlphaBeta hydrolase & Carboxymethylbutenolide lactonase (V) & unassigned & $\mathrm{V}$ \\
\hline EstC55-88 & Lipase_2 & Bacterial_lipase (I) & I & I \\
\hline EstC55-90 & Lipase_2 & Bacterial_lipase (I) & I & I \\
\hline \multirow{2}{*}{$\begin{array}{l}\text { EstC55-95 } \\
\text { EstC55-96 }\end{array}$} & Polyesterase-lipase-cutinase & Polyesterase-lipase-cutinase (III) & III & III \\
\hline & ABHD11-Acetyl_transferase & Carboxymethylbutenolide_lactonase (V) & $\begin{array}{l}\text { nonlipolytic } \alpha / \beta \\
\text { hydorlase }\end{array}$ & $\mathrm{V}$ \\
\hline EstC55-100 & 6_AlphaBeta_hydrolase & Carboxymethylbutenolide_lactonase (V) & unassigned & $\mathrm{V}$ \\
\hline EstC55-102 & Lipase_bact____lipase & Chlorophyllase (EstGS) & unassigned & new family (this study) \\
\hline EstC55-105 & Lipase_2 - - & Bacterial_lipase (I) & I & I \\
\hline
\end{tabular}




\begin{tabular}{|c|c|c|c|c|}
\hline EstC55-118 & Carb_B_Bacteria & Carb_B_Bacteria (VII) & VII & VII \\
\hline EstC55-145 & Hormone-sensitive_lipase_like & Hormone_sensitive_lipase_like (IV) & IV & IV \\
\hline EstC55-151 & Lipase_2 & Bacterial_lipase (I) & I & I \\
\hline EstC55-154 & Fungal-Bact_LIP & Fungal_Bact_LIP (X-2/XVII/XIX) & XVII & XVII \\
\hline EstC55-156 & Tannase & Tannase (Tannase) & Tannase & Tannase \\
\hline EstC55-159 & 6_AlphaBeta_hydrolase & ABHD6-Lip (V) & unassigned & $\mathrm{V}$ \\
\hline EstC55-165 & 5 AlphaBeta hydrolase & A85-Feruloyl-Esterase (EstSt7/Rlip1) & unassigned & FLS18 \\
\hline EstC55-169 & Tannase & Tannase (Tannase) & Tannase & Tannase \\
\hline EstC55-186 & Carb_B_Bacteria & Carb_B_Bacteria (VII) & VII & VII \\
\hline EstC55-188 & AlphāBeta_hydrolase & Lysophospholipase_carboxylesterase (VI) & unassigned & new family (this study) \\
\hline EstC55-197 & Aclacinomycin-methylesterase_RdmC & Carboxymethylbutenolide_lactonase (V) & unassigned & $\mathrm{V}$ \\
\hline EstC55-213 & PGAP1 & Bacterial_lipase (I) & unassigned & $\begin{array}{l}\text { new subfamily (this } \\
\text { study) }\end{array}$ \\
\hline EstC55-215 & 6_AlphaBeta_hydrolase & Carboxymethylbutenolide_lactonase (V) & unassigned & $\mathrm{V}$ \\
\hline EstC55-229 & Hormone-sensitive lipase_like & Hormone_sensitive lipase_like (IV) & IV & IV \\
\hline EstC55-231 & 6_AlphaBeta_hydrolase & Carboxymethylbutenolide lactonase (V) & unassigned & $\mathrm{V}$ \\
\hline EstC55-234 & Tannase & Tannase (Tannase) & Tannase & Tannase \\
\hline EstC55-235 & Lipase_2 & Bacterial_lipase (I) & I & I \\
\hline EstC55-241 & 5_AlphaBeta_hydrolase & A85-Feruloyl-Esterase (EstSt7/Rlip1) & unassigned & FLS18 \\
\hline EstC55-244 & 6_AlphaBeta_hydrolase & Carboxymethylbutenolide_lactonase (V) & unassigned & $\mathrm{V}$ \\
\hline EstC55-247 & Hormone-sensitive lipase like & Hormone sensitive lipase like (IV) & IV & IV \\
\hline EstC55-253 & Hormone-sensitive_lipase_like & Hormone_sensitive_lipase_like (IV) & IV & IV \\
\hline EstC55-256 & 6_AlphaBeta_hydrolase & Carboxymethylbutenolide_lactonase (V) & unassigned & $\mathrm{V}$ \\
\hline EstC55-268 & Hormone-sensitive_lipase_like & Hormone_sensitive_lipase_like (IV) & IV & IV \\
\hline EstC76-28_1 & 6_AlphaBeta_hydrolase & Carboxymethylbutenolide_lactonase (V) & unassigned & $\mathrm{V}$ \\
\hline EstC76-28_2 & 6_AlphaBeta_hydrolase & ABHD6-Lip (V) & unassigned & $\mathrm{V}$ \\
\hline EstC76-36 & ĀlphaBeta_hydrolase & Lysophospholipase carboxylesterase (VI) & unassigned & new family (this study) \\
\hline EstC76-135 & Hormone-sensitive_lipase_like & Hormone_sensitive_lipase_like (IV) & IV & IV \\
\hline EstC76-136 & Carb_B_Bacteria & Carb_B_Bacteria (V̄II) & VII & VII \\
\hline EstC76-137 & 5_AlphaBeta_hydrolase & A85-Feruloyl-Esterase (EstSt7/Rlip1) & unassigned & FLS18 \\
\hline EstC76-177 & 6_AlphaBeta_hydrolase & Lipase_2 (I) & unassigned & $\begin{array}{l}\text { new subfamily (this } \\
\text { study) }\end{array}$ \\
\hline EstC76-179 & Pectinacetylesterase-Notum & Pectinacetylesterase-Notum (LipT) & LipT & LipT \\
\hline EstC76-202 & $\mathrm{BioH}$ & Carboxymethylbutenolide_lactonase (V) & unassigned & $\mathrm{V}$ \\
\hline EstC76-218 & Pectinacetylesterase-Notum & Pectinacetylesterase-Notum (LipT) & LipT & LipT \\
\hline EstC76-221 & AlphaBeta_hydrolase & Bacterial_EstLip_FamX (X) & unassigned & new family (this study) \\
\hline EstC76-248 & 6_AlphaBeta_hydrolase & Carboxymethylbutenolide_lactonase (V) & unassigned & $\mathrm{V}$ \\
\hline EstC76-263 & 6_AlphaBeta_hydrolase & Carboxymethylbutenolide_lactonase (V) & unassigned & $\mathrm{V}$ \\
\hline EstC76-266 & 6_AlphaBeta_hydrolase & Carboxymethylbutenolide_lactonase (V) & unassigned & $\mathrm{V}$ \\
\hline EstC55-227 & $\mathrm{ND}^{\mathrm{d}}$ & $\mathrm{ND}^{\mathrm{d}}$ & $\mathrm{ND}^{\mathrm{d}}$ & new family (this study) \\
\hline EstC55-97 & $\mathrm{ND}^{\mathrm{d}}$ & $\mathrm{ND}^{\mathrm{d}}$ & $\mathrm{ND}^{\mathrm{d}}$ & new family (this study) \\
\hline EstC55-167 & $\mathrm{ND}^{\mathrm{d}}$ & $\mathrm{ND}^{\mathrm{d}}$ & $\mathrm{ND}^{\mathrm{d}}$ & new family (this study) \\
\hline \multicolumn{5}{|l|}{ Dataset 4} \\
\hline $\begin{array}{l}\text { L7VEQ3 } \\
\text { A0A0U0R506 }\end{array}$ & $\begin{array}{l}\text { Carbon-carbon_bond_hydrolase } \\
\text { Carbon-carbon bond hydrolase }\end{array}$ & $\begin{array}{l}\text { ABHD6-Lip (V) } \\
\text { ABHD6-Lip (V) }\end{array}$ & $\begin{array}{l}\text { Non-lipolytic protein } \\
\text { Non-lipolytic protein }\end{array}$ & $\begin{array}{l}\mathrm{ND}^{\mathrm{d}} \\
\mathrm{ND}^{\mathrm{d}}\end{array}$ \\
\hline
\end{tabular}


A0A2X1S8R4

A0A0T9VGE3

A0A1S7N7P8

A0A1S7T7D7

A0A1S7T6S9

A0A1S7N8N9

A0A1S7N8S2

A0A1S7S3Y6

A0A1S7NDN6

A0A2Z5YBG9

A0A2N9APQ2

G4SWJ2

A0A0N7H8D0

A0A102S299

A0A375DNQ7

A0A375EC98

A0A375FEC6

A0A375D6M7

A0A375IUU4

A0A1S7RCN8

A0A1S7R0G9

A0A1S7RQ23

A0A1S7N1P6

A0A1S7R2U2

A0A1S7R186

A0A1S7S3E0

A0A1S7TAY0

A0A379M130

A0A1S7TZP1

A0A375DP21

A0A1S7RZV4

A0A1S7QF97

A0A1S7QYM3

A0A1S7UBZ3

A0A1X2DNB5

A0A1S7R6I3

A0A2X4UF21

A0A1S7N0Y4

A0A1S7RYV9

A0A1S7SHR8

A0A378W1U8

A0A1S7S3D1

A0A1S7MUS0

A0A1S7MPM6

A0A375EBJ1
Carbon-carbon bond hydrolase Carbon-carbon_bond_hydrolase Haloperoxidase

Haloperoxidase

Haloperoxidase

Haloperoxidase

Haloperoxidase

Haloperoxidase

Haloperoxidase

6_AlphaBeta_hydrolase

Haloperoxidase

Haloperoxidase

6_AlphaBeta_hydrolase

Haloperoxidase

Haloperoxidase

Haloperoxidase

Haloperoxidase

Haloperoxidase

Haloperoxidase

Haloperoxidase

Haloperoxidase

Haloperoxidase

Haloperoxidase

Haloperoxidase

Haloperoxidase

Haloperoxidase

Haloperoxidase

Haloperoxidase

Haloperoxidase

Haloperoxidase

Haloperoxidase

Haloperoxidase

Haloperoxidase

Haloperoxidase

6_AlphaBeta_hydrolase

Haloperoxidase

Haloperoxidase

Haloperoxidase

Haloperoxidase

Haloperoxidase

6 AlphaBeta_hydrolase

Haloperoxidase

Haloperoxidase

Haloperoxidase

Haloperoxidase
ABHD6-Lip (V)

ABHD6-Lip (V)

Carboxymethylbutenolide_lactonase (V)

Carboxymethylbutenolide lactonase (V)

Carboxymethylbutenolide lactonase (V)

Carboxymethylbutenolide_lactonase (V)

Carboxymethylbutenolide lactonase (V)

Carboxymethylbutenolide lactonase (V)

Carboxymethylbutenolide_lactonase (V)

Carboxymethylbutenolide_lactonase (V)

Carboxymethylbutenolide lactonase (V)

Carboxymethylbutenolide lactonase (V)

Carboxymethylbutenolide_lactonase (V)

Carboxymethylbutenolide lactonase (V)

Carboxymethylbutenolide lactonase (V)

Carboxymethylbutenolide lactonase (V)

Carboxymethylbutenolide_lactonase (V)

Carboxymethylbutenolide_lactonase (V)

Carboxymethylbutenolide lactonase (V)

Carboxymethylbutenolide_lactonase (V)

Carboxymethylbutenolide_lactonase (V)

Carboxymethylbutenolide lactonase (V)

Carboxymethylbutenolide lactonase (V)

Carboxymethylbutenolide_lactonase (V)

Carboxymethylbutenolide lactonase (V)

Carboxymethylbutenolide lactonase (V)

Carboxymethylbutenolide_lactonase (V)

Carboxymethylbutenolide_lactonase (V)

Carboxymethylbutenolide lactonase (V)

Carboxymethylbutenolide lactonase (V)

Carboxymethylbutenolide_lactonase (V)

Carboxymethylbutenolide lactonase (V)

Carboxymethylbutenolide lactonase (V)

Carboxymethylbutenolide_lactonase (V)

Carboxymethylbutenolide_lactonase (V)

Carboxymethylbutenolide lactonase (V)

ABHD6-Lip (V)

Carboxymethylbutenolide_lactonase (V)

Carboxymethylbutenolide lactonase (V)

Carboxymethylbutenolide lactonase (V)

Carboxymethylbutenolide_lactonase (V)

Carboxymethylbutenolide_lactonase (V)

Carboxymethylbutenolide lactonase (V)

Carboxymethylbutenolide lactonase (V)

Carboxymethylbutenolide_lactonase (V)
Non-lipolytic protein

Non-lipolytic protein

Non-lipolytic protein

Non-lipolytic protein

Non-lipolytic protein

Non-lipolytic protein

Non-lipolytic protein

Non-lipolytic protein

Non-lipolytic protein

Non-lipolytic protein

Non-lipolytic protein

Non-lipolytic protein

Non-lipolytic protein

Non-lipolytic protein

Non-lipolytic protein

Non-lipolytic protein

Non-lipolytic protein

Non-lipolytic protein

Non-lipolytic protein

Non-lipolytic protein

Non-lipolytic protein

Non-lipolytic protein

Non-lipolytic protein

Non-lipolytic protein

Non-lipolytic protein

Non-lipolytic protein

Non-lipolytic protein

Non-lipolytic protein

Non-lipolytic protein

Non-lipolytic protein

Non-lipolytic protein

Non-lipolytic protein

Non-lipolytic protein

Non-lipolytic protein

unassigned

Non-lipolytic protein

Non-lipolytic protein

Non-lipolytic protein

Non-lipolytic protein

Non-lipolytic protein

unassigned

Non-lipolytic protein

Non-lipolytic protein

Non-lipolytic protein

Non-lipolytic protein
$\mathrm{ND}^{\mathrm{d}}$

ND

$\mathrm{ND}^{\mathrm{d}}$

ND $^{d}$

$\mathrm{ND}^{\mathrm{d}}$

$\mathrm{ND}^{\mathrm{d}}$

$\mathrm{ND}^{\mathrm{d}}$

$\mathrm{ND}^{\mathrm{d}}$

$\mathrm{ND}^{\mathrm{d}}$

$\mathrm{ND}^{\mathrm{d}}$

$\mathrm{ND}^{\mathrm{d}}$

$\mathrm{ND}^{\mathrm{d}}$

$\mathrm{ND}^{\mathrm{d}}$

$\mathrm{ND}^{\mathrm{d}}$

$\mathrm{ND}^{\mathrm{d}}$

$\mathrm{ND}^{\mathrm{d}}$

$\mathrm{ND}^{\mathrm{d}}$

$\mathrm{ND}^{\mathrm{d}}$

$\mathrm{ND}^{\mathrm{d}}$

$\mathrm{ND}^{\mathrm{d}}$

$\mathrm{ND}^{\mathrm{d}}$

$\mathrm{ND}^{\mathrm{d}}$

$\mathrm{ND}^{\mathrm{d}}$

$\mathrm{ND}^{\mathrm{d}}$

$\mathrm{ND}^{\mathrm{d}}$

$\mathrm{ND}^{\mathrm{d}}$

$\mathrm{ND}^{\mathrm{d}}$

$\mathrm{ND}^{\mathrm{d}}$

$\mathrm{ND}^{\mathrm{d}}$

$\mathrm{ND}^{\mathrm{d}}$

$\mathrm{ND}^{\mathrm{d}}$

$\mathrm{ND}^{\mathrm{d}}$

$\mathrm{ND}^{\mathrm{d}}$

$\mathrm{ND}^{\mathrm{d}}$

$\mathrm{ND}^{\mathrm{d}}$

$\mathrm{ND}^{\mathrm{d}}$

$\mathrm{ND}^{\mathrm{d}}$

$\mathrm{ND}^{\mathrm{d}}$

$\mathrm{ND}^{\mathrm{d}}$

$\mathrm{ND}^{\mathrm{d}}$

ND $^{d}$

$\mathrm{ND}^{\mathrm{d}}$

$\mathrm{ND}^{\mathrm{d}}$

ND $^{d}$

$\mathrm{ND}^{\mathrm{d}}$ 


\begin{tabular}{|c|c|c|c|c|}
\hline F8JH88 & Haloperoxidase & Carboxymethylbutenolide lactonase (V) & Non-lipolytic protein & $\mathrm{ND}^{\mathrm{d}}$ \\
\hline F8JA98 & Haloperoxidase & Carboxymethylbutenolide_lactonase (V) & Non-lipolytic protein & $\mathrm{ND}^{\mathrm{d}}$ \\
\hline A0A2N9AMF4 & Haloperoxidase & Carboxymethylbutenolide lactonase (V) & Non-lipolytic protein & $\mathrm{ND}^{\mathrm{d}}$ \\
\hline ALO47455 & Epoxide_hydrolase & ABHD6-Lip (V) & unassigned & $\mathrm{ND}^{\mathrm{d}}$ \\
\hline PNG95674 & Epoxide hydrolase & Carboxymethylbutenolide lactonase (V) & Non-lipolytic protein & $\mathrm{ND}^{\mathrm{d}}$ \\
\hline PNG97285 & Epoxide hydrolase & ABHD6-Lip (V) & Non-lipolytic protein & $\mathrm{ND}^{\mathrm{d}}$ \\
\hline KDN82212 & Epoxide hydrolase & ABHD6-Lip (V) & unassigned & $\mathrm{ND}^{\mathrm{d}}$ \\
\hline KIE51135 & Epoxide hydrolase & Carboxymethylbutenolide lactonase (V) & unassigned & $\mathrm{ND}^{\mathrm{d}}$ \\
\hline KIE52625 & Epoxide hydrolase & ABHD6-Lip (V) & Non-lipolytic protein & $\mathrm{ND}^{\mathrm{d}}$ \\
\hline
\end{tabular}

${ }^{\text {a }}$ Protein sequences were searched against the whole ESTHER database

${ }^{\mathrm{b}}$ Lipolytic family assignment using the strategy developed in this study; unassigned, could not assigned to any lipolytic family

${ }^{\mathrm{c}}$ Lipolytic family assignment reported in literatures (or this study) using pylogenetic-related methods

${ }^{d}$ No data 
Supplementary Table S13 Validation of the liplolytic family assignment strategy with LEs affiliated to family II, VIII and patatin-like-protein

\begin{tabular}{|c|c|c|c|c|}
\hline Protein name & CATH HMMs annotation & pHMM-pfam annotation & $\begin{array}{l}\text { Lipolytic family (This } \\
\text { study) }\end{array}$ & $\begin{array}{l}\text { Lipolytic family } \\
\text { (literatures or } \\
\text { database) }^{\mathrm{b}}\end{array}$ \\
\hline \multicolumn{5}{|l|}{ Dataset 1} \\
\hline A0A1B0QVN6 & Beta-lactamase (Penicillin-binding protein) (Penicillinase) & Beta-lactamase (VIII) & VIII & VIII \\
\hline A0A248RGG8 & Beta-lactamase (Penicillin-binding protein) (Penicillinase) & Beta-lactamase (VIII) & VIII & VIII \\
\hline A0A2C9EHI5 & Beta-lactamase (Penicillin-binding protein) (Penicillinase) & Beta-lactamase (VIII) & VIII & VIII \\
\hline B5TWC2 & Beta-lactamase (Penicillin-binding protein) (Penicillinase) & Beta-lactamase (VIII) & VIII & VIII \\
\hline D4N4E9 & Beta-lactamase (Penicillin-binding protein) (Penicillinase) & Beta-lactamase (VIII) & VIII & VIII \\
\hline F4MYP0 & Beta-lactamase (Penicillin-binding protein) (Penicillinase) & Beta-lactamase (VIII) & VIII & VIII \\
\hline F7Q845 & Beta-lactamase (Penicillin-binding protein) (Penicillinase) & Beta-lactamase (VIII) & VIII & VIII \\
\hline I7CDN7 & Beta-lactamase (Penicillin-binding protein) (Penicillinase) & Beta-lactamase (VIII) & VIII & VIII \\
\hline J2YNH3 & Beta-lactamase (Penicillin-binding protein) (Penicillinase) & Beta-lactamase (VIII) & VIII & VIII \\
\hline K4HQE7 & Beta-lactamase (Penicillin-binding protein) (Penicillinase) & Beta-lactamase (VIII) & VIII & VIII \\
\hline Q48LQ9 & Beta-lactamase (Penicillin-binding protein) (Penicillinase) & Beta-lactamase (VIII) & VIII & VIII \\
\hline Q4KH73 & Beta-lactamase (Penicillin-binding protein) (Penicillinase) & Beta-lactamase (VIII) & VIII & VIII \\
\hline Q56CZ4 & Beta-lactamase (Penicillin-binding protein) (Penicillinase) & Beta-lactamase (VIII) & VIII & VIII \\
\hline Q9KX30 & Beta-lactamase (Penicillin-binding protein) (Penicillinase) & Beta-lactamase (VIII) & VIII & VIII \\
\hline S5Y3D1 & Beta-lactamase (Penicillin-binding protein) (Penicillinase) & Beta-lactamase (VIII) & VIII & VIII \\
\hline A0A024H3L8 & Class $\mathrm{C}$ beta-lactamase CMY-10 & Beta-lactamase (VIII) & VIII & VIII \\
\hline A0A031ILE0 & Class C beta-lactamase CMY-10 & Beta-lactamase (VIII) & VIII & VIII \\
\hline A0A0G2RKR9 & Class $\mathrm{C}$ beta-lactamase CMY-10 & Beta-lactamase (VIII) & VIII & VIII \\
\hline $\mathrm{A} 0 \mathrm{~A} 0 \mathrm{H} 3 \mathrm{C} 4 \mathrm{U} 3$ & Class C beta-lactamase CMY-10 & Beta-lactamase (VIII) & VIII & VIII \\
\hline A0A0N7CSD6 & Class $\mathrm{C}$ beta-lactamase CMY-10 & Beta-lactamase (VIII) & VIII & VIII \\
\hline A0A0N9R483 & Class C beta-lactamase CMY-10 & Beta-lactamase (VIII) & VIII & VIII \\
\hline A1RB78 & Class C beta-lactamase CMY-10 & Beta-lactamase (VIII) & VIII & VIII \\
\hline A4F8E6 & Class C beta-lactamase CMY-10 & Beta-lactamase (VIII) & VIII & VIII \\
\hline A4FFW1 & Class $\mathrm{C}$ beta-lactamase CMY-10 & Beta-lactamase (VIII) & VIII & VIII \\
\hline A9WQD8 & Class C beta-lactamase CMY-10 & Beta-lactamase (VIII) & VIII & VIII \\
\hline B0M0H4 & Class C beta-lactamase CMY-10 & Beta-lactamase (VIII) & VIII & VIII \\
\hline D5P454 & Class $\mathrm{C}$ beta-lactamase CMY-10 & Beta-lactamase (VIII) & VIII & VIII \\
\hline E1VYY4 & Class C beta-lactamase CMY-10 & Beta-lactamase (VIII) & VIII & VIII \\
\hline G8PW82 & Class C beta-lactamase CMY-10 & Beta-lactamase (VIII) & VIII & VIII \\
\hline I4KBV5 & Class $\mathrm{C}$ beta-lactamase CMY-10 & Beta-lactamase (VIII) & VIII & VIII \\
\hline I4KTB2 & Class C beta-lactamase CMY-10 & Beta-lactamase (VIII) & VIII & VIII \\
\hline I4KYM0 & Class $\mathrm{C}$ beta-lactamase CMY-10 & Beta-lactamase (VIII) & VIII & VIII \\
\hline $\mathrm{J} 2 \mathrm{~F} 4 \mathrm{~F} 4$ & Class C beta-lactamase CMY-10 & Beta-lactamase (VIII) & VIII & VIII \\
\hline K9NGV2 & Class $\mathrm{C}$ beta-lactamase CMY-10 & Beta-lactamase (VIII) & VIII & VIII \\
\hline Q8VU79 & Class C beta-lactamase CMY-10 & Beta-lactamase (VIII) & VIII & VIII \\
\hline
\end{tabular}


A0A068QRN7 A0A068QRU1 A0A068R3C8 A0A068R3W3

A0A068R5Z7 A0A077P070

A0A077P6A6

A0A077PR36

A0A077QJI8

A0A077QNW8

A0A085G4J8

A0A0A8NSM3

A0A0B7DJ26

A0A0D5XSS9

A0A0F7Y552

A0A0G3SQ37

A0A0G5NCG5

A0A0H5LZ72

A0A0R4FM75

A0A0S4IA49

A0A0T9KAX4

A0A0X8XW81

A0A109KKX4

A0A126VD94

A0A145P5E7

A0A168FS98

A0A1B8YLL8

A0A1C0U1I1

A0A1C3HL68

A0A1N6MX70

A0A1N6MXA7

A0A1W5DEF9

A0A240A646

A0A2H1L984

A8G7T5

B1JE37

B4STS4

B6VK93

D2BTP5

D3V7P0

D3VEQ2

D4DZP7

G8Q0A2

I4JZT9

I4L7G6
Autotransporting lipase, GDSL family Autotransporting lipase, GDSL family Autotransporting lipase, GDSL family Autotransporting lipase, GDSL family Autotransporting lipase, GDSL family Autotransporting lipase, GDSL family Autotransporting lipase, GDSL family Autotransporting lipase, GDSL family Autotransporting lipase, GDSL family Autotransporting lipase, GDSL family Autotransporting lipase, GDSL family Autotransporting lipase, GDSL family Autotransporting lipase, GDSL family Autotransporting lipase, GDSL family Autotransporting lipase, GDSL family Autotransporting lipase, GDSL family Autotransporting lipase, GDSL family Autotransporting lipase, GDSL family Autotransporting lipase, GDSL family Autotransporting lipase, GDSL family Autotransporting lipase, GDSL family Autotransporting lipase, GDSL family Autotransporting lipase, GDSL family Autotransporting lipase, GDSL family Autotransporting lipase, GDSL family Autotransporting lipase, GDSL family Autotransporting lipase, GDSL family Autotransporting lipase, GDSL family Autotransporting lipase, GDSL family Autotransporting lipase, GDSL family Autotransporting lipase, GDSL family Autotransporting lipase, GDSL family Autotransporting lipase, GDSL family Autotransporting lipase, GDSL family Autotransporting lipase, GDSL family Autotransporting lipase, GDSL family Autotransporting lipase, GDSL family Autotransporting lipase, GDSL family Autotransporting lipase, GDSL family Autotransporting lipase, GDSL family Autotransporting lipase, GDSL family Autotransporting lipase, GDSL family Autotransporting lipase, GDSL family Autotransporting lipase, GDSL family Autotransporting lipase, GDSL family
Lipase GDSL (II)

Lipase_GDSL (II)

Lipase_GDSL (II)

Lipase GDSL (II)

Lipase GDSL (II)

Lipase_GDSL (II)

Lipase GDSL (II)

Lipase GDSL (II)

Lipase_GDSL (II)

Lipase_GDSL (II)

Lipase GDSL (II)

Lipase GDSL (II)

Lipase_GDSL (II)

Lipase GDSL (II)

Lipase GDSL (II)

Lipase GDSL (II)

Lipase_GDSL (II)

Lipase GDSL (II)

Lipase GDSL (II)

Lipase_GDSL (II)

Lipase_GDSL (II)

Lipase GDSL (II)

Lipase GDSL (II)

Lipase_GDSL (II)

Lipase GDSL (II)

Lipase GDSL (II)

Lipase GDSL (II)

Lipase_GDSL (II)

Lipase GDSL (II)

Lipase GDSL (II)

Lipase_GDSL (II)

Lipase GDSL (II)

Lipase GDSL (II)

Lipase_GDSL (II)

Lipase_GDSL (II)

Lipase GDSL (II)

Lipase GDSL (II)

Lipase_GDSL (II)

Lipase GDSL (II)

Lipase GDSL (II)

Lipase_GDSL (II)

Lipase_GDSL (II)

Lipase_GDSL (II)

Lipase GDSL (II)

Lipase_GDSL (II) 
J2MF23

N1NPB9

P40601

S2F0I5

W1IPR6

W1ITZ2

W1IUL6

W6VSR6

A0A011NHS5

A0A011NNZ6

A0A177W0K3

A0A1J5Q6R0

A0A1J5QST6

A0A1R4EFW9

A0A1R4GRG6

A0A1T0A9C3

A0A1U6GL33

A0A1Y5Q328

A0A2H4UKY2

A5WHX6

A7IIP4

D5VAI8

E6WQ95

G6FMT2

K9XFG2

Q3JVI5

Q7X4K7

A0A024HQ86

A0A080VLY1

A0A0D0T6T3

A0A0F6UI63

A0A0H3YNS8

A0A0K0Q1Y2

A0A0P8X538

A0A0S4HWF7

A0A120G8H3

A0A127N102

A0A157V5L0

A0A193SIR7

A0A1Y6JH94

E3VST9

I4KAN1

J2FAM4

K9NS61
Autotransporting lipase, GDSL family Autotransporting lipase, GDSL family Autotransporting lipase, GDSL family Autotransporting lipase, GDSL family Autotransporting lipase, GDSL family Autotransporting lipase, GDSL family Autotransporting lipase, GDSL family Autotransporting lipase, GDSL family

GDSL esterase/lipase APG

GDSL esterase/lipase APG

GDSL esterase/lipase APG

GDSL esterase/lipase APG

GDSL esterase/lipase APG

GDSL esterase/lipase APG

GDSL esterase/lipase APG

GDSL esterase/lipase APG

GDSL esterase/lipase APG

GDSL esterase/lipase APG

GDSL esterase/lipase APG

GDSL esterase/lipase APG

GDSL esterase/lipase APG

GDSL esterase/lipase APG

GDSL esterase/lipase APG

GDSL esterase/lipase APG

GDSL esterase/lipase APG

GDSL esterase/lipase APG

GDSL esterase/lipase APG

GDSL esterase/lipase APG

Autotransporting lipase, GDSL family

Autotransporting lipase, GDSL family

Autotransporting lipase, GDSL family

Autotransporting lipase, GDSL family

Autotransporting lipase, GDSL family

Autotransporting lipase, GDSL family

Autotransporting lipase, GDSL family

Autotransporting lipase, GDSL family

Autotransporting lipase, GDSL family

Autotransporting lipase, GDSL family

Autotransporting lipase, GDSL family

Autotransporting lipase, GDSL family

Autotransporting lipase, GDSL family

Autotransporting lipase, GDSL family

Autotransporting lipase, GDSL family

Autotransporting lipase, GDSL family

Autotransporting lipase, GDSL family
Lipase GDSL (II)

Lipase_GDSL (II)

Lipase_GDSL (II)

Lipase GDSL (II)

Lipase GDSL (II)

Lipase_GDSL (II)

Lipase GDSL (II)

Lipase GDSL (II)

Lipase_GDSL (II)

Lipase_GDSL (II)

Lipase GDSL (II)

Lipase GDSL (II)

Lipase_GDSL (II)

Lipase GDSL (II)

Lipase GDSL (II)

Lipase GDSL (II)

Lipase_GDSL (II)

Lipase GDSL (II)

Lipase GDSL (II)

Lipase_GDSL (II)

Lipase_GDSL (II)

Lipase GDSL (II)

Lipase GDSL (II)

Lipase_GDSL (II)

Lipase GDSL (II)

Lipase GDSL (II)

Lipase_GDSL (II)

Lipase_GDSL (II)

Lipase GDSL (II)

Lipase GDSL (II)

Lipase_GDSL (II)

Lipase_GDSL (II)

Lipase GDSL (II)

Lipase_GDSL (II)

Lipase_GDSL (II)

Lipase GDSL (II)

Lipase GDSL (II)

Lipase_GDSL (II)

Lipase GDSL (II)

Lipase GDSL (II)

Lipase GDSL (II)

Lipase_GDSL (II)

Lipase GDSL (II)

Lipase GDSL (II)

Lipase_GDSL (II) 
O33407

Q6B6R8

Q938A9

V6ANT9

W0HFT8

A0A0S2KQT2

A0A1V6JD04

A0A246KHV9

G0HD65

Q3JQU3

A0A0P0FMI4

A0A135YTB5

A0A139JRQ6

A0A143PQQ6

A0A173Z3Y1

A0A174CNS8

A0A174H821

A0A174NAV5

A0A174UHE3

A0A1E8F0P5

A0A1P8WFW9

A0A1V5F586

A0A1V5G6A1

A0A1V5HGJ2

A0A1V5UHU4

A0A1V5V5J1

A0A1V6JUN0

A0A2A3N2S7

F3Y895

S5XQR0

A0A009FQY3

A0A009GH23

A0A009HL94

A0A009L278

A0A009MNS1

A0A009RMH5

A0A009YG53

A0A010IZF7

A0A010L6U0

A0A010WGV4

A0A011K3K9

A0A011M4E2

A0A011NRW2

A0A011P1E4

A0A011P216
Autotransporting lipase, GDSL family

Autotransporting lipase, GDSL family

Autotransporting lipase, GDSL family

Autotransporting lipase, GDSL family

Autotransporting lipase, GDSL family

GDSL esterase/lipase APG

GDSL esterase/lipase APG

GDSL esterase/lipase APG

GDSL esterase/lipase APG

GDSL esterase/lipase APG

Isoamyl acetate-hydrolyzing esterase 1 homolog

Isoamyl acetate-hydrolyzing esterase 1 homolog

Isoamyl acetate-hydrolyzing esterase 1 homolog

Isoamyl acetate-hydrolyzing esterase 1 homolog

Isoamyl acetate-hydrolyzing esterase 1 homolog

Isoamyl acetate-hydrolyzing esterase 1 homolog

Isoamyl acetate-hydrolyzing esterase 1 homolog

Isoamyl acetate-hydrolyzing esterase 1 homolog

Isoamyl acetate-hydrolyzing esterase 1 homolog

Isoamyl acetate-hydrolyzing esterase 1 homolog

Isoamyl acetate-hydrolyzing esterase 1 homolog

Isoamyl acetate-hydrolyzing esterase 1 homolog

Isoamyl acetate-hydrolyzing esterase 1 homolog

Isoamyl acetate-hydrolyzing esterase 1 homolog

Isoamyl acetate-hydrolyzing esterase 1 homolog

Isoamyl acetate-hydrolyzing esterase 1 homolog Isoamyl acetate-hydrolyzing esterase 1 homolog Isoamyl acetate-hydrolyzing esterase 1 homolog Isoamyl acetate-hydrolyzing esterase 1 homolog Isoamyl acetate-hydrolyzing esterase 1 homolog Acyl-CoA thioesterase I

Acyl-CoA thioesterase I

Acyl-CoA thioesterase I

Acyl-CoA thioesterase I

Acyl-CoA thioesterase I

Acyl-CoA thioesterase I

Acyl-CoA thioesterase I

Acyl-CoA thioesterase I

Acyl-CoA thioesterase I

Acyl-CoA thioesterase I

Acyl-CoA thioesterase I

Acyl-CoA thioesterase I

Acyl-CoA thioesterase I

Acyl-CoA thioesterase I

Acyl-CoA thioesterase I
Lipase GDSL (II)

Lipase_GDSL (II)

Lipase_GDSL (II)

Lipase_GDSL (II)

Lipase GDSL (II)

Lipase_GDSL_2 (II)

Lipase GDSL 2 (II)

Lipase GDSL 2 (II)

Lipase_GDSL_2 (II)

Lipase_GDSL_2 (II)

Lipase GDSL_2 (II)

Lipase GDSL 2 (II)

Lipase_GDSL_2 (II)

Lipase_GDSL 2 (II)

Lipase GDSL 2 (II)

Lipase GDSL 2 (II)

Lipase_GDSL_2 (II)

Lipase GDSL 2 (II)

Lipase GDSL 2 (II)

Lipase_GDSL_2 (II)

Lipase_GDSL_2 (II)

Lipase GDSL 2 (II)

Lipase GDSL 2 (II)

Lipase_GDSL_2 (II)

Lipase GDSL 2 (II)

Lipase GDSL 2 (II)

Lipase GDSL 2 (II)

Lipase_GDSL_2 (II)

Lipase GDSL 2 (II)

Lipase GDSL 2 (II)

Lipase_GDSL_2 (II)

Lipase_GDSL 2 (II)

Lipase GDSL 2 (II)

Lipase_GDSL_2 (II)

Lipase_GDSL_2 (II)

Lipase GDSL 2 (II)

Lipase GDSL 2 (II)

Lipase_GDSL_2 (II)

Lipase_GDSL 2 (II)

Lipase GDSL 2 (II)

Lipase GDSL 2 (II)

Lipase_GDSL_2 (II)

Lipase GDSL 2 (II)

Lipase GDSL 2 (II)

Lipase_GDSL_2 (II) 
A0A011PQC4 A0A011Q348 A0A013S8D5 A0A013TPS8 A0A014B4I3 A0A014BP77 A0A014BVI3

A0A014CG41 A0A014DCG5 A0A014DNG3 A0A014DW77 A0A014F485 A0A022I9R1 A0A022J7T0

A0A022KIF1

A0A024HK50 A0A061D1S0 A0A062BV08

A0A062GRT9 A0A062LUI1

A0A062N102

A0A062SY13

A0A078BGB8

A0A080LZ83

A0A080M659

A0A098G8E8

A0A0A8RH78

A0A0B5FA02

A0A0B7DFD2

A0A0C6F7I8

A0A0D0T8I6

A0A0D6H809

A0A0F7XZS5

A0A0G6AHG2

A0A0H4WD71

A0A0J6CCW4

A0A0K1J6Z2

A0A0K2G755

A0A0K2ZPA4

A0A0K2ZYS

A0A0K2ZZJ3

A0A0M2WGX5

A0A0M7HJ86

A0A0M9IS63

A0A0N1JS35
Acyl-CoA thioesterase I Acyl-CoA thioesterase I Acyl-CoA thioesterase I Acyl-CoA thioesterase I Acyl-CoA thioesterase I Acyl-CoA thioesterase I Acyl-CoA thioesterase I Acyl-CoA thioesterase I Acyl-CoA thioesterase I Acyl-CoA thioesterase I Acyl-CoA thioesterase I Acyl-CoA thioesterase I Acyl-CoA thioesterase I Acyl-CoA thioesterase I Acyl-CoA thioesterase I Acyl-CoA thioesterase I Acyl-CoA thioesterase I Acyl-CoA thioesterase I Acyl-CoA thioesterase I Acyl-CoA thioesterase I Acyl-CoA thioesterase I Acyl-CoA thioesterase I Acyl-CoA thioesterase I Acyl-CoA thioesterase I Acyl-CoA thioesterase I Acyl-CoA thioesterase I Acyl-CoA thioesterase I Acyl-CoA thioesterase I Acyl-CoA thioesterase I Acyl-CoA thioesterase I Acyl-CoA thioesterase I Acyl-CoA thioesterase I Acyl-CoA thioesterase I Acyl-CoA thioesterase I Acyl-CoA thioesterase I Acyl-CoA thioesterase I Acyl-CoA thioesterase I Acyl-CoA thioesterase I Acyl-CoA thioesterase I Acyl-CoA thioesterase I Acyl-CoA thioesterase I Acyl-CoA thioesterase Acyl-CoA thioesterase Acyl-CoA thioesterase Acyl-CoA thioesterase I
Lipase GDSL 2 (II)

Lipase GDSL 2 (II)

Lipase_GDSL_2 (II)

Lipase GDSL 2 (II)

Lipase_GDSL_2 (II)

Lipase_GDSL_2 (II)

Lipase_GDSL 2 (II)

Lipase GDSL 2 (II)

Lipase_GDSL_2 (II)

Lipase_GDSL_2 (II)

Lipase GDSL 2 (II)

Lipase GDSL 2 (II)

Lipase_GDSL_2 (II)

Lipase_GDSL_2 (II)

Lipase GDSL 2 (II)

Lipase GDSL 2 (II)

Lipase_GDSL_2 (II)

Lipase GDSL 2 (II)

Lipase_GDSL_2 (II)

Lipase_GDSL_2 (II)

Lipase_GDSL_2 (II)

Lipase GDSL 2 (II)

Lipase_GDSL 2 (II)

Lipase_GDSL_2 (II)

Lipase GDSL 2 (II)

Lipase GDSL 2 (II)

Lipase_GDSL_2 (II)

Lipase_GDSL_2 (II)

Lipase GDSL 2 (II)

Lipase GDSL 2 (II)

Lipase_GDSL_2 (II)

Lipase_GDSL 2 (II)

Lipase GDSL 2 (II)

Lipase_GDSL_2 (II)

Lipase_GDSL_2 (II)

Lipase_GDSL_2 (II)

Lipase GDSL 2 (II)

Lipase_GDSL_2 (II)

Lipase_GDSL 2 (II)

Lipase GDSL 2 (II)

Lipase GDSL 2 (II)

Lipase_GDSL_2 (II)

Lipase GDSL 2 (II)

Lipase GDSL 2 (II)

Lipase_GDSL_2 (II) 
A0A0P0M8K2

A0A0P0MG90

A0A0P9MUM9

A0A0Q0MJ90

A0A0Q9YFU6

A0A0Q9YN66

A0A0S4HZN8

A0A0S4I4I4

A0A0S4KTX7

A0A0S4L7Q0

A0A0T7QZN4

A0A0T8L2J5

A0A0W0S4P5

A0A0W0UTZ0

A0A0W0XMR7

A0A0W0YWH7

A0A0W1AA95

A0A0X8HBX8

A0A0X8X153

A0A109KRK3

A0A109LCQ0

A0A119A1M5

A0A127MOK8

A0A150HXT3

A0A150I107

A0A157KKJ6

A0A157R8P7

A0A157SW77

A0A157WLJ5

A0A177YYG5

A0A193SRH1

A0A1A8TGJ1

A0A1A8TLQ4

A0A1B8NWA4

A0A1C3JU89

A0A1C9W8B0

A0A1D3JUB8

A0A1D8AVV5

A0A1E2ZHS6

A0A1E3GPF1

A0A1E7VQY7

A0A1E7WA72

A0A1E7WRQ8

A0A1E7X2W6

A0A1G5SHI5
Acyl-CoA thioesterase I Acyl-CoA thioesterase I Acyl-CoA thioesterase I Acyl-CoA thioesterase I Acyl-CoA thioesterase I Acyl-CoA thioesterase I Acyl-CoA thioesterase I Acyl-CoA thioesterase I Acyl-CoA thioesterase I Acyl-CoA thioesterase I Acyl-CoA thioesterase I Acyl-CoA thioesterase I Acyl-CoA thioesterase I Acyl-CoA thioesterase I Acyl-CoA thioesterase I Acyl-CoA thioesterase I Acyl-CoA thioesterase I Acyl-CoA thioesterase I Acyl-CoA thioesterase I Acyl-CoA thioesterase I Acyl-CoA thioesterase I Acyl-CoA thioesterase I Acyl-CoA thioesterase I Acyl-CoA thioesterase I Acyl-CoA thioesterase I Acyl-CoA thioesterase I Acyl-CoA thioesterase I Acyl-CoA thioesterase I Acyl-CoA thioesterase I Acyl-CoA thioesterase I Acyl-CoA thioesterase I Acyl-CoA thioesterase I Acyl-CoA thioesterase I Acyl-CoA thioesterase I Acyl-CoA thioesterase I Acyl-CoA thioesterase I Acyl-CoA thioesterase I Acyl-CoA thioesterase I Acyl-CoA thioesterase I Acyl-CoA thioesterase I Acyl-CoA thioesterase I Acyl-CoA thioesterase I Acyl-CoA thioesterase I Acyl-CoA thioesterase I Acyl-CoA thioesterase I
Lipase GDSL 2 (II)

Lipase GDSL 2 (II)

Lipase_GDSL_2 (II)

Lipase GDSL 2 (II)

Lipase_GDSL_2 (II)

Lipase_GDSL_2 (II)

Lipase_GDSL 2 (II)

Lipase GDSL 2 (II)

Lipase_GDSL_2 (II)

Lipase_GDSL_2 (II)

Lipase_GDSL_2 (II)

Lipase GDSL 2 (II)

Lipase_GDSL_2 (II)

Lipase_GDSL_2 (II)

Lipase GDSL 2 (II)

Lipase_GDSL_2 (II)

Lipase_GDSL_2 (II)

Lipase GDSL 2 (II)

Lipase_GDSL_2 (II)

Lipase_GDSL_2 (II)

Lipase_GDSL_2 (II)

Lipase GDSL 2 (II)

Lipase_GDSL_2 (II)

Lipase_GDSL_2 (II)

Lipase GDSL 2 (II)

Lipase GDSL 2 (II)

Lipase_GDSL_2 (II)

Lipase_GDSL_2 (II)

Lipase GDSL 2 (II)

Lipase_GDSL_2 (II)

Lipase_GDSL_2 (II)

Lipase_GDSL 2 (II)

Lipase GDSL 2 (II)

Lipase_GDSL_2 (II)

Lipase_GDSL_2 (II)

Lipase_GDSL_2 (II)

Lipase_GDSL_2 (II)

Lipase_GDSL_2 (II)

Lipase_GDSL 2 (II)

Lipase GDSL 2 (II)

Lipase_GDSL_2 (II)

Lipase_GDSL_2 (II)

Lipase GDSL 2 (II)

Lipase GDSL 2 (II)

Lipase_GDSL_2 (II) 
A0A1J5Q6Y2 A0A1J5R2D9 A0A1J5RJY5 A0A1J5S9V8

A0A1J5SBR3 A0A1J5SZP4 A0A1J5TMA6 A0A1M9K390 A0A1N7SPK8 A0A1Q9QUR5 A0A1R4H672 A0A1R7Q8F7 A0A1V5FB52 A0A1V5FZ99

A0A1V5QVA5 A0A1W1I3M1 A0A1Y0N3S6 A0A1Y6JN14 A0A221V155 A0A222P1J4

A0A238DU51

A0A239RYM4

A0A239SIU4

A0A2H5XLT0

A0A2H6A0X2

K1LKT4

M7MWS1

Q9HZY8

V4XZF9

V5BQM9

V6ACN8

W5YPF5

W7W0N3

W7WJE7

A0A089X6P7

A0A0K2APQ7

A0A0K2YGH5

A0A0N1FSK5

A0A0N1G8S1

A0A0N1NB57

A0A0N1NV23

A0A0T9M857

A0A0T9MCT6

A0A0U5LEC0

A0A0U5LH42
Acyl-CoA thioesterase I Acyl-CoA thioesterase I Acyl-CoA thioesterase I Acyl-CoA thioesterase I Acyl-CoA thioesterase I Acyl-CoA thioesterase I Acyl-CoA thioesterase I Acyl-CoA thioesterase I Acyl-CoA thioesterase I Acyl-CoA thioesterase I Acyl-CoA thioesterase I Acyl-CoA thioesterase I Acyl-CoA thioesterase I Acyl-CoA thioesterase I Acyl-CoA thioesterase I Acyl-CoA thioesterase I Acyl-CoA thioesterase I Acyl-CoA thioesterase I Acyl-CoA thioesterase I Acyl-CoA thioesterase I Acyl-CoA thioesterase I Acyl-CoA thioesterase I Acyl-CoA thioesterase I Acyl-CoA thioesterase I Acyl-CoA thioesterase I Acyl-CoA thioesterase I Acyl-CoA thioesterase I Acyl-CoA thioesterase I Acyl-CoA thioesterase I Acyl-CoA thioesterase I Acyl-CoA thioesterase I Acyl-CoA thioesterase I Acyl-CoA thioesterase I Acyl-CoA thioesterase I Putative secreted hydrolase Putative secreted hydrolase Putative secreted hydrolase Putative secreted hydrolase Putative secreted hydrolase Putative secreted hydrolase Putative secreted hydrolase Putative secreted hydrolase Putative secreted hydrolase Putative secreted hydrolase Putative secreted hydrolase
Lipase GDSL 2 (II)

Lipase GDSL 2 (II)

Lipase_GDSL_2 (II)

Lipase GDSL 2 (II)

Lipase_GDSL_2 (II)

Lipase_GDSL_2 (II)

Lipase_GDSL_2 (II)

Lipase GDSL 2 (II)

Lipase_GDSL_2 (II)

Lipase_GDSL_2 (II)

Lipase_GDSL_2 (II)

Lipase GDSL 2 (II)

Lipase_GDSL_2 (II)

Lipase_GDSL_2 (II)

Lipase GDSL 2 (II)

Lipase_GDSL_2 (II)

Lipase_GDSL_2 (II)

Lipase GDSL_2 (II)

Lipase_GDSL_2 (II)

Lipase_GDSL_2 (II)

Lipase_GDSL_2 (II)

Lipase GDSL 2 (II)

Lipase_GDSL_2 (II)

Lipase_GDSL_2 (II)

Lipase GDSL 2 (II)

Lipase GDSL 2 (II)

Lipase_GDSL_2 (II)

Lipase_GDSL_2 (II)

Lipase GDSL 2 (II)

Lipase_GDSL_2 (II)

Lipase_GDSL_2 (II)

Lipase_GDSL 2 (II)

Lipase GDSL 2 (II)

Lipase_GDSL_2 (II)

Lipase_GDSL_2 (II)

Lipase_GDSL_2 (II)

Lipase GDSL 2 (II)

Lipase_GDSL_2 (II)

Lipase_GDSL 2 (II)

Lipase GDSL 2 (II)

Lipase_GDSL_2 (II)

Lipase_GDSL_2 (II)

Lipase_GDSL_2 (II)

Lipase GDSL 2 (II)

Lipase_GDSL_2 (II) 
A0A0U5LIQ2 A0A0U5LZ22 A0A100JAY0 A0A100JN53

A0A101RXH9 A0A117EEV0 A0A124C2F1

A0A161I105

A0A 177 HFK 3

A0A177HFS2

A0A177HPT5

A0A177HYI6

A0A178X1Y4

A0A178XEM1

A0A1A9GGR0

A0A1A9GRX0

A0A1B2H079

A0A1B9EN57

A0A1B9EP94

A0A1B9EUB5

A0A1D2IIH5

A0A1D8C1F8

A0A1D8FY73

A0A1K2FJY5

A0A1K2FK87

A0A1K2FWE4

A0A1K2G0G8

A0A1P8Y9C1

A0A1Q2ZMI7

A0A1Q2ZNA0

A0A1U2G8U4

A0A1U2UMR2

A0A1V2MND5

A0A1V2MS28

A0A1V2MTK7

A0A1V2RA89

A0A1V2RFC3

A0A1Y2MP54

A0A1Y2N1Y0

A0A1Y2NGJ5

A0A1Y2NN23

A0A221W7P5

A0A222TGI3

A0A222TK45

A0A222TQN6
Putative secreted hydrolase Putative secreted hydrolase Putative secreted hydrolase Putative secreted hydrolase Putative secreted hydrolase Putative secreted hydrolase Putative secreted hydrolase

Putative secreted hydrolase Putative secreted hydrolase Putative secreted hydrolase Putative secreted hydrolase Putative secreted hydrolase Putative secreted hydrolase Putative secreted hydrolase Putative secreted hydrolase Putative secreted hydrolase Putative secreted hydrolase Putative secreted hydrolase Putative secreted hydrolase Putative secreted hydrolase Putative secreted hydrolase Putative secreted hydrolase Putative secreted hydrolase Putative secreted hydrolase Putative secreted hydrolase Putative secreted hydrolase Putative secreted hydrolase Putative secreted hydrolase Putative secreted hydrolase Putative secreted hydrolase Putative secreted hydrolase Putative secreted hydrolase Putative secreted hydrolase Putative secreted hydrolase Putative secreted hydrolase Putative secreted hydrolase Putative secreted hydrolase Putative secreted hydrolase Putative secreted hydrolase Putative secreted hydrolase Putative secreted hydrolase Putative secreted hydrolase Putative secreted hydrolase Putative secreted hydrolase Putative secreted hydrolase

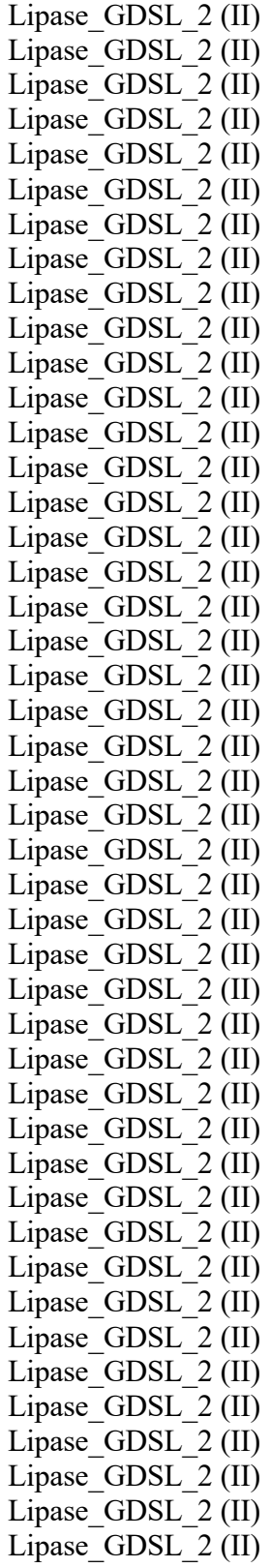

II
II
II
II
II
II
II
II
II
II
II
II
II
II
II
II
II
II
II
II
II
II
II
II
II
II
II
II
II
II
II
II
II
II
II
II
II
II
II
II
II
II
II
II
II




\begin{tabular}{|c|c|c|c|c|}
\hline A0A231GU95 & Putative secreted hydrolase & Lipase_GDSL_2 (II) & II & II \\
\hline A0A250VES8 & Putative secreted hydrolase & Lipase_GDSL_2 (II) & II & II \\
\hline A0ACN1 & Putative secreted hydrolase & Lipase_GDSL_2 (II) & II & II \\
\hline A3KIR8 & Putative secreted hydrolase & Lipase_GDSL_2 (II) & II & II \\
\hline D1A8A5 & Putative secreted hydrolase & Lipase_GDSL_2 (II) & II & II \\
\hline D2AXW6 & Putative secreted hydrolase & Lipase_GDSL_2 (II) & II & II \\
\hline D2PMS0 & Putative secreted hydrolase & Lipase_GDSL_2 (II) & II & II \\
\hline D3CSD1 & Putative secreted hydrolase & Lipase_GDSL_2 (II) & II & II \\
\hline D3EZZ4 & Putative secreted hydrolase & Lipase_GDSL_2 (II) & II & II \\
\hline D3FC90 & Putative secreted hydrolase & Lipase_GDSL_2 (II) & II & II \\
\hline D3PZ28 & Putative secreted hydrolase & Lipase_GDSL_2 (II) & II & II \\
\hline D3Q6I6 & Putative secreted hydrolase & Lipase_GDSL_2 (II) & II & II \\
\hline D3Q953 & Putative secreted hydrolase & Lipase_GDSL_2 (II) & II & II \\
\hline D6EML8 & Putative secreted hydrolase & Lipase_GDSL_2 (II) & II & II \\
\hline D6EUD8 & Putative secreted hydrolase & Lipase_GDSL_2 (II) & II & II \\
\hline D9T622 & Putative secreted hydrolase & Lipase_GDSL_2 (II) & II & II \\
\hline D9T737 & Putative secreted hydrolase & Lipase_GDSL_2 (II) & II & II \\
\hline E8W5N2 & Putative secreted hydrolase & Lipase_GDSL_2 (II) & II & II \\
\hline E8WF38 & Putative secreted hydrolase & Lipase_GDSL_2 (II) & II & II \\
\hline F6FXF1 & Putative secreted hydrolase & Lipase_GDSL_2 (II) & II & II \\
\hline G0Q5X5 & Putative secreted hydrolase & Lipase_GDSL_2 (II) & II & II \\
\hline G0Q684 & Putative secreted hydrolase & Lipase_GDSL_2 (II) & II & II \\
\hline G2NLA7 & Putative secreted hydrolase & Lipase_GDSL_2 (II) & II & II \\
\hline G8S919 & Putative secreted hydrolase & Lipase_GDSL_2 (II) & II & II \\
\hline G8SAJ2 & Putative secreted hydrolase & Lipase_GDSL_2 (II) & II & II \\
\hline K0K0J4 & Putative secreted hydrolase & Lipase_GDSL_2 (II) & II & II \\
\hline K0KD57 & Putative secreted hydrolase & Lipase_GDSL_2 (II) & II & II \\
\hline K4RCW1 & Putative secreted hydrolase & Lipase_GDSL_2 (II) & II & II \\
\hline N0CT46 & Putative secreted hydrolase & Lipase_GDSL_2 (II) & II & II \\
\hline N0CWZ8 & Putative secreted hydrolase & Lipase_GDSL_2 (II) & II & II \\
\hline Q93J06 & Putative secreted hydrolase & Lipase_GDSL_2 (II) & II & II \\
\hline Q93MW7 & Putative secreted hydrolase & Lipase_GDSL_2 (II) & II & II \\
\hline Q9S2A5 & Putative secreted hydrolase & Lipase_GDSL_2 (II) & II & II \\
\hline W5W553 & Putative secreted hydrolase & Lipase_GDSL_2 (II) & II & II \\
\hline A0A066WSZ1 & YMR313Cp-like protein & Patatin & patatin-like-protein & patatin-like-protein \\
\hline A0A0L8V990 & YMR313Cp-like protein & Patatin & patatin-like-protein & patatin-like-protein \\
\hline A0A1S5VH73 & YMR313Cp-like protein & Patatin & patatin-like-protein & patatin-like-protein \\
\hline W2UM92 & YMR313Cp-like protein & Patatin & patatin-like-protein & patatin-like-protein \\
\hline A0A0A3W7A6 & Patatin-like phospholipase domain-containing protein & Patatin & patatin-like-protein & patatin-like-protein \\
\hline A0A0X8R679 & Patatin-like phospholipase domain-containing protein & Patatin & patatin-like-protein & patatin-like-protein \\
\hline B5JS88 & Patatin-like phospholipase domain-containing protein & Patatin & patatin-like-protein & patatin-like-protein \\
\hline B8KFQ3 & Patatin-like phospholipase domain-containing protein & Patatin & patatin-like-protein & patatin-like-protein \\
\hline B8KQY9 & Patatin-like phospholipase domain-containing protein & Patatin & patatin-like-protein & patatin-like-protein \\
\hline B8KXA8 & Patatin-like phospholipase domain-containing protein & Patatin & patatin-like-protein & patatin-like-protein \\
\hline
\end{tabular}

Dataset 2 


\begin{tabular}{|c|c|c|c|c|}
\hline AcXE2 & Isoamyl acetate-hydrolyzing esterase 1 homolog & Lipase_GDSL_2 (II) & II & II \\
\hline Est01 & Beta-lactamase (Penicillin-binding protein) (Penicillinase) & Beta-lactamase (VIII) & VIII & VIII \\
\hline Est2 & Class $\mathrm{C}$ beta-lactamase CMY-10 & Beta-lactamase (VIII) & VIII & VIII \\
\hline Est22_2 & Beta-lactamase (Penicillin-binding protein) (Penicillinase) & Beta-lactamase (VIII) & VIII & VIII \\
\hline Est7K & Beta-lactamase (Penicillin-binding protein) (Penicillinase) & Beta-lactamase (VIII) & VIII & VIII \\
\hline EstA3_2 & Beta-lactamase (Penicillin-binding protein) (Penicillinase) & Beta-lactamase (VIII) & VIII & VIII \\
\hline EstBL $^{-}$ & Beta-lactamase (Penicillin-binding protein) (Penicillinase) & Beta-lactamase (VIII) & VIII & VIII \\
\hline EstC & Beta-lactamase (Penicillin-binding protein) (Penicillinase) & Beta-lactamase (VIII) & VIII & VIII \\
\hline EstCE1 & Beta-lactamase (Penicillin-binding protein) (Penicillinase) & Beta-lactamase (VIII) & VIII & VIII \\
\hline EstF4K & Beta-lactamase (Penicillin-binding protein) (Penicillinase) & Beta-lactamase (VIII) & VIII & VIII \\
\hline EstHE1 & Acyl-CoA thioesterase I & Lipase_GDSL_2 (II) & II & II \\
\hline EstM-N1 & Beta-lactamase (Penicillin-binding protein) (Penicillinase) & Beta-lactamase (VIII) & VIII & VIII \\
\hline EstM-N2 & Beta-lactamase (Penicillin-binding protein) (Penicillinase) & Beta-lactamase (VIII) & VIII & VIII \\
\hline EstQE & Beta-lactamase (Penicillin-binding protein) (Penicillinase) & Beta-lactamase (VIII) & VIII & VIII \\
\hline EstSL3 & Acyl-CoA thioesterase I & Lipase_GDSL_2 (II) & II & II \\
\hline EstSTR1 & Class $\mathrm{C}$ beta-lactamase CMY-10 & Beta-lactamase (VIII) & VIII & VIII \\
\hline EstU1 & Beta-lactamase (Penicillin-binding protein) (Penicillinase) & Beta-lactamase (VIII) & VIII & VIII \\
\hline LAE2 & Acyl-CoA thioesterase I & Lipase_GDSL_2 (II) & II & II \\
\hline LAE5 & Isoamyl acetate-hydrolyzing esterase 1 homolog & Lipase_GDSL_2 (II) & II & II \\
\hline LAE7 & Acyl-CoA thioesterase I & Lipase_GDSL_2 (II) & II & II \\
\hline LipA9 & Beta-lactamase (Penicillin-binding protein) (Penicillinase) & Beta-lactamase (VIII) & VIII & VIII \\
\hline LipBL & Beta-lactamase (Penicillin-binding protein) (Penicillinase) & Beta-lactamase (VIII) & VIII & VIII \\
\hline Lipo12B & Beta-lactamase (Penicillin-binding protein) (Penicillinase) & Beta-lactamase (VIII) & VIII & VIII \\
\hline Lipo13 & Isoamyl acetate-hydrolyzing esterase 1 homolog & Lipase_GDSL_2 (II) & II & II \\
\hline Lipo4B & YMR313Cp-like protein & $\begin{array}{l}\text { Patatin (patatin-like- } \\
\text { protein) }\end{array}$ & patatin-like-protein & patatin-like-protein \\
\hline Lipo4C & $\mathrm{ND}^{\mathrm{c}}$ & $\begin{array}{l}\text { Patatin (patatin-like- } \\
\text { protein) }\end{array}$ & unassigned & patatin-like-protein \\
\hline Lipo8 & Acyl-CoA thioesterase I & Lipase_GDSL_2 (II) & II & II \\
\hline Lpc53E1 & Beta-lactamase (Penicillin-binding protein) (Penicillinase) & Beta-lactamase (VIII) & VIII & VIII \\
\hline MGS0010 & Beta-lactamase (Penicillin-binding protein) (Penicillinase) & Beta-lactamase (VIII) & VIII & VIII \\
\hline MGS0105 & Beta-lactamase (Penicillin-binding protein) (Penicillinase) & Beta-lactamase (VIII) & VIII & VIII \\
\hline PLP & YMR313Cp-like protein & $\begin{array}{l}\text { Patatin (patatin-like- } \\
\text { protein) }\end{array}$ & patatin-like-protein & patatin-like-protein \\
\hline SBLip1 & Beta-lactamase (Penicillin-binding protein) (Penicillinase) & Beta-lactamase (VIII) & VIII & VIII \\
\hline \multicolumn{5}{|l|}{ Dataset 3} \\
\hline \multirow{2}{*}{$\begin{array}{l}\text { EstC55-4_2 } \\
\text { EstC55-6 }\end{array}$} & Beta-lactamase (Penicillin-binding protein) (Penicillinase) & \multirow{2}{*}{$\begin{array}{l}\text { Beta-lactamase (VIII) } \\
\text { Patatin (patatin-like- } \\
\text { protein) }\end{array}$} & VIII & \multirow{2}{*}{$\begin{array}{l}\text { VIII } \\
\text { patatin-like-protein }\end{array}$} \\
\hline & $\mathrm{ND}^{\mathrm{c}}$ & & unassigned & \\
\hline EstC55-7 & Beta-lactamase (Penicillin-binding protein) (Penicillinase) & \multirow{2}{*}{$\begin{array}{l}\text { Peta-lactamase (VIII) } \\
\text { patin (patatin-like- } \\
\text { protein) }\end{array}$} & VIII & \multirow{2}{*}{$\begin{array}{l}\text { VIII } \\
\text { patatin-like-protein }\end{array}$} \\
\hline EstC55-10 & $\mathrm{ND}^{\mathrm{c}}$ & & unassigned & \\
\hline EstC55-26 & YMR313Cp-like protein & $\begin{array}{l}\text { Patatin (patatin-like- } \\
\text { protein) }\end{array}$ & patatin-like-protein & patatin-like-protein \\
\hline EstC55-40 & Beta-lactamase (Penicillin-binding protein) (Penicillinase) & Beta-lactamase (VIII) & VIII & VIII \\
\hline
\end{tabular}




\begin{tabular}{|c|c|c|c|c|}
\hline EstC55-46 & Beta-lactamase (Penicillin-binding protein) (Penicillinase) & Beta-lactamase (VIII) & VIII & VIII \\
\hline EstC55-53 & Beta-lactamase (Penicillin-binding protein) (Penicillinase) & Beta-lactamase (VIII) & VIII & VIII \\
\hline EstC55-61 & Beta-lactamase (Penicillin-binding protein) (Penicillinase) & Beta-lactamase (VIII) & VIII & VIII \\
\hline EstC55-63 & YMR313Cp-like protein & $\begin{array}{l}\text { Patatin (patatin-like- } \\
\text { protein) }\end{array}$ & patatin-like-protein & patatin-like-protein \\
\hline EstC55-65 & Beta-lactamase (Penicillin-binding protein) (Penicillinase) & Beta-lactamase (VIII) & VIII & VIII \\
\hline EstC55-66 & Beta-lactamase (Penicillin-binding protein) (Penicillinase) & Beta-lactamase (VIII) & VIII & VIII \\
\hline EstC55-73 & Beta-lactamase (Penicillin-binding protein) (Penicillinase) & Beta-lactamase (VIII) & VIII & VIII \\
\hline EstC55-80 & Class $\mathrm{C}$ beta-lactamase $\mathrm{CMY}-10$ & Beta-lactamase (VIII) & VIII & VIII \\
\hline EstC55-110 & Beta-lactamase (Penicillin-binding protein) (Penicillinase) & Beta-lactamase (VIII) & VIII & VIII \\
\hline EstC55-111 & GDSL esterase/lipase APG & Lipase_GDSL (II) & II & II \\
\hline EstC55-113 & Beta-lactamase (Penicillin-binding protein) (Penicillinase) & Beta-lactamase (VIII) & VIII & VIII \\
\hline EstC55-131 & YMR313Cp-like protein & $\begin{array}{l}\text { Patatin (patatin-like- } \\
\text { protein) }\end{array}$ & patatin-like-protein & patatin-like-protein \\
\hline EstC55-147 & Beta-lactamase (Penicillin-binding protein) (Penicillinase) & Beta-lactamase (VIII) & VIII & VIII \\
\hline EstC55-150 & GDSL esterase/lipase APG & Lipase_GDSL_2 (II) & II & II \\
\hline EstC55-163 & $\mathrm{ND}^{\mathrm{c}}$ & $\begin{array}{l}\text { Patatin (patatin-like- } \\
\text { protein) }\end{array}$ & unassigned & patatin-like-protein \\
\hline EstC55-164 & Beta-lactamase (Penicillin-binding protein) (Penicillinase) & Beta-lactamase (VIII) & VIII & VIII \\
\hline EstC55-168 & Beta-lactamase (Penicillin-binding protein) (Penicillinase) & Beta-lactamase (VIII) & VIII & VIII \\
\hline EstC55-239 & Beta-lactamase (Penicillin-binding protein) (Penicillinase) & Beta-lactamase (VIII) & VIII & VIII \\
\hline EstC55-245 & Beta-lactamase (Penicillin-binding protein) (Penicillinase) & Beta-lactamase (VIII) & VIII & VIII \\
\hline EstC55-251 & YMR313Cp-like protein & $\begin{array}{l}\text { Patatin (patatin-like- } \\
\text { protein) }\end{array}$ & patatin-like-protein & patatin-like-protein \\
\hline EstC55-258 & Class C beta-lactamase CMY-10 & Beta-lactamase (VIII) & VIII & VIII \\
\hline EstC76-21 & $\mathrm{ND}^{\mathrm{c}}$ & $\begin{array}{l}\text { Patatin (patatin-like- } \\
\text { protein) }\end{array}$ & unassigned & patatin-like-protein \\
\hline EstC76-98 & Beta-lactamase (Penicillin-binding protein) (Penicillinase) & Beta-lactamase (VIII) & VIII & VIII \\
\hline EstC76-123 & Beta-lactamase (Penicillin-binding protein) (Penicillinase) & Beta-lactamase (VIII) & VIII & VIII \\
\hline EstC76-174 & Beta-lactamase (Penicillin-binding protein) (Penicillinase) & Beta-lactamase (VIII) & VIII & VIII \\
\hline EstC76-222 & YMR313Cp-like protein & $\begin{array}{l}\text { Patatin (patatin-like- } \\
\text { protein) }\end{array}$ & patatin-like-protein & patatin-like-protein \\
\hline EstC76-250 & YMR313Cp-like protein & $\begin{array}{l}\text { Patatin (patatin-like- } \\
\text { protein) }\end{array}$ & patatin-like-protein & patatin-like-protein \\
\hline EstC76-261 & $\mathrm{ND}^{\mathrm{c}}$ & $\begin{array}{l}\text { Patatin (patatin-like- } \\
\text { protein) }\end{array}$ & unassigned & patatin-like-protein \\
\hline EstC76-262 & Beta-lactamase (Penicillin-binding protein) (Penicillinase) & Beta-lactamase (VIII) & VIII & VIII \\
\hline EstC76-269 & Neuropathy target esterase sws & $\begin{array}{l}\text { Patatin (patatin-like- } \\
\text { protein) }\end{array}$ & patatin-like-protein & patatin-like-protein \\
\hline
\end{tabular}

${ }^{a}$ Lipolytic family assignment using the strategy developed in this study; unassigned, could not assigned to any lipolytic family

${ }^{\mathrm{b}}$ Lipolytic family assignment reported in literatures (or this study) using pylogenetic-related methods 
${ }^{c}$ No data 
Supplementary Table S14 Phylogenetic origin (at genus level) of assigned lipolytic genes and their corresponding contigs for compost55 and compost76

\begin{tabular}{|c|c|c|c|c|}
\hline \multirow[t]{2}{*}{ Bacterial genus $^{\text {a }}$} & \multicolumn{2}{|c|}{ Genes } & \multicolumn{2}{|c|}{$\begin{array}{c}\begin{array}{c}\text { Contigs harboring corresponding } \\
\text { genes }\end{array} \\
\end{array}$} \\
\hline & compost55 & compost76 & compost55 & compost76 \\
\hline $\begin{array}{l}\text { P_Actinobacteria|C_Actinobacteria|O_Streptosporangiales } \mid F_{-} \text {_Thermomonosporaceae } \\
\mid G_{-} \text {Thermomonospora }\end{array}$ & $22(1.99 \%)$ & $21(3.04 \%)$ & $30(2.48 \%)$ & $44(5.99 \%)$ \\
\hline $\begin{array}{l}\text { P_Actinobacteria|C_Actinobacteria|O_Streptomycetales } \mid \text { F_Streptomycetaceae } \\
\mid G_{-} \_ \text {Streptomyces }\end{array}$ & $40(3.62 \%)$ & $7(1.01 \%)$ & $24(1.99 \%)$ & $3(0.41 \%)$ \\
\hline P_Acidobacteria|C_unclassified_Acidobacteria & $34(3.08 \%)$ & $9(1.3 \%)$ & $40(3.31 \%)$ & $3(0.41 \%)$ \\
\hline $\begin{array}{l}\text { P_Actinobacteria|C_Actinobacteria } \mid{ }_{-} \_ \text {Micromonosporales } \mid \text { F_Micromonosporaceae } \\
\mid G_{-} \_ \text {Micromonospora }\end{array}$ & $40(3.62 \%)$ & $7(1.01 \%)$ & $37(3.06 \%)$ & $2(0.27 \%)$ \\
\hline uncultured_bacterium & $19(1.72 \%)$ & $11(1.59 \%)$ & $35(2.89 \%)$ & $9(1.22 \%)$ \\
\hline $\begin{array}{l}\text { P_Actinobacteria } \mid C_{-} \text {Actinobacteria } \mid O_{-} \text {Corynebacteriales } \mid F_{-} \text {Mycobacteriaceae } \\
\mid G_{-} \text {Mycobacterium }\end{array}$ & $23(2.08 \%)$ & $11(1.59 \%)$ & $18(1.49 \%)$ & $9(1.22 \%)$ \\
\hline $\begin{array}{l}\text { P_Actinobacteria|C_Actinobacteria|O_Pseudonocardiales } \mid \text { F_Pseudonocardiaceae } \\
\mid \bar{G} \_ \text {Pseudonocardia }\end{array}$ & $27(2.45 \%)$ & 0 & $38(3.14 \%)$ & $1(0.14 \%)$ \\
\hline $\begin{array}{l}\text { P_Actinobacteria|C_Actinobacteria } \mid \mathrm{O}_{-} \text {_Streptosporangiales } \mid \mathrm{F} \_ \text {Thermomonosporaceae } \\
\mid \overline{\mathrm{G}} \_ \text {Actinomadura }\end{array}$ & $22(1.99 \%)$ & $9(1.3 \%)$ & $10(0.83 \%)$ & $5(0.68 \%)$ \\
\hline P_Firmicutes $\mid$ C_Bacilli $\mid$ O_Bacillales $\mid$ F_Paenibacillaceae $\mid$ G_Paenibacillus & $11(1 \%)$ & $15(2.17 \%)$ & $3(0.25 \%)$ & $6(0.82 \%)$ \\
\hline 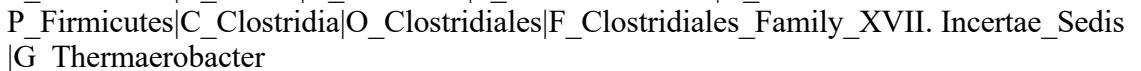 & $15(1.36 \%)$ & $13(1.88 \%)$ & $16(1.32 \%)$ & $13(1.77 \%)$ \\
\hline P_Chloroflexi|C_unclassified_Chloroflexi & $10(0.91 \%)$ & $1(0.14 \%)$ & $37(3.06 \%)$ & $3(0.41 \%)$ \\
\hline P Firmicutes $\mid \mathrm{C}$ Bacilli $\mid \mathrm{O}$ Bacillales $\mid \mathrm{F}$ Bacillaceae $\mid \mathrm{G}$ Bacillus & $13(1.18 \%)$ & $13(1.88 \%)$ & $5(0.41 \%)$ & $6(0.82 \%)$ \\
\hline P_Bacteroidetes $\mid$ C_unclassified_Bacteroidetes & $18(1.63 \%)$ & $8(1.16 \%)$ & $10(0.83 \%)$ & $6(0.82 \%)$ \\
\hline $\begin{array}{l}\text { P_Proteobacteria } \mid \bar{C} \_ \text {Deltaproteobacteria } \mid \text { O_Myxococcales } \mid \text { F_Nannocystaceae } \\
\mid \bar{G} \_ \text {Nannocystis }\end{array}$ & 0 & $10(1.45 \%)$ & 0 & $15(2.04 \%)$ \\
\hline P_Proteobacteria|C_Betaproteobacteria|O_unclassified_Betaproteobacteria & $15(1.36 \%)$ & $5(0.72 \%)$ & $22(1.82 \%)$ & $2(0.27 \%)$ \\
\hline $\begin{array}{l}\text { P_Proteobacteria|C_Gammaproteobacteria } \mid \text { O_Methylococcales } \mid \text { F_Methylococcaceae } \\
\mid \bar{G} \_ \text {Methylocaldum }\end{array}$ & $4(0.36 \%)$ & $5(0.72 \%)$ & $5(0.41 \%)$ & $8(1.09 \%)$ \\
\hline $\begin{array}{l}\text { P_Chloroflexi|C_Thermomicrobia } \mid \text { O_Sphaerobacterales } \mid \text { F_Sphaerobacteraceae } \\
\mid \bar{G} \_ \text {Sphaerobacter }\end{array}$ & $6(0.54 \%)$ & $1(0.14 \%)$ & $20(1.65 \%)$ & $1(0.14 \%)$ \\
\hline 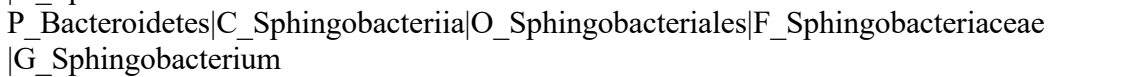 & 0 & $13(1.88 \%)$ & 0 & $17(2.31 \%)$ \\
\hline P_Bacteroidetes $\mid$ C_Chitinophagia|O_Chitinophagales $\mid$ F_Chitinophagaceae $\mid G_{-}$Niastella & $3(0.27 \%)$ & $7(1.01 \%)$ & $2(0.17 \%)$ & $15(2.04 \%)$ \\
\hline $\begin{array}{l}\text { P_Bacteroidetes } \mid \mathrm{C}_{-} \text {Chitinophagia } \mid \mathrm{O}_{-} \text {Chitinophagales } \mid \mathrm{F}_{-} \text {Chitinophagaceae } \\
\mid \mathrm{G}_{-} \text {Flavihumibacter }\end{array}$ & 0 & $8(1.16 \%)$ & 0 & $12(1.63 \%)$ \\
\hline P_Firmicutes $\mid$ C_Clostridia $\left|\mathrm{O} \_C l o s t r i d i a l e s\right|$ F_Clostridiaceae $\mid$ G_Clostridium & $8(0.72 \%)$ & $10(1.45 \%)$ & $2(0.17 \%)$ & $8(1.09 \%)$ \\
\hline $\begin{array}{l}\text { P_Actinobacteria|C_Actinobacteria|O_Streptosporangiales } \mid \text { F_Streptosporangiaceae } \\
\mid G_{-} \_ \text {Nonomuraea }\end{array}$ & $10(0.91 \%)$ & $2(0.29 \%)$ & $10(0.83 \%)$ & 0 \\
\hline P_Gemmatimonadetes $\mid$ C_unclassified_Gemmatimonadetes & $12(1.09 \%)$ & 0 & $18(1.49 \%)$ & 0 \\
\hline
\end{tabular}


P Actinobacteria|C Actinobacteria|G Thermobispora

P_Proteobacteria|C_Gammaproteobacteria|O_unclassified_Gammaproteobacteria

P_Actinobacteria $\mid C_{-}$Actinobacteria $\mid O_{-}$Propionibacteriales $\mid$F_Nocardioidaceae

G Actinopolymorpha

P Actinobacteria $\mid$ C_Actinobacteria $\mid$ O_Pseudonocardiales $\mid$ F_Pseudonocardiaceae

$\mid \bar{G} \_$Thermocrispum

$\mathrm{P}$ Firmicutes $\mid \mathrm{C}$ Clostridia $\mid \mathrm{O}$ Clostridiales $\mid \mathrm{F}$ unclassified Clostridiales

$\mathrm{P}$ Actinobacteria $\mid \mathrm{C}$ Actinobacteria $\mid \mathrm{O}$ Pseudonocardiales $\mid \mathrm{F}$ Pseudonocardiaceae

$\mid \bar{G} \_$Amycolatopsis

P_Proteobacteria|C_Gammaproteobacteria|O_Pseudomonadales $\mid$ F_Pseudomonadaceae

G Pseudomonas

P Proteobacteria|C Gammaproteobacteria|O Xanthomonadales $\mid \mathrm{F}$ Xanthomonadaceae

P_Proteobacteria|C_Alphaproteobacteria|O_Rhizobiales $\mid$ F_Beijerinckiaceae

G Chelatococcus

P_Actinobacteria $\mid$ C_Actinobacteria $\mid$ O_Streptosporangiales $\mid$ F_Nocardiopsaceae

$\mid \bar{G}$ Thermobifida

P Firmicutes $\mid \mathrm{C}$ Clostridia $\mid \mathrm{O}$ Clostridiales $\mid \mathrm{F}$ Ruminococcaceae $\mid \mathrm{G}$ Ruminiclostridium

$\mathrm{P}$ Bacteroidetes $\mid \mathrm{C}$ Flavobacteriia $\mid \mathrm{O}$ Flavobacteriales $\mid \mathrm{F}$ Flavobacteriaceae

$\mid \bar{G}$ Flavobacterium

P_Firmicutes $\mid$ C_Bacilli $\mid \mathrm{O} \_$Bacillales $\mid \mathrm{F} \_$Bacillaceae $\mid \mathrm{G}$ _unclassified_Bacillaceae

$\mathrm{P}$ Bacteroidetes $\mid \mathrm{O}$ Bacteroidetes Order II. Incertae sedis $\mid \mathrm{F}$ Rhodothermaceae

G Rhodothermus

P_Actinobacteria|C_Actinobacteria|O_unclassified_Actinobacteria

$\mathrm{P}$ Proteobacteria|C Alphaproteobacteria $\mid \mathrm{O}$ unclassified Alphaproteobacteria

P Proteobacteria $\mid \mathrm{C}$ Gammaproteobacteria $\mid \mathrm{O}$ Nevskiales $\mid \mathrm{F}$ Sinobacteraceae

P_Actinobacteria|C_Actinobacteria|O_Micromonosporales $\mid$ F_Micromonosporaceae

G Actinoplanes

P Firmicutes $\mid \mathrm{C}$ Bacilli $\mid \mathrm{O}$ Bacillales $\mid \mathrm{F}$ Bacillaceae $\mid \mathrm{G}$ Calditerricola

P_Deinococcus-Thermus $\mid \bar{C} \_$Deinococci $\mid \mathrm{O}_{-}$Thermales $\mid \mathrm{F} \_$Thermaceae $\mid \mathrm{G}_{-}$Thermus

P Gemmatimonadetes $\mid$ C_Gemmatimonadetes $\mid \mathrm{O}$ Gemmatimonadales

F Gemmatimonadaceae

G- Gemmatimonas

P_Actinobacteria $\mid$ C_Actinobacteria $\mid \mathrm{O}_{-}$Corynebacteriales $\mid \mathrm{F} \_$Nocardiaceae $\mid \mathrm{G} \_$Nocardia

$\mathrm{P}$ Firmicutes $\mid \mathrm{C}$ Bacilli $\mid \mathrm{O}$ Bacillales $\mid \mathrm{F}$ Thermoactinomycetaceae $\mid \mathrm{G}$ Planifilum

$\mathrm{P}$ Proteobacteria $\mid \mathrm{C}$ Deltaproteobacteria $\mid \mathrm{O}$ Myxococcales $\mid \mathrm{F}$ unclassified Myxococcales

P_Firmicutes $\mid$ C_Clostridia|O_Clostridiales $\mid$ F_Caldicoprobacteraceae $\mid G_{-}$Caldicoprobacter

P_Firmicutes $\mid$ C_Bacilli $\mid \mathrm{O} \_$Bacillales $\mid \mathrm{F} \_$Alicyclobacillaceae $\mid \mathrm{G} \_$Alicyclobacillus

Proteobacteria|C Alphaproteobacteria|O Rhizobiales $\mid \mathrm{F}$ Bradyrhizobiaceae $\overline{\text { G }}$ Bradyrhizobium $\mid \overline{\mathrm{G}}$ Pseudoxanthomonas

$\mid \bar{G} \_$Steroidobacter

\begin{tabular}{|c|c|c|c|}
\hline $10(0.91 \%)$ & $2(0.29 \%)$ & $19(1.57 \%)$ & $6(0.82 \%)$ \\
\hline $13(1.18 \%)$ & $5(0.72 \%)$ & $20(1.65 \%)$ & $2(0.27 \%)$ \\
\hline $7(0.63 \%)$ & 0 & $21(1.74 \%)$ & 0 \\
\hline $11(1 \%)$ & 0 & $18(1.49 \%)$ & 0 \\
\hline $3(0.27 \%)$ & $8(1.16 \%)$ & $2(0.17 \%)$ & $5(0.68 \%)$ \\
\hline $13(1.18 \%)$ & $3(0.43 \%)$ & $6(0.5 \%)$ & 0 \\
\hline $5(0.45 \%)$ & $9(1.3 \%)$ & $5(0.41 \%)$ & $5(0.68 \%)$ \\
\hline $1(0.09 \%)$ & $11(1.59 \%)$ & $1(0.08 \%)$ & $17(2.31 \%)$ \\
\hline $1(0.09 \%)$ & $10(1.45 \%)$ & $3(0.25 \%)$ & $10(1.36 \%)$ \\
\hline 0 & $8(1.16 \%)$ & $1(0.08 \%)$ & $12(1.63 \%)$ \\
\hline $2(0.18 \%)$ & $9(1.3 \%)$ & $3(0.25 \%)$ & $15(2.04 \%)$ \\
\hline $3(0.27 \%)$ & $8(1.16 \%)$ & $2(0.17 \%)$ & $7(0.95 \%)$ \\
\hline $3(0.27 \%)$ & $4(0.58 \%)$ & $4(0.33 \%)$ & $6(0.82 \%)$ \\
\hline $13(1.18 \%)$ & 0 & $18(1.49 \%)$ & 0 \\
\hline $\begin{array}{l}3(0.27 \%) \\
6(0.54 \%)\end{array}$ & $\begin{array}{l}1(0.14 \%) \\
2(0.29 \%)\end{array}$ & $\begin{array}{l}9(0.74 \%) \\
4(0.33 \%)\end{array}$ & $\begin{array}{l}1(0.14 \%) \\
0\end{array}$ \\
\hline $4(0.36 \%)$ & $5(0.72 \%)$ & $4(0.33 \%)$ & $16(2.18 \%)$ \\
\hline $8(0.72 \%)$ & $1(0.14 \%)$ & $13(1.08 \%)$ & 0 \\
\hline $4(0.36 \%)$ & $\begin{array}{l}7(1.01 \%) \\
6(0.87 \%)\end{array}$ & $\begin{array}{l}0 \\
4(0.33 \%)\end{array}$ & $\begin{array}{l}9(1.22 \%) \\
4(0.54 \%)\end{array}$ \\
\hline $10(0.91 \%)$ & $1(0.14 \%)$ & $22(1.82 \%)$ & $1(0.14 \%)$ \\
\hline $4(0.36 \%)$ & $1(0.14 \%)$ & $4(0.33 \%)$ & $2(0.27 \%)$ \\
\hline $1(0.09 \%)$ & $4(0.58 \%)$ & $2(0.17 \%)$ & $4(0.54 \%)$ \\
\hline $5(0.45 \%)$ & $2(0.29 \%)$ & $3(0.25 \%)$ & $5(0.68 \%)$ \\
\hline 0 & $7(1.01 \%)$ & 0 & $6(0.82 \%)$ \\
\hline 0 & $1(0.14 \%)$ & $3(0.25 \%)$ & $5(0.68 \%)$ \\
\hline $10(0.91 \%)$ & $2(0.29 \%)$ & $7(0.58 \%)$ & $1(0.14 \%)$ \\
\hline
\end{tabular}

${ }^{a}$ Only genera from abundant orders (greater than $1 \%$ ) are given. In the case the order or genus could not be assigned, the taxonomic name at the highest determined taxonomic resolution is given in parenthesis. 
Supplementary Table S15 Mapping coverage of FA-identified lipolytic genes by metagenome short reads

\begin{tabular}{|c|c|c|c|c|}
\hline Gene name & $\begin{array}{l}\text { Gene length } \\
\text { (bp) }\end{array}$ & $\begin{array}{l}\text { Nr. of reads } \\
\text { mapped on gene } \\
\text { (read depth) }\end{array}$ & $\begin{array}{l}\text { Total number of covered } \\
\text { bases (with }>=1 \mathrm{X} \\
\text { coverage depth) }\end{array}$ & $\begin{array}{l}\text { Breadth of coverage } \\
(\% \text {, at } 1 \mathrm{X} \text { coverage } \\
\text { depth })\end{array}$ \\
\hline estC55-2 & 876 & 41 & 876 & 100 \\
\hline estC55-3 & 1542 & 815 & 1542 & 100 \\
\hline estC55-4_1 & 816 & 102 & 816 & 100 \\
\hline estC55-4_2 & 1173 & 157 & 1173 & 100 \\
\hline estC55-5 & 933 & 442 & 933 & 100 \\
\hline estC55-6 & 1038 & 83 & 1038 & 100 \\
\hline estC55-7 & 1293 & 35 & 1068 & 82.6 \\
\hline estC55-8_1 & 957 & 69 & 954 & 99.7 \\
\hline estC55-8_2 & 813 & 74 & 813 & 100 \\
\hline estC55-10 & 867 & 579 & 867 & 100 \\
\hline estC55-12 & 1155 & 1419 & 1155 & 100 \\
\hline estC55-13 & 1068 & 93 & 1067 & 99.9 \\
\hline estC55-15 & 762 & 853 & 762 & 100 \\
\hline estC55-18 & 804 & 9 & 723 & 90 \\
\hline estC55-19_1 & 780 & 131 & 780 & 100 \\
\hline estC55-19_2 & 957 & 147 & 957 & 100 \\
\hline estC55-20 & 1053 & 181 & 1053 & 100 \\
\hline estC55-23 & 969 & 43 & 968 & 99.9 \\
\hline estC55-24 & 978 & 63 & 978 & 100 \\
\hline estC55-25 & 1104 & 170 & 1104 & 100 \\
\hline estC55-26 & 954 & 843 & 954 & 100 \\
\hline estC55-31 & 798 & 43 & 796 & 99.7 \\
\hline estC55-34 & 873 & 939 & 873 & 100 \\
\hline estC55-38 & 990 & 4 & 439 & 44.3 \\
\hline estC55-40 & 1335 & 119 & 1335 & 100 \\
\hline estC55-42 & 1122 & 1449 & 1122 & 100 \\
\hline estC55-46 & 1248 & 24 & 638 & 51.1 \\
\hline estC55-51 & 807 & 858 & 807 & 100 \\
\hline estC55-52 & 1512 & 90 & 1512 & 100 \\
\hline estC55-53 & 1221 & 11 & 1106 & 90.6 \\
\hline estC55-56 & 945 & 32 & 944 & 99.9 \\
\hline estC55-57 & 816 & 36 & 774 & 95 \\
\hline estC55-60 & 1023 & 26 & 971 & 95 \\
\hline estC55-61 & 1167 & 28 & 982 & 84.1 \\
\hline estC55-62 & 1587 & 135 & 1586 & 99.9 \\
\hline estC55-63 & 954 & 488 & 954 & 100 \\
\hline estC55-65 & 1230 & 92 & 1230 & 100 \\
\hline estC55-66 & 1290 & 78 & 1290 & 100 \\
\hline estC55-71 & 954 & 73 & 951 & 99.7 \\
\hline estC55-72 & 897 & 20 & 879 & 98 \\
\hline estC55-73 & 1158 & 47 & 1154 & 99.6 \\
\hline estC55-76 & 1122 & 90 & 1117 & 99.5 \\
\hline estC55-77 & 837 & 13 & 591 & 70.6 \\
\hline estC55-78 & 960 & 104 & 957 & 99.7 \\
\hline estC55-80 & 1227 & 308 & 1227 & 100 \\
\hline estC55-81 & 849 & 61 & 846 & 99.6 \\
\hline estC55-88 & 954 & 56 & 953 & 99.9 \\
\hline estC55-90 & 861 & 55 & 860 & 99.9 \\
\hline
\end{tabular}




\begin{tabular}{|c|c|c|c|c|}
\hline estC55-95 & 867 & 60 & 866 & 99.9 \\
\hline estC55-96 & 804 & 1313 & 804 & 100 \\
\hline estC55-97 & 723 & 8 & 720 & 99.6 \\
\hline estC55-100 & 1131 & 4 & 365 & 32.3 \\
\hline estC55-102 & 2124 & 41 & 1979 & 93.2 \\
\hline estC55-105 & 672 & 730 & 672 & 100 \\
\hline estC55-110 & 1305 & 32 & 1304 & 99.9 \\
\hline estC55-111 & 1179 & 2088 & 1179 & 100 \\
\hline estC55-113 & 1230 & 20 & 874 & 71.1 \\
\hline estC55-118 & 1494 & 16 & 1230 & 82.3 \\
\hline estC55-131 & 756 & 32 & 755 & 99.9 \\
\hline estC55-145 & 918 & 955 & 918 & 100 \\
\hline estC55-147 & 1161 & 186 & 1161 & 100 \\
\hline estC55-150 & 1182 & 1469 & 1182 & 100 \\
\hline estC55-151 & 936 & 22 & 935 & 99.9 \\
\hline estC55-154 & 1185 & 50 & 1183 & 99.9 \\
\hline estC55-156 & 1554 & 37 & 1519 & 97.7 \\
\hline estC55-159 & 855 & 7 & 634 & 74.1 \\
\hline estC55-163 & 912 & 46 & 912 & 100 \\
\hline estC55-164 & 1293 & 0 & 0 & 0 \\
\hline estC55-165 & 834 & 1783 & 834 & 100 \\
\hline estC55-167 & 747 & 24 & 747 & 100 \\
\hline estC55-168 & 1542 & 1254 & 1542 & 100 \\
\hline estC55-169 & 1602 & 96 & 1581 & 98.6 \\
\hline estC55-186 & 1548 & 84 & 1540 & 99.5 \\
\hline estC55-188 & 714 & 37 & 714 & 100 \\
\hline estC55-197 & 876 & 584 & 876 & 100 \\
\hline estC55-213 & 861 & 1711 & 861 & 100 \\
\hline estC55-215 & 792 & 123 & 792 & 100 \\
\hline estC55-227 & 1419 & 1616 & 1419 & 100 \\
\hline estC55-229 & 936 & 28 & 929 & 99.2 \\
\hline estC55-231 & 1101 & 61 & 1071 & 97.3 \\
\hline estC55-234 & 1674 & 17 & 1380 & 82.4 \\
\hline estC55-235 & 981 & 540 & 981 & 100 \\
\hline estC55-239 & 1146 & 3222 & 1146 & 100 \\
\hline estC55-241 & 1704 & 1707 & 1704 & 100 \\
\hline estC55-244 & 768 & 46 & 747 & 97.3 \\
\hline estC55-245 & 1389 & 27 & 1309 & 94.2 \\
\hline estC55-247 & 1107 & 3 & 499 & 45.1 \\
\hline estC55-251 & 954 & 358 & 954 & 100 \\
\hline estC55-253 & 894 & 72 & 894 & 100 \\
\hline estC55-256 & 813 & 166 & 813 & 100 \\
\hline estC55-258 & 1170 & 23 & 1153 & 99 \\
\hline estC55-268 & 912 & 489 & 912 & 100 \\
\hline estC76-21 & 867 & 14 & 740 & 85 \\
\hline estC76-28_1 & 774 & 9 & 212 & 27 \\
\hline estC76-28_2 & 732 & 135 & 732 & 100 \\
\hline estC76-36 & 714 & 155 & 714 & 100 \\
\hline estC76-98 & 1278 & 8 & 781 & 61 \\
\hline estC76-123 & 1353 & 284 & 1353 & 100 \\
\hline estC76-135 & 933 & 71 & 932 & 100 \\
\hline estC76-136 & 1548 & 360 & 1548 & 100 \\
\hline estC76-137 & 738 & 512 & 738 & 100 \\
\hline estC76-174 & 1167 & 197 & 1167 & 100 \\
\hline estC76-177 & 798 & 699 & 798 & 100 \\
\hline estC76-179 & 990 & 65 & 879 & 89 \\
\hline
\end{tabular}




\begin{tabular}{lllll} 
estC76-202 & 804 & 71 & 804 & 100 \\
estC76-218 & 990 & 48 & 838 & 85 \\
estC76-221 & 774 & 454 & 774 & 100 \\
estC76-222 & 783 & 1154 & 783 & 100 \\
estC76-248 & 774 & 12 & 614 & 79 \\
estC76-250 & 756 & 190 & 756 & 100 \\
estC76-261 & 1038 & 58 & 1038 & 100 \\
estC76-262 & 1248 & 1 & 61 & 5 \\
estC76-263 & 1131 & 109 & 1131 & 100 \\
estC76-266 & 774 & 153 & 774 & 100 \\
estC76-269 & 783 & 418 & 783 & 100 \\
\hline
\end{tabular}


Supplementary Table S16 BLAST-based comparison between putative lipolytic genes identified by sequencebased screening against these identified by function-driven screening

\begin{tabular}{|c|c|c|c|c|c|c|c|c|}
\hline Query $^{a}$ & $\begin{array}{l}\text { query } \\
\text { length }\end{array}$ & Subject ${ }^{b}$ & Subject length & Identity $(\%)$ & E-value & Bit score & Coverage & Alignment length \\
\hline EstC55-2 & 291 & LLBHCHNC 108683 & 291 & 99.656 & 0 & 588 & 100 & 291 \\
\hline EstC55-3 & 513 & LLBHCHNC $^{-} 04031$ & 509 & 94.303 & 0 & 927 & 99 & 509 \\
\hline EstC55-4_1 & 271 & LLBHCHNC_60489 & 271 & 100 & 0 & 539 & 100 & 271 \\
\hline EstC55-4_2 & 390 & LLBHCHNC_60488 & 390 & 100 & 0 & 795 & 100 & 390 \\
\hline EstC55-5 & 310 & LLBHCHNC_10894 & 310 & 100 & 0 & 615 & 100 & 310 \\
\hline EstC55-6 & 345 & LLBHCHNC_24927 & 345 & 99.71 & 0 & 669 & 100 & 345 \\
\hline EstC55-7 & 430 & LLBHCHNC 198339 & 355 & 99.437 & 0 & 732 & 83 & 355 \\
\hline EstC55-8 1 & 318 & LLBHCHNC $^{-} 170023$ & 318 & 99.686 & 0 & 645 & 100 & 318 \\
\hline EstC55-8_2 & 270 & LLBHCHNC_170022 & 270 & 100 & 0 & 539 & 100 & 270 \\
\hline EstC55-10 & 288 & LLBHCHNC_-150671 & 279 & 100 & 0 & 551 & 97 & 279 \\
\hline EstC55-12 & 384 & LLBHCHNC 52061 & 384 & 100 & 0 & 760 & 100 & 384 \\
\hline EstC55-13 & 355 & LLBHCHNC_01691 & 355 & 100 & 0 & 709 & 100 & 355 \\
\hline EstC55-15 & 253 & LLBHCHNC_23859 & 265 & 100 & 3.67E-178 & 492 & 99 & 252 \\
\hline EstC55-18 & 267 & LLBHCHNC_236409 & 247 & 37.402 & $5.02 \mathrm{E}-41$ & 144 & 93 & 254 \\
\hline EstC55-19_1 & 317 & LLBHCHNC_149694 & 317 & 100 & 0 & 639 & 100 & 317 \\
\hline EstC55-19-2 & 270 & LLBHCHNC_-149695 & 270 & 99.63 & 0 & 539 & 100 & 270 \\
\hline EstC55-20 & 350 & LLBHCHNC 54608 & 350 & 100 & 0 & 703 & 100 & 350 \\
\hline EstC55-23 & 322 & LLBHCHNC_76053 & 322 & 100 & 0 & 650 & 100 & 322 \\
\hline EstC55-24 & 325 & LLBHCHNC_164893 & 325 & 100 & 0 & 637 & 100 & 325 \\
\hline EstC55-25 & 367 & LLBHCHNC_31406 & 358 & 99.721 & 0 & 707 & 98 & 358 \\
\hline EstC55-26 & 317 & LLBHCHNC_-200149 & 317 & 96.845 & 0 & 627 & 100 & 317 \\
\hline EstC55-31 & 265 & LLBHCHNC 90973 & 265 & 100 & 0 & 542 & 100 & 265 \\
\hline EstC55-34 & 290 & LLBHCHNC_296992 & 290 & 98.276 & 0 & 568 & 100 & 290 \\
\hline EstC55-38 & 329 & LLBHCHNC_417537 & 329 & 99.392 & 0 & 672 & 100 & 329 \\
\hline EstC55-40 & 444 & LLBHCHNC 161203 & 442 & 99.774 & 0 & 888 & 99 & 442 \\
\hline EstC55-42 & 373 & LLBHCHNC_68310 & 427 & 56.919 & $2.95 \mathrm{E}-140$ & 408 & 100 & 383 \\
\hline EstC55-46 & 415 & LLBHCHNC_180099 & 436 & 82.339 & 0 & 716 & 100 & 436 \\
\hline EstC55-51 & 268 & LLBHCHNC_181078 & 286 & 61.024 & $9.53 \mathrm{E}-98$ & 290 & 95 & 254 \\
\hline EstC55-52 & 503 & LLBHCHNC_276493 & 505 & 86.558 & 0 & 822 & 97 & 491 \\
\hline EstC55-53 & 406 & LLBHCHNC_208903 & 404 & 52.12 & $2.26 \mathrm{E}-135$ & 396 & 99 & 401 \\
\hline EstC55-56 & 314 & LLBHCHNC_-147899 & 314 & 96.166 & 0 & 605 & 99 & 313 \\
\hline EstC55-57 & 271 & LLBHCHNC_422494 & 271 & 100 & 0 & 560 & 100 & 271 \\
\hline EstC55-60 & 340 & LLBHCHNC_114902 & 303 & 100 & 0 & 597 & 89 & 303 \\
\hline EstC55-61 & 388 & LLBHCHNC 499519 & 340 & 98.824 & 0 & 654 & 88 & 340 \\
\hline EstC55-62 & 528 & LLBHCHNC 23442 & 528 & 100 & 0 & 1077 & 100 & 528 \\
\hline EstC55-63 & 317 & LLBHCHNC 200149 & 317 & 97.161 & 0 & 629 & 100 & 317 \\
\hline EstC55-65 & 409 & LLBHCHNC_225424 & 408 & 83.99 & 0 & 701 & 99 & 406 \\
\hline
\end{tabular}




\begin{tabular}{|c|c|c|c|c|c|c|c|c|}
\hline EstC55-66 & 429 & LLBHCHNC_233808 & 451 & 97.203 & 0 & 839 & 100 & 429 \\
\hline EstC55-71 & 317 & LLBHCHNC_-228249 & 317 & 97.792 & 0 & 634 & 100 & 317 \\
\hline EstC55-72 & 298 & LLBHCHNC 523008 & 297 & 51.701 & $7.09 \mathrm{E}-89$ & 269 & 98 & 294 \\
\hline EstC55-73 & 385 & LLBHCHNC_146194 & 385 & 99.481 & 0 & 764 & 100 & 385 \\
\hline EstC55-76 & 373 & LLBHCHNC_58044 & 373 & 100 & 0 & 736 & 100 & 373 \\
\hline EstC55-77 & 278 & LLBHCHNC_517812 & 267 & 40.58 & $1.29 \mathrm{E}-44$ & 154 & 95 & 276 \\
\hline EstC55-78 & 319 & LLBHCHNC_93396 & 319 & 100 & 0 & 637 & 100 & 319 \\
\hline EstC55-80 & 408 & LLBHCHNC_-225424 & 408 & 99.755 & 0 & 828 & 100 & 408 \\
\hline EstC55-81 & 282 & LLBHCHNC_53848 & 312 & 100 & 0 & 561 & 100 & 282 \\
\hline EstC55-88 & 317 & LLBHCHNC_228249 & 317 & 97.792 & 0 & 631 & 100 & 317 \\
\hline EstC55-90 & 286 & LLBHCHNC_-112431 & 286 & 100 & 0 & 589 & 100 & 286 \\
\hline EstC55-95 & 288 & LLBHCHNC_71403 & 371 & 99.653 & 0 & 578 & 100 & 288 \\
\hline EstC55-96 & 267 & LLBHCHNC_432831 & 267 & 99.625 & 0 & 536 & 100 & 267 \\
\hline EstC55-97 & 240 & LLBHCHNC_354884 & 753 & 38.068 & $1.16 \mathrm{E}-21$ & 96.3 & 72 & 176 \\
\hline EstC55-100 & 376 & LLBHCHNC_-195970 & 378 & 53.704 & $1.13 \mathrm{E}-133$ & 389 & 95 & 378 \\
\hline EstC55-102 & 707 & LLBHCHNC_174663 & 719 & 60.734 & 0 & 790 & 99 & 736 \\
\hline EstC55-105 & 223 & LLBHCHNC_309242 & 223 & 100 & $8.96 \mathrm{E}-166$ & 458 & 100 & 223 \\
\hline EstC55-110 & 434 & LLBHCHNC_266798 & 434 & 99.77 & 0 & 891 & 100 & 434 \\
\hline EstC55-111 & 392 & LLBHCHNC_- 445504 & 392 & 100 & 0 & 795 & 100 & 392 \\
\hline EstC55-113 & 409 & LLBHCHNC_232836 & 442 & 39.225 & 4.19E-85 & 269 & 91 & 413 \\
\hline EstC55-118 & 497 & LLBHCHNC 276493 & 505 & 76.578 & 0 & 730 & 99 & 491 \\
\hline EstC55-131 & 251 & LLBHCHNC_- 493354 & 330 & 97.211 & $1.24 \mathrm{E}-168$ & 471 & 100 & 251 \\
\hline EstC55-145 & 305 & LLBHCHNC 59760 & 305 & 99.672 & 0 & 573 & 100 & 305 \\
\hline EstC55-147 & 386 & LLBHCHNC_53082 & 386 & 99.741 & 0 & 775 & 100 & 386 \\
\hline EstC55-150 & 393 & LLBHCHNC_- 445504 & 392 & 92.875 & 0 & 694 & 100 & 393 \\
\hline EstC55-151 & 311 & LLBHCHNC 364509 & 311 & 99.678 & 0 & 640 & 100 & 311 \\
\hline EstC55-154 & 394 & LLBHCHNC 502793 & 394 & 100 & 0 & 791 & 100 & 394 \\
\hline EstC55-156 & 517 & LLBHCHNC_37415 & 520 & 79.31 & 0 & 828 & 94 & 493 \\
\hline EstC55-159 & 284 & LLBHCHNC_- 338455 & 395 & 100 & 0 & 544 & 100 & 284 \\
\hline EstC55-163 & 303 & LLBHCHNC_311877 & 558 & 99.34 & 0 & 589 & 100 & 303 \\
\hline EstC55-164 & 430 & LLBHCHNC_32909 & 424 & 51.741 & $3.85 \mathrm{E}-123$ & 366 & 90 & 402 \\
\hline EstC55-165 & 277 & LLBHCHNC_-112340 & 276 & 52.049 & $1.08 \mathrm{E}-78$ & 242 & 87 & 244 \\
\hline EstC55-167 & 248 & LLBHCHNC_-90003 & 248 & 100 & 0 & 511 & 100 & 248 \\
\hline EstC55-168 & 513 & LLBHCHNC_291282 & 513 & 99.805 & 0 & 1028 & 100 & 513 \\
\hline EstC55-169 & 533 & LLBHCHNC_-116580 & 533 & 100 & 0 & 1088 & 100 & 533 \\
\hline EstC55-186 & 515 & LLBHCHNC_353867 & 504 & 39.6 & $2.85 \mathrm{E}-83$ & 270 & 94 & 500 \\
\hline EstC55-188 & 237 & LLBHCHNC_239154 & 97 & 100 & $1.08 \mathrm{E}-62$ & 193 & 41 & 97 \\
\hline EstC55-197 & 292 & LLBHCHNC_21317 & 294 & 99.656 & 0 & 577 & 99 & 291 \\
\hline EstC55-213 & 286 & LLBHCHNC_206366 & 268 & 97.015 & 0 & 524 & 94 & 268 \\
\hline EstC55-215 & 263 & LLBHCHNC_-65857 & 263 & 99.24 & 0 & 525 & 100 & 263 \\
\hline EstC55-227 & 459 & LLBHCHNC_-119846 & 472 & 99.564 & 0 & 908 & 100 & 459 \\
\hline EstC55-229 & 311 & LLBHCHNC_- 315562 & 325 & 100 & 0 & 633 & 100 & 311 \\
\hline EstC55-231 & 366 & LLBHCHNC_25121 & 366 & 100 & 0 & 737 & 100 & 366 \\
\hline EstC55-234 & 557 & LLBHCHNC_37415 & 520 & 40.301 & $2.73 \mathrm{E}-120$ & 367 & 92 & 531 \\
\hline EstC55-235 & 326 & LLBHCHNC_51521 & 326 & 96.599 & 0 & 580 & 90 & 294 \\
\hline
\end{tabular}




\begin{tabular}{|c|c|c|c|c|c|c|c|c|}
\hline EstC55-239 & 381 & LLBHCHNC 156698 & 968 & 99.213 & 0 & 773 & 100 & 381 \\
\hline EstC55-241 & 567 & LLBHCHNC_129990 & 567 & 95.767 & 0 & 1071 & 100 & 567 \\
\hline EstC55-244 & 255 & LLBHCHNC_-100331 & 255 & 100 & 0 & 526 & 100 & 255 \\
\hline EstC55-245 & 462 & LLBHCHNC_507289 & 231 & 98.701 & $9.06 \mathrm{E}-164$ & 463 & 50 & 231 \\
\hline EstC55-247 & 368 & LLBHCHNC_400362 & 352 & 53.977 & $3.80 \mathrm{E}-121$ & 356 & 96 & 352 \\
\hline EstC55-251 & 317 & LLBHCHNC_200149 & 317 & 98.423 & 0 & 636 & 100 & 317 \\
\hline EstC55-253 & 304 & LLBHCHNC_96988 & 297 & 100 & $5.27 \mathrm{E}-165$ & 462 & 77 & 233 \\
\hline EstC55-256 & 270 & LLBHCHNC_152981 & 270 & 98.519 & 0 & 545 & 100 & 270 \\
\hline EstC55-258 & 389 & LLBHCHNC_167603 & 394 & 56.633 & $1.80 \mathrm{E}-147$ & 426 & 98 & 392 \\
\hline EstC55-268 & 303 & LLBHCHNC_244305 & 303 & 64.726 & $2.48 \mathrm{E}-125$ & 362 & 96 & 292 \\
\hline EstC76-21 & 288 & FKLAIAFA_ $\overline{4} 37586$ & 279 & 97.849 & 0 & 539 & 97 & 279 \\
\hline EstC76-28_1 & 257 & FKLAIAFA_63857 & 257 & 91.829 & $1.56 \mathrm{E}-164$ & 457 & 100 & 257 \\
\hline EstC76-28_2 & 243 & FKLAIAFA_63856 & 243 & 100 & $5.52 \mathrm{E}-176$ & 485 & 100 & 243 \\
\hline EstC76-36 & 237 & FKLAIAFA_40146 & 238 & 98.734 & $3.29 \mathrm{E}-162$ & 450 & 100 & 237 \\
\hline EstC76-98 & 425 & FKLAIAFA_43605 & 437 & 40.92 & $1.08 \mathrm{E}-102$ & 314 & 99 & 435 \\
\hline EstC76-123 & 450 & FKLAIAFA_91696 & 450 & 99.333 & 0 & 903 & 100 & 450 \\
\hline EstC76-135 & 310 & FKLAIAFA 98475 & 317 & 71.613 & $8.65 \mathrm{E}-167$ & 468 & 100 & 310 \\
\hline EstC76-136 & 515 & FKLAIAFA_43393 & 515 & 100 & 0 & 1009 & 100 & 515 \\
\hline EstC76-137 & 277 & FKLAIAFA_218044 & 221 & 43.231 & $2.10 \mathrm{E}-54$ & 177 & 81 & 229 \\
\hline EstC76-174 & 388 & FKLAIAFA_18366 & 388 & 99.485 & 0 & 750 & 100 & 388 \\
\hline EstC76-179 & 329 & FKLAIAFA_34048 & 329 & 97.568 & 0 & 664 & 100 & 329 \\
\hline EstC76-202 & 267 & FKLAIAFA_18975 & 267 & 100 & 0 & 538 & 100 & 267 \\
\hline EstC76-218 & 329 & FKLAIAFA_34048 & 329 & 98.784 & 0 & 669 & 100 & 329 \\
\hline EstC76-221 & 257 & FKLAIAFA_399351 & 257 & 99.611 & 0 & 524 & 100 & 257 \\
\hline EstC76-222 & 260 & FKLAIAFA_58163 & 260 & 99.231 & 0 & 523 & 100 & 260 \\
\hline EstC76-248 & 257 & FKLAIAFA_63857 & 257 & 93.385 & $8.27 \mathrm{E}-169$ & 468 & 100 & 257 \\
\hline EstC76-250 & 251 & FKLAIAFA 107970 & 251 & 98.805 & $2.16 \mathrm{E}-174$ & 482 & 100 & 251 \\
\hline EstC76-261 & 345 & FKLAIAFA_06843 & 345 & 99.71 & 0 & 669 & 100 & 345 \\
\hline EstC76-262 & 415 & FKLAIAFA 69458 & 411 & 57.039 & $2.80 \mathrm{E}-151$ & 437 & 97 & 412 \\
\hline EstC76-263 & 376 & FKLAIAFA 04547 & 376 & 99.734 & 0 & 752 & 100 & 376 \\
\hline EstC76-266 & 257 & FKLAIAFA_63857 & 257 & 100 & 0 & 501 & 100 & 257 \\
\hline EstC76-269 & 260 & FKLAIAFA 60368 & 260 & 98.462 & 0 & 513 & 100 & 260 \\
\hline
\end{tabular}

${ }^{a}$ Lipolytic genes identifies through function-driven approach in this study

${ }^{\mathrm{b}}$ Putative lipolytic genes identifies through sequence-based approach in this study 
Supplementary Table S17 Pseudo-value of ANOSIM test for group dissimilarity of lipolytic family profiles across different habitats (LPGM was $\log 10$ transformed. The distance is Bray-Curtis, permutation $\mathrm{N}=9999$, overall $\mathrm{R}$ value $=0.6168, p=0.0001$ )

\begin{tabular}{|c|c|c|c|c|c|c|c|c|c|c|c|c|c|c|c|}
\hline Habitat & ADAS & $\mathrm{COM}$ & AS & GS & HG & $\mathrm{HM}$ & HRE & HS & LL & MS & MW & OR & RW & TFS & WB \\
\hline ADAS & $\mathrm{NA}^{\mathrm{a}}$ & 0.705 & 0.468 & 0.781 & 0.999 & 0.949 & 0.936 & 0.358 & 0.389 & 0.318 & 0.67 & 0.962 & 0.999 & 0.937 & 1 \\
\hline $\mathrm{COM}$ & 0.705 & $\mathrm{NA}^{\mathrm{a}}$ & 0.726 & 0.786 & 0.852 & 0.365 & 0.84 & 0.733 & 0.796 & 0.641 & 0.52 & 0.799 & 0.471 & 0.686 & 0.194 \\
\hline AS & 0.468 & 0.726 & $\mathrm{NA}^{\mathrm{a}}$ & 0.511 & 0.708 & 0.175 & 0.336 & 0.438 & 0.627 & 0.442 & 0.466 & 0.631 & 0.443 & 0.563 & 0.62 \\
\hline GS & 0.781 & 0.786 & 0.511 & $\mathrm{NA}^{\mathrm{a}}$ & 1 & 0.885 & 0.968 & 0.384 & 0.46 & 0.334 & 0.779 & 0.995 & 0.961 & 0.883 & 0.972 \\
\hline HG & 0.999 & 0.852 & 0.708 & 1 & $\mathrm{NA}^{\mathrm{a}}$ & 0.995 & 0.939 & 0.617 & 0.844 & 0.687 & 0.987 & 0.982 & 1 & 0.952 & 0.999 \\
\hline HM & 0.949 & 0.365 & 0.175 & 0.885 & 0.995 & $\mathrm{NA}^{\mathrm{a}}$ & 0.773 & 0.403 & 0.495 & 0.324 & 0.631 & 0.875 & 0.655 & 0.843 & 0.865 \\
\hline HRE & 0.936 & 0.84 & 0.336 & 0.968 & 0.939 & 0.773 & $\mathrm{NA}^{\mathrm{a}}$ & 0.75 & 0.899 & 0.606 & 0.905 & 0.929 & 0.893 & 0.904 & 0.9 \\
\hline HS & 0.358 & 0.733 & 0.438 & 0.384 & 0.617 & 0.403 & 0.75 & $\mathrm{NA}^{\mathrm{a}}$ & 0.514 & 0.252 & 0.507 & 0.637 & 0.516 & 0.604 & 0.668 \\
\hline LL & 0.389 & 0.796 & 0.627 & 0.46 & 0.844 & 0.495 & 0.899 & 0.514 & $\mathrm{NA}^{\mathrm{a}}$ & 0.263 & 0.484 & 0.792 & 0.685 & 0.838 & 0.754 \\
\hline MS & 0.318 & 0.641 & 0.442 & 0.334 & 0.687 & 0.324 & 0.606 & 0.252 & 0.263 & $\mathrm{NA}^{\mathrm{a}}$ & 0.397 & 0.65 & 0.504 & 0.597 & 0.584 \\
\hline MW & 0.67 & 0.52 & 0.466 & 0.779 & 0.987 & 0.631 & 0.905 & 0.507 & 0.484 & 0.397 & $\mathrm{NA}^{\mathrm{a}}$ & 0.894 & 0.783 & 0.892 & 0.81 \\
\hline OR & 0.962 & 0.799 & 0.631 & 0.995 & 0.982 & 0.875 & 0.929 & 0.637 & 0.792 & 0.65 & 0.894 & $\mathrm{NA}^{\mathrm{a}}$ & 0.918 & 0.977 & 0.871 \\
\hline RW & 0.999 & 0.471 & 0.443 & 0.961 & 1 & 0.655 & 0.893 & 0.516 & 0.685 & 0.504 & 0.783 & 0.918 & $\mathrm{NA}^{\mathrm{a}}$ & 0.875 & 0.95 \\
\hline TFS & 0.937 & 0.686 & 0.563 & 0.883 & 0.952 & 0.843 & 0.904 & 0.604 & 0.838 & 0.597 & 0.892 & 0.977 & 0.875 & $\mathrm{NA}^{\mathrm{a}}$ & 0.83 \\
\hline WB & 1 & 0.194 & 0.62 & 0.972 & 0.999 & 0.865 & 0.9 & 0.668 & 0.754 & 0.584 & 0.81 & 0.871 & 0.95 & 0.83 & $\mathrm{NA}^{\mathrm{a}}$ \\
\hline Mean $^{\text {b }}$ & 0.748 & 0.686 & 0.511 & 0.764 & 0.897 & 0.659 & 0.827 & 0.527 & 0.631 & 0.471 & 0.695 & 0.851 & 0.761 & 0.813 & 0.787 \\
\hline
\end{tabular}

${ }^{\mathrm{a}}$ No data

${ }^{\mathrm{b}}$ Averaged $\mathrm{R}$ values 
Supplementary Table S19 Rseudo-value of ANOSIM test for group dissimilarity of phylogenetic distribution (at genus level) of assigned PLPs across habitats (Only genus with a mean LPGM $\geq 0.5$ across all the samples were used. The distance is Bray-Curtis, permutation $\mathrm{N}=9999$, overall $\mathrm{R}$ value $=0.8199, p=0.0001$ )

\begin{tabular}{|c|c|c|c|c|c|c|c|c|c|c|c|c|c|c|c|}
\hline Habitat & $\mathrm{AS}$ & ADAS & $\mathrm{COM}$ & GS & $\mathrm{HG}$ & $\mathrm{HM}$ & HRE & $\mathrm{HS}$ & $\mathrm{LL}$ & MS & MW & $\mathrm{OR}$ & RW & TFS & WB \\
\hline AS & $\mathrm{NA}^{\mathrm{a}}$ & 0.609 & 0.628 & 0.596 & 0.88 & 0.506 & 0.567 & 0.682 & 0.613 & 0.683 & 0.639 & 0.791 & 0.646 & 0.435 & 0.69 \\
\hline ADAS & 0.609 & $\mathrm{NA}^{\mathrm{a}}$ & 0.588 & 0.993 & 1 & 1 & 0.991 & 0.669 & 0.638 & 0.573 & 0.694 & 0.997 & 1 & 1 & 1 \\
\hline $\mathrm{COM}$ & 0.628 & 0.588 & $\mathrm{NA}^{\mathrm{a}}$ & 0.568 & 0.868 & 0.572 & 0.864 & 0.752 & 0.759 & 0.727 & 0.53 & 0.837 & 0.546 & 0.595 & 0.232 \\
\hline GS & 0.596 & 0.993 & 0.568 & $\mathrm{NA}^{\mathrm{a}}$ & 1 & 0.993 & 0.995 & 0.738 & 0.947 & 0.726 & 0.866 & 1 & 1 & 0.977 & 0.994 \\
\hline $\mathrm{HG}$ & 0.88 & 1 & 0.868 & 1 & $\mathrm{NA}^{\mathrm{a}}$ & 1 & 0.937 & 0.916 & 1 & 0.995 & 1 & 1 & 1 & 1 & 1 \\
\hline HM & 0.506 & 1 & 0.572 & 0.993 & & $\mathrm{NA}^{\mathrm{a}}$ & 0.971 & 0.736 & 1 & 0.943 & 0.904 & 1 & 0.973 & 0.999 & 0.978 \\
\hline HRE & 0.567 & 0.991 & 0.864 & 0.995 & 0.937 & 0.971 & $\mathrm{NA}^{\mathrm{a}}$ & 0.811 & 0.998 & 0.994 & 0.99 & 0.98 & 0.983 & 0.949 & 0.956 \\
\hline HS & 0.682 & 0.669 & 0.752 & 0.738 & 0.916 & 0.736 & 0.811 & $\mathrm{NA}^{\mathrm{a}}$ & 0.724 & 0.704 & 0.732 & 0.874 & 0.651 & 0.864 & 0.791 \\
\hline LL & 0.613 & 0.638 & 0.759 & 0.947 & 1 & 1 & 0.998 & 0.724 & $\mathrm{NA}^{\mathrm{a}}$ & 0.401 & 0.799 & 0.996 & 1 & 1 & 0.993 \\
\hline MS & 0.683 & 0.573 & 0.727 & 0.726 & 0.995 & 0.943 & 0.994 & 0.704 & 0.401 & $\mathrm{NA}^{\mathrm{a}}$ & 0.622 & 0.991 & 0.962 & 0.992 & 0.948 \\
\hline MW & 0.639 & 0.694 & 0.53 & 0.866 & 1 & 0.904 & 0.99 & 0.732 & 0.799 & 0.622 & $\mathrm{NA}^{\mathrm{a}}$ & 0.997 & 0.947 & 0.992 & 0.937 \\
\hline OR & 0.791 & 0.997 & 0.837 & 1 & 1 & 1 & 0.98 & 0.874 & 0.996 & 0.991 & 0.997 & $\mathrm{NA}^{\mathrm{a}}$ & 1 & 1 & 0.999 \\
\hline RW & 0.646 & 1 & 0.546 & 1 & 1 & 0.973 & 0.983 & 0.651 & 1 & 0.962 & 0.947 & 1 & $\mathrm{NA}^{\mathrm{a}}$ & 1 & 1 \\
\hline TFS & 0.435 & 1 & 0.595 & 0.977 & 1 & 0.999 & 0.949 & 0.864 & 1 & 0.992 & 0.992 & 1 & 1 & $\mathrm{NA}^{\mathrm{a}}$ & 0.967 \\
\hline WB & 0.69 & 1 & 0.232 & 0.994 & 1 & 0.978 & 0.956 & 0.791 & 0.993 & 0.948 & 0.937 & 0.999 & 1 & 0.967 & $\mathrm{NA}^{\mathrm{a}}$ \\
\hline Mean $^{b}$ & 0.640 & 0.839 & 0.647 & 0.885 & 0.969 & 0.898 & 0.928 & 0.760 & 0.848 & 0.804 & 0.832 & 0.961 & 0.908 & 0.912 & 0.892 \\
\hline
\end{tabular}

${ }^{\text {a }}$ No data

${ }^{\mathrm{b}}$ Averaged R values 
Supplementary Table S21 Pseudo-values of ANOSIM test for group dissimilarity of phylogenetic distributions (at genus level) of total PLPs across habitats (Only genera with a mean LPGM $>0.5$ across all the samples were used. The distance is Bray-Curtis, permutation $\mathrm{N}=9999$, overall $\mathrm{R}$ value $=0.821, p=0.0001$ )

\begin{tabular}{|c|c|c|c|c|c|c|c|c|c|c|c|c|c|c|c|}
\hline Habitat & ADAS & $\mathrm{COM}$ & $\mathrm{AS}$ & GS & $\mathrm{HG}$ & HM & HRE & HS & LL & MS & MW & OR & RW & TFS & WB \\
\hline ADAS & $\mathrm{NA}^{\mathrm{a}}$ & 0.605 & 0.862 & 1 & 1 & 0.994 & 0.989 & 0.577 & 0.636 & 0.537 & 0.657 & 0.995 & 1 & 1 & 0.999 \\
\hline COM & 0.605 & $\mathrm{NA}^{\mathrm{a}}$ & 0.677 & 0.448 & 0.907 & 0.47 & 0.849 & 0.752 & 0.791 & 0.689 & 0.527 & 0.853 & 0.495 & 0.551 & 0.218 \\
\hline AS & 0.862 & 0.677 & $\mathrm{NA}^{\mathrm{a}}$ & 0.804 & 0.95 & 0.79 & 0.791 & 0.867 & 0.866 & 0.879 & 0.86 & 0.914 & 0.798 & 0.494 & 0.854 \\
\hline GS & 1 & 0.448 & 0.804 & $\mathrm{~N}^{\mathrm{a}}$ & 1 & 0.968 & 0.978 & 0.615 & 0.938 & 0.607 & 0.757 & 1 & 1 & 0.962 & 0.964 \\
\hline $\mathrm{HG}$ & 1 & 0.907 & 0.95 & 1 & $\mathrm{NA}^{\mathrm{a}}$ & 1 & 0.938 & 0.987 & 1 & 0.995 & 1 & 1 & 1 & 1 & 1 \\
\hline HM & 0.994 & 0.47 & 0.79 & 0.968 & 1 & $\mathrm{NA}^{\mathrm{a}}$ & 0.922 & 0.765 & 1 & 0.873 & 0.859 & 1 & 0.952 & 0.983 & 0.956 \\
\hline HRE & 0.989 & 0.849 & 0.791 & 0.978 & 0.938 & 0.922 & $\mathrm{NA}^{\mathrm{a}}$ & 0.975 & 0.998 & 0.986 & 0.97 & 0.984 & 0.967 & 0.931 & 0.939 \\
\hline HS & 0.577 & 0.752 & 0.867 & 0.615 & 0.987 & 0.765 & 0.975 & $\mathrm{NA}^{\mathrm{a}}$ & 0.776 & 0.589 & 0.682 & 0.952 & 0.673 & 0.908 & 0.805 \\
\hline LL & 0.636 & 0.791 & 0.866 & 0.938 & 1 & 1 & 0.998 & 0.776 & $\mathrm{NA}^{\mathrm{a}}$ & 0.369 & 0.778 & 0.993 & 0.999 & 0.999 & 0.994 \\
\hline MS & 0.537 & 0.689 & 0.879 & 0.607 & 0.995 & 0.873 & 0.986 & 0.589 & 0.369 & $\mathrm{NA}^{\mathrm{a}}$ & 0.541 & 0.972 & 0.94 & 0.977 & 0.912 \\
\hline MW & 0.657 & 0.527 & 0.86 & 0.757 & 1 & 0.859 & 0.97 & 0.682 & 0.778 & 0.541 & $\mathrm{NA}^{\mathrm{a}}$ & 0.986 & 0.923 & 0.972 & 0.922 \\
\hline OR & 0.995 & 0.853 & 0.914 & 1 & 1 & 1 & 0.984 & 0.952 & 0.993 & 0.972 & 0.986 & $\mathrm{NA}^{\mathrm{a}}$ & 1 & 1 & 1 \\
\hline RW & 1 & 0.495 & 0.798 & 1 & 1 & 0.952 & 0.967 & 0.673 & 0.999 & 0.94 & 0.923 & 1 & $\mathrm{NA}^{\mathrm{a}}$ & 0.994 & 0.998 \\
\hline TFS & 1 & 0.551 & 0.494 & 0.962 & 1 & 0.983 & 0.931 & 0.908 & 0.999 & 0.977 & 0.972 & 1 & 0.994 & $\mathrm{NA}^{\mathrm{a}}$ & 0.95 \\
\hline WB & 0.999 & 0.218 & 0.854 & 0.964 & 1 & 0.956 & 0.939 & 0.805 & 0.994 & 0.912 & 0.922 & 1 & 0.998 & 0.95 & $\mathrm{NA}^{\mathrm{a}}$ \\
\hline Mean $^{\mathrm{b}}$ & 0.846 & 0.631 & 0.815 & 0.860 & 0.984 & 0.895 & 0.944 & 0.780 & 0.867 & 0.776 & 0.817 & 0.975 & 0.910 & 0.909 & 0.894 \\
\hline
\end{tabular}

a No data

${ }^{\mathrm{b}}$ Averaged R values 


\section{Chapter III}

\section{Biochemical profiles of two thermostable and organic solvent-tolerant esterases derived from a compost metagenome}

Mingji Lu, Amélie Dukunde, Rolf Daniel

Doi: https://doi.org/10.1007/s00253-019-09695-1

https://link.springer.com/article/10.1007/s00253-019-09695-1 


\section{Biochemical profiles of two thermostable and organic solvent-tolerant esterases derived from a compost metagenome}

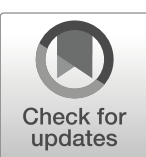

Mingji Lu ${ }^{1} \cdot$ Amélie Dukunde $^{1} \cdot$ Rolf Daniel $^{1}$

Received: 1 December 2018 /Revised: 11 February 2019 / Accepted: 12 February 2019

(C) Springer-Verlag GmbH Germany, part of Springer Nature 2019

Abstract

Owing to the functional versatility and potential applications in industry, interest in lipolytic enzymes tolerant to organic solvents is increasing. In this study, functional screening of a compost soil metagenome resulted in identification of two lipolytic genes, est 1 and est2, encoding 270 and 389 amino acids, respectively. The two genes were heterologously expressed and characterized. Est1 and Est2 are thermostable enzymes with optimal enzyme activities at 80 and $70{ }^{\circ} \mathrm{C}$, respectively. A second-order rotatable design, which allows establishing the relationship between multiple variables with the obtained responses, was used to explore the combined effects of temperature and $\mathrm{pH}$ on esterase stability. The response curve indicated that Est1, and particularly Est2, retained high stability within a broad range of temperature and $\mathrm{pH}$ values. Furthermore, the effects of organic solvents on Est 1 and Est2 activities and stabilities were assessed. Notably, Est2 activity was significantly enhanced (two- to tenfold) in the presence of ethanol, methanol, isopropanol, and 1-propanol over a concentration range between 6 and 30\% (v/v). For the short-term stability ( $2 \mathrm{~h}$ of incubation), Est2 exhibited high tolerance against $60 \%(v / v)$ of ethanol, methanol, isopropanol, DMSO, and acetone, while Est1 activity resisted these solvents only at lower concentrations (below 30\%, v/v). Est2 also displayed high stability towards some water-immiscible organic solvents, such as ethyl acetate, diethyl ether, and toluene. With respect to long-term stability, Est2 retained most of its activity after 26 days of incubation in the presence of 30\% $(v / v)$ ethanol, methanol, isopropanol, DMSO, or acetone. All of these features indicate that Est1 and Est2 possess application potential.

Keywords Carboxylesterases $\cdot$ Metagenomic library $\cdot$ Second-order rotatable design $\cdot$ Thermophilic $\cdot$ Organic solvent tolerance

\section{Introduction}

Extreme environments exhibiting elevated temperatures, extreme $\mathrm{pH}$ values, and exposure to organic solvents or high salinity are used to recover novel robust bioactive molecules that can be applied under industrial conditions (Antranikian and Egorova 2007). The targeted environments such as hot springs, compost, oil fields, and deep-sea marine sediments are reservoirs for extremophilic microorganisms that could produce potentially relevant industrial enzymes (Auernik

Electronic supplementary material The online version of this article (https://doi.org/10.1007/s00253-019-09695-1) contains supplementary material, which is available to authorized users.

$\triangle$ Rolf Daniel

rdaniel@gwdg.de

1 Department of Genomic and Applied Microbiology, Göttingen Genomics Laboratory, Institute of Microbiology and Genetics, Georg-August-University of Göttingen, Grisebachstraße 8, 37077 Göttingen, Germany

Published online: 26 February 2019 et al. 2008). Culture-independent metagenomic approaches are alternatives to conventional culture-based screening methods. Recently, some extremozymes, such as amylases, amidases, proteases, cellulases, and esterases, have been successfully identified through metagenomic approaches (Daniel 2005; Simon and Daniel 2011; González-González et al. 2017; Jayanath et al. 2018; Martínez-Martínez et al. 2018).

Lipolytic enzymes, which catalyze the hydrolysis and synthesis of acylglycerols, are considered as one of the most important groups of biocatalysts. Lipolytic enzymes include esterases (EC 3.1.1.1, carboxylesterases) and true lipases (EC 3.1.1.3, triacylglycerol acyl hydrolases) and are widespread in bacteria, archaea, and eukaryotes (Hasan et al. 2006). Due to their broad substrate, $\mathrm{pH}$, and temperature spectra combined with high regio- and enantioselectivity, lipolytic enzymes are of interest for food, paper, medical, detergent, and pharmaceutical industries (Hita et al. 2009; Romdhane et al. 2010; Ferrer et al. 2015; Sarmah et al. 2018). In particular, lipolytic enzymes that function in non-aqueous solvents have attracted considerable attention, as they offer new possibilities for 
bioprocesses, such as shifting of thermodynamic equilibrium in favor of synthesis (esterification and transesterification), controlling substrate specificity and solubility by solvent engineering, and suppressing water-dependent side reactions (Secundo and Carrea 2002; Hun et al. 2003; Ahmed et al. 2010). However, the inhibition or inactivation of enzyme activity resulting from organic solvents has restricted the use of many lipolytic enzymes (Klibanov 2001; Jin et al. 2012). To overcome this limitation, some organic solvent-tolerant (OST) lipolytic enzymes have been isolated, including enzymes from Bacillus licheniformis S-86 (Torres et al. 2009), Streptomyces coelicolor A3(2) (Brault et al. 2012), Psychrobacter celer 3Pb1 (Wu et al. 2013), Alcanivorax dieselolei B-5(T) (Zhang et al. 2014), and Acetomicrobium hydrogeniformans (Kumagai et al. 2018), as well as from metagenomes of seawater (Chu et al. 2008), compost (Kang et al. 2011), lipid-contaminated soil (Glogauer et al. 2011), mountain soil (Jin et al. 2012), swamp sediment (Seo et al. 2014), and deep-sea hydrothermal vents (Yang et al. 2018). However, these enzymes only show tolerance towards specific organic solvents.

Industrially required versatile lipolytic enzymes that exhibit satisfactory activity and stability in both water-miscible and water-immiscible organic solvents are rare (Doukyu and Ogino 2010). It has been shown that the thermostability of an enzyme in water is correlated to its tolerance against organic solvents (Kumar et al. 2016). Thus, it is straightforward to screen naturally evolved OST enzymes from thermostable ones (Lotti and Alberghina 2007; Ahmed et al. 2010). Most industrial processes utilizing lipolytic enzymes are carried out at higher temperatures (above $45^{\circ} \mathrm{C}$ ); it is required that the enzymes exhibit activity and stability optima around $50{ }^{\circ} \mathrm{C}$ (Sharma et al. 2002). Thus, thermostable lipolytic enzymes exhibiting organic solvent tolerance are of high importance with respect to industrial applications.

Composting is the process of biological, aerobic decomposition of organic waste by microorganisms (Ryckeboer et al. 2003). During the thermophilic phase of composting, heat generated by microbial succession can raise temperatures to above $50{ }^{\circ} \mathrm{C}$ (Dougherty et al. 2012). Correspondingly, compost is a potential source for recovery of thermostable enzymes. Recently, lipolytic enzymes have been isolated from compost (Lämmle et al. 2007; Tirawongsaroj et al. 2008; Kim et al. 2010; Ohlhoff et al. 2015; Woo Lee et al. 2016), but only one (EstCS2) of the isolated enzymes was moderately thermostable (optimum $55{ }^{\circ} \mathrm{C}$ ) and showed resistance to certain water-miscible organic solvents (Kang et al. 2011).

In this study, two genes encoding lipolytic enzymes (est 1 and est2) were identified from a thermophilic compost metagenome. The corresponding enzymes were purified and characterized. Enzyme characterizations are usually conducted as one-factor-at-a-time for comparison with reported enzyme features from other studies.
However, this methodology ignores interacting effects between factors, which may result in misleading conclusions, especially when at least two requirements must be fulfilled simultaneously. An alternative is to employ design of experiments (DOE) methodologies, i.e., secondorder rotatable design approach, which use statistical and mathematical approaches to evaluate the combined effect of factors. DOE has been successfully applied in different aspects related to lipolytic enzymes such as the growth condition optimization and enzyme activity or stability measurements (Kamimura et al. 2001; Shieh et al. 2003; Benaiges et al. 2010). In this study, the combined effect of $\mathrm{pH}$ and temperature on the stability of Est1and Est2 was evaluated by the second-order rotatable design approach. Analysis of the recovered two metagenome-derived enzymes showed that they are thermophilic and tolerant towards organic solvents. In addition, Est2 was remarkably resistant to both water-miscible and immiscible organic solvents.

\section{Materials and methods}

\section{Strains and plasmids}

For the construction of metagenomic plasmid libraries, Escherichia coli TOP10 and pFLD (Invitrogen $\mathrm{GmbH}$, Karlsruhe, Germany) were used as host and vector, respectively. Escherichia coli BL21 Star (DE3) and the pET101/DTOPO ${ }^{\circledR}$ vector (Invitrogen $\mathrm{GmbH}$ ) were used for heterologous expression of the recovered lipolytic genes.

\section{Sample collection and DNA extraction}

Compost samples were collected at a composting company (Göttingen GmbH, Göttingen, Germany, 51 $34^{\circ} 25.1^{\prime \prime} \mathrm{N} 9^{\circ}$ $54^{\prime} 33.0^{\prime \prime} \mathrm{E}$ ). The sampling pile was the fermentation product of household waste and fresh tree branches. To ensure using mainly thermophilic microorganisms as a source for metagenomic library construction, compost at the core zone of compost pile was collected. The temperature at the sampling spot was $55^{\circ} \mathrm{C}$. The compost soil sample $(50 \mathrm{~g})$ was collected in sterile plastic bags and stored at $-20{ }^{\circ} \mathrm{C}$ until required.

Metagenomic DNA of the compost sample was extracted following a phenol-chloroform method according to Zhou et al. (1996). In addition, DNA was also isolated with MoBio Power Soil DNA extraction kit following the protocol of the manufacturer (MoBio Laboratories, Carlsbad, CA, USA). DNA obtained from these two methods was pooled and stored at $-20^{\circ} \mathrm{C}$ until required. 


\section{Metagenomic library construction and screening for lipolytic activity}

To construct a metagenomic plasmid library, DNA was sheared, and fragments from 6 to $12 \mathrm{~kb}$ were recovered by gel extraction with the peqGold gel extraction kit (Peqlab Biotechnologie GmbH, Erlangen, Germany). End-repaired DNA fragments and Pmll-digested $\mathrm{pFLD}$ vector were ligated by employing T4 DNA ligase at $16{ }^{\circ} \mathrm{C}$, overnight as recommended by the manufacturer (Thermo Scientific, Bremen, Germany). To screen for lipolytic activity, metagenomic library-bearing cells were plated onto LB agar plates containing $100 \mu \mathrm{g} / \mathrm{ml}$ ampicillin and $1 \%(v / v)$ emulsified tributyrin (Sigma, Germany) and subsequently incubated at $30^{\circ} \mathrm{C} 1$ to 7 days. Lipolytic-positive clones were identified by the formation of clear zones (halos) around individual colonies. The phenotype of positive clones was confirmed by the isolation of recombinant plasmids from positive strains, transformation of the isolated plasmids into the host, and rescreening on indicator agar plates.

\section{Sequence analysis and homology modeling}

Lipolytic genes (est 1 and est2) were initially predicted by using the ORF Finder program (http://www.ncbi.nlm.nih. gov/gorf/gorf.html), and verified by Clone Manager and FramePlot analysis (Ishikawa and Hotta 1999). Similarity searches of the deduced amino acid sequences were performed by BLASTP program against the public GenBank database (Ye et al. 2006). Signal peptides were detected by using the SignalP 4.0 server (Bendtsen et al. 2004). The deduced amino acid sequences of est 1 and est 2 and reference sequences retrieved from GenBank were used to construct a phylogenetic tree with the neighbor-joining method by using MEGA version 6 (Tamura et al. 2013). Bootstrapping of 1000 replicates was used to estimate the confidence level.

Based on the deduced amino acid sequence, secondary structure and tertiary structure predictions were performed with I-TASSER (Zhang 2008). The identified structural analogs were used for multiple-sequence alignment using the Expresso webserver (Notredame et a1. 2000). Figures showing secondary structure alignments were exported by ESPript3 (Robert and Gouet 2014). The analog with the highest TM score was also selected for structural superimposition.

\section{Expression and purification of recombinant proteins Est1 and Est2}

The primer pairs 5'-CACCATGCCCCTGGCCCGAG TGGA-3' and 5'-GGCGCCCACCGGC ACCTGAGTC-3' and 5'-CACCATGACCGAGCTGCCGGTGGGAG-3' and 5'-GCG TCTTAGCGCGCGGTACAC-3 were used to amplify est 1 and est2, respectively. The resulting PCR products were purified and ligated into expression vector pET101/ D-TOPO $®$ according to the protocol of the manufacturer (Invitrogen). To produce $\mathrm{His}_{6}$-tagged Est1 and Est2, recombinant plasmid DNA was transformed into E. coli BL21 (DE3) cells and plated on LB agar plates with $100 \mu \mathrm{g} / \mathrm{ml}$ ampicillin. A single colony was picked and grown overnight at $30^{\circ} \mathrm{C}$ in 60-ml LB medium containing $100 \mu \mathrm{g} / \mathrm{ml}$ ampicillin. Subsequently, this pre-culture was added to $600-\mathrm{ml} \mathrm{LB}$ medium with $100 \mu \mathrm{g} / \mathrm{ml}$ ampicillin and grown with shaking at $30{ }^{\circ} \mathrm{C}$. At an optical density $\left(\mathrm{OD}_{600}\right)$ of 0.6 , isopropyl-beta$\mathrm{D}$ thiogalactopyranoside (IPTG) was added to a final concentration of $0.5 \mathrm{mM}$. After a $6-\mathrm{h}$ induction at $30^{\circ} \mathrm{C}$, cells were harvested by centrifugation $\left(7000 \times g, 4^{\circ} \mathrm{C}, 10 \mathrm{~min}\right)$. Cell pellets were washed with $100 \mathrm{ml} \mathrm{LEW} \mathrm{buffer}\left(50 \mathrm{mM} \mathrm{NaH}_{2} \mathrm{PO}_{4}\right.$, $300 \mathrm{mM} \mathrm{NaCl}, \mathrm{pH} 8$ ) and stored at $-20^{\circ} \mathrm{C}$ until required.

To purify Est1 and Est2, Protino® Ni-TED 2000 packed column (Macherey-Nagel, Germany) was used following the manufacturer's protocol, however, with the modified LEW buffer $\left(50 \mathrm{mM} \mathrm{NaH} \mathrm{PO}_{4}, 1 \mathrm{M} \mathrm{NaCl}, 10 \% v / v\right.$ glycerol, $0.05 \% v / v$ Triton $\mathrm{X}-100, \mathrm{pH}$ 8). Protein concentration was measured by the Bradford method (Bradford 1976). Purity and molecular mass of the purified proteins were determined by sodium dodecyl sulfate-polyacrylamide gel electrophoresis (SDS-PAGE) using the procedure of Laemmli (1970). Fractions derived from affinity chromatography showing a single band with the estimated molecular mass of the targeted proteins were pooled, dialyzed with $50 \mathrm{mM}$ sodium phosphate buffer ( $\mathrm{pH} 8)$, and stored in $50 \%(\mathrm{v} / \mathrm{v})$ glycerol at $-20^{\circ} \mathrm{C}$ until use.

\section{Standard enzyme assays}

Esterase activity was measured by a spectrophotometric method (Jaeger et al. 1999) using $p$-nitrophenyl ( $p$-NP) acyl esters (Sigma) as substrates. To minimize substrate auto-hydrolysis at high temperatures, $p$-NP caprylate (C8) was used as a standard substrate. Unless otherwise indicated, Est1 activity was measured at $80{ }^{\circ} \mathrm{C}$ in $1 \mathrm{ml}$ containing $50 \mathrm{mM}$ sodium phosphate assay buffer ( $\mathrm{pH} 8), 1 \mathrm{mM} p$-NP caprylate (C8), and $1 \%$ $(v / v)$ isopropanol, while Est2 activity was measured at $70{ }^{\circ} \mathrm{C}$ in $1 \mathrm{ml}$ containing $50 \mathrm{mM}$ TAPS (3-(2, 4 dinitrostyrl)-(6R,7R7-(2-thienylacetamido)-ceph-3-em-4-carboxylic acid) assay buffer ( $\mathrm{pH} 9), 1 \mathrm{mM} p$-NP caprylate (C8), and 1\% (v/v) isopropanol. The assay buffer was initially incubated in a screwed-cap test tube for $10 \mathrm{~min}$ at assay temperature. Then, the reaction was initiated by adding enzyme and substrate to the buffer. The amount of $p$-nitrophenol released by esterasecatalyzed hydrolysis was continuously monitored at a wavelength of $410 \mathrm{~nm}$ against an enzyme-free blank. One unit (U) of enzyme activity was defined as the amount of enzyme that released $1 \mu \mathrm{mol}$ of $p$-nitrophenol per minute. All experiments 
were performed in at least triplicate. Results are shown as mean values \pm standard deviation $(\mathrm{SD})$.

Substrate specificities of Est1 and Est2 were checked towards the following $p$-NP acyl esters of different chain lengths: $p$-NP acetate (C2), $p$-NP butyrate (C4), $p$-NP valerate (C5), $p$-NP caproate (C6), $p$-NP caprylate (C8), $p$-NP caprate (C10), $p$-NP laurate (C12), $p$-NP myristate (C14), and $p$-NP palmitate (C16). Considering the instability of short-chain substrates, the assay temperature was decreased to $50{ }^{\circ} \mathrm{C}$. Initial rates of reaction for $p$-NP butyrate and $p$-NP valerate were calculated by estimating Est1 and Est2 activities with different substrate concentrations ranging from 1 to $2000 \mu \mathrm{M}$. Values for $K_{\mathrm{m}}$ and $V_{\max }$ were determined by employing the Lineweaver-Burk plots (Lineweaver and Burk 1934). Lipolytic activity towards different triacylglycerides was also measured qualitatively by incubating Est1 and Est2 on agar plates emulsified with tributyrin (C4), tricaproin (C6), tricaprylin (C8), tricaprin (C10), trilaurin (C12), trimyristin (C14), or tripalmitin (C16). Formation of clearing zones (halos) on agar plates indicated lipolytic activity. Beta-lactamase activity of Est2 was tested spectrophotometrically at $486 \mathrm{~nm}$, under standard assay conditions with $1 \mathrm{mM}$ nitrocefin (E-isomer) as substrate.

\section{Effect of temperature and $\mathrm{pH}$}

The effect of $\mathrm{pH}$ on Est1 and Est2 activities was measured at $348 \mathrm{~nm}$ (the $\mathrm{pH}$-independent isosbestic wavelength) under standard assay conditions (Glogauer et al. 2011). The following overlapping buffer systems were used: $50 \mathrm{mM}$ acetate buffer (pH 3.0 to 6.0 ), $50 \mathrm{mM}$ sodium phosphate buffer ( $\mathrm{pH} 6.0$ to 8.0), $50 \mathrm{mM}$ TAPS buffer (pH 8.0 to 9.0), and $50 \mathrm{mM}$ CHES ( $N$-cyclohexyl-2-aminoethanesulfonic acid) buffer (pH 9.0 to 10.0). Temperature optima for Est1 and Est2 activities were measured in a temperature range of 20 to $100{ }^{\circ} \mathrm{C}$. Thermostability of enzyme activity was determined by incubating Est1 and Est2 in their optimal buffers at various temperatures $\left(50\right.$ to $\left.80^{\circ} \mathrm{C}\right)$ for up to 6 days. Subsequently, Est1 and Est2 activities were determined under standard assay conditions.

\section{Combined effect of pH and temperature on the stabilities of Est1 and Est2}

Second-order rotatable design was applied to study the combined effect of $\mathrm{pH}$ and temperature on the stabilities of Est1and Est2. The design was based on five levels and two variables (Table S2). Experimental data were fitted to the empirical model using Eq. (1):

$Z(\%)=b_{0}+b_{1} X+b_{2} Y+b_{12} X Y+b_{11} X^{2}+b_{22} Y^{2}$

in which $Z$ was residual relative activity, presented as the percentage of activity measured before incubation and under standard assay conditions; $X$ and $Y$ were code values of $\mathrm{pH}$ and temperature shown in Table $S 2 ; b_{0}, b_{1}, b_{2}, b_{12}, b_{11}$, and $b_{22}$ were regression coefficients. Significance of regression coefficients was checked by Student's $t$ test $(\alpha=0.05)$. Statistically non-significant coefficients were removed, and best-fit parameters were recalculated (Lazić 2004). The consistency of regression models was checked by Fisher's test $(\alpha=0.05)$. The ratios of the following mean squares were compared with the $F$-criterion tabular values. Based on the following mean square ratios (Box et al. 2005), models were accepted if:

$F_{1}=$ Model $/$ Experimental error $\quad \mathrm{F}_{1} \geq F_{\text {den }}^{\text {num }}$

$F_{2}=$ Lack of fitting/Experimental error $\quad F_{2} \leq F_{\text {den }}^{\text {num }}$

Est 1 and Est 2 were incubated under conditions described in Table $\mathrm{S} 1$ for $2 \mathrm{~h}$, and residual activity was subsequently measured under the respective standard assay conditions.

\section{Effect of miscible and immiscible organic solvents}

The following organic solvents with different $\log p$ values were used in this study: water-miscible organic solvents of DMSO (-1.3), methanol $(-0.75)$, ethanol $(-0.24)$, acetone $(-0.24)$, isopropanol (0.074), and 1-propanol (0.28), as well as the water-immiscible organic solvents of ethyl acetate (0.68), diethyl ether (0.85), chloroform (2.0), and toluene (2.5). The effects of water-miscible organic solvents on Est1 and Est2 activities were measured by adding each organic solvent into the assay buffer to obtain a final concentration ranging from 6 to $30 \%(v / v)$ under standard assay conditions. Enzyme activity measured in organic solvent-free assay buffer was regarded as $100 \%$. Appropriate controls were also set to eliminate changes in extinction coefficients due to the presence of solvents.

To evaluate short-term stability towards water-miscible and water-immiscible organic, Est1 and Est2 were incubated in $100-\mu \mathrm{l}$ aliquots with different amounts of water-miscible organic solvents ( 0 to $75 \%, v / v$ for Est1; 0 to $95 \%, v / v$ for Est2) or water-immiscible organic solvents $(15$ and $30 \%, v / v)$ at $30{ }^{\circ} \mathrm{C}$ for $2 \mathrm{~h}$ with vigorous shaking ( $\left.300 \mathrm{rpm}\right)$. The longterm stability towards water-miscible solvents was only measured for Est2. In the presence of 30 or $60 \%(v / v)$ organic solvents, Est2 was incubated at $30{ }^{\circ} \mathrm{C}$ with constant shaking in a screwed-cap test tube for up to 26 or 13 days, respectively. Enzyme activities in either the aqueous phase (for waterimmiscible solvents) or the mixture (for water-miscible solvents) were measured. Each water-miscible organic solvent was equalized to the same final concentration in the assay buffer and the residual activity was measured under standard 
assay conditions. A blank reference was prepared by using the same buffer solution without enzyme containing and the same amount and type of organic solvent (Shao et al. 2013). Residual activity was subsequently measured under the respective standard assay conditions. The activity measured at the start of the experiment was taken as $100 \%$.

\section{Effect of additives on Est1 and Est2 activities}

The effects of metal ions on Est1 and Est2 activities were examined in $50 \mathrm{mM}$ sodium phosphate buffer $(\mathrm{pH} 8)$ at $50{ }^{\circ} \mathrm{C}$, in the presence of $1 \mathrm{mM}$ and $10 \mathrm{mM} \mathrm{KCl}, \mathrm{CaCl}_{2}$, $\mathrm{MnCl}_{2}, \mathrm{MgCl}_{2}, \mathrm{ZnSO}_{4}, \mathrm{FeSO}_{4}, \mathrm{CuCl}_{2}, \mathrm{NiSO}_{4}, \mathrm{FeCl}_{3}$, and $\mathrm{AlCl}_{3}$. The inhibitory effect on enzyme activity was measured under standard assay conditions with the following known esterase effectors (each $1 \mathrm{mM}$ and $10 \mathrm{mM}$ ): phenylmethylsulfonyl fluoride (PMSF), dithiothreitol (DTT), and ethylenediaminetetraacetic acid (EDTA). In addition, the effect of the following detergents (each 0.1 and $1 \%, v / v$ ), Triton X-100, Tween 20, Tween 80 , and SDS, was determined. Furthermore, the effect of $\mathrm{NaCl}$ and $\mathrm{KCl}$ on enzyme activity in a range between 0.5 and $4 \mathrm{M}$ was assessed. Activity measured in additive-free assay buffer was regarded as $100 \%$ activity, while reactions that included corresponding additive but no enzyme were used as blanks.

\section{Accession number}

The gene sequences are available at the GenBank database under accession numbers KR149567.1 (Est1) and KR149568.1 (Est2).

\section{Results}

\section{Metagenomic library screening and analysis of two novel esterase-encoding genes}

To isolate novel lipolytic enzymes, a compost sample at the thermophilic stage $\left(55^{\circ} \mathrm{C}\right)$ was used for constructing a metagenomic plasmid library. The library consisted of approximately 675,200 clones with an average insert size of $5.3 \mathrm{~kb}$ and comprised a total size of $3.58 \mathrm{~GB}$. Among the 279 lipolytic-positive clones, two $E$. coli clones harboring the plasmids pFLD_Est1 and pFLD_Est2 showed strong lipolytic activity (large halos) on indicator plates and were selected for further characterization.

Sequence analyses of the plasmids pFLD_Est1 and pFLD_Est2 revealed that each contained one putative esterase-encoding gene, est $1(813 \mathrm{bp})$ and est2 (1170 bp), respectively. The deduced proteins comprised 270 (Est1) and 389 (Est2) amino acids. Putative signal peptides indicating extracellular localization were not detected in the deduced protein sequences. Sequence similarity searches showed that Est1 exhibited $49 \%$ identity to a hypothetical protein from Candidatus Entotheonella (GenBank: ETW96815) and 43\% identity to Est28 from a grassland soil metagenomic library (Nacke et al. 2011). Est2 showed 53\% sequence identity to a beta-lactamase from Streptomyces lavenduligriseus (GenBank: WP_030784121) and 52\% sequence identity to a putative esterase from Streptomyces bottropensis (GenBank: EMF58012).

Sequence analysis and the subsequently constructed phylogenetic tree revealed that Est1 belonged to family $\mathrm{V}$ and Est2 to family VIII of lipolytic enzymes (Fig. S1). The tertiary structure predicted by I-TASSER obtained the $C$-scores of 1.03 for Est1 and 1.27 for Est2, which indicated a significant confidence of good quality.

The two conserved family V motifs G-X-S-X-G-G and P$\mathrm{T}-\mathrm{L}$ were present in the Est1 protein sequence at amino acid positions 92 to 97 and 208 to 210, respectively (Fig. S2a). The tertiary structure of Est1 was composed of a cap domain with five $\alpha$-helices ( $\alpha 4$ to $\alpha 8$, Fig. S2a) and an $\alpha / \beta$-hydrolase fold core domain (Fig. 1a). The core domain consists of six helices surrounded by eight $\beta$-strands that form parallel structures, in which $\mathrm{Ser}^{94}$ is located between $\beta 5$ and $\alpha 3, \mathrm{Asp}^{217}$ after $\beta 7$, and $\mathrm{His}^{268}$ between $\beta 8$ and $\alpha 10$. The overall structure of Est1 superimposed on MGS-M2 (TM score 0.96; RMSD 0.94) (Alcaide et al. 2015), with a global amino acid sequence identity of $21.9 \%$ (Fig. 1c)

Due to the high sequence similarity between family VIII lipolytic enzymes and class $C$ beta-lactamases/penicillin-binding proteins, amino acid sequences in the two categories were aligned (Fig. S2b). All the aligned sequences shared the same catalytic triad of serine, lysin, and tyrosine. Conserved family VIII motifs including $\mathrm{S}^{70}-\mathrm{X}-\mathrm{X}-\mathrm{K}^{73}, \mathrm{G}^{154}-\mathrm{X}-\mathrm{X}-\mathrm{X}-\mathrm{X}-\mathrm{H}^{159}$, and $\mathrm{H} / \mathrm{W}^{341}-\mathrm{X}-\mathrm{G}^{343}$ were detected in Est2 sequences. However, classical $\beta$-lactamase motifs such as Y-A-N and L-S/T-G (KTG-box) were absent in the Est2 sequence (Fig. S2b). Similar to Est1, the tertiary structure of Est2 also consists of two domains: a large $\alpha / \beta$ domain and a small $\alpha$-helical domain. The small $\alpha$-helix domain contains four helices and two $3_{10}$ helices. For the large $\alpha / \beta$ domain, a central eight-stranded antiparallel $\beta$-sheet is flanked by six helices on one face and two on the opposite face (Fig. 1c). The deduced catalytic residues of $\mathrm{Ser}^{70}$, $\mathrm{Lys}^{73}$, and $\mathrm{Tyr}^{159}$ are located in the large cavity between the two domains (Fig. 1b). The overall structure of Est2 superimposed well on CcEstA (TM score 0.95; RMSD 0.46 ) (Woo Lee et al. 2016), with a global amino acid sequence identity of $39.6 \%$ (Fig. 1d)

\section{Purification of Est1 and Est2 and substrate specificity towards $p$-NP acyl esters}

The esterase-encoding genes est 1 and est 2 were successfully cloned in expression vectors and expressed in E. coli BL21(DE3). The produced gene products Est1 and Est2 were 
Fig. 1 The modeled threedimensional structure of Est1 and Est2. a A 3D structure model of Est 1 . The overall structure is composed of two domains: cap domain and catalytic domain. $\mathbf{b} \mathrm{A}$ 3D structure model of Est2. The overall structure harbors two domains: a large $\alpha / \beta$ domain and a small $\alpha$-helix domain. $\alpha$-helices and $\beta$-strands are colored in cyan and magenta, respectively. The catalytic triad of Est1 (residues $\mathrm{Ser}^{94}, \mathrm{Asp}^{215}$, and $\mathrm{His}^{245}$ ) and that of Est2 (residues $\mathrm{Ser}^{70}$, Lys ${ }^{73}$, and Try $^{160}$ ) are indicated in stick representation. The cap domain for Estl and the small $\alpha$-helix domain for Est2 are presented as transparent surface. c Structural superposition of Estl (pink) onto its structural homolog MGS-M2 (yellow; PDB: 4Q3L). d Structural superposition of Est2 (pink) onto its structural homolog CcEstA (yellow; PDB:5GKV)
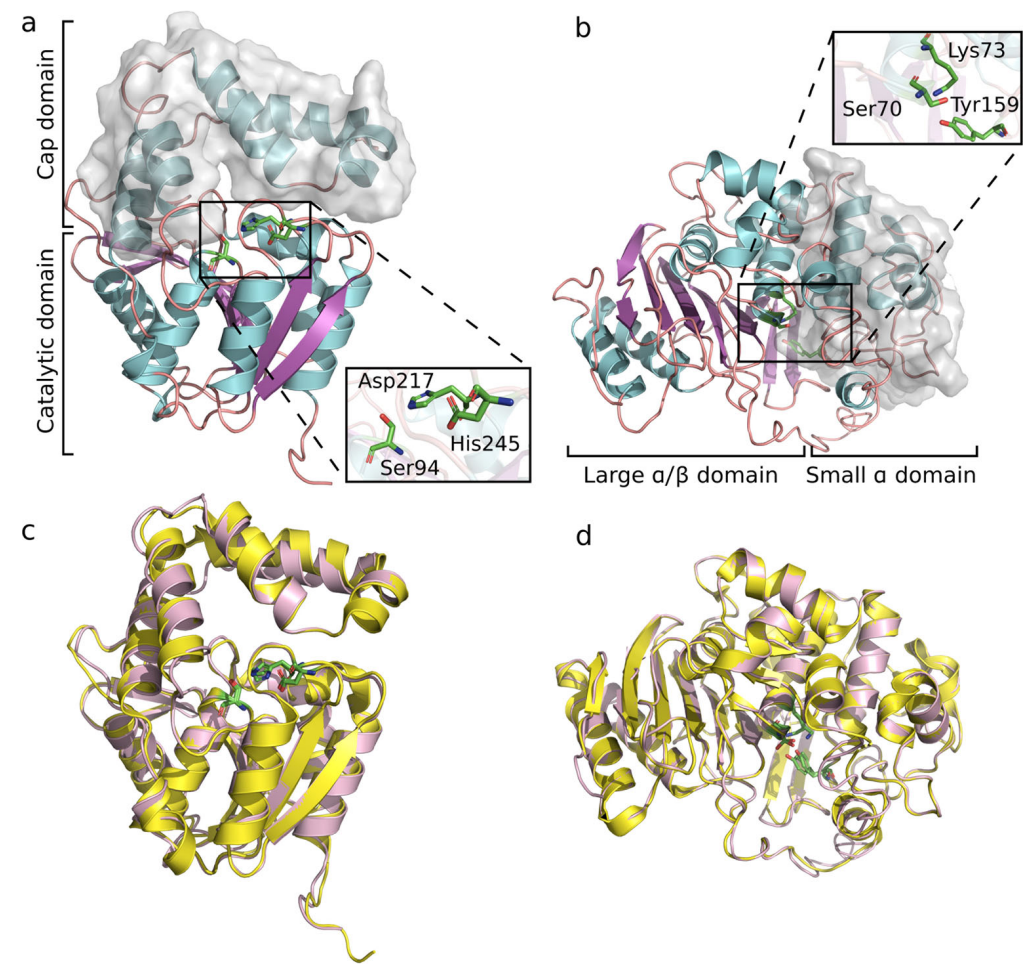

d

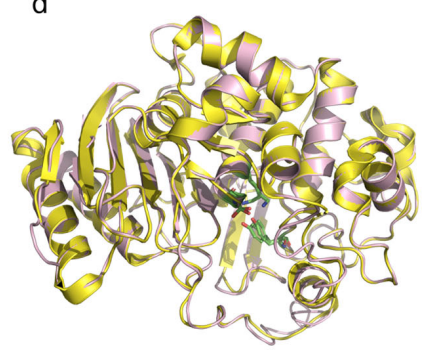

subsequently purified by Ni-TED affinity chromatography, yielding 28.9-fold and 16.9-fold purification values and specific activities of 22.2 and $7.3 \mathrm{U} / \mathrm{mg}$, respectively (Table S2). SDS-PAGE analysis revealed single bands with molecular masses of approximately $35 \mathrm{kDa}$ (Est1) and $48 \mathrm{kDa}$ (Est2) (Fig. S3), which were in accordance with the calculated masses including during cloning added V5 epitope and $\mathrm{His}_{6}{ }^{-}$ tag.

Est1 and Est2 exhibited a substrate preference for esters with short-chain fatty acids. The maximal activities were detected with $p$-NP butyrate (C4) for Est1 and $p$-NP valerate (C5) for Est2 as substrates (Fig. 2). Correspondingly, $p$-NP butyrate and $p$-NP valerate were used for calculating the $K_{\mathrm{m}}$ and $V_{\max }$ values. The $K_{\mathrm{m}}$ and $V_{\max }$ values of Est1 were $3.0 \mu \mathrm{M}$ and $31.2 \mathrm{U} / \mathrm{mg}$, respectively, and that of Est2 $2.7 \mu \mathrm{M}$ and $19.0 \mathrm{U} / \mathrm{mg}$, respectively. With respect to esters with longchain fatty acids as substrates ( $\mathrm{C} 10$ to $\mathrm{C} 16)$, Estl was active with $p$-NP caprate (C10) retaining 45\% activity, but barely with other substrates. In comparison, Est2 hydrolyzes more acyl esters with long-chain fatty acids. It showed $80 \%, 45 \%$, $12 \%$, and $30 \%$ activity towards $p$-NP caprate (C10), laurate (C12), $p$-NP myristate (C14), and $p$-NP palmitate (C16), respectively. Chain length selectivity towards triacylglycerides was recorded for the substrates tributyrin $\left(\mathrm{C}_{4}\right)$ and tricaproin $\left(\mathrm{C}_{6}\right)$ for both enzymes. The preference for short substrates (< C10) indicates that Est1 and Est2 are esterases, rather than "true" lipases which are commonly found in family I of lipolytic enzymes (Arpigny and Jaeger 1999). Moreover, betalactamase activity of Est2 with nitrocefin as substrate was tested, but no significant beta-lactamase activity was detected (data not shown).

\section{Effect of pH and temperature on Est1 and Est2 activities}

Est 1 and Est 2 were active over the entire tested $\mathrm{pH}$ range (3 to 10), with the exception of Est1 at pH 3 (Fig. 3a, d). Est1 exhibited maximal activity at $\mathrm{pH} 7$ and retained more than $95 \%$ activity between $\mathrm{pH} 6$ and 8 . Est 2 activity increased with $\mathrm{pH}$ and peaked at $\mathrm{pH} 9$. With respect to the temperature dependence of enzyme activity, Est1 and Est2 displayed a similar ascending trend along the temperature gradient, showing a maximal activity at 80 and $70{ }^{\circ} \mathrm{C}$, respectively. At higher temperatures $\left(90\right.$ and $100{ }^{\circ} \mathrm{C}$ ), approximately $80 \%$ activity was retained (Fig. 3b, e). 

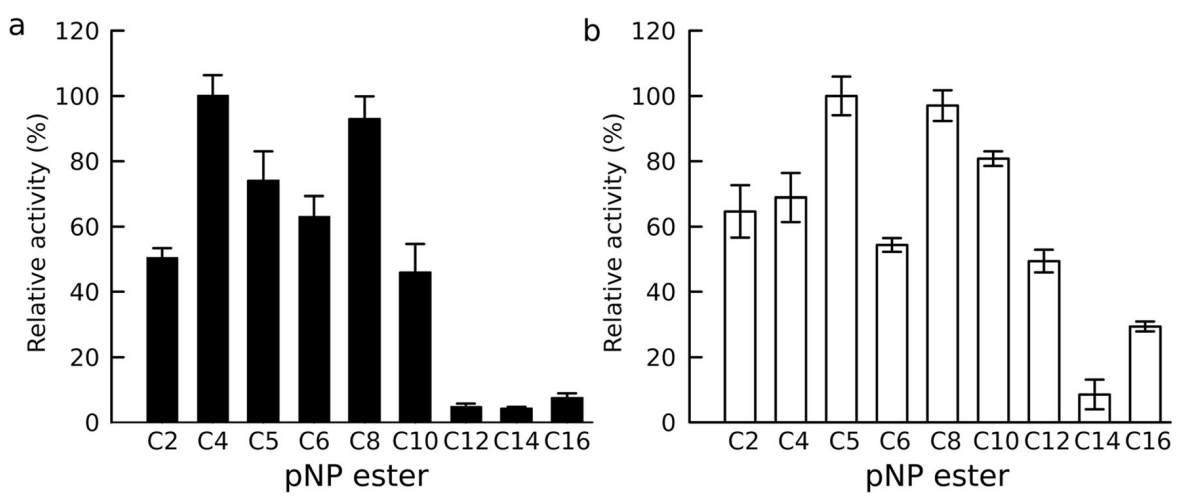

Fig. 2 Substrate specificity towards $p$-NP acyl esters with different chain lengths. a Est1, the maximal activity (23.3 U/mg) measured towards $p$-NP butyrate (C4) was taken as $100 \%$. b Est2, the maximal activity $(10.7 \mathrm{U} / \mathrm{mg})$ measured towards $p$-NP valerate (C5) was taken as $100 \%$

Despite that the activity maximum of Est 1 was at $80^{\circ} \mathrm{C}$, stability at $70{ }^{\circ} \mathrm{C}$ was low as a rapid drop of activity to $50 \%$ was detected after $15 \mathrm{~min}$ of incubation. Similar results were obtained for Est2 at $80^{\circ} \mathrm{C}$ with $35 \%$ residual activity after 30 min. Strikingly, Est2 showed significant stability at $70{ }^{\circ} \mathrm{C}$, with $50 \%$ residual activity after $12 \mathrm{~h}$ of incubation. At lower temperatures $\left(50\right.$ or $\left.60^{\circ} \mathrm{C}\right)$, the activities of both enzymes were remarkably stable for extended incubation times; nonetheless, Est1 displayed a higher stability than Est2 (Fig. 3c). Est1 retained more than $80 \%$ activity at $50{ }^{\circ} \mathrm{C}$ over the entire incubation period (7 days), whereas $52 \%$ residual activity was observed for Est2 after 5 days. When incubated at $60^{\circ} \mathrm{C}$, Est1 exhibited a half-life of 2 days, which is nearly twice as that of Est2 (Fig. 3f).
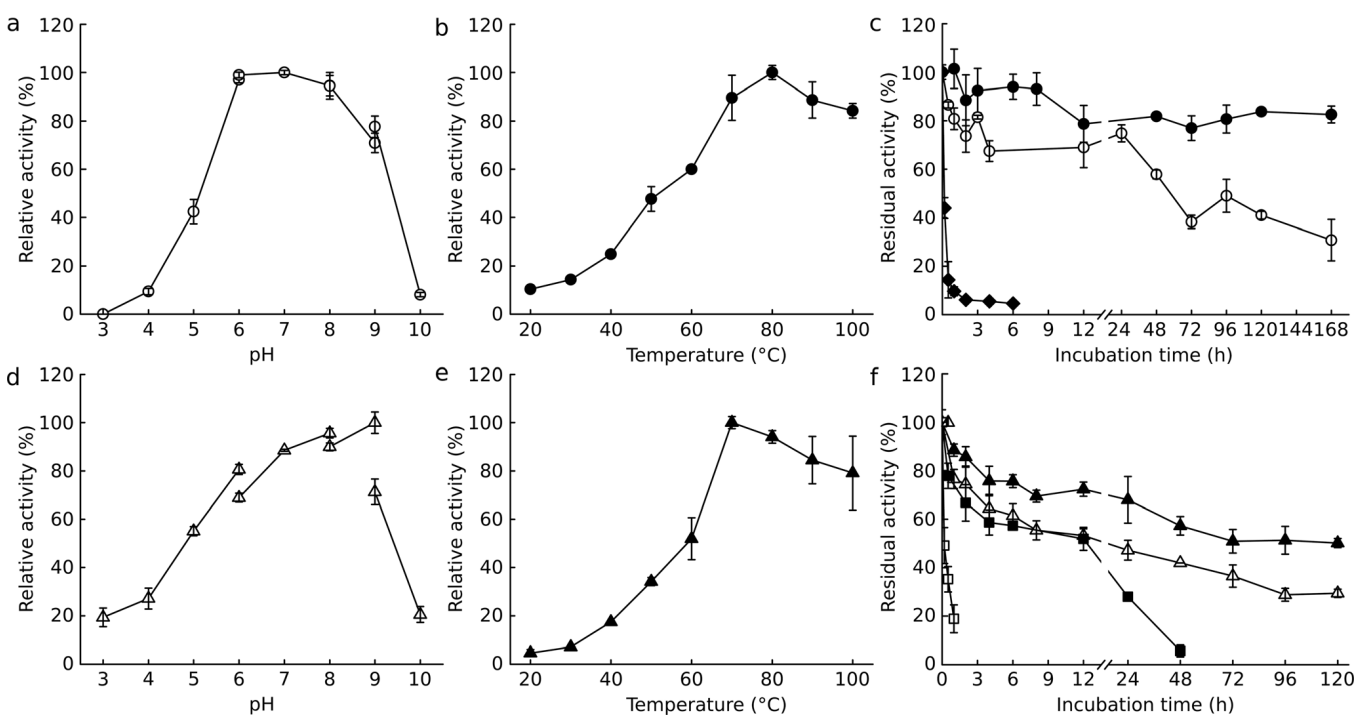

Fig. 3 Effect of $\mathrm{pH}$ and temperature on esterase activity. a Effect of $\mathrm{pH}$ on Estl activity. Maximal activity at $\mathrm{pH} 7(45.8 \mathrm{U} / \mathrm{mg})$ was taken as $100 \%$. b Effect of temperature on Est1 activity. Maximal activity at $80^{\circ} \mathrm{C}(46.1 \mathrm{U} /$ $\mathrm{mg}$ ) was taken as $100 \%$. c Thermostability of Est 1 at $50{ }^{\circ} \mathrm{C}$ (closed circle), $60{ }^{\circ} \mathrm{C}$ (open circle), and $70{ }^{\circ} \mathrm{C}$ (closed diamond); activity measured before incubation $(40.4 \mathrm{U} / \mathrm{mg})$ was taken as $100 \%$. d Effect

of $\mathrm{pH}$ on Est2 activity. Maximal activity at $\mathrm{pH} 9(14.4 \mathrm{U} / \mathrm{mg})$ was taken as $100 \%$. e Effect of temperature on Est2 activity. Maximal activity at $70^{\circ} \mathrm{C}$ $(15.2 \mathrm{U} / \mathrm{mg})$ was taken as $100 \%$. f Thermostability of Est2 at $50{ }^{\circ} \mathrm{C}$ (closed triangle), $60{ }^{\circ} \mathrm{C}$ (open triangle), $70{ }^{\circ} \mathrm{C}$ (closed square), and $80{ }^{\circ} \mathrm{C}$ (open square); activity measured before incubation $(12.0 \mathrm{U} / \mathrm{mg})$ was taken as $100 \%$ 


\section{Combined effect of $\mathrm{pH}$ and temperature on Est1 and Est2 stabilities}

The combined effect of temperature and $\mathrm{pH}$ on the stability of Est1 and Est2 was visualized by response surfaces (Fig. 4). After removing the insignificant terms and refitting to the empirical model, the following equations for Est1 (4) and Est2 (5) stability under different conditions were obtained:

Residual activity $(\%)=76.33$

$+13.56 \mathrm{pH}-31.89 T-11.19 \mathrm{pH} T-19.09 \mathrm{pH}^{2}-17.82 T^{2}$

Residual activity $(\%)=84.1$

$$
+14.1 \mathrm{pH}-19.3 \mathrm{~T}-6.3 \mathrm{pH}^{2}-23.8 \mathrm{~T}^{2}
$$

Variance analysis implied that the models for Est 1 and Est2 significantly fitted to the experimental data $\left(F_{1} \geq \mathrm{F}_{\text {den }}^{\text {num }}, F_{2} \leq \mathrm{F}_{\text {den }}^{\text {num }}, \alpha=0.05\right.$; Table S3). In equations (4) and (5), the negative coefficients for $T, \mathrm{pH}^{2}$ and $T^{2}$ indicate the existence of an absolute maximum of residual activity. The calculated optimal conditions were located at pH 8.2 and $39.1{ }^{\circ} \mathrm{C}$ (Est1) and pH 8.7 and $48.9{ }^{\circ} \mathrm{C}$ (Est2). It was predicted that Est1 and Est2 retain 98.6 and $96.3 \%$ residual activity, respectively, under these conditions. Moreover, according to the elliptical contour plot on the $\mathrm{pH}-T(x-y)$ dimension, the $\mathrm{pH}$ and temperature ranges in which Est 1 and Est 2 retain more than $80 \%$ residual activity were $\mathrm{pH} 7.2$ to 9.2 and 23.6 to $54.6{ }^{\circ} \mathrm{C}$ for Est 1 and pH 7.0 to 10.4 and 37.4 to $60.4{ }^{\circ} \mathrm{C}$ for Est2 (Fig. 4). In conclusion, Est1 and Est2 are stable under thermophilic and alkaline conditions.

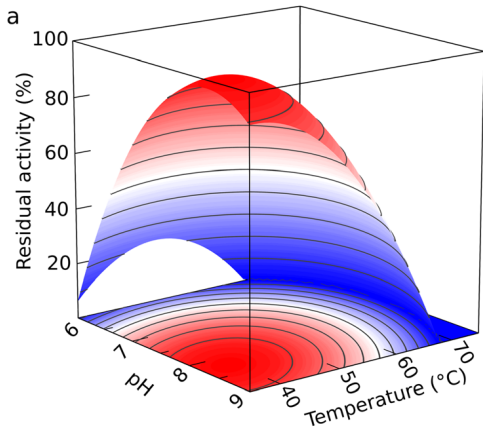

Fig. 4 Response surface corresponding to the combined effect of $\mathrm{pH}$ and temperature on Est1 (a) and Est2 (b) stabilities. Residual activity was expressed as a percentage of the initial activity measured before

\section{Effect of water-miscible organic solvents on Est1 and Est2 activities}

Est 1 activity increased to approximately $150 \%$ in the presence of low concentrations of 1-propanol (below 6\%,v/v), and ethanol and isopropanol $(<12 \%, v / v)$. At higher concentration, Est1 activity decreased rapidly and was not detectable at $18 \%(v / v)$ 1-propanol, $30 \%(v / v)$ ethanol, and $24 \%(v / v)$ isopropanol (Fig. 5a). Addition of DMSO, methanol, and acetone inhibited Est1 activity over the tested concentration range, retaining $50 \%$ activity at $30 \%(v / v)$ DMSO, or less than $10 \%$ activity at $30 \%(v / v)$ methanol and acetone.

Ethanol, methanol, isopropanol, and 1-propanol had a stimulatory effect on Est2 activity almost at all tested concentrations (Fig. 5b). In the presence of ethanol and methanol, Est2 activity increased continually with raising concentrations. At $30 \%(v / v)$ ethanol and methanol, Est2 activity increased 8.8fold and 5.4-fold, respectively. For isopropanol and 1propanol, optimal Est2 activity was recorded at concentrations of 18 and $12 \%(v / v)$, respectively (Fig. $5 b)$. In the presence of DMSO, Est2 retained more than $70 \%$ activity at all tested concentrations, with the maximal activity at $6 \%(v / v)$. Acetone caused an activity decrease to $34.1 \%$ at $30 \%(v / v)$.

\section{Effect of water-miscible organic solvents on Est1 and Est2 stabilities}

Est1 retained almost unchanged residual activity after $2 \mathrm{~h}$ of incubation in the presence of 15 and $30 \%(v / v)$ of all tested organic solvents, with the exception of 1-propanol (Fig. 6a). Est1 residual activity rapidly dropped to $17.0 \%$ at $30 \%(v / v)$ 1-propanol. Decreased tolerance was also detected after exposure to $45 \%(v / v)$ isopropanol, methanol, acetone, and DMSO for $2 \mathrm{~h}$ (Fig. 6a). At $60 \%(v / v)$ solvent concentration, Est1 exhibited moderate tolerance to DMSO (60.5\% residual activity), but lost most of its activity (less than $10 \%$

\section{金 Springer}

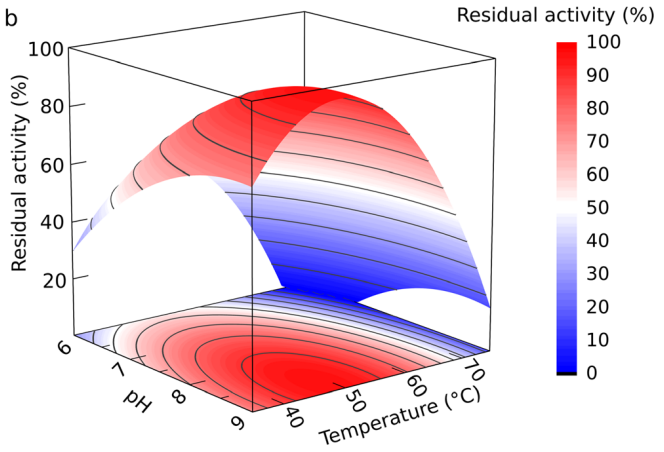

incubation under standard assay conditions. The elliptical contour plot on the $\mathrm{pH}-T$ dimension demonstrates the change of residual activity (\%) 


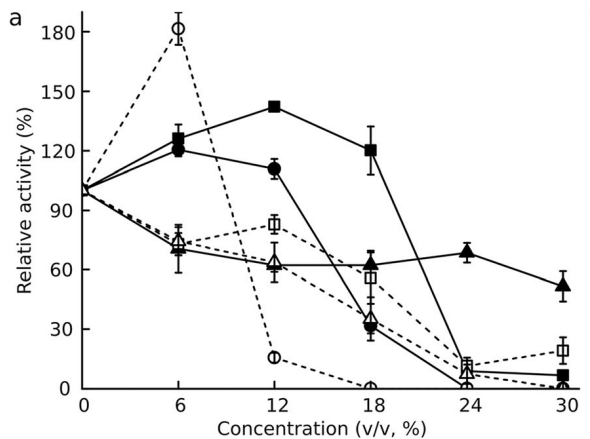

Fig. 5 Effects of water-miscible organic solvents on of Est1 (a) and Est2 (b) activities. Catalytic activity was measured under standard assay conditions in the presence of different amounts of ethanol (closed square), methanol (open square), isopropanol (closed circle), 1-propanol

residual activity) in the presence of the other tested organic solvents. Est 1 activity was almost inactivated by all the tested organic solvents at $75 \%(v / v)$.

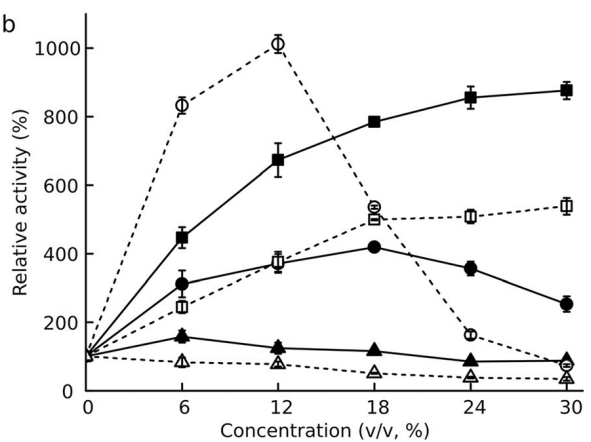

(open circle), DMSO (closed triangle), and acetone (open triangle) Specific activities corresponding to $100 \%$ relative activity were $21.9 \mathrm{U} /$ $\mathrm{mg}$ (Est1) and $5.1 \mathrm{U} / \mathrm{mg}$ (Est2)

In comparison with Est1, Est2 displayed higher resistance towards water-miscible solvents (Fig. 6b). In the organic solvent concentration range of 0 and $60 \%(v / v)$, Est2 retained its

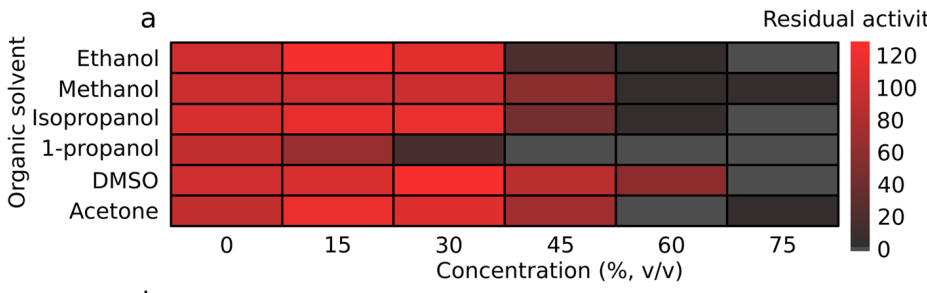

b $30 \quad 45$
Concentration $(\%, v / v)$

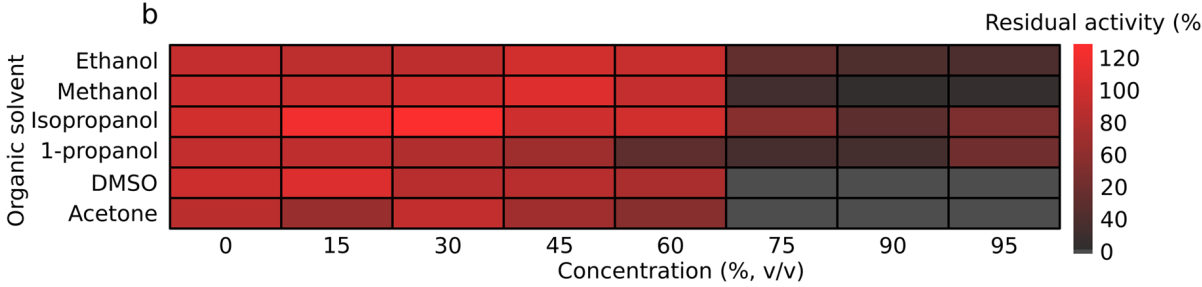

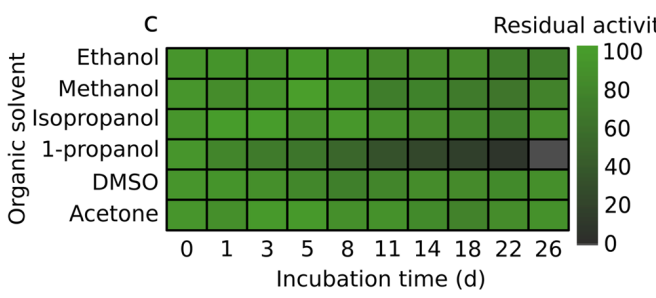

Fig. 6 Heatmap displaying the effect of enzyme stability towards watermiscible organic solvents. Short-term stability of Est1 (a) and Est2 (b) towards different concentrations of organic solvents at $30^{\circ} \mathrm{C}$ for $2 \mathrm{~h}$. The specific activity expressed as percentages of Est1 reference reactions $(100 \%)$ are $23.3,17.5,24.3,6.6,15.3$, and $13.6 \mathrm{U} / \mathrm{mg}$ for reactions in the presence of ethanol, methanol, isopropanol, 1-propanol, DMSO, and acetone, respectively, and that of Est2 are 32.7,14.4, 17.7, 42.7, 4.34, and

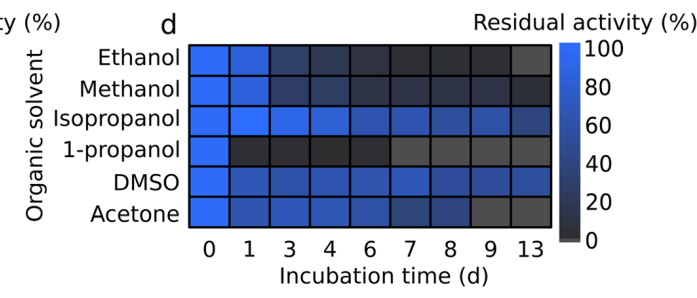

$3.1 \mathrm{U} / \mathrm{mg}$, respectively. Long-term stability of Est2 towards $30 \%(v / v ; \mathbf{c})$ and $60 \%(v / v ; \mathbf{d})$ organic solvents for prolonged time periods at $30{ }^{\circ} \mathrm{C}$. The specific activity values expressed as percentages of Est 2 reference reactions $(100 \%)$ are $14.3,7.1,9.2,25.9,5.0$, and $4.1 \mathrm{U} / \mathrm{mg}$ for reactions in the presence of ethanol, methanol, isopropanol, 1-propanol, DMSO, and acetone, respectively 
full activity in the presence of isopropanol, methanol, and ethanol and had a slight activity loss by the addition of DMSO and acetone. On the contrary, Est2 stability decreased along the rising concentration of 1-propanol, retaining 32.4\% residual activity at $60 \%(v / v)$. Incubating with concentrations above $60 \%(v / v)$ of isopropanol, 1-propanol ethanol, or methanol led to a rapid decline of Est2 residual activity (Fig. 6b). Exposure to $75 \%(v / v)$ DMSO and acetone led to inactivation of Est2 activity.

Est2 exhibited high stability in the presence of $30 \%(v / v)$ ethanol, methanol, isopropanol, DMSO, and acetone, as its residual activity was above $70 \%$ over the extended incubation period of up to 26 days (Fig. 6c). This residual activity was even higher than that Est2 exhibited during incubation without additions (Fig. S4). However, a continuous drop in Est2 residual activity was observed when exposed to 1-propanol. Est2 also displayed decreased tolerance towards $60 \%(v / v)$ organic solvents (Fig. 6d). Incubation with 1-propanol, ethanol, and methanol reduced Est2 activity rapidly $(<10 \%$ residual activity). Nonetheless, Est2 exhibited substantial tolerance against isopropanol, DMSO, and acetone, retaining approximately $40 \%$ residual activity after the 13 -day incubation.

\section{Effect of water-immiscible organic solvents on Est1 and Est2 stabilities}

Incubation with water-immiscible organic solvents of diethyl ether, chloroform, and toluene resulted in deleterious effects on Est1 enzyme activity, which was not detectable at the tested concentrations (Table 1). However, Est1 activity displayed some resistance towards ethyl acetate, with 46.6 and $21.1 \%$ residual activities at the tested concentrations of 15 and $30 \%$ $(v / v)$, respectively. In contrast, Est2 was tolerant to ethyl acetate, diethyl ether, and toluene. Est2 retained its activity after incubation with ethyl acetate and diethyl ether. In the presence of toluene, Est2 retained 87.0 and $68.8 \%$ activities at the tested concentrations of 15 and $30 \%(v / v)$, respectively. In addition, Est2 was as Est1 inactivated by chloroform.

\section{Effect of other additives on Est1 and Est2 activities}

Metal ions, inhibitors, detergents, and salts were also analyzed for their effects on Est1 and Est2 activities. Est1 and Est2 are generally resistant to various metal ions (Table S4). The addition of tested metal ions at $1 \mathrm{mM}$ concentration had minor effects on Est1 and Est2 activities as approximately $90 \%$ of the activity was retained. Moreover, both enzymes exhibited substantial tolerance (above 50\% activity) towards some metal ions such as $\mathrm{Ca}^{2+}, \mathrm{Zn}^{2+}, \mathrm{Cu}^{2+}, \mathrm{Mn}^{2+}, \mathrm{Fe}^{3+}$, and $\mathrm{Al}^{3+}$ at a concentration of $10 \mathrm{mM}$. Enzyme activity was slightly enhanced in the presence of $10 \mathrm{mM} \mathrm{Mg}{ }^{2+}$ (Est1) and $\mathrm{Zn}^{2+}$ (Est2). The presence of the chelating agent EDTA did not affect Est1 activity but decreased Est2 activity to less than
$70 \%$. The latter results indicated that Est 1 activity is independent of metal ions, whereas Est2 might be a metalloenzyme (Mohamed et al. 2013).

With respect to the detergents, Est1 activity was enhanced in the presence of $0.1 \%(v / v)$ Tween 20 and 0.1 and $1 \%(v / v)$ Tween 80. Est2 activity was less resistant than that of Est1 towards the tested detergents. Est2 retained more than 70\% activity after addition of $1 \mathrm{mM}$ Triton-100, Tween 20 , and Tween 80 . The anionic detergent SDS inhibited the activity of both enzymes entirely (Table S4). The activity of Est1 and Est2 was substantially decreased at $10 \mathrm{mM}$ DTT and PMSF. DEPC displayed detrimental effects on Est1 activity, while Est2 activity was almost unaffected even at a concentration of $10 \mathrm{mM}$. The inhibition of enzyme activity by SDS and PMSF indicates that Est1 and Est2 belong to the serine hydrolases (Peng et al. 2011). In addition, Est1 and Est2 were active in the presence of 0 to $4 \mathrm{M} \mathrm{NaCl}$ and $\mathrm{KCl}$. Both enzymes retained more than $50 \%$ of activity up to $2.5 \mathrm{M} \mathrm{NaCl}$ and $\mathrm{KCl}$, which is indicative of halotolerance (Table S5).

\section{Discussion}

Extreme environments such as compost have been used for mining biocatalysts, which are likely to be adapted to harsh industrial reaction conditions (Ryckeboer et al. 2003). In this study, compost derived from the thermophilic core of the pile was used as DNA source to construct a metagenomic library, whereby two putative genes encoding lipolytic enzymes were identified. The low identities of the deduced amino acid sequences to known proteins (Est1 43\% and Est2 53\%) indicate that Est1and Est2 are novel lipolytic enzymes. Est1 is most related to the characterized esterase EstPS5, which was derived from a screening of a peat-swamp forest soil metagenome (Bunterngsook et al. 2010). Est2 shared the highest sequence identity with a beta-lactamase from Streptomyces achromogenes. In addition, most of the enzyme sequences similar to Est2 were derived from members of the Streptomyces genus. Streptomyces strains are predominantly found in soil and decaying vegetation and well-known as important natural sources for antibiotics (Raja and Prabakarana 2011).

Phylogenetic analyses indicate that Est 1 and Est 2 belong to family V and family VIII of lipolytic enzymes, respectively (Fig. S1). For most of the known lipolytic enzymes, the catalytic activity generally relies on the typical catalytic triad of Ser, Asp/Gly, and His (Ollis et al. 1992; Jaeger et al. 1999). This triad is also present in the Est1 amino acid sequence (Fig. S2a) and its tertiary structure (Fig. 1a). However, another catalytic triad consisting of $\mathrm{Ser}^{70}, \mathrm{Lys}^{73}$, and $\mathrm{Tyr}^{159}$ (Sakai et al. 1999) is responsible for Est2 catalytic activity (Fig. 1c, Fig. $\mathrm{S} 2 \mathrm{~b})$. This triad is conserved among family VIII lipolytic enzymes, class $\mathrm{C} \beta$-lactamases, and penicillin-binding proteins (Arpigny and Jaeger 1999; Hausmann and Jaeger 2010; Biver 
Appl Microbiol Biotechnol

Table 1 Short-term stabilities of Est1 and Est2 towards waterimmiscible organic solvents.

\begin{tabular}{|c|c|c|c|}
\hline \multirow[t]{2}{*}{ Water-immiscible organic solvent $(\log P)$} & \multicolumn{3}{|c|}{ Residual activity $(\%)^{\mathrm{a}}$} \\
\hline & $\begin{array}{l}\text { Concentration } \\
(v / v)\end{array}$ & Est1 & Est2 \\
\hline None $^{b}$ & - & $100 \pm 2.6$ & $100 \pm 1.5$ \\
\hline \multirow[t]{2}{*}{ Ethyl acetate $(0.68)$} & 15 & $46.6 \pm 5.7$ & $95.5 \pm 4.2$ \\
\hline & 30 & $21.1 \pm 3.0$ & $101.5 \pm 8.9$ \\
\hline \multirow[t]{2}{*}{ Diethyl ether (0.85) } & 15 & $\mathrm{ND}^{\mathrm{c}}$ & $116.0 \pm 3.8$ \\
\hline & 30 & $\mathrm{ND}^{\mathrm{c}}$ & $96.5 \pm 4.2$ \\
\hline \multirow[t]{2}{*}{ Chloroform (2.0) } & 15 & $\mathrm{ND}^{\mathrm{c}}$ & $\mathrm{ND}^{\mathrm{c}}$ \\
\hline & 30 & $\mathrm{ND}^{\mathrm{c}}$ & $\mathrm{ND}^{\mathrm{c}}$ \\
\hline \multirow[t]{2}{*}{ Toluene (2.5) } & 15 & $\mathrm{ND}^{\mathrm{c}}$ & $87.0 \pm 6.0$ \\
\hline & 30 & $\mathrm{ND}^{\mathrm{c}}$ & $68.8 \pm 8.0$ \\
\hline
\end{tabular}

${ }^{a}$ Aliquots of enzyme were shortly centrifuged; the aqueous layer was used for activity measurements under standard assay conditions

${ }^{\mathrm{b}}$ Specific activity corresponding to $100 \%$ residual activities were $21.9 \mathrm{U} / \mathrm{mg}$ (Est1) and $11.2 \mathrm{U} / \mathrm{mg}$ (Est2)

${ }^{\mathrm{c}}$ Not detectable and Vandenbol 2013; Popovic et al. 2017). Despite the same catalytic triad and high amino acid sequence identity to $\beta$ lactamases, some family VIII esterases show promiscuous $\beta$-lactamase activity. Similar to Est2, the family VIII esterases EstB (Petersen et al. 2001), Lip8 (Ogino et al. 2004), Est2K (Kim et al. 2010), and Est7K (Woo Lee et al. 2016) showed negligible or no detectable $\beta$-lactamase activity, whereas EstC (Rashamuse et al. 2009), EstM-N1 (Yu et al. 2011), and PBS2 (Boyineni et al. 2014) exhibited moderate or high $\beta$ lactamase activity. Yu et al. (2011) suggested that those promiscuous $\beta$-lactamase activities could be a result of family VIII esterases evolving from class $C \beta$-lactamases or vice versa.

According to the predicted tertiary structure (Fig. 1), the active sites of Est 1 and Est 2 are protected by an $\alpha$-helix domain (cap domain). This domain acts as a shield for the catalytic site of many lipolytic enzymes and appears to play a key role in several functional aspects, such as activity, substrate specificity, and thermostability (Gall et al. 2014; Li et al. 2015; Kim 2017). Est 2 was capable of utilizing acyl esters with long-chain fatty acids as substrate (C10, C12, and C16) (Fig. b). In addition, the Est 2 structural homolog Est-Y29 (Ngo et al. 2013) was also reported to hydrolyze a wide variety of hydrophobic compounds, as a result of the deep hydrophobic patch between the large $\alpha / \beta$ domain in which the small $\alpha$ helix domain defines a wide active site (Ngo et al. 2014).

Est1 and Est 2 were generally thermoalkaline esterases (Fig. 3). In terms of thermophilicity, defined as increased enzymatic activity along a temperature gradient (Georis et al. 2000), Est1 and Est 2 have great advantages in comparison with its homologs from other sources. Although esterases from Fervidobacterium nodosum (Yu et al. 2010), Anoxybacillus gonensis (Faiz et al. 2007), Sulfolobus tokodaii
(Angkawidjaja et al. 2012), and a compost metagenome (Riedel et al. 2015) displayed optimal activities above $70{ }^{\circ} \mathrm{C}$, Estland Est 2 exhibited higher activities (above $80 \%$ ) at 90 and $100{ }^{\circ} \mathrm{C}$. Moreover, Est1 and Est2 displayed unprecedented thermostability at high temperatures, with a half-life of more than 7 days at $50^{\circ} \mathrm{C}$ and 2 days at $60^{\circ} \mathrm{C}$ for Est1 (Fig. 4c) and 5 days at $50^{\circ} \mathrm{C}, 1$ day at $60^{\circ} \mathrm{C}$, and $12 \mathrm{~h}$ at $70^{\circ} \mathrm{C}$ for Est2 (Fig. 4f). This remarkable feature distinguished the two enzymes from their thermophilic counterparts, such as esterases from the thermophilic microorganisms Archaeoglobus fulgidus $\left(40 \%\right.$ residual activity after $30 \mathrm{~min}$ at $60{ }^{\circ} \mathrm{C}$; D'Auria et al. 2000), Thermogutta terrifontis (75\% residual activity after $30 \mathrm{~min}$ at $70{ }^{\circ} \mathrm{C}$; Sayer et al. 2015a), and Thermoanaerobacter tengcongensis (half-life of $2 \mathrm{~h}$ at $70{ }^{\circ} \mathrm{C}$; Cook et al. 1996), as well as those from metagenomes of compost (approx. $90 \%$ residual activity after $1 \mathrm{~h}$ at $60{ }^{\circ} \mathrm{C}$; Kang et al. 2011) and hot spring (half-life of $6 \mathrm{~h}$ at $60{ }^{\circ} \mathrm{C}$; Zarafeta et al. 2016).

In general, thermostability is dependent on the structural rigidity, which is an accumulation of various features, including but not limited to amino acid composition, ion pairing, hydrogen bonds, hydrophobic interactions, and sulfide bridges (Sadeghi et al. 2006; Jochens et al. 2010; Ebrahimi et al. 2011; Pezzullo et al. 2013). Interestingly, the two characterized structural homologs of Est1, MGS-M2 (Alcaide et al. 2015) and TtEst (Sayer et al. 2015b), were reported as thermostable esterases (Alcaide et al. 2013; Sayer et al. 2015a). The thermostability of Est 1 and Est 2 is advantageous for industrial applications, as higher reactivity, stability, and process yields, as well as lower viscosity and fewer contamination problems, can be achieved at an elevated operation temperature (Lima et al. 2004; Panda and Gowrishankar 2005; Doukyu and Ogino 2010; Sood et al. 2016). 
Temperature and $\mathrm{pH}$ play important roles in enzymecatalyzed reactions and the two factors have to be considered together for optimization of enzyme reactions. Est1 and Est2 are most stable at conditions which are close to that of the original compost habitat (Fig. 4a, b). Generally, enzymes are stable at conditions similar to their original habitats (Elend et al. 2006; Kovacic et al. 2016), but show maximal activities at higher or lower temperatures (Hardeman and Sjoling 2007; Hu et al. 2010), which is also the case for Est1 and Est2 (Fig. 3). Est1 and Est 2 were predicted to retain more than $80 \%$ residual activity over a broad temperature and $\mathrm{pH}$ range (Fig. 4). Thus, together with the feature of broad substrate specificity (Fig. 2), the two enzymes, particularly Est2, could be potentially utilized in detergents, in which high enzyme stability is required during washing conditions of $\mathrm{pH} 8$ to 11 and temperatures of 30 to $60{ }^{\circ} \mathrm{C}$, as well as versatile substrate specificity (Nerurkar et al. 2013; Bora 2014).

Water-miscible organic solvents are generally detrimental to enzymes. In contrast, Est 2 activity was significantly enhanced by the addition of ethanol, isopropanol, methanol, and 1propanol (Fig. 5b). The stimulated catalytic activity of esterase from Burkholderia cepacia was also observed in the presence of DMSO, DMF, methanol, ethanol, 2-propanol, and acetone (Takeda et al. 2006). Similar results were obtained for family VIII esterases Est2K, lpc53E1, and Est7K in the presence of isopropanol and methanol (Kim et al. 2010; Selvin et al. 2012; Woo Lee et al. 2016). The significant activating effect could be attributed to the uniform water phase formed by water-miscible solvents (Ogino and Ishikawa 2001) or the high diffusion rate of substrate in the presence of water-miscible solvents (Metin et al. 2006), which enables substrates quick and easy access to the active site. Moreover, Est2 activity generally showed a bellshaped dependence on water-miscible organic solvent concentration (Fig. 5b), which indicated an optimal water activity for its hydrolytic activity (Léonard-Nevers et al. 2009; Adlercreutz 2013). Other esterases, including Est1 (Fig. 5a), commonly showed slightly increased (Hotta et al. 2002; Schütte and Fetzner 2007; Faulds et al. 2011; Kang et al. 2017), or decreased (Li and Yu 2013; Monsef Shokri et al. 2014; Dukunde et al. 2017) activity towards water-miscible organic solvents. For these enzymes, water-miscible solvents strip off the crucial water monolayer around the enzyme surface and compete for hydrogen bonds, which at higher solvent concentrations finally leads to denaturation (Ó’Fágáin 2003; Doukyu and Ogino 2010; Monsef Shokri et al. 2014).

In addition, Est1 was stable in the presence of $30 \%(\mathrm{v} / \mathrm{v})$ water-miscible organic solvents (Fig. 6a). Similar or even lower solvent tolerance was found for most of the reported OST esterases (Doukyu and Ogino 2010; Kang et al. 2011; Brault et al. 2012; Xing et al. 2012; Zhang et al. 2014; Kang et al. 2017; Kumagai et al. 2018). Thus, in comparison, Est 2 exhibited superior stability against higher concentrations of the analyzed water-miscible organic solvents (Fig. 6b). Among the rare counterparts, a hyper-thermophilic archaeal esterase (Hotta et al. 2002) and EstB from Alcanivorax dieselolei B-5(T) (Zhang et al. 2014) displayed substantial stability towards certain water-miscible organic solvents at high concentrations. An increase of hydrophobic interactions and hydrogen bonds are essential in enhancing esterase tolerance against water-miscible organic solvents (Song and Rhee 2001; Kawata and Ogino 2009; Park et al. 2012). Est2 also showed considerable tolerance towards $30 \%(v / v)$ ethanol, isopropanol, DMSO, methanol, and acetone for up to 26 days (Fig. 6c) and towards $60 \%(v / v)$ isopropanol, DMSO, and acetone up to 13 days (Fig. 6d). The preservation of high esterase activity over an extended period has been rarely described previously (Sana et al. 2007; Jin et al. 2012; Li and Yu 2013). This feature could allow to apply Est2 as an immobilized biocatalyst in non-aqueous-based continuous bioprocesses (Sana et al. 2007; Yang et al. 2011).

Overall, both enhanced activity and adequate stability towards water-miscible organic solvents are pre-requisite properties for applications of esterases in organic synthesis (Panda and Gowrishankar 2005; Lopez-Lopez et al. 2014). In nonaqueous media, such enzymes could be exploited for ester synthesis and transesterification reactions and a variety of other chemical reactions (Salihu and Alam 2015; Sood et al. 2016; Sarmah et al. 2018). Thus, Est2 could be advantageous for use in biodiesel production, as the acyl acceptors methanol or ethanol are added in esterase/lipase-catalyzed transesterification reactions (Srimhan et al. 2011; Nasaruddin et al. 2014; Wang et al. 2017). In addition, Est1 and Est2 activities responded differently to the presence of polar protic solvents (methanol, ethanol, isopropanol, and 1-propanol) and polar aprotic solvents (acetone and DMSO), which further illustrates that the nature of the solvent influences esterase activity (Torres and Castro 2004). However, the relationship between the corresponding organic solvent $\log P_{\text {ow }}$ value and esterase activity/stability remains uncertain.

Comparing with water-miscible organic solvents, waterimmiscible organic solvents are less deleterious for enzymesolvent interactions (Rahman et al. 2005). However, most of the reported esterases were similar to Est1 (Table 1), showing a significant decrease of activity or inactivation after the incubation with water-immiscible organic solvents (Schütte and Fetzner 2007; Berlemont et al. 2013; Jin et al. 2012). In contrast, lipolytic enzymes of family I, also known as true lipases, are commonly resistant to water-immiscible organic solvents. In the presence of a water-solvent (hydrophobic) interface, the hydrophobic amino acid residues in the lid/flap region stabilize lipases in a flexible, open conformation (Dandavate et al. 2009; Yang et al. 2011; Kamal et al. 2013). However, the cap domain for most esterases is not intrinsically flexible and does not provide open and closed conformations (Bornscheuer 2002; Gall et al. 2014; Kim 2017). This could be the reason that water-immiscible tolerant esterases are rare. To the best of

\section{Springer}


our knowledge, only Est2 and the esterases EstC23 (Jin et al. 2012), LipBL (Pérez et al. 2012), Lpc53E1 (Selvin et al. 2012), RBest1 (Berlemont et al. 2013), Pf Est (Mandelli et al. 2016), EST4 (Gao et al. 2016), and LipA9 (Park et al. 2018) show substantial resistance towards certain waterimmiscible organic solvents. This feature further expands the application potential of Est2 to synthetic reactions in the presence of water-immiscible solvents (Gao et al. 2016; Sarmah et al. 2018).

Est1 and Est2 are to some extent resistant to metal ions (Table S4), which is an important feature in the bioremediation of environmental waste (Brault et al. 2012). Est1 and Est2 were also active at a salinity range of up to $4 \mathrm{M}$ (Fig. S5), suggesting halotolerance (Jeon et al. 2012). Halotolerant enzymes are desirable in processes in which water activity is low (Delgado-García et al. 2012). In combination with the tolerance of Est1 and Est2 to organic solvents, it can be further confirmed that halotolerance is somehow positively correlated with organic solvent tolerance (Berlemont et al. 2013).

In conclusion, the characterization of Est1 and Est2 revealed that both, especially Est2, exhibit several applicationrelevant features, such as a broad substrate range, thermostability, halotolerance, and resistance to various organic solvents. In addition, as revealed by second-order rotatable design, Est1 and Est2 were predicted to be stable at a broad crossed range of temperature and $\mathrm{pH}$. To the best of our knowledge, this is the first time to report an esterase, Est2, which simultaneously exhibits a significantly enhanced activity and unprecedented high stability towards water-miscible organic solvents and substantial tolerance towards waterimmiscible organic solvents.

Acknowledgments We thank Dr Silja Brady and Sarah Zachmann for providing assistance. We acknowledge the support of Mingji Lu by "Erasmus Mundus Action 2 - Lotus I Project."

Author contributions The manuscript was written through the contributions of all authors. All authors have given approval to the final version of the manuscript.

Compliance with ethical standards This article does not contain any studies with human participants or animals performed by any of the authors.

Conflict of interest The authors declare that they have no conflict of interest.

Publisher's note Springer Nature remains neutral with regard to jurisdictional claims in published maps and institutional affiliations.

\section{References}

Adlercreutz P (2013) Immobilisation and application of lipases in organic media. Chem Soc Rev 42:6406-6436. https://doi.org/10.1039/ c $3 \operatorname{cs} 35446 f$
Ahmed EH, Raghavendra T, Madamwar D (2010) An alkaline lipase from organic solvent-tolerant Acinetobacter sp. EH28: application for ethyl caprylate synthesis. Bioresour Technol 101:3628-3634. https://doi.org/10.1016/J.BIORTECH.2009.12.107

Alcaide M, Tornés J, Stogios PJ, Xu X, Gertler C, Di Leo R, Bargiela R, Lafraya Á, Guazzaroni M-E, López-Cortés N, Chernikova TN, Golyshina OV, Nechitaylo TY, Plumeier I, Pieper DH, Yakimov MM, Savchenko A, Golyshin PN, Ferrer M (2013) Single residues dictate the co-evolution of dual esterases: MCP hydrolases from the $\alpha / \beta$ hydrolase family. Biochem J 454:157-166. https://doi.org/10. 1042/BJ20130552

Alcaide M, Stogios PJ, Lafraya Á, Tchigvintsev A, Flick R, Bargiela R, Chernikova TN, Reva ON, Hai T, Leggewie CC, Katzke N, La Cono V, Matesanz R, Jebbar M, Jaeger K-E, Yakimov MM, Yakunin AF, Golyshin PN, Golyshina OV, Savchenko A, Ferrer M, MAMBA Consortium (2015) Pressure adaptation is linked to thermal adaptation in salt-saturated marine habitats. Environ Microbiol 17:332-345. https://doi.org/10.1111/1462-2920.12660

Angkawidjaja C, Koga Y, Takano K, Kanaya S (2012) Structure and stability of a thermostable carboxylesterase from the thermoacidophilic archaeon Sulfolobus tokodaii. FEBS J 279: 3071-3084. https://doi.org/10.1111/j.1742-4658.2012.08687.x

Antranikian G, Egorova K (2007) Extremophiles, a unique resource of biocatalysts for industrial biotechnology. In: Physiology and biochemistry of extremophiles. American Society of Microbiology, Washington DC, pp 361-406

Arpigny JL, Jaeger K-E (1999) Bacterial lipolytic enzymes: classification and properties. Biochem J 343:177. https://doi.org/10.1042/0264 6021:3430177

Auernik KS, Cooper CR, Kelly RM (2008) Life in hot acid: pathway analyses in extremely thermoacidophilic archaea. Curr Opin Biotechnol 19:445-453. https://doi.org/10.1016/j.copbio.2008.08. 001

Benaiges MD, Alarcón M, Fuciños P, Ferrer P, Rua M, Valero F (2010) Recombinant Candida rugosa lipase 2 from Pichia pastoris: immobilization and use as biocatalyst in a stereoselective reaction Biotechnol Prog 26:1252-1258. https://doi.org/10.1002/btpr.444

Bendtsen JD, Nielsen H, Von Heijne G, Brunak S (2004) Improved prediction of signal peptides: SignalP 3.0. J Mol Biol 340:783-795. https://doi.org/10.1016/j.jmb.2004.05.028

Berlemont R, Spee O, Delsaute M, Lara Y, Schuldes J, Simon C, Power P, Daniel R, Galleni M (2013) Novel organic solvent-tolerant esterase isolated by metagenomics: insights into the lipase/esterase classification. Rev Argent Microbiol 45:3-12

Biver S, Vandenbol M (2013) Characterization of three new carboxylic ester hydrolases isolated by functional screening of a forest soil metagenomic library. J Ind Microbiol Biotechnol 40:191-200. https://doi.org/10.1007/s10295-012-1217-7

Bora L (2014) Purification and characterization of highly alkaline lipase from Bacillus licheniformis MTCC 2465: and study of its detergen compatibility and applicability. J Surfactant Deterg 17:889-898. https://doi.org/10.1007/s11743-013-1517-6

Bornscheuer UT (2002) Microbial carboxyl esterases: classification, properties and application in biocatalysis. FEMS Microbiol Rev 26:73-81. https://doi.org/10.1111/j.1574-6976.2002.tb00599.x

Box GEP, Hunter JS, Hunter WG (2005) Statistics for experimenters: design, innovation and discovery, 2nd edn. Wiley-Interscience, Hoboken

Boyineni J, Kim J, Kang BS, Lee C, Jang SH (2014) Enhanced catalytic site thermal stability of cold-adapted esterase EstK by a W208Y mutation. Biochim Biophys Acta, Proteins Proteomics 1844:10761082. https://doi.org/10.1016/j.bbapap.2014.03.009

Bradford MM (1976) A rapid and sensitive method for the quantitation of microgram quantities of protein utilizing the principle of protein-dye binding. Anal Biochem 72:248-254 
Brault G, Shareck F, Hurtubise Y, Lépine F, Doucet N (2012) Isolation and characterization of EstC, a new cold-active esterase from Streptomyces coelicolor A3(2). PLoS One 7:e32041. https://doi. org/10.1371/journal.pone.0032041

Bunterngsook B, Kanokratana P, Thongaram T, Tanapongpipat S, Uengwetwanit T, Rachdawong S, Vichitsoonthonkul T, Eurwilaichitr L (2010) Identification and characterization of lipolytic enzymes from a peat-swamp forest soil metagenome. Biosci Biotechnol Biochem 74:1848-1854. https://doi.org/10.1271/bbb. 100249

Chu X, He H, Guo C, Sun B (2008) Identification of two novel esterases from a marine metagenomic library derived from South China Sea. Appl Microbiol Biotechnol 80:615-625. https://doi.org/10.1007/ s00253-008-1566-3

Cook GM, Rainey FA, Patel BKC, Morgan HW (1996) Characterization of a new obligately anaerobic thermophile, Thermoanaerobacter wiegelii sp. nov. Int J Syst Bacteriol 46:123-127. https://doi.org/ 10.1099/00207713-46-1-123

D’Auria S, Herman P, Lakowicz J, Bertoli E, Tanfani F, Rossi M, Manco G (2000) The thermophilic esterase from Archaeoglobus fulgidus: structure and conformational dynamics at high temperature. Proteins Struct Funct Genet 38:351-360

Dandavate V, Jinjala J, Keharia H, Madamwar D (2009) Production, partial purification and characterization of organic solvent-tolerant lipase from Burkholderia multivorans V2 and its application for ester synthesis. Bioresour Technol 100:3374-3381. https://doi.org/ 10.1016/j.biortech.2009.02.011

Daniel R (2005) The metagenomics of soil. Nat Rev Microbiol 3:470 478. https://doi.org/10.1038/nrmicro 1160

Delgado-García M, Valdivia-Urdiales B, Aguilar-González CN, Contreras-Esquivel JC, Rodríguez-Herrera R (2012) Halophilic hydrolases as a new tool for the biotechnological industries. J Sci Food Agric 92:2575-2580. https://doi.org/10.1002/jsfa.5860

Dougherty MJ, D'haeseleer P, Hazen TC, Simmons BA, Adams PD, Hadi MZ (2012) Glycoside hydrolases from a targeted compost metagenome, activity screening and functional characterization. BMC Biotechnol 12:38. https://doi.org/10.1186/1472-6750-12-38

Doukyu N, Ogino H (2010) Organic solvent-tolerant enzymes. Biochem Eng J 48:270-282. https://doi.org/10.1016/J.BEJ.2009.09.009

Dukunde A, Schneider D, Lu M, Brady S, Daniel R (2017) A novel, versatile family IV carboxylesterase exhibits high stability and activity in a broad pH spectrum. Biotechnol Lett 39:577-587. https:// doi.org/10.1007/s10529-016-2282-1

Ebrahimi M, Lakizadeh A, Agha-Golzadeh P, Ebrahimie E, Ebrahimi M (2011) Prediction of thermostability from amino acid attributes by combination of clustering with attribute weighting: a new vista in engineering enzymes. PLoS One 6:e23146. https://doi.org/10.1371/ journal.pone. 0023146

Elend C, Schmeisser C, Leggewie C, Babiak P, Steele HL, Reymond J, Jaeger K, Streit R, Carballeira JD, Streit WR (2006) Isolation and biochemical characterization of two novel metagenome-derived esterases. Appl Environ Microbiol 72:3637-3645. https://doi.org/10. 1128/AEM.72.5.3637

Faiz O, Colak A, Saglam N, Canakçi S, Beldüz AO (2007) Determination and characterization of thermostable esterolytic activity from a novel thermophilic bacterium Anoxybacillus gonensis A4. J Biochem Mol Biol 40:588-594. https://doi.org/10.5483/BMBRep.2007.40.4.588

Faulds CB, Pérez-Boada M, Martínez ÁT (2011) Influence of organic cosolvents on the activity and substrate specificity of feruloyl esterases. Bioresour Technol 102:4962-4967. https://doi.org/10.1016/j. biortech.2011.01.088

Ferrer M, Bargiela R, Martínez-Martínez M, Mir J, Koch R, Golyshina OV, Golyshin PN (2015) Biodiversity for biocatalysis: a review of the $\alpha / \beta$-hydrolase fold superfamily of esterases-lipases discovered in metagenomes. Biocatal Biotransformation 33:235-249. https:// doi.org/10.3109/10242422.2016.1151416
Gall MG, Nobili A, Pavlidis IV, Bornscheuer UT (2014) Improved thermostability of a Bacillus subtilis esterase by domain exchange. App Microbiol Biotechnol 98:1719-1726. https://doi.org/10.1007/ s00253-013-5053-0

Gao W, Wu K, Chen L, Fan H, Zhao Z, Gao B, Wang H, Wei D (2016) A novel esterase from a marine mud metagenomic library for biocatalytic synthesis of short-chain flavor esters. Microb Cell Factories 15: 41. https://doi.org/10.1186/s12934-016-0435-5

Georis J, Esteves FDL, Lamotte-Brasseur J, Bougnet V, Giannotta F, Frère J-M, Devreese B, Granier B (2000) An additional aromatic interaction improves the thermostability and thermophilicity of a mesophilic family 11 xylanase: structural basis and molecular study. Protein Sci 9:466-475. https://doi.org/10.1110/ps.9.3.466

Glogauer A, Martini VP, Faoro H, Couto GH, Müller-Santos M, Monteiro RA, Mitchell DA, de Souza EM, Pedrosa FO, Krieger N (2011) Identification and characterization of a new true lipase isolated through metagenomic approach. Microb Cell Factories 10:54. https://doi.org/10.1186/1475-2859-10-54

González-González R, Fuciños P, Rúa ML (2017) An overview on extremophilic esterases. In: Extremophilic enzymatic processing of lignocellulosic feedstocks to bioenergy. Springer International Publishing, Cham, pp 181-204

Hardeman F, Sjoling S (2007) Metagenomic approach for the isolation of a novel low-temperature-active lipase from uncultured bacteria of marine sediment. FEMS Microbiol Ecol 59:524-534. https://doi. org/10.1111/j.1574-6941.2006.00206.x

Hasan F, Shah AA, Hameed A (2006) Industrial applications of microbial lipases. Enzym Microb Technol 39:235-251. https://doi.org/10. 1016/J.ENZMICTEC.2005.10.016

Hausmann S, Jaeger K-E (2010) Lipolytic enzymes from bacteria. In: Handbook of Hydrocarbon and Lipid Microbiology. Springer Berlin Heidelberg, Berlin, pp 1099-1126

Hita E, Robles A, Camacho B, González PA, Esteban L, Jiménez MJ, Muñío MM, Molina E (2009) Production of structured triacylglycerols by acidolysis catalyzed by lipases immobilized in a packed bed reactor. Biochem Eng J 46:257-264. https://doi.org/10.1016/J.BEJ. 2009.05.015

Hotta Y, Ezaki S, Atomi H, Imanaka T (2002) Extremely stable and versatile carboxylesterase from a hyperthermophilic archaeon. Appl Environ Microbiol 68:3925-3931. https://doi.org/10.1128/ AEM.68.8.3925-3931.2002

Hu X, Thumarat U, Zhang X, Tang M, Kawai F (2010) Diversity of polyester-degrading bacteria in compost and molecular analysis of a thermoactive esterase from Thermobifida alba AHK119. Appl Microbiol Biotechnol 87:771-779. https://doi.org/10.1007/s00253$010-2555-x$

Hun CJ, Rahman RNZA, Salleh AB, Basri M (2003) A newly isolated organic solvent-tolerant Bacillus sphaericus 205y producing organic solvent-stable lipase. Biochem Eng J 15:147-151. https://doi.org/ 10.1016/S1369-703X(02)00185-7

Ishikawa J, Hotta K (1999) FramePlot: a new implementation of the frame analysis for predicting protein-coding regions in bacterial DNA with a high G + C content. FEMS Microbiol Lett 174:251-263

Jaeger K-E, Dijkstra BW, Reetz MT (1999) Bacterial biocatalysts: molecular biology, three-dimensional structures, and biotechnologica applications of lipases. Annu Rev Microbiol 53:315-351. https:/ doi.org/10.1146/annurev.micro.53.1.315

Jayanath G, Mohandas SP, Kachiprath B, Solomon S, Sajeevan TP, Bright Singh IS, Philip R (2018) A novel solvent-tolerant esterase of GDSGG motif subfamily from solar saltern through metagenomic approach: recombinant expression and characterization. Int J Biol Macromol 119:393-401. https://doi.org/10.1016/j.ijbiomac.2018. 06.057

Jeon JH, Lee HS, Kim JT, Kim SJ, Choi SH, Kang SG, Lee JH (2012) Identification of a new subfamily of salt-tolerant esterases from a metagenomic library of tidal flat sediment. 
Appl Microbiol Biotechnol 93:623-631. https://doi.org/10. 1007/s00253-011-3433-x

Jin P, Pei X, Du P, Yin X, Xiong X, Wu H, Zhou X, Wang Q (2012) Overexpression and characterization of a new organic solventtolerant esterase derived from soil metagenomic DNA. Bioresour Technol 116:234-240. https://doi.org/10.1016/j.biortech.2011.10. 087

Jochens H, Aerts D, Bornscheuer UT (2010) Thermostabilization of an esterase by alignment-guided focussed directed evolution. Protein Eng Des Sel 23:903-909. https://doi.org/10.1093/protein/gzq071

Kamal Z, Yedavalli P, Deshmukh MV, Rao NM (2013) Lipase in aqueous-polar organic solvents: activity, structure, and stability. Protein Sci 22:904-915. https://doi.org/10.1002/pro.2271

Kamimura ES, Medieta O, Rodrigues MI, Maugeri F (2001) Studies on lipase-affinity adsorption using response surface analysis Biotechnol Appl Biochem 33:153-159

Kang CH, Oh KH, Lee MH, Oh TK, Kim BH, Yoon JH (2011) A novel family VII esterase with industrial potential from compost metagenomic library. Microb Cell Factories 10:41. https://oi.org/ 10.1186/1475-2859-10-41

Kang LJ, Meng ZT, Hu C, Zhang Y, Guo HL, Li Q, Li M (2017) Screening, purification, and characterization of a novel organic solvent-tolerant esterase, Lip2, from Monascus purpureus strain M7. Extremophiles 21:345-355. https://doi.org/10.1007/s00792016-0907-x

Kawata T, Ogino H (2009) Enhancement of the organic solvent-stability of the LST-03 lipase by directed evolution. Biotechnol Prog 25: 1605-1611. https://doi.org/10.1002/btpr.264

Kim TD (2017) Bacterial hormone-sensitive lipases (bHSLs): Emerging enzymes for biotechnological applications. J Microbiol Biotechnol 27:1907-1915. https://doi.org/10.4014/jmb.1708.08004

Kim YH, Kwon EJ, Kim SK, Jeong YS, Kim J, Yun HD, Kim H (2010) Molecular cloning and characterization of a novel family VIII alkaline esterase from a compost metagenomic library. Biochem Biophys Res Commun 393:45-49. https://doi.org/10.1016/j.bbrc. 2010.01.070

Klibanov AM (2001) Improving enzymes by using them in organic solvents. Nature 409:241-246. https://doi.org/10.1038/35051719

Kovacic F, Mandrysch A, Poojari C, Strodel B, Jaeger K-E (2016) Structural features determining thermal adaptation of esterases. Protein Eng Des Sel 29:65-76. https://doi.org/10.1093/protein/ gzv061

Kumagai PS, Gutierrez RF, Lopes JLS, Martins JM, Jameson DM, Castro AM, Martins LF, DeMarco R, Bossolan NRS, Wallace BA, Araujo APU (2018) Characterization of esterase activity from an Acetomicrobium hydrogeniformans enzyme with high structural stability in extreme conditions. Extremophiles 22:781-793. https://doi. org/10.1007/s00792-018-1038-3

Kumar A, Dhar K, Singh Kanwar S, Arora PK (2016) Lipase catalysis in organic solvents: advantages and applications. Biol Proced Online 18:2. https://doi.org/10.1186/s12575-016-0033-2

Laemmli UK (1970) Cleavage of structural proteins during the assembly of the head of bacteriophage T4. Nature 227:680-685. https://doi. org/10.1038/227680a0

Lämmle K, Zipper H, Breuer M, Hauer B, Buta C, Brunner H, Rupp S (2007) Identification of novel enzymes with different hydrolytic activities by metagenome expression cloning. J Biotechnol 127 : 575-592. https://doi.org/10.1016/j.jbiotec.2006.07.036

Lazić ŽR (2004) Design of experiments in chemical engineering. Wiley$\mathrm{VCH}$ Verlag $\mathrm{GmbH} \& \mathrm{Co}$. KGaA, Weinheim

Léonard-Nevers V, Marton Z, Lamare S, Hult K, Graber M (2009) Understanding water effect on Candida antarctica lipase B activity and enantioselectivity towards secondary alcohols. J Mol Catal B Enzym 59:90-95. https://doi.org/10.1016/J.MOLCATB.2009.01. 008
Li X, Yu H (2013) Purification and characterization of an extracellular esterase with organic solvent tolerance from a halotolerant isolate, Salimicrobium sp. LY19. BMC Biotechnol 13:108

Li P, Chen X, Ji P, Li C, Wang P, Zhang Y, Xie B, Qin Q, Su H, Zhou B, Zhang Y, Zhang X (2015) Interdomain hydrophobic interactions modulate the thermostability of microbial esterases from the hormone-sensitive lipase family. J Biol Chem 290:11188-11,198. https://doi.org/10.1074/jbc.M115.646182

Lima VMG, Krieger N, Mitchell DA, Baratti JC, de FI, Fontana JD (2004) Evaluation of the potential for use in biocatalysis of a lipase from a wild strain of Bacillus megaterium. J Mol Catal B Enzym 31: 53-61. https://doi.org/10.1016/J.MOLCATB.2004.07.005

Lineweaver H, Burk D (1934) The determination of enzyme dissociation constants. J Am Chem Soc 56:658-666. https://doi.org/10.1021/ ja01318a036

Lopez-Lopez O, Cerdan M, Siso M (2014) New extremophilic lipases and esterases from metagenomics. Curr Protein Pept Sci 15:445455. https://doi.org/10.2174/1389203715666140228153801

Lotti M, Alberghina L (2007) Lipases: molecular structure and function. In: Industrial Enzymes. Springer Netherlands, Dordrecht, pp 263 281

Mandelli F, Goncalves TA, Gandin CA, Oliveira ACP, Oliveira Neto M, Squina FM (2016) Characterization and low-resolution structure of an extremely thermostable esterase of potential biotechnological interest from Pyrococcus furiosus. Mol Biotechnol 58:757-766. https://doi.org/10.1007/s12033-016-9975-5

Martínez-Martínez M, Coscolín C, Santiago G, Chow J, Stogios PJ, Bargiela R, Gertler C, Navarro-Fernández J, Bollinger A, Thies S, Méndez-García C, Popovic A, Brown G, Chernikova TN, GarcíaMoyano A, Bjerga GEK, Pérez-García P, Hai T, Del Pozo M V. Stokke R, Steen IH, Cui H, Xu X, Nocek BP, Alcaide M, Distaso M, Mesa V, Peláez AI, Sánchez J, Buchholz PCF, Pleiss J, FernándezGuerra A, Glöckner FO, Golyshina O V., Yakimov MM, Savchenko A, Jaeger K-E, Yakunin AF, Streit WR, Golyshin PN, Guallar V, Ferrer M, The INMARE Consortium TI (2018) Determinants and prediction of esterase substrate promiscuity patterns. ACS Chem Biol 13:225-234. doi: https://doi.org/10.1021/acschembio.7b00996

Metin K, Burcu Bakir Ateslier Z, Basbulbul G, Halil Biyik H (2006) Characterization of esterase activity in Geobacillus sp. HBB-4. J Basic Microbiol 46:400-409. https://doi.org/10.1002/jobm. 200510121

Mohamed YM, Ghazy MA, Sayed A, Ouf A, El-Dorry H, Siam R (2013) Isolation and characterization of a heavy metal-resistant, thermophilic esterase from a Red Sea Brine Pool. Sci Rep 3:3358. https:// doi.org/10.1038/srep03358

Monsef Shokri M, Ahmadian S, Akbari N, Khajeh K (2014) Hydrophobic substitution of surface residues affects lipase stability in organic solvents. Mol Biotechnol 56:360-368. https://doi.org/10. 1007/s12033-013-9716-y

Nacke H, Will C, Herzog S, Nowka B, Engelhaupt M, Daniel R (2011) Identification of novel lipolytic genes and gene families by screening of metagenomic libraries derived from soil samples of the German Biodiversity Exploratories. FEMS Microbiol Ecol 78: 188-201. https://doi.org/10.1111/j.1574-6941.2011.01088.x

Nasaruddin RR, Alam MZ, Jami MS (2014) Evaluation of solvent system for the enzymatic synthesis of ethanol-based biodiesel from sludge palm oil (SPO). Bioresour Technol 154:155-161. https://doi.org/10. 1016/j.biortech.2013.11.095

Nerurkar M, Joshi M, Pariti S, Adivarekar R (2013) Application of lipase from marine bacteria Bacillus sonorensis as an additive in detergent formulation. J Surfactant Deterg 16:435-443. https://doi.org/10. 1007/s11743-012-1434-0

Ngo TD, Ryu BH, Ju H, Jang E, Park K, Kim KK, Kim TD (2013) Structural and functional analyses of a bacterial homologue of hormone-sensitive lipase from a metagenomic library. Acta 
Crystallogr Sect D Biol Crystallogr 69:1726-1737. https://doi.org/ 10.1107/S0907444913013425

Ngo TD, Ryu BH, Ju H, Jang EJ, Kim KK, Kim TD (2014) Crystallographic analysis and biochemical applications of a novel penicillin-binding protein/ $\beta$-lactamase homologue from a metagenomic library. Acta Crystallogr Sect D Biol Crystallogr 70: 2455-2466. https://doi.org/10.1107/S1399004714015272

Notredame C, Higgins DG, Heringa J (2000) T-coffee: a novel method for fast and accurate multiple sequence alignment. J Mol Biol 302:205217. https://doi.org/10.1006/JMBI.2000.4042

Ó’Fágáin C (2003) Enzyme stabilization-recent experimental progress. Enzym Microb Technol 33:137-149. https://doi.org/10.1016/ S0141-0229(03)00160-1

Ogino H, Ishikawa H (2001) Enzymes which are stable in the presence of organic solvents. J Biosci Bioeng 91:109-116

Ogino H, Mimitsuka T, Muto T, Matsumura M, Yasuda M, Ishimi K, Ishikawa H (2004) Cloning, expression, and characterization of a lipolytic enzyme gene (lip8) from Pseudomonas aeruginosa LST03. J Mol Microbiol Biotechnol 7:212-223. https://doi.org/10.1159/ 000079830

Ohlhoff CW, Kirby BM, Van Zyl L, Mutepfa DLR, Casanueva A, Huddy RJ, Bauer R, Cowan DA, Tuffin M (2015) An unusual feruloyl esterase belonging to family VIII esterases and displaying a broad substrate range. J Mol Catal B Enzym 118:79-88. https://doi.org/10. 1016/J.MOLCATB.2015.04.010

Ollis DL, Cheah E, Cygler M, Dijkstra B, Frolow F, Franken SM, Harel M, Remington SJ, Silman I, Schrag J, Sussman JL, Verschueren KHG, Goldman A (1992) The $\alpha / \beta$ hydrolase fold. Protein Eng Des Sel 5:197-211. https://doi.org/10.1093/protein/5.3.197

Panda T, Gowrishankar BS (2005) Production and applications of esterases. Appl Microbiol Biotechnol 67:160-169. https://doi.org/10. 1007/s00253-004-1840-y

Park HJ, Joo JC, Park K, Yoo YJ (2012) Stabilization of Candida antarctica lipase $\mathrm{B}$ in hydrophilic organic solvent by rational design of hydrogen bond. Biotechnol Bioprocess Eng 17:722-728. https:// doi.org/10.1007/s12257-012-0092-4

Park SH, Kim S, Park S, Kim HK (2018) Characterization of organic solvent-tolerant lipolytic enzyme from Marinobacter lipolyticus isolated from the Antarctic Ocean. Appl Biochem Biotechnol. https:// doi.org/10.1007/s12010-018-2865-5

Peng Q, Zhang X, Shang M, Wang X, Wang G, Li B, Guan G, Li Y, Wang Y (2011) A novel esterase gene cloned from a metagenomic library from neritic sediments of the South China Sea. Microb Cell Factories 10:95. https://doi.org/10.1186/1475-2859-10-95

Pérez D, Kovačic F, Wilhelm S, Jaeger K-E, García MT, Ventosa A, Mellado EN (2012) Identification of amino acids involved in the hydrolytic activity of lipase LipBL from Marinobacter lipolyticus. Microbiology 158:2192-2203. doi: https://doi.org/10.1099/mic.0. 058792-0

Petersen EI, Valinger G, Sölkner B, Stubenrauch G, Schwab H (2001) A novel esterase from Burkholderia gladioli which shows high deacetylation activity on cephalosporins is related to betalactamases and DD-peptidases. J Biotechnol 89:11-25

Pezzullo M, Del Vecchio P, Mandrich L, Nucci R, Rossi M, Manco G (2013) Comprehensive analysis of surface charged residues involved in thermal stability in Alicyclobacillus acidocaldarius esterase 2. Protein Eng Des Sel 26:47-58. https://doi.org/10.1093/ protein/gzs066

Popovic A, Hai T, Tchigvintsev A, Hajighasemi M, Nocek B, Khusnutdinova AN, Brown G, Glinos J, Flick R, Skarina T, Chernikova TN, Yim V, Brüls T, Le PD, Yakimov MM, Joachimiak A, Ferrer M, Golyshina OV, Savchenko A, Golyshin PN, Yakunin AF (2017) Activity screening of environmental metagenomic libraries reveals novel carboxylesterase families. Sci Rep 7:44103. https://doi.org/10.1038/srep44103
Rahman RNZRA, Baharum SN, Basri M, Salleh AB (2005) High-yield purification of an organic solvent-tolerant lipase from Pseudomonas sp. strain S5. Anal Biochem 341:267-274. https://doi.org/10.1016/j. ab.2005.03.006

Raja A, Prabakarana P (2011) Actinomycetes and drug-an overview. Am J Drug Discov Dev 1:75-84. https://doi.org/10.3923/ajdd.2011.75. 84

Rashamuse K, Magomani V, Ronneburg T, Brady D (2009) A novel family VIII carboxylesterase derived from a leachate metagenome library exhibits promiscuous $\beta$-lactamase activity on nitrocefin. Appl Microbiol Biotechnol 83:491-500. https://doi.org/10.1007/ s00253-009-1895-x

Riedel K, Sutherland R, Donato JJ, Liebl W, Leis B, Angelov A, Mientus M, Li H, Pham VTT, Lauinger B, Bongen P, Pietruszka J, Gonçalves LG, Santos H (2015) Identification of novel esterase-active enzymes from hot environments by use of the host bacterium Thermus thermophilus. Front Microbiol 6:27. https://doi.org/10.3389/fmicb. 2015.00275

Robert X, Gouet P (2014) Deciphering key features in protein structures with the new ENDscript server. Nucleic Acids Res 42:320-324. https://doi.org/10.1093/nar/gku316

Romdhane IB-B, Fendri A, Gargouri Y, Gargouri A, Belghith H (2010) A novel thermoactive and alkaline lipase from Talaromyces thermophilus fungus for use in laundry detergents. Biochem Eng J 53:112-120. https://doi.org/10.1016/J.BEJ.2010.10.002

Ryckeboer J, Mergaert J, Vaes K, Klammer S, Clercq D, Coosemans J, Insam H, Swings J (2003) A survey of bacteria and fungi occurring during composting and self-heating processes. Ann Microbiol 53: 349-410

Sadeghi M, Naderi-Manesh H, Zarrabi M, Ranjbar B (2006) Effective factors in thermostability of thermophilic proteins. Biophys Chem 119:256-270. https://doi.org/10.1016/j.bpc.2005.09.018

Sakai Y, Ishikawa J, Fukasaka S, Yurimoto H, Mitsui R, Yanase H, Kato $\mathrm{N}$ (1999) A new carboxylesterase from Brevibacterium linens IFO 12171 responsible for the conversion of 1,4-butanediol diacrylate to 4-hydroxybutyl acrylate: purification, characterization, gene cloning, and gene expression in Escherichia coli. Biosci Biotechnol Biochem 63:688-697. https://doi.org/10.1271/bbb.63.688

Salihu A, Alam MZ (2015) Solvent tolerant lipases: a review. Process Biochem 50:86-96. https://doi.org/10.1016/j.procbio.2014.10.019

Sana B, Ghosh D, Saha M, Mukherjee J (2007) Purification and characterization of an extremely dimethylsulfoxide tolerant esterase from a salt-tolerant Bacillus species isolated from the marine environment of the Sundarbans. Process Biochem 42:1571-1578. https://doi.org/ 10.1016/j.procbio.2007.05.026

Sarmah N, Revathi D, Sheelu G, Yamuna Rani K, Sridhar S, Mehtab V, Sumana C (2018) Recent advances on sources and industrial applications of lipases. Biotechnol Prog 34:5-28. https://doi.org/10.1002/ btpr. 2581

Sayer C, Isupov MN, Bonch-Osmolovskaya E, Littlechild JA (2015a) Structural studies of a thermophilic esterase from a new Planctomycetes species, Thermogutta terrifontis. FEBS J 282: 2846-2857. https://doi.org/10.1111/febs.13326

Sayer C, Szabo Z, Isupov MN, Ingham C, Littlechild JA (2015b) The structure of a novel thermophilic esterase from the Planctomycetes species, Thermogutta terrifontis reveals an open active site due to a minimal "Cap" domain. Front Microbiol 6:1294. https://doi.org/10. 3389/fmicb.2015.01294

Schütte M, Fetzner S (2007) EstA from Arthrobacter nitroguajacolicus Ru61a, a thermo- and solvent-tolerant carboxylesterase related to class C b-Lactamases. Curr Microbiol 54:230-236. https://doi.org/ 10.1007/s00284-006-0438-2

Secundo F, Carrea G (2002) Lipase activity and conformation in neat organic solvents. J Mol Catal B Enzym 19:93-102. https://doi.org/ 10.1016/S1381-1177(02)00155-8 
Selvin J, Kennedy J, Lejon DPH, Kiran GS, Dobson ADW (2012) Isolation identification and biochemical characterization of a novel halo-tolerant lipase from the metagenome of the marine sponge Haliclona simulans. Microb Cell Factories 11:72. https://doi.org/ 10.1186/1475-2859-11-72

Seo S, Lee YS, Yoon SH, Kim SJ, Cho JY, Hahn BS, Koo BS, Lee CM (2014) Characterization of a novel cold-active esterase isolated from swamp sediment metagenome. World J Microbiol Biotechnol 30: 879-886. https://doi.org/10.1007/s11274-013-1496-9

Shao H, Xu L, Yan Y (2013) Isolation and characterization of a thermostable esterase from a metagenomic library. J Ind Microbiol Biotechnol 40:1211-1222. https://doi.org/10.1007/s10295-013$1317-\mathrm{z}$

Sharma R, Soni S, Vohra R, Gupta L, Gupta J (2002) Purification and characterisation of a thermostable alkaline lipase from a new thermophilic Bacillus sp. RSJ-1. Process Biochem 37:1075-1084. https://doi.org/10.1016/S0032-9592(01)00316-8

Shieh C-J, Liao H-F, Lee C-C (2003) Optimization of lipase-catalyzed biodiesel by response surface methodology. Bioresour Technol 88 : 103-116

Simon C, Daniel R (2011) Metagenomic analyses: past and future trends. Appl Environ Microbiol 77:1153-1161. https://doi.org/10.1128/ AEM.02345-10

Song JK, Rhee JS (2001) Enhancement of stability and activity of phospholipase A1 in organic solvents by directed evolution. Biochim Biophys Acta Protein Struct Mol Enzymol 1547:370-378. https:// doi.org/10.1016/S0167-4838(01)00204-7

Sood S, Sharma A, Sharma N, Kanwar SS (2016) Carboxylesterases: sources, characterization and broader applications. Insights Enzym Res 1:1. https://doi.org/10.21767/2573-4466.100002

Srimhan P, Kongnum K, Taweerodjanakarn S, Hongpattarakere T (2011) Selection of lipase producing yeasts for methanol-tolerant biocatalyst as whole cell application for palm-oil transesterification. Enzym Microb Technol 48:293-298. https://doi.org/10.1016/j.enzmictec. 2010.12.004

Takeda Y, Aono R, Doukyu N (2006) Purification, characterization, and molecular cloning of organic-solvent-tolerant cholesterol esterase from cyclohexane-tolerant Burkholderia cepacia strain ST-200. Extremophiles 10:269-277. https://doi.org/10.1007/s00792-0050494-8

Tamura K, Stecher G, Peterson D, Filipski A, Kumar S (2013) MEGA6: molecular evolutionary genetics analysis version 6.0. Mol Biol Evol 30:2725-2729. https://doi.org/10.1093/molbev/mst197

Tirawongsaroj P, Sriprang R, Harnpicharnchai P, Thongaram T, Champreda V, Tanapongpipat S, Pootanakit K, Eurwilaichitr L (2008) Novel thermophilic and thermostable lipolytic enzymes from a Thailand hot spring metagenomic library. J Biotechnol 133:42-49. https://doi.org/10.1016/J.JBIOTEC.2007.08.046

Torres S, Castro GR (2004) Non-aqueous biocatalysis in homogeneous solvent systems. Crit Rev Biotechnol 42:271-277

Torres S, Martínez MA, Pandey A, Castro GR (2009) An organicsolvent-tolerant esterase from thermophilic Bacillus licheniformis
S-86. Bioresour Technol 100:896-902. https://doi.org/10.1016/j. biortech.2008.07.009

Wang X, Qin X, Li D, Yang B, Wang Y (2017) One-step synthesis of high-yield biodiesel from waste cooking oils by a novel and highly methanol-tolerant immobilized lipase. Bioresour Technol 235:18 24. https://doi.org/10.1016/J.BIORTECH.2017.03.086

Woo Lee H, Kyeong Jung W, Ho Kim Y, Han Ryu B, Doohun Kim T, Kim J, Kim H (2016) Characterization of a novel alkaline family VIII esterase with s-enantiomer preference from a compost metagenomic library. J Microbiol Biotechnol 26:315-325. https:// doi.org/10.4014/jmb.1509.09081

Wu G, Zhang S, Zhang H, Zhang S, Liu Z (2013) A novel esterase from a psychrotrophic bacterium Psychrobacter celer 3Pb1 showed coldadaptation and salt tolerance. J Mol Catal B Enzym 98:119-126. https://doi.org/10.1016/j.molcatb.2013.10.012

Xing MN, Zhang XZ, Huang H (2012) Application of metagenomic techniques in mining enzymes from microbial communities for biofuel synthesis. Biotechnol Adv 30:920-929. https://doi.org/10.1016/ j.biotechadv.2012.01.021

Yang Y, Yu Y, Zhang Y, Liu C, Shi W, Li Q (2011) Lipase/esterasecatalyzed ring-opening polymerization: a green polyester synthesis technique. Process Biochem 46:1900-1908. https://doi.org/10. 1016/j.procbio.2011.07.016

Yang X, Wu L, Xu Y, Ke C, Hu F, Xiao X, Huang J (2018) Identification and characterization of a novel alkalistable and salt-tolerant esterase from the deep-sea hydrothermal vent of the East Pacific Rise. Microbiology 7:e00601. https://doi.org/10.1002/mbo3.601

Ye J, McGinnis S, Madden TL (2006) BLAST: improvements for better sequence analysis. Nucleic Acids Res 34:W6-W9. https://doi.org/ 10.1093/nar/gkl164

Yu S, Zheng B, Zhao X, Feng Y (2010) Gene cloning and characterization of a novel thermophilic esterase from Fervidobacterium nodosum Rt17-B1. Acta Biochim Biophys Sin Shanghai 42:288295. https://doi.org/10.1093/abbs/gmq020

Yu EY, Kwon MA, Lee M, Oh JY, Choi JE, Lee JY, Song BK, Hahm DH, Song JK (2011) Isolation and characterization of cold-active family VIII esterases from an arctic soil metagenome. Appl Microbiol Biotechnol 90:573-581. https://doi.org/10.1007/s00253-011-3132-7

Zarafeta D, Moschidi D, Ladoukakis E, Gavrilov S, Chrysina ED, Chatziioannou A, Kublanov I, Skretas G, Kolisis FN (2016) Metagenomic mining for thermostable esterolytic enzymes uncovers a new family of bacterial esterases. Sci Rep 6:38886. https://doi.org/10.1038/srep38886

Zhang Y (2008) I-TASSER server for protein 3D structure prediction. BMC Bioinformatics 23:40. https://doi.org/10.1186/1471-2105-9 40

Zhang S, Wu G, Liu Z, Shao Z, Liu Z (2014) Characterization of EstB, a novel cold-active and organic solvent-tolerant esterase from marine microorganism Alcanivorax dieselolei B-5(T). Extremophiles 18: 251-259. https://doi.org/10.1007/s00792-013-0612-y

Zhou J, Bruns MA, Tiedje JM (1996) DNA recovery from soils of diverse composition. Appl Environ Microbiol 62:316-322 


\section{Supplemental information for chapter III}

\section{Content}

Figure S1 Unrooted phylogenetic tree constructed by neighbor-joining method with Est1, Est2 and reference lipolytic enzymes from different families.

Figure S2 Multiple sequence alignments of Est1 (a) and Est2 (b) with other esterase sequences. Figure S3 SDS-PAGE of purified Est1 and Est2.

Figure S4 Thermostability of Est 2 incubated at $30{ }^{\circ} \mathrm{C}$ for 26 days.

Figure $\mathbf{S} 5$ Effect of $\mathrm{NaCl}$ and $\mathrm{KCl}$ on esterase activity.

Table S1 Second-order rotable design for the combined effect of temperature and $\mathrm{pH}$ on Est1 and Est2 stability.

Table S2 Purification of recombinant Est1 and Est2.

Table S3 Analysis of variance (ANOVA) for the response surface models.

Table S4 Effect of metal ions on Est1 and Est2 activity

Table S5 Effect of inhibitors and detergent on Est1 and Est2 activity 


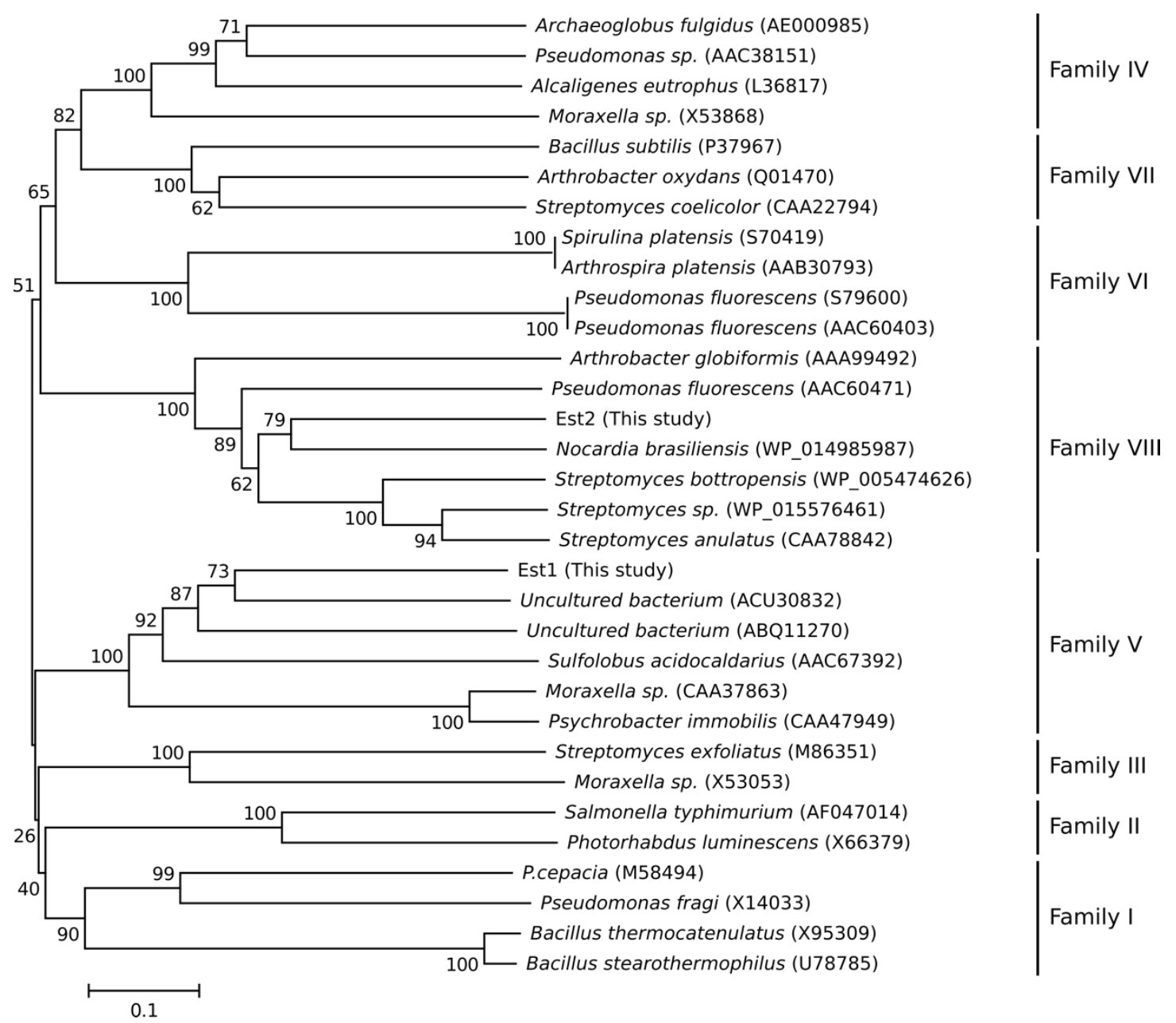

Figure S1 Unrooted phylogenetic tree constructed by the neighbor-joining method with Est1, Est2 and reference lipolytic enzymes from different families. With the exception of Est 1 and Est2, other sequences were retrieved from GenBank. The full organism names and accession numbers are given for reference sequences. Only bootstrap values above 50\% are shown. Scale represents the number of amino acid substations per site 
a

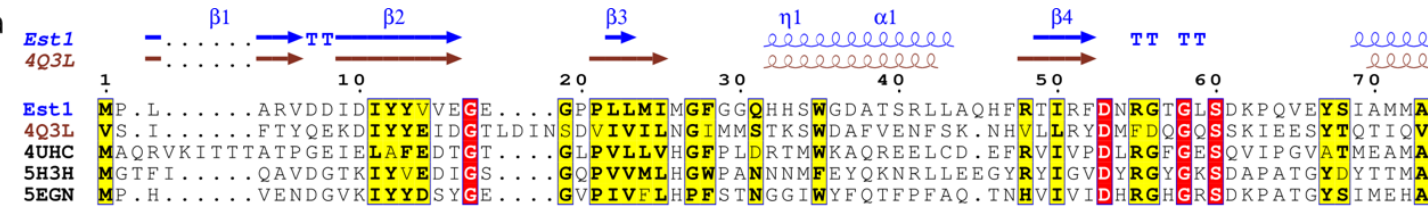

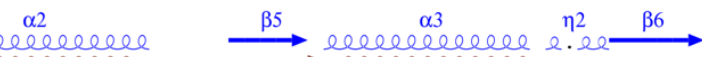
Est1 elebeleed $4 Q 3 L$ elebele 80
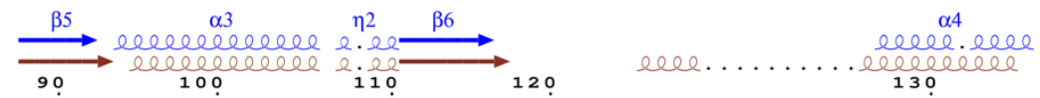

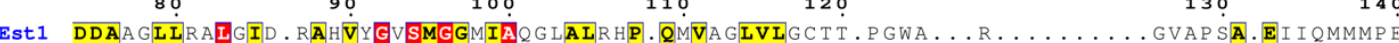

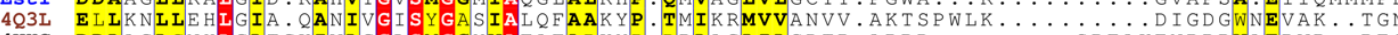
4UHC DDLAGLCNHLGLTGKIVIGGLSMGGYVAFAFARKYR. DRLAGLILCDTR. ARPD . . . . .

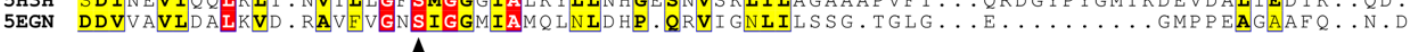
Est1
ele

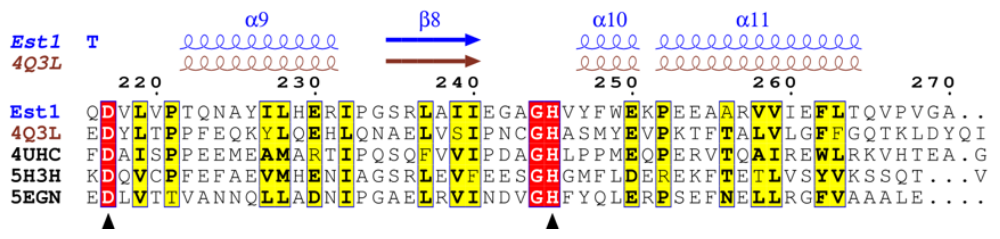


b Est2 Est2
5 GKV
4P6B
4IVK
3ZYT
P05364
P00811
Q9F3Z2

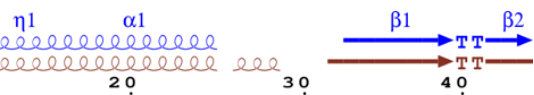
. MTE..... LPVGGTCD...PREADVRDAFLENFARHG. EVGAAVCVVVENRVV

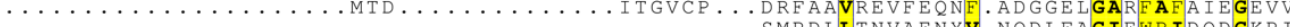
MKTSAKELSFAVSFVLLIIASTSEAEGPVTATK

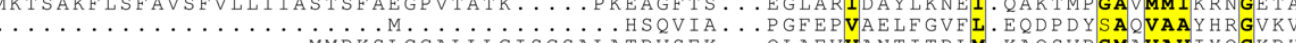

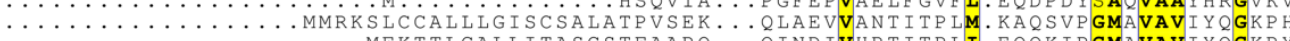

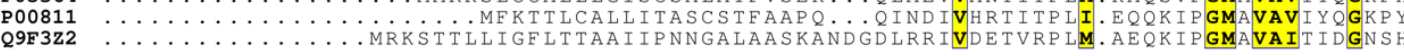
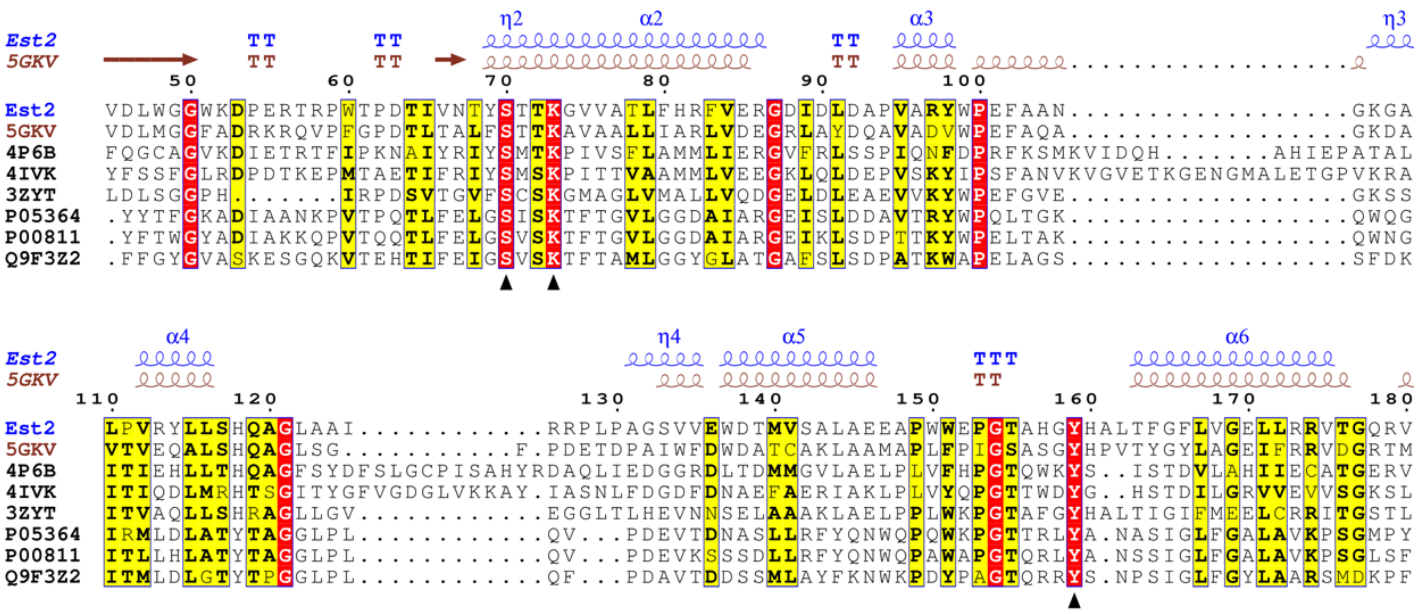

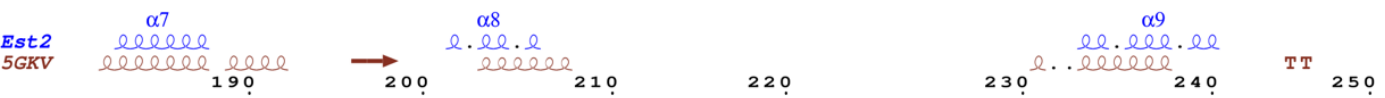

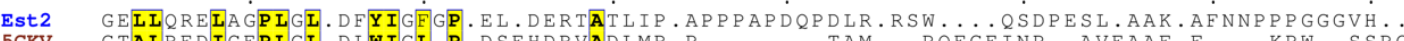

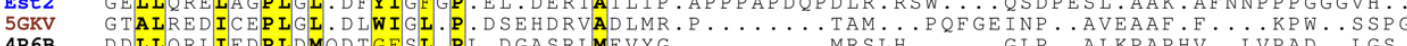

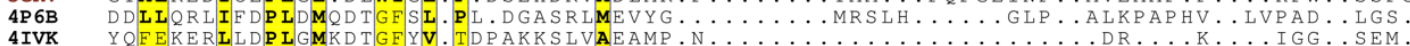

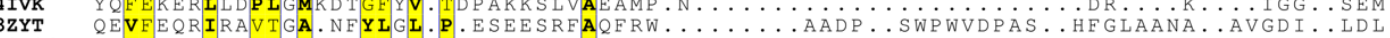

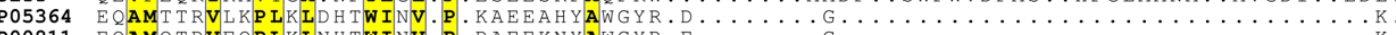

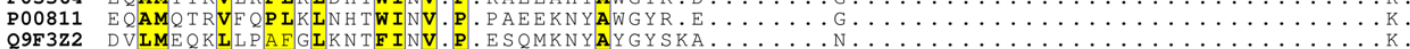

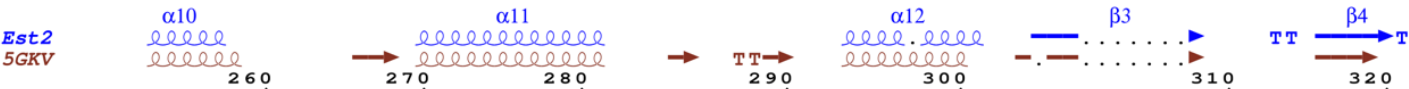

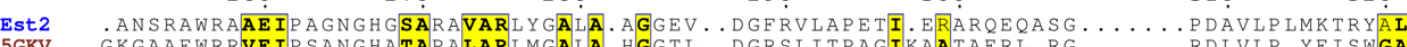
5GKV GKGAAEWRRVEIPSANGHATAPALARLMGLA. HGGTL..DGRSLITPAGIKAATAERL.RG.....RDLVLP.YEISWGA 4P6B . SHP TDDPDFRRGGHGLYSTLDYMAFANMLI. SGQTP. EGETLLSPAVLKLALAPRV. HFGARGMR. INDEPF. AGYSWNI

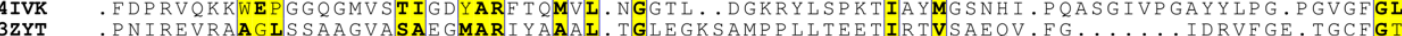
205364. AVRVSPGMI P.

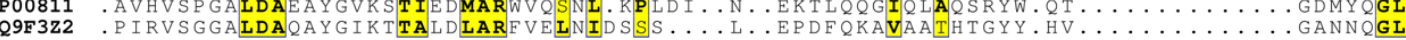

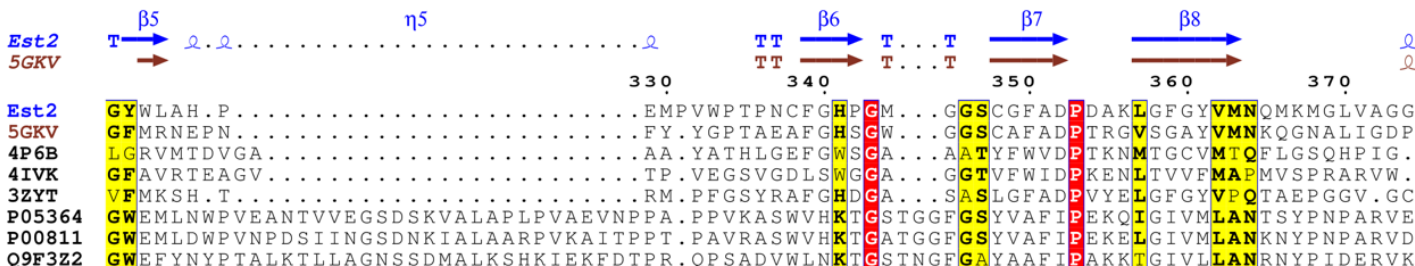

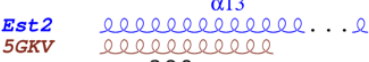

$\begin{array}{ll} & \mathbf{3 8 0} \\ \text { Est2 } & \text { TGQRIIEAVYRAL ....RR } \\ \text { 5GKV } & \text { RSVRIIEAAYASL ..... }\end{array}$

4P6B $\quad \ldots$ SDMQAAAMSMLG.

4IVK
3ZYT RNTLRNIVYGAFDRLE.

05364 AAYHILEALO.

P00811 AAWQILNALQ ...............

Q9F3Z2 AAYRILQALDNKQ $\ldots \ldots$ 
Figure S2 Multiple sequence alignments of Est1 (a) and Est2 (b) with other esterase sequences. Secondary structures of Est 1 and Est 2 were predicted by I-TASSER. Sequences were aligned using structural information derived from EPRESSO. Identical residues are shaded. Stars underneath residues indicate the catalytic triad. Secondary structures are presented as $\alpha$-helices and $\beta$-strands on top of sequences. a, Alignment of Est1 and its structural analogs. Secondary structures of Est1 and MGS-M2 (PDB: 4Q3L) are presented. b, Alignment of Est2, its esterase structural analogs and class C beta-lactamases (GenBank: P05364, P00811 and Q9F3Z2). Secondary structures of Est2 and CcEstA (PDB: 5GKV) are presented 


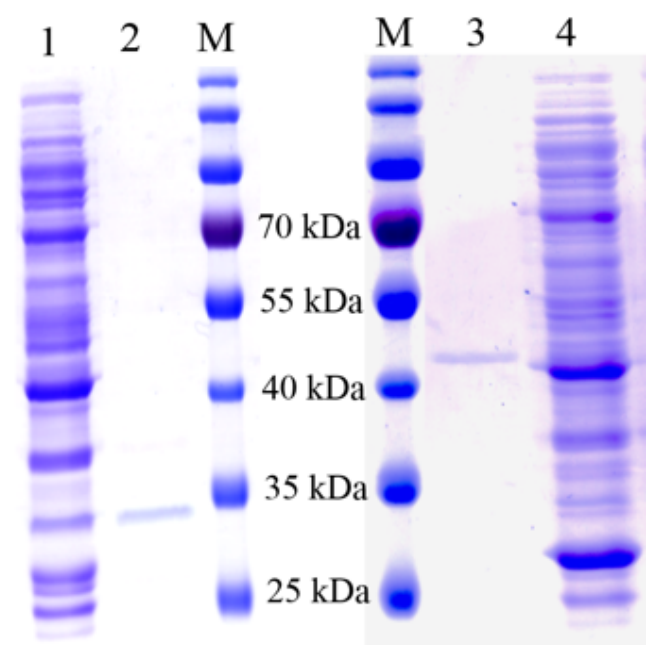

Figure S3 SDS-PAGE of purified Est1 and Est2. Lane M, standard molecular weight marker; Lane 1, soluble fraction of induced Est1 cell lysate; Lane 2, purified Est1; Lane 3, purified Est2; Lane 4, soluble fraction of induced Est 2 cell lysate 


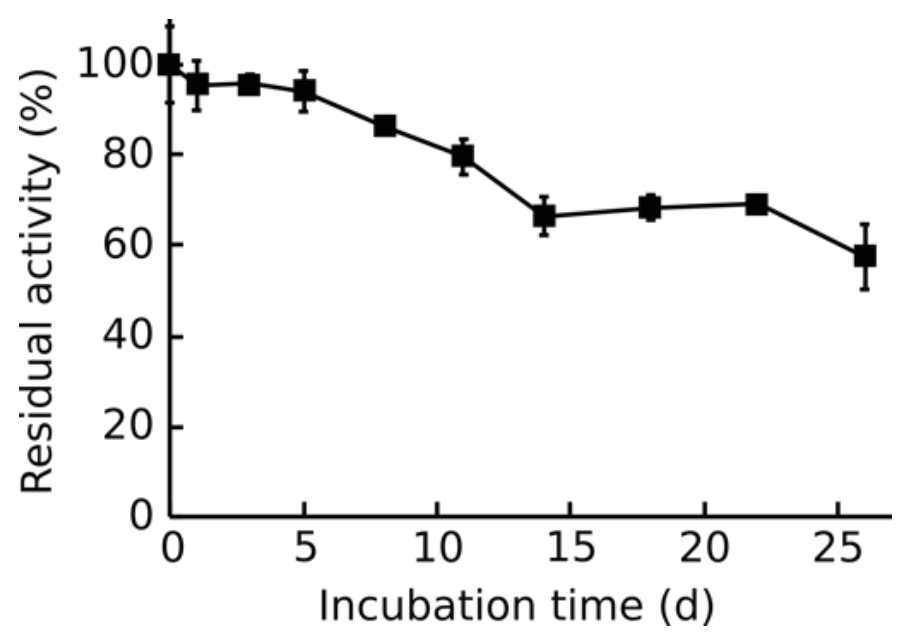

Figure S4 Thermostability of Est 2 incubated at $30{ }^{\circ} \mathrm{C}$ for 26 days. Activity measured before incubation and under standard assay condition (14.8 U/mg) was regarded as $100 \%$. 

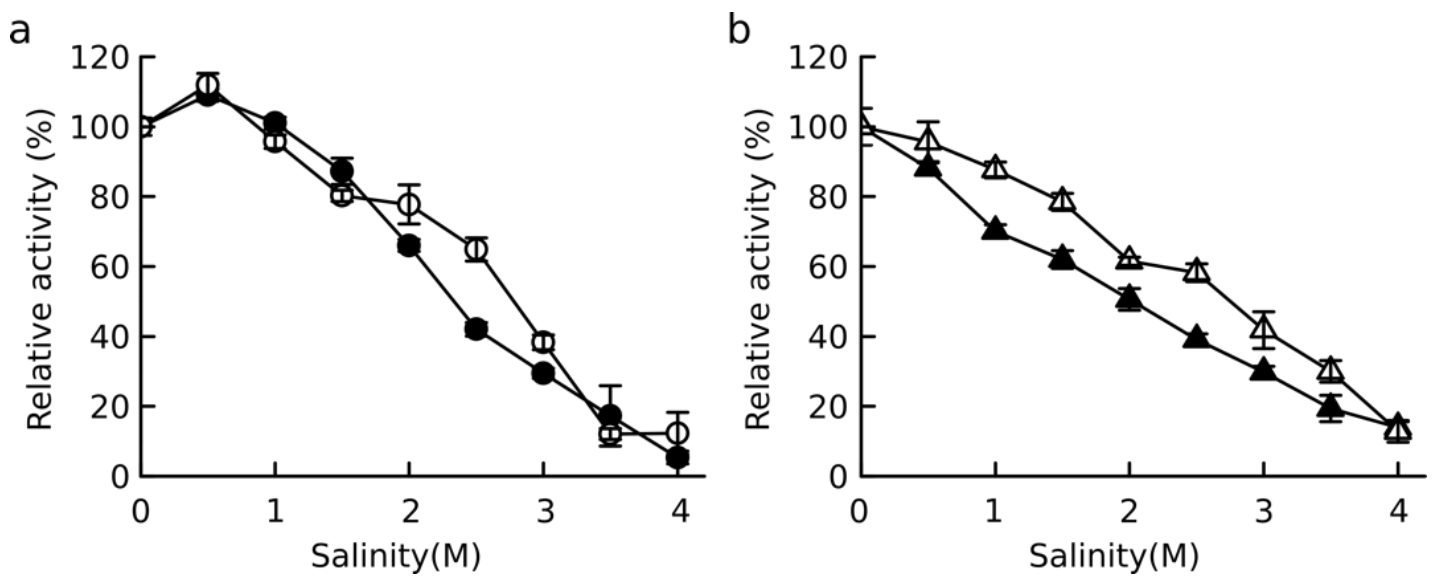

Figure S5 Effect of $\mathrm{NaCl}$ and $\mathrm{KCl}$ on esterase activity. a, Effect of $\mathrm{NaCl}$ (closed circle) and $\mathrm{KCl}$ (open circle) on Estl activity. b, Effect of $\mathrm{NaCl}$ (closed triangle) and $\mathrm{KCl}$ (open triangle) on Est 2 activity. Specific activity corresponding to $100 \%$ relative activity is $30.7 \mathrm{U} / \mathrm{mg}$ for Est1 and 13.5 U/mg for Est2. 
Table S1 Second-order rotable design for the combined effect of temperature and $\mathrm{pH}$ on Est1 and Est2 stability

\begin{tabular}{lllllllll}
\hline $\begin{array}{l}\text { Number } \\
\text { of trials (N) }\end{array}$ & \multicolumn{3}{c}{ Coded values } & \multicolumn{3}{c}{ Nature values } & \multicolumn{3}{c}{ Residual activity (\%) } \\
\cline { 2 - 8 } & $\mathrm{pH}$ & $\mathrm{T}$ & $\mathrm{pH}$ & $\mathrm{T}\left({ }^{\circ} \mathrm{C}\right)$ & $\begin{array}{l}\text { Obtained } \\
(\text { Est1) }\end{array}$ & $\begin{array}{l}\text { Predicted } \\
(\text { Est1) }\end{array}$ & $\begin{array}{l}\text { Obtained } \\
(\text { Est2) }\end{array}$ & $\begin{array}{l}\text { Predicted } \\
(\text { Est2) }\end{array}$ \\
\hline 1 & 1 & 1 & 8.5 & 69.1 & 1.8 & 9.8 & 52.5 & 48.8 \\
2 & -1 & 1 & 6.5 & 69.1 & 4.3 & 5.2 & 19.4 & 20.6 \\
3 & 1 & -1 & 8.5 & 40.9 & 98.2 & 96.0 & 90.4 & 87.4 \\
4 & -1 & -1 & 6.5 & 40.9 & 56.0 & 46.6 & 53.9 & 59.2 \\
5 & $-\sqrt{ } 2$ & 0 & 6 & 55 & 13.3 & 19.1 & 56.1 & 51.6 \\
6 & $\sqrt{ } 2$ & 0 & 9 & 55 & 61.9 & 57.2 & 86.8 & 91.4 \\
7 & 0 & $-\sqrt{ } 2$ & 7.5 & 35 & 78.0 & 85.8 & 65.6 & 63.8 \\
8 & 0 & $\sqrt{2}$ & 7.5 & 75 & 2.3 & -4.4 & 7.6 & 9.2 \\
9 & 0 & 0 & 7.5 & 55 & 69.6 & 76.3 & 87.26 & 84.1 \\
10 & 0 & 0 & 7.5 & 55 & 79.5 & 76.3 & 82.95 & 84.1 \\
11 & 0 & 0 & 7.5 & 55 & 74.9 & 76.3 & 80.21 & 84.1 \\
12 & 0 & 0 & 7.5 & 55 & 81.2 & 76.3 & 82.27 & 84.1 \\
13 & 0 & 0 & 7.5 & 55 & 76.4 & 76.3 & 87.95 & 84.1 \\
\hline
\end{tabular}


Table S2 Purification of recombinant Est1 and Est2.

\begin{tabular}{lllllll}
\hline Esterase $^{\mathrm{a}}$ & $\begin{array}{l}\text { Purification } \\
\text { step }\end{array}$ & $\begin{array}{l}\text { Total } \\
\text { activity } \\
(\mathrm{U})\end{array}$ & $\begin{array}{l}\text { Total protein } \\
(\mathrm{mg})\end{array}$ & $\begin{array}{l}\text { Specific } \\
\text { activity } \\
(\mathrm{U} / \mathrm{mg})\end{array}$ & $\begin{array}{l}\text { Purification } \\
(\text { fold })\end{array}$ & $\begin{array}{l}\text { Yield } \\
(\%)\end{array}$ \\
\hline Est1 & Crude enzyme & 143.0 & 190.6 & 0.77 & 1 & 100 \\
& Ni-TED & 23.1 & 1.0 & 22.2 & 28.9 & 16.2 \\
Est2 & Crude enzyme & 52.1 & 111.3 & 0.43 & 1 & 100 \\
& Ni-TED & 5.6 & 0.78 & 7.3 & 16.9 & 10.8 \\
\hline
\end{tabular}

a, Est 1 and Est2 activity were measured using $1 \mathrm{mM} p$-NP acyl caprylate (C8) under respective standard assay conditions. 
Table S3 Analysis of variance (ANOVA) for the response surface models.

\begin{tabular}{|c|c|c|c|c|c|}
\hline Esterase & $\begin{array}{l}\text { Source of } \\
\text { variance }\end{array}$ & $\mathrm{DF}^{\mathrm{a}}$ & $\mathrm{SS}^{\mathrm{b}}$ & $\mathrm{MS}^{\mathrm{c}}$ & Mean square ratios $(\alpha=0.05)^{\mathrm{d}}$ \\
\hline \multirow[t]{4}{*}{$\begin{array}{l}\text { Est1 }\left(R_{a d j}^{2}=\right. \\
0.953)^{\mathrm{e}}\end{array}$} & Model & 5 & 14291.3 & 2858.3 & \multirow[t]{2}{*}{$F_{1}=\frac{M S M}{M S E e}=146.1>F_{4}^{5}=6.26$} \\
\hline & Error & 7 & 399.7 & 57.1 & \\
\hline & $\begin{array}{l}\text { Experimental } \\
\text { error }\end{array}$ & 4 & 80.5 & 20.1 & \multirow[t]{2}{*}{$F_{2}=\frac{M S L F}{M S E e}=5.28<F_{4}^{3}=6.59$} \\
\hline & Lack of fit Model & 3 & 319.3 & 106.4 & \\
\hline \multirow[t]{4}{*}{$\begin{array}{l}\text { Est2 }\left(R_{a d j}^{2}=\right. \\
0.975)^{\mathrm{e}}\end{array}$} & Model & 4 & 8577.7 & 2144.4 & $F_{1}=\frac{M S M}{M S E e}=192.45>F_{4}^{4}=6.39$ \\
\hline & Error & 8 & 144.7 & 18.1 & \multirow{3}{*}{$F_{2}=\frac{M S L F}{M S E e}=2.25<F_{4}^{4}=6.39$} \\
\hline & $\begin{array}{l}\text { Experimental } \\
\text { error }\end{array}$ & 4 & 44.6 & 11.1 & \\
\hline & Lack of fit Model & 4 & 100.2 & 25.0 & \\
\hline
\end{tabular}

${ }^{a}$ Degrees of Freedom;

${ }^{\mathrm{b}}$ Sum of Squares;

${ }^{\mathrm{c}}$ Mean Squares;

${ }^{\mathrm{d}}$ MSM, mean squares of the model; MSMLF, mean squares for the model lack of fit; MSEe, mean squares for experimental error;

${ }^{\mathrm{e}} \boldsymbol{R}_{a d j}^{2}$, adjusted $\mathrm{R}^{2}$, indication of model quality. 
Table S4 Effect of metal ions on Est1 and Est2 activity

\begin{tabular}{lll}
\hline Variable & Est1 relative activity (\%) & Est2 relative activity (\%) \\
\hline None $^{\mathrm{a}}$ & $100 \pm 4.1$ & $100 \pm 2.5$ \\
$\mathrm{~K}+1 \mathrm{mM}$ & $121.8 \pm 0.8$ & $100.3 \pm 1.6$ \\
$\mathrm{~K}+10 \mathrm{mM}$ & $111.3 \pm 2.9$ & $100.3 \pm 1.6$ \\
$\mathrm{Mg} 2+1 \mathrm{mM}$ & $98.7 \pm 3.3$ & $92.8 \pm 5.9$ \\
$\mathrm{Mg} 2+10 \mathrm{mM}$ & $114.5 \pm 8.7$ & $94.5 \pm 1.2$ \\
$\mathrm{Ca} 2+1 \mathrm{mM}$ & $90.4 \pm 6.0$ & $98.3 \pm 7.4$ \\
$\mathrm{Ca} 2+10 \mathrm{mM}$ & $80.4 \pm 1.3$ & $106.2 \pm 7.2$ \\
$\mathrm{Zn} 2+1 \mathrm{mM}$ & $84.0 \pm 6.0$ & $98.4 \pm 2.0$ \\
$\mathrm{Zn} 2+10 \mathrm{mM}$ & $60.7 \pm 5.1$ & $132.5 \pm 9.8$ \\
$\mathrm{Cu} 2+1 \mathrm{mM}$ & $83.5 \pm 3.3$ & $93.5 \pm 4.3$ \\
$\mathrm{Cu} 2+10 \mathrm{mM}$ & $56.2 \pm 8.6$ & $93.0 \pm 9.1$ \\
$\mathrm{Ni2}+1 \mathrm{mM}$ & $87.7 \pm 1.6$ & $87.8 \pm 0.7$ \\
$\mathrm{Ni} 2+10 \mathrm{mM}$ & $27.4 \pm 3.7$ & $23.0 \pm 4.5$ \\
$\mathrm{Mn} 2+1 \mathrm{mM}$ & $94.9 \pm 3.5$ & $93.8 \pm 3.9$ \\
$\mathrm{Mn} 2+10 \mathrm{mM}$ & $53.6 \pm 1.0$ & $57.6 \pm 2.7$ \\
$\mathrm{Fe} 2+1 \mathrm{mM}$ & $86.0 \pm 6.2$ & $101.0 \pm 6.6$ \\
$\mathrm{Fe} 2+10 \mathrm{mM}$ & $34.3 \pm 8.8$ & $20.8 \pm 0.7$ \\
$\mathrm{Fe} 3+1 \mathrm{mM}$ & $86.9 \pm 7.8$ & $92.5 \pm 5.3$ \\
$\mathrm{Fe} 3+10 \mathrm{mM}$ & $58.2 \pm 8.7$ & $66.4 \pm 6.8$ \\
$\mathrm{Al3}+1 \mathrm{mM}$ & $85.6 \pm 0.3$ & $108.5 \pm 4.9$ \\
$\mathrm{Al} 3+10 \mathrm{mM}$ & $56.4 \pm 4.2$ & $54.9 \pm 2.3$ \\
\hline
\end{tabular}

a Assay of metal ion effect for Est1 and Est2 was measured at $50^{\circ} \mathrm{C}$ in phosphate buffer $(\mathrm{pH}=8)$. Specific activity corresponding to $100 \%$ relative activity is: $23.0 \mathrm{U} / \mathrm{mg}$ for Est1, $10.4 \mathrm{U} / \mathrm{mg}$ for Est2. 
Table S5 Effect of inhibitors and detergent on Est1 and Est2 activity

\begin{tabular}{|c|c|c|c|c|c|}
\hline Variable & $\begin{array}{l}\text { Est1 relative } \\
\text { activity }(\%)\end{array}$ & $\begin{array}{l}\text { Est2 relative } \\
\text { activity }(\%)\end{array}$ & Variable & $\begin{array}{l}\text { Est1 relative } \\
\text { activity (\%) }\end{array}$ & $\begin{array}{l}\text { Est2 relative } \\
\text { activity (\%) }\end{array}$ \\
\hline$\overline{\text { None }^{\mathrm{a}}}$ & $100 \pm 3.2$ & $100 \pm 1.6$ & None $^{\mathrm{a}}$ & $100 \pm 3.2$ & $100 \pm 1.6$ \\
\hline $\begin{array}{l}\text { Triton X-100 } \\
(0.1 \%)\end{array}$ & $67.6 \pm 1.0$ & $70.4 \pm 2.0$ & $\begin{array}{l}\text { EDTA } \\
1 \mathrm{mM}\end{array}$ & $118.7 \pm 8.3$ & $77.2 \pm 9.5$ \\
\hline $\begin{array}{l}\text { Triton X-100 } \\
(1 \%)\end{array}$ & $30.3 \pm 3.8$ & $40.8 \pm 5.1$ & $\begin{array}{l}\text { EDTA } \\
10 \mathrm{mM}\end{array}$ & $111.3 \pm 7.9$ & $64.4 \pm 5.9$ \\
\hline Tween $20(0.1 \%)$ & $124.1 \pm 2.0$ & $77.5 \pm 1.0$ & $\begin{array}{l}\text { DTT } \\
1 \mathrm{mM}\end{array}$ & $91.7 \pm 8.9$ & $96.9 \pm 0.2$ \\
\hline Tween $20(1 \%)$ & $61.0 \pm 4.3$ & $35.1 \pm 3.0$ & $\begin{array}{l}\text { DTT } \\
10 \mathrm{mM}\end{array}$ & $21.3 \pm 0.6$ & $32.4 \pm 1.7$ \\
\hline Tween $80(0.1 \%)$ & $144.7 \pm 4.3$ & $85.2 \pm 1.9$ & $\begin{array}{l}\text { PMSF } \\
1 \mathrm{mM}\end{array}$ & $84.8 \pm 8.2$ & $89.1 \pm 13.7$ \\
\hline Tween $80(1 \%)$ & $122.9 \pm 5.8$ & $36.5 \pm 1.5$ & $\begin{array}{l}\text { PMSF } \\
10 \mathrm{mM}\end{array}$ & $30.4 \pm 11.0$ & $57.7 \pm 2.8$ \\
\hline $\operatorname{SDS}(0.1 \%)$ & $\mathrm{ND}^{\mathrm{b}}$ & $\mathrm{ND}^{\mathrm{b}}$ & $\begin{array}{l}\text { DEPC } \\
1 \mathrm{mM} \\
\end{array}$ & $41.7 \pm 4.1$ & $74.9 \pm 6.7$ \\
\hline
\end{tabular}

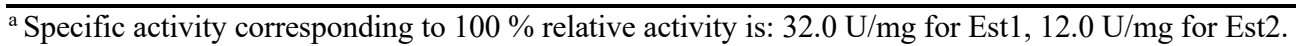

${ }^{b}$ Not detectable 


\section{Chapter IV}

\section{A novel caboxylesterase derived from a compost metagenome exhibiting high stability and activity towards high salinity}

Mingji Lu, Rolf Daniel

Doi: https://doi.org/10.3390/genes12010122

https://www.mdpi.com/2073-4425/12/1/122 


\title{
A Novel Carboxylesterase Derived from a Compost Metagenome Exhibiting High Stability and Activity towards High Salinity
}

\author{
Mingji Lu and Rolf Daniel *(B)
}

check for

Citation: Lu, M.; Daniel, R. A Novel Carboxylesterase Derived from a Compost Metagenome Exhibiting High Stability and Activity towards High Salinity. Genes 2021, 12, 122. https://doi.org/10.3390/ genes 12010122

Received: 21 December 2020 Accepted: 13 January 2021 Published: 19 January 2021

Publisher's Note: MDPI stays neutral with regard to jurisdictional claims in published maps and institutional affiliations.

\section{(c) (i)}

Copyright: () 2021 by the authors Licensee MDPI, Basel, Switzerland. This article is an open access article distributed under the terms and conditions of the Creative Commons Attribution (CC BY) license (https:// creativecommons.org/licenses/by/ $4.0 /)$.
Department of Genomic and Applied Microbiology and Göttingen Genomics Laboratory, Institute of Microbiology and Genetics, Georg-August-University of Göttingen, Grisebachstr. 8 37077 Göttingen, Germany; mingji.lu@biologie.uni-goettingen.de

* Correspondence: rdaniel@gwdg.de; Tel.: +49-551-393-3827

\begin{abstract}
Halotolerant lipolytic enzymes have gained growing interest, due to potential applications under harsh conditions, such as hypersalinity and presence of organic solvents. In this study, a lipolytic gene, est56, encoding 287 amino acids was identified by functional screening of a compost metagenome. Subsequently, the gene was heterologously expressed, and the recombinant protein (Est56) was purified and characterized. Est56 is a mesophilic $\left(\mathrm{T}_{\mathrm{opt}} 50^{\circ} \mathrm{C}\right)$ and moderate alkaliphilic $\left(\mathrm{pH}_{\text {opt }} 8\right)$ enzyme, showing high thermostability at 30 and $40^{\circ} \mathrm{C}$. Strikingly, Est56 is halotolerant as it exhibited high activity and stability in the presence of up to $4 \mathrm{M} \mathrm{NaCl}$ or $\mathrm{KCl}$. Est56 also displayed enhanced stability against high temperatures $\left(50\right.$ and $\left.60^{\circ} \mathrm{C}\right)$ and urea $(2,4$, and $6 \mathrm{M})$ in the presence of $\mathrm{NaCl}$. In addition, the recently reported halotolerant lipolytic enzymes were summarized. Phylogenetic analysis grouped these enzymes into 13 lipolytic protein families. The majority (45\%) including Est56 belonged to family IV. To explore the haloadaptation of halotolerant enzymes, the amino acid composition between halotolerant and halophilic enzymes was statistically compared. The most distinctive feature of halophilic from non-halophilic enzymes are the higher content of acidic residues (Asp and Glu), and a lower content of lysine, aliphatic hydrophobic (Leu, Met and Ile) and polar (Asn) residues. The amino acid composition and 3-D structure analysis suggested that the high content of acidic residues (Asp and Glu, 12.2\%) and low content of lysine residues ( $0.7 \%$ ), as well as the excess of surface-exposed acidic residues might be responsible for the haloadaptation of Est56.
\end{abstract}

Keywords: carboxylesterases; metagenome; compost; lipolytic enzymes; halotolerance; halophilic; haloadaptation

\section{Introduction}

Halophilic and halotolerant enzymes, which show resistance to salinity, are one of the major groups of extremozymes with industrial relevance. Microorganisms growing optimally at high salt concentrations are reservoirs for halophilic and halotolerant enzymes. They are generally divided into halophiles and halotolerant organisms [1,2]. To survive in high salinity, osmotic balance between cell cytoplasm and the external medium has to be maintained [3]. Halophilic archaea of the Halobacteriales order primarily adopt the "salt-in" strategy by accumulating equimolar concentrations of inorganic ions such as potassium and chloride ions $[4,5]$. This mechanism of osmoregulation results in intracellular enzymes, which evolve to halophilic types [6,7], which intrinsically show high activity and stability towards increasing salinity. In contrast, halophilic methanogenic archaea, as well as most halophilic and halotolerant bacteria largely employ the "salt-out" strategy by excluding salt from the cell inside and synthesizing and/or accumulating compatible organic osmolytes [8]. Some show also a combination of "salt-in" and "salt-out" strategies $[9,10]$. Halotolerant enzymes identified from "salt-out" microorganisms usually show 
different levels of salinity resistance [11-13]. Unlike most halophilic enzymes, which are inactive under low salt concentrations, halotolerant enzymes are still active in the absence of $\mathrm{NaCl}[14]$.

Haloadaptation of halophilic enzymes has been extensively studied by amino acid sequence and 3D structure comparison [15-17]. The presence of an unusually high proportion of acid residues and a drastic reduction of lysine residues on the surface of proteins play a key role in haloadaptation of enzymes [18,19]. Comparative analyses of halotolerant and halophilic enzymes with respect to amino acid compositions or 3D structures are rare. Given that scrutiny of protein and genome sequences may not unravel small differences during sequence-based comparative analysis [2,20], homologous enzymes sharing relatively high sequence similarity and conserved 3D structure would be promising in unveiling differences potentially related to shared strategies of adaptation to high salt environments.

Lipolytic enzymes, including esterases (EC 3.1.1.1, carboxylesterase) and true lipases (EC 3.1.1.3, triacylglycerol acyl hydrolase), are involved in catalyzing the cleavage and formation of ester bonds. Esterases prefer short-chain substrates with an acyl chain length of less than 10 carbon atoms, while lipases mainly catalyze the hydrolysis of long-chain triacylglycerols ( $\geq 10$ carbon atoms) [21]. A distinguishing feature of lipolytic enzyme sequences is the conserved catalytic triad composed of a serine residue, which is located in the GXSXG consensus sequence, an aspartate or glutamate, and a histidine residue [22] Most lipolytic enzymes also exhibit a similar core topology that typically consists of parallel $\beta$-pleated strands connected by $\alpha$-helices [21]. Based on amino acid sequences and biological properties of lipolytic enzymes, 19 families (family I-XIX) have been identified [23]. Subsequently, new families such as EstLiu [24], Em3L4 [25], FLS18 [26], and Est9x [27] were added. Halophilic and halotolerant lipolytic enzymes have been detected in different families but the majority belonged to family IV [11,28-30]. Family IV esterases share high amino acid sequence similarity with mammalian hormone-sensitive lipases (HSL) and hence have also been referred to as the HSL family [21]. Previous studies on members of this family have predominantly explored the thermostability [31-33] and substrate specificity [34,35] However, studies on salt tolerance of HSL family members are often missing.

Halophilic lipolytic enzymes are rare, among them, only LipC [36], MGS-B1 [37], and LipS2 [38] were characterized. In contrast, an increasing number of halotolerant lipolytic enzymes have been identified, through culture-dependent [28,39] and metagenomic approaches [29,40-42]. Apart from general features, such as broad substrate spectrum, chemo-, regio-, and enantio-selectivity and nonrequirement of cofactors, halophilic and halotolerant enzymes also tend to be resistant to organic solvents [15,31,43]. High salinity or presence of organic solvents both result in reduced water activity. Thus, halophilic and halotolerant lipolytic enzymes are advantageous in applications involving nonaqueous and aqueous/organic media, such as the degradation of organic pollutants in saline wastewater, bioremediation of oil spills, production of inter-esterification substances in food industry and nonaqueous synthesis of non-natural chemical compounds $[14,44,45]$.

Composting is a process of decomposition and humification of organic matter [46]. During composting, enzymes secreted by microorganisms play key roles in degrading various organic substances. Recently, extremophilic enzymes, such as thermophilic, organic solvent tolerant, and alkaliphilic lipolytic enzymes, have been successfully identified from compost metagenomes [47-49]. However, there are no current reports of halophilic or halotolerant lipolytic enzymes from compost. In our previous study, a compost sample at the thermophilic stage $\left(55^{\circ} \mathrm{C}\right)$ was used to construct a metagenomic plasmid library [50] of which the identified esterase-encoding gene est56 was selected for characterization Est56 was a novel member of lipolytic family IV and further characterized as halotolerant. The haloadaptation mechanism of Est56 was also explored by comparing its amino acid composition with other halotolerant and halophilic enzymes, and subsequent analysis of homology-modelled 3D structures. 


\section{Materials and Methods}

\subsection{Bacterial Strains and Plasmids}

The lipolytic recombinant plasmid (pFLD56) harboring the putative lipolytic gene est56 was derived from the functional screen of the metagenomic compost library as described in our previous study [50]. The plasmid pFLD56 was used as template for amplification of est56. E. coli strain BL21(DE3) and the plasmid pET101/D-TOPO ${ }^{\circledR}$ (Invitrogen, Karlsruhe, Germany) were used as expression host and vector, respectively.

\subsection{Identification and Analysis of Est56 Sequence}

Putative open reading frames (ORF) encoding lipolytic enzymes were initially predicted using the ORF Finder program (http:/ / www.ncbi.nlm.nih.gov/gorf/gorf.html), and further verified using FramePlot analysis [51]. Potential signal peptides were detected using SignalP 4.0 [52]. Amino acid sequence similarity searches were performed using the BLASTP program against the public GenBank database [53]. Amino acid sequences of esterases homologous to the deduced gene product of est56 were aligned by employing clustalW. Secondary structure prediction was performed with I-TASSER [54]. Annotation of aligned sequences was performed with ESPript 3.0 [55].

\subsection{Cloning and Expression of Est56}

The putative lipolytic gene est56 was amplified using the following primers: $5^{\prime}-$

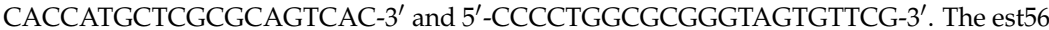
PCR product was cloned into $\mathrm{pET101/D-TOPO}{ }^{\circledR}$ vector (Invitrogen, Germany), following the manufacturer's instructions. The resulting recombinant plasmid was transformed into E. coli BL21 (DE3) cells. For expression of est56, a $6 \mathrm{~mL}$ preculture, incubated overnight, was used to inoculate $600 \mathrm{~mL} \mathrm{LB}$ medium containing $100 \mu \mathrm{g} / \mathrm{mL}$ ampicillin. The culture was incubated overnight with shaking at $30^{\circ} \mathrm{C}$ to an $\mathrm{OD}_{600}$ of 0.6. Expression was induced by adding IPTG (isopropyl-b-D thiogalactopyranoside) to a final concentration of $0.5 \mathrm{mM}$. After incubation for $6 \mathrm{~h}$ at $30^{\circ} \mathrm{C}$, cells were harvested by centrifugation $(7000 \times g, 10 \mathrm{~min}$, $\left.4{ }^{\circ} \mathrm{C}\right)$, suspended in lysis-equilibration-wash buffer ( $50 \mathrm{mM} \mathrm{NaH}_{2} \mathrm{PO}_{4}, 300 \mathrm{mM} \mathrm{NaCl}, \mathrm{pH}$ ), and then lysed on ice by sonication using a UPS200S homogenizer (Hielscher Ultrasonics $\mathrm{GmbH}$, Teltow, Germany).

\subsection{Purification of Recombinant Est56}

To purify $\mathrm{His}_{6}$-tagged Est56, Protino ${ }^{\circledR}$ Ni-TED 2000 packed columns (Macherey-Nagel, Düren, Germany) were used as recommended by the manufacturer. SDS-PAGE was performed to determine the purity and molecular mass of Est56. Protein concentration was measured by the Bradford method [56]. Finally, fractions containing the purified enzyme were pooled and dialyzed against $50 \mathrm{mM}$ sodium phosphate buffer $(\mathrm{pH} 8)$ at $4{ }^{\circ} \mathrm{C}$.

\subsection{Esterase Standard Assay}

Unless otherwise mentioned, Est56 activity was measured in $1.0 \mathrm{~mL}$ assay buffer containing $50 \mathrm{mM}$ sodium phosphate $(\mathrm{pH} 8)$ and $1 \mathrm{mM} p$-nitrophenyl ( $p$-NP) butyrate (Sigma-Aldrich, Munich, Germany), at $50^{\circ} \mathrm{C}$. The amount of $p$-NP released by enzymecatalyzed hydrolysis was continuously monitored for at least $2 \mathrm{~min}$ at a wavelength of $410 \mathrm{~nm}$ against an enzyme-free reference. One unit of enzymatic activity was defined as the amount of Est56 needed to release $1 \mu \mathrm{mol}$ of $p$-NP per minute under the assay conditions. All experiments were performed in at least triplicate, and extinction coefficients of $p$-NP under every assay condition were determined. Results are shown as mean values \pm standard deviation (SD). 


\subsection{Characterization of Est56}

\subsubsection{Substrate Specificity}

Substrate specificity of Est56 was assessed under standard assay conditions using $1 \mathrm{mM} p$-NP esters (Sigma-Aldrich, Munich, Germany) of different chain lengths as substrates: $p$-NP acetate (C2), $p$-NP butyrate (C4), $p$-NP valerate (C5), $p$-NP caproate (C6), $p$-NP caprylate (C8), $p$-NP caprate (C10), $p$-NP laurate (C12), $p$-NP myristate (C14), and $p$ $\mathrm{NP}$ palmitate (C16). All substrates were prepared as a $0.1 \mathrm{M}$ stock solution dissolved in isopropanol. For long chain substrates $(>\mathrm{C} 10)$, the stock solution was first heated $\left(50^{\circ} \mathrm{C}\right)$ for a short time until the formation of clear transparent solution [40]. Initial reaction rates were calculated by estimating Est56 activity with different substrate concentrations ranging from 5 to $5000 \mu \mathrm{M}$. Michaelis-Menten constant $\left(\mathrm{K}_{\mathrm{m}}\right)$ and the maximal velocity $\left(\mathrm{V}_{\max }\right)$ were determined by employing Lineweaver-Burk plots [57].

\subsubsection{Effect of Temperature and $\mathrm{pH}$}

Optimum temperature of Est56 activity was determined between 20 and $70{ }^{\circ} \mathrm{C}$. To assess protein thermostability, Est56 was preincubated in assay buffer at 30, 40, 50, and $60{ }^{\circ} \mathrm{C}$ for different time periods, and subsequently, residual activity was measured under standard assay condition.

Due to $\mathrm{pH}$-dependent absorption of $\mathrm{p}-\mathrm{NP}$ in acidic buffers [58], the effect of $\mathrm{pH}$ on Est56 activity was determined between $\mathrm{pH}$ values 3 and 10 at $348 \mathrm{~nm}$ (the $\mathrm{pH}$-independent isosbestic wavelength). The overlapping buffer systems used comprised $50 \mathrm{mM}$ acetate buffer ( $\mathrm{pH} 3.0$ to 6.0 ), $50 \mathrm{mM}$ sodium phosphate buffer ( $\mathrm{pH} 6.0$ to 8.0$), 50 \mathrm{mM}$ TAPS (3-(2,4 dinitrostyrl)-(6R,7R-7-(2-thienylacetamido)-ceph-3-em-4-carboxylic acid) buffer ( $\mathrm{pH} 8.0$ 9.0), and $50 \mathrm{mM}$ CHES (N-cyclohexyl-2-aminoethanesulfonic acid) buffer (pH 9.0 to 10.0). The effect of $\mathrm{pH}$ on Est56 stability was examined by preincubating the enzyme at the respective $\mathrm{pH}$ value at $10^{\circ} \mathrm{C}$ for $24 \mathrm{~h}$. Subsequently, residual activity was measured under standard assay condition.

\subsubsection{Effect of Salinity}

The effect of salinity on Est56 activity was measured by adding 0.5 to $4 \mathrm{M} \mathrm{NaCl}$ or $\mathrm{KCl}$ to the standard reaction assay mixture. Est56 stability against salt was determined by incubating the enzyme in assay buffer containing $\mathrm{NaCl}(0.5$ to $4 \mathrm{M})$ at $10^{\circ} \mathrm{C}$ for $24 \mathrm{~h}$. Residual activity was measured under standard assay conditions.

Salt has been reported to protect halophilic proteins against denaturants (such as high temperature and urea) [18]. In this study, the protective effect of $\mathrm{NaCl}$ was investigated by adding different amounts of $\mathrm{NaCl}(0-4 \mathrm{M})$ to the incubation buffer, in which Est56 was incubated at different temperatures or in the presence of different amounts of urea. Specifically, Est56 was incubated at high temperatures $\left(50\right.$ or $\left.60{ }^{\circ} \mathrm{C}\right)$ for $30 \mathrm{~min}$. For urea impact, Est56 was incubated with different amounts of urea $(2,4$, and $6 \mathrm{M})$ at $10^{\circ} \mathrm{C}$ for $24 \mathrm{~h}$. Each additive was equalized to the same final concentration in the assay buffer, and the residual activity was measured under standard assay conditions. A blank reference was prepared using the buffer solution without enzyme but containing the same amount and type of additive. Activity measured before incubation was taken as $100 \%$.

\subsubsection{Effect of Organic Solvents}

The effect of organic solvents on Est56 stability was assayed by incubating Est56 in the presence of $15 \%$ and $30 \%(v / v)$ water-miscible organic solvents (DMSO, methanol, ethanol, acetone, isopropanol, and 1-propanol) or water-immiscible organic solvents (ethyl acetate, diethyl ether, chloroform, and toluene) at $10{ }^{\circ} \mathrm{C}$ for $24 \mathrm{~h}$ under constant shaking. Residual activity was measured under standard assay conditions.

\subsubsection{Effect of Other Additives}

The effect of metal ions including $\mathrm{K}^{+}, \mathrm{Ca}^{2+}, \mathrm{Mn}^{2+}, \mathrm{Mg}^{2+}, \mathrm{Zn}^{2+}, \mathrm{Fe}^{2+}, \mathrm{Cu}^{2+}, \mathrm{Ni}^{2+}, \mathrm{Fe}^{3+}$, and $\mathrm{Al}^{3+}$ and inhibitors including phenylmethyl-sulfonyl fluoride (PMSF), dithiothreitol 
(DTT), and ethylenediaminetetraacetic acid (EDTA) was measured at concentrations of 1 and $10 \mathrm{mM}$. In addition, the impact of detergents such as Triton X-100, Tween 20, Tween 80, and SDS at concentrations of $0.1 \%, 1 \%$ and $5 \%(v / v)$ on enzyme activity was investigated. The catalytic activity of Est 56 was measured under standard reaction conditions by directly adding each additive to the standard assay mixture. Activity measured in additive-free assay buffer was regarded as $100 \%$ activity, while reactions that included corresponding additive but no enzyme were used as blanks.

\subsection{Sequence Analysis of Halotolerant Enzymes}

Phylogenetic trees were constructed with the neighbor-joining method using MEGA version 7 [59]. For this purpose, Est56, other reported halotolerant, and reference lipolytic enzyme sequences retrieved from GenBank were employed (Table S1). A bootstrap value of 500 replicates was used to estimate the confidence level [60]. The phylogenetic tree was subsequently visualized by GraPhlAn [61]. In addition, a tree containing only family IV esterases was constructed.

Characterized halophilic lipolytic enzymes are rare but enzymes from halophilic microorganism that adopt the "salt-in" strategy were evolved to be halophilic [6]. Thus, we retrieved 22 putative halophilic lipolytic enzymes originating from archaea of the Halobacteriales order from GenBank (Table S2). The halophilic feature of each archaeal strain was checked on HaloDom webserver (http:/ /www.halodom.bio.auth.gr) [62]. We summarized experimentally confirmed halophilic proteins as controls (Table S3). Thereafter, we used HT_Lip, HP_Lip, and HP_Enz to refer to the 40 halotolerant lipolytic enzymes, 22 putative halophilic lipolytic enzymes, and 16 experimentally confirmed halophilic proteins, respectively. Amino acid composition, theoretical pI values, and molecular weight of each protein were calculated by the ProtParam tool at Expasy (www.expasy.org) [63].

Differences in amino acid compositions among HT_Lip, HP_Lip, and HP_Enz were statistically compared. Individual amino acids were analyzed as variants for each enzyme Nonparametric Kruskal-Wallis (KW) and Mann-Whitney (MW) pairwise post hoc tests were used to evaluate median differences among univariate groups. Analysis of similarities (ANOSIM) test was used to pairwise compare the overall differences between multivariate groups based on Bray-Curtis distance, with 9999 permutations. A high $\mathrm{R}$ value generated by the ANOSIM test indicates a high dissimilarity between groups. Similarity percentage (SIMPER) tool calculates the average contribution of individual amino acids to the average dissimilarity between groups based on Bray-Curtis similarity. Statistical analyses were performed with R (http: / / www.r-project.org) using the "vegan" package [64].

\subsection{Homology Modeling and Putative Structure Analysis}

Based on deduced amino acid sequence, the tertiary structure prediction of Est56 was performed by I-TASSER [54]. PyMOL (PyMOL molecular graphics system, DeLano Scientific, Palo Alto, CA, USA; http:/ /www.pymol.org) was used to visualize the predicted model. The analog with the highest TM score was also selected for structural superimposition. The surface electrostatic potential was calculated by the APBS plugin [65] and visualized by PyMOL.

\subsection{Accession Numbers}

The amino acid sequence of Est56 is available in the GenBank database under accession number KR149569.1. The compost metagenome sequences are available in the NCBI sequence read archive (SRA) under the accession number SRR13115019.

\section{Results}

\subsection{Identification and Sequence Analysis of a Novel Lipolytic Gene}

A metagenomic plasmid library derived from compost using Escherichia coli as host was constructed and function-based screened for genes conferring lipolytic activity as previously described [50]. An E. coli clone showing strong lipolytic activity (large halos) 
on indicator plates was selected for further characterization. Sequence analysis of the plasmid insert ( $6.1 \mathrm{~kb}$, Figure S1) revealed a putative lipolytic gene (est56, $864 \mathrm{bp})$ encoding 287 amino acids. Putative signal peptides indicating extracytoplasmic localization were not detected in the deduced protein sequence. Similarity searches showed that protein sequences similar to Est56 were mainly identified during metagenome screenings. These comprised ELP45 isolated from a forest topsoil [66], EstC23 from mountain soil [67], Est06 from forest soil [68], and EstMY from activated sludge [69], which showed $62 \%, 61 \%, 60 \%$, and $60 \%$ amino acid identity to Est 56 , respectively.

Multiple sequence alignments of Est56 with other esterases revealed that Est56 belongs to the HSL group (family IV) of lipolytic proteins. The conserved family IV motif H-G-G was present in the Est56 protein at amino acid sequence positions from 62 to 64 . This motif plays an essential role in the stabilization of the oxyanion hole and catalysis. The catalytic triad composed of Ser ${ }^{132}$, Glu ${ }^{226}$, and $\mathrm{His}^{256}$, as well as another conserved motif E-X-L-XD-D (amino acid residues from 226 to 231), was also detected in Est56 amino acid sequence (Figure S2).

\subsection{Purification of Recombinant Est56 and Substrate Specificity}

Est56 was heterologously produced in E. coli BL21 (DE3). After purification by Ni-TED affinity chromatography, Est56 was purified 73-fold with a specific activity of $90.44 \mathrm{U} / \mathrm{mg}$ (Table S4). SDS-PAGE revealed a single band with a molecular mass of approximately $34.0 \mathrm{kDa}$ (Figure S3). This is consistent with the calculated protein mass including the sequences for the V5 epitope and $\mathrm{His}_{6}$-tag, which were added during cloning of est56.

Assays with $p$-NP esters showed that Est56 exhibited a substrate preference for esters with short-chain fatty acids such as $p$-NP acetate (C2), $p$-NP butyrate (C4), $p$-NP valerate (C5), and $p$-NP caproate (C6). The enzyme did show little or no significant activity by employing $p$-NP esters with long-chain fatty acids (C8-C16) as substrates (Figure S4). This indicates that Est56 is an esterase and not a lipase [21]. The maximal specific activity was detected with $p$-NP butyrate (C4). The $\mathrm{K}_{\mathrm{m}}$ and $\mathrm{V}_{\max }$ values with this substrate were $128.0 \mu \mathrm{M}$ and $102.0 \mathrm{U} / \mathrm{mg}$, respectively.

\subsection{Effect of Temperature and $p H$}

Est56 retained high activity (above $40 \%$ ) over the entire tested temperature range from 20 to $70{ }^{\circ} \mathrm{C}$ with maximal activity at $50{ }^{\circ} \mathrm{C}$ (Figure 1a). Despite the high activity (above $80 \%$ ) of Est56 at 50 and $60{ }^{\circ} \mathrm{C}$, thermostability at 50 and $60^{\circ} \mathrm{C}$ was low. Est56 retained $50 \%$ activity after $30 \mathrm{~min}$ of incubation at $50{ }^{\circ} \mathrm{C}$ and $12.4 \%$ after $10 \mathrm{~min}$ at $60^{\circ} \mathrm{C}$. However, Est56 showed high stability for extended incubation times at 30 and $40^{\circ} \mathrm{C}$, with a half-life of 192 and $16 \mathrm{~h}$, respectively (Figure $1 \mathrm{c}$ ).

Est56 exhibited more than $80 \%$ activity between $\mathrm{pH} 6$ and 8, with maximal activity at pH 8 (Figure 1b). Similarly, Est56 was most stable from pH 6 to 8, retaining more than $90 \%$ residual activity after $24 \mathrm{~h}$ incubation at $10^{\circ} \mathrm{C}$ (Figure 1d).

\subsection{Effect of Salinity}

Addition of salt $(\mathrm{NaCl}$ or $\mathrm{KCl})$ produced a stimulatory effect on Est56 activity, with an enhanced activity at $\mathrm{NaCl}$ and $\mathrm{KCl}$ concentrations of up to $2.5 \mathrm{M}$. Maximal activities compared to the reference without addition were recorded in the presence of $1.5 \mathrm{M} \mathrm{NaCl}$ $(130.4 \%)$ and $1 \mathrm{M} \mathrm{KCl}(141.6 \%)$. At higher concentrations, Est56 activity decreased gradually with increasing concentration of $\mathrm{NaCl}$ and $\mathrm{KCl}$. Notably, Est56 still retained approximately $90 \%$ activity at $3 \mathrm{M} \mathrm{NaCl}$ and $3.5 \mathrm{M} \mathrm{KCl}$ and $40 \%$ activity at $4 \mathrm{M} \mathrm{NaCl}$ and $\mathrm{KCl}$ (Figure 2a).

As for the stability, Est56 was stable over the tested $\mathrm{NaCl}$ and $\mathrm{KCl}$ concentration ranges $(0-4 \mathrm{M})$, with almost unaltered activity after $24 \mathrm{~h}$ incubation at $10^{\circ} \mathrm{C}$ (Figure $2 \mathrm{~b}$ ). 

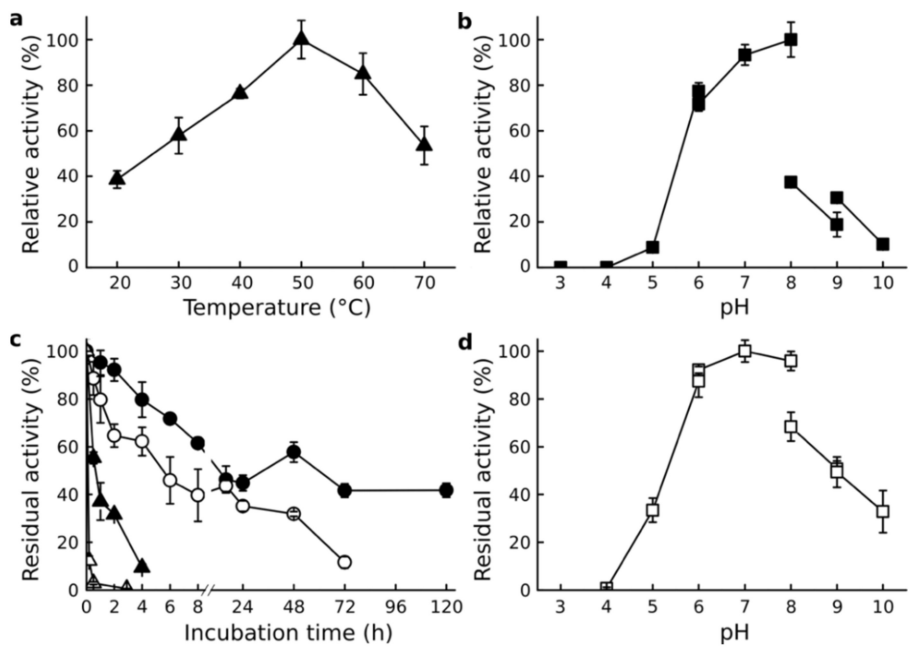

Figure 1. Effect of temperature and $\mathrm{pH}$ on Est56 activity. (a) Effect of temperature on Est56 activity; the maximal activity $\left(153.9 \mathrm{U} / \mathrm{mg}\right.$ ) at $50{ }^{\circ} \mathrm{C}$ was taken as $100 \%$. (b) Effect of $\mathrm{pH}$ on Est56 activity; the maximal activity $(90.4 \mathrm{U} / \mathrm{mg})$ at $\mathrm{pH} 8$ was taken as $100 \%$. (c) Thermostability of Est56 at $30{ }^{\circ} \mathrm{C}$ (closed circle), $40^{\circ} \mathrm{C}$ (open circle), $50^{\circ} \mathrm{C}$ (closed triangle), and $60^{\circ} \mathrm{C}$ (open triangle); specific activity $(87.2 \mathrm{U} / \mathrm{mg})$ measured at the start of the incubation and under standard assay conditions was taken as 100\%. (d) Effect of $\mathrm{pH}$ on Est56 stability was measured by incubating Est56 at $4{ }^{\circ} \mathrm{C}$ for $24 \mathrm{~h}$, the maximal residual activity $(33.1 \mathrm{U} / \mathrm{mg})$ at $\mathrm{pH} 7$ was taken as $100 \%$.
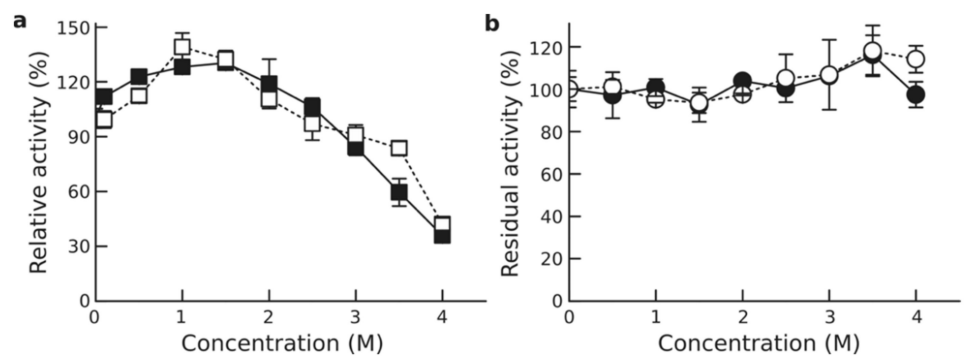

Figure 2. Effect of salinity on Est56 activity and stability. (a) Effect of $\mathrm{NaCl}$ (closed square) and $\mathrm{KCl}$ (open square) on Est56 activity. (b) Effect of $\mathrm{NaCl}$ (closed circle) and $\mathrm{KCl}$ (open circle) on Est56 stability. Specific activities corresponding to $100 \%$ activity were 93.1 and $94.1 \mathrm{U} / \mathrm{mg}$ for graph (a) and (b), respectively.

\subsection{Protective Effect of $\mathrm{NaCl}$ against Denaturants}

The presence of $\mathrm{NaCl}$ enhanced stability of Est56 to temperature (Table 1). At $50{ }^{\circ} \mathrm{C}$, the addition of 1,2, and $3 \mathrm{M} \mathrm{NaCl}$ significantly $(p<0.05)$ increased Est56 residual activity (above $70 \%$ ) compared to that incubated in the salt-free assay. The best stabilization effect was detected at $2 \mathrm{M} \mathrm{NaCl}$. However, incubation with $4 \mathrm{M} \mathrm{NaCl}$ significantly $(p<0.05)$ decreased Est56 stability. At $60^{\circ} \mathrm{C}$, Est56 was almost inactivated after 30-min without $\mathrm{NaCl}$ addition, whereas a significant increase in residual activity was detected by adding $1,2,3$, or $4 \mathrm{M} \mathrm{NaCl}$. 
Table 1. Role of $\mathrm{NaCl}$ in protecting Est56 against denaturants.

\begin{tabular}{|c|c|c|c|c|c|}
\hline & \multicolumn{5}{|c|}{ Residual Relative Activity (\%) ${ }^{a}$} \\
\hline & $\mathbf{0} \mathbf{M}$ & $1 \mathrm{M}$ & $2 \mathrm{M}$ & $3 \mathbf{M}$ & $4 \mathrm{M}$ \\
\hline \multicolumn{6}{|c|}{ Temperature $^{b}$} \\
\hline $50^{\circ} \mathrm{C}$ & $54.9 \pm 2.4$ & $71.3 \pm 8.1 *$ & $82.8 \pm 7.4^{* *}$ & $72.9 \pm 4.2 *$ & $38.9 \pm 3.1 * *$ \\
\hline $60^{\circ} \mathrm{C}$ & $1.4 \pm 0.7$ & $12.7 \pm 2.2 * *$ & $11.8 \pm 1.6^{* *}$ & $8.0 \pm 0.7^{* *}$ & $10.6 \pm 0.6^{* *}$ \\
\hline \multicolumn{6}{|l|}{ Urea $^{c}$} \\
\hline $2 \mathrm{M}$ & $36.3 \pm 4.7$ & $36.2 \pm 4.0$ & $43.0 \pm 4.7$ & $54.0 \pm 2.4 * *$ & $41.5 \pm 9.5$ \\
\hline $4 \mathrm{M}$ & $19.5 \pm 3.5$ & $31.6 \pm 2.5^{* *}$ & $33.6 \pm 2.2 * *$ & $29.3 \pm 4.0 *$ & $\mathrm{ND}^{\mathrm{d}}$ \\
\hline $6 \mathrm{M}$ & $17.2 \pm 2.4$ & $28.6 \pm 2.2 * *$ & $\mathrm{ND}^{\mathrm{d}}$ & $\mathrm{ND}^{\mathrm{d}}$ & $\mathrm{ND}^{\mathrm{d}}$ \\
\hline
\end{tabular}

${ }^{a}$ Residual activity was measured after incubation with different amounts of $\mathrm{NaCl}$. Two-sample $t$ test was performed between residual activities incubated with and without $\mathrm{NaCl}$. ${ }^{*} \alpha=0.05$; ${ }^{* *} \alpha=0.01$. ${ }^{b}$ Est56 was incubated at 50 or $60^{\circ} \mathrm{C}$ for $30 \mathrm{~min}$ in the presence of different concentrations of $\mathrm{NaCl}$. Specific activity $(41.9 \mathrm{U} / \mathrm{mg})$ measured before incubation was taken as $100 \%$. ${ }^{\mathrm{c}}$ Est56 was incubated at $10^{\circ} \mathrm{C}$ for $24 \mathrm{~h}$ in the presence of different combinations of $\mathrm{NaCl}$ and urea. Specific activity $(45.8 \mathrm{U} / \mathrm{mg})$ measured before incubation was taken as $100 \% .{ }^{d}$ Not detectable.

Urea is another denaturant that can cause inactivation of an enzyme directly and indirectly [70]. Addition of $3 \mathrm{M} \mathrm{NaCl}$ significantly $(p<0.05)$ enhanced Est56 tolerance against $2 \mathrm{M}$ urea, with the residual activity increasing from $36.3 \%$ without $\mathrm{NaCl}$ addition to $54.0 \%$ in the presence of $3 \mathrm{M} \mathrm{NaCl}$. A stabilizing effect of $\mathrm{NaCl}(p<0.05)$ on Est56 activity was also observed in the presence of 4 or $6 \mathrm{M}$ urea (Table 1 ).

\subsection{Effect of Organic Solvents}

Generally, Est56 exhibited enhanced activity after incubating with $15 \%$ and $30 \%(v / v)$ water-miscible organic solvents (Table 2), with the exception of $30 \%(v / v) 1$-propanol, which caused a considerable loss of Est56 residual activity (23.0\%). The highest stimulation of residual activity $(286 \%)$ was observed after incubation with $30 \%(v / v)$ isopropanol. In the presence of water-immiscible organic solvents, Est 56 was inhibited by $15 \%$ and 30\% $(v / v)$ ethyl acetate, chloroform, and toluene (Table 3$)$. Est56 retained its full activity after incubation with $15 \%$ and $30 \%(v / v)$ diethyl ether.

Table 2. Effect of water-miscible organic solvents on Est56 stability.

\begin{tabular}{ccc}
\hline \multirow{2}{*}{ Organic Solvent } & \multicolumn{2}{c}{ Residual Activity (\%) ${ }^{\text {a }}$} \\
\cline { 2 - 3 } & $\mathbf{1 5 \% ( \boldsymbol { v } / \boldsymbol { v } )}$ & $\mathbf{3 0 \% ( \boldsymbol { v } / \boldsymbol { v } )}$ \\
\hline DMSO & $135.0 \pm 11.8$ & $135.4 \pm 4.7$ \\
Methanol & $116.1 \pm 6.7$ & $96.6 \pm 7.6$ \\
Ethanol & $116.2 \pm 8.7$ & $107.3 \pm 4.2$ \\
Acetone & $138.3 \pm 8.9$ & $115.3 \pm 14.8$ \\
Isopropanol & $136.3 \pm 10.1$ & $286.6 \pm 9.0$ \\
1-Propanol & $114.0 \pm 3.7$ & $23.0 \pm 1.0$ \\
\hline
\end{tabular}

${ }^{\text {a }}$ Specific activity $(33.4 \mathrm{U} / \mathrm{mg})$ incubated in the organic solvent-free assay buffer was taken as $100 \%$.

Table 3. Effect of water-immiscible organic solvents on Est56 stability.

\begin{tabular}{ccc}
\hline \multirow{2}{*}{ Organic Solvent } & \multicolumn{2}{c}{ Residual Activity (\%) ${ }^{\text {a }}$} \\
\cline { 2 - 3 } & $\mathbf{1 5 \% ( v / v )}$ & $\mathbf{3 0 \% ( v / v )}$ \\
\hline Ethyl acetate & $55.3 \pm 8.1$ & $50.2 \pm 3.5$ \\
Diethyl ether & $110.8 \pm 5.7$ & $106.7 \pm 10.8$ \\
Chloroform & $46.5 \pm 0.5$ & $6.3 \pm 2.5$ \\
Toluene & $50.0 \pm 2.2$ & $54.9 \pm 0.7$ \\
\hline
\end{tabular}

a Specific activity $(53.4 \mathrm{U} / \mathrm{mg})$ incubated in the organic solvent-free assay buffer was taken as $100 \%$. 


\subsection{Effect of Other Additives}

Metal ions exhibited different effects on Est56 activity (Table S5). The addition of $\mathrm{Al}^{3+}$ and $\mathrm{Ca}^{2+}$ at 1 and $10 \mathrm{mM}$ had a stimulatory effect (approximately 130\%). Est56 retained its full activity in the presence of 1 and $10 \mathrm{mM} \mathrm{Mg}^{2+}$. In contrast, Est56 activity slightly decreased to approximately $80 \%$ in the presence of $1 \mathrm{mM} \mathrm{Fe}^{2+}, 10 \mathrm{mM} \mathrm{Mn}^{2+}$, and 1 and $10 \mathrm{mM} \mathrm{Zn}^{2+}$. The additives $\mathrm{Cu}^{2+}$ and $\mathrm{Ni}^{2+}$ at 1 and $10 \mathrm{mM}$ and $\mathrm{Fe}^{2+}$ and $\mathrm{Fe}^{3+}$ at $10 \mathrm{mM}$ were deleterious to Est 56 , as enzyme activity dropped to approximately $20 \%$. EDTA did not affect Est56 activity, which indicated that Est56 activity is independent of divalent cations.

The nonionic detergents Triton X-100, Tween 20, and Tween 80 at $0.1 \%(v / v)$ significantly enhanced Est56 activity to $156.8 \%, 154.3 \%$, and $112.7 \%$, respectively. Est 56 activity was inhibited or inactivated in the presence of $1 \%$ and $5 \%(v / v)$ of the other tested detergents (Table S6). The addition of the inhibitors DTT, PMSF, and DEPC at 1 and $10 \mathrm{mM}$ had detrimental effects on Est56 activity (Table S7). The inhibition of enzyme activity by PMSF and DEPC indicated that serine and histidine residues, respectively, are part of the Est56 catalytic triad [71].

\subsection{Sequence Analysis of Halotolerant Lipolytic Enzymes}

A phylogenetic tree was constructed to group 40 halotolerant lipolytic enzymes (Table S1) into families based on Arpigny and Jaeger [21] classification. As shown in Figure 3, these enzymes covered nine Arpigny and Jaeger families including family I, II, IV, V, VI, VII, VIII, XV, and XVII, as well as four new families including Est9x [27], EstLiu [24], lp_3505 [72], and EM3L4 [25]. Most of the analyzed halotolerant enzymes (18 enzymes) including Est56 belonged to Family IV. Family IV can be further divided into two sub-families based on the conserved GXSXG motif [34]. Est56 and other 10 halotolerant lipolytic enzymes belong to the GDSAG motif subfamily and the remaining 7 enzymes grouped into the GTSAG motif subfamily (Figure S5).

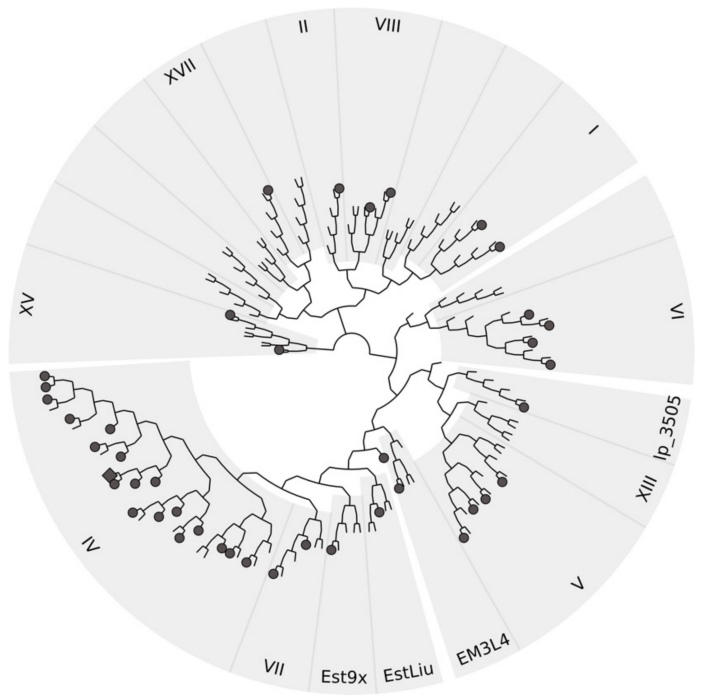

Figure 3. Phylogenetic classification of Est56 (closed diamond) and reported halotolerant (closed circle) lipolytic enzymes by neighbor-joining method. With the exception of Est56, other sequences were retrieved from GenBank.

Generally, the overall amino acid compositions among 3 different groups of selected and characterized halotolerant or halophilic enzymes were analyzed. The halotolerant lipolytic enzymes (HT_Lip; Table S1), the halophilic lipolytic enzymes (HP_Lip; Table S2), 
and other characterized halophilic enzymes (HP_Enz; Table S3) are pairwise different (ANOSIM test, $p<0.01$ in all three cases). The R values between HT_Lip and HP_Lip (0.4254) and HT_Lip and HP_Enz (0.4251) were higher than that of HP_Lip and HP_Enz (0.2065). This result indicated a high separation of amino acid composition between halotolerant (HT_Lip) and halophilic (HP_Lip and HP_Enz) enzymes, rather than within halophilic enzymes. This result was also consistent with the average dissimilarity revealed by SIMPER analysis (Table S8). The KW test identified that 12 residues (Asp, Glu, Lys, Arg, His, Leu, Met, Ile, Ala, Ser, Asn, and Gln) (Figure 4a) and theoretical pI values (Figure $4 \mathrm{~b}$ ) were significantly different $(p<0.05)$ among the three groups. The groups of halophilic enzymes HP_Lip and HP_Enz obtained significantly higher content of aspartic acid, glutamic acid, and arginine residues and lower content of lysine, leucine, methionine, isoleucine, and asparagine residues and theoretical $\mathrm{pI}$ values than those in halotolerant lipolytic enzymes HT_Lip (MW post hoc pairwise test, $p<0.05$ ). As reported above, the most notable feature for the halophilic adaptation of halophilic enzymes is the excessive number of acidic residues (Asp and Glu) compared to lysine residues. This is reflected by low theoretical pI values [15]. However, halotolerant lipolytic enzymes exhibited broad range of theoretical $\mathrm{pI}$ values (4.59 to 9.44) (Figure 4b). Among them, Est56 obtains high content of acidic residues (Asp and Glu, 12.12\%) and particularly low content of lysine residues $(0.7 \%)$, as well as a relatively low theoretical pI value of 4.97 .

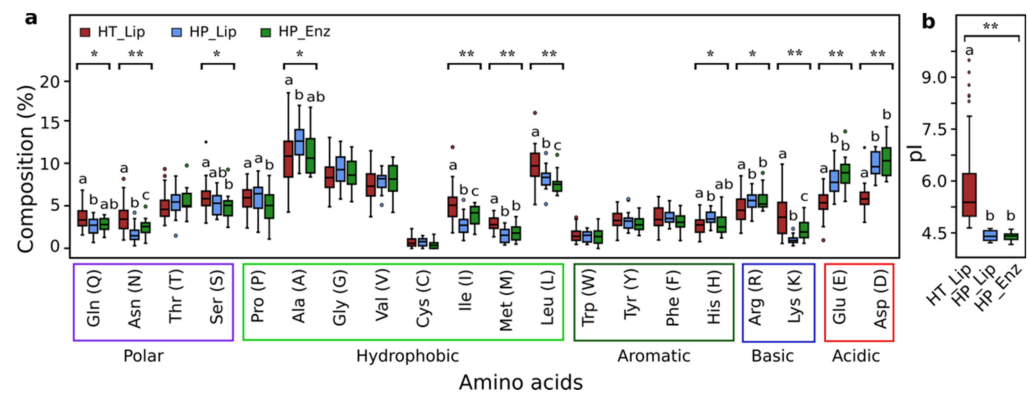

Figure 4. Comparison between halotolerant and halophilic enzymes. Box plots of (a) amino acid composition and (b) theoretical pI value of enzymes in groups HT_Lip (red), HP_Lip (blue), and HP_Enz (green). Statistical comparison was conducted by Kruskal-Wallis (KW) test $\left({ }^{* *}, p<0.01 ;{ }^{*}, p<0.05\right)$ among three groups and Mann-Whitney (MW) post hoc test between pairwise groups (medians sharing a letter above boxes indicate no significant difference in the pairwise test).

\subsection{Structural Modeling of Est56}

The tertiary structure of Est56 is composed of a cap domain and an $\alpha / \beta$-hydrolase fold core domain. The cap domain of Est56 consists of $\alpha$-helices at the $\mathrm{N}$-terminal side $(\alpha 1$ to $\alpha 2$ ) and between $\beta 6$ and $\beta 7$ ( $\alpha 6$ to $\alpha 7$ ) (Figure 5a). The core domain comprises six helices surrounded by eight $\beta$-strands that form parallel structures. The catalytic triad of Est56 consists of Ser ${ }^{132}$ located between $\beta 5$ and $\alpha 5$, Glu ${ }^{226}$ after $\beta 7$, and $\mathrm{His}^{256}$ between $\beta 8$ and $\alpha 9$ (Figure 5a). The overall structure of Est56 superimposed well (TM-score 0.984; RMSD 0.48) on E40 [73], with a global amino acid sequence identity of 53\% (Figure 5b). The electrostatic potential of Est56 was calculated and described. The distribution of charges revealed that Est56 had negative charges on the surface (Figure $5 c, d$ ). 

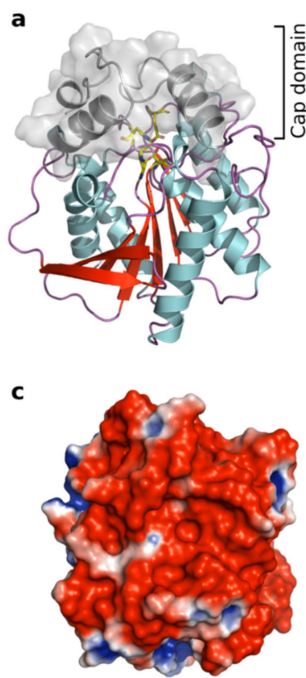

b

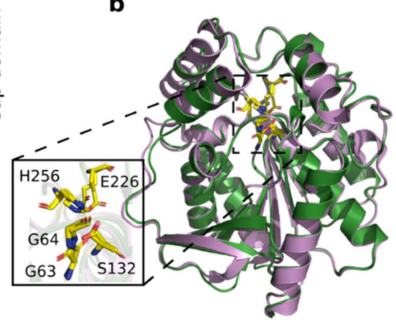

d

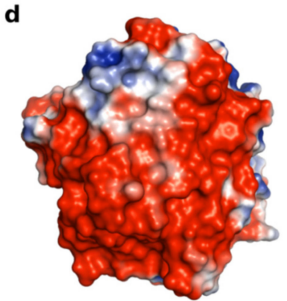

Figure 5. The modelled three-dimensional structure of Est56. (a) Ribbon representation of the Est56 monomer colored according to secondary structure elements. The overall structure is composed of two domains: cap domain and catalytic domain. (b) Superposition of Est56 (pink) onto its structural homolog E40 (PDB: 4xvc). The residues involved in stabilizing the oxyanion hole (Gly63 and Gly64) and catalytic triad (Ser94, Asp215 and His245) were indicated in stick representation (the sequence number is based on Est56 amino acid sequence). (c) Structural electrostatic potential of Est56. The most negative and most positive electrostatic potentials are indicated by red and blue, respectively, from -1 (red) to $+1 \mathrm{kT} / \mathrm{e}$ (blue). (d) The $180^{\circ}$ rotated view of (c).

\section{Discussion}

In this study, we identified a halotolerant esterase, Est56, from a compost metagenome and compared it to recently reported halotolerant enzymes (Table S1). In total, the 46 halotolerant lipolytic enzymes were grouped into 13 lipolytic families, which suggested a high diversity of halotolerant lipolytic enzymes (Figure 3). Including Est56, 29 halotolerant lipolytic enzymes were derived from metagenomes and correspondingly from uncultured microorganisms, which confirms the efficiency of metagenomic approaches in exploring novel enzymes $[74,75]$. The remaining 17 halotolerant enzymes were derived from individual microorganisms of which most thrive in saline environments, i.e., Zunongwangia profunda was derived from a surface seawater [24], Erythrobacter seohaensis from a tidal flat [76], Psychrobacter celer from a deep-sea sediment [77], and Alkalibacterium sp. SL3 from a soda lake sediment [78]. Some of the metagenome-derived halotolerant enzymes also originated from saline environments, such as marine water $[27,37,41,79]$, deep sea sponges [42,80], deep-sea sediments [25,81], and deep-sea shrimps [82]. Recently, compost was reported as an habitat for halophilic and halotolerant microorganisms [83,84]. To our knowledge, Est56 is the first halotolerant esterase identified from a compost metagenome.

Est56 activity exhibited a $\mathrm{T}_{\mathrm{opt}}$ at $50{ }^{\circ} \mathrm{C}$ and high stability at $30^{\circ} \mathrm{C}$ (Figure 2). This is also the case for the majority of halotolerant esterases, which show a temperature optimum of enzyme activity between 35 and $50{ }^{\circ} \mathrm{C}$ and high stability at low temperatures (below $30^{\circ} \mathrm{C}$ in most cases) $[34,85-87]$. Nevertheless, psychrophilic and thermophilic halotolerant lipolytic enzymes were also detected. For example, 7N9 [42], lp_3505 [72], EstS [11], Est10 [88], and EstLiu [24] displayed a temperature optimum ranging from 0 to $30^{\circ} \mathrm{C}$, whereas E69 [76], Est9x [27], BmEST [89], and LipJ2 [39] exhibit their optimal activity between 60 and $80^{\circ} \mathrm{C}$. With respect to $\mathrm{pH}$, most of the halotolerant enzymes including Est56 are moderate alkaliphilic, with the optimum pH between 7.5 and 9 (Table S1). 
Generally, halotolerant lipolytic enzymes could be classified into three groups, according to their NaCl-dependent catalytic activity (Table S1). In the first group comprising Lpc53E1 [80], PE10 [86], Lip3 [90], MGS-RG1 [82], LipC12 [40], Est9x [27], and Est700 [91] $\mathrm{NaCl}$ stimulated esterase activity was recorded over the tested concentration range (0-4 M in most cases). The second group harbors enzymes for which esterase activity continuously decreases with increasing $\mathrm{NaCl}$ concentration. Members of this group are 7N9 [42], ABO_1251 [79], Esth [92], ABO_1197 [79], EstS [11], and EstLiu [24]. For the third group, $\mathrm{NaCl}$ serves as an activator at low or moderate concentrations and as inhibitor at higher concentrations. Est56 belonged to this group, as its activity increased in the presence of $\mathrm{NaCl}$ up to a concentration of $1.5 \mathrm{M}$ and decreased at higher concentrations (Figure 2). Moreover, almost $50 \%$ of the described halotolerant esterases such as E69 [76], ThaEst2349 [85], E25 [34], lp_3505 [72], and Est10 [88] follow this pattern.

Evaluation of enzyme stability towards salts revealed that Est56 retained its full activity after $24 \mathrm{~h}$ of incubation with $\mathrm{NaCl}$ or $\mathrm{KCl}$ concentrations ranging from 0 to $4 \mathrm{M}$ at $10{ }^{\circ} \mathrm{C}$ (Figure 2b). Similarly, almost unaltered residual activity was also detected for esterases such as Lpc53E1 [80], EaEST [93], Esth [92], EstSL3 [78], LipC12 [40], Est12 [77], Est-OKK [94], and Est700 [91]. Nevertheless, esterases such as EstSP [30], EstS [11], EstLiu [24], and H8 [81] were inhibited by high salt concentrations. Noteworthy are the stabilities of EM3L4 [25], ThaEst2349 [85], Lip3 [90], and Est10 [88], which were enhanced by addition of $\mathrm{NaCl}$. Although potassium ions are preferable for some halophilic enzymes [15,95], $\mathrm{NaCl}$ and $\mathrm{KCl}$ exhibited similar effects on Est56 activity and stability. This was tested only for a few other enzymes and similar results were obtained [29].

To shed a light on the mechanism of how halotolerant lipolytic enzymes resist salts, we compared the amino acid compositions among the groups HT_Lip, HP_Lip, and HP_Enz (Figure 4). The amino acid composition between halophilic and halotolerant proteins are significantly different, although enzymes in all groups were salt resistant. Our results were generally consistent with previous comparisons between halophilic and nonhalophilic homologs [9,96-99]. The most crucial feature of halophilic enzymes (HP_Lip and HP_Enz) compared to halotolerant lipolytic enzymes (HT_Lip) is the higher content of acidic residues (Asp and Glu) accompanied by a lower content of lysine residues (Figure 4a). This feature was also reflected by the lower $\mathrm{pI}$ values of halophilic enzymes (Figure $4 \mathrm{~b}$ ). Another consistent feature for halophilic enzymes is the low hydrophobicity $[4,15]$, which was presented by a low content of aliphalic hydrophobic residues (Leu, Met and Ile) in this study (Figure 4a). Significant difference in aromatic (Phe, Try, and Trp), small (Gly and Vla), and borderline (Ser and Thr) hydrophobic residues was not detected between HT_Lip and HP_Lip (Figure 4a). On the contrary, different results were reported for certain residues therein by comparing halophilic and non-halophilic homologs [97,99-102]. Moreover, a significantly lower content of polar amino acids (Gln and Asn) was recorded for HP_Lip compared to HT_Lip enzymes (Figure 4a), which has not been observed in other comparative analyses. In general, high acidity and low hydrophobicity reflected by the amino acid composition of halophilic enzymes is a distinctive feature for their "halophilic adaptation," which simultaneously enables them to resist salinity. Nonetheless, we could not find clear patterns in amino acid composition among halotolerant lipolytic enzymes, except the broad range of theoretical $\mathrm{pI}$ values (Figure $4 \mathrm{~b}$ ).

Recently, Dassarma and Dassarma [2] proposed a correlation between the halophilic character and acidic nature of proteins by reviewing the proteomes of different halophilic and halotolerant bacteria. Further studies on halophilic protein structure also confirmed that excessive surface-exposed acidic residues are the basis for halophilic adaptation [5,103-106]. Thus, given the low pI values of halophilic enzymes, we assume that halotolerant lipolytic enzymes with relatively low $\mathrm{pI}$ values would follow a similar haloadaptation as halophilic enzymes. Est56 exhibits a low pI value (4.97), with predominantly acidic residues located at its surface (Figure $5 c, d$ ). This is an indication that Est56 applies a salt resistance strategy similar to that of halophilic enzymes. Moreover, the distinctive feature of enhanced Est56 stability against denaturants (high temperatures and 
urea) mediated by $\mathrm{NaCl}$ suggests that Est 56 possess a halophilic character caused by the high acidic amino acid content. Another family IV esterase, ThaEst2349 (theoretical pI value 4.94), was also reported as halotolerant due to the high ratio of surface-exposed acidic residues [85]. Acidic amino acids were reported to have a greater capacity than other amino acids in keeping proteins hydralated, which is important for the solubility of protein under salt stress $[95,107,108]$. Interestingly, several reports on the haloadaptation of enzymes with higher $\mathrm{pI}$ values than Est56 indicated that instead of acidic residues, basic residues at protein surfaces played a key role in their halotolerance $[91,109,110]$. By site-directed mutagenesis, Zhang et al. [81] identified two basic residues $\mathrm{Arg}^{195}$ and $\mathrm{Arg}^{236}$ located on the surface of $\mathrm{H} 8$ (theoretical pI value 9.09), which were essential in the salt tolerance. Additional mechanisms were reported to contribute to the haloadaptation of halotolerant enzymes. By introducing hydrophobic residues in the cap and catalytic domain, the halotolerance of E40 (a family IV esterase) was significantly improved [31]. However, this adaptation hardly applies to all of the family IV esterases, since the cap domain is the most variable region [111]. The cap domain was even not observed for the family IV enzyme MGS-M1, which was also reported to resist high salinity [37]. Thus, the underlying mechanism for the haloadaptation of different lipolytic enzymes remains unclear.

To some extent, the hydration characteristics of halotolerant/halophilic enzymes may extend their function to nonaqueous environments $[15,43]$. In this study, Est56 was stable towards the tested water-miscible organic solvents (Table 2), and moderately tolerant to some water-immiscible organic solvents (Table 3). Some halotolerant lipolytic enzymes, such as LipC12 [40], estHIJ [112], EstSP [30], Est12 [77], and H8 [81] were also reported to resist the presence of organic solvents but to different degrees. The stability towards organic solvents could broaden Est56 application in organic solvent-mediated catalytic processes, such as flavor production in food industry, synthesis of antibiotics and antiinflammatory compounds in pharmaceutical industry, and production of pesticides for agricultural applications [113-116].

Additionally, other properties, such as the effect of metal ions, inhibitors, and detergents on Est56 activity were also studied. Est56 activity was enhanced by $\mathrm{Ca}^{2+}$ and $\mathrm{Al}^{3+}$ at 1 and $10 \mathrm{mM}$. Several esterases and lipases are also reported to be activated by $\mathrm{Ca}^{2+}$ ions $[117,118]$. However, it is rare for esterases to show an increased activity in the presence of $\mathrm{Al}^{3+}$ ions. Est56 activity was enhanced at a low concentration $(0.1 \%, v / v)$ of nonionic detergents such as Triton X-100, Tween 20, and Tween 80 , while suppressed at high concentrations $(1 \%$ and $5 \%, v / v)$. This similar concentration-dependent effect of detergents on esterases was also found in other studies $[40,88,119]$.

\section{Conclusions}

A functional screening of a compost metagenome yielded an esterase, showing activity and stability over a salinity range of $0-4 \mathrm{M}$. The recently reported halotolerant lipolytic enzymes (40 in total) were also summarized and used for phylogenetic analysis in this study. To explore the haloadaptation of halotolerant lipolytic enzymes, their amino acid compositions were statistically compared with halophilic counterparts. However, no clear pattern was found in the amino acid composition in the halotolerant lipolytic enzymes. For Est56, the excessive content of acidic residues over lysine residues, as well as the predominantly negatively charged surface indicated that it applies a haloadaptation similar to that of halophilic enzymes. In addition, Est56 exhibits a tolerance toward various organic solvents and enhanced activity in the presence of $\mathrm{Ca}^{2+}$ and $\mathrm{Al}^{3+}$ ions and a low concentration $(0.1 \%, v / v)$ of nonionic detergents. Thus, Est56 is a novel biocatalyst with application potential, particularly under high salinity and in nonaqueous environments.

Supplementary Materials: The following are available online at https:/ / www.mdpi.com/2073-4 425/12/1/122/s1, Figure S1: Genetic organization of the insert harboring est56 and BLAST search results for the predicted open reading frames (ORFs); Figure S2: Multiple sequence alignment of Est56 and its homologs; Figure S3: SDS-PAGE analysis of the purification of recombinant Est56 (including $\mathrm{His}_{6}$-tag); Figure S4: Substrate specificity of Est56 towards $p$-NP esters of different chain 
length; Figure S5: Unrooted phylogenetic tree of family IV esterases using neighbor-joining method; Table S1: Features of characterized halotolerant lipolytic enzymes; Table S2: Lipolytic enzymes derived from halophilic archaea adapting the "salt in" strategy; Table S3: Characterized halophilic enzymes from other studies; Table S4: Purification of recombinant Est56; Table S5: Effect of metal ions and ethylenediaminetetraacetic acid (EDTA) on Est56 activity; Table S6: Effect of detergents on Est56 activity; Table S7: Effect of inhibitors on Est56 activity; Table S8: Amino acid composition comparison between halotolerant and halophilic enzymes.

Author Contributions: R.D. developed the concept and supervised the work. M.L. performed microbiological and molecular experiments and analysis of the sequences. All authors interpreted the results, contributed to the writing of the final version of the manuscript, and approved submission. All authors have read and agreed to the published version of the manuscript.

Funding: We acknowledge the support of Mingji Lu by "Erasmus Mundus Action 2-Lotus I Project." We acknowledge support by the Open Access Publication Funds of the University of Göttingen.

Institutional Review Board Statement: Not applicable.

Informed Consent Statement: Not applicable.

Data Availability Statement: All datasets are publicly available stored in or derived from NCBI databases (https://www.ncbi.nlm.nih.gov). The amino acid sequence of Est56 is available in the GenBank database under accession number KR149569.1. The compost metagenome sequences are available in the NCBI sequence read archive (SRA) under the accession number SRR13115019.

Acknowledgments: We thank Silja Brady and Mechthild Bömeke for providing technical assistance. We also thank Sarah Zachmann for assistance.

Conflicts of Interest: The authors declare that the research was conducted in the absence of any commercial or financial relationships that could be construed as a potential conflict of interest.

\section{References}

1. Yadav, A.N.; Saxena, A.K. Biodiversity and biotechnological applications of halophilic microbes for sustainable agriculture. J. Appl. Biol. Biotechnol. 2018, 6, 48-55. [CrossRef]

2. Dassarma, S.; Dassarma, P. Halophiles and their enzymes: Negativity put to good use. Curr. Opin. Microbiol. 2015, 25, 120-126. [CrossRef] [PubMed]

3. Salma, M.; Abdulla, M.K.; Samina, M. Osmoadaptation in halophilic bacteria and archaea. Res. J. Biotechnol. 2020, 15, 154-161.

4. Siglioccolo, A.; Paiardini, A.; Piscitelli, M.; Pascarella, S. Structural adaptation of extreme halophilic proteins through decrease of conserved hydrophobic contact surface. BMC Struct. Biol. 2011, 11, 50. [CrossRef] [PubMed]

5. Ishibashi, M.; Hayashi, T.; Yoshida, C.; Tokunaga, M. Increase of salt dependence of halophilic nucleoside diphosphate kinase caused by a single amino acid substitution. Extremophiles 2013, 17, 585-591. [CrossRef] [PubMed]

6. Moshfegh, M.; Shahverdi, A.R.; Zarrini, G.; Faramarzi, M.A. Biochemical characterization of an extracellular polyextremophilic $\alpha$-amylase from the halophilic archaeon Halorubrum xinjiangense. Extremophiles 2013, 17, 677-687. [CrossRef] [PubMed]

7. Cycil, L.M.; DasSarma, S.; Pecher, W.; McDonald, R.; AbdulSalam, M.; Hasan, F. Metagenomic insights into the diversity of halophilic microorganisms indigenous to the Karak Salt Mine, Pakistan. Front. Microbiol. 2020, 11, 1567. [CrossRef]

8. Oren, A. Bioenergetic aspects of halophilism. Microbiol. Mol. Biol. Rev. 1999, 63, 334-348. [CrossRef]

9. Ventosa, A.; Nieto, J.J.; Oren, A. Biology of moderately halophilic aerobic bacteria. Microbiol. Mol. Biol. Rev. 1998, 62, 504-544. [CrossRef]

10. Corral, P.; Amoozegar, M.A.; Ventosa, A. Halophiles and their biomolecules: Recent advances and future applications in biomedicine. Mar. Drugs 2020, 18, 33. [CrossRef]

11. Jiang, H.; Zhang, S.; Gao, H.; Hu, N. Characterization of a cold-active esterase from Serratia sp. and improvement of thermostability by directed evolution. BMC Biotechnol. 2016, 16. [CrossRef] [PubMed]

12. Bai, W.; Xue, Y.; Zhou, C.; Ma, Y. Cloning, expression, and characterization of a novel alkali-tolerant xylanase from alkaliphilic Bacillus sp. SN5. Biotechnol. Lett. 2012, 34, 2093-2099. [CrossRef] [PubMed]

13. Kumar, S.; Paul, D.; Bhushan, B.; Wakchaure, G.C.; Meena, K.K.; Shouche, Y. Traversing the "Omic" landscape of microbial halotolerance for key molecular processes and new insights. Crit. Rev. Microbiol. 2020, 46, 631-653. [CrossRef] [PubMed]

14. Delgado-García, M.; Valdivia-Urdiales, B.; Aguilar-González, C.N.; Contreras-Esquivel, J.C.; Rodríguez-Herrera, R. Halophilic hydrolases as a new tool for the biotechnological industries. J. Sci. Food Agric. 2012, 92, 2575-2580. [CrossRef] [PubMed]

15. Munawar, N.; Engel, P.C. Halophilic enzymes: Characteristics, structural adaptation and potential applications for biocatalysis. Curr. Biotechnol. 2013, 2, 334-344. [CrossRef]

16. Ortega, G.; Laín, A.; Tadeo, X.; López-Méndez, B.; Castaño, D.; Millet, O. Halophilic enzyme activation induced by salts. Sci. Rep 2011, 1, 6. [CrossRef] 
17. Edbeib, M.F.; Aksoy, H.M.; Kaya, Y.; Wahab, R.A.; Huyop, F. Haloadaptation: Insights from comparative modeling studies between halotolerant and non-halotolerant dehalogenases. J. Biomol. Struct. Dyn. 2020, 38, 3452-3461. [CrossRef]

18. Sinha, R.; Khare, S.K. Protective role of salt in catalysis and maintaining structure of halophilic proteins against denaturation. Front. Microbiol. 2014, 5, 165. [CrossRef]

19. Vogler, M.; Karan, R.; Renn, D.; Vancea, A.; Vielberg, M.-T.; Grötzinger, S.W.; DasSarma, P.; DasSarma, S.; Eppinger, J.; Groll, M.; et al. Crystal structure and active site engineering of a halophilic $\gamma$-carbonic anhydrase. Front. Microbiol. $2020,11,742$. [CrossRef]

20. Bardavid, R.E.; Oren, A. The amino acid composition of proteins from anaerobic halophilic bacteria of the order Halanaerobiales. Extremophiles 2012, 16, 567-572. [CrossRef]

21. Arpigny, J.L.; Jaeger, K.-E. Bacterial lipolytic enzymes: Classification and properties. Biochem. J. 1999, 343, 177-183. [CrossRef] [PubMed]

22. Bornscheuer, U.T. Microbial carboxyl esterases: Classification, properties and application in biocatalysis. FEMS Microbiol. Rev. 2002, 26, 73-81. [CrossRef] [PubMed]

23. Kovacic, F.; Babic, N.; Krauss, U.; Jaeger, K. Classification of lipolytic enzymes from bacteria. In Aerobic Utilization of Hydrocarbons, Oils and Lipids. Handbook of Hydrocarbon and Lipid Microbiology; Rojo, F., Ed.; Springer: Cham, Switzerland, 2019; pp. 1-35, ISBN 9783319397825.

24. Rahman, M.A.; Culsum, U.; Tang, W.; Zhang, S.W.; Wu, G.; Liu, Z. Characterization of a novel cold active and salt tolerant esterase from Zunongwangia profunda. Enzyme Microb. Technol. 2016, 85, 1-11. [CrossRef] [PubMed]

25. Lee, J.H.; Jeon, J.H.; Kim, J.T.; Lee, H.S.; Kim, S.J.; Kang, S.G.; Choi, S.H. Novel lipolytic enzymes identified from metagenomic library of deep-sea sediment. Evid. Based Complement. Altern. Med. 2011, 2011. [CrossRef]

26. Hu, Y.; Fu, C.; Huang, Y.; Yin, Y.; Cheng, G.; Lei, F.; Lu, N.; Li, J.; Ashforth, E.J.; Zhang, L.; et al. Novel lipolytic genes from the microbial metagenomic library of the South China Sea marine sediment. FEMS Microbiol. Ecol. 2010, 72, 228-237. [CrossRef] [PubMed]

27. Fang, Z.; Li, J.; Wang, Q.; Fang, W.; Peng, H.; Zhang, X.; Xiao, Y. A novel esterase from a marine metagenomic library exhibiting salt tolerance ability. J. Microbiol. Biotechnol. 2014, 24, 771-780. [CrossRef] [PubMed]

28. Asyari, M.; Aditiawati, P.; Akhmaloka; Hertadi, R. Cloning and sequence analysis of lipase gene of halophilic bacteria isolated from mud crater of Bledug Kuwu, Central Java, Indonesia. Biosci. Biotechnol. Res. Asia 2015, 12, 1903-1912. [CrossRef]

29. Jeon, J.H.; Lee, H.S.; Kim, J.T.; Kim, S.J.; Choi, S.H.; Kang, S.G.; Lee, J.H. Identification of a new subfamily of salt-tolerant esterases from a metagenomic library of tidal flat sediment. Appl. Microbiol. Biotechnol. 2012, 93, 623-631. [CrossRef]

30. Jayanath, G.; Mohandas, S.P.; Kachiprath, B.; Solomon, S.; Sajeevan, T.P.; Singh, I.S.B.; Philip, R. A novel solvent tolerant esterase of GDSGG motif subfamily from solar saltern through metagenomic approach: Recombinant expression and characterization. Int. J. Biol. Macromol. 2018, 119, 393-401. [CrossRef]

31. Li, P.-Y.; Zhang, Y.; Xie, B.-B.; Zhang, Y.-Q.; Hao, J.; Wang, Y.; Wang, P.; Li, C.-Y.; Qin, Q.-L.; Zhang, X.-Y.; et al. Structural and mechanistic insights into the improvement of the halotolerance of a marine microbial esterase by increasing intra- and interdomain hydrophobic interactions. Appl. Environ. Microbiol. 2017, 83, e01286-17. [CrossRef]

32. Mandrich, L.; de Pascale, D. An overview on thermal adaptation of esterases and lipases belonging to the HSL family: New insight on the computational analysis. Curr. Chem. Biol. 2011, 5, 17-28. [CrossRef]

33. Mandrich, L.; Menchise, V.; Alterio, V.; De Simone, G.; Pedone, C.; Rossi, M.; Manco, G. Functional and structural features of the oxyanion hole in a thermophilic esterase from Alicyclobacillus acidocaldarius. Proteins Struct. Funct. Genet. 2008, 71, 1721-1731. [CrossRef]

34. Li, P.Y.; Ji, P.; Li, C.Y.; Zhang, Y.; Wang, G.L.; Zhang, X.Y.; Xie, B.B.; Qin, Q.L.; Chen, X.L.; Zhou, B.C.; et al. Structural basis for dimerization and catalysis of a novel sterase from the GTSAG motif subfamily of the bacterial hormone-sensitive lipase family. J. Biol. Chem. 2014, 289, 19031-19041. [CrossRef] [PubMed]

35. Chahinian, H.; Ali, Y.B.; Abousalham, A.; Petry, S.; Mandrich, L.; Manco, G.; Canaan, S.; Sarda, L. Substrate specificity and kinetic properties of enzymes belonging to the hormone-sensitive lipase family: Comparison with non-lipolytic and lipolytic carboxylesterases. Biochim. Biophys. Acta 2005, 1738, 29-36. [CrossRef]

36. Rao, L.; Zhao, X.; Li, Y.; Xue, Y.; Ma, Y.; Lu, J.R. Solution behavior and activity of a halophilic esterase under high salt concentration PLoS ONE 2009, 4, e6980. [CrossRef] [PubMed]

37. Alcaide, M.; Stogios, P.J.; Lafraya, Á.; Tchigvintsev, A.; Flick, R.; Bargiela, R.; Chernikova, T.N.; Reva, O.N.; Hai, T.; Leggewie, C.C.; et al. Pressure adaptation is linked to thermal adaptation in salt-saturated marine habitats. Environ. Microbiol. 2015, 17, 332-345. [CrossRef] [PubMed]

38. Ai, L.; Huang, Y.; Wang, C. Purification and characterization of halophilic lipase of Chromohalobacter sp. from ancient salt well. J. Basic Microbiol. 2018, 58, 647-657. [CrossRef] [PubMed]

39. Castilla, A.; Panizza, P.; Rodríguez, D.; Bonino, L.; Díaz, P.; Irazoqui, G.; Giordano, S.R. A novel thermophilic and halophilic esterase from Janibacter sp. R02, the first member of a new lipase family (Family XVII). Enzyme Microb. Technol. 2017, 98, 86-95. [CrossRef] [PubMed]

40. Glogauer, A.; Martini, V.P.; Faoro, H.; Couto, G.H.; Müller-Santos, M.; Monteiro, R.A.; Mitchell, D.A.; de Souza, E.M.; Pedrosa, F.O.; Krieger, N. Identification and characterization of a new true lipase isolated through metagenomic approach. Microb. Cell Fact. 2011, 10, 54. [CrossRef] 
41. Mohamed, Y.M.; Ghazy, M.A.; Sayed, A.; Ouf, A.; El-Dorry, H.; Siam, R. Isolation and characterization of a heavy metal-resistant, thermophilic esterase from a Red Sea Brine Pool. Sci. Rep. 2013, 3, 3358. [CrossRef]

42. Borchert, E.; Selvin, J.; Kiran, S.G.; Jackson, S.A.; O'Gara, F.; Dobson, A.D.W. A novel cold active esterase from a deep sea sponge Stelletta normani metagenomic library. Front. Mar. Sci. 2017, 4, 1-13. [CrossRef]

43. Marhuenda-Egea, F.C.; Bonete, M.J. Extreme halophilic enzymes in organic solvents. Curr. Opin. Biotechnol. $2002,13,385-389$. [CrossRef]

44. Pérez, D.; Martín, S.; Fernández-Lorente, G.; Filice, M.; Guisán, J.M.; Ventosa, A.; García, M.T.; Mellado, E. A novel halophilic lipase, LipBL, showing high efficiency in the production of eicosapentaenoic acid (EPA). PLoS ONE 2011, 6, e23325. [CrossRef]

45. Oren, A. Industrial and environmental applications of halophilic microorganisms. Environ. Technol. 2010, 31, 825-834. [CrossRef] [PubMed]

46. Ryckeboer, J.; Mergaert, J.; Vaes, K.; Klammer, S.; Clercq, D.; Coosemans, J.; Insam, H.; Swings, J. A survey of bacteria and fungi occurring during composting and self-heating processes. Ann. Microbiol. 2003, 53, 349-410.

47. Kang, C.H.; Oh, K.H.; Lee, M.H.; Oh, T.K.; Kim, B.H.; Yoon, J.H. A novel family VII esterase with industrial potential from compost metagenomic library. Microb. Cell Fact. 2011, 10, 41. [CrossRef]

48. Ilmberger, N.; Meske, D.; Juergensen, J.; Schulte, M.; Barthen, P.; Rabausch, U.; Angelov, A.; Mientus, M.; Liebl, W.; Schmitz, R.A.; et al. Metagenomic cellulases highly tolerant towards the presence of ionic liquids—Linking thermostability and halotolerance. Appl. Microbiol. Biotechnol. 2012, 95, 135-146. [CrossRef] [PubMed]

49. Dougherty, M.J.; D’haeseleer, P.; Hazen, T.C.; Simmons, B.A.; Adams, P.D.; Hadi, M.Z. Glycoside hydrolases from a targeted compost metagenome, activity-screening and functional characterization. BMC Biotechnol. 2012, 12, 38. [CrossRef]

50. Lu, M.; Dukunde, A.; Daniel, R. Biochemical profiles of two thermostable and organic solvent-tolerant esterases derived from a compost metagenome. Appl. Microbiol. Biotechnol. 2019, 103, 3421-3437. [CrossRef]

51. Ishikawa, J.; Hotta, K. FramePlot: A new implementation of the frame analysis for predicting protein-coding regions in bacterial DNA with a high G + C content. FEMS Microbiol. Lett. 1999, 174, 251-263. [CrossRef]

52. Bendtsen, J.D.; Nielsen, H.; Von Heijne, G.; Brunak, S. Improved prediction of signal peptides: SignalP 3.0. J. Mol. Biol. 2004, 340, 783-795. [CrossRef] [PubMed]

53. Ye, J.; McGinnis, S.; Madden, T.L. BLAST: Improvements for better sequence analysis. Nucleic Acids Res. 2006, 34, W6-W9. [CrossRef] [PubMed]

54. Zhang, Y. I-TASSER server for protein 3D structure prediction. BMC Bioinform. 2008, 9, 40. [CrossRef] [PubMed]

55. Robert, X.; Gouet, P. Deciphering key features in protein structures with the new ENDscript server. Nucleic Acids Res. 2014, 42, 320-324. [CrossRef] [PubMed]

56. Bradford, M.M. A rapid and sensitive method for the quantitation of microgram quantities of protein utilizing the principle of protein-dye binding. Anal. Biochem. 1976, 72, 248-254. [CrossRef]

57. Lineweaver, H.; Burk, D. The determination of enzyme dissociation constants. J. Am. Chem. Soc. 1934, 56, 658-666. [CrossRef]

58. Fitter, J.; Haber-Pohlmeier, S. Structural stability and unfolding properties of thermostable bacterial $\alpha$-amylases: A comparative study of homologous enzymes. Biochemistry 2004, 43, 9589-9599. [CrossRef]

59. Tamura, K.; Stecher, G.; Peterson, D.; Filipski, A.; Kumar, S. MEGA6: Molecular evolutionary genetics analysis version 6.0. Mol. Biol. Evol. 2013, 30, 2725-2729. [CrossRef]

60. Felsenstein, J. Confidence limits on phylogenies: An approach using the bootstrap. Evolution 1985, 39, 783-791. [CrossRef]

61. Asnicar, F.; Weingart, G.; Tickle, T.L.; Huttenhower, C.; Segata, N. Compact graphical representation of phylogenetic data and metadata with GraPhlAn. Peer] 2015, 3, e1029. [CrossRef]

62. Loukas, A.; Kappas, I.; Abatzopoulos, T.J. HaloDom: A new database of halophiles across all life domains. J. Biol. Res. 2018, 25, 2. [CrossRef] [PubMed]

63. Gasteiger, E.; Hoogland, C.; Gattiker, A.; Duvaud, S.; Wilkins, M.R.; Appel, R.D.; Bairoch, A. Protein identification and analysis tools on the ExPASy server. In The Proteomics Protocols Handbook; Humana Press: Totowa, NJ, USA, 2005; pp. 571-607.

64. Oksanen, J.; Blanchet, F.G.; Friendly, M.; Kindt, R.; Legendre, P.; Mcglinn, D.; Minchin, P.R.; O’hara, R.B.; Simpson, G.L.; Solymos, P.; et al. Vegan: Community Ecology Package. Available online: https://cran.r-project.org/web/packages/vegan/ vegan.pdf (accessed on 30 October 2018).

65. Baker, N.A.; Sept, D.; Joseph, S.; Holst, M.J.; McCammon, J.A. Electrostatics of nanosystems: Application to microtubules and the ribosome. Proc. Natl. Acad. Sci. USA 2001, 98, 10037-10041. [CrossRef]

66. Lee, S.W.; Won, K.; Lim, H.K.; Kim, J.C.; Choi, G.J.; Cho, K.Y. Screening for novel lipolytic enzymes from uncultured soil microorganisms. Appl. Microbiol. Biotechnol. 2004, 65, 720-726. [CrossRef]

67. Jin, P.; Pei, X.; Du, P.; Yin, X.; Xiong, X.; Wu, H.; Zhou, X.; Wang, Q. Overexpression and characterization of a new organic solvent-tolerant esterase derived from soil metagenomic DNA. Bioresour. Technol. 2012, 116, 234-240. [CrossRef] [PubMed]

68. Dukunde, A.; Schneider, D.; Lu, M.; Brady, S.; Daniel, R. A novel, versatile family IV carboxylesterase exhibits high stability and activity in a broad pH spectrum. Biotechnol. Lett. 2017, 39, 577-587. [CrossRef] [PubMed]

69. Li, J.; Zhang, K.; Han, W. Cloning and biochemical characterization of a novel lipolytic gene from activated sludge metagenome, and its gene product. Microb. Cell Fact. 2010, 9, 83. [CrossRef]

70. Lindgren, M.; Westlund, P.-O. On the stability of chymotrypsin inhibitor 2 in a $10 \mathrm{M}$ urea solution. The role of interaction energies for urea-induced protein denaturation. Phys. Chem. Chem. Phys. 2010, 12, 9358. [CrossRef] 
71. Peng, Q.; Zhang, X.; Shang, M.; Wang, X.; Wang, G.; Li, B.; Guan, G.; Li, Y.; Wang, Y. A novel esterase gene cloned from a metagenomic library from neritic sediments of the South China Sea. Microb. Cell Fact. 2011, 10, 95. [CrossRef]

72. Esteban-Torres, M.; Santamaría, L.; de las Rivas, B.; Muñoz, R. Characterisation of a cold-active and salt-tolerant esterase from Lactobacillus plantarum with potential application during cheese ripening. Int. Dairy J. 2014, 39, 312-315. [CrossRef]

73. Li, P.; Chen, X.; Ji, P.; Li, C.; Wang, P.; Zhang, Y.; Xie, B.; Qin, Q.; Su, H.; Zhou, B.; et al. Interdomain hydrophobic interactions modulate the thermostability of microbial esterases from the hormone-sensitive lipase family. J. Biol. Chem. 2015, 290, 11188-11198. [CrossRef]

74. Khan, M.; Sathya, T.A. Extremozymes from metagenome: Potential applications in food processing. Crit. Rev. Food Sci. Nutr. 2018, 58, 2017-2025. [CrossRef] [PubMed]

75. Simon, C.; Daniel, R. Metagenomic analyses: Past and future trends. Appl. Environ. Microbiol. 2011, 77, 1153-1161. [CrossRef] [PubMed]

76. Huo, Y.Y.; Rong, Z.; Jian, S.L.; Xu, C.D.; Li, J.; Xu, X.W. A novel halotolerant thermoalkaliphilic esterase from marine bacterium Erythrobacter seohaensis SW-135. Front. Microbiol. 2017, 8, 2315. [CrossRef]

77. Wu, G.; Zhang, S.; Zhang, H.; Zhang, S.; Liu, Z. A novel esterase from a psychrotrophic bacterium Psychrobacter celer 3Pb1 showed cold-adaptation and salt-tolerance. J. Mol. Catal. B Enzym. 2013, 98, 119-126. [CrossRef]

78. Wang, G.; Wang, Q.; Lin, X.; Ng, T.B.; Yan, R.; Lin, J.; Ye, X. A novel cold-adapted and highly salt-tolerant esterase from Alkalibacterium sp. SL3 from the sediment of a soda lake. Sci. Rep. 2016, 6, 19494. [CrossRef] [PubMed]

79. Tchigvintsev, A.; Tran, H.; Popovic, A.; Kovacic, F.; Brown, G.; Flick, R.; Hajighasemi, M.; Egorova, O.; Somody, J.C.; Tchigvintsev, D.; et al. The environment shapes microbial enzymes: Five cold-active and salt-resistant carboxylesterases from marine metagenomes. Appl. Microbiol. Biotechnol. 2015, 99, 2165-2178. [CrossRef] [PubMed]

80. Selvin, J.; Kennedy, J.; Lejon, D.P.H.; Kiran, G.S.; Dobson, A.D.W. Isolation identification and biochemical characterization of a novel halo-tolerant lipase from the metagenome of the marine sponge Haliclona simulans. Microb. Cell Fact. 2012, 11, 72. [CrossRef]

81. Zhang, Y.; Hao, J.; Zhang, Y.Q.; Chen, X.L.; Xie, B.B.; Shi, M.; Zhou, B.C.; Zhang, Y.Z.; Li, P.Y. Identification and characterization of a novel salt-tolerant esterase from the deep-sea sediment of the South China Sea. Front. Microbiol. 2017, 8, 441. [CrossRef]

82. Alcaide, M.; Tchigvintsev, A.; Martínez-Martínez, M.; Popovic, A.; Reva, O.N.; Lafraya, Á.; Bargiela, R.; Nechitaylo, T.Y.; Matesanz, R.; Cambon-Bonavita, M.-A.; et al. Identification and characterization of carboxyl esterases of gill chamber-associated microbiota in the deep-sea shrimp Rimicaris exoculata by using functional metagenomics. Appl. Environ. Microbiol. 2015, 81, 2125-2136. [CrossRef]

83. Oliveira, L.C.G.; Ramos, P.L.; Marem, A.; Kondo, M.Y.; Rocha, R.C.S.; Bertolini, T.; Silveira, M.A.; da Cruz, J.B.; de Vasconcellos, S.P.; Juliano, L.; et al. Halotolerant bacteria in the São Paulo Zoo composting process and their hydrolases and bioproducts. Braz. J. Microbiol. 2015, 46, 347-354. [CrossRef]

84. Chandna, P.; Mayilraj, S.; Kuhad, R.C. Bacillus pseudoflexus sp. nov., a moderately halophilic bacterium isolated from compost. Ann. Microbiol. 2016, 66, 895-905. [CrossRef]

85. De Santi, C.; Leiros, H.K.S.; Di Scala, A.; de Pascale, D.; Altermark, B.; Willassen, N.P. Biochemical characterization and structural analysis of a new cold-active and salt-tolerant esterase from the marine bacterium Thalassospira sp. Extremophiles 2016, 20, 323-336. [CrossRef] [PubMed]

86. Jiang, X.; Huo, Y.; Cheng, H.; Zhang, X.; Zhu, X.; Wu, M. Cloning, expression and characterization of a halotolerant esterase from a marine bacterium Pelagibacterium halotolerans B2T. Extremophiles 2012, 16, 427-435. [CrossRef] [PubMed]

87. Leite, A.E.T.; Briganti, L.; de Araújo, E.A.; Pellegrini, V.D.; Camilo, C.M.; Polikarpov, I. Low-resolution molecular shape, biochemical characterization and emulsification properties of a halotolerant esterase from Bacillus licheniformis. Eur. Biophys. J. 2020, 49, 435-447. [CrossRef]

88. Wu, G.; Wu, G.; Zhan, T.; Shao, Z.; Liu, Z. Characterization of a cold-adapted and salt-tolerant esterase from a psychrotrophic bacterium Psychrobacter pacificensis. Extremophiles 2013, 17, 809-819. [CrossRef]

89. Adıgüzel, A.O. Production and characterization of thermo-, halo- and solvent-stable esterase from Bacillus mojavensis TH309. Biocatal. Biotransform. 2020, 38, 210-226. [CrossRef]

90. De Santi, C.; Altermark, B.; Pierechod, M.M.; Ambrosino, L.; de Pascale, D.; Willassen, N.-P. Characterization of a cold-active and salt tolerant esterase identified by functional screening of Arctic metagenomic libraries. BMC Biochem. 2016, 17. [CrossRef] [PubMed]

91. Zhang, W.; Xu, H.; Wu, Y.; Zeng, J.; Guo, Z.; Wang, L.; Shen, C.; Qiao, D.; Cao, Y. A new cold-adapted, alkali-stable and highly salt-tolerant esterase from Bacillus licheniformis. Int. J. Biol. Macromol. 2018, 111, 1183-1193. [CrossRef]

92. Hang, Y.; Ran, S.; Wang, X.; Jiao, J.; Wang, S.; Liu, Z. Mutational analysis and stability characterization of a novel esterase of lipolytic enzyme family VI from Shewanella sp. Int. J. Biol. Macromol. 2016, 93, 655-664. [CrossRef]

93. Lee, C.W.; Kwon, S.; Park, S.H.; Kim, B.Y.; Yoo, W.; Ryu, B.H.; Kim, H.W.; Shin, S.C.; Kim, S.; Park, H.; et al. Crystal structure and functional characterization of an esterase (EaEST) from Exiguobacterium antarcticum. PLoS ONE 2017, 12, e0169540. [CrossRef]

94. Yang, X.; Wu, L.; Xu, Y.; Ke, C.; Hu, F.; Xiao, X.; Huang, J. Identification and characterization of a novel alkalistable and salt-tolerant esterase from the deep-sea hydrothermal vent of the East Pacific Rise. Microbiology 2018, 7, e00601. [CrossRef]

95. Dennis, P.P.; Shimmin, L.C. Evolutionary divergence and salinity-mediated selection in halophilic archaea. Microbiol. Mol. Biol. Rev. 1997, 61, 90-104. [CrossRef] [PubMed] 
96. Margesin, R.; Schinner, F. Potential of halotolerant and halophilic microorganisms for biotechnology. Extremophiles 2001, 5, 73-83. [CrossRef] [PubMed]

97. Lanyi, J.K. Salt-dependent properties of proteins from extremely halophilic bacteria. Bacteriol. Rev. 1974, 38, 272-290. [CrossRef]

98. Madern, D.; Ebel, C.; Zaccai, G. Halophilic adaptation of enzymes. Extremophiles 2000, 4, 91-98. [CrossRef]

99. Hutcheon, G.W.; Vasisht, N.; Bolhuis, A. Characterisation of a highly stable $\alpha$-amylase from the halophilic archaeon Haloarcula hispanica. Extremophiles 2005, 9, 487-495. [CrossRef] [PubMed]

100. Kastritis, P.L.; Papandreou, N.C.; Hamodrakas, S.J. Haloadaptation: Insights from comparative modeling studies of halophilic archaeal DHFRs. Int. J. Biol. Macromol. 2007, 41, 447-453. [CrossRef]

101. Madern, D.; Pfister, C.; Zaccai, G. Mutation at a single acidic amino acid enhances the halophilic behaviour of malate dehydrogenase from Haloarcula marismortui in physiological salts. Eur. J. Biochem. 1995, 230, 1088-1095. [CrossRef]

102. Paul, S.; Bag, S.K.; Das, S.; Harvill, E.T.; Dutta, C. Molecular signature of hypersaline adaptation: Insights from genome and proteome composition of halophilic prokaryotes. Genome Biol. 2008, 9, R70. [CrossRef]

103. Winter, J.A.; Christofi, P.; Morroll, S.; Bunting, K.A. The crystal structure of Haloferax volcanii proliferating cell nuclear antigen reveals unique surface charge characteristics due to halophilic adaptation. BMC Struct. Biol. 2009, 9, 1-15. [CrossRef]

104. Tadeo, X.; Ló Pez-Mé Ndez, B.; Trigueros, T.; Laín, A.; Castañ, D.; Millet, O. Structural basis for the amino acid composition of proteins from halophilic Archea. PLoS Biol. 2009, 7, e1000257. [CrossRef] [PubMed]

105. Munawar, N.; Engel, P.C. Overexpression in a non-native halophilic host and biotechnological potential of NAD+-dependent glutamate dehydrogenase from Halobacterium salinarum strain NRC-36014. Extremophiles 2012, 16, 463-476. [CrossRef] [PubMed]

106. Coquelle, N.; Talon, R.; Juers, D.H.; Girard, É.; Kahn, R.; Madern, D. Gradual adaptive changes of a protein facing high salt concentrations. J. Mol. Biol. 2010, 404, 493-505. [CrossRef] [PubMed]

107. Oren, A.; Gavrieli, I.; Gavrieli, J.; Kohen, M.; Lati, J.; Aharoni, M. Microbial communities in the Dead Sea-Past, present and future. In Adaptation to Life at High Salt Concentrations in Archaea, Bacteria, and Eukarya. Cellular Origin, Life in Extreme Habitats and Astrobiology; Gunde-Cimerman, N., Oren, A., Plemenitaš, A., Eds.; Springer: Berlin/Heidelberg, Germany, 2005; Volume 9, pp. 27-39, ISBN 1402036337.

108. Bieger, B.; Essen, L.-O.; Oesterhelt, D. Crystal structure of halophilic dodecin: A novel, dodecameric flavin binding protein from Halobacterium salinarum. Structure 2003, 11, 375-385. [CrossRef]

109. Altermark, B.; Helland, R.; Moe, E.; Willassen, N.P.; Smalås, A.O. Structural adaptation of endonuclease I from the cold-adapted and halophilic bacterium Vibrio salmonicida. Acta Crystallogr. Sect. D Biol. Crystallogr. 2008, 64, 368-376. [CrossRef] [PubMed]

110. Sivakumar, N.; Li, N.; Tang, J.W.; Patel, B.K.C.; Swaminathan, K. Crystal structure of AmyA lacks acidic surface and provide insights into protein stability at poly-extreme condition. FEBS Lett. 2006, 580, 2646-2652. [CrossRef]

111. Kim, T.D. Bacterial hormone-sensitive lipases (bHSLs): Emerging enzymes for biotechnological applications. J. Microbiol. Biotechnol. 2017, 27, 1907-1915. [CrossRef] [PubMed]

112. Noby, N.; Hussein, A.; Saeed, H.; Embaby, A.M. Recombinant cold-adapted halotolerant, organic solvent-stable esterase (estHIJ) from Bacillus halodurans. Anal. Biochem. 2020, 591, 113554. [CrossRef]

113. Panda, T.; Gowrishankar, B.S. Production and applications of esterases. Appl. Microbiol. Biotechnol. 2005, 67, 160-169. [CrossRef]

114. Hasan, F.; Shah, A.A.; Hameed, A. Industrial applications of microbial lipases. Enzyme Microb. Technol. 2006, 39, 235-251. [CrossRef]

115. Karan, R.; Capes, M.D.; DasSarma, S. Function and biotechnology of extremophilic enzymes in low water activity. Aquat. Biosyst. 2012, 8, 4. [CrossRef] [PubMed]

116. Lopez-Lopez, O.; Cerdan, M.; Siso, M. New extremophilic lipases and esterases from metagenomics. Curr. Protein Pept. Sci. 2014, 15, 445-455. [CrossRef] [PubMed]

117. Rathi, P.; Bradoo, S.; Saxena, R.K.; Gupta, R. A hyper-thermostable, alkaline lipase from Pseudomonas sp. with the property of thermal activation. Biotechnol. Lett. 2000, 22, 495-498. [CrossRef]

118. Castro-Ochoa, L.D.; Rodríguez-Gómez, C.; Valerio-Alfaro, G.; Oliart Ros, R. Screening, purification and characterization of the thermoalkalophilic lipase produced by Bacillus thermoleovorans CCR11. Enzyme Microb. Technol. 2005, 37, 648-654. [CrossRef]

119. Kamarudin, N.H.A.; Rahman, R.N.Z.R.A.; Ali, M.S.M.; Leow, T.C.; Basri, M.; Salleh, A.B. A new cold-adapted, organic solvent stable lipase from mesophilic Staphylococcus epidermidis AT2. Protein J. 2014, 33, 296-307. [CrossRef] [PubMed] 


\section{Supplemental information for chapter IV}

\section{Content}

Figure S1. Strand, location and BLAST searching results for predicted open reading frames (ORFs) on insert sequence that harboring lipolytic gene est56.

Figure S2. Multiple sequence alignment of Est56 and its homologs.

Figure S3. SDS-PAGE analysis of the purification of recombinant Est56 (including His 6 -tag).

Figure S4. Substrate specificity of Est56 towards p-NP esters of different chain length

Figure S5. Unrooted phylogenetic tree of family IV esterases using neighbor-joining method.

Table S1. Features of characterized halotolerant lipolytic enzymes.

Table S2. Lipolytic enzymes derived from halophilic archaea adapting the "salt in" strategy.

Table S3. Characterized halophilic enzymes from other studies.

Table S4. Purification of recombinant Est56.

Table S5. Effect of metal ions and EDTA on Est56 activity.

Table S6. Effect of detergents on Est56 activity.

Table S7. Effect of inhibitors on Est56 activity.

Table S8. Amino acid composition comparison between halotolerant and halophilic enzymes. 


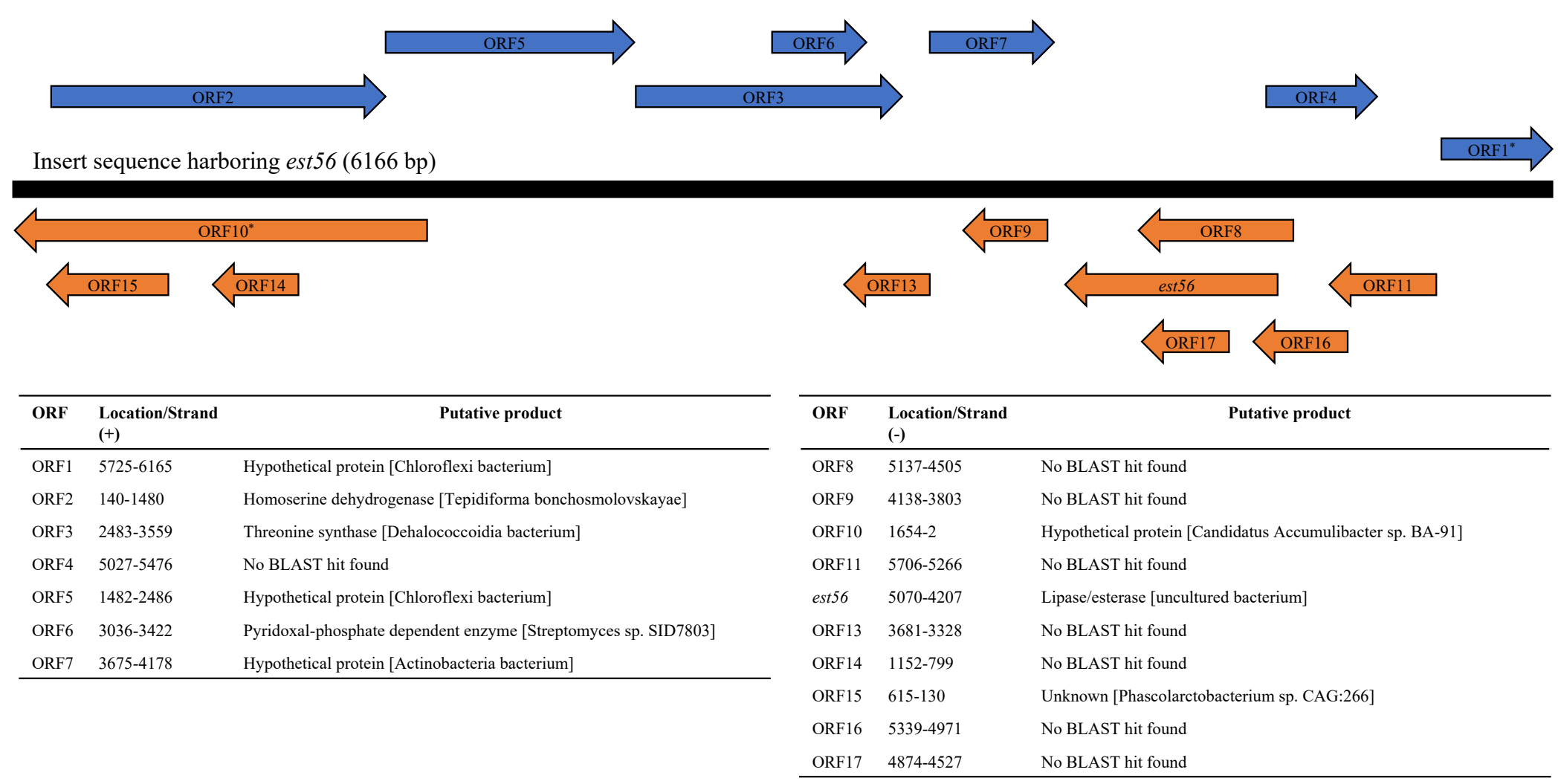

Figure S1. Strand, location and BLAST searching results for predicted open reading frames (ORFs) on insert sequence that harboring lipolytic gene est56. Only amino acid sequence length $\geq 100$ aa were mentioned. The ORF prediction was conducted by ORFfinder (https://www.ncbi.nlm.nih.gov/orffinder/). The closest hit for each ORF was searched using BLASTP. * Partial ORFs. 


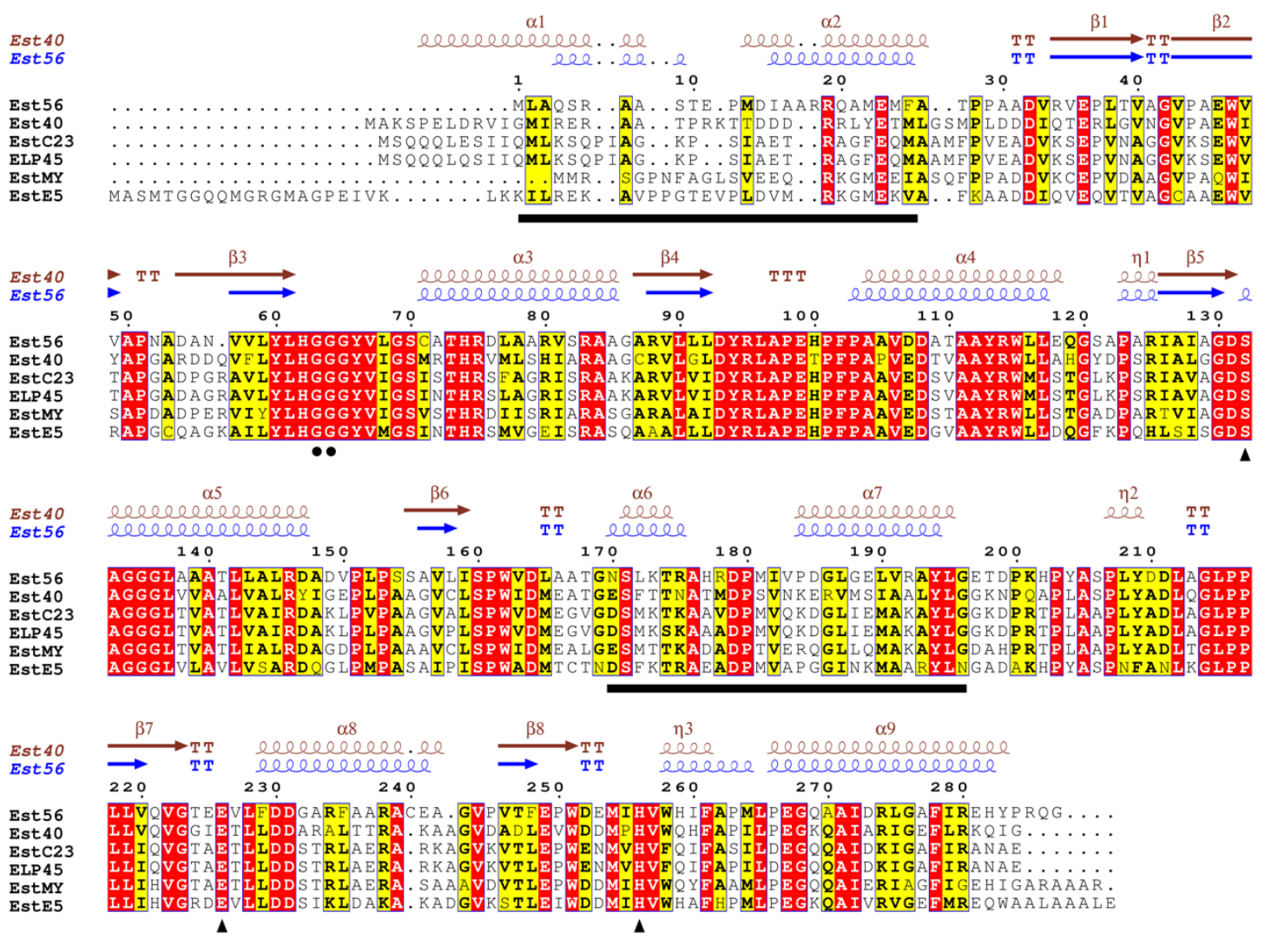

Figure S2. Multiple sequence alignment of Est56 and its homologs. Partially conserved residues are in frames. Identical residues are shaded. Triangles underneath residues indicate the catalytic triad and circles represent residues involved in the oxyanion hole. The secondary structures of Est56 and its structural analog Est40 ( $\mathrm{Li}$ et al. 2015b) are presented as: squiggles for $\alpha$ helices, arrows for $\beta$ strands, by TT letters for turns, and $\eta$ letters for 310 -helices. The square bar represents regions of the cap domain. The reference esterases EstC23 (Jin et al. 2012), ELP45 (Lee et al. 2004), EstMY (Li et al. 2010) and EstE5 (Nam et al. 2009) were derived from GenBank. 


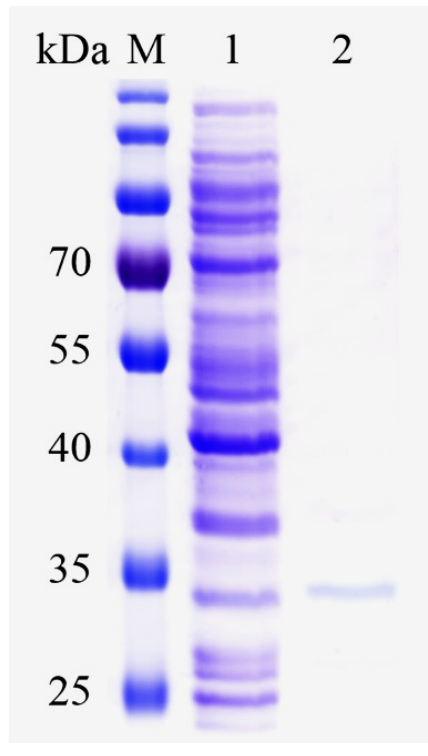

Figure S3. SDS-PAGE analysis of purification of recombinant Est56 (including His6-tag). Lane M, standard molecular weight marker; Lane 1, cell lysate (21.5 $\mu \mathrm{g})$; Lane 2, purified Est56 (1.0 $\mu \mathrm{g})$. 


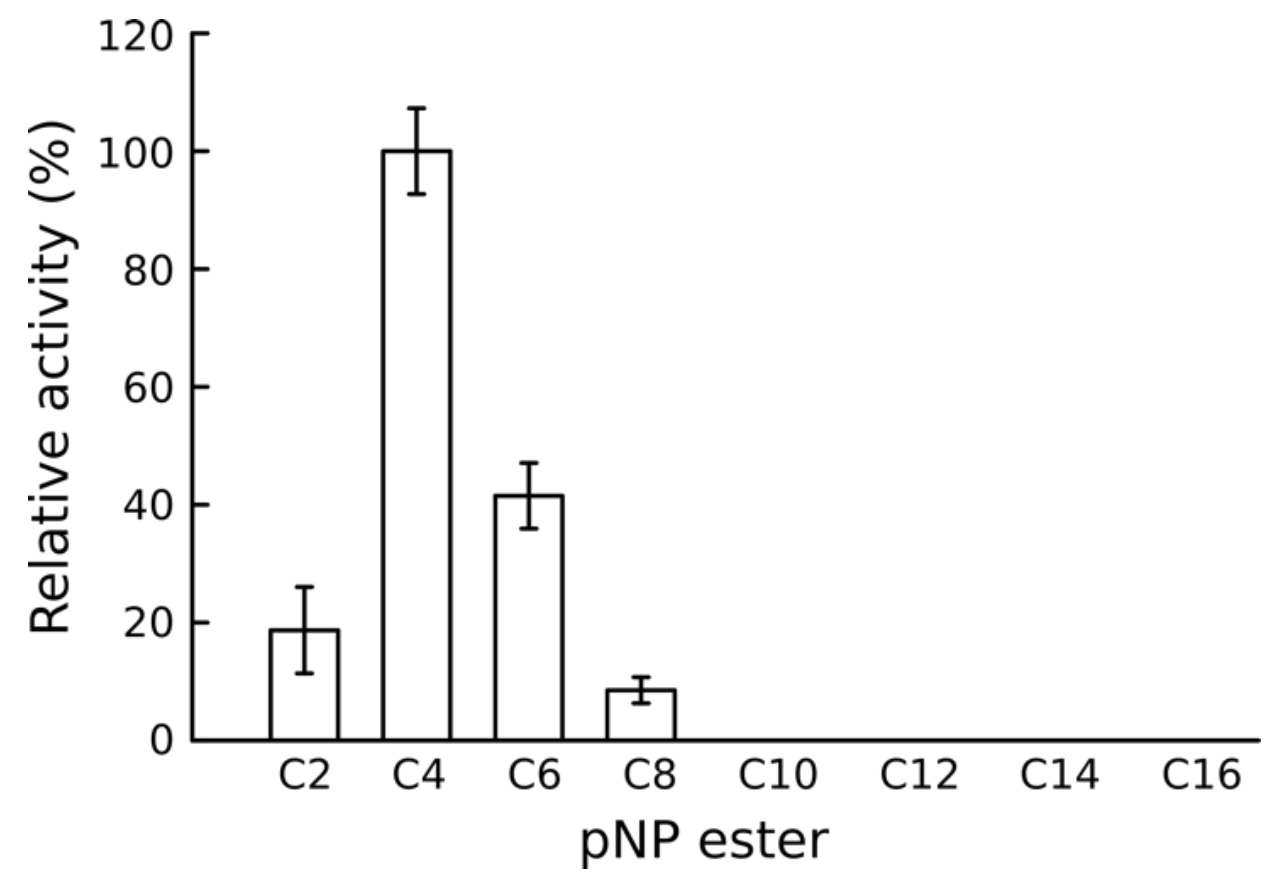

Figure S4. Substrate specificity of Est56 towards p-NP esters of different chain length. The maximal activity (189.5 U/mg) measured with p-NP butyrate (C4) was taken as $100 \%$. 


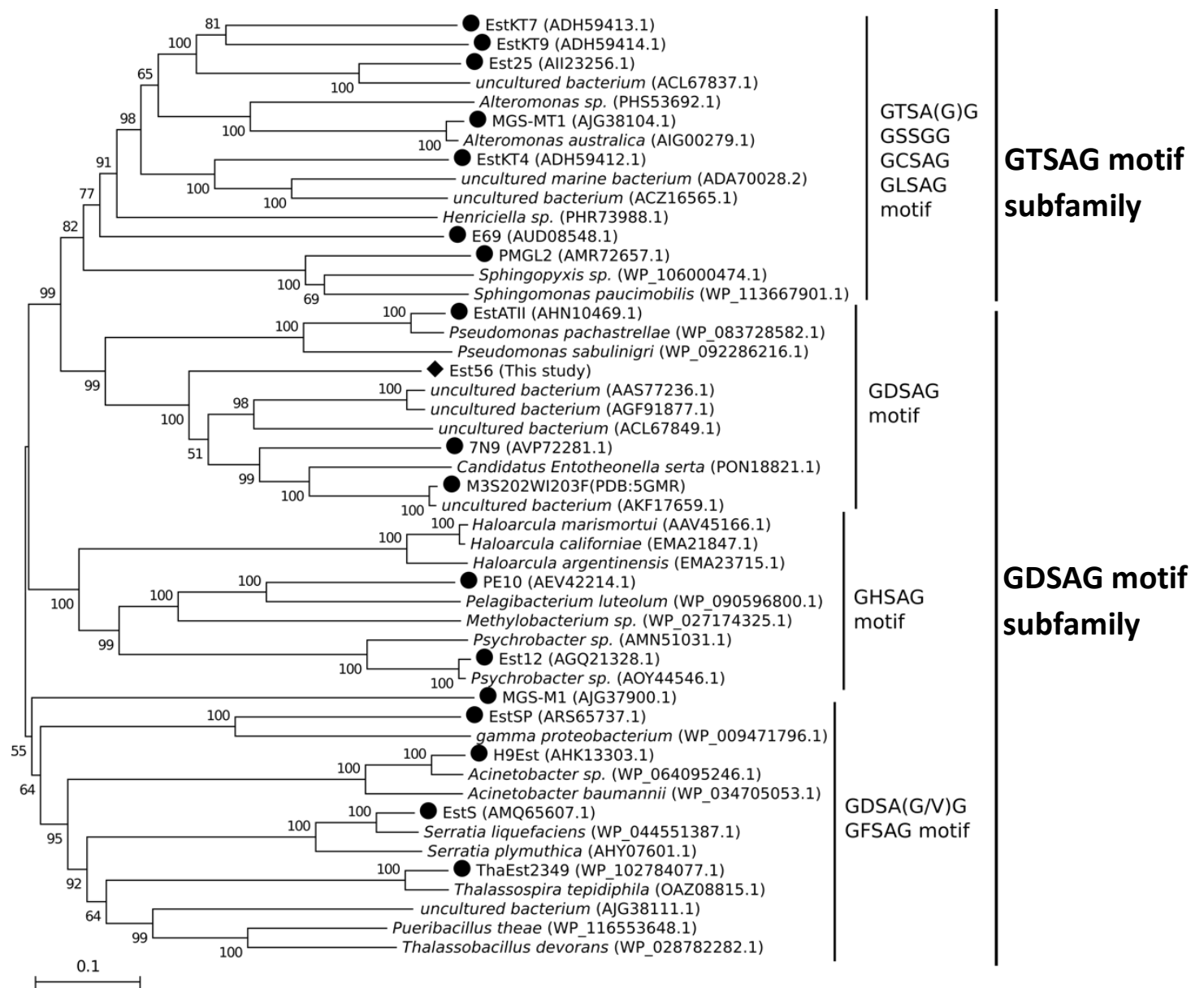

Figure S5. Unrooted phylogenetic tree of family IV esterases using neighbor-joining method. Est56 (closed diamond) and characterized halotolerant (closed circles) are depicted. With the exception of Est56, other sequences were retrieved from GenBank, with accession numbers in parentheses. Only bootstrap values greater than $50 \%$ are shown. Scale represents the number of amino acid substitutions per site. 
Table S1. Features of characterized halotolerant lipolytic enzymes (HT_LIP)

\begin{tabular}{|c|c|c|c|c|c|c|c|c|c|c|}
\hline \multirow{3}{*}{$\begin{array}{l}\text { Lipolytic } \\
\text { enzyme }\end{array}$} & \multirow{3}{*}{ Organism } & \multirow{3}{*}{$\mathrm{pI}$} & \multirow{3}{*}{$\mathrm{pH}_{\mathrm{opt}}$} & \multirow{3}{*}{$\begin{array}{l}\mathrm{T}_{\mathrm{opt}} \\
\left({ }^{\circ} \mathrm{C}\right)\end{array}$} & \multicolumn{5}{|c|}{$\begin{array}{l}\text { Salinity } \\
\end{array}$} & \multirow{3}{*}{ Reference } \\
\hline & & & & & \multicolumn{3}{|c|}{ Effect on enzyme activity } & \multicolumn{2}{|c|}{ Effect on enzyme stability } & \\
\hline & & & & & $\begin{array}{l}\text { Salt } \\
\text { range }\end{array}$ & Maximum activity $(\%)^{\mathrm{a}}$ & Minimum activity $(\%)^{\mathrm{a}}$ & Incubation condition & Residual activity $(\%)^{\mathrm{b}}$ & \\
\hline $7 \mathrm{~N} 9$ & Uncultured bacterium & 4.59 & 8 & $0-30$ & $0-24 \%$ & $100 \%$ at $0 \%$ & $\sim 55 \%$ at $24 \%$ & $\mathrm{ND}^{\mathrm{d}}$ & $\mathrm{ND}^{\mathrm{d}}$ & (Borchert et \\
\hline Lpc53E1 & Uncultured bacterium & 4.61 & 7 & 40 & $0-4 \mathrm{M}$ & $234 \%$ at $5 \mathrm{M}$ & $100 \%$ at $0 \mathrm{M}$ & $4^{\circ} \mathrm{C}, 24 \mathrm{~h}$ & $\sim 100 \%$, over $0-5 \mathrm{M}$ & (Selvin et \\
\hline EM3L4 & Uncultured bacterium & 4.61 & 7.5 & 35 & $0-4 \mathrm{M}$ & $\mathrm{ND}^{\mathrm{d}}$ & $\mathrm{ND}^{\mathrm{d}}$ & $35^{\circ} \mathrm{C}, 30 \mathrm{~min}$ & $>100 \%$, over $0-4 \mathrm{M}$ & (Lee et al. \\
\hline PE10 & Pelagibacterium halotolerans & 4.65 & 7.5 & 45 & $0-4 \mathrm{M}$ & $\sim 160 \%$ at $3 \mathrm{M}$ & $100 \%$ at $0 \mathrm{M}$ & $\mathrm{ND}^{\mathrm{d}}$ & $\mathrm{ND}^{\mathrm{d}}$ & (Jiang et al. \\
\hline EstSP & Uncultured bacterium & 4.65 & 8 & 40 & $0-5 \mathrm{M}$ & $155 \%$ at $1 \mathrm{M}$ & $\sim 10 \%$ at $5 \mathrm{M}$ & $25^{\circ} \mathrm{C}, 24 \mathrm{~h}$ & $>70 \%$, over $0-5 \mathrm{M}$ & (Jayanath et \\
\hline ABO_1251 & Alcanivorax borkumensis & 4.74 & $\mathrm{ND}^{\mathrm{d}}$ & 35 & $0-3.5 \mathrm{M}$ & $100 \%$ at $0 \mathrm{M}$ & $\sim 40 \%$ at $3.5 \mathrm{M} \mathrm{NaCl} / \mathrm{KCl}$ & $\mathrm{ND}^{\mathrm{d}}$ & $\mathrm{ND}^{\mathrm{d}}$ & (Tchigvints \\
\hline E69 & Erythrobacter seohaensis & 4.76 & 10.5 & 60 & $0-3 \mathrm{M}$ & $\sim 150 \%$ at $0.5 \mathrm{M}$ & $\sim 40 \%$ at $3 \mathrm{M}$ & $\mathrm{ND}^{\mathrm{d}}$ & $\mathrm{ND}^{\mathrm{d}}$ & (Huo et al. \\
\hline MGS-K1 & Uncultured bacterium & 4.89 & 7 & 30 & $0-4 \mathrm{M}$ & $0.8 \mathrm{M} \mathrm{NaCl}^{\mathrm{c}}$ & $\mathrm{ND}^{\mathrm{d}}$ & $\mathrm{ND}^{\mathrm{d}}$ & $\mathrm{ND}^{\mathrm{d}}$ & (Alcaide et \\
\hline estHIJ & Bacillus halodurans & 4.90 & 7 & 28 & $0-4 \mathrm{M}$ & $100 \%$ at $0 \mathrm{M}$ & $\sim 70 \%$ at $4 \mathrm{M}$ & $25^{\circ} \mathrm{C}, 6 \mathrm{~h}$ & $\sim 100 \%$ at $4 \mathrm{M}$ & (Noby et al. \\
\hline EaEST & Exiguobacterium antarcticum & 4.91 & 8 & 40 & $0-5 \mathrm{M}$ & $\mathrm{ND}^{\mathrm{d}}$ & $\mathrm{ND}^{\mathrm{d}}$ & $25^{\circ} \mathrm{C}, 1 \mathrm{~h}$ & $\sim 100 \%$, over $0-5 \mathrm{M}$ & (Lee et al. \\
\hline Esth & Shewanella & 4.93 & 8 & 30 & $0-5 \mathrm{M}$ & $100 \%$ at $0 \mathrm{M}$ & $\sim 50 \%$ at $5 \mathrm{M}$ & $4{ }^{\circ} \mathrm{C}, 24 \mathrm{~h}$ & $\sim 100 \%$, over $0-5 \mathrm{M}$ & (Hang et al. \\
\hline ABO_1197 & Alcanivorax borkumensis & 4.93 & $\mathrm{ND}^{\mathrm{d}}$ & 30 & $0-3.5 \mathrm{M}$ & $100 \%$ at $0 \mathrm{M}$ & $\sim 30 \%$ at $3.5 \mathrm{M} \mathrm{NaCl} / \mathrm{KCl}$ & $\mathrm{ND}^{\mathrm{d}}$ & $\mathrm{ND}^{\mathrm{d}}$ & (Tchigvints \\
\hline ThaEst2349 & Thalassospira sp. & 4.94 & 8.5 & 45 & $0-4 \mathrm{M}$ & $283 \%$ at $3 \mathrm{M}$ & $40 \%$ at $4 \mathrm{M}$ & $4^{\circ} \mathrm{C}, 24 \mathrm{~h}$ & $>280 \%$, over $1-3 \mathrm{M}$ & (De Santi et \\
\hline Est56 & Uncultured bacterium & 4.97 & 8 & 50 & $0-4 \mathrm{M}$ & $\begin{array}{l}\sim 140 \% \text { at } 1.5 \mathrm{M} \mathrm{NaCl} \\
\text { or } 1 \mathrm{M} \mathrm{KCl}\end{array}$ & $\sim 40 \%$ at $4 \mathrm{M} \mathrm{NaCl} / \mathrm{KCl}$ & $10^{\circ} \mathrm{C}, 24 \mathrm{~h}$ & $\begin{array}{l}\sim 100 \% \text {, over } 0-4 \mathrm{M} \\
\mathrm{NaCl} / \mathrm{KCl}\end{array}$ & This study \\
\hline Lip3 & Uncultured bacterium & 4.98 & 8 & 35 & $0-4 \mathrm{M}$ & $675 \%$ at $3 \mathrm{M} \mathrm{NaCl}$ & $100 \%$ at $0 \mathrm{M}$ & $4{ }^{\circ} \mathrm{C}, 24 \mathrm{~h}$ & $>100 \%$, over $0-3 \mathrm{M}$ & (De Santi et \\
\hline EstS & Serratia sp. & 5.05 & 8.5 & 10 & $0-4 \mathrm{M}$ & $100 \%$ at $0 \mathrm{M}$ & $94 \%$ at $4 \mathrm{M}$ & $4{ }^{\circ} \mathrm{C}, 24 \mathrm{~h}$ & $>80 \%$, over $0-4 \mathrm{M}$ & (Jiang et al. \\
\hline MGS-RG1 & Uncultured bacterium & 5.07 & 8 & 45 & $0-4 \mathrm{M}$ & $\sim 250 \%$ at $3.2 \mathrm{M}$ & $100 \%$ at $0 \mathrm{M}$ & $\mathrm{ND}^{\mathrm{d}}$ & $\mathrm{ND}^{\mathrm{d}}$ & (Alcaide et \\
\hline E25 & Uncultured bacterium & 5.16 & 8.5 & 50 & $0-4 \mathrm{M}$ & $\sim 130 \%$ at $1 \mathrm{M}$ & $\sim 50 \%$ at $4 \mathrm{M}$ & $\mathrm{ND}^{\mathrm{d}}$ & $\mathrm{ND}^{\mathrm{d}}$ & (Li et al. \\
\hline $\begin{array}{l}\text { M3S202WI } \\
203 F\end{array}$ & Uncultured bacterium & 5.17 & $\mathrm{ND}^{\mathrm{d}}$ & 55 & $0-4 \mathrm{M}$ & $\sim 110 \%$ at $0.5 \mathrm{M}$ & $\sim 60 \%$ at $4 \mathrm{M}$ & $20^{\circ} \mathrm{C}, 1 \mathrm{~h}$ & $>70 \%$, over $0-4 \mathrm{M}$ & $\begin{array}{l}\text { (Li et al. } \\
\text { 2017a) }\end{array}$ \\
\hline $\mathrm{YbfF}$ & Halomonas elongata & 5.25 & 8 & $\mathrm{ND}^{\mathrm{d}}$ & $0-4 \mathrm{M}$ & $100 \%$ at $2 \mathrm{M}$ & $\sim 15 \%$ at $0 \mathrm{M}$ & $\mathrm{ND}^{\mathrm{d}}$ & $\mathrm{ND}^{\mathrm{d}}$ & (Yoo et al. \\
\hline EstSL3 & Alkalibacterium sp. & 5.28 & 9 & 30 & $0-4 \mathrm{M}$ & $\sim 105 \%$ at $2 \mathrm{M}$ & $98 \%$ at $4 \mathrm{M}$ & $37^{\circ} \mathrm{C}, 2 \mathrm{~h}$ & $\sim 100 \%$, over $0-4 \mathrm{M}$ & (Wang et \\
\hline MGS-RG2 & Uncultured bacterium & 5.31 & 8 & 50 & $0-4 \mathrm{M}$ & $\sim 250 \%$ at $3.6 \mathrm{M}$ & $100 \%$ at $0 \mathrm{M}$ & $\mathrm{ND}^{\mathrm{d}}$ & $\mathrm{ND}^{\mathrm{d}}$ & (Alcaide et \\
\hline BlEst1 & Bacillus licheniformis & 5.33 & 7 & 40 & $0-5 \mathrm{M}$ & $100 \%$ at $1 \mathrm{M}$ & $\sim 70 \%$ at $5 \mathrm{M}$ & $\mathrm{ND}^{\mathrm{d}}$ & $\mathrm{ND}^{\mathrm{d}}$ & (Nakamura \\
\hline Est10 & Psychrobacter pacificensis & 5.35 & 7.5 & 25 & $0-5 \mathrm{M}$ & $143.2 \%$ at $2 \mathrm{M}$ & $\sim 80 \%$ at $5 \mathrm{M}$ & $4{ }^{\circ} \mathrm{C}, 6.5 \mathrm{~h}$ & $>100 \%$, over $0-5 \mathrm{M}$ & (Wu et al. \\
\hline MGS0010 & Uncultured bacterium & 5.4 & $\mathrm{ND}^{\mathrm{d}}$ & 30 & $0-3.5 \mathrm{M}$ & $\sim 250 \%$ at $3.5 \mathrm{M}$ & $100 \%$ at $0 \mathrm{M}$ & $\mathrm{ND}^{\mathrm{d}}$ & $\mathrm{ND}^{\mathrm{d}}$ & (Tchigvints \\
\hline BlEstA & Bacillus licheniformis & 5.54 & 9 & 30 & $0-3 \mathrm{M}$ & $165 \%$ at $2 \mathrm{M}$ & $100 \%$ at $0 \mathrm{M}$ & $\mathrm{ND}^{\mathrm{d}}$ & $\mathrm{ND}^{\mathrm{d}}$ & $\begin{array}{l}\text { (Leite et al. } \\
\text { m }\end{array}$ \\
\hline
\end{tabular}




\begin{tabular}{|c|c|c|c|c|c|c|c|c|c|c|}
\hline EstWSD & Uncultured bacterium & 5.61 & 7 & 50 & $0-5 \mathrm{M}$ & $\sim 140 \%$ at $1 \mathrm{M}$ & $\sim 50 \%$ at $5 \mathrm{M}$ & $\mathrm{ND}^{\mathrm{d}}$ & $\mathrm{ND}^{\mathrm{d}}$ & (Wang et \\
\hline EstSHJ2 & Chromohalobacter canadensis & 5.71 & 8 & 50 & $0-5 \mathrm{M}$ & $100 \%$ at $2.5 \mathrm{M}$ & $\sim 10 \%$ at $0 \mathrm{M}$ & $\mathrm{ND}^{\mathrm{d}}$ & $\mathrm{ND}^{\mathrm{d}}$ & (Wang et \\
\hline PMGL2 & Uncultured bacterium & 5.72 & 8.5 & 45 & $0-1.75 \mathrm{M}$ & $165 \%$ at $0.25 \mathrm{M}$ & $84 \%$ at $1.75 \mathrm{M}$ & $\mathrm{ND}^{\mathrm{d}}$ & $\mathrm{ND}^{\mathrm{d}}$ & (Petrovskay \\
\hline MGS-M1 & Uncultured bacterium & 5.77 & 8 & 25 & $0-4 \mathrm{M}$ & $3.6 \mathrm{M} \mathrm{NaCl}^{\mathrm{c}}$ & $\mathrm{ND}^{\mathrm{d}}$ & $\mathrm{ND}^{\mathrm{d}}$ & $\mathrm{ND}^{\mathrm{d}}$ & (Alcaide et \\
\hline EstKT4 & Uncultured bacterium & 5.81 & 8.5 & 40 & $0-4 \mathrm{M}$ & $\mathrm{ND}^{\mathrm{d}}$ & $\mathrm{ND}^{\mathrm{d}}$ & $35^{\circ} \mathrm{C}, 30 \mathrm{~min}$ & $>50 \%$, over $0-3.5 \mathrm{M}$ & (Jeon et al. \\
\hline EstKT7 & Uncultured bacterium & 5.84 & 8 & 35 & $0-4 \mathrm{M}$ & $\mathrm{ND}^{\mathrm{d}}$ & $\mathrm{ND}^{\mathrm{d}}$ & $35^{\circ} \mathrm{C}, 30 \mathrm{~min}$ & $>50 \%$, over $0-3 \mathrm{M}$ & (Jeon et al. \\
\hline LipC12 & Uncultured bacterium & 5.98 & 9 & 30 & $0-4 \mathrm{M}$ & $1501 \%$ at $1.5 \mathrm{M}$ & $100 \%$ at $0 \mathrm{M}$ & $4^{\circ} \mathrm{C}, 24 \mathrm{~h}$ & $\sim 100 \%$, over $0-3.7 \mathrm{M}$ & (Ĝlogauer \\
\hline EstKT9 & Uncultured bacterium & 6.1 & 8.5 & 45 & $0-4 \mathrm{M}$ & $\mathrm{ND}^{\mathrm{d}}$ & $\mathrm{ND}^{\mathrm{d}}$ & $35^{\circ} \mathrm{C}, 30 \mathrm{~min}$ & $>50 \%$, over $0-3.5 \mathrm{M}$ & (Jeon et al. \\
\hline lp_3505 & Lactobacillus plantarum & 6.12 & 6 & 5 & $0-25 \%$ & $\sim 250 \%$ at $5 \%$ & $\sim 70 \%$ at $25 \%$ & $\mathrm{ND}^{\mathrm{d}}$ & $\mathrm{ND}^{\mathrm{d}}$ & (Esteban- \\
\hline Est9x & Uncultured bacterium & 6.17 & 8 & 65 & $0-4 \mathrm{M}$ & $\sim 190 \%$ at $4 \mathrm{M}$ & $100 \%$ at $0 \mathrm{M}$ & $\mathrm{ND}^{\mathrm{d}}$ & $\mathrm{ND}^{\mathrm{d}}$ & (Fang et al. \\
\hline Est12 & Psychrobacter celer & 6.5 & 7.5 & 35 & $0-4.5 \mathrm{M}$ & $\mathrm{ND}^{\mathrm{d}}$ & $\mathrm{ND}^{\mathrm{d}}$ & $25^{\circ} \mathrm{C}, 12 \mathrm{~h}$ & $\sim 100 \%$, over $0-4.5 \mathrm{M}$ & (Wu et al. \\
\hline EstATII & Uncultured bacterium & 7.11 & 8.5 & 65 & $0-4 \mathrm{M}$ & $\mathrm{ND}^{\mathrm{d}}$ & $\sim 50 \%$ at $4 \mathrm{M}$ & $\mathrm{ND}^{\mathrm{d}}$ & $\mathrm{ND}^{\mathrm{d}}$ & (Mohamed \\
\hline Est-OKK & Uncultured bacterium & 7.82 & 9 & 50 & $0-3 \mathrm{M}$ & $\sim 130 \%$ at $1.5 \mathrm{M}$ & $\sim 100 \%$ at $3 \mathrm{M}$ & $\mathrm{RT}, 4 \mathrm{~h}$ & $\sim 100 \%$, over $0-3 \mathrm{M}$ & (Yang et al. \\
\hline LipJ2 & Janibacter sp. & 8.25 & 9 & 80 & $1,10 \mathrm{mM}$ & $\sim 250 \%$ at $0.1 \mathrm{mM} \mathrm{NaCl}$ & $100 \%$ at $0 \mathrm{M}$ & $\mathrm{ND}^{\mathrm{d}}$ & $\mathrm{ND}^{\mathrm{d}}$ & (Castilla et \\
\hline MGS-MT1 & Uncultured bacterium & 8.4 & 8.5 & 50 & $0-4 \mathrm{M}$ & & $\mathrm{ND}^{\mathrm{d}}$ & $\mathrm{ND}^{\mathrm{d}}$ & $\mathrm{ND}^{\mathrm{d}}$ & (Alcaide et \\
\hline EstLiu & Zunongwangia profunda & 8.42 & 8 & 30 & $0-4.5 \mathrm{M}$ & $100 \%$ at $0 \mathrm{M}$ & $57 \%$ at $4.5 \mathrm{M}$ & $4{ }^{\circ} \mathrm{C}, 12 \mathrm{~h}$ & $>80 \%$, over $0-4.5 \mathrm{M}$ & (Rahman et \\
\hline H9Est & Uncultured bacterium & 8.72 & 8 & 40 & $0-2.5 \mathrm{M}$ & $\sim 150 \%$ at $1 \mathrm{M}$ & $\sim 50 \%$ at $2.5 \mathrm{M}$ & $\mathrm{ND}^{\mathrm{d}}$ & $\mathrm{ND}^{\mathrm{d}}$ & (Santi et al. \\
\hline $\mathrm{H} 8$ & Uncultured bacterium & 9.09 & 10 & 35 & $0-5 \mathrm{M}$ & $\sim 105 \%$ at $4 \mathrm{M}$ & $\sim 10 \%$ at $5 \mathrm{M}$ & $0{ }^{\circ} \mathrm{C}, 1 \mathrm{~h}$ & $>80 \%$, over $0-4.7 \mathrm{M}$ & (Zhang et \\
\hline Est700 & Bacillus licheniformis & 9.44 & 8 & 30 & $0-5 \mathrm{M}$ & $588 \%$ at $3.5 \mathrm{M}$ & $100 \%$ at $0 \mathrm{M}$ & $4{ }^{\circ} \mathrm{C}, 1 \mathrm{~h}$ & $\sim 100 \%$, over $0-5 \mathrm{M}$ & (Zhang et \\
\hline BmEST & Bacillus mojavensis & $N D^{d}$ & 8 & 80 & $0-25 \%$ & $\sim 300 \%$ at $20 \%$ & $100 \%$ at $0 \%$ & $80^{\circ} \mathrm{C}, 1 \mathrm{~h}$ & $>60 \%$, over $0-25 \%$ & (Ad̂̃igüzel \\
\hline
\end{tabular}

a The activity measured without salt $(\mathrm{NaCl}$, if not mentioned) was taken as $100 \%$

${ }^{\mathrm{b}}$ The activity measured after incubating in salt-free ( $\mathrm{NaCl}$, unless stated otherwise) buffer was defined as $100 \%$

${ }^{c}$ The activity at Salt ${ }_{\mathrm{opt}}$ was set as $100 \%$

${ }^{\mathrm{d}}$ No data 
Table S2. Lipolytic enzymes (HP_Lip) derived from halophilic archaea adapting the "salt in" strategy

\begin{tabular}{|c|c|c|c|c|c|c|c|}
\hline $\begin{array}{l}\text { Lipolytic } \\
\text { enzyme }\end{array}$ & Length (aa) & $\mathrm{pI}$ & Microorganism & $\begin{array}{l}\text { GeneBank/PDB } \\
\text { Accession Nr. }\end{array}$ & Family & Halophile & Growth condition $(\mathrm{NaCl}, \%)$ \\
\hline HP_Lip_1 & 285 & 4.45 & Halococcus thailandensis & EMA51434.1 & Halobacteriaceae & Extreme & $20 \%-30 \%$ \\
\hline HP_Lip_2 & 292 & 4.55 & Halococcus morrhuae & EMA45705.1 & Halobacteriaceae & Extreme & $>12 \%, 23.3 \%$ \\
\hline HP_Lip_3 & 320 & 4.16 & Halococcus saccharolyticus & EMA45019.1 & Halobacteriaceae & Extreme & $15 \%$ - satr, optimum $25 \%$ \\
\hline HP_Lip_4 & 285 & 4.5 & Halococcus hamelinensis & EMA39292.1 & Halobacteriaceae & Moderate & $12.5 \%$ - $30 \%$, optimum $15 \%$ \\
\hline HP_Lip_5 & 263 & 4.4 & Halosimplex carlsbadense & ELZ28160.1 & Halobacteriaceae & Extreme & $20 \%-30 \%$ (optimum $25 \%$ ) \\
\hline HP_Lip_6 & 261 & 4.54 & Halosimplex carlsbadense & ELZ24957.1 & Halobacteriaceae & Extreme & $20 \%-30 \%$ (optimum $25 \%$ ) \\
\hline HP_Lip_7 & 330 & 4.17 & Haladaptatus sp. & KZN24148.1 & Halobacteriaceae & Extreme & $\mathrm{ND}^{\mathrm{b}}$ \\
\hline HP_Lip_9 & 340 & 4.34 & Halorhabdus utahensis & ACV10409.1 & Halobacteriaceae & Extreme & $9 \%-30 \%$, optimum $27 \%$ \\
\hline HP_Lip_10 & 258 & 4.57 & Haloarcula marismortui & AAV45777.1 & Halobacteriaceae & Extreme & optimum $20 \%-23 \%$ \\
\hline HP_Lip_11 & 318 & 4.22 & Haloarcula hispanica & AHB65276.1 & Halobacteriaceae & Moderate & $>12 \%$ \\
\hline HP_Lip_12 & 377 & 4.32 & Haloarcula japonica & WP_004591147.1 & Halobacteriaceae & Extreme & $15 \%-30 \%$, optimum $20 \%$ \\
\hline HP_Lip_13 & 318 & 4.18 & Haloarcula japonica & EMA29911.1 & Halobacteriaceae & Extreme & $15 \%-30 \%$, optimum $20 \%$ \\
\hline HP_Lip_14 & 318 & 4.19 & Haloarcula vallismortis & EMA07756.1 & Halobacteriaceae & Extreme & $>15 \%$, optimum $25 \%$ \\
\hline HP_Lip_15 & 376 & 4.3 & Haloarcula vallismortis & WP_004515030.1 & Halobacteriaceae & Extreme & $>15 \%$, optimum $25 \%$ \\
\hline HP_Lip_16 & 314 & 4.23 & Haloprofundus marisrubri & KTG11548.1 & Haloferacaceae & Extreme & $\min 7 \%$ \\
\hline HP_Lip_18 & 217 & 4.44 & Halogeometricum pallidum & ELZ32922.1 & Haloferacaceae & Moderate & optimum $18.1 \%$ \\
\hline HP_Lip_19 & 275 & 4.48 & Halogeometricum pallidum & ELZ27142.1 & Haloferacaceae & Moderate & optimum $18.1 \%$ \\
\hline HP_Lip_20 & 215 & 4.53 & Halogeometricum borinquense & ELY30686.1 & Haloferacaceae & Extreme & $\min 8 \%$, optimum $20 \%$ - \\
\hline HP_Lip_21 & 344 & 4.27 & Natronolimnobius baerhuensis & OVE85190.1 & Natrialbaceae & Extreme & $\begin{array}{l}\text { Nen } \\
\text { optimum } 20 \%\end{array}$ \\
\hline HP_Lip_22 & 455 & 4.54 & Haloterrigena mahii & OAQ52820.1 & Natrialbaceae & Extreme & optimum $20.4 \%-29.2 \%$ \\
\hline
\end{tabular}


Table S3. Characterized halophilic enzymes (HP_Enz) from other studies

\begin{tabular}{|c|c|c|c|c|c|c|c|c|}
\hline Protein & $\begin{array}{l}\text { Length } \\
\text { (aa) }\end{array}$ & $\mathrm{pI}$ & Organism & Domain & Family & Halophile & Growth condition $(\mathrm{NaCl}, \%)$ & Reference \\
\hline Malate dehydrogenase & 303 & 4.2 & Haloarcula marismortui & Archaea & Halobacteriaceae & Extreme & $10 \%-30 \%$, optimum $20 \%-23 \%$ & (Richard et \\
\hline Malate dehydrogenase & 304 & 4.2 & Haloarcula marismortui & Archaea & Halobacteriaceae & Extreme & $10 \%-30 \%$, optimum $20 \%-23 \%$ & $\begin{array}{l}\text { f1 } \\
\text { (Fioravanti }\end{array}$ \\
\hline Catalase-peroxidase & 731 & 4.32 & Haloarcula marismortui & Archaea & Halobacteriaceae & Extreme & $10 \%-30 \%$, optimum $20 \%-23 \%$ & $\begin{array}{l}\text { of } 1 \text { t } 7 \mathrm{nn}^{2} \\
\text { (Yamada }\end{array}$ \\
\hline Esterase & 327 & 4.24 & Haloarcula marismortui & Archaea & Halobacteriaceae & Extreme & $10 \%-30 \%$, optimum $20 \%-23 \%$ & $\begin{array}{l}\text { et al } 20 \mathrm{n} \text { ) } \\
\text { (Rao et al. }\end{array}$ \\
\hline $\begin{array}{l}\text { Nucleoside diphosphate } \\
\text { kinase }\end{array}$ & 161 & 4.37 & Halobacterium salinarum & Archaea & Halobacteriaceae & Moderate & $>12 \%, \mathrm{NaCl}$ saturation & $\begin{array}{l}\text { 2nnoal } \\
\text { (Besir et } \\
\text { al. 2005) }\end{array}$ \\
\hline $\begin{array}{l}\text { Nucleoside diphosphate } \\
\text { kinase }\end{array}$ & 164 & 4.42 & Halobacterium salinarum & Archaea & Halobacteriaceae & Moderate & $>12 \%, \mathrm{NaCl}$ saturation & $\begin{array}{l}\text { (Besir et } \\
\text { al. 2005) }\end{array}$ \\
\hline RNase H1 & 199 & 4.36 & Halobacterium salinarum & Archaea & Halobacteriaceae & Moderate & $>12 \%, \mathrm{NaCl}$ saturation & (Tannous \\
\hline Phosphatase & 431 & 4.35 & Halobacterium salinarum & Archaea & Halobacteriaceae & Moderate & $>12 \%, \mathrm{NaCl}$ saturation & (Wende et \\
\hline Dehydrogenase & 435 & 4.39 & Halobacterium salinarum & Archaea & Halobacteriaceae & Moderate & $>12 \%, \mathrm{NaCl}$ saturation & $\begin{array}{l}\text { al } 701 \mathrm{n} \\
\text { (Munawar }\end{array}$ \\
\hline DNA protecting protein & 182 & 4.3 & Halobacterium salinarum & Archaea & Halobacteriaceae & Moderate & $>12 \%, \mathrm{NaCl}$ saturation & $\begin{array}{l}\text { nnd Enmal } \\
\text { (Zeth et al. }\end{array}$ \\
\hline Beta-galactosidase & 663 & 4.54 & Haloferax lucentense & Archaea & Haloferacaceae & Extreme & $10.5 \%-29.8 \%$ (optimum $25.1 \%$ ) & (Holmes et \\
\hline DNA ligase & 699 & 4.34 & Haloferax volcanii & Archaea & Haloferacaceae & Extreme & $6 \%-29 \%$, optimum $10 \%-15 \%$ & $\begin{array}{l}\text { al } 1997 \text { ) } \\
\text { (Poidevin }\end{array}$ \\
\hline Dihydrofolate reductase & 162 & 4.45 & Haloferax volcanii & Archaea & Haloferacaceae & Extreme & $6 \%-29 \%$, optimum $10 \%-15 \%$ & (Pieper et \\
\hline Glucose dehydrogenase & 357 & 4.55 & Haloferax mediterranei & Archaea & Haloferacaceae & Moderate & $>12 \%$ & (Britton et \\
\hline Alpha-amylase & 504 & 4.11 & Natronococcus $s p$. & Archaea & Natrialbaceae & Moderate & $>12 \%$ & $\begin{array}{l}\text { 21 } 200 \text { na } \\
\text { (Kobayashi }\end{array}$ \\
\hline Esterase & 316 & 4.38 & unclutured bacterium & Bacterium & $\mathrm{ND}^{\mathrm{b}}$ & $\mathrm{ND}^{\mathrm{b}}$ & $\mathrm{ND}^{\mathrm{b}}$ & (Alcaide et \\
\hline
\end{tabular}

${ }^{\mathrm{a}}$ no data 
Table S4. Purification of recombinant Est56.

\begin{tabular}{llllll}
\hline Purification step & $\begin{array}{l}\text { Total activity } \\
(\mathrm{U})\end{array}$ & $\begin{array}{l}\text { Total protein } \\
(\mathrm{mg})\end{array}$ & $\begin{array}{l}\text { Specific activity } \\
(\mathrm{U} / \mathrm{mg})\end{array}$ & $\begin{array}{l}\text { Purification } \\
(\text { fold })\end{array}$ & $\begin{array}{l}\text { Yield } \\
(\%)\end{array}$ \\
\hline Crude extract & 239.8 & 193.7 & 1.24 & 1.0 & 100 \\
Ni-TED & 108.8 & 1.2 & 90.44 & 73.0 & 45.4 \\
\hline
\end{tabular}


Table S5. Effect of metal ions and EDTA on Est56 activity

\begin{tabular}{lll}
\hline Metal ions & Concentration $(\mathrm{mM})$ & Relative activity $(\%)^{\text {a }}$ \\
\hline $\mathrm{Al}^{3+}$ & 1 & $127.5 \pm 3.0$ \\
$\mathrm{Al}^{3+}$ & 10 & $142.8 \pm 6.3$ \\
$\mathrm{Ca}^{2+}$ & 1 & $126.5 \pm 10.3$ \\
$\mathrm{Ca}^{2+}$ & 10 & $136.9 \pm 9.3$ \\
$\mathrm{Mg}^{2+}$ & 1 & $106.1 \pm 0.9$ \\
$\mathrm{Mg}^{2+}$ & 10 & $105.5 \pm 5.6$ \\
$\mathrm{Mn}^{2+}$ & 1 & $113.2 \pm 2.5$ \\
$\mathrm{Mn}^{2+}$ & 10 & $82.4 \pm 3.5$ \\
$\mathrm{Zn}^{2+}$ & 1 & $75.4 \pm 8.2$ \\
$\mathrm{Zn2}^{+}$ & 10 & $83.6 \pm 7.3$ \\
$\mathrm{Fe}^{2+}$ & 1 & $82.4 \pm 6.6$ \\
$\mathrm{Fe}^{2+}$ & 10 & $13.2 \pm 1.9$ \\
$\mathrm{Fe}^{3+}$ & 1 & $60.9 \pm 5.0$ \\
$\mathrm{Fe}^{3+}$ & 10 & $19.5 \pm 6.3$ \\
$\mathrm{Ni}^{2+}$ & 1 & $13.3 \pm 4.1$ \\
$\mathrm{Ni}^{2+}$ & 10 & $22.0 \pm 4.4$ \\
$\mathrm{Cu}^{2+}$ & 1 & $16.7 \pm 8.7$ \\
$\mathrm{Cu}^{2+}$ & 10 & $23.2 \pm 2.2$ \\
$\mathrm{EDTA}^{2+}$ & 1 & $119.4 \pm 2.5$ \\
$\mathrm{EDTA}$ & 10 & $93.8 \pm 10.5$ \\
\hline
\end{tabular}

a The effects of metal ions on Est56 activity were measured under standard assay conditions. Specific activity $(93.1 \mathrm{U} / \mathrm{mg})$ assayed without additive was taken as $100 \%$.

${ }^{\mathrm{b}}$ Not detectable. 
Table S6. Effect of detergents on Est56 activity

\begin{tabular}{lll}
\hline Detergent & Concentration $(\mathrm{v} / \mathrm{v}, \%)$ & Relative activity $(\%)^{\mathrm{a}}$ \\
\hline Triton X-100 & 0.1 & $156.8 \pm 3.4$ \\
Triton X-100 & 1 & $44.6 \pm 2.8$ \\
Triton X-100 & 5 & $18.3 \pm 3.6$ \\
Tween 20 & 0.1 & $154.3 \pm 2.3$ \\
Tween 20 & 1 & $53.6 \pm 3.0$ \\
Tween 20 & 5 & $\mathrm{ND}^{\mathrm{b}}$ \\
Tween 80 & 0.1 & $112.7 \pm 1.7$ \\
Tween 80 & 1 & $36.6 \pm 1.8$ \\
Tween 80 & 5 & $11.4 \pm 1.6$ \\
\hline
\end{tabular}

a The effects of detergents on Est56 activity were measured under standard assay conditions. Specific activity (93.1 U/mg) assayed without additive was taken as $100 \%$.

${ }^{\mathrm{b}}$ Not detectable. 
Table S7. Effect of inhibitors on Est56 activity

\begin{tabular}{lll}
\hline Inhibitor & Concentration $(\mathrm{mM})$ & Relative activity $(\%)^{\mathrm{a}}$ \\
\hline DTT & 1 & $102.2 \pm 9.3$ \\
DTT & 10 & $46.0 \pm 1.4$ \\
PMSF & 1 & $69.9 \pm 0.7$ \\
PMSF & 10 & $10.1 \pm 1.2$ \\
DEPC & 1 & $1.9 \pm 1.1$ \\
DEPC & 10 & $\mathrm{ND}^{\mathrm{b}}$ \\
\hline
\end{tabular}

${ }^{\text {a }}$ The effects of inhibitors on Est56 activity were measured under standard assay conditions. Specific activity (93.1 $\mathrm{U} / \mathrm{mg}$ ) assayed without additive was taken as $100 \%$.

${ }^{\mathrm{b}}$ Not detectable. 
Table S8. Amino acid composition comparison between halotolerant and halophilic enzymes

\begin{tabular}{|c|c|c|c|c|c|c|}
\hline \multirow[b]{2}{*}{ Amino acid } & \multirow[b]{2}{*}{$\operatorname{HT}(\%)^{\mathrm{a}}$} & \multirow[b]{2}{*}{ HP_Lip $(\%)^{\mathrm{a}}$} & \multirow[b]{2}{*}{ HP_Enz $(\%)^{\mathrm{a}}$} & \multicolumn{3}{|c|}{ SIMPER analysis $^{\mathrm{b}}$} \\
\hline & & & & $\begin{array}{l}\text { HT vs HP_Lip } \\
\text { Contribution }(\%)^{\mathrm{c}}\end{array}$ & $\begin{array}{l}\text { HT vs HP_Enz } \\
\text { Contribution }(\%)^{\mathrm{d}}\end{array}$ & $\begin{array}{l}\text { HP_Lip vs HP_Enz } \\
\text { Contribution }(\%)^{\mathrm{e}}\end{array}$ \\
\hline Asp (D) & $5.92 \pm 1.39$ & $9.87 \pm 1.39$ & $10.47 \pm 1.99$ & $10.44(1)$ & $11.58(1)$ & $6.32(5)$ \\
\hline Lys (K) & $3.74 \pm 2.30$ & $1.01 \pm 0.48$ & $2.16 \pm 1.26$ & $7.34(3)$ & $5.77(5)$ & $4.24(14)$ \\
\hline Glu (E) & $5.27 \pm 1.47$ & $7.86 \pm 1.59$ & $8.87 \pm 1.98$ & $7.13(4)$ & $9.26(2)$ & $6.97(3)$ \\
\hline Ile (I) & $5.14 \pm 1.97$ & $2.80 \pm 1.16$ & $3.86 \pm 1.16$ & $6.75(5)$ & $4.92(8)$ & $5.20(9)$ \\
\hline $\operatorname{Asn}(\mathrm{N})$ & $3.51 \pm 1.56$ & $1.66 \pm 0.94$ & $2.49 \pm 1.07$ & $5.41(8)$ & $4.12(13)$ & $4.34(12)$ \\
\hline $\operatorname{Thr}(\mathrm{T})$ & $4.93 \pm 1.61$ & $5.41 \pm 1.61$ & $5.56 \pm 1.50$ & $4.71(9)$ & $4.3(12)$ & $5.39(6)$ \\
\hline $\operatorname{Arg}(\mathrm{R})$ & $4.73 \pm 1.72$ & $5.60 \pm 1.09$ & $5.70 \pm 1.31$ & $4.68(10)$ & $4.67(11)$ & $4.27(13)$ \\
\hline Pro (P) & $5.98 \pm 1.50$ & $6.11 \pm 1.70$ & $4.83 \pm 1.93$ & $4.59(11)$ & $5.31(6)$ & $7.393(2)$ \\
\hline Ser (S) & $5.93 \pm 1.68$ & $5.21 \pm 1.33$ & $4.88 \pm 1.62$ & $4.39(12)$ & $4.77(10)$ & $5.371(7)$ \\
\hline Phe $(F)$ & $3.61 \pm 1.21$ & $3.65 \pm 0.81$ & $3.13 \pm 1.20$ & $3.03(17)$ & $3.45(15)$ & $3.94(15)$ \\
\hline Tyr (Y) & $3.34 \pm 0.98$ & $3.24 \pm 1.05$ & $2.91 \pm 0.87$ & $2.9(18)$ & $2.73(18)$ & $3.47(16)$ \\
\hline $\operatorname{Trp}(\mathrm{W})$ & $1.54 \pm 0.76$ & $1.40 \pm 0.63$ & $1.46 \pm 0.99$ & $2.02(19)$ & $2.46(19)$ & 2.99 (19) \\
\hline Cys (C) & $0.76 \pm 0.65$ & $0.72 \pm 0.51$ & $0.41 \pm 0.47$ & $1.68(20)$ & $1.65(20)$ & $1.99(20)$ \\
\hline
\end{tabular}

${ }^{a}$ Data shown are averages with the standard deviation per group.

${ }^{\mathrm{b}}$ SIMPER analysis the contribution of each amino acid to the dissimilarity between groups, with permutations 9999 . Data shown are contributions (\%) with the rank in the brackets.

${ }^{\mathrm{c}}$ SIMPER-revealed average dissimilarity was 19.38 .

${ }^{\mathrm{d}}$ SIMPER-revealed average dissimilarity was 20.03 .

e SIMPER-revealed average dissimilarity was 15.31 . 


\section{References}

Abd Rahman RNZR, Ahmad Kamarudin NH, Yunus J, Salleh AB, Basri M (2010) Expression of an organic solvent stable lipase from Staphylococcus epidermidis AT2. Int J Mol Sci 11:3195-3208 . doi: 10.3390/ijms11093195

Abdel-Fattah YR, Gaballa AA (2008) Identification and over-expression of a thermostable lipase from Geobacillus thermoleovorans Toshki in Escherichia coli. Microbiol Res 163:13-20 . doi: 10.1016/j.micres.2006.02.004

Adigüzel AO (2020) Production and characterization of thermo-, halo- and solvent-stable esterase from Bacillus mojavensis TH309. Biocatal Biotransformation 38:210-226 . doi: 10.1080/10242422.2020.1715370

Adlercreutz P (2013) Immobilisation and application of lipases in organic media. Chem Soc Rev 42:6406-6436 . doi: $10.1039 / \mathrm{c} 3 \operatorname{cs} 35446 \mathrm{f}$

Ahmed EH, Raghavendra T, Madamwar D (2010) An alkaline lipase from organic solvent tolerant Acinetobacter sp. EH28: Application for ethyl caprylate synthesis. Bioresour Technol 101:3628-3634 . doi: 10.1016/J.BIORTECH.2009.12.107

Ai L, Huang Y, Wang C (2018) Purification and characterization of halophilic lipase of Chromohalobacter sp . from ancient salt well. J Basic Microbiol 58:647-657 . doi: 10.1002/jobm.201800116

Akoh CC, Lee GC, Liaw YC, Huang TH, Shaw JF (2004) GDSL family of serine esterases/lipases. Prog Lipid Res 43:534-552 . doi: 10.1016/j.plipres.2004.09.002

Albers S V., Jonuscheit M, Dinkelaker S, Urich T, Kletzin A, Tampé R, Driessen AJM, Schleper C (2006) Production of recombinant and tagged proteins in the hyperthermophilic archaeon Sulfolobus solfataricus. Appl Environ Microbiol 72:102-111 . doi: 10.1128/AEM.72.1.102-111.2006

Alcaide M, Stogios PJ, Lafraya Á, Tchigvintsev A, Flick R, Bargiela R, Chernikova TN, Reva ON, Hai T, Leggewie CC, Katzke N, La Cono V, Matesanz R, Jebbar M, Jaeger K-E, Yakimov MM, Yakunin AF, Golyshin PN, Golyshina O V., Savchenko A, Ferrer M, MAMBA Consortium (2015a) Pressure adaptation is linked to thermal adaptation in salt-saturated marine habitats. Environ Microbiol 17:332-345 . doi: 10.1111/14622920.12660

Alcaide M, Tchigvintsev A, Martínez-Martínez M, Popovic A, Reva ON, Lafraya Á, Bargiela R, Nechitaylo TY, Matesanz R, Cambon-Bonavita M-A, Jebbar M, Yakimov MM, Savchenko A, Golyshina O V, Yakunin AF, Golyshin PN, Ferrer M, MAMBA Consortium (2015b) Identification and characterization of carboxyl esterases of gill chamber-associated microbiota in the deep-sea shrimp Rimicaris exoculata by using functional metagenomics. Appl Environ Microbiol 81:2125-2136 . doi: 10.1128/AEM.03387-14

Alcaide M, Tornés J, Stogios PJ, Xu X, Gertler C, Di Leo R, Bargiela R, Lafraya Á, Guazzaroni M-E, López-Cortés N, Chernikova TN, Golyshina OV, Nechitaylo TY, Plumeier I, Pieper DH, Yakimov MM, Savchenko A, Golyshin PN, Ferrer M (2013) Single residues dictate the co-evolution of dual esterases: MCP hydrolases from the $\alpha / \beta$ hydrolase family. Biochem J 454:157-166 . doi: 10.1042/BJ20130552

Alcock BP, Raphenya AR, Lau TTY, Tsang KK, Bouchard M, Edalatmand A, Huynh W, Nguyen AL V., Cheng AA, Liu S, Min SY, Miroshnichenko A, Tran HK, Werfalli RE, Nasir JA, Oloni M, Speicher DJ, Florescu A, Singh B, Faltyn M, Hernandez-Koutoucheva A, Sharma AN, Bordeleau E, Pawlowski AC, Zubyk HL, Dooley D, Griffiths E, Maguire F, Winsor GL, Beiko RG, Brinkman FSL, Hsiao WWL, Domselaar G V., McArthur AG (2020) CARD 2020: Antibiotic resistome surveillance with the comprehensive antibiotic resistance database. Nucleic Acids Res 48:D517-D525 . doi: 10.1093/nar/gkz935

Almeida A, Mitchell AL, Boland M, Forster SC, Gloor GB, Tarkowska A, Lawley TD, Finn RD (2019) A new genomic blueprint of the human gut microbiota. Nature 568:499-504 . doi: 10.1038/s41586-019-0965-1

Altermark B, Helland R, Moe E, Willassen NP, Smalås AO (2008) Structural adaptation of endonuclease I from the cold-adapted and halophilic bacterium Vibrio salmonicida. Acta Crystallogr Sect D Biol Crystallogr 64:368376 . doi: 10.1107/S0907444908000097

Andualema B, Gessesse A (2012) Microbial lipases and their industrial applications: Review. Biotechnology 11:100-118 . doi: 10.3923/biotech.2012.100.118

Angelov A, Mientus M, Liebl S, Liebl W (2009) A two-host fosmid system for functional screening of (meta)genomic libraries from extreme thermophiles. Syst Appl Microbiol 32:177-185 . doi: 10.1016/j.syapm.2008.01.003

Antunes LP, Martins LF, Pereira RV, Thomas AM, Barbosa D, Lemos LN, Silva GMM, Moura LMS, Epamino GWC, Digiampietri LA, Lombardi KC, Ramos PL, Quaggio RB, De Oliveira JCF, Pascon RC, Da Cruz JB, Da Silva AM, Setubal JC (2016) Microbial community structure and dynamics in thermophilic composting viewed through metagenomics and metatranscriptomics. Sci Rep 6:38915 . doi: 10.1038/srep38915 
Armour CR, Nayfach S, Pollard KS, Sharpton TJ (2019) A metagenomic meta-analysis reveals functional signatures of health and disease in the human gut microbiome. mSystems 4:e00332-18 . doi: 10.1128/msystems.0033218

Arpigny JL, Jaeger K-E (1999) Bacterial lipolytic enzymes: classification and properties. Biochem J 343:177-183 . doi: 10.1042/0264-6021:3430177

Awasthi MK, Duan Y, Liu T, Awasthi SK, Zhang Z (2020a) Relevance of biochar to influence the bacterial succession during pig manure composting. Bioresour Technol 304:122962 . doi: 10.1016/j.biortech.2020.122962

Awasthi MK, Ravindran B, Sarsaiya S, Chen H, Wainaina S, Singh E, Liu T, Kumar S, Pandey A, Singh L, Zhang Z (2020b) Metagenomics for taxonomy profiling : tools and approaches. Bioengineered 11:356-374 . doi: 10.1080/21655979.2020.1736238

Aylward FO, Burnum KE, Scott JJ, Suen G, Tringe SG, Adams SM, Barry KW, Nicora CD, Piehowski PD, Purvine SO, Starrett GJ, Goodwin LA, Smith RD, Lipton MS, Currie CR (2012) Metagenomic and metaproteomic insights into bacterial communities in leaf-cutter ant fungus gardens. ISME J 6:1688-1701 . doi: 10.1038/ismej.2012.10

Bailey TL, Boden M, Buske FA, Frith M, Grant CE, Clementi L, Ren J, Li WW, Noble WS (2009) MEME SUITE: tools for motif discovery and searching. Nucleic Acids Res 37:W202-W208 . doi: 10.1093/nar/gkp335

Barriuso J, Jesús Martínez M (2015) In silico metagenomes mining to discover novel esterases with industrial application by sequential search strategies. J Microbiol Biotechnol 25:732-737 . doi: 10.4014/jmb.1406.06049

Bassegoda A, Pastor FIJ, Diaz P (2012) Rhodococcus sp. strain CR-53 LipR, the first member of a new bacterial lipase family (family X) displaying an unusual Y-type oxyanion hole, similar to the Candida antarctica lipase clan. Appl Environ Microbiol 78:1724-1732 . doi: 10.1128/AEM.06332-11

Bayer S, Kunert A, Ballschmiter M, Greiner-Stoeffele T (2010) Indication for a new lipolytic enzyme family: Isolation and characterization of two esterases from a metagenomic library. J Mol Microbiol Biotechnol 18:181-187 . doi: 10.1159/000315459

Bell PJL, Sunna A, Gibbs MD, Curach NC, Nevalainen H, Bergquist PL (2002) Prospecting for novel lipase genes using PCR. Microbiology 148:2283-2291 . doi: 10.1099/00221287-148-8-2283

Bender JM, Bard JDi (2018) Metagenomics in pediatrics: Using a shotgun approach to diagnose infections. Curr Opin Pediatr 30:125-130 . doi: 10.1097/MOP.0000000000000577

Berini F, Casciello C, Marcone GL, Marinelli F (2017) Metagenomics: novel enzymes from non-culturable microbes. FEMS Microbiol Lett 364: . doi: 10.1093/femsle/fnx211

Berlemont R, Spee O, Delsaute M, Lara Y, Schuldes J, Simon C, Power P, Daniel R, Galleni M (2013) Novel organic solvent-tolerant esterase isolated by metagenomics: insights into the lipase/esterase classification. Rev Argent Microbiol 45:3-12

Besir H, Zeth K, Bracher A, Heider U, Ishibashi M, Tokunaga M, Oesterhelt D (2005) Structure of a halophilic nucleoside diphosphate kinase from Halobacterium salinarum. Febs Lett 579:6595-6600 . doi: 10.2210/PDB2AZ1/PDB

Bharagava RN, Purchase D, Saxena G, Mulla SI (2018) Applications of metagenomics in microbial bioremediation of pollutants: from genomics to environmental cleanup. In: Das S, Dash HR (eds) Microbial Diversity in the Genomic Era. Elsevier Inc., Oxford, UK, pp 459-477

Biver S, Vandenbol M (2013) Characterization of three new carboxylic ester hydrolases isolated by functional screening of a forest soil metagenomic library. J Ind Microbiol Biotechnol 40:191-200 . doi: 10.1007/s10295012-1217-7

Boeva V (2016) Analysis of genomic sequence motifs for deciphering transcription factor binding and transcriptional regulation in Eukaryotic cells. Front. Genet. 7:24

Boni I V. (2006) Diverse molecular mechanisms of translation initiation in prokaryotes. Mol Biol 40:587-596 . doi: 10.1134/S002689330604011X

Bora L (2014) Purification and characterization of highly alkaline lipase from bacillus licheniformis MTCC 2465: And study of its detergent compatibility and applicability. J Surfactants Deterg 17:889-898 . doi: 10.1007/s11743-013-1517-6

Borchert E, Selvin J, Kiran SG, Jackson SA, O’Gara F, Dobson ADW (2017) A novel cold active esterase from a deep sea sponge Stelletta normani metagenomic library. Front Mar Sci 4:1-13 . doi: $10.3389 /$ fmars.2017.00287

Bornscheuer UT (2002) Microbial carboxyl esterases: classification, properties and application in biocatalysis. FEMS Microbiol Rev 26:73-81 . doi: 10.1111/j.1574-6976.2002.tb00599.x 
Brady SF (2007) Construction of soil environmental DNA cosmid libraries and screening for clones that produce biologically active small molecules. Nat Protoc 2:1297-1305 . doi: 10.1038/nprot.2007.195

Britton KL, Baker PJ, Fisher M, Ruzheinikov S, Gilmour DJ, Bonete M-J, Ferrer J, Pire C, Esclapez J, Rice DW (2006) Analysis of protein solvent interactions in glucose dehydrogenase from the extreme halophile Haloferax mediterranei. Proc Natl Acad Sci U S A 103:4846-4851 . doi: 10.1073/pnas.0508854103

Brumlik MJ, Buckley JT (1996) Identification of the catalytic triad of the lipase/acyltransferase from Aeromonas hydrophila. J Bacteriol 178:2060-2064 . doi: 10.1128/jb.178.7.2060-2064.1996

Bzhalava Z, Hultin E, Dillner J (2018) Extension of the viral ecology in humans using viral profile hidden Markov models. PLoS One 13:e0190938 . doi: 10.1371/journal.pone.0190938

Cai J, Xie Y, Song B, Wang Y, Zhang Z, Feng Y (2011) Fervidobacterium changbaicum Lip1: Identification, cloning, and characterization of the thermophilic lipase as a new member of bacterial lipase family V. Appl Microbiol Biotechnol 89:1463-1473 . doi: 10.1007/s00253-010-2971-y

Cammarota MC, Rosa DR, Duarte ICS, Saavedra NK, Varesche MBA, Zaiat M, Freire DMG (2013) The effect of enzymatic pre-hydrolysis of dairy wastewater on the granular and immobilized microbial community in anaerobic bioreactors. Environ Technol 34:417-428 . doi: 10.1080/09593330.2012.698649

Cantarel BL, Coutinho PM, Rancurel C, Bernard T, Lombard V, Henrissat B (2009) The Carbohydrate-Active EnZymes database (CAZy): an expert resource for Glycogenomics. Nucleic Acids Res 37:233-238

Castilla A, Panizza P, Rodríguez D, Bonino L, Díaz P, Irazoqui G, Rodríguez Giordano S (2017) A novel thermophilic and halophilic esterase from Janibacter sp. R02, the first member of a new lipase family (Family XVII). Enzyme Microb Technol 98:86-95 . doi: 10.1016/J.ENZMICTEC.2016.12.010

Chandel C, Kumar A, Kanwar SS (2011) Enzymatic synthesis of butyl ferulate by silica-immobilized lipase in a non-aqueous medium. J Biomater Nanobiotechnol 2:400-408 . doi: 10.4236/jbnb.2011.24049

Charbonneau DM, Beauregard M (2013) Role of key salt bridges in thermostability of G. thermodenitrificans EstGtA2: distinctive patterns within the new bacterial lipolytic enzyme family XV. PLoS One 8:e76675 . doi: 10.1371/journal.pone.0076675

Chauhan M, Garlapati VK (2013) Production and characterization of a halo-, solvent-, thermo-tolerant alkaline lipase by staphylococcus arlettae JPBW-1, isolated from rock salt mine. Appl Biochem Biotechnol 171:14291443 . doi: 10.1007/s12010-013-0433-6

Chen IMA, Markowitz VM, Chu K, Palaniappan K, Szeto E, Pillay M, Ratner A, Huang J, Andersen E, Huntemann M, Varghese N, Hadjithomas M, Tennessen K, Nielsen T, Ivanova NN, Kyrpides NC (2017) IMG/M: Integrated genome and metagenome comparative data analysis system. Nucleic Acids Res 45:507-516 . doi: 10.1093/nar/gkw929

Choi JE, Kwon MA, Na HY, Hahm DH, Song JK (2013) Isolation and characterization of a metagenome-derived thermoalkaliphilic esterase with high stability over a broad $\mathrm{pH}$ range. Extremophiles 17:1013-1021 . doi: 10.1007/s00792-013-0583-Z

Chow J, Kovacic F, Dall Antonia Y, Krauss U, Fersini F, Schmeisser C, Lauinger B, Bongen P, Pietruszka J, Schmidt M, Menyes I, Bornscheuer UT, Eckstein M, Thum O, Liese A, Mueller-Dieckmann J, Jaeger KE, Streit WR (2012) The metagenome-derived enzymes LipS and LipT increase the diversity of known lipases. PLoS One 7:e47665 . doi: 10.1371/journal.pone.0047665

Chu X, He H, Guo C, Sun B (2008) Identification of two novel esterases from a marine metagenomic library derived from South China Sea. Appl Microbiol Biotechnol 80:615-625 . doi: 10.1007/s00253-008-1566-3

Consortium TU (2018) UniProt: The universal protein knowledgebase. Nucleic Acids Res 45:D158-D169 . doi: 10.1093/nar/gkw1099

Coquelle N, Talon R, Juers DH, Girard É, Kahn R, Madern D (2010) Gradual adaptive changes of a protein facing high salt concentrations. J Mol Biol 404:493-505 . doi: 10.1016/j.jmb.2010.09.055

Coughlan LM, Cotter PD, Hill C, Alvarez-Ordóñez A (2015) Biotechnological applications of functional metagenomics in the food and pharmaceutical industries. Front Microbiol 6:672 . doi: $10.3389 /$ fmicb. 2015.00672

Craig JW, Chang FY, Kim JH, Obiajulu SC, Brady SF (2010) Expanding small-molecule functional metagenomics through parallel screening of broad-host-range cosmid environmental DNA libraries in diverse proteobacteria. Appl Environ Microbiol 76:1633-1641 . doi: 10.1128/AEM.02169-09

Dandavate V, Jinjala J, Keharia H, Madamwar D (2009) Production, partial purification and characterization of organic solvent tolerant lipase from Burkholderia multivorans V2 and its application for ester synthesis. Bioresour Technol 100:3374-3381 . doi: 10.1016/j.biortech.2009.02.011

Daniel R (2005) The metagenomics of soil. Nat Rev Microbiol 3:470-478 . doi: 10.1038/nrmicro1160 
De Filippis F, Parente E, Ercolini D (2017) Metagenomics insights into food fermentations. Microb Biotechnol 10:91-102 . doi: 10.1111/1751-7915.12421

de Pascale D, Cusano AM, Autore F, Parrilli E, di Prisco G, Marino G, Tutino ML (2008) The cold-active Lip1 lipase from the Antarctic bacterium Pseudoalteromonas haloplanktis TAC125 is a member of a new bacterial lipolytic enzyme family. Extremophiles 12:311-323 . doi: 10.1007/s00792-008-0163-9

De Santi C, Altermark B, Pierechod MM, Ambrosino L, de Pascale D, Willassen N-P (2016a) Characterization of a cold-active and salt tolerant esterase identified by functional screening of Arctic metagenomic libraries. BMC Biochem 17: . doi: 10.1186/s12858-016-0057-x

De Santi C, Leiros HKS, Di Scala A, de Pascale D, Altermark B, Willassen NP (2016b) Biochemical characterization and structural analysis of a new cold-active and salt-tolerant esterase from the marine bacterium Thalassospira sp. Extremophiles 20:323-336 . doi: 10.1007/s00792-016-0824-z

DeCastro ME, Rodríguez-Belmonte E, González-Siso MI (2016) Metagenomics of thermophiles with a focus on discovery of novel thermozymes. Front Microbiol 7:1521 . doi: 10.3389/fmicb.2016.01521

Delgado-García M, Valdivia-Urdiales B, Aguilar-González CN, Contreras-Esquivel JC, Rodríguez-Herrera R (2012) Halophilic hydrolases as a new tool for the biotechnological industries. J Sci Food Agric 92:25752580 . doi: $10.1002 /$ jsfa. 5860

Devi SG, Fathima AA, Radha S, Arunraj R, Curtis WR, Ramya M (2015) A rapid and economical method for efficient DNA extraction from diverse soils suitable for metagenomic applications. PLoS One 10:e 0132441 . doi: 10.1371/journal.pone.0132441

Donato JJ, Moe LA, Converse BJ, Smart KD, Berklein EC, McManus PS, Handelsman J (2010) Metagenomic analysis of apple orchard soil reveals antibiotic resistance genes encoding predicted bifunctional proteins. Appl Environ Microbiol 76:4396-4401 . doi: 10.1128/AEM.01763-09

Dornelas JCM, Figueiredo JEF, De Abreu CS, Lana UGP, Oliveira CA, Marriel IE (2017) Characterization and phylogenetic affiliation of actinobacteria from tropical soils with potential uses for agro-industrial processes. Genet Mol Res 16:1-16 . doi: 10.4238/gmr16039703

Dougherty MJ, D'haeseleer P, Hazen TC, Simmons BA, Adams PD, Hadi MZ (2012) Glycoside hydrolases from a targeted compost metagenome, activity-screening and functional characterization. BMC Biotechnol 12:38 . doi: 10.1186/1472-6750-12-38

Drö Ge J, Gregor I, Mchardy AC (2015) Taxator-tk: precise taxonomic assignment of metagenomes by fast approximation of evolutionary neighborhoods. Bioinformatics 31:817-824 . doi: 10.1093/bioinformatics/btu745

Dukunde A, Schneider D, Lu M, Brady S, Daniel R (2017) A novel, versatile family IV carboxylesterase exhibits high stability and activity in a broad pH spectrum. Biotechnol Lett 39:577-587 . doi: 10.1007/s10529-0162282-1

Dulanto Chiang A, Dekker JP (2020) From the pipeline to the bedside: advances and challenges in clinical metagenomics. J Infect Dis 221:S331-S340 . doi: 10.1093/infdis/jiz151

Ebrahimie E, Ebrahimi M, Sarvestani R, Ebrahimi M (2011) Protein attributes contribute to halo-stability, bioinformatics approach. Saline Systems 7:1 . doi: 10.1186/1746-1448-7-1

Ebrahimpour A, Rahman RNZRA, Basri M, Salleh AB (2011) High level expression and characterization of a novel thermostable, organic solvent tolerant, 1,3-regioselective lipase from Geobacillus sp. strain ARM. Bioresour Technol 102:6972-6981 . doi: 10.1016/j.biortech.2011.03.083

Egelkamp R, Zimmermann T, Schneider D, Hertel R, Daniel R (2019) Impact of nitriles on bacterial communities. Front Environ Sci 7:103 . doi: 10.3389/fenvs.2019.00103

Esteban-Torres M, Santamaría L, de las Rivas B, Muñoz R (2014) Characterisation of a cold-active and salt-tolerant esterase from Lactobacillus plantarum with potential application during cheese ripening. Int Dairy J 39:312315 . doi: 10.1016/j.idairyj.2014.08.004

Fang Y, Wang S, Liu S, Jiao Y (2015) Discovery a novel organic solvent tolerant esterase from Salinispora arenicola CNP193 through genome mining. Int J Biol Macromol 80:334-340 . doi: 10.1016/j.ijbiomac.2015.06.045

Fang Z, Li J, Wang Q, Fang W, Peng H, Zhang X, Xiao Y (2014) A novel esterase from a marine metagenomic library exhibiting salt tolerance ability. J Microbiol Biotechnol 24:771-780 . doi: 10.4014/jmb.1311.11071

Fawal N, Li Q, Savelli B, Brette M, Passaia G, Fabre M, Mathé C, Dunand C (2013) PeroxiBase: A database for large-scale evolutionary analysis of peroxidases. Nucleic Acids Res 41:441-444 . doi: 10.1093/nar/gks1083

Feng Y, Joh Y-G, Ishikawa K, Ishida H, Ando S, Yamagaki T, Nakanishi H, Cao S, Matsui I, Kosugi Y (2000) Thermophilic phospholipase A2 in the cytosolic fraction from the archaeon Pyrococcus horikoshii. J Am Oil Chem Soc 77:1147-1152 . doi: 10.1007/s11746-000-0180-8 
Ferrer M, Martínez-Martínez M, Bargiela R, Streit WR, Golyshina O V., Golyshin PN (2016) Estimating the success of enzyme bioprospecting through metagenomics: Current status and future trends. Microb. Biotechnol. 9:22-34

Ferrer M, Martínez-Martínez M, Bargiela R, Streit WR, Golyshina O V, Golyshin PN (2015) Estimating the success of enzyme bioprospecting through metagenomics: current status and future trends. Microb Biotechnol 9:2234 . doi: 10.1111/1751-7915.12309

Finn RD, Bateman A, Clements J, Coggill P, Eberhardt RY, Eddy SR, Heger A, Hetherington K, Holm L, Mistry J, Sonnhammer ELL, Tate J, Punta M (2014) Pfam: the protein families database. Nucleic Acids Res 42:D222D230 . doi: 10.1093/nar/gkt1223

Fioravanti E, Vellieux FMD, Amara P, Madern D, Weik M (2007) Specific radiation damage to acidic residues and its relation to their chemical and structural environment. J Synchrotron Radiat 14:84-91 . doi: $10.1107 /$ S0909049506038623

Fischer M, Pleiss J (2003) The Lipase Engineering Database: a navigation and analysis tool for protein families. Nucleic Acids Res 31:319-321

Forbes JD, Knox NC, Ronholm J, Pagotto F, Reimer A (2017) Metagenomics: The next culture-independent game changer. Front Microbiol 8:1069 . doi: 10.3389/fmicb.2017.01069

Fu C, Hu Y, Xie F, Guo H, Ashforth EJ, Polyak SW, Zhu B, Zhang L (2011) Molecular cloning and characterization of a new cold-active esterase from a deep-sea metagenomic library. Appl Microbiol Biotechnol 90:961-970 . doi: 10.1007/s00253-010-3079-0

Fu J, Leiros HKS, De Pascale D, Johnson KA, Blencke HM, Landfald B (2013) Functional and structural studies of a novel cold-adapted esterase from an Arctic intertidal metagenomic library. Appl Microbiol Biotechnol 97:3965-3978 . doi: 10.1007/s00253-012-4276-9

Gabor EM, Alkema WBL, Janssen DB (2004) Quantifying the accessibility of the metagenome by random expression cloning techniques. Environ Microbiol 6:879-886 . doi: 10.1111/j.1462-2920.2004.00640.x

Gaida SM, Sandoval NR, Nicolaou SA, Chen Y, Venkataramanan KP, Papoutsakis ET (2015) Expression of heterologous sigma factors enables functional screening of metagenomic and heterologous genomic libraries. Nat Commun 6:1-10 . doi: 10.1038/ncomms8045

Gao W, Wu K, Chen L, Fan H, Zhao Z, Gao B, Wang H, Wei D (2016) A novel esterase from a marine mud metagenomic library for biocatalytic synthesis of short-chain flavor esters. Microb Cell Fact 15:41 . doi: 10.1186/s12934-016-0435-5

Garlapati VK, Banerjee R (2013) Solvent-free synthesis of flavour esters through immobilized lipase mediated transesterification. Enzyme Res 2013: . doi: 10.1155/2013/367410

Garrido-Cardenas JA, Manzano-Agugliaro F (2017) The metagenomics worldwide research. Curr Genet 63:819829 . doi: 10.1007/s00294-017-0693-8

Gaur R, Khare SK (2011) Solvent tolerant Pseudomonads as a source of novel lipases for applications in nonaqueous systems. Biocatal Biotransformation 29:161-171 . doi: 10.3109/10242422.2011.609588

Gerlt JA, Bouvier JT, Davidson DB, Imker HJ, Sadkhin B, Slater DR, Whalen KL (2015) Enzyme Function Initiative-Enzyme Similarity Tool (EFI-EST): A web tool for generating protein sequence similarity networks. Biochim Biophys Acta 1854:1019-1037 . doi: 10.1002/cncr.27633.Percutaneous

Glogauer A, Martini VP, Faoro H, Couto GH, Müller-Santos M, Monteiro RA, Mitchell DA, de Souza EM, Pedrosa FO, Krieger N (2011) Identification and characterization of a new true lipase isolated through metagenomic approach. Microb Cell Fact 10:54 . doi: 10.1186/1475-2859-10-54

Gotor-Fernández V, Brieva R, Gotor V (2006) Lipases: Useful biocatalysts for the preparation of pharmaceuticals. J Mol Catal B Enzym 40:111-120 . doi: 10.1016/j.molcatb.2006.02.010

Guerrand D (2017) Lipases industrial applications: Focus on food and agroindustries. OCL 24:D403 . doi: $10.1051 /$ ocl/2017031

Guo J, Quensen JF, Sun Y, Wang Q, Brown CT, Cole JR, Tiedje JM (2019) Review, evaluation, and directions for gene-targeted assembly for ecological analyses of metagenomes. Front Genet 10:957 . doi: 10.3389/fgene.2019.00957

Handrick R, Reinhardt S, Focarete ML, Scandola M, Adamus G, Kowalczuk M, Jendrossek D (2001) A new type of thermoalkalophilic hydrolase of Paucimonas lemoignei with high specificity for amorphous polyesters of short chain-length hydroxyalkanoic acids. J Biol Chem 276:36215-36224 . doi: 10.1074/jbc.M101106200

Hang Y, Ran S, Wang X, Jiao J, Wang S, Liu Z (2016) Mutational analysis and stability characterization of a novel esterase of lipolytic enzyme family VI from Shewanella sp. Int J Biol Macromol 93:655-664 . doi: 10.1016/j.ijbiomac.2016.09.032 
Hao T, Xie Z, Wang M, Liu L, Zhang Y, Wang W, Zhang Z, Zhao X, Li P, Guo Z, Gao S, Lou C, Zhang G, Merritt J, Horsman GP, Chen Y (2019) An anaerobic bacterium host system for heterologous expression of natural product biosynthetic gene clusters. Nat Commun 10:3665 . doi: 10.1038/s41467-019-11673-0

Hasan F, Shah AA, Hameed A (2006) Industrial applications of microbial lipases. Enzyme Microb Technol 39:235251 . doi: 10.1016/J.ENZMICTEC.2005.10.016

Hausmann S, Jaeger K-E (2010) Lipolytic enzymes from bacteria. In: Timmis KN (ed) Handbook of Hydrocarbon and Lipid Microbiology. Springer Berlin Heidelberg, Berlin, Heidelberg, pp 1099-1126

Heinz S, Benner C, Spann N, Bertolino E, Lin YC, Laslo P, Cheng JX, Murre C, Singh H, Glass CK (2010) Simple combinations of lineage-determining transcription factors prime cis-regulatory elements required for macrophage and B cell identities. Mol Cell 38:576-589 . doi: 10.1016/j.molcel.2010.05.004

Hitch TCA, Clavel T (2019) A proposed update for the classification and description of bacterial lipolytic enzymes. PeerJ 7:e7249 . doi: 10.7717/peerj.7249

Holmes ML, Scopes RK, Moritz RL, Simpson RJ, Englert C, Pfeifer F, Dyall-Smith ML (1997) Purification and analysis of an extremely halophilic beta-galactosidase from Haloferax alicantei. Biochim Biophys Acta 1337:276-286

Horne I, Harcourt RL, Sutherland TD, Russell RJ, Oakeshott JG (2002) Isolation of a Pseudomonas monteilli strain with a novel phosphotriesterase. FEMS Microbiol Lett 206:51-55 . doi: 10.1111/j.1574-6968.2002.tb10985.x

Hotta Y, Ezaki S, Atomi H, Imanaka T (2002) Extremely stable and versatile carboxylesterase from a hyperthermophilic Archaeon. Appl Environ Microbiol 68:3925-3931 . doi: 10.1128/AEM.68.8.39253931.2002

Hu P, Tom L, Singh A, Thomas BC, Baker BJ, Piceno YM, Andersen GL, Banfield JF (2016) Genome-resolved metagenomic analysis reveals roles for candidate phyla and other microbial community members in biogeochemical transformations in oil reservoirs. MBio 7:e01669-15 . doi: 10.1128/mBio.01669-15

Hu Y, Fu C, Huang Y, Yin Y, Cheng G, Lei F, Lu N, Li J, Ashforth EJ, Zhang L, Zhu B (2010) Novel lipolytic genes from the microbial metagenomic library of the South China Sea marine sediment. FEMS Microbiol Ecol 72:228-37 . doi: 10.1111/j.1574-6941.2010.00851.x

Huang Y, Niu B, Gao Y, Fu L, Li W (2010) CD-HIT Suite: a web server for clustering and comparing biological sequences. Bioinformatics 26:680-692 . doi: 10.1093/bioinformatics/btq003

Huerta-Cepas J, Szklarczyk D, Heller D, Hernández-Plaza A, Forslund SK, Cook H, Mende DR, Letunic I, Rattei T, Jensen LJ, Mering C von, Bork P (2019) eggNOG 5.0: a hierarchical, functionally and phylogenetically annotated orthology resource based on 5090 organisms and 2502 viruses. Nucleic Acids Res 47:D309-D314

Hugenholtz P, Tyson GW (2008) Microbiology: Metagenomics. Nature 455:481-483

Hun CJ, Rahman RNZA, Salleh AB, Basri M (2003) A newly isolated organic solvent tolerant Bacillus sphaericus 205y producing organic solvent-stable lipase. Biochem Eng J 15:147-151 . doi: 10.1016/S1369703X(02)00185-7

Huo YY, Rong Z, Jian SL, Xu C Di, Li J xi, Xu XW (2017) A novel halotolerant thermoalkaliphilic esterase from marine bacterium Erythrobacter seohaensis SW-135. Front Microbiol 8:2315 . doi: 10.3389/fmicb.2017.02315

Huson DH, Beier S, Flade I, Górska A, El-Hadidi M, Mitra S, Ruscheweyh H-J, Tappu R (2016) MEGAN community edition - interactive exploration and analysis of large-scale microbiome sequencing data. PLOS Comput Biol 12:e1004957 . doi: 10.1371/journal.pcbi.1004957

Ikeda M, Clark DS (1998) Molecular cloning of extremely thermostable esterase gene from hyperthermophilic archaeon Pyrococcus furiosus in Escherichia coli. Biotechnol Bioeng 57:624-629 . doi: 10.1002/(SICI)10970290(19980305)57:5<624::AID-BIT15>3.0.CO;2-B

Ishibashi M, Hayashi T, Yoshida C, Tokunaga M (2013) Increase of salt dependence of halophilic nucleoside diphosphate kinase caused by a single amino acid substitution. Extremophiles 17:585-591 . doi: 10.1007/s00792-013-0541-9

Jaeger K-E, Dijkstra BW, Reetz MT (1999) Bacterial biocatalysts: Molecular biology, three-dimensional structures, and biotechnological applications of lipases. Annu Rev Microbiol 53:315-351 . doi: 10.1146/annurev.micro.53.1.315

Jaeger KE, Eggert T (2002) Lipases for biotechnology. Curr Opin Biotechnol 13:390-397 . doi: 10.1016/S09581669(02)00341-5

Jaeger KE, Reetz MT (1998) Microbial lipases form versatile tools for biotechnology. Trends Biotechnol 16:396403 . doi: 10.1016/S0167-7799(98)01195-0

Jang E, Ryu BH, Kim TD (2014) Identification, characterization, and immobilization of an organic solvent-stable alkaline hydrolase (PA27) from Pseudomonas aeruginosa MH38. Molecules 19:14396-14405 . doi: 10.3390/molecules 190914396 
Jara-Samaniego J, Pérez-Murcia MD, Bustamante MA, Pérez-Espinosa A, Paredes C, López M, López-Lluch DB, Gavilanes-Terán I, Moral R (2017) Composting as sustainable strategy for municipal solid waste management in the Chimborazo Region, Ecuador: Suitability of the obtained composts for seedling production. J Clean Prod 141:1349-1358 . doi: 10.1016/j.jclepro.2016.09.178

Jayanath G, Mohandas SP, Kachiprath B, Solomon S, Sajeevan TP, Bright Singh IS, Philip R (2018) A novel solvent tolerant esterase of GDSGG motif subfamily from solar saltern through metagenomic approach: Recombinant expression and characterization. Int J Biol Macromol 119:393-401 . doi: 10.1016/j.ijbiomac.2018.06.057

Jensen MB V., Horsfall LE, Wardrope C, Togneri PD, Marles-Wright J, Rosser SJ (2016) Characterisation of a new family of carboxyl esterases with an OsmC domain. PLoS One 11:e0166128 . doi: 10.1371/journal.pone.0166128

Jeon JH, Kim JT, Kim YJ, Kim HK, Lee HS, Kang SG, Kim SJ, Lee JH (2009) Cloning and characterization of a new cold-active lipase from a deep-sea sediment metagenome. Appl Microbiol Biotechnol 81:865-874 . doi: 10.1007/s00253-008-1656-2

Jeon JH, Kim S-J, Lee HS, Cha S-S, Lee JH, Yoon S-H, Koo B-S, Lee C-M, Choi SH, Lee SH, Kang SG, Lee J-H (2011) Novel metagenome-derived carboxylesterase that hydrolyzes $\beta$-lactam antibiotics. Appl Environ Microbiol 77:7830-6 . doi: 10.1128/AEM.05363-11

Jeon JH, Lee HS, Kim JT, Kim SJ, Choi SH, Kang SG, Lee JH (2012) Identification of a new subfamily of salttolerant esterases from a metagenomic library of tidal flat sediment. Appl Microbiol Biotechnol 93:623-631 . doi: 10.1007/s00253-011-3433-x

Jiang H, Zhang S, Gao H, Hu N (2016) Characterization of a cold-active esterase from Serratia sp. and improvement of thermostability by directed evolution. BMC Biotechnol 16: . doi: 10.1186/s12896-016-0235-3

Jiang X, Huo Y, Cheng H, Zhang X, Zhu X, Wu M (2012) Cloning, expression and characterization of a halotolerant esterase from a marine bacterium Pelagibacterium halotolerans B2T. Extremophiles 16:427-435 . doi: 10.1007/s00792-012-0442-3

Jin P, Pei X, Du P, Yin X, Xiong X, Wu H, Zhou X, Wang Q (2012) Overexpression and characterization of a new organic solvent-tolerant esterase derived from soil metagenomic DNA. Bioresour Technol 116:234-240 . doi: 10.1016/j.biortech.2011.10.087

Jochens H, Aerts D, Bornscheuer UT (2010) Thermostabilization of an esterase by alignment-guided focussed directed evolution. Protein Eng Des Sel 23:903-909 . doi: 10.1093/protein/gzq071

Jünemann S, Kleinbölting N, Jaenicke S, Henke C, Hassa J, Nelkner J, Stolze Y, Albaum SP, Schlüter A, Goesmann A, Sczyrba A, Stoye J (2017) Bioinformatics for NGS-based metagenomics and the application to biogas research. J Biotechnol 261:10-23 . doi: 10.1016/j.jbiotec.2017.08.012

Kanehisa M, Sato Y, Kawashima M, Furumichi M, Tanabe M (2016) KEGG as a reference resource for gene and protein annotation. Nucleic Acids Res 44:D457-D462 . doi: 10.1093/nar/gkv1070

Kang CH, Oh KH, Lee MH, Oh TK, Kim BH, Yoon JH (2011) A novel family VII esterase with industrial potential from compost metagenomic library. Microb Cell Fact 10:41 . doi: 10.1186/1475-2859-10-41

Kang HY, Kim JF, Kim MH, Park SH, Oh TK, Hur CG (2006) MELDB: A database for microbial esterases and lipases. FEBS Lett 580:2736-2740 . doi: 10.1016/j.febslet.2006.04.034

Kang LJ, Meng ZT, Hu C, Zhang Y, Guo HL, Li Q, Li M (2017) Screening, purification, and characterization of a novel organic solvent-tolerant esterase, Lip2, from Monascus purpureus strain M7. Extremophiles 21:345355 . doi: 10.1007/s00792-016-0907-X

Karadzic I, Masui A, Zivkovic LI, Fujiwara N (2006) Purification and characterization of an alkaline lipase from Pseudomonas aeruginosa isolated from putrid mineral cutting oil as component of metalworking fluid. J Biosci Bioeng 102:82-89 . doi: 10.1263/jbb.102.82

Karan R, Capes MD, DasSarma S (2012) Function and biotechnology of extremophilic enzymes in low water activity. Aquat Biosyst 8:4 . doi: 10.1186/2046-9063-8-4

Keegan KP, Glass EM, Meyer F (2016) MG-RAST, a metagenomics service for analysis of microbial community structure and function. In: Methods in Molecular Biology. Humana Press Inc., pp 207-233

Kent WJ (2002) BLAT---The BLAST-Like Alignment Tool. Genome Res 12:656-664 . doi: 10.1101/gr.229202

Khan FI, Lan D, Durrani R, Huan W, Zhao Z, Wang Y (2017) The lid domain in lipases: structural and functional determinant of enzymatic properties. Front Bioeng Biotechnol 5:16 . doi: 10.3389/fbioe.2017.00016

Khan M, Sathya TA (2018) Extremozymes from metagenome: Potential applications in food processing. Crit Rev Food Sci Nutr 58:2017-2025 . doi: 10.1080/10408398.2017.1296408

Kim MK, Kang TH, Kim J, Kim H, Yun HD (2012) Cloning and identification of a new group esterase (Est5S) from noncultured rumen bacterium. J Microbiol Biotechnol 22:1044-1053 
Kim S, Joo S, Yoon S, Kim S, Moon J, Ryu Y, Kim KK, Kim TD (2009) Purification, crystallization and preliminary crystallographic analysis of Est-Y29: A novel oligomeric $\beta$-lactamase. Acta Crystallogr Sect F Struct Biol Cryst Commun 65:310-312 . doi: 10.1107/S1744309109005442

Kim TD (2017) Bacterial hormone-sensitive lipases (bHSLs): emerging enzymes for biotechnological applications. J Microbiol Biotechnol 27:1907-1915 . doi: 10.4014/jmb.1708.08004

Kim YH, Kwon EJ, Kim SK, Jeong YS, Kim J, Yun HD, Kim H (2010) Molecular cloning and characterization of a novel family VIII alkaline esterase from a compost metagenomic library. Biochem Biophys Res Commun 393:45-49 . doi: 10.1016/j.bbrc.2010.01.070

Kim YJ, Kim H, Kim SH, Rha E, Choi SL, Yeom SJ, Kim HS, Lee SG (2016) Improved metagenome screening efficiency by random insertion of T7 promoters. J Biotechnol 230:47-53 . doi: 10.1016/j.jbiotec.2016.05.018

Kiran GS, Lipton AN, Kennedy J, Dobson ADW, Selvin J (2014) A halotolerant thermostable lipase from the marine bacterium Oceanobacillus sp. PUMB02 with an ability to disrupt bacterial biofilms. Bioengineered 5:305-318 . doi: 10.4161/bioe.29898

Kixmüller D, Greie JC (2012) Construction and characterization of a gradually inducible expression vector for Halobacterium salinarum, based on the kdp promoter. Appl Environ Microbiol 78:2100-2105 . doi: 10.1128/AEM.07155-11

Knudsen M, Wiuf C (2010) The CATH database. Hum Genomics 4:207-212 . doi: 10.1186/1479-7364-4-3-207

Kobayashi T, Kanai H, Aono R, Horikoshi K, Kudo T (1994) Cloning, expression, and nucleotide sequence of the alpha-amylase gene from the haloalkaliphilic archaeon Natronococcus sp. strain Ah-36. J Bacteriol 176:51315144

Koseki T, Fushinobu S, Ardiansyah, Shirakawa H, Komai M (2009) Occurrence, properties, and applications of feruloyl esterases. Appl Microbiol Biotechnol 84:803-810 . doi: 10.1007/s00253-009-2148-8

Kovacic F, Babic N, Krauss U, Jaeger K (2019) Classification of Lipolytic Enzymes from Bacteria. In: Rojo F (ed) Aerobic Utilization of Hydrocarbons, Oils and Lipids. Handbook of Hydrocarbon and Lipid Microbiology. Springer, Cham, pp 1-35

Kuddus M (2018) Cold-active enzymes in food biotechnology: An updated mini review. J Appl Biol Biotechnol 6:58-63 . doi: 10.7324/JABB.2018.60310

Kumar A, Dhar K, Singh Kanwar S, Arora PK (2016) Lipase catalysis in organic solvents: advantages and applications. Biol Proced Online 18:2 . doi: 10.1186/s12575-016-0033-2

Kumar S, Krishnani KK, Bhushan B, Brahmane MP (2015) Metagenomics: Retrospect and prospects in high throughput age. Biotechnol Res Int 2015:1-13 . doi: 10.1155/2015/121735

Lam KN, Cheng J, Engel K, Neufeld JD, Charles TC (2015) Current and future resources for functional metagenomics. Front Microbiol 6:1196 . doi: 10.3389/fmicb.2015.01196

Lämmle K, Zipper H, Breuer M, Hauer B, Buta C, Brunner H, Rupp S (2007) Identification of novel enzymes with different hydrolytic activities by metagenome expression cloning. J Biotechnol 127:575-592 . doi: 10.1016/j.jbiotec.2006.07.036

Lanyi JK (1974) Salt-dependent properties of proteins from extremely halophilic bacteria. Bacteriol Rev 38:272290

Laudadio I, Fulci V, Stronati L, Carissimi C (2019) Next-generation metagenomics: methodological challenges and opportunities. Omi A J Integr Biol 23:327-333 . doi: 10.1089/omi.2019.0073

Lee CW, Kwon S, Park SH, Kim BY, Yoo W, Ryu BH, Kim HW, Shin SC, Kim S, Park H, Kim TD, Lee JH (2017) Crystal structure and functional characterization of an esterase (EaEST) from Exiguobacterium antarcticum. PLoS One 12:e0169540 . doi: 10.1371/journal.pone.0169540

Lee D-W, Koh Y-S, Kim K-J, Kim B-C, Choi H-J, Kim D-S, Suhartono MT, Pyun Y-R (1999) Isolation and characterization of a thermophilic lipase from Bacillus thermoleovorans ID-1. FEMS Microbiol Lett 179:393400 . doi: 10.1111/j.1574-6968.1999.tb08754.x

Lee JH, Jeon JH, Kim JT, Lee HS, Kim SJ, Kang SG, Choi SH (2011) Novel lipolytic enzymes identified from metagenomic library of deep-sea sediment. Evidence-based Complement Altern Med 2011: . doi: $10.1155 / 2011 / 271419$

Lee M-H, Oh K-H, Kang C-H, Kim J-H, Oh T-K, Ryu C-M, Yoon J-H (2012) Novel metagenome-derived, coldadapted alkaline phospholipase with superior lipase activity as an intermediate between phospholipase and lipase. Appl Environ Microbiol 78:4959-4966 . doi: 10.1128/aem.00260-12

Lee MH, Hong KS, Malhotra S, Park J-H, Hwang EC, Choi HK, Kim YS, Tao W, Lee S-W (2010) A new esterase EstD2 isolated from plant rhizosphere soil metagenome. Appl Microbiol Biotechnol 88:1125-1134 . doi: $10.1007 / \mathrm{s} 00253-010-2729-6$ 
Lee MH, Lee CH, Oh TK, Song JK, Yoon JH (2006) Isolation and characterization of a novel lipase from a metagenomic library of tidal flat sediments: Evidence for a new family of bacterial lipases. Appl Environ Microbiol 72:7406-7409 . doi: 10.1128/AEM.01157-06

Lee SW, Won K, Lim HK, Kim JC, Choi GJ, Cho KY (2004) Screening for novel lipolytic enzymes from uncultured soil microorganisms. Appl Microbiol Biotechnol 65:720-726 . doi: 10.1007/s00253-004-1722-3

Lei J, Miao Y, Lan Y, Han X, Liu H, Gan Y, Niu L, Wang Y, Zheng Z (2018) A novel complementation assay for quick and specific screen of genes encoding glycerol-3-phosphate acyltransferases. Front Plant Sci 9:353 . doi: 10.3389/fpls.2018.00353

Leis B, Angelov A, Mientus M, Li H, Pham VTT, Lauinger B, Bongen P, Pietruszka J, Gonçalves LG, Santos H, Liebl W (2015) Identification of novel esterase-active enzymes from hot environments by use of the host bacterium Thermus thermophilus. Front Microbiol 6:275 . doi: 10.3389/fmicb.2015.00275

Leite AET, Briganti L, de Araújo EA, Pellegrini V de OA, Camilo CM, Polikarpov I (2020) Low-resolution molecular shape, biochemical characterization and emulsification properties of a halotolerant esterase from Bacillus licheniformis. Eur Biophys J 49:435-447 . doi: 10.1007/s00249-020-01448-7

Lenfant N, Hotelier T, Velluet E, Bourne Y, Marchot P, Chatonnet A (2013) ESTHER, the database of the $\alpha / \beta$ hydrolase fold superfamily of proteins: tools to explore diversity of functions. Nucleic Acids Res 41:423-429 . doi: $10.1093 /$ nar/gks1154

Levisson M, Van Der Oost J, Kengen SWM (2007) Characterization and structural modeling of a new type of thermostable esterase from Thermotoga maritima. FEBS J 274:2832-2842 . doi: 10.1111/j.17424658.2007.05817.x

Li D, Liu CM, Luo R, Sadakane K, Lam TW (2015a) MEGAHIT: An ultra-fast single-node solution for large and complex metagenomics assembly via succinct de Bruijn graph. Bioinformatics 31:1674-1676 . doi: 10.1093/bioinformatics/btv033

Li H, Han X, Qiu W, Xu D, Wang Y, Yu M, Hu X, Zhuo R (2019) Identification and expression analysis of the GDSL esterase/lipase family genes, and the characterization of SaGLIP8 in Sedum alfredii Hance under cadmium stress. PeerJ 7:e6741 . doi: 10.7717/peerj.6741

Li J, Zhang K, Han W (2010) Cloning and biochemical characterization of a novel lipolytic gene from activated sludge metagenome, and its gene product. Microb Cell Fact 9:83 . doi: 10.1186/1475-2859-9-83

Li LL, McCorkle SR, Monchy S, Taghavi S, van der Lelie D (2009) Bioprospecting metagenomes: Glycosyl hydrolases for converting biomass. Biotechnol Biofuels 2:1-11 . doi: 10.1186/1754-6834-2-10

Li M, Yang LR, Xu G, Wu JP (2016) Cloning and characterization of a novel lipase from Stenotrophomonas maltophilia GS11: The first member of a new bacterial lipase family XVI. J Biotechnol 228:30-36 . doi: 10.1016/j.jbiotec.2016.04.034

Li M, Yang LR, Xu G, Wu JP (2013) Screening, purification and characterization of a novel cold-active and organic solvent-tolerant lipase from Stenotrophomonas maltophilia CGMCC 4254. Bioresour Technol 148:114-120 . doi: 10.1016/j.biortech.2013.08.101

Li P-Y, Zhang Y, Xie B-B, Zhang Y-Q, Hao J, Wang Y, Wang P, Li C-Y, Qin Q-L, Zhang X-Y, Su H-N, Shi M, Zhang Y-Z, Chen X-L (2017a) Structural and mechanistic insights into the improvement of the halotolerance of a marine microbial esterase by increasing intra- and interdomain hydrophobic interactions. Appl Environ Microbiol 83:e01286-17 . doi: 10.1128/AEM.01286-17

Li P, Chen X, Ji P, Li C, Wang P, Zhang Y, Xie B, Qin Q, Su H, Zhou B, Zhang Y, Zhang X (2015b) Interdomain hydrophobic interactions modulate the thermostability of microbial esterases from the hormone-sensitive lipase family. J Biol Chem 290:11188-11198 . doi: 10.1074/jbc.M115.646182

Li PY, Ji P, Li CY, Zhang Y, Wang GL, Zhang XY, Xie B Bin, Qin QL, Chen XL, Zhou BC, Zhang YZ (2014) Structural basis for dimerization and catalysis of a novel sterase from the GTSAG motif subfamily of the bacterial hormone-sensitive lipase family. J Biol Chem 289:19031-19041 . doi: 10.1074/jbc.M114.574913

Li PY, Yao QQ, Wang P, Zhang Y, Li Y, Zhang YQ, Hao J, Zhou BC, Chen XL, Shi M, Zhang YZ, Zhang XY (2017b) A novel subfamily esterase with a homoserine transacetylase-like fold but no transferase activity. Appl Environ Microbiol 83:e0131-17 . doi: 10.1128/AEM.00131-17

Liebl W, Angelov A, Juergensen J, Chow J, Loeschcke A, Drepper T, Classen T, Pietruzska J, Ehrenreich A, Streit WR, Jaeger KE (2014) Alternative hosts for functional (meta)genome analysis. Appl. Microbiol. Biotechnol. 98:8099-8109

Lima VMG, Krieger N, Mitchell DA, Fontana JD (2004) Activity and stability of a crude lipase from Penicillium aurantiogriseum in aqueous media and organic solvents. Biochem Eng J 18:65-71 . doi: 10.1016/S1369$703 \times(03) 00165-7$ 
Liu K, Wang J, Bu D, Zhao S, McSweeney C, Yu P, Li D (2009) Isolation and biochemical characterization of two lipases from a metagenomic library of China Holstein cow rumen. Biochem Biophys Res Commun 385:605611 . doi: 10.1016/j.bbrc.2009.05.110

Liu SP, Liu RX, Zhang L, Shi GY (2015) Sequence-based screening and characterization of cytosolic mandelate oxidase using oxygen as electron acceptor. Enzyme Microb Technol 69:24-30 . doi: 10.1016/j.enzmictec.2014.11.001

Liu YF, Galzerani DD, Mbadinga SM, Zaramela LS, Gu JD, Mu BZ, Zengler K (2018) Metabolic capability and in situ activity of microorganisms in an oil reservoir. Microbiome 6:5 . doi: 10.1186/s40168-017-0392-1

Lopez-Lopez O, Cerdan M, Siso M (2014) New extremophilic lipases and esterases from metagenomics. Curr Protein Pept Sci 15:445-455 . doi: 10.2174/1389203715666140228153801

López-López O, Knapik K, Cerdán ME, González-Siso MI (2015) Metagenomics of an alkaline hot spring in Galicia (Spain): Microbial diversity analysis and screening for novel lipolytic enzymes. Front Microbiol 6:1291 . doi: 10.3389/fmicb.2015.01291

Lu M, Daniel R (2021) A novel carboxylesterase derived from a compost metagenome exhibiting high stability and activity towards high salinity. Genes (Basel) 12:1-18 . doi: 10.3390/genes 12010122

Lu M, Dukunde A, Daniel R (2019) Biochemical profiles of two thermostable and organic solvent-tolerant esterases derived from a compost metagenome. Appl Microbiol Biotechnol 103:3421-3437 . doi: 10.1007/s00253-01909695-1

Lu M, Schneider D, Daniel R (2021) Metagenomic screening for lipolytic genes reveals an ecology-clustered distribution pattern. bioRxiv 2021.02.01.429111 . doi: 10.1101/2021.02.01.429111

Luan Z-J, Yu H-L, Ma B-D, Qi Y-K, Chen Q, Xu J-H (2016) Dramatically improved performance of an esterase for cilastatin synthesis by cap domain engineering. Ind Eng Chem Res 55:12167-12172 . doi: 10.1021/acs.iecr.6b02440

Ma S, Fang C, Sun X, Han L, He X, Huang G (2018) Bacterial community succession during pig manure and wheat straw aerobic composting covered with a semi-permeable membrane under slight positive pressure. Bioresour Technol 259:221-227 . doi: 10.1016/j.biortech.2018.03.054

Madhavan A, Sindhu R, Parameswaran B, Sukumaran RK, Pandey A (2017) Metagenome analysis: a powerful tool for enzyme bioprospecting. Appl Biochem Biotechnol 183:636-651 . doi: 10.1007/s12010-017-2568-3

Mahapatra GP, Raman S, Nayak S, Gouda S, Das G, Patra JK (2020) Metagenomics approaches in discovery and development of new bioactive compounds from marine Actinomycetes. Curr Microbiol 77:645-656 . doi: 10.1007/s00284-019-01698-5

Mandrich L, Menchise V, Alterio V, De Simone G, Pedone C, Rossi M, Manco G (2008) Functional and structural features of the oxyanion hole in a thermophilic esterase from Alicyclobacillus acidocaldarius. Proteins Struct Funct Genet 71:1721-1731 . doi: 10.1002/prot.21877

Mandrich L, Merone L, Pezzullo M, Cipolla L, Nicotra F, Rossi M, Manco G (2005) Role of the N terminus in enzyme activity, stability and specificity in thermophilic esterases belonging to the HSL family. J Mol Biol 345:501-512 . doi: 10.1016/j.jmb.2004.10.035

Mao H, Lv Z, Sun H, Li R, Zhai B, Wang Z, Awasthi MK, Wang Q, Zhou L (2018) Improvement of biochar and bacterial powder addition on gaseous emission and bacterial community in pig manure compost. Bioresour Technol 258:195-202 . doi: 10.1016/j.biortech.2018.02.082

Markel U, Essani KD, Besirlioglu V, Schiffels J, Streit WR, Schwaneberg U (2020) Advances in ultrahighthroughput screening for directed enzyme evolution. Chem Soc Rev 49:233-262 . doi: 10.1039/c8cs00981c

Maruthamuthu M, Jiménez DJ, Stevens P, van Elsas JD (2016) A multi-substrate approach for functional metagenomics-based screening for (hemi)cellulases in two wheat straw-degrading microbial consortia unveilsnovel thermoalkaliphilic enzymes. BMC Genomics 17:1-16 . doi: 10.1186/s12864-016-2404-0

McArthur AG, Waglechner N, Nizam F, Yan A, Azad MA, Baylay AJ, Bhullar K, Canova MJ, De Pascale G, Ejim L, Kalan L, King AM, Koteva K, Morar M, Mulvey MR, O’Brien JS, Pawlowski AC, Piddock LJV, Spanogiannopoulos P, Sutherland AD, Tang I, Taylor PL, Thaker M, Wang W, Yan M, Yu T, Wright GD (2013) The comprehensive antibiotic resistance database. Antimicrob Agents Chemother 57:3348-3357 . doi: 10.1128/AAC.00419-13

McMahon MD, Guan C, Handelsman J, Thomas MG (2012) Metagenomic analysis of Streptomyces lividans reveals host-dependent functional expression. Appl Environ Microbiol 78:3622-3629 . doi: 10.1128/AEM.00044-12

Meng Q, Yang W, Men M, Bello A, Xu X, Xu B, Deng L, Jiang X, Sheng S, Wu X, Han Y, Zhu H (2019) Microbial community succession and response to environmental variables during cow manure and corn straw composting. Front Microbiol 10:529 . doi: 10.3389/fmicb.2019.00529 
Meng Y, Li S, Yuan H, Zou D, Liu Y, Zhu B, Li X (2015) Effect of lipase addition on hydrolysis and biomethane production of Chinese food waste. Bioresour Technol 179:452-459 . doi: 10.1016/j.biortech.2014.12.015

Menzel P, Ng KL, Krogh A (2016) Fast and sensitive taxonomic classification for metagenomics with Kaiju. Nat Commun 7:11257 . doi: 10.1038/ncomms11257

Metin K, Burcu Bakir Ateslier Z, Basbulbul G, Halil Biyik H (2006) Characterization of esterase activity in Geobacillus sp. HBB-4. J Basic Microbiol 46:400-409 . doi: 10.1002/jobm.200510121

Miller DN, Bryant JE, Madsen EL, Ghiorse WC (1999) Evaluation and optimization of DNA extraction and purification procedures for soil and sediment samples. Appl Environ Microbiol 65:4715-4724 . doi: 10.1128/aem.65.11.4715-4724.1999

Minegishi H, Shimane Y, Echigo A, Ohta Y, Hatada Y, Kamekura M, Maruyama T, Usami R (2013) Thermophilic and halophilic $\beta$-agarase from a halophilic archaeon Halococcus sp. 197A. Extremophiles 17:931-939 . doi: 10.1007/s00792-013-0575-z

Mirete S, Morgante V, González-Pastor JE (2016) Functional metagenomics of extreme environments. Curr Opin Biotechnol 38:143-149 . doi: 10.1016/j.copbio.2016.01.017

Misawa E, Chan Kwo Chion CKC, Archer I V., Woodland MP, Zhou N-Y, Carter SF, Widdowson DA, Leak DJ (1998) Characterisation of a catabolic epoxide hydrolase from a Corynebacterium sp. Eur J Biochem 253:173183 . doi: 10.1046/j.1432-1327.1998.2530173.x

Mohamed YM, Ghazy MA, Sayed A, Ouf A, El-Dorry H, Siam R (2013) Isolation and characterization of a heavy metal-resistant, thermophilic esterase from a Red Sea Brine Pool. Sci Rep 3:3358 . doi: 10.1038/srep03358

Mølgaard A, Kauppinen S, Larsen S (2000) Rhamnogalacturonan acetylesterase elucidates the structure and function of a new family of hydrolases. Structure 8:373-383 . doi: 10.1016/S0969-2126(00)00118-0

Montoro-García S, Martínez-Martínez I, Navarro-Fernández J, Takami H, García-Carmona F, Sánchez-Ferrer Á (2009) Characterization of a novel thermostable carboxylesterase from Geobacillus kaustophilus HTA426 shows the existence of a new carboxylesterase family. J Bacteriol 191:3076-3085 . doi: 10.1128/JB.01060-08

Moreno-Indias I, Tinahones FJ (2020) Metagenomics. Princ Nutr Nutr 81-87 . doi: 10.1016/b978-0-12-8045725.00011-2

Moriya T, Hikota T, Yumoto I, Ito T, Terui Y, Yamagishi A, Oshima T (2011) Calditerricola satsumensis gen. nov., sp. nov. and Calditerricola yamamurae sp. nov., extreme thermophiles isolated from a high-temperature compost. Int J Syst Evol Microbiol 61:631-636 . doi: 10.1099/ijs.0.018416-0

Munawar N, Engel PC (2013) Halophilic enzymes: Characteristics, structural adaptation and potential applications for biocatalysis. Curr Biotechnol 2:334-344 . doi: 10.2174/18722083113076660033

Munawar N, Engel PC (2012) Overexpression in a non-native halophilic host and biotechnological potential of NAD+-dependent glutamate dehydrogenase from Halobacterium salinarum strain NRC-36014. Extremophiles 16:463-476 . doi: 10.1007/s00792-012-0446-z

Musa H, Kasim FH, Gunny AAN, Gopinath SCB, Ahmad MA (2018) Biosecretion of higher halophilic lipase by a novel Bacillus amyloliquefaciens AIKK2 using agro-waste as supporting substrate. Process Biochem 72:5562 . doi: 10.1016/j.procbio.2018.06.022

Nacke H, Will C, Herzog S, Nowka B, Engelhaupt M, Daniel R (2011) Identification of novel lipolytic genes and gene families by screening of metagenomic libraries derived from soil samples of the German Biodiversity Exploratories. FEMS Microbiol Ecol 78:188-201 . doi: 10.1111/j.1574-6941.2011.01088.x

Nagamine S, Liu C, Nishishita J, Kozaki T, Sogahata K, Sato Y, Minami A, Ozaki T, Schmidt-Dannert C, Maruyama J ichi, Oikawa H (2019) Ascomycete Aspergillus oryzae is an efficient expression host for production of basidiomycete terpenes by using genomic DNA sequences. Appl Environ Microbiol 85:e040919. doi: 10.1128/AEM.00409-19

Nakamura AM, Kadowaki MAS, Godoy A, Nascimento AS, Polikarpov I (2018) Low-resolution envelope, biophysical analysis and biochemical characterization of a short-chain specific and halotolerant carboxylesterase from Bacillus licheniformis. Int J Biol Macromol 120:1893-1905 . doi: 10.1016/j.ijbiomac.2018.10.003

Nam KH, Kim MY, Kim SJ, Priyadarshi A, Lee WH, Hwang KY (2009) Structural and functional analysis of a novel EstE5 belonging to the subfamily of hormone-sensitive lipase. Biochem Biophys Res Commun 379:553-556 . doi: 10.1016/j.bbrc.2008.12.085

Namiki T, Hachiya T, Tanaka H, Sakakibara Y (2012) MetaVelvet: An extension of Velvet assembler to de novo metagenome assembly from short sequence reads. Nucleic Acids Res 40:e155 . doi: 10.1093/nar/gks678

Neher DA, Weicht TR, Bates ST, Leff JW, Fierer N (2013) Changes in bacterial and fungal communities across compost recipes, preparation methods, and composting times. PLoS One 8:e79512 . doi: 10.1371/journal.pone.0079512 
Nerurkar M, Joshi M, Pariti S, Adivarekar R (2013) Application of lipase from marine bacteria Bacillus sonorensis as an additive in detergent formulation. J Surfactants Deterg 16:435-443 . doi: 10.1007/s11743-012-1434-0

Neves Petersen MT, Fojan P, Petersen SB (2001) How do lipases and esterases work: The electrostatic contribution. J Biotechnol 85:115-147 . doi: 10.1016/S0168-1656(00)00360-6

Ngara TR, Zhang H (2018) Recent advances in function-based metagenomic screening. Genomics, Proteomics Bioinforma. 16:405-415

Noby N, Hussein A, Saeed H, Embaby AM (2020) Recombinant cold-adapted halotolerant, organic solvent-stable esterase (estHIJ) from Bacillus halodurans. Anal Biochem 591:113554 . doi: 10.1016/j.ab.2019.113554

Nurk S, Meleshko D, Korobeynikov A, Pevzner PA (2017) MetaSPAdes: A new versatile metagenomic assembler. Genome Res 27:824-834 . doi: 10.1101/gr.213959.116

Ogino H, Ishikawa H (2001) Enzymes which are stable in the presence of organic solvents. J Biosci Bioeng 91:109116

Ogino H, Mimitsuka T, Muto T, Matsumura M, Yasuda M, Ishimi K, Ishikawa H (2004) Cloning, expression, and characterization of a lipolytic enzyme gene (lip8) from Pseudomonas aeruginosa LST-03. J Mol Microbiol Biotechnol 7:212-223 . doi: 10.1159/000079830

Ollis DL, Cheah E, Cygler M, Dijkstra B, Frolow F, Franken SM, Harel M, Remington SJ, Silman I, Schrag J, Sussman JL, Verschueren KHG, Goldman A (1992) The $\alpha / \beta$ hydrolase fold. Protein Eng Des Sel 5:197-211 . doi: 10.1093/protein/5.3.197

Palaniveloo K, Amran MA, Norhashim NA, Mohamad-Fauzi N, Peng-Hui F, Hui-Wen L, Kai-Lin Y, Jiale L, Chian-Yee MG, Jing-Yi L, Gunasekaran B, Razak SA (2020) Food waste composting and microbial community structure profiling. Processes 8:723 . doi: 10.3390/pr8060723

Panda T, Gowrishankar BS (2005) Production and applications of esterases. Appl Microbiol Biotechnol 67:160169 . doi: 10.1007/s00253-004-1840-y

Parapouli M, Foukis A, Stergiou P-Y, Koukouritaki M, Magklaras P, Gkini OA, Papamichael EM, Afendra A-S, Hatziloukas E (2018) Molecular, biochemical and kinetic analysis of a novel, thermostable lipase (LipSm) from Stenotrophomonas maltophilia Psi-1, the first member of a new bacterial lipase family (XVIII). J Biol Res 25:4 . doi: 10.1186/s40709-018-0074-6

Park SY, Kim JT, Kang SG, Woo JH, Lee JH, Choi HT, Kim SJ (2007) A new esterase showing similarity to putative dienelactone hydrolase from a strict marine bacterium, Vibrio sp. GMD509. Appl Microbiol Biotechnol 77:107-115 . doi: 10.1007/s00253-007-1134-2

Patel U, Chandpura J, Chauhan K, Gupte S (2018) Screening and isolation of an organic solvent tolerant lipase producing bacteria from various oil contaminated sites. Indian J Appl Microbiol 21:22-36

Pehrsson EC, Tsukayama P, Patel S, Mejía-Bautista M, Sosa-Soto G, Navarrete KM, Calderon M, Cabrera L, Hoyos-Arango W, Bertoli MT, Berg DE, Gilman RH, Dantas G (2016) Interconnected microbiomes and resistomes in low-income human habitats. Nature 533:212-216 . doi: 10.1038/nature17672

Peng Y, Leung HCM, Yiu SM, Chin FYL (2011) Meta-IDBA: A de Novo assembler for metagenomic data. Bioinformatics 27:94-101 . doi: 10.1093/bioinformatics/btr216

Peng Y, Leung HCM, Yiu SM, Chin FYL (2012) IDBA-UD: A de novo assembler for single-cell and metagenomic sequencing data with highly uneven depth. Bioinformatics 28:1420-1428 . doi: 10.1093/bioinformatics/bts 174

Pereira MR, Mercaldi GF, Maester TC, Balan A, de Macedo Lemos EG (2015) Est16, a new esterase isolated from a metagenomic library of a microbial consortium specializing in diesel oil degradation. PLoS One 10:e0133723 . doi: 10.1371/journal.pone.0133723

Petrovskaya LE, Novototskaya-Vlasova KA, Spirina E V., Durdenko E V., Lomakina GY, Zavialova MG, Nikolaev EN, Rivkina EM (2016) Expression and characterization of a new esterase with GCSAG motif from a permafrost metagenomic library. FEMS Microbiol Ecol 92:fiw046 . doi: 10.1093/femsec/fiw046

Pezzullo M, Del Vecchio P, Mandrich L, Nucci R, Rossi M, Manco G (2013) Comprehensive analysis of surface charged residues involved in thermal stability in Alicyclobacillus acidocaldarius esterase 2. Protein Eng Des Sel 26:47-58 . doi: 10.1093/protein/gzs066

Pieper U, Kapadia G, Mevarech M, Herzberg O (1998) Structural features of halophilicity derived from the crystal structure of dihydrofolate reductase from the Dead Sea halophilic archaeon, Haloferax volcanii. Structure 6:75-88 . doi: 10.2210/PDB1VDR/PDB

Poidevin L, MacNeill SA (2006) Biochemical characterisation of LigN, an NAD+-dependent DNA ligase from the halophilic euryarchaeon Haloferax volcanii that displays maximal in vitro activity at high salt concentrations. BMC Mol Biol 7:44 . doi: 10.1186/1471-2199-7-44

Pratama R, Schneider D, Böer T, Daniel R (2019) First insights into bacterial gastrointestinal tract communities of the Eurasian beaver (Castor fiber). Front Microbiol 10:1646 . doi: 10.3389/fmicb.2019.01646 
Qin Q-L, Zhao D-L, Wang J, Chen X-L, Dang H-Y, Li T-G, Zhang Y-Z, Gao P-J (2007) Wangia profunda gen. nov., sp. nov., a novel marine bacterium of the family Flavobacteriaceae isolated from southern Okinawa Trough deep-sea sediment. FEMS Microbiol Lett 271:53-58 . doi: 10.1111/j.1574-6968.2007.00694.X

Quast C, Pruesse E, Yilmaz P, Gerken J, Schweer T, Yarza P, Peplies J, Glöckner FO (2013) The SILVA ribosomal RNA gene database project: Improved data processing and web-based tools. Nucleic Acids Res 41:D590D596 . doi: 10.1093/nar/gks1219

Quince C, Walker AW, Simpson JT, Loman NJ, Segata N (2017) Shotgun metagenomics, from sampling to analysis. Nat Biotechnol 35:833-844 . doi: 10.1038/nbt.3935

Rabausch U, Juergensen J, Ilmberger N, Böhnke S, Fischer S, Schubach B, Schulte M, Streit W V. (2013) Functional screening of metagenome and genome libraries for detection of novel flavonoid-modifying enzymes. Appl Environ Microbiol 79:4551-4563 . doi: 10.1128/AEM.01077-13

Rahman MA, Culsum U, Tang W, Zhang SW, Wu G, Liu Z (2016) Characterization of a novel cold active and salt tolerant esterase from Zunongwangia profunda. Enzyme Microb Technol 85:1-11 . doi: 10.1016/j.enzmictec.2015.12.013

Rajendran A, Palanisamy A, Thangavelu V (2009) Lipase catalyzed ester synthesis for food processing industries. Brazilian Arch Biol Technol 52:207-219 . doi: 10.1590/S1516-89132009000100026

Ramnath L, Sithole B, Govinden R (2016) Classification of lipolytic enzymes and their biotechnological applications in the pulping industry. Can J Microbiol 63:179-192 . doi: 10.1139/cjm-2016-0447

Rao L, Xue Y, Zhou C, Tao J, Li G, Lu JR, Ma Y (2011) A thermostable esterase from Thermoanaerobacter tengcongensis opening up a new family of bacterial lipolytic enzymes. Biochim Biophys Acta - Proteins Proteomics 1814:1695-1702 . doi: 10.1016/j.bbapap.2011.08.013

Rao L, Zhao X, Li Y, Xue Y, Ma Y, Lu JR (2009a) Solution behavior and activity of a halophilic esterase under high salt concentration. PLoS One 4:e6980 . doi: 10.1371/journal.pone.0006980

Rao L, Zhao X, Pan F, Li Y, Xue Y, Ma Y, Lu JR (2009b) Solution behavior and activity of a halophilic esterase under high salt concentration. PLoS One 4:e6980 . doi: 10.1371/journal.pone.0023325

Rao L, Zhao X, Pan F, Li Y, Xue Y, Ma Y, Lu JR (2009c) Solution behavior and activity of a halophilic esterase under high salt concentration. PLoS One 4:e6980 . doi: 10.1371/journal.pone.0006980

Rastogi M, Nandal M, Khosla B (2020) Microbes as vital additives for solid waste composting. Heliyon 6:e03343 . doi: 10.1016/j.heliyon.2020.e03343

Rathi P, Bradoo S, Saxena RK, Gupta R (2000) A hyper-thermostable, alkaline lipase from Pseudomonas sp. with the property of thermal activation. Biotechnol Lett 22:495-498 . doi: 10.1023/A:1005604617440

Rebollido R, Martínez J, Aguilera Y, Melchor K, Koerner I, Stegmann R (2008) Microbial populations during composting process of organic fraction of municipal solid waste. Appl Ecol Environ Res 6:61-67

Reyes-Duarte D, Ferrer M, García-Arellano H (2012) Functional-based screening methods for lipases, esterases, and phospholipases in metagenomic libraries. Methods Mol Biol 861:101-113 . doi: 10.1007/978-1-61779-60056

Reyes A, Alves JMP, Durham AM, Gruber A (2017) Use of profile hidden Markov models in viral discovery: current insights. Adv Genomics Genet 7:29-45 . doi: 10.2147/AGG.S136574

Rhee J, Ahn D, Kim Y, Oh J (2005) New thermophilic and thermostable esterase with sequence similarity to the hormone-sensitive lipase family, cloned from a metagenomic library. Appl Environ Microbiol 71:817-825 . doi: 10.1128/AEM.71.2.817

Richard SB, Madern D, Garcin E, Zaccai G (2000) Halophilic adaptation: novel solvent protein interactions observed in the 2.9 and 2.6 A resolution structures of the wild type and a mutant of malate dehydrogenase from Haloarcula marismortui. Biochemistry 39:992-1000

Rigoldi F, Donini S, Redaelli A, Parisini E, Gautieri A (2018) Review: Engineering of thermostable enzymes for industrial applications. APL Bioeng 2:011501 . doi: 10.1063/1.4997367

Rodríguez MC, Loaces I, Amarelle V, Senatore D, Iriarte A, Fabiano E, Noya F (2015) Est10: A novel alkaline esterase isolated from bovine rumen belonging to the new family XV of lipolytic enzymes. PLoS One 10:e0126651 . doi: 10.1371/journal.pone.0126651

Romdhane IB-B, Fendri A, Gargouri Y, Gargouri A, Belghith H (2010) A novel thermoactive and alkaline lipase from Talaromyces thermophilus fungus for use in laundry detergents. Biochem Eng J 53:112-120 . doi: 10.1016/J.BEJ.2010.10.002

Rong Z, Huo Y-Y, Jian S-L, Wu Y-H, Xu X-W (2018) Characterization of a novel alkaline esterase from Altererythrobacter epoxidivorans CGMCC 1.7731 T. Prep Biochem Biotechnol 48:113-120 . doi: $10.1080 / 10826068.2017 .1387559$ 
Rosenstein R, Götz F (2000) Staphylococcal lipases: Biochemical and molecular characterization. Biochimie 82:1005-1014

Ryckeboer J, Mergaert J, Vaes K, Klammer S, Clercq D, Coosemans J, Insam H, Swings J (2003) A survey of bacteria and fungi occurring during composting and self-heating processes. Ann Microbiol 53:349-410

Sabri S, Rahman RNZRA, Leow TC, Basri M, Salleh AB (2009) Secretory expression and characterization of a highly Ca2+-activated thermostable L2 lipase. Protein Expr Purif 68:161-166 . doi: 10.1016/j.pep.2009.08.002

Sadeghi M, Naderi-Manesh H, Zarrabi M, Ranjbar B (2006) Effective factors in thermostability of thermophilic proteins. Biophys Chem 119:256-270 . doi: 10.1016/j.bpc.2005.09.018

Salihu A, Alam MZ (2015) Solvent tolerant lipases: A review. Process Biochem 50:86-96 . doi: 10.1016/j.procbio.2014.10.019

Samoylova Y V., Sorokina KN, Piligaev A V., Parmon VN (2019) Application of bacterial thermostable lipolytic enzymes in the modern biotechnological processes: A review. Catal Ind 11:168-178 . doi: $10.1134 / \mathrm{S} 2070050419020107$

Samoylova Y V., Sorokina KN, Romanenko M V., Parmon VN (2018) Cloning, expression and characterization of the esterase estUT1 from Ureibacillus thermosphaericus which belongs to a new lipase family XVIII. Extremophiles 22:271-285 . doi: 10.1007/s00792-018-0996-9

Santi C De, Zhai L, Ambrosino L, Tedesco P, Zhou C, Xue Y, Ma Y, Pascale D de (2015) Identification and characterization of a novel salt-tolerant esterase from a Tibetan glacier metagenomic library. Biotechnol Prog 31:890-899 . doi: 10.1002/btpr.

Sarmah N, Revathi D, Sheelu G, Yamuna Rani K, Sridhar S, Mehtab V, Sumana C (2018) Recent advances on sources and industrial applications of lipases. Biotechnol Prog 34:5-28 . doi: 10.1002/btpr.2581

Satyanarayana SDV, Krishna MSR, Kumar PP (2017) Optimization of high-yielding protocol for DNA extraction from the forest rhizosphere microbes. 3 Biotech 7:91 . doi: 10.1007/s13205-017-0737-2

Sayali K, Sadichha P, Surekha S (2013) Microbial esterases : An overview. Int J Curr Microbiol Appl Sci 2:135-146

Sayer C, Finnigan W, Isupov MN, Levisson M, Kengen SWM, van der Oost J, Harmer NJ, Littlechild JA (2016) Structural and biochemical characterisation of Archaeoglobus fulgidus esterase reveals a bound CoA molecule in the vicinity of the active site. Sci Rep 6:25542 . doi: 10.1038/srep25542

Schloss PD, Hay AG, Wilson DB, Walker LP (2003) Tracking temporal changes of bacterial community fingerprints during the initial stages of composting. FEMS Microbiol Ecol 46:1-9 . doi: 10.1016/S01686496(03)00153-3

Schmidt-Dannert C, Rua ML, Atomi H, Schmid RD (1996) Thermoalkalophilic lipase of Bacillus thermocatenulatus. I. Molecular cloning, nucleotide sequence, purification and some properties. Measurement 1301:105-114

Schulz G, Schneider D, Brinkmann N, Edy N, Daniel R, Polle A, Scheu S, Krashevska V (2019) Changes in trophic groups of protists with conversion of rainforest into rubber and oil palm plantations. Front Microbiol 10:240 . doi: $10.3389 /$ fmicb.2019.00240

Secundo F, Carrea G (2002) Lipase activity and conformation in neat organic solvents. J Mol Catal B Enzym 19:93102 . doi: $10.1016 / \mathrm{S} 1381-1177(02) 00155-8$

Selvin J, Kennedy J, Lejon DPH, Kiran GS, Dobson ADW (2012) Isolation identification and biochemical characterization of a novel halo-tolerant lipase from the metagenome of the marine sponge Haliclona simulans. Microb Cell Fact 11:72 . doi: 10.1186/1475-2859-11-72

Seo S, Lee YS, Yoon SH, Kim SJ, Cho JY, Hahn BS, Koo BS, Lee CM (2014) Characterization of a novel coldactive esterase isolated from swamp sediment metagenome. World J Microbiol Biotechnol 30:879-886 . doi: 10.1007/s11274-013-1496-9

Seshadri R, Kravitz SA, Smarr L, Gilna P, Frazier M (2007) CAMERA: A community resource for metagenomics. PLoS Biol 5:e75 . doi: 10.1371/journal.pbio.0050075

Shannon P, Markiel A, Ozier O, Baliga NS, Wang JT, Ramage D, Amin N, Schwikowski B, Ideker T (2003) Cytoscape: A software environment for integrated models of biomolecular interaction networks. Genome Res 13:2498-2504 . doi: 10.1101/gr.1239303

Shao H, Xu L, Yan Y (2013) Isolation and characterization of a thermostable esterase from a metagenomic library. J Ind Microbiol Biotechnol 40:1211-1222 . doi: 10.1007/s10295-013-1317-z

Silva CC, Hayden H, Sawbridge T, Mele P, Kruger RH, Rodrigues MVN, Costa GGL, Vidal RO, Sousa MP, Torres APR, Santiago VMJ, Oliveira VM (2012) Phylogenetic and functional diversity of metagenomic libraries of phenol degrading sludge from petroleum refinery wastewater treatment system. AMB Express 2:18 . doi: $10.1186 / 2191-0855-2-18$ 
Simon C, Daniel R (2009) Achievements and new knowledge unraveled by metagenomic approaches. Appl Microbiol Biotechnol 85:265-276 . doi: 10.1007/s00253-009-2233-z

Simon C, Daniel R (2011) Metagenomic analyses: Past and future trends. Appl Environ Microbiol 77:1153-1161 . doi: 10.1128/AEM.02345-10

Sivakumar N, Li N, Tang JW, Patel BKC, Swaminathan K (2006) Crystal structure of AmyA lacks acidic surface and provide insights into protein stability at poly-extreme condition. FEBS Lett 580:2646-2652 . doi: 10.1016/J.FEBSLET.2006.04.017

Skewes-Cox P, Sharpton TJ, Pollard KS, DeRisi JL (2014) Profile Hidden Markov Models for the detection of viruses within metagenomic sequence data. PLoS One 9:e105067 . doi: 10.1371/journal.pone.0105067

Sood S, Sharma A, Sharma N, Kanwar SS (2016) Carboxylesterases: Sources, characterization and broader applications. Insights Enzym Res 1:1-11 . doi: 10.21767/2573-4466.100002

Stergiou PY, Foukis A, Filippou M, Koukouritaki M, Parapouli M, Theodorou LG, Hatziloukas E, Afendra A, Pandey A, Papamichael EM, Moh B, Salameh DA, Gupta R, Gupta N, Rathi P, Levisson M, Mrugesh D, Togneri PD, Anobom CD, Pinheiro AS, De-Andrade RA, Aguieiras ECG, Andrade GC, Moura M V, Almeida R V, Freire DM, Nagazawa AA, Nalder TD, Bornscheuer UT, Dheeman DS, Henehan PG, Lopez-Lopez O, Cerdan M, Siso M, Alex D, Udatha G (2014) Biochemical and Structural Analysis of Thermostable Esterases. Biotechnol Adv 31:445-455 . doi: 10.1016/S0168-6445(01)00075-4

Tadeo X, Ló Pez-Mé Ndez B, Trigueros T, Laín A, Castañ D, Millet O (2009) Structural basis for the aminoacid composition of proteins from halophilic Archea. PLoS Biol 7:e1000257 . doi: 10.1371/journal.pbio. 1000257

Tannous E, Kanaya S (2014) Divalent metal ion-induced folding mechanism of RNase H1 from extreme halophilic archaeon Halobacterium sp. NRC-1. PLoS One 9:e109016 . doi: 10.1371/journal.pone.0109016

Tasse L, Bercovici J, Pizzut-Serin S, Robe P, Tap J, Klopp C, Cantarel BL, Coutinho PM, Henrissat B, Leclerc M, Doré J, Monsan P, Remaud-Simeon M, Potocki-Veronese G (2010) Functional metagenomics to mine the human gut microbiome for dietary fiber catabolic enzymes. Genome Res 20:1605-1612 . doi: $10.1101 /$ gr. 108332.110

Tatusov RL, Galperin MY, Natale DA, Koonin E V (2000) The COG database: a tool for genome-scale analysis of protein functions and evolution

Tchigvintsev A, Tran H, Popovic A, Kovacic F, Brown G, Flick R, Hajighasemi M, Egorova O, Somody JC, Tchigvintsev D, Khusnutdinova A, Chernikova TN, Golyshina O V., Yakimov MM, Savchenko A, Golyshin PN, Jaeger KE, Yakunin AF (2015) The environment shapes microbial enzymes: five cold-active and saltresistant carboxylesterases from marine metagenomes. Appl Microbiol Biotechnol 99:2165-2178 . doi: 10.1007/s00253-014-6038-3

Techtmann SM, Hazen TC (2016) Metagenomic applications in environmental monitoring and bioremediation. J. Ind. Microbiol. Biotechnol. 43:1345-1354

Thai QK, Bös F, Pleiss J (2009) The lactamase engineering database: A critical survey of TEM sequences in public databases. BMC Genomics 10:390 . doi: 10.1186/1471-2164-10-390

Thakur S (2012) Lipases, its sources, properties and applications: A review. Int J Sci Eng Res 3:1-29

Tirawongsaroj P, Sriprang R, Harnpicharnchai P, Thongaram T, Champreda V, Tanapongpipat S, Pootanakit K, Eurwilaichitr L (2008) Novel thermophilic and thermostable lipolytic enzymes from a Thailand hot spring metagenomic library. J Biotechnol 133:42-49 . doi: 10.1016/J.JBIOTEC.2007.08.046

Tripathi NK, Shrivastava A (2019) Recent developments in bioprocessing of recombinant proteins: expression hosts and process development. Front Bioeng Biotechnol 7:420 . doi: 10.3389/fbioe.2019.00420

Troy SM, Nolan T, Kwapinski W, Leahy JJ, Healy MG, Lawlor PG (2012) Effect of sawdust addition on composting of separated raw and anaerobically digested pig manure. J Environ Manage 111:70-77 . doi: 10.1016/j.jenvman.2012.06.035

Turnbaugh PJ, Gordon JI (2009) The core gut microbiome, energy balance and obesity. J Physiol 587:4153-4158 . doi: 10.1113/jphysiol.2009.174136

Turnbaugh PJ, Hamady M, Yatsunenko T, Cantarel BL, Ley RE, Sogin ML, Jones WJ, Roe B a, Jason P, Egholm M, Henrissat B, Heath AC, Knight R, Gordon JI, Rey FE, Manary MJ, Trehan I, Dominguez-Bello MG, Contreras M, Magris M, Hidalgo G, Baldassano RN, Anokhin AP, Heath AC, Warner B, Reeder J, Kuczynski J, Caporaso JG, Lozupone C a., Lauber C, Clemente JC, Knights D, Knight R, Gordon JI (2009) A core gut microbiome between lean and obesity twins. Nature 457:480-484 . doi: 10.1038/nature07540

Uchiyama T, Miyazaki K (2009) Functional metagenomics for enzyme discovery: challenges to efficient screening. Curr Opin Biotechnol 20:616-622 . doi: 10.1016/j.copbio.2009.09.010

Ufarté L, Laville É, Duquesne S, Potocki-Veronese G (2015a) Metagenomics for the discovery of pollutant degrading enzymes. Biotechnol Adv 33:1845-1854 . doi: 10.1016/j.biotechadv.2015.10.009 
Ufarté L, Potocki-Veronese G, Laville É (2015b) Discovery of new protein families and functions: New challenges in functional metagenomics for biotechnologies and microbial ecology. Front. Microbiol. 6:563

Uritskiy G, Di Ruggiero J (2019) Applying genome-resolved metagenomics to deconvolute the halophilic microbiome. Genes (Basel) 10:220 . doi: 10.3390/genes10030220

Vargas-Albores F, Martínez-Córdova LR, Martínez-Porchas M, Calderón K, Lago-Lestón A (2019) Functional metagenomics: a tool to gain knowledge for agronomic and veterinary sciences. Biotechnol Genet Eng Rev 35:69-91 . doi: 10.1080/02648725.2018.1513230

Varma VS, Dhamodharan K, Kalamdhad AS (2018) Characterization of bacterial community structure during invessel composting of agricultural waste by $16 \mathrm{~S}$ rRNA sequencing. 3 Biotech 8:301 . doi: 10.1007/s13205-0181319-7

Ventosa A, Nieto JJ, Oren A (1998) Biology of moderately halophilic aerobic bacteria. Microbiol Mol Biol Rev 62:504-544

Verma N, Thakur S, Bhatt AK (2012) Microbial lipases: Industrial applications and properties (a review). Int Res J Biol Sci Int Res J Biol Sci 1:2278-3202 . doi: 10.4172/2375-4338.1000156

Verschueren KHG, Seljee F, Rozeboom HJ, Kalk KH, Dijkstra BW (1993) Crystallographic analysis of the catalytic mechanism of haloalkane dehalogenase. Nature 363:693-698 . doi: 10.1038/363693a0

Vieille C, Zeikus GJ (2001) Hyperthermophilic enzymes: sources, uses, and molecular mechanisms for thermostability. Microbiol Mol Biol Rev 65:1-43 . doi: 10.1128/MMBR.65.1.1-43.2001

Vigneron A, Alsop EB, Lomans BP, Kyrpides NC, Head IM, Tsesmetzis N (2017) Succession in the petroleum reservoir microbiome through an oil field production lifecycle. ISME J 11:2141-2154 . doi: 10.1038/ismej.2017.78

Wagner UG, Petersen EI, Schwab H, Kratky C (2002) EstB from Burkholderia gladioli: A novel esterase with a $\beta$ lactamase fold reveals steric factors to discriminate between esterolytic and $\beta$-lactam cleaving activity. Protein Sci 11:467-478 . doi: 10.1110/ps.33002

Wang C, Dong D, Wang H, Müller K, Qin Y, Wang H, Wu W (2016a) Metagenomic analysis of microbial consortia enriched from compost : new insights into the role of Actinobacteria in lignocellulose decomposition. Biotechnol Biofuels 9:22 . doi: 10.1186/s13068-016-0440-2

Wang G, Wang Q, Lin X, Ng TB, Yan R, Lin J, Ye X (2016b) A novel cold-adapted and highly salt-tolerant esterase from Alkalibacterium sp. SL3 from the sediment of a soda lake. Sci Rep 6:19494 . doi: 10.1038/srep19494

Wang H, Hart DJ, An Y (2019) Functional metagenomic technologies for the discovery of novel enzymes for biomass degradation and biofuel production. Bioenergy Res 12:457-470 . doi: 10.1007/s12155-019-10005-w

Wang M, Ai L, Zhang M, Wang F, Wang C (2020) Characterization of a novel halotolerant esterase from Chromohalobacter canadensis isolated from salt well mine. 3 Biotech 10:430 . doi: 10.1007/s13205-02002420-0

Wang S, Wang K, Li L, Liu Y (2013) Isolation and characterization of a novel organic solvent-tolerant and halotolerant esterase from a soil metagenomic library. J Mol Catal B Enzym 95:1-8 . doi: 10.1016/j.molcatb.2013.05.015

Wang X, Qin X, Li D, Yang B, Wang Y (2017) One-step synthesis of high-yield biodiesel from waste cooking oils by a novel and highly methanol-tolerant immobilized lipase. Bioresour Technol 235:18-24 . doi: 10.1016/j.biortech.2017.03.086

Wei H, Tucker MP, Baker JO, Harris M, Luo Y, Xu Q, Himmel ME, Ding SY (2012) Tracking dynamics of plant biomass composting by changes in substrate structure, microbial community, and enzyme activity. Biotechnol Biofuels 5:1-14 . doi: 10.1186/1754-6834-5-20

Wei H, Wang L, Hassan M, Xie B (2018) Succession of the functional microbial communities and the metabolic functions in maize straw composting process. Bioresour Technol 256:333-341 . doi: 10.1016/j.biortech.2018.02.050

Wei T, Feng S, Shen Y, He P, Ma G, Yu X, Zhang F, Mao D (2013) Characterization of a novel thermophilic pyrethroid-hydrolyzing carboxylesterase from Sulfolobus tokodaii into a new family. J Mol Catal B Enzym 97:225-232 . doi: $10.1016 /$ j.molcatb.2013.07.022

Wende A, Johansson P, Vollrath R, Dyall-Smith M, Oesterhelt D, Grininger M (2010) Structural and biochemical characterization of a halophilic archaeal alkaline phosphatase. J Mol Biol 400:52-62 . doi: 10.1016/J.JMB.2010.04.057

Wilke A, Harrison T, Wilkening J, Field D, Glass EM, Kyrpides N, Mavrommatis K, Meyer F (2012) The M5nr: A novel non-redundant database containing protein sequences and annotations from multiple sources and associated tools. BMC Bioinformatics 13:141 . doi: 10.1186/1471-2105-13-141 
Wu C, Sun B (2009) Identification of novel esterase from metagenomic library of Yangtze River. J Microbiol Biotechnol 19:187-193 . doi: 10.4014/jmb.0804.292

Wu G, Wu G, Zhan T, Shao Z, Liu Z (2013a) Characterization of a cold-adapted and salt-tolerant esterase from a psychrotrophic bacterium Psychrobacter pacificensis. Extremophiles 17:809-819 . doi: 10.1007/s00792-0130562-4

Wu G, Zhang S, Zhang H, Zhang S, Liu Z (2013b) A novel esterase from a psychrotrophic bacterium Psychrobacter celer 3Pb1 showed cold-adaptation and salt-tolerance. J Mol Catal B Enzym 98:119-126 . doi: 10.1016/j.molcatb.2013.10.012

Xiao F, Li Z, Pan L (2017) Application of microbial lipase and its research progress. Prog Appl Microbiol 8-14

Yamada Y, Fujiwara T, Sato T, Igarashi N, Tanaka N (2002) The 2.0 A crystal structure of catalase-peroxidase from Haloarcula marismortui. Nat Struct Biol 9:691-695 . doi: 10.1038/nsb834

Yang X, Wu L, Xu Y, Ke C, Hu F, Xiao X, Huang J (2018) Identification and characterization of a novel alkalistable and salt-tolerant esterase from the deep-sea hydrothermal vent of the East Pacific Rise. Microbiology 7:e00601 . doi: 10.1002/mbo3.601

Yoo W, Kim B, Jeon S, Kim KK, Kim TD (2020) Identification, characterization, and immobilization of a novel YbfF esterase from Halomonas elongata. Int J Biol Macromol 165:1139-1148 . doi: 10.1016/j.ijbiomac.2020.09.247

Zarafeta D, Moschidi D, Ladoukakis E, Gavrilov S, Chrysina ED, Chatziioannou A, Kublanov I, Skretas G, Kolisis FN (2016) Metagenomic mining for thermostable esterolytic enzymes uncovers a new family of bacterial esterases. Sci Rep 6:38886 . doi: 10.1038/srep38886

Zeth K, Offermann S, Essen L-O, Oesterhelt D (2004) Iron-oxo clusters biomineralizing on protein surfaces: structural analysis of Halobacterium salinarum DpsA in its low- and high-iron states. Proc Natl Acad Sci U S A 101:13780-5 . doi: 10.1073/pnas.0401821101

Zhang S, Lin X (2019) CARMA3: Scaffold protein involved in NF-אB signaling. Front Immunol 10:176 . doi: 10.3389/fimmu.2019.00176

Zhang W, Xu H, Wu Y, Zeng J, Guo Z, Wang L, Shen C, Qiao D, Cao Y (2018a) A new cold-adapted, alkali-stable and highly salt-tolerant esterase from Bacillus licheniformis. Int J Biol Macromol 111:1183-1193 . doi: 10.1016/J.IJBIOMAC.2018.01.152

Zhang Y, Hao J, Zhang YQ, Chen XL, Xie B Bin, Shi M, Zhou BC, Zhang YZ, Li PY (2017) Identification and characterization of a novel salt-tolerant esterase from the deep-sea sediment of the South China Sea. Front Microbiol 8:441 . doi: 10.3389/fmicb.2017.00441

Zhang Y, Ji F, Wang J, Pu Z, Jiang B, Bao Y (2018b) Purification and characterization of a novel organic solventtolerant and cold-adapted lipase from Psychrobacter sp. ZY124. Extremophiles 22:287-300 . doi: 10.1007/s00792-018-0997-8

Zhang Y, Pengjun S, Wanli L, Kun M, Yingguo B, Guozeng W, Zhichun Z, Bin Y (2009) Lipase diversity in glacier soil based on analysis of metagenomic DNA fragments and cell culture. J Microbiol Biotechnol 19:888-897 . doi: 10.4014 jmb.0812.695

Zhou G, Xu X, Qiu X, Zhang J (2019) Biochar influences the succession of microbial communities and the metabolic functions during rice straw composting with pig manure. Bioresour Technol 272:10-18 . doi: 10.1016/j.biortech.2018.09.135

Zhou H, Gu W, Sun W, Hay AG (2018) A microbial community snapshot of windrows from a commercial composting facility. Appl Microbiol Biotechnol 102:8069-8077 . doi: 10.1007/s00253-018-9201-4

Zhu Y, Li J, Cai H, Ni H, Xiao A, Hou L (2013) Characterization of a new and thermostable esterase from a metagenomic library. Microbiol Res 168:589-597 . doi: 10.1016/J.MICRES.2013.04.004 
Chapter V

General discussion 
Owing to the functional versatility, LEs are one of the most industrially interested groups of biocatalysts. Particularly, LEs of thermophilic origin gain more attention due to their intrinsic thermal and chemical stability (Stergiou et al. 2014). This thesis describes the results of applying function-driven and sequence-based metagenomic approaches to explore novel LEs in microbial consortia of thermophilic composts. The information obtained in this study illustrated an insight of the genetic diversity of LEs, which have broadened the spectrum of LEs, with the aim to provide more potentials for industrial application.

\subsection{Microbial composition in compost consortia}

Firstly, this thesis presents the phylogenetic profile of microbial consortia in the two compost samples. Generally, the microbial communities revealed from the pyrotag sequencing data and shotgun whole metagenomic sequencing data were accordance with those in other studies: members in phyla Actinobacteria, Proteobacteria, Firmicutes and Bacteroidetes were dominant in compost microbial consortia (Antunes et al. 2016; Ma et al. 2018; Palaniveloo et al. 2020). The incisive taxonomic disparity were found among compost samples (Fig. 5-1), which was the combination of many factors, such as $\mathrm{C} / \mathrm{N}$ ratio, $\mathrm{pH}$, moisture content, aeration/O2 supply, temperature and particle size (Rastogi et al. 2020). Under aerobic conditions, temperature and the availability of various organic matters are the major selective factors for the microbial population (Ryckeboer et al. 2003). Particularly, the temperature determines the rate metabolic activity (Ma et al. 2018; Rastogi et al. 2020). Antunes et al. (2016) tracked the dynamic change of microbial community throughout the composting process at metagenomic and metatranscriptomic level. Significantly, the rising temperature favors for members in Firmicutes that outcompeted other organisms, and were dominant over the thermophilic stage. This was found even in different composting systems feed with various organic wastes (Fig. 
5-1). During the thermophilic phase, many microbes in Firmicutes are able to form endospore-like structures to resist the heat (Wei et al. 2018; Zhou et al. 2019; Awasthi et al. 2020a). They are also reported to hydrolyze sugar, protein and lignocellulose that resulted in a variety of acids, alcohols and lipids (Mao et al. 2018). For members in Actinobacteria, they are important degraders for natural polymers and hard to degrade debris, e.g. woody stems, bark or newspaper, and optimally grow at temperature between 45 to $55^{\circ} \mathrm{C}$ (Ryckeboer et al. 2003; Wei et al. 2012). Wang et al. (2016) verified that members of the Actinobacteria play an essential role in decomposing cellulose and hemicellulose originating from the rice straw. In this study, the excessive heat $\left(76^{\circ} \mathrm{C}\right)$ by the compost pile (Pile_2) with household waste as feedstock, could be associated with high sugar content and water-soluble carbon (Troy et al. 2012; Jara-Samaniego et al. 2017). The other pile (Pile_1) was mainly supplemented with wood chips (i.e. high $\mathrm{C} / \mathrm{N}$ ratio). A higher $\mathrm{C} / \mathrm{N}$ ratio (compare to Pile_2) could slowed down composting, with simultaneously reach a lower temperature $\left(55{ }^{\circ} \mathrm{C}\right)$ during the compost process. Therefore, under the same composting operation (i.e. turning regime), the temperature and feedstock composition primarily shape the microbial community in the two compost samples (Fig. 5-1). 


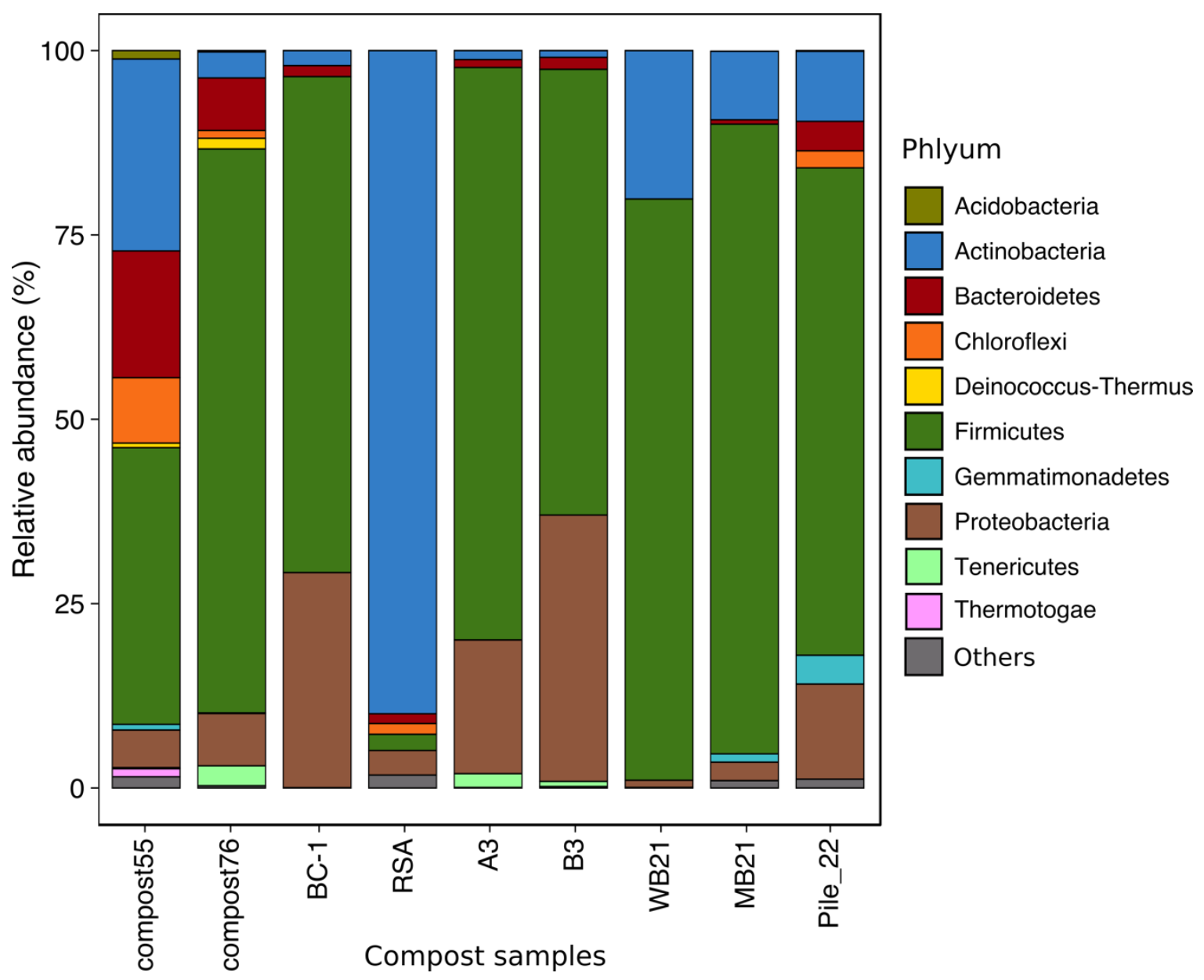

Fig. 5-1. Phylogenetic distribution of dominant bacterial groups (at phylum level) in compost samples investigated in this thesis and other surveys. Compost samples of compost55 (feedstock: green waste) and compost76 (household waste) were from this thesis and at thermophilic stage; $\mathrm{BC}-1$ (manure and sawdust) was at thermophilic stage with temperature at $67.8^{\circ} \mathrm{C}$ (Mao et al. 2018); RSA was at maturing stage with temperature at $30{ }^{\circ} \mathrm{C}$ (Wang et al. 2016a); A3 (pig manure and wheat straw) and B3 (pig manure and wheat straw covered with a semi-permeable membrane) were at thermophilic stage with temperature at 58 and $57^{\circ} \mathrm{C}$, respectively (Ma et al. 2018); WB21 and MB21 were at cooling/second mesophilic phase stage with temperature at 44 and $45^{\circ} \mathrm{C}$, respectively (Zhou et al. 2019); Pile_22 (manure/silage and hay) was at thermophilic stage (Neher et al. 2013).

Proteobacteria were the major group for decomposing plant straw (Awasthi et al., 2017), and some members of the Proteobacteria are known to be involved in sulfur and nitrogen cycling (Zhong et al., 2017; Wei et al., 2018). Many microbes in Bacteroidetes were important degraders for organic matter, as they have the ability 
to break down cellulose, hemicellulose and lignin (Dodd et al., 2011; Zhong et al., 2017).

At genus level, genera such as Brockia, Thermobispora, Thermomonospora, Longispora, Salinispora, Geobacillus, Thermaerobacter, Filomicrobium, Rhodothermus, Symbiobacterium, Caldicoprobacter and Calditerricola were among major bacterial groups previously identified during composting (Ryckeboer et al. 2003; Antunes et al. 2016; Rong et al. 2018; Zhou et al. 2018). Among them, Calditerricola, Geobacillus, Thermobispora, Thermus and Thermomonospora were typical bacterial thermophiles. At temperatures above $60{ }^{\circ} \mathrm{C}$, the degradation of organic compounds such as cellulose, hemicellulose and chitin is performed essentially by these thermophilic bacteria (Moriya et al. 2011; Awasthi et al. 2020a).

\subsection{Screening for lipolytic enzymes through metagenomic approaches}

Functional metagenomics is activity-based screening of metagenomic libraries that constructed by cloning environmental DNA into expression vectors and propagating them in the appropriate hosts (Daniel 2005). This technique is widely used for novel biocatalyst identification, due to no requiring the cultivation of the native microorganisms or sequence information of the genes a priori (DeCastro et al. 2016). Attributing to the functional versatility, lipolytic enzymes are one of the most important groups in industrial applications (Rosenstein and Götz 2000). As summarized by Ferrer et al. (2015) of recent reported 256 metagenomic libraries, $68 \%$ of the 6,038 clones that were active against different tested substrates were lipases and/or esterases. Among them, 4,034 were selected by functional screening of metagenomes from various habitats, such as soil, marine and freshwater environments, microbiota from several hosts, and waste treatments (Lopez-Lopez et al. 2014; Berini et al. 2017). The thermophilic compost samples harbor divergent thermophiles, from which the enzymes are intrinsic thermophilic. Thus, this thesis 
used the function-based approach for identifying potential extremophilic lipolytic genes in two thermophilic compost metagenomes. Overall, approximately 4.89 and $2.56 \mathrm{~Gb}$ of cloned compost DNA were screened, generating 199 and 51 positive clones for compost55 and compost76, respectively. The targeting probability towards a LE in our study (between 16.1 and 43.6 per $\mathrm{Gb}$ ) is generally consistent with those from other compost metagenomic libraries (Lämmle et al. 2007; Kim et al. 2010; Leis et al. 2015). The hit rates were affected by many factors, such as the source of the metagenomic DNA, the size of the gene of interest, its abundance in the metagenome, biases in cell lysis during DNA extraction, homegroups expression host and vector, and the expression condition (Uchiyama and Miyazaki 2009).

To date, E. coli has been chosen as the cloning host for the vast majority of metagenomic libraries (Uchiyama and Miyazaki 2009), as it possesses a number of desirable attributes, e.g. high transformation efficiency, lacks genes for restriction modification and can deal with diverse translation signals (Boni 2006). Despite these advantages, E. coli is unable to express all foreign DNA due to the incompatibility with the heterologous expression system (Lam et al. 2015). As Gabor et al. (2004) estimated, on averagely, only $30 \%-40 \%$ of environmental bacterial genes could be efficiently expressed in E. coli, the value even drops to $7 \%$ for high $\mathrm{G}+\mathrm{C}$ DNA from bacterial strains. Moreover, gene product from extremophiles were normally active under its original extreme conditions (Munawar and Engel 2013), whereas, mesophiles, like the expression host E. coli, do not survive under these conditions. In the future, to enhance the hit rate, the functional screening can be improved by modifying the E. coli-based expression system, such as introducing heterologous sigma factors (Gaida et al. 2015), employing T7 RNA polymerase and forming hybrid ribosomes (Gaida et al. 2015). Alternatively, various hosts (Tripathi and Shrivastava 2019) such as Haloferax volcanii (Kixmüller and Greie 2012), 
Halobacterium salinarum (Kixmüller and Greie 2012), Thermus thermophilus (Leis et al. 2015) and Bacillus subtilis (Mahapatra et al. 2020) can be chosen for production of extremophilic gene products .

These functionally derived LEs were further classified into different families according to the gold standard classification system initiated by Arpigny \& Jaeger (1999), which has extended to nineteen families nowadays. Until recently, there are still claims of identifying novel families, however, they are not integrated into the classification system. Without comparison of these so-called novel families, the possibility exists that different publications may describe members of the same 'novel' family under different names (Hitch and Clavel 2019). In this study, after extensive literature search, we integrated 29 so-called novel families into the classification system, resulting in a total of 48 lipolytic family (subfamilies in one family were counted as one). According to the phylogenetic tree, the functionalderived LEs were detected in 12 known families and 7 novel families.

Sequence-based screening relies on direct sequencing of environmental DNA, either with or without cloning prior to sequencing, and then subjecting the sequences to bioinformatic analyses. In comparison to the function-based screening, this strategy avoids the cloning-related disadvantages. In addition, sequence-based screening involves less labor-intensive operations than function-based screening (Ngara and Zhang 2018). The sequence-driven approach highly depends on the accuracy and completeness of the reference databases, from which the functions of newly discovered genes are inferred. Thus, if the novel genes have weak sequence similarity to sequences recruited in the database, or the sequence similarity does not correspond to a functional relationship, this approach may return false annotation. Interestingly, in practice, function-based approach was generally more favorable than sequence-based approach. According to Ferrer et al. (2015), 6,100 of the 
metagenomic identified bioactive compounds (from 2,192 different sites), only $3.5 \%$ were derived from sequence-based approach.

In the specific case of lipases, only a few studies used sequence-based approaches for LEs identification. For example, Lipases were identified by PCR amplification of metagenomic DNA from a hot spring (Bell et al. 2002) and glacier soil (Zhang et al. 2009), using primers targeting the consensus sequences of the oxyanion hole and regions of active sites. Zarafeta et al. (2016) sequenced the whole-genome of microbial consortia in a water sludge, they found an esterase (EstDZ2) by screening the processed data against the conserved motifs at the active sites, oxyanion hole and signal peptide. Verma et al. (2019) revealed a total 21 unique sequences of new alkaliphilic lipases by searching against the reconstructed ancestral sequence of an alkaliphilic lipase.

In this thesis, a LE-specific profile HMM database was built for sequence-based screening of putative lipolytic genes. Profile HMMs have been widely adopted for improved annotation of general functions in microbial genomes and metagenomes (Skewes-Cox et al. 2014; Reyes et al. 2017; Bzhalava et al. 2018). However, they have not yet been specifically applied to LEs in microbial genomes/metagenomes. In this study, to gain high recall (sensitivity), we compared 4 sets of profile HMMs constructed using different multiple sequence alignment algorithms. The high specificity was achieved by a subsequent BLAST searching after the scan against the selected profile HMM database. This strategy for putative lipolytic gene identification and subsequent family assignment was further validated on different datasets. Using this strategy, we successfully identified 4,157 and 2,234 putative lipolytic proteins (PLPs), among them, 1,234 and 759 were further assigned into 28 and 26 lipolytic families in the assembled metagenomes of compost55 and compost76, respectively. The assigned PLPs generally originated from Bacteria 
(>95\%), and mainly affiliated to the phyla Actinobacteria, Proteobacteria, Firmicutes and Bacteroidetes. Moreover, we identified a link between microbial taxa and their functional traits (lipolytic genes), which was also reported in studies of profiling CAZyme encoding genes (Wang et al. 2016a) and resistomes (Pehrsson et al. 2016) through sequence-based approaches.

Furthermore, we compared LEs identified in the plasmid library with putative lipolytic proteins derived from the sequence-based screening of the assembled compost55 and compost76 metagenomes. All in all, function-based approach and sequence-based approach has its respective advantages and disadvantages (Table 51). The most distinctive advantage of the function-based screening is the certainty that novel compound is correctly biosynthesized by the host cell, and hence, discovery of novel enzymes with no sequence information is required (Ngara and Zhang 2018). However, since this approach is activity-directed, one of the main limitations is the low expression level of heterologous genes in the host (Mirete et al. 2016), which normally led to a low hit rate (Simon and Daniel 2011). Sequencingbased screening is effective in identifying targeted biomolecules in metagenomes, with similarities to known biomolecules. However, sequence-based screening largely relies on the search algorithms, and quality and completeness of reference databases, which are used to infer the functions of newly discovered genes (Lam et al. 2015). Thus, the best way to explore novel molecules is to combine the two approaches (Barriuso and Jesús Martínez 2015). The information obtained from functional metagenomics can broaden the database for future annotation, thus, complements sequence-based metagenomics. 


\section{Table 5-1 Comparison of function-driven and sequence-based screening for lipolytic genes}

\begin{tabular}{|c|c|c|c|c|c|}
\hline $\begin{array}{l}\text { Metagenomic } \\
\text { approach }\end{array}$ & Method & Advantages & Limitations & Future prospects & References \\
\hline $\begin{array}{l}\text { Function- } \\
\text { driven }\end{array}$ & $\begin{array}{l}\text { Hydrolysis of natural } \\
\text { triacylglycerols: tributyrin, } \\
\text { tricaprylin, triolein, olive } \\
\text { oil, formation of hydrolysis } \\
\text { halos } \\
\text { - Hydrolysis of 1-naphthyl } \\
\text { palmitate, } p \text {-nitrophenyl } \\
\text { esters, formation of colored } \\
\text { colonies }\end{array}$ & $\begin{array}{l}\text { - } \text { Culture-independent } \\
\text { - No requirement of } \\
\text { sequence information } a \\
\text { priori } \\
\text { - Activity-based, thus, direct } \\
\text { detection of bioactive LEs }\end{array}$ & $\begin{array}{l}\text { - Low hit rate } \\
\text { - Labor-intensive operations } \\
\text { - Only qualitative } \\
\text { - Low/no level of gene } \\
\text { expression in the most } \\
\text { widely used host E. coli }\end{array}$ & $\begin{array}{l}\text { - Enrichment of environmental } \\
\text { samples under conditions } \\
\text { mimicking the application } \\
\text { - High-throughput screening } \\
\text { strategies, such as fluorescence- } \\
\text { activated cell sorting (FACS), } \\
\text { substrate induced gene expression } \\
\text { (SIGEX), genetic enzyme } \\
\text { screening system (GESS) and } \\
\text { microfluidics-driven screening } \\
\text { - Modification of expression hots E. } \\
\text { coli, such as random insertion of } \\
\text { T7 promoters; introducing } \\
\text { heterologous sigma factors; } \\
\text { forming hybrid ribosomes } \\
\text { - Alternative expression systems, } \\
\text { such as Agrobacterium, Bacillus, } \\
\text { Rhodococcus, Streptomyces and } \\
\text { Pseudomonas, as well as a few } \\
\text { archaea }\end{array}$ & $\begin{array}{l}\text { Gabor et al. } \\
\text { 2004; Liebl et al. } \\
\text { 2014; Gaida et } \\
\text { al. 2015; Kim et } \\
\text { al. 2016; Berini } \\
\text { et al. 2017; } \\
\text { Ngara and Zhang } \\
\text { 2018; Mahapatra } \\
\text { et al. 2020; } \\
\text { Markel et al. } \\
\text { 2020 }\end{array}$ \\
\hline $\begin{array}{l}\text { Sequence- } \\
\text { based }\end{array}$ & $\begin{array}{l}\text { - Target sequencing: PCR } \\
\text { amplification with primers } \\
\text { specific for conserved } \\
\text { regions of genes of interest } \\
\text { - Shotgun sequencing: such } \\
\text { as motif- and homology- } \\
\text { based searching against } \\
\text { database }\end{array}$ & $\begin{array}{l}\text { - Culture-independent } \\
\text { - No requirement of cloning } \\
\text { a priori } \\
\text { - Easy access to } \\
\text { metagenomic sequencing } \\
\text { data nowadays } \\
\text { - Plenty of software and } \\
\text { platforms for data } \\
\text { analyzing }\end{array}$ & $\begin{array}{l}\text { - Highly dependent on the } \\
\text { accuracy and completeness } \\
\text { of the refence database } \\
\text { - Relying on the algorithms } \\
\text { available } \\
\text { - Genes predicted in silico } \\
\text { require experimental } \\
\text { confirmation } \\
\text { - Tend to find proteins with } \\
\text { high homologies with } \\
\text { known amino acid } \\
\text { sequences }\end{array}$ & $\begin{array}{l}\text { - Synthetic metagenomics: } \\
\text { identified genes of interest are } \\
\text { subjected to high-throughput } \\
\text { expression system for further } \\
\text { characterization } \\
\text { - Constant improvement of database } \\
\text { and database annotations }\end{array}$ & $\begin{array}{l}\text { Simon and } \\
\text { Daniel 2011; } \\
\text { Ufarté et al. } \\
\text { 2015a; Ferrer et } \\
\text { al. 2016; Quince } \\
\text { et al. 2017; } \\
\text { Awasthi et al. } \\
\text { 2020b }\end{array}$ \\
\hline
\end{tabular}




\subsection{Comparative analysis of distribution of putative lipolytic genes across various habitats}

This thesis retrieved 175 assembled metagenomes representing microbial communities of various habitats from IMG/M database. In total, we have screened approx. 1.23 billion genes in $65 \mathrm{Gbp}$ of assembled metagenomes, with approx. 0.22 million (absolute counts) PLP-encoding genes were identified. Among them, the assigned putative lipolytic proteins were account for $34 \%$ (inferred from the absolute counts). The abundance of putative lipolytic proteins in each sample was normalized to LPGM values for comparative analysis. Generally, putative lipolytic proteins were selectively abundant in samples subjected to certain enrichment processes (Fig. 5-2), such as samples from hydrocarbon resource environments and oil reservoir, which are enriched with oil-degrading microbes (Liu et al. 2015; Hu et al. 2016; Vigneron et al. 2017; Liu et al. 2018); composts and wastewater bioreactor, which are reservoirs of various microbes for decomposing organic compounds (Dougherty et al. 2012; Silva et al. 2012; Antunes et al. 2016; Berini et al. 2017). The unequally distribution of PLPs was also observed across different lipolytic families. Families of Hormone-sensitive_lipase_like and patatin-like-protein were most abundant (average LPGM values across samples $>2000$ ), followed by A85EsteraseD-FGH, VIII and Bacterial_lip_FamI.1 (average LPGM values > 700). The lipolytic family profile and taxonomic profile revealed from the assigned putative lipolytic proteins were clustered by habitats. Thus, putative lipolytic proteins were distributed ecologically across samples. 


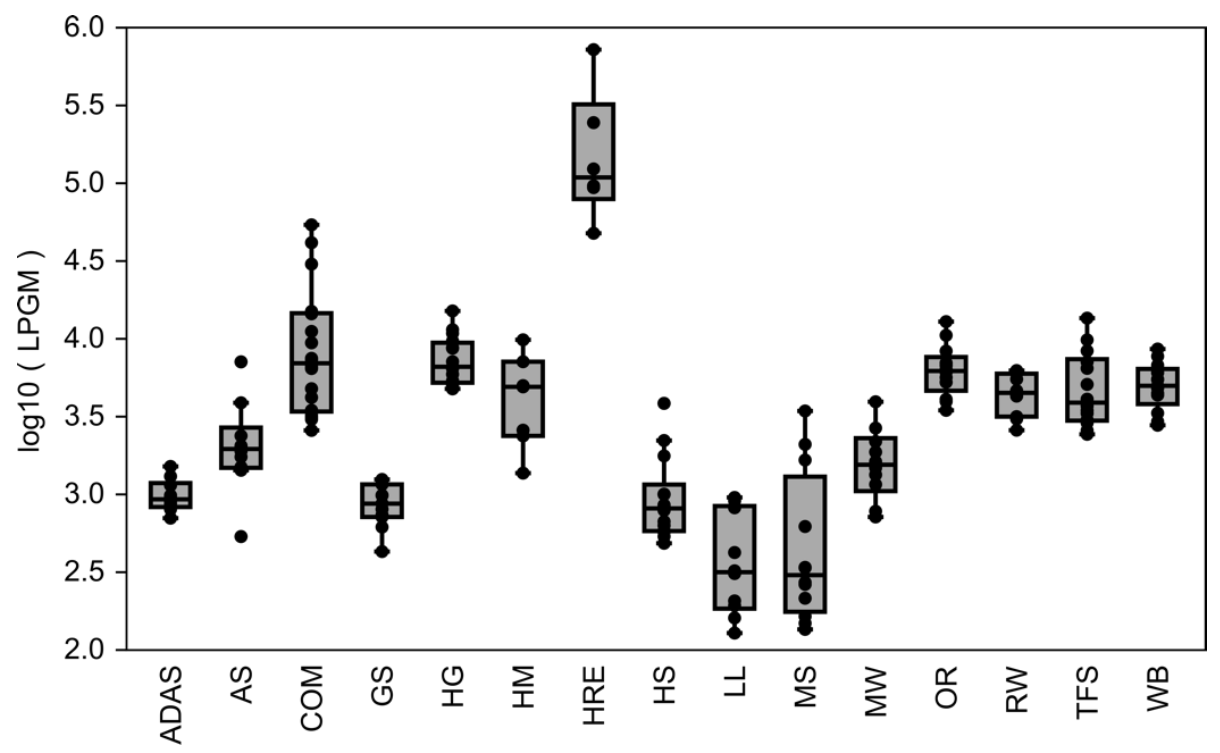

Fig. 5-2: Distribution of assigned putative lipolytic proteins across habitats. LPGM values were $\log _{10}$ transformed. Abbreviations of habitats: ADAS, anaerobic digestor active sludge; AS, agricultural soil; COM, compost; GS grassland soil; HG, human gut; HM, hypersaline mat; HRE, hydrocarbon resource environments; HS, hot spring; LL, landfill leachate; MS, marine sediment; MW, marine water; OR, oil reservoir; RW, river water; TFS, tropical forest soil; WB, wastewater bioreactor.

\subsection{Characterization of extremophilic LEs}

Finally, three lipolytic genes obtained through functional screening were selected for biochemical characterization. Among them, Est1 and Est2 are thermoalkaline enzymes with optimal enzyme activities at 80 and $70{ }^{\circ} \mathrm{C}$, respectively. Est1 and Est2 also displayed unprecedented thermostability at high temperatures, with a half-life of more than 7 (Est1) and 5 days (Est2) at $50{ }^{\circ} \mathrm{C}, 2$ (Est1) and 1 (Est2) days at $60{ }^{\circ} \mathrm{C}$. Generally, thermostability is dependent on the structural rigidity, which is an accumulation of various features, including but not limited to amino acid composition, ion-pairing, hydrogen bonds, hydrophobic interactions, and sulfide bridges (Sadeghi et al. 2006; Jochens et al. 2010; Ebrahimie et al. 2011; Pezzullo et al. 2013). The modeled three-dimensional structures of Est1 and Est2 were homologous to thermostable esterases, which to some extent, structurally proved their thermostable features (Alcaide et al. 2013; Sayer et al. 2016). As described 
before, the thermostability of an enzyme could somehow, extend to its tolerance towards organic-solvent (Khan and Sathya 2018). This is also the case for Est1 and Est2. Particularly for Est2, its activity was significantly enhanced (2- to 10-fold) in the presence of ethanol, methanol, isopropanol and 1-propanol over a concentration range between $6 \%$ and $30 \%(\mathrm{v} / \mathrm{v})$. The significant activating effect could be attributed to the uniform water phase formed by water-miscible solvents (Ogino and Ishikawa 2001) or the high diffusion rate of substrate in the presence of watermiscible solvents (Metin et al. 2006), which enables substrates quick and easy access to the active site. With respect to the short-term stability ( $2 \mathrm{~h}$ incubation), Est2 exhibited high tolerance against $60 \%(\mathrm{v} / \mathrm{v})$ of ethanol, methanol, isopropanol, DMSO and acetone, while Est 1 resisted these solvents only at lower concentrations (below $30 \%, v / v)$. For the long-term stability, Est2 retained most of its activity after 26 days incubation in the presence of $30 \%(\mathrm{v} / \mathrm{v})$ ethanol, methanol, isopropanol, DMSO or acetone. Thus, all these features allows for Est1 and Est2, especially Est2, potentially applying in variety of industrial relevant processes, where the reactions are normally carried out at elevated temperature and mediated with different organic solvents (Panda and Gowrishankar 2005; López-López et al. 2015; Sood et al. 2016; Sarmah et al. 2018).

The other esterase (Est56) was tolerant to high salinity. Addition of salt $(\mathrm{NaCl}$ or $\mathrm{KCl})$ produced a stimulatory effect on Est56 activity, with an enhanced activity at $\mathrm{NaCl}$ and $\mathrm{KCl}$ up to $2.5 \mathrm{M}$. Est56 was stable over the tested $\mathrm{NaCl}$ and $\mathrm{KCl}$ concentration ranges ( 0 to $4 \mathrm{M})$, with almost unaltered activity after $24 \mathrm{~h}$ incubation at $10{ }^{\circ} \mathrm{C}$. To shed light on the mechanism of haloadapation of Est56, we summarized the recently reported halotolerant lipolytic enzymes (40 in total), and their amino acid compositions were statistically compared with the halophilic counterparts. However, no clear pattern was found in the amino acid composition of halotolerant 
LEs. Actually, LEs with different theoretical pI values apply different mechanisms for haloadapation (Sivakumar et al. 2006; Altermark et al. 2008; De Santi et al. 2016b). Est56 has a low theoretical pI value of 4.97, which is an indication that Est56 apply a salt resistence stategy similar to that of halophilic enzymes (Ishibashi et al. 2013; Munawar and Engel 2013). Similar to other halophilic enzymes, the increased content of acidic residues over lysine residues and a predominantly negatively charged surface were also observed for Est56 (Ventosa et al. 1998; Tadeo et al. 2009; Coquelle et al. 2010; Munawar and Engel 2012; Ishibashi et al. 2013). Moreover, Est56 displayed enhanced stability against high temperatures $\left(50\right.$ and $\left.60{ }^{\circ} \mathrm{C}\right)$ and urea $(2,4$, and $6 \mathrm{M})$ in the presence of $\mathrm{NaCl}$. This distinctive feature of enhanced Est56 stability against denaturants (high temperatures and urea) mediated by $\mathrm{NaCl}$ also suggests that Est56 possess a halophilic character caused by the high acidic amino acid content (Coquelle et al. 2010). 


\subsection{Reference}

Alcaide M, Tornés J, Stogios PJ, Xu X, Gertler C, Di Leo R, Bargiela R, Lafraya Á, Guazzaroni M-E, López-Cortés N, Chernikova TN, Golyshina OV, Nechitaylo TY, Plumeier I, Pieper DH, Yakimov MM, Savchenko A, Golyshin PN, Ferrer M (2013) Single residues dictate the co-evolution of dual esterases: MCP hydrolases from the $\alpha / \beta$ hydrolase family. Biochem $\mathrm{J}$ 454:157-166 . doi: 10.1042/BJ20130552

Altermark B, Helland R, Moe E, Willassen NP, Smalås AO (2008) Structural adaptation of endonuclease I from the cold-adapted and halophilic bacterium Vibrio salmonicida. Acta Crystallogr Sect D Biol Crystallogr 64:368-376 . doi: 10.1107/S0907444908000097

Antunes LP, Martins LF, Pereira RV, Thomas AM, Barbosa D, Lemos LN, Silva GMM, Moura LMS, Epamino GWC, Digiampietri LA, Lombardi KC, Ramos PL, Quaggio RB, De Oliveira JCF, Pascon RC, Da Cruz JB, Da Silva AM, Setubal JC (2016) Microbial community structure and dynamics in thermophilic composting viewed through metagenomics and metatranscriptomics. Sci Rep 6:38915 . doi: 10.1038/srep38915

Awasthi MK, Duan Y, Liu T, Awasthi SK, Zhang Z (2020a) Relevance of biochar to influence the bacterial succession during pig manure composting. Bioresour Technol 304:122962 . doi: 10.1016/j.biortech.2020.122962

Awasthi MK, Ravindran B, Sarsaiya S, Chen H, Wainaina S, Singh E, Liu T, Kumar S, Pandey A, Singh L, Zhang Z (2020b) Metagenomics for taxonomy profiling: tools and approaches. Bioengineered 11:356-374 . doi: 10.1080/21655979.2020.1736238

Barriuso J, Jesús Martínez M (2015) In silico metagenomes mining to discover novel esterases with industrial application by sequential search strategies. J Microbiol Biotechnol 25:732737 . doi: 10.4014/jmb.1406.06049

Bell PJL, Sunna A, Gibbs MD, Curach NC, Nevalainen H, Bergquist PL (2002) Prospecting for novel lipase genes using PCR. Microbiology 148:2283-2291 . doi: 10.1099/00221287-148$8-2283$

Berini F, Casciello C, Marcone GL, Marinelli F (2017) Metagenomics: novel enzymes from nonculturable microbes. FEMS Microbiol Lett 364. doi: 10.1093/femsle/fnx211 
Boni I V. (2006) Diverse molecular mechanisms of translation initiation in prokaryotes. Mol Biol 40:587-596 . doi: 10.1134/S002689330604011X

Bzhalava Z, Hultin E, Dillner J (2018) Extension of the viral ecology in humans using viral profile hidden Markov models. PLoS One 13:e0190938 . doi: 10.1371/journal.pone.0190938

Coquelle N, Talon R, Juers DH, Girard É, Kahn R, Madern D (2010) Gradual adaptive changes of a protein facing high salt concentrations. J Mol Biol 404:493-505 . doi: 10.1016/j.jmb.2010.09.055

Daniel R (2005) The metagenomics of soil. Nat Rev Microbiol 3:470-478 . doi: 10.1038/nrmicro1160

De Santi C, Leiros HKS, Di Scala A, de Pascale D, Altermark B, Willassen NP (2016) Biochemical characterization and structural analysis of a new cold-active and salt-tolerant esterase from the marine bacterium Thalassospira sp. Extremophiles 20:323-336 . doi: 10.1007/s00792016-0824-z

DeCastro ME, Rodríguez-Belmonte E, González-Siso MI (2016) Metagenomics of thermophiles with a focus on discovery of novel thermozymes. Front Microbiol 7:1521 . doi: 10.3389/fmicb.2016.01521

Dougherty MJ, D’haeseleer P, Hazen TC, Simmons BA, Adams PD, Hadi MZ (2012) Glycoside hydrolases from a targeted compost metagenome, activity-screening and functional characterization. BMC Biotechnol 12:38 . doi: 10.1186/1472-6750-12-38

Ebrahimie E, Ebrahimi M, Sarvestani R, Ebrahimi M (2011) Protein attributes contribute to halostability, bioinformatics approach. Saline Systems 7:1 . doi: 10.1186/1746-1448-7-1

Ferrer M, Martínez-Martínez M, Bargiela R, Streit WR, Golyshina O V., Golyshin PN (2016) Estimating the success of enzyme bioprospecting through metagenomics: Current status and future trends. Microb. Biotechnol. 9:22-34

Gabor EM, Alkema WBL, Janssen DB (2004) Quantifying the accessibility of the metagenome by random expression cloning techniques. Environ Microbiol 6:879-886 . doi: 10.1111/j.14622920.2004.00640.x 
Gaida SM, Sandoval NR, Nicolaou SA, Chen Y, Venkataramanan KP, Papoutsakis ET (2015) Expression of heterologous sigma factors enables functional screening of metagenomic and heterologous genomic libraries. Nat Commun 6:1-10 . doi: 10.1038/ncomms8045

Hitch TCA, Clavel T (2019) A proposed update for the classification and description of bacterial lipolytic enzymes. PeerJ 7:e7249 . doi: 10.7717/peerj.7249

Hu P, Tom L, Singh A, Thomas BC, Baker BJ, Piceno YM, Andersen GL, Banfield JF (2016) Genome-resolved metagenomic analysis reveals roles for candidate phyla and other microbial community members in biogeochemical transformations in oil reservoirs. MBio 7:e0166915 . doi: 10.1128/mBio.01669-15

Ishibashi M, Hayashi T, Yoshida C, Tokunaga M (2013) Increase of salt dependence of halophilic nucleoside diphosphate kinase caused by a single amino acid substitution. Extremophiles 17:585-591 . doi: 10.1007/s00792-013-0541-9

Jara-Samaniego J, Pérez-Murcia MD, Bustamante MA, Pérez-Espinosa A, Paredes C, López M, López-Lluch DB, Gavilanes-Terán I, Moral R (2017) Composting as sustainable strategy for municipal solid waste management in the Chimborazo Region, Ecuador: Suitability of the obtained composts for seedling production. J Clean Prod 141:1349-1358 . doi: 10.1016/j.jclepro.2016.09.178

Jochens H, Aerts D, Bornscheuer UT (2010) Thermostabilization of an esterase by alignmentguided focussed directed evolution. Protein Eng Des Sel 23:903-909 . doi: 10.1093/protein/gzq071

Khan M, Sathya TA (2018) Extremozymes from metagenome: Potential applications in food processing. Crit Rev Food Sci Nutr 58:2017-2025 . doi: 10.1080/10408398.2017.1296408

Kim YH, Kwon EJ, Kim SK, Jeong YS, Kim J, Yun HD, Kim H (2010) Molecular cloning and characterization of a novel family VIII alkaline esterase from a compost metagenomic library. Biochem Biophys Res Commun 393:45-49 . doi: 10.1016/j.bbrc.2010.01.070

Kim YJ, Kim H, Kim SH, Rha E, Choi SL, Yeom SJ, Kim HS, Lee SG (2016) Improved metagenome screening efficiency by random insertion of T7 promoters. J Biotechnol 230:4753 . doi: 10.1016/j.jbiotec.2016.05.018 
Kixmüller D, Greie JC (2012) Construction and characterization of a gradually inducible expression vector for Halobacterium salinarum, based on the kdp promoter. Appl Environ Microbiol 78:2100-2105 . doi: 10.1128/AEM.07155-11

Lam KN, Cheng J, Engel K, Neufeld JD, Charles TC (2015) Current and future resources for functional metagenomics. Front Microbiol 6:1196 . doi: 10.3389/fmicb.2015.01196

Lämmle K, Zipper H, Breuer M, Hauer B, Buta C, Brunner H, Rupp S (2007) Identification of novel enzymes with different hydrolytic activities by metagenome expression cloning. J Biotechnol 127:575-592 . doi: 10.1016/j.jbiotec.2006.07.036

Leis B, Angelov A, Mientus M, Li H, Pham VTT, Lauinger B, Bongen P, Pietruszka J, Gonçalves LG, Santos H, Liebl W (2015) Identification of novel esterase-active enzymes from hot environments by use of the host bacterium Thermus thermophilus. Front Microbiol 6:275 . doi: $10.3389 /$ fmicb.2015.00275

Liebl W, Angelov A, Juergensen J, Chow J, Loeschcke A, Drepper T, Classen T, Pietruzska J, Ehrenreich A, Streit WR, Jaeger KE (2014) Alternative hosts for functional (meta)genome analysis. Appl. Microbiol. Biotechnol. 98:8099-8109

Liu SP, Liu RX, Zhang L, Shi GY (2015) Sequence-based screening and characterization of cytosolic mandelate oxidase using oxygen as electron acceptor. Enzyme Microb Technol 69:24-30 . doi: 10.1016/j.enzmictec.2014.11.001

Liu YF, Galzerani DD, Mbadinga SM, Zaramela LS, Gu JD, Mu BZ, Zengler K (2018) Metabolic capability and in situ activity of microorganisms in an oil reservoir. Microbiome 6:5 . doi: $10.1186 / \mathrm{s} 40168-017-0392-1$

Lopez-Lopez O, Cerdan M, Siso M (2014) New extremophilic lipases and esterases from metagenomics. Curr Protein Pept Sci 15:445-455 . doi: $10.2174 / 1389203715666140228153801$

López-López O, Knapik K, Cerdán ME, González-Siso MI (2015) Metagenomics of an alkaline hot spring in Galicia (Spain): Microbial diversity analysis and screening for novel lipolytic enzymes. Front Microbiol 6:1291 . doi: 10.3389/fmicb.2015.01291

Ma S, Fang C, Sun X, Han L, He X, Huang G (2018) Bacterial community succession during pig manure and wheat straw aerobic composting covered with a semi-permeable membrane under 
slight positive pressure. Bioresour Technol 259:221-227 . doi: 10.1016/j.biortech.2018.03.054

Mahapatra GP, Raman S, Nayak S, Gouda S, Das G, Patra JK (2020) Metagenomics approaches in discovery and development of new bioactive compounds from marine Actinomycetes. Curr Microbiol 77:645-656 . doi: 10.1007/s00284-019-01698-5

Mao H, Lv Z, Sun H, Li R, Zhai B, Wang Z, Awasthi MK, Wang Q, Zhou L (2018) Improvement of biochar and bacterial powder addition on gaseous emission and bacterial community in pig manure compost. Bioresour Technol 258:195-202 . doi: 10.1016/j.biortech.2018.02.082

Markel U, Essani KD, Besirlioglu V, Schiffels J, Streit WR, Schwaneberg U (2020) Advances in ultrahigh-throughput screening for directed enzyme evolution. Chem Soc Rev 49:233-262 . doi: $10.1039 / \mathrm{c} 8 \mathrm{cs} 00981 \mathrm{c}$

Metin K, Burcu Bakir Ateslier Z, Basbulbul G, Halil Biyik H (2006) Characterization of esterase activity in Geobacillus sp. HBB-4. J Basic Microbiol 46:400-409 . doi: $10.1002 /$ jobm. 200510121

Mirete S, Morgante V, González-Pastor JE (2016) Functional metagenomics of extreme environments. Curr Opin Biotechnol 38:143-149 . doi: 10.1016/j.copbio.2016.01.017

Moriya T, Hikota T, Yumoto I, Ito T, Terui Y, Yamagishi A, Oshima T (2011) Calditerricola satsumensis gen. nov., sp. nov. and Calditerricola yamamurae sp. nov., extreme thermophiles isolated from a high-temperature compost. Int J Syst Evol Microbiol 61:631-636 . doi: 10.1099/ijs.0.018416-0

Munawar N, Engel PC (2013) Halophilic enzymes: Characteristics, structural adaptation and potential applications for biocatalysis. Curr Biotechnol 2:334-344 . doi: $10.2174 / 18722083113076660033$

Munawar N, Engel PC (2012) Overexpression in a non-native halophilic host and biotechnological potential of NAD+-dependent glutamate dehydrogenase from Halobacterium salinarum strain NRC-36014. Extremophiles 16:463-476 . doi: 10.1007/s00792-012-0446-z

Neher DA, Weicht TR, Bates ST, Leff JW, Fierer N (2013) Changes in bacterial and fungal communities across compost recipes, preparation methods, and composting times. PLoS One 8:e79512 . doi: 10.1371/journal.pone.0079512 
Ngara TR, Zhang H (2018) Recent advances in function-based metagenomic screening. Genomics, Proteomics Bioinforma. 16:405-415

Ogino H, Ishikawa H (2001) Enzymes which are stable in the presence of organic solvents. J Biosci Bioeng 91:109-116

Palaniveloo K, Amran MA, Norhashim NA, Mohamad-Fauzi N, Peng-Hui F, Hui-Wen L, Kai-Lin Y, Jiale L, Chian-Yee MG, Jing-Yi L, Gunasekaran B, Razak SA (2020) Food waste composting and microbial community structure profiling. Processes 8:723 . doi: 10.3390/pr8060723

Panda T, Gowrishankar BS (2005) Production and applications of esterases. Appl Microbiol Biotechnol 67:160-169 . doi: 10.1007/s00253-004-1840-y

Pehrsson EC, Tsukayama P, Patel S, Mejía-Bautista M, Sosa-Soto G, Navarrete KM, Calderon M, Cabrera L, Hoyos-Arango W, Bertoli MT, Berg DE, Gilman RH, Dantas G (2016) Interconnected microbiomes and resistomes in low-income human habitats. Nature 533:212216 . doi: $10.1038 /$ nature 17672

Pezzullo M, Del Vecchio P, Mandrich L, Nucci R, Rossi M, Manco G (2013) Comprehensive analysis of surface charged residues involved in thermal stability in Alicyclobacillus acidocaldarius esterase 2. Protein Eng Des Sel 26:47-58 . doi: 10.1093/protein/gzs066

Quince C, Walker AW, Simpson JT, Loman NJ, Segata N (2017) Shotgun metagenomics, from sampling to analysis. Nat Biotechnol 35:833-844 . doi: 10.1038/nbt.3935

Rastogi M, Nandal M, Khosla B (2020) Microbes as vital additives for solid waste composting. Heliyon 6:e03343 . doi: 10.1016/j.heliyon.2020.e03343

Reyes A, Alves JMP, Durham AM, Gruber A (2017) Use of profile hidden Markov models in viral discovery: current insights. Adv Genomics Genet 7:29-45 . doi: 10.2147/AGG.S136574

Rong Z, Huo YY, Jian SL, Wu YH, Xu XW (2018) Characterization of a novel alkaline esterase from Altererythrobacter epoxidivorans CGMCC 1.7731 T. Prep Biochem Biotechnol 48:113-120 . doi: 10.1080/10826068.2017.1387559

Rosenstein R, Götz F (2000) Staphylococcal lipases: Biochemical and molecular characterization. Biochimie 82:1005-1014 
Ryckeboer J, Mergaert J, Vaes K, Klammer S, Clercq D, Coosemans J, Insam H, Swings J (2003) A survey of bacteria and fungi occurring during composting and self-heating processes. Ann Microbiol 53:349-410

Sadeghi M, Naderi-Manesh H, Zarrabi M, Ranjbar B (2006) Effective factors in thermostability of thermophilic proteins. Biophys Chem 119:256-270 . doi: 10.1016/j.bpc.2005.09.018

Sarmah N, Revathi D, Sheelu G, Yamuna Rani K, Sridhar S, Mehtab V, Sumana C (2018) Recent advances on sources and industrial applications of lipases. Biotechnol Prog 34:5-28 . doi: $10.1002 /$ btpr. 2581

Sayer C, Finnigan W, Isupov MN, Levisson M, Kengen SWM, van der Oost J, Harmer NJ, Littlechild JA (2016) Structural and biochemical characterisation of Archaeoglobus fulgidus esterase reveals a bound CoA molecule in the vicinity of the active site. Sci Rep 6:25542 . doi: $10.1038 / \operatorname{srep} 25542$

Silva CC, Hayden H, Sawbridge T, Mele P, Kruger RH, Rodrigues MVN, Costa GGL, Vidal RO, Sousa MP, Torres APR, Santiago VMJ, Oliveira VM (2012) Phylogenetic and functional diversity of metagenomic libraries of phenol degrading sludge from petroleum refinery wastewater treatment system. AMB Express 2:18 . doi: 10.1186/2191-0855-2-18

Simon C, Daniel R (2011) Metagenomic analyses: Past and future trends. Appl Environ Microbiol 77:1153-1161 . doi: 10.1128/AEM.02345-10

Sivakumar N, Li N, Tang JW, Patel BKC, Swaminathan K (2006) Crystal structure of AmyA lacks acidic surface and provide insights into protein stability at poly-extreme condition. FEBS Lett 580:2646-2652 . doi: 10.1016/J.FEBSLET.2006.04.017

Skewes-Cox P, Sharpton TJ, Pollard KS, DeRisi JL (2014) Profile Hidden Markov Models for the detection of viruses within metagenomic sequence data. PLoS One 9:e105067 . doi: 10.1371/journal.pone.0105067

Sood S, Sharma A, Sharma N, Kanwar SS (2016) Carboxylesterases: Sources, characterization and broader applications. Insights Enzym Res 1:1-11 . doi: 10.21767/2573-4466.100002

Stergiou PY, Foukis A, Filippou M, Koukouritaki M, Parapouli M, Theodorou LG, Hatziloukas E, Afendra A, Pandey A, Papamichael EM, Moh B, Salameh DA, Gupta R, Gupta N, Rathi P, Levisson M, Mrugesh D, Togneri PD, Anobom CD, Pinheiro AS, De-Andrade RA, Aguieiras 
ECG, Andrade GC, Moura M V, Almeida R V, Freire DM, Nagazawa AA, Nalder TD, Bornscheuer UT, Dheeman DS, Henehan PG, Lopez-Lopez O, Cerdan M, Siso M, Alex D, Udatha G (2014) Biochemical and Structural Analysis of Thermostable Esterases. Biotechnol Adv 31:445-455 . doi: 10.1016/S0168-6445(01)00075-4

Tadeo X, Ló Pez-Mé Ndez B, Trigueros T, Laín A, Castañ D, Millet O (2009) Structural basis for the aminoacid composition of proteins from halophilic Archea. PLoS Biol 7:e1000257 doi: 10.1371/journal.pbio. 1000257

Tripathi NK, Shrivastava A (2019) Recent developments in bioprocessing of recombinant proteins: expression hosts and process development. Front Bioeng Biotechnol 7:420 . doi: 10.3389/fbioe. 2019.00420

Troy SM, Nolan T, Kwapinski W, Leahy JJ, Healy MG, Lawlor PG (2012) Effect of sawdust addition on composting of separated raw and anaerobically digested pig manure. J Environ Manage 111:70-77 . doi: 10.1016/j.jenvman.2012.06.035

Uchiyama T, Miyazaki K (2009) Functional metagenomics for enzyme discovery: challenges to efficient screening. Curr Opin Biotechnol 20:616-622 . doi: 10.1016/j.copbio.2009.09.010

Ufarté L, Laville É, Duquesne S, Potocki-Veronese G (2015) Metagenomics for the discovery of pollutant degrading enzymes. Biotechnol Adv 33:1845-1854 . doi: 10.1016/j.biotechadv.2015.10.009

Ventosa A, Nieto JJ, Oren A (1998) Biology of moderately halophilic aerobic bacteria. Microbiol Mol Biol Rev 62:504-44

Vigneron A, Alsop EB, Lomans BP, Kyrpides NC, Head IM, Tsesmetzis N (2017) Succession in the petroleum reservoir microbiome through an oil field production lifecycle. ISME J 11:2141-2154 . doi: 10.1038/ismej.2017.78

Wang C, Dong D, Wang H, Müller K, Qin Y, Wang H, Wu W (2016) Metagenomic analysis of microbial consortia enriched from compost : new insights into the role of Actinobacteria in lignocellulose decomposition. Biotechnol Biofuels 9:22 . doi: 10.1186/s13068-016-0440-2

Wei H, Tucker MP, Baker JO, Harris M, Luo Y, Xu Q, Himmel ME, Ding SY (2012) Tracking dynamics of plant biomass composting by changes in substrate structure, microbial community, and enzyme activity. Biotechnol Biofuels 5:1-14 . doi: 10.1186/1754-6834-5-20 
Wei H, Wang L, Hassan M, Xie B (2018) Succession of the functional microbial communities and the metabolic functions in maize straw composting process. Bioresour Technol 256:333-341 . doi: 10.1016/j.biortech.2018.02.050

Zhang Y, Pengjun S, Wanli L, Kun M, Yingguo B, Guozeng W, Zhichun Z, Bin Y (2009) Lipase diversity in glacier soil based on analysis of metagenomic DNA fragments and cell culture. $\mathrm{J}$ Microbiol Biotechnol 19:888-897 . doi: 10.4014/jmb.0812.695

Zhou G, Xu X, Qiu X, Zhang J (2019) Biochar influences the succession of microbial communities and the metabolic functions during rice straw composting with pig manure. Bioresour Technol 272:10-18 . doi: 10.1016/j.biortech.2018.09.135

Zhou H, Gu W, Sun W, Hay AG (2018) A microbial community snapshot of windrows from a commercial composting facility. Appl Microbiol Biotechnol 102:8069-8077 . doi: 10.1007/s00253-018-9201-4 


\section{Summary}

Lipolytic enzymes, including lipases and esterases, have a wide spectrum of applications in various industrial fields, due to their functional versatility. Despite an increasing number of lipolytic enzymes that have been reported recently, only a small portion were experimentally verified and studied. Until recently, the demand for new lipolytic enzymes, particularly the extremophilic ones that can survive in extreme environments, is still high.

In this thesis, two compost samples (compost55 and compost76) at thermophilic stage were collected for exploring potential extremophilic lipolytic enzymes. Firstly, we investigated the microbial composition in the two composts. The 16S rRNA genes and transcripts data as well as metagenomic data revealed that Actinobacteria, Proteobacteria, Firmicutes, Bacteroidetes and Chloroflexi were dominant in the microbial consortia of both samples. The taxonomic disparity between compost55 and compost76 was mainly attributed to the different feedstock composition and composting conditions. In general, analysis of the metagenome data of compost55 and compost76 showed that both share similar metabolic pattern. Nevertheless, genes involved in lipid transport and metabolism as well as the categories fatty acids, lipids, and isoprenoids were more abundant in the compost55 than in compost76 community, suggesting a higher possibility to identify lipolytic genes in the compost55 metagenome.

Four metagenomic libraries were prepared to probe the diversity of LEs from compost microbes by function-based approaches. Overall, 199 and 51 positive clones for compost55 and compost 76 , respectively, were recovered. The inserts of the recovered plasmids with a confirmed phenotype were sequenced, and putative lipolytic genes were identified. Clustering the amino acid sequences deduced from 
the corresponding lipolytic genes yielded 115 unique and full-length lipolytic enzymes. Then, the family assignment of these enzymes was conducted by analyzing the phylogenetic relationship and protein sequence similarity network according to an integral classification system. To the best of our knowledge, the system included all the reported lipolytic enzymes so far (46 families in total) to avoid artificial inflation of the number of families during classification. The functional screeningderived lipolytic enzymes were affiliated to 12 lipolytic families. Seven LEs were not assigned to any known families, indicating new branches of lipolytic families. Subsequently, the multiple sequence alignment further showed the catalytic residues and conserved motif in each family.

For sequence-based screening, we have developed a searching and subsequent annotation strategy specific for putative lipolytic genes in metagenomes. The profile hidden Markov models-based searching methods was highly sensitive and accurate for lipolytic enzymes. With this sequence-based screening and annotation strategy, 4,157 and 2,234 putative lipolytic proteins (PLPs) were initially identified in the assembled metagenomes of compost55 and compost76, respectively. Among them, 1,234 (compost55) and 759 (compost76) of these were further assigned into 28 and 26 families, respectively. The enrichments were observed in families, such as VIII, hormone-sensitive lipase-like, patatin-like proteins, II, A85-Feruloyl-Esterase, Carb_B_Bacteria and homoserine transacetylase. Analysis of the phylogenetic origin of the assigned PLPs indicated a potential link between microbial taxa and their functional traits. By comparing the lipolytic hits identified by function-driven and sequence-based screening indicated that the activity-directed selection complements sequence-based selection, and vice versa.

In addition, comparative analysis of the distribution of lipolytic genes in metagenomes from various ecological niches were explored using the sequence- 
based approach developed in this thesis. The lipolytic family assignment (functional profile) and phylogenetic origin (taxonomic profile) of assigned PLPs for each sample were driven by the ecological factor (habitat). Moreover, the habitat also determined the conserved and distinctive microbial groups harboring the putative lipolytic genes.

Finally, three lipolytic genes (est 1, est 2, and est56) belonging to different lipolytic families and showing low sequence identity to known lipolytic enzymes were selected for biochemical characterization. The three genes were heterologously expressed and characterized.

The gene product Est1 (est1) and Est2 (est2) are thermostable enzymes with optimal enzyme activities at 80 and $70{ }^{\circ} \mathrm{C}$, respectively. The two enzymes, particularly Est2, were also proved as organic solvent tolerant. Est2 activity was significantly enhanced (two- to tenfold) in the presence of ethanol, methanol, isopropanol, and 1-propanol over a concentration range ranging from 6 to $30 \%(\mathrm{v} / \mathrm{v})$. Moreover, Est2 exhibited short-term ( $2 \mathrm{~h}$ of incubation) and long-term (up to 26 days) stability towards various water-miscible organic solvents at different concentrations. Est2 also displayed high stability towards water-immiscible organic solvents of ethyl acetate, diethyl ether, and toluene. All of these features indicated that Est 1 and Est2 possess application potential.

The other lipolytic enzyme, Est56 (gene product of est56), was halotolerant. It exhibited high activity and stability towards up to $4 \mathrm{M} \mathrm{NaCl}$ and $\mathrm{KCl}$. In the presence of $\mathrm{NaCl}$, Est56 also displayed enhanced stability against denaturants including high temperatures $\left(50\right.$ and $\left.60^{\circ} \mathrm{C}\right)$ and urea $(2,4$, and $6 \mathrm{M})$. The amino acid composition of recently reported halotolerant lipolytic enzymes (40 in total) and halophilic enzymes was statistically compared to reveal the potential salt resistance mechanism for Est56. The results indicate that the haloadaptation of Est56 was mainly attributed 
to the high content of acidic residues (Asp and Glu, $12.2 \%$ ), the low content of lysine residues $(0.7 \%)$, and the excess of surface-exposed acidic residue. 


\section{Acknowledgements}

First of all, I want to show my deep and sincere appreciation to my doctoral advisor Prof. Rolf Daniel, for giving me the opportunity to join in his excellent team, providing me with this interesting topic, and giving me all the wise supervision, advises and help. He is very kind, knowledgeable, empathetic and brilliant, I appreciated his support throughout the years very much.

I am also grateful to Prof. Michael Hoppert for being my second supervisor and giving me all the insightful suggestions.

It is my honor that Prof. Dr. Kai Heimel, Prof. Dr. Stefanie Pöggeler, Prof. Dr. Gerhard Braus and Prof. Dr. Dieter Heineke agree to join in my examination board.

Many thanks to my friends and also colleagues, Amelie, Martin, Dominik and Silja. Thank you very much for your company, support, encouragement and help. Special thanks to Amelie, I miss and cherish the nice talks, letters, meetings and emails, which helped me in going through the dark times.

I am also thankful to have worked with all these great people: Amelie, Dominik, Silja, Mechthild, Heiko, Martin, Birgit, Bernd, Ines, Sarah, Kristin, Henis, Rhandi, Dirk and Tanja. It was such a wonderful and unforgettable experience.

I am much obliged to my family, in particular to my parents and parents-in-law. They unconditionally support all my decisions, understand my situation, offer me warm hugs and comforts. They are always my solid backing. 
Finally, I want to give all my heart to my husband and daughter. Despite all the difficulties, thank you for always holding my hands, support me, encourage me, trust me, and love me. I am so lucky to have such a wonderful and responsible man. For my daughter, she is the most precious and great gift from the god. Thank you for being my daughter and making my life full of sunshine, laughter and joy. 